Check for updates

Cite this: RSC Adv., 2019, 9, 8778

\title{
A review on graphene-based nanocomposites for electrochemical and fluorescent biosensors
}

\author{
Siva Kumar Krishnan, (D) a Eric Singh, (DD b Pragya Singh, (DD c Meyya Meyyappan*d \\ and Hari Singh Nalwa (D) *e
}

Biosensors with high sensitivity, selectivity and a low limit of detection, reaching nano/picomolar concentrations of biomolecules, are important to the medical sciences and healthcare industry for evaluating physiological and metabolic parameters. Over the last decade, different nanomaterials have been exploited to design highly efficient biosensors for the detection of analyte biomolecules. The discovery of graphene has spectacularly accelerated research on fabricating low-cost electrode materials because of its unique physical properties, including high specific surface area, high carrier mobility, high electrical conductivity, flexibility, and optical transparency. Graphene and its oxygenated derivatives, including graphene oxide (GO) and reduced graphene oxide ( $\mathrm{rGO}$ ), are becoming an important class of nanomaterials in the field of biosensors. The presence of oxygenated functional groups makes GO nanosheets strongly hydrophilic, facilitating chemical functionalization. Graphene, GO and rGO nanosheets can be easily combined with various types of inorganic nanoparticles, including metals, metal oxides, semiconducting nanoparticles, quantum dots, organic polymers and biomolecules, to create a diverse range of graphene-based nanocomposites with enhanced sensitivity for biosensor applications. This review summarizes the advances in two-dimensional (2D) and threedimensional (3D) graphene-based nanocomposites as emerging electrochemical and fluorescent biosensing platforms for the detection of a wide range of biomolecules with enhanced sensitivity, selectivity and a low limit of detection. The biofunctionalization and nanocomposite formation processes of graphene-based materials and their unique properties, surface functionalization, enzyme immobilization strategies, covalent immobilization, physical adsorption, biointeractions and direct electron transfer (DET) processes are discussed in connection with the design and fabrication of biosensors. The enzymatic and nonenzymatic reactions on graphene-based nanocomposite surfaces for glucose- and cholesterol-related electrochemical biosensors are analyzed. This review covers a very broad range of graphene-based electrochemical and fluorescent biosensors for the detection of glucose, cholesterol, hydrogen peroxide $\left(\mathrm{H}_{2} \mathrm{O}_{2}\right)$, nucleic acids (DNA/RNA), genes, enzymes, cofactors nicotinamide adenine dinucleotide (NADH) and adenosine triphosphate (ATP), dopamine (DA), ascorbic acid (AA), uric acid (UA), cancer biomarkers, pathogenic microorganisms, food toxins, toxic heavy metal ions, mycotoxins, and pesticides. The sensitivity and selectivity of graphene-based electrochemical and fluorescent biosensors are also examined with respect to interfering analytes present in biological systems. Finally, the future outlook for the development of graphene based biosensing technology is outlined.

Received 21st November 2018 Accepted 15th February 2019

DOI: $10.1039 / \mathrm{c} 8 \mathrm{ra09577a}$

rsc.li/rsc-advances
${ }^{a}$ CONACYT-Instituto de Física, Benemérita Universidad Autónoma de Puebla, Apdo. Postal J-48, Puebla 72570, Mexico

${ }^{b}$ Department of Computer Science, Stanford University, Stanford, California 94305, USA

'Department of Electrical Engineering and Computer Science, National Chiao Tung University, Hsinchu 30010, Taiwan

${ }^{d}$ Center for Nanotechnology, NASA Ames Research Center, Moffett Field, Mountain View, California 94035, USA. E-mail: m.meyyappan@nasa.gov

${ }^{e}$ Advanced Technology Research, 26650 The Old Road, Valencia, California 91381, USA. E-mail: nalwa@mindspring.com

\section{Introduction}

Biosensors with high sensitivity, able to detect femto- or picomolar concentrations of analyte molecules, are of paramount importance not only in biomedical applications such as glucose monitoring and clinical diagnostics ${ }^{1}$ but also in the agriculture ${ }^{2}$ and food industries ${ }^{3}$ and environmental monitoring., ${ }^{4,5}$ The development of highly sensitive devices and new approaches that can provide efficient point-of-care testing with high 
accuracy and low cost is an urgent need in the healthcare industry. ${ }^{6-8}$ In addition, in vivo biosensors have received attention since they enable the long-term monitoring of target analytes within live cells with high sensitivity, selectivity and biocompatibility. ${ }^{\mathbf{9} 10}$ Notably, biosensor research is considered to be an important field since it covers a wide range of sensing capabilities, including pulse, heart rate, blood pressure, body motions, blood oxygen level, glucose, cholesterol, antibodies, nucleic acids, proteins, cancer cells, toxins in food products, and heavy metals in drinking water. ${ }^{11-13}$ Numerous approaches have been explored, including colorimetric biosensors, ${ }^{14}$ potentiometric biosensors, ${ }^{15}$ electrochemical biosensors, ${ }^{16}$ fluorescent biosensors, ${ }^{17}$ and Raman spectroscopy-based platforms. ${ }^{18}$ Compared with other detection methods, an electrochemistry- ${ }^{\mathbf{1 9}, 20}$ and fluorescence-based ${ }^{\mathbf{2 1}}$ approach offers a much less expensive, more facile and highly sensitive detection method, which enables the monitoring of different analytes, fast response-recovery times and very low detection limits. ${ }^{22}$

After the discovery of buckminsterfullerene $\left(\mathrm{C}_{60}\right)$ molecules in 1985, the field of nanotechnology focused intently on developing new materials and devices within the 1-100 nm scale because nanoscale materials show unique chemical and physical properties compared to their counterpart bulk materials. ${ }^{23}$ A wide variety of nanoscale materials have been developed, including zero-dimensional (0D) nanoparticles (such as metallic and semiconducting nanoparticles), ${ }^{\mathbf{2 4 2 5}}$ onedimensional (1D) nanostructures (nanowires, nanorods, nanotubes), ${ }^{26}$ and two-dimensional (2D) nanostructures including graphene nanosheets (GNs), transition metal dichalcogenides (TMDs), etc. ${ }^{27}$ with substantial progress on their synthesis, processing, characterization and potential applications. Over the past two decades, these nanoscale materials have been used in many applications, including light-emitting diodes, memory devices, communication devices, magnetic disks, solar cells, batteries, fuel cells, supercapacitors, and catalysts. ${ }^{28,29}$ Interfacing with various probe biomolecules has been studied in order to develop highly sensitive biosensors with significantly enhanced sensitivity. ${ }^{30}$ Due to the size and unprecedented physical properties of these nanomaterials, the development of biosensors with extremely small dimensions and substantially improved performance is possible, introducing new opportunities in the development and commercialization of nextgeneration biosensors for biomedicine and healthcare fields. ${ }^{31}$

Since the initial isolation of graphene from bulk graphite and characterization in 2004 by Geim and Novoselov, ${ }^{32}$ intensive research efforts have been directed toward 2D graphene nanomaterials and their potential applications. ${ }^{33-36}$ Graphene (GR) is a 2D sheet of carbons with atomic thickness that exhibits unique electrical, optical, mechanical and thermal properties. ${ }^{37}$ Graphene nanosheets can be easily exfoliated from earthabundant graphite and are considered allotropically similar to fullerenes and carbon nanotubes. Graphene can be easily processed into single-layer, few-layer or multi-layer nanosheets, ${ }^{38}$ stretchable ultrathin films, ${ }^{39}$ papers, ${ }^{\mathbf{4 0}}$ nanoribbons, ${ }^{\mathbf{4 1}}$ and foams. ${ }^{\mathbf{4 2 , 4 3}}$ Single-layer graphene nanosheets exhibit a high mechanical strength with a Young's modulus of $1.1 \mathrm{TPa},{ }^{40}$ thermal conductivity of $\sim 5000 \mathrm{~W} \mathrm{~m} \mathrm{~K}^{-1},{ }^{44}$ high carrier mobility
(200000 $\left.\mathrm{cm}^{2} \mathrm{~V}^{-1} \mathrm{~s}^{-1}\right),{ }^{45}$ high optical transparency toward visible light $(\sim 2.3 \% \text { absorption })^{46}$ and a large specific surface area $\left(2630 \mathrm{~m}^{2} \mathrm{~g}^{-1}\right) .{ }^{47}$ As a result, graphene-based materials (GBMs) have been explored for a wide range of applications, including bulk-heterojunction ${ }^{48}$ and dye-sensitized solar cells, ${ }^{49}$ energy storage devices, ${ }^{50}$ electronic skin and touchscreen-panel devices, ${ }^{51}$ field effect transistors, ${ }^{52}$ light-emitting diodes (LEDs), ${ }^{53}$ gas and chemical sensors, ${ }^{54}$ nanomedicine, ${ }^{37}$ drug delivery, ${ }^{55}$ and many other applications. ${ }^{56}$ Graphene and its oxidized derivatives, such as graphene oxide (GO), which contain various oxygen functional groups (hydroxyl, carboxyl and epoxy functional groups), have emerged for potential use in biosensors. ${ }^{57}$ The presence of these functional groups makes GO sheets strongly hydrophilic and allows the integration of various types of inorganic nanoparticles, including noble metals, metal oxides, semiconducting nanoparticles, quantum dots (QDs), and nanoclusters (NCs), to enhance the performance of sensors based on them.$^{58,59}$ Moreover, the reduction of GO into reduced GO (rGO) results in a high density of defects that leads to high electrochemical activity compared with that of CVD-grown graphene, which is particularly useful for developing electrochemical biosensors. Graphene-based nanocomposites also inherit unique morphological structures and properties useful for sensing. ${ }^{60}$ The $3 \mathrm{D}$ interconnected hierarchical structures of graphene nanocomposites facilitate the diffusion of different types of biomolecules and preserve their biocatalytic functions to optimize biosensing functionality. ${ }^{61-63}$ Graphene-based hybrids with polymers ${ }^{64,65}$ and surface-decorated metal nanoparticles ${ }^{58}$ have been explored for biosensing due to their excellent biocompatibility, high surface area, and site-selective conjugation with biomolecules.

Various nanostructures have been explored for biosensors, including the detection of glucose and hydrogen peroxide, ${ }^{66}$ cancer biomarkers, ${ }^{67-69}$ nucleic acids, ${ }^{11}$ antibodies,${ }^{70}$ heavy metals, ${ }^{71,72}$ pathogenic bacteria, ${ }^{73}$ and many other targets ${ }^{19,74-77}$ However, no comprehensive review focused on a wide range of electrochemical and fluorescent biosensors utilizing graphenebased nanocomposites is yet available in the literature. There are no reviews on graphene-based fluorescent biosensors. Therefore, this review is intended to summarize the recent advances in both electrochemical and fluorescent biosensors based on graphene nanocomposites, including graphene, GO, rGO/polymer nanocomposites, graphene/inorganic NP nanocomposites, 3D graphene integrated with various metal/metal oxide nanoparticles, and polymer hydrogel networks. Biosensor systems to detect glucose, hydrogen peroxide $\left(\mathrm{H}_{2} \mathrm{O}_{2}\right)$, cholesterol, dopamine (DA), ascorbic acid (AA), uric acid (UA), nucleic acids (NAs), cofactors nicotinamide adenine dinucleotide (NADH) and adenosine triphosphate (ATP), cancer biomarkers, pathogens, food toxins, metal ions and pesticides are discussed. The discussion focuses primarily on advances in enzymatic and nonenzymatic electrochemical platforms as well as conceptual advances in fluorescent biosensing and amplified detection techniques. The selectivity of graphene-based electrochemical and fluorescent biosensors are also examined in biological systems with respect to interfering analytes. This review will provide a single reference source for researchers in 
biosensors, graphene, materials science, nanotechnology, chemistry, and electrochemistry. Therefore, this review will attract a wide range of audiences from diverse research areas and stimulate further interest in graphene-based biosensors for future sensor industries.

\section{Graphene-based nanocomposites}

Numerous strategies have been developed for the synthesis of graphene..$^{37,57,78-81}$ The synthesis of graphene and its graphene oxide (GO) and reduced graphene oxide (rGO) derivatives is illustrated in Fig. 1A. Four different routes have been commonly used to prepare graphene, namely, epitaxial growth on SiC or metals, ${ }^{82}$ chemical exfoliation of graphite, ${ }^{83}$ liquid-phase ultrasonic exfoliation of graphite powder, ${ }^{83}$ and chemical vapor deposition (CVD). ${ }^{84}$ In addition to these approaches, graphene nanosheets have been prepared by the electrochemical exfoliation of ionic liquid-functionalized graphite ${ }^{85}$ and by electrical arc discharge between two graphitic electrodes ${ }^{86,87}$ Though the CVD process is considered a primary route to synthesize highquality, large-area graphene nanosheets of different layers and sizes, CVD-grown graphene nanosheets exhibit relatively low electrocatalytic activity due to the low number of structural defects. The physicochemical properties and surface features of graphene can be effectively altered by using nitrogen $(\mathrm{N})$, boron (B), phosphorus (P), and sulfur (S) heteroatom doping. ${ }^{\mathbf{8}}$ Numerous approaches have been used for chemical doping, including in situ doping, where the synthesis and doping of graphene are achieved simultaneously, for example, in CVD, ball milling, and bottom-up synthetic techniques. The highly controlled S-doped graphene (S-GR) nanostructure prepared by the ball milling technique is shown in Fig. 1B.

Graphene oxide (GO) consists of hydrophobic $\mathrm{sp}^{2}$ - and $\mathrm{sp}^{3}$ bonded carbon and different oxygen-containing functional

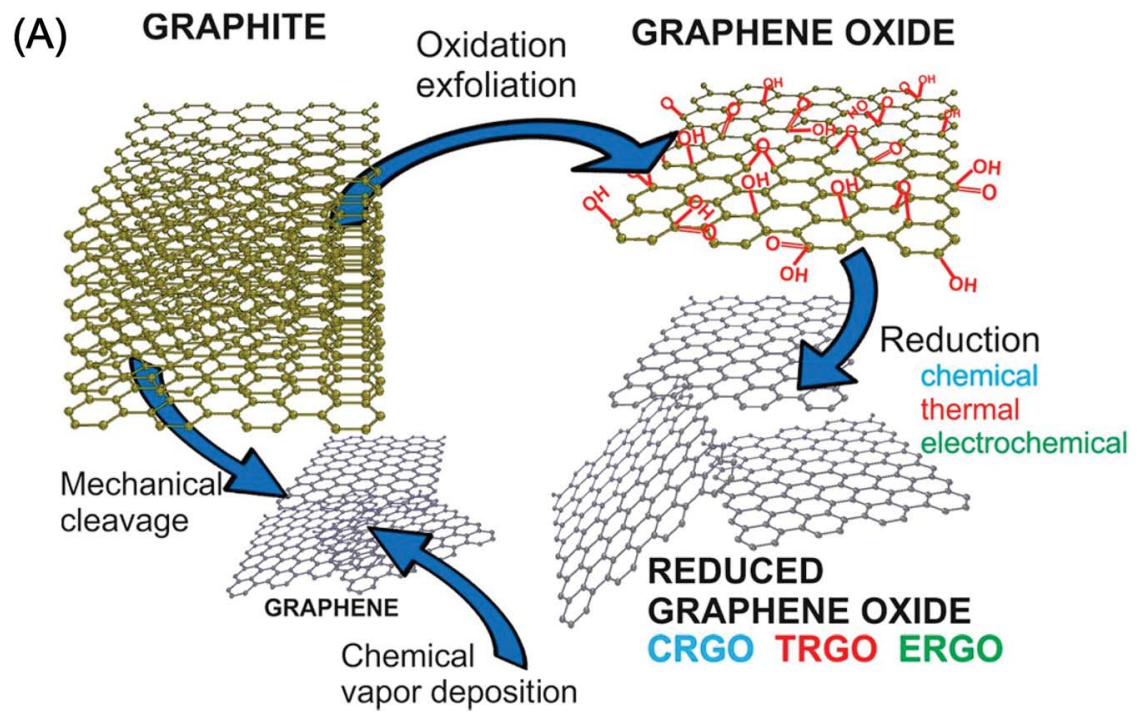

(B)

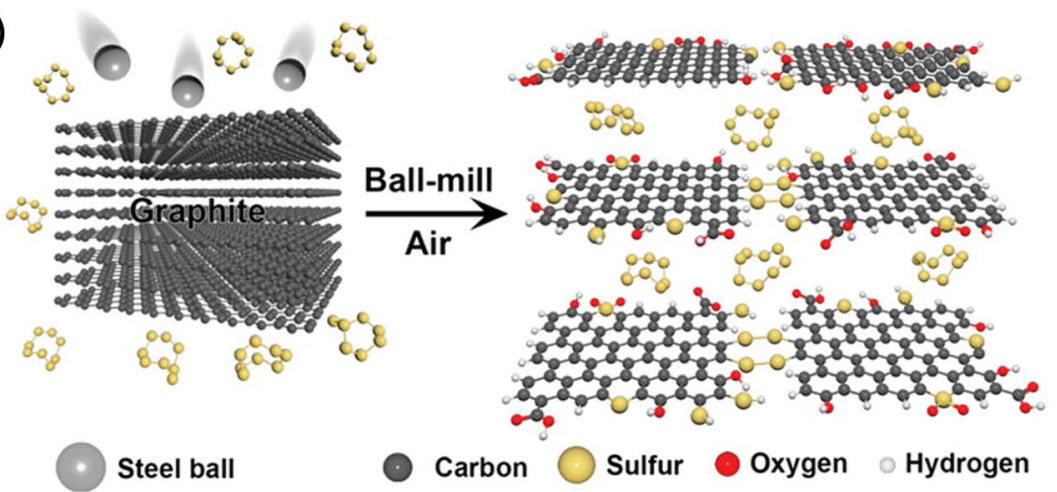

Fig. 1 (A) Schematic illustration of possible methods for the preparation of graphene, GO and rGO from graphite using mechanical cleavage, exfoliation, CVD and reduction methods including chemical, thermal and electrochemical methods. [Reprinted with permission from ref. 89, J. Filip and J. Tkac, Is Graphene Worth Using in Biofuel Cells?, Electrochim. Acta, 2014, 136, 340-354. Copyright $\odot$ Elsevier.] (B) A schematic representation of the physical cracking of graphite-flake into functionalized graphene derivatives using a ball milling technique. [Reprinted with permission from ref. 90, J. Xu, J. Shui, J. Wang, M. Wang, H.-K. Liu, S. X. Dou, I.-Y. Jeon, J.-M. Seo, J.-B. Baek and L. Dai, Sulfur-Graphene Nanostructured Cathodes via Ball-Milling for High-Performance Lithium-Sulfur Batteries, ACS Nano, 2014, 8, 10920-10930. Copyright@ American Chemical Society.] 
groups including hydroxyl (-OH), carbonyl, epoxy and carboxyl $(-\mathrm{COOH})$ groups on the basal plane as well as at the edges of GO nanosheet. ${ }^{91,92}$ Similar to graphene, GO also exhibits unique electronic, electrochemical, thermal, and mechanical properties and can be developed into flexible, transparent, and biocompatible nanosheets due to its hydrophilic nature. The availability of functional groups on GO nanosheets facilitates their interaction with a wide range of biomolecules, which is highly advantageous for developing biosensors. ${ }^{78,93,94}$ GO nanosheets have been synthesized by the Brodie, Staudenmaier, and Hummers methods and by modified versions thereof. ${ }^{95,96}$ Furthermore, the reduction of GO into reduced $\mathrm{GO}(\mathrm{rGO})$ can be accomplished by reducing the GO employing chemical, electrochemical, high-temperature thermal annealing, and ultraviolet irradiation methods. ${ }^{91,96-99}$ The rGO also exhibits excellent properties; including solvent dispersibility; electrical, optical, and mechanical properties; and thermal stability comparable to those of graphene and GO nanosheets. Graphene quantum dots (GQDs) have also appeared as a novel class of zero-dimensional (0D) graphene derivatives possessing unique properties with quantum confinement and edge effects for biosensor applications. ${ }^{100-108}$

The hybridization of GBMs with different types of organic polymers, metal nanoparticles and surface functionalities with diverse enzymes and proteins is quite useful in developing new biosensors. ${ }^{64,109,110}$ In particular, both GO and rGO have been extensively used for developing nanocomposite-based biosensors because of their reactive oxygen functional groups and surface defects, which permit the controlled nucleation and growth of metal, metal oxides and semiconductor nanoparticles. Liu et al. ${ }^{63}$ developed bioconjugate assemblies of various nanoparticles such as AuNPs, AgNPs, PtNPs, PdNPs and

\section{(A)}

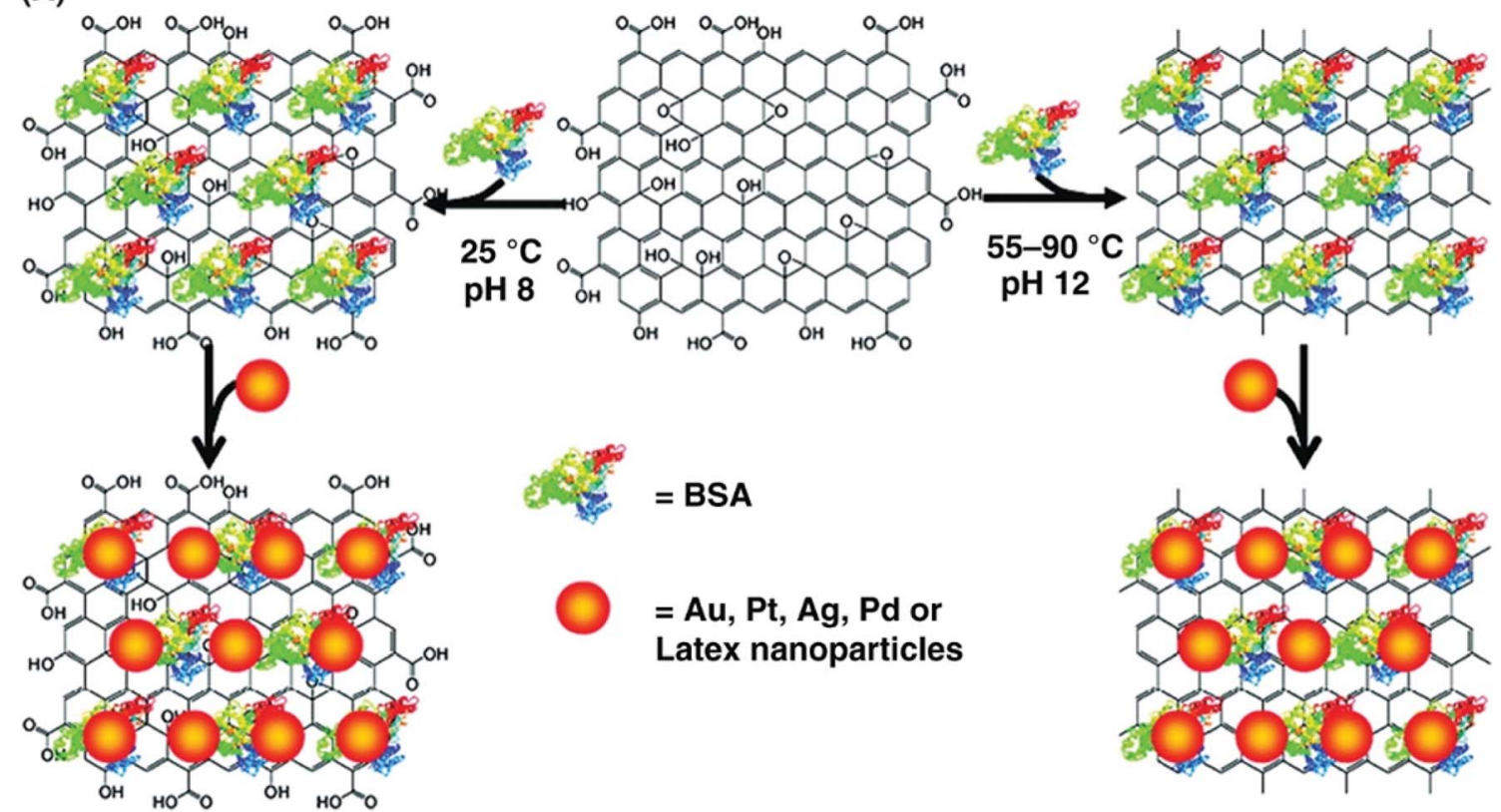

(B)
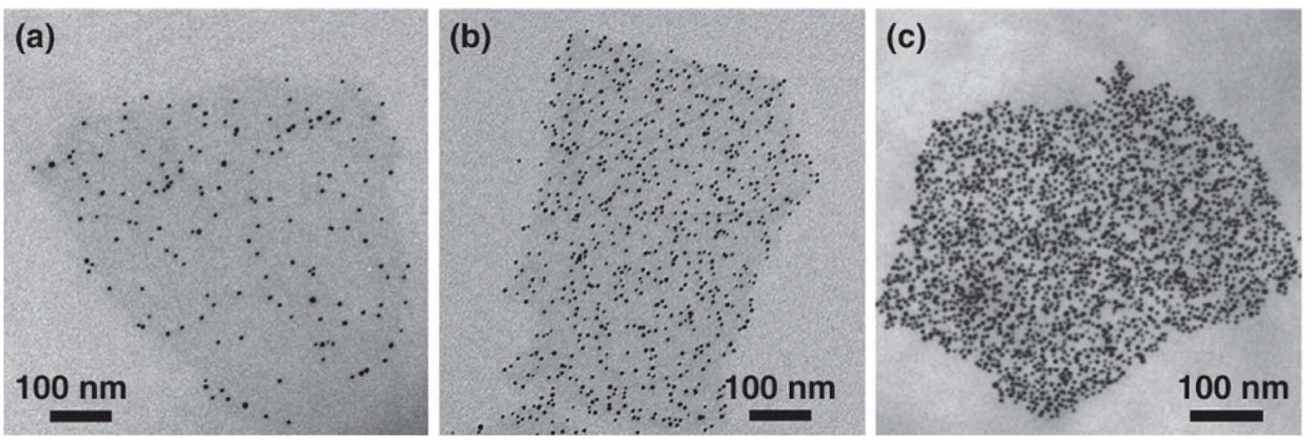

Fig. 2 (A) Schematic illustration of the reduction and decoration of GO nanosheets using BSA protein to develop a new platform for biosensing. (B) TEM images show AuNPs-decorated BSA-GO nanosheets with well-controlled AuNPs densities by increasing the concentration of BSA protein from $0.5 \mathrm{mg} \mathrm{mL}^{-1}$ to $20 \mathrm{mg} \mathrm{mL}^{-1}$ during BSA-GO hybrid formation. In (c) the density of AuNPs was further increased by the addition of $0.1 \mathrm{M} \mathrm{NaCl}$ to the BSA-GO assembly in (b). NaCl was not used for (a) and (b). [Reprinted with permission from ref. 63, J. Liu, S. Fu, B. Yuan, Y. Li and Z. Deng, Toward a Universal "Adhesive Nanosheet" for the Assembly of Multiple Nanoparticles Based on a Protein-Induced Reduction/Decoration of Graphene Oxide, J. Am. Chem. Soc., 2010, 132, 7279-7281. Copyright@ American Chemical Society.] 
latex using the bovine serum albumin (BSA)-based reduction and decoration of GO nanosheets (Fig. 2). The density of AuNPs in the BSA-GO nanocomposite was controlled by adjusting the concentration of BSA protein. Various synthetic approaches, including chemical methods and hydrothermal, electrochemical, and ex situ strategies, have been developed to prepare graphene- and nanoparticle-based nanocomposites by fine tuning the nanoparticle size, shape, distribution, and functionality over graphene, GO and rGO..$^{58}$

\section{Graphene-based electrochemical biosensors}

\subsection{Glucose biosensors}

Diabetes is one of the most common diseases, affecting millions of people worldwide. In 2017, according to the International Diabetes Federation (IDF), 425 million adults were affected by diabetes and this number is expected to rise to 629 million cases of diabetes by $2045 .{ }^{111}$ The physiological level of blood glucose after a minimum of 8 hours of fasting (pre-meal) determines the prevalence of diabetes; fasting blood glucose level in the 70 to 99 milligrams per deciliter $\left(\mathrm{mg} \mathrm{dL}^{-1}\right.$ ) (from $3.9 \mathrm{mmol} \mathrm{L}^{-1}$ to $5.4 \mathrm{mmol} \mathrm{L}^{-1}$ ) range is considered normal, while blood glucose level from $100 \mathrm{mg} \mathrm{dL} \mathrm{L}^{-1}$ to $125 \mathrm{mg} \mathrm{dL}^{-1}$ (from $5.6 \mathrm{mmol} \mathrm{L}^{-1}$ to $6.9 \mathrm{mmol} \mathrm{L}^{-1}$ ) is considered prediabetes, and a blood glucose level of $126 \mathrm{mg} \mathrm{dL}^{-1}\left(7.0 \mathrm{mmol} \mathrm{L}^{-1}\right)$ or higher indicates the existence of the diabetes (https:// medlineplus.gov/ency/article/003482.htm). The accurate and rapid detection of blood glucose is of critical importance for continuous point-of-care glucose monitoring and clinical diagnosis of diabetes. ${ }^{6}$ Since the first report of a glucose oxide (GOx)based enzyme electrode by Clark and Lyons et al. ${ }^{112}$ for monitoring blood glucose levels, there has been a growing demand for reliable devices for diabetes control; the need for the fabrication of electrochemical biosensors that are capable of labelfree real-time monitoring of blood glucose levels with high sensitivity and resolution has been documented in the literature. ${ }^{113}$

The development of electrochemical glucose sensors to date can be categorized into three generations. ${ }^{114}$ In the first generation of glucose enzyme electrodes, the measurements relied on the oxygen consumed by the enzyme-catalyzed reaction. Specifically, an enzymatic reaction occurred between glucose and the GOx enzyme electrode in the presence of oxygen, producing hydrogen peroxide $\left(\mathrm{H}_{2} \mathrm{O}_{2}\right)$, and the glucose level was monitored through the amount of enzymatically generated $\mathrm{H}_{2} \mathrm{O}_{2} \cdot{ }^{115}$ The second generation witnessed the utilization of electrical mediators to facilitate electron transfer process between the flavin adenine dinucleotide (FAD) active site of GOx and the electrode's surface, a process called mediated electron transfer (MET). ${ }^{115}$ However, MET-based glucose sensors have several disadvantages; for example, the mediator lowers the total energy of the system and affects the operational stability. Ultimately, third-generation enzymatic biosensors have been developed with no mediator to achieve direct electron transfer (DET) between FAD-GOx and the electrode surface. However, several challenges still exist related to the achievement of DET for accurate and reliable glucose monitoring with operational stability. ${ }^{113}$ Numerous nanostructured materials have been employed as electrode materials for the fabrication of electrochemical glucose biosensors with greatly enhanced DET processes due to their unique optical and electronic properties. ${ }^{116-119}$ In particular, carbon nanotubes (CNTs) ${ }^{\mathbf{1 1 6 , 1 2 0 - 1 2 3}}$ carbon nanodots (CNDs), ${ }^{117}$ porous carbon, ${ }^{\mathbf{1 2 4}}$ graphene, ${ }^{\mathbf{1 2 5}}$ and GQDs $^{\mathbf{1 2 6}}$ are preferred as ideal nanostructures for the effective immobilization of enzymes and for the improvement of DET characteristics. Among them, graphene and GO are particularly attractive as promising electrode materials for the immobilization of enzymes and electrochemical glucose biosensing. ${ }^{127,128}$ Graphene is highly advantageous compared to other materials due to its high specific surface area $\left(2630 \mathrm{~m}^{2} \mathrm{~g}^{-1}\right.$ for single-layer graphene), excellent electronic properties and biocompatibility. ${ }^{57,129,130}$

3.1.1 Surface functionalization. The surface functionalization of graphene often plays a key role in the precise control of the electronic surface states, which could help bringing the detection targets onto the graphene surface through specific molecular interactions and widen its applicability in biosensing. ${ }^{127}$ The surface functionalization of graphene significantly affects its electrical properties. Moreover, the chemical reduction of GO into rGO with different defect densities, numbers of layers and oxygen concentrations influences the electrochemical behavior and glucose biosensor performance. ${ }^{\mathbf{1 3 1}}$ Doping with semiconducting nitrogen (N), boron (B), sulfur (S), fluorine (F) and hydrogen $(\mathrm{H})$ has a profound impact on the physical and chemical properties of graphene, resulting in improved sensitivity. ${ }^{75,132,133}$

3.1.2 Enzyme immobilization strategies on graphene. The immobilization of enzymes on a solid support is one of the key steps in the fabrication of enzymatic biosensors with high sensitivity, selectivity, stability and confined electron transport between the enzymes and the electrode surface. ${ }^{79}$ The enzyme immobilization method can affect the enzyme loading, biocatalytic activity and operational stability. ${ }^{\mathbf{1 3 4}}$ Different nanostructured materials have been successfully used for the effective immobilization of glucose oxidase (GOx) and their glucose-sensing ability. CNTs and graphene have been extensively studied due to their unique catalytic and electronic properties. ${ }^{\mathbf{1 3 5}}$ The high specific surface area and availability of abundant functional groups in graphene and GO make them ideal substrates for modulating enzyme activity and stability. ${ }^{136,137}$ Different immobilization strategies have been developed for effectively immobilizing enzymes on graphenebased materials. ${ }^{\mathbf{1 2 7}, \mathbf{1 3 8}}$ There are two main approaches for achieving efficient enzyme immobilization: simple physical adsorption (noncovalent) and covalent immobilization. ${ }^{79}$

3.1.3 Physical adsorption. Noncovalent immobilization on GO or the simple physical adsorption of enzymes on graphene occurs through physical forces such as van der Waals, ionic and hydrophobic interactions. The immobilization procedure consists of simple deposition of the enzyme onto the graphene surface; thus, the $\mathrm{sp}^{2}$-hybridized carbon network is not affected, and the electrical properties of GO are preserved. Specifically, 
the supramolecular interaction of aromatic molecules can be achieved by the $\pi-\pi$ stacking of pyrene derivatives for noncovalent immobilization. However, due to the weak physical interaction between the enzyme and the graphene surface, the electron transfer characteristics, catalytic function and operational stability are highly affected. ${ }^{76}$

3.1.4 Covalent immobilization. Covalent immobilization can be achieved by modifying GO with different organic linker molecules containing reactive functional groups, which enables the direct covalent conjugation of the enzymes to the GO surface. The covalent immobilization reactions are formed in two main steps. The functionalization of amine groups ${ }^{\mathbf{1 3 9 , 1 4 0}}$ and carboxylic groups ${ }^{\mathbf{1 4 1}, \mathbf{1 4 2}}$ on graphene results in covalent bonding between the organic functional groups and the oxygenated groups of GO, which is followed by the conjugation of amine groups of the enzyme with chemically activated amine or carboxylic groups in the second step through bifunctional crosslinker molecules. In general, either 1-ethyl-3-(3-

Table 1 Enzymatic electrochemical glucose biosensors developed from graphene nanocomposite-based electrodes and their sensitivity, concentration range, LOD, and heterogeneous electron transfer rate constant $\left(K_{\mathrm{s}}\right)$ between GOx enzyme and the modified graphene electrodes ${ }^{a}$

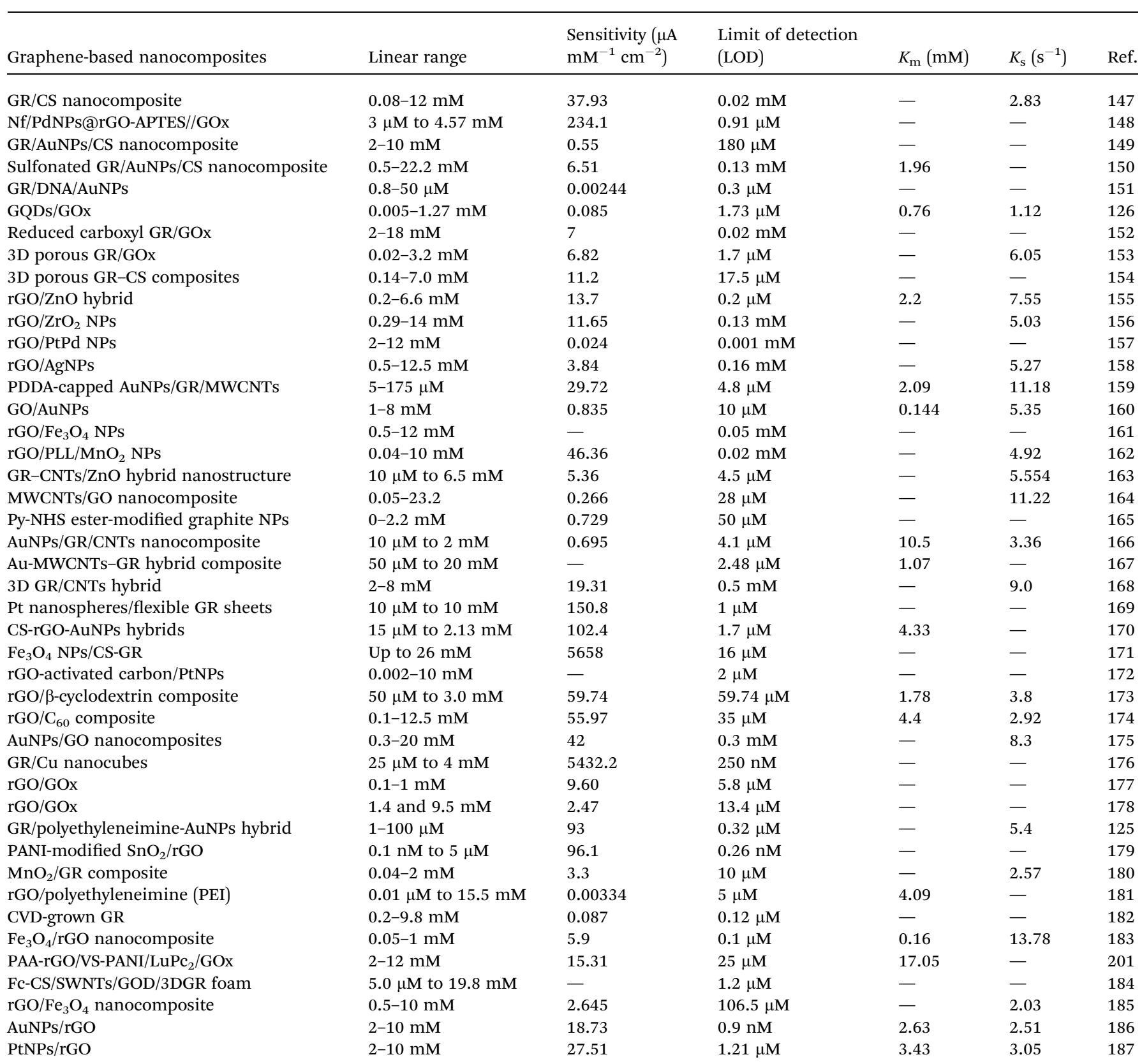

a Abbreviations used: APTES: (3-aminopropyl)triethoxysilane, PEDOT: poly(3,4-ethylenedioxythiophene), PAA: polyacrylic acid, PEI: polyethyleneimine, PANI: polyaniline, VS-PANI: vinyl-substituted polyaniline, MWCNTs: multiwalled carbon nanotubes, CS: chitosan, Nf: Nafion, QDs: quantum dots. PDDA: poly(diallyldimethylammonium chloride), Py-NHS: 1-pyrenebutyric acid $N$-hydroxysuccinimide ester. LuPc ${ }_{2}$ : lutetium phthalocyanine, Fc: ferrocene. 
dimethylaminopropyl)carbodiimide hydrochloride (EDC) or $\mathrm{N}$ hydroxysuccinimide (NHS) is used for carboxylated graphene, while glutaraldehyde is utilized for amino-functionalized graphene. ${ }^{127}$ The covalent immobilization of the enzyme prevents the inactivation of catalytic functions that might occur in the case of direct physical adsorption and electrically wires the active cofactor of the enzyme to the GO surface. However, covalent immobilization strategies significantly alter the native graphene lattice structure by converting carbon bonding from $\mathrm{sp}^{2}$ to $\mathrm{sp}^{3}$, which markedly decreases the electronic properties of graphene. ${ }^{79}$ The electroactive enzyme surface density $(T, \mathrm{~mol}$ $\mathrm{cm}^{-2}$ ) on the modified electrode can be evaluated by: ${ }^{\mathbf{1 4 3}}$

$$
\Gamma=Q / n F A
$$

where $Q$ is the charge involved in the reaction (obtained by integrating the anodic peak and dividing by the scan rate), $n$ is the number of transferred electrons $(n=2), F$ is the Faraday constant, and $A$ represents the geometric area of an electrode.

3.1.5 Graphene-based electrochemical enzymatic glucose biosensors. Enzyme-based electrochemical glucose sensors have attracted significant attention over the past 40 years because of their high selectivity, simplicity, and sensitivity. ${ }^{\mathbf{1 6}}$ Most of the clinically available systems for monitoring blood glucose levels depend on bioelectrodes based on the glucose oxidase enzyme (GOx). ${ }^{\mathbf{1 4 4}}$ Enhanced electrical contact of redox enzymes with the electrode surface is of fundamental interest for the development of mediator-free third-generation electrochemical glucose biosensors with high sensitivity and selectivity. ${ }^{\mathbf{1 3 4 , 1 4 5}}$ Depending on the graphene derivative, the conductivity and electrocatalytic activity can differ significantly. For example, the electrical properties of graphene can change upon reduction to GO or rGO as well as upon chemical functionalization. ${ }^{\mathbf{1 4 6}}$ Graphene-based materials provide a large specific surface area, excellent electrochemical properties, biocompatibility and plentiful oxygenated functional groups such as hydroxyl, carbonyl, carboxyl and epoxy groups, all of which facilitate the effective immobilization of redox enzymes through either physical adsorption or covalent conjugation for subsequent glucose sensing. ${ }^{79}$ Graphene-based nanocomposites have been widely used to fabricate enzymatic glucose biosensors, as summarized in Table 1 in terms of their sensitivity, linear detection range and the lowest limit of detection (LOD).$^{147-187}$

An example of the enzymatic glucose biosensor is discussed here. Qi et al. ${ }^{\mathbf{1 7 5}}$ used AuNPs decorated GO nanosheet for developing a glucose sensor by attaching AuNPs on to the surface of GO nanosheet via a benzene (Ph) bridge employing aryldiazonium salt chemistry. The schematic biosensor fabrication process is depicted in Fig. 3. GO-Ph-AuNPs nanocomposite was attached to 4-aminophenyl modified glassy carbon electrode (GCE). Thereafter, the GCE/GO-Ph-AuNPs nanocomposite was functionalized with 4-carboxyphenyl (CP) and GOx was covalently attached to form the GCE/GO-PhAuNPs-CP/GOx based glucose sensor. The sensor showed a linear range of $0.3-20 \mathrm{mM}$, and sensitivity of $42 \mu \mathrm{AmM}$ $\mathrm{cm}^{-2}$ for glucose detection as well as fast electron transfer and enzyme turnover rates of 8.3 and $112 / \mathrm{s}$, respectively. The GCE/GO-Ph-AuNPs-CP/GOx sensor also exhibited a high

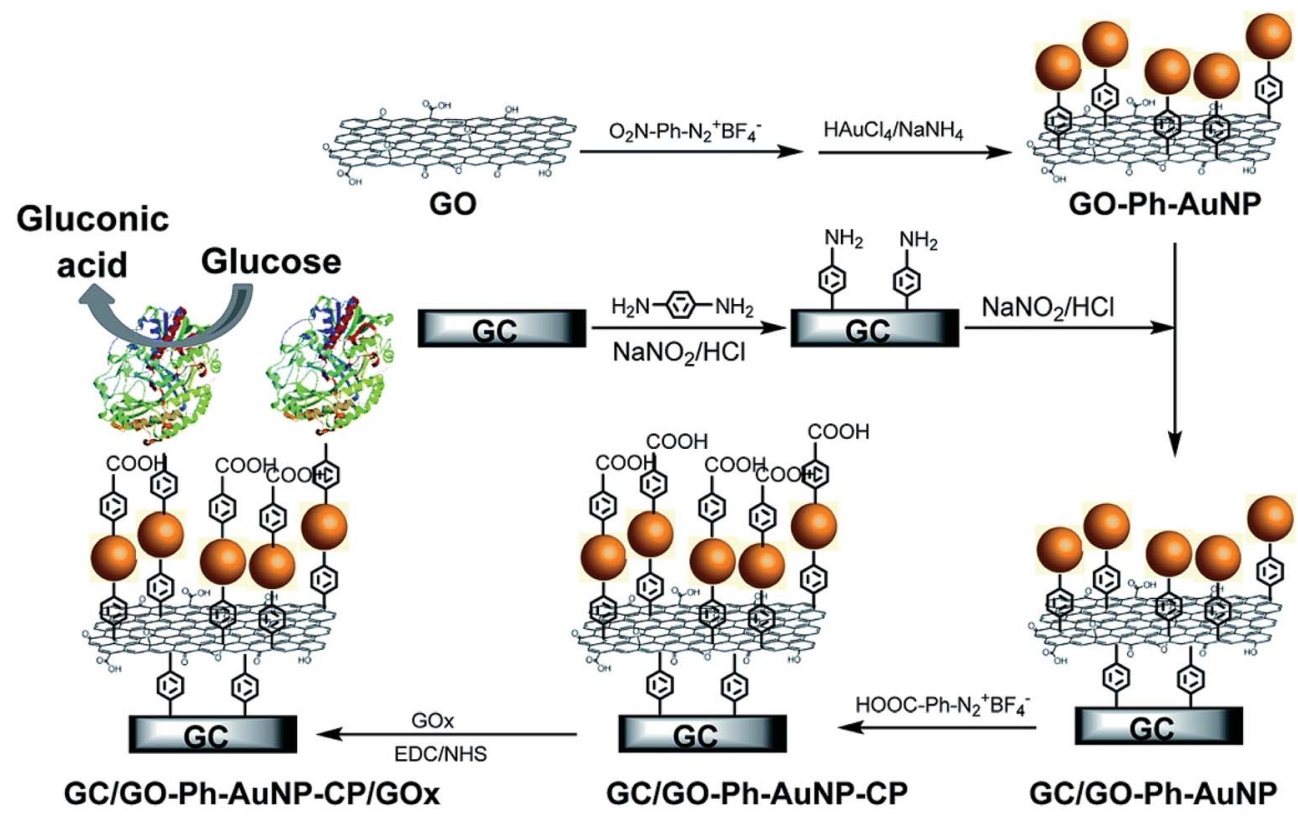

Fig. 3 The schematic fabrication of glucose biosensor using AuNP-decorated GO nanosheet. AuNPs were decorated onto GO nanosheet via a benzene bridge using aryldiazonium salt chemistry (GO-Ph-AuNPs) which was thereafter attached to 4-aminophenyl modified GC electrode. The GC/GO-Ph-AuNPs was further functionalized with 4-carboxyphenyl (CP) before covalently attaching GOx via amide bonds to form GC/GOPh-AuNPs-CP/GOx based glucose sensor. [Reprinted with permission from ref. 175, M. Qi, Y. Zhang, C. Cao, Y. Lu and G. Liu, Increased Sensitivity of Extracellular Glucose Monitoring Based on AuNP Decorated GO Nanocomposites, RSC Adv., 2016, 6, 39180-39187. Copyright@ The Royal Society of Chemistry.] 
selectivity toward glucose detection because almost no amperometric current was noticed for $100 \mathrm{mM}$ of dopamine (DA), ascorbic acid (AA), uric acid (UA), acetaminophen (AP), fructose, lactose, and galactose interfering analytes compared to $10 \mathrm{mM}$ of glucose concentration. The GCE/GO-Ph-AuNP-CP/ GOx-based sensor was also used to detect in real time the glucose changes occurring during growth of two cell lines, where glucose uptake rate of 8.8 fmol per min per cell for human umbilical vein endothelial cells (HUVECs) and 15.5 fmol per min per cell for human cervical cancer HeLa cell lines was measured. The GCE/GO-Ph-AuNP-CP/GOx sensor showed long-term stability by retaining $90.3 \%$ initial current density after 10 days storage at $4{ }^{\circ} \mathrm{C}$ due to the strongly entrapped GOx on AuNPs and covalent bonding between the sensor interfaces.

3.1.6 Direct electron transfer (DET). Accomplishing DET in amperometric glucose biosensors is of significant interest for the fabrication of third-generation electrochemical biosensors without mediators. The direct electrical wiring of immobilized GOx enables the detection of glucose at low potentials that are little more positive than the redox potential of GOx. ${ }^{188}$ Significant work has been performed on the effective immobilization of the GOx enzyme on graphene-based materials for the development of highly sensitive enzymatic glucose biosensors with DET characteristics. ${ }^{74,152,153}$ However, establishing efficient DET between the biocatalyst and the electrode surface is a key challenge that arises due to the large and complex structure of the GOx enzyme; with the FAD redox active center deeply embedded within the protein, accomplishing DET is challenging. ${ }^{189}$ The location of the active site FAD within the GOx structure creates a high electron tunneling distance between the electron donor (FAD) and the electrode surface, thus significantly limiting the electron transfer rate. Efficient electrical communication between the redox enzyme and the electrode surface significantly minimizes the electron tunneling distance, leading to highly enhanced biosensor sensitivity. ${ }^{\mathbf{1 6 8}}$ Liang et al. ${ }^{190}$ demonstrated the challenge associated with the DET process in GOx-immobilized GR electrodes with and without mediators for sensing glucose, as shown in Fig. 4A. The corresponding cyclic voltammograms (CVs) of the GCE are shown in Fig. 4B. A pair of well-defined redox peaks at $-0.48 \mathrm{~V}$ with a $40 \mathrm{mV}$ separation of peak potential is associated with the redox potential of FAD-GOx on the modified electrode surface; however, no redox peaks are observed when GOx is directly adsorbed onto the bare GCE, indicating that the rGO/GOxmodified GCE can promote DET, which occurs primarily via contact between the rGO and FAD/GOx. They observed that DET and the catalytic activity of GOx cannot occur simultaneously.

The DET process between FAD-GOx and GO can be described as follows:

$$
\begin{aligned}
\text { D-glucose }+ \text { GOx }(\text { FAD }) \rightarrow & \text { D-glucono-1,5-lactone } \\
& + \text { GOx }\left(\text { FADH }_{2}\right), \\
\text { GOx }\left(\text { FADH }_{2}\right) \leftrightarrow & \text { GOx }(\text { FAD })+2 \mathrm{H}^{+}+2 \mathrm{e}^{-}
\end{aligned}
$$

where $\mathrm{FAD}$ and $\mathrm{FADH}_{2}$ are the oxidized and reduced forms of the redox center of the GOx enzyme. GOx is a homodimer containing its cofactor bound to its two identical $80-\mathrm{kDa}$ subunits, which can easily undergo enzymatic reactions. FAD is an electroactive center that exhibits a two-electron and twoproton redox reaction, such as $\mathrm{GOx}(\mathrm{FAD})+2 \mathrm{H}^{+}+2 \mathrm{e}^{-} \leftrightarrow$ $\mathrm{GOx}\left(\mathrm{FADH}_{2}\right)$. Glucose, when added, is oxidized by the GOx enzyme into D-glucono-1-5-lactone and $\mathrm{GOx}\left(\mathrm{FADH}_{2}\right)$ by the transfer of two electrons and two protons to FAD-GOx. If $\mathrm{FADH}_{2}$ can be oxidized back to FAD without any electrochemical mediators or oxygen, then the process is referred to as DET. ${ }^{118}$ The rate constant of the electron transfer $\left(K_{\mathrm{s}}\right)$ between the GOx-
(A)

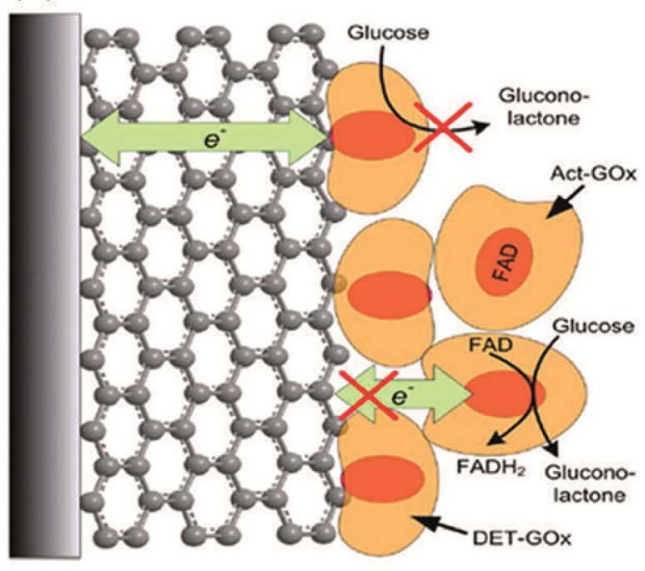

(B) $\mathrm{GO}_{\mathrm{x}}(\mathrm{FAD})+2 \mathrm{H}^{+}+2 \mathrm{e}^{-} \leftrightarrow \mathrm{GO}_{\mathrm{x}}\left(\mathrm{FADH}_{2}\right)$

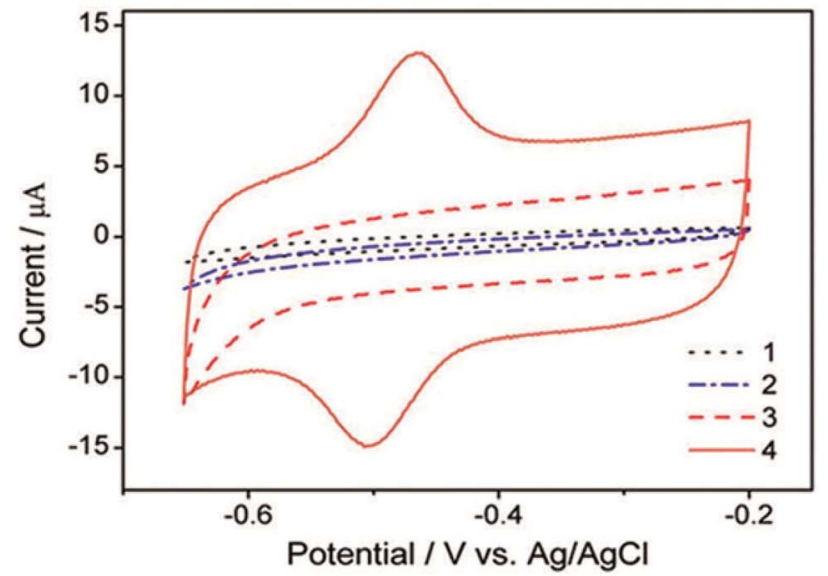

Fig. 4 (A) Schematic illustration of DET with GOx adsorption on the ERCGR/GOx/GCE. (B) CVs of the different modified GCEs; (1) bare GCE, (2) GOx/GCE, (3) ERGO/GCE, and (4) GOx/ERGO/GCE in N2-saturated phosphate buffer solution (PBS). [Reprinted with permission from ref. 190, B. Liang, X. Guo, L. Fang, Y. Hu, G. Yang, Q. Zhu, J. Wei and X. Ye, Study of Direct Electron Transfer and Enzyme Activity of Glucose Oxidase on Graphene Surface, Electrochem. Commun., 2015, 50, 1-5. Copyright@ Elsevier.] 
and GO-modified electrodes was calculated using the Laviron equation. ${ }^{191}$

$$
\begin{aligned}
\log K_{\mathrm{s}}= & \alpha \log (1-\alpha)+(1-\alpha) \log \alpha-\log (R T / n F v) \\
& -\alpha(1-\alpha) n F \Delta E_{\mathrm{p}} / 2.3
\end{aligned}
$$

where $\alpha$ is the charge transfer coefficient, $R$ is the gas constant (8.314 $\mathrm{J} \mathrm{mol}^{-1} \mathrm{~K}^{-1}$ ), $T$ is the room temperature, $\Delta E_{\mathrm{p}}$ is the peak separation of the $\mathrm{FAD} / \mathrm{FADH}_{2}$ redox couple, and $n$ is the number of electrons transferred $(n=2)$.

Considerable efforts have been made to overcome the limitation of long electron tunneling distances and accomplish DET between the immobilized enzyme and the electrode surface. Among different nanostructured materials, GR has been extensively investigated for DET characteristics and glucosesensing performance. ${ }^{\mathbf{1 4 1}, 192}$ For example, Wang et al. ${ }^{\mathbf{1 4 0}}$ showed a direct electrochemical reduction of single-layer GO and subsequent functionalization with (3-aminopropyl)triethoxysilane (APTES) for covalent immobilization with GOx and application of developed GCE-APTES-rGO-GOx electrode in glucose sensing with a linear range of 0 to $24 \mathrm{mM}$. Additionally, Guler et al. ${ }^{193}$ fabricated a glucose sensor by immobilizing GOx onto APTES-functionalized rGO deposited on GCE. The functionalization of APTES provides an $-\mathrm{NH}_{2}$-terminated surface, which enables covalent conjugation of the $-\mathrm{NH}_{2}$ groups of GOx via bifunctional cross-linker glutaraldehyde (GA) molecules. The enzymatic glucose biosensor also consisted of the Nafion (Nf) protective membrane. The Nf/rGO-APTES/GOx/GCE composite-based based biosensor showed the sensitivity of $75.26 \mu \mathrm{A} \mathrm{mM}^{-1} \mathrm{~cm}^{-2}$ and $124.87 \mu \mathrm{A} \mathrm{mM}^{-1} \mathrm{~cm}^{-2}$, a wide linear range of 0.02-4.34 $\mathrm{mM}$ and $0.05-15.25 \mathrm{mM}$ with LOD values of 9 $\mu \mathrm{M}$ and $0.017 \mathrm{mM}$ for glucose and $\mathrm{H}_{2} \mathrm{O}_{2}$ detection, respectively. Unnikrishnan et al. ${ }^{\mathbf{1 9 4}}$ demonstrated a simple one-step immobilization of GOx onto rGO and subsequent glucose sensing. The resulting rGO-GOx-GCE sensor exhibited DET characteristics with a sensitivity of $1.85 \mu \mathrm{A} \mathrm{mM}^{-1} \mathrm{~cm}^{-2}$ and a linear range of 0.1-27 mM. Osikoya et al. ${ }^{182}$ used CVD-grown graphene to construct an electrochemical biosensor that displayed electrocatalytic activities and exhibited a linear response from 0.2 to $9.8 \mathrm{mM}$ with a sensitivity of $0.087 \mu \mathrm{A} \mu \mathrm{M}^{-1} \mathrm{~cm}^{-2}$ and an LOD of $0.12 \mu \mathrm{M}$. Ravenna et al. ${ }^{195}$ reported a glucose sensor using rGO films with adsorbed phenothiazone to study electron transfer process between the FAD-dependent glucose dehydrogenase and the used electrodes. The DET results showed sensitivity of $42 \mathrm{~mA} \mathrm{M}^{-1} \mathrm{~cm}^{-2}$ and a linear range of $0.5-12 \mathrm{mM}$ for glucose detection as well as high selectivity against different interfering analytes. Liu et $a l .{ }^{196}$ reported the fabrication of a GR-GOx enzyme electrode by the alternate layer-by-layer (LBL) selfassembly of GOx over single-layer or multilayer graphene through noncovalent $\pi-\pi$ stacking interactions, as shown in Fig. 5. Each pyrene-functionalized GOx enzyme contained 5.4 pyrene functional groups and maintained over $76 \%$ of the biocatalytic activity of GOx. The graphene-bridged GOx enzyme electrode-based biosensor showed a linear range between 0.2 and $40 \mathrm{mM}$ and an LOD as low as $0.154 \mathrm{mM}$ for glucose sensing. The sensitivity towards glucose increased with increasing layers

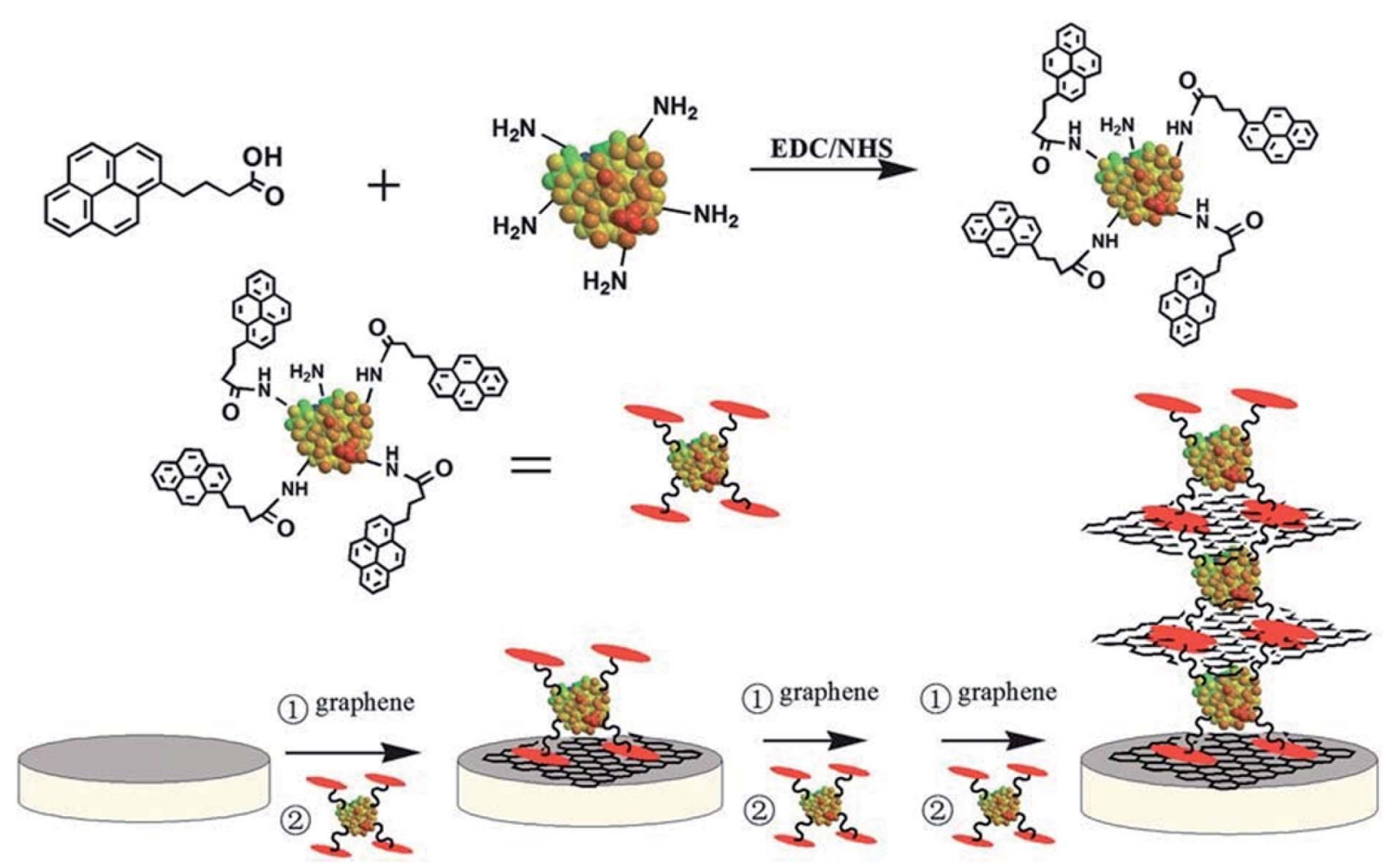

Fig. 5 Schematic illustration of the immobilization of GOx enzymes on graphene via pyrene and the subsequent fabrication of single- and multilayered enzyme electrodes. [Reprinted with permission from ref. 196, J. Liu, N. Kong, A. Li, X. Luo, L. Cui, R. Wang and S. Feng, Graphene Bridged Enzyme Electrodes for Glucose Biosensing Application, Analyst, 2013, 138, 2567-2575. Copyright@ Royal Society of Chemistry.] 
of graphene and GOx but was insignificant at 4 enzyme layers. The GOx enzyme electrode showed a very low response after adding $0.2 \mathrm{mM}$ of ascorbic acid and uric acid, and also retained $82.2 \%$ biocatalytic activity after 4 weeks of storage at $4{ }^{\circ} \mathrm{C}$.

The chemical reduction of GO into rGO with different amounts of defect density, layers and oxygen concentrations influences the electrochemical behavior and the glucose sensor performance. ${ }^{\mathbf{1 3 1}}$ Chemical doping with foreign atoms is a promising approach to intrinsically tune the electrical properties and regulate electron transport to influence glucose sensor performance. ${ }^{133}$ Wang et $a l .{ }^{133}$ demonstrated that a glucose sensor using $\mathrm{N}$-doped graphene that exhibited strong electrochemical activity and fast DET kinetics of GOx with a linear range of $0.1-1.1 \mathrm{mM}$ and LOD of $0.01 \mathrm{mM}$. The enhanced performance of the biosensor is due to the high electronic state density and the plenty of free electrons available in N-doped GR, which facilitates $\mathrm{H}_{2} \mathrm{O}_{2}$ electrochemical reduction by breaking of the $\mathrm{O}-\mathrm{O}$ bond in $\mathrm{H}_{2} \mathrm{O}_{2}$. Nitrogen doping modifies the density of states around the Fermi level of graphene, which considerably enhances the electron transfer rate. Liang et al. ${ }^{152}$ reported the DET of self-assembled GOx on the surface of electrochemically reduced carboxyl graphene on a GC electrode. Their biosensor showed distinct and quasi-reversible redox peaks at a potential of $-0.467 \mathrm{~V}$, promoting DET process with an improved linear range from 2-18 $\mathrm{mM}$ and a low LOD of $0.02 \mathrm{mM}$.

The direct electrochemistry of GOx immobilized on threedimensional (3D) porous graphene-modified electrodes and the effect of graphene defect structure on glucose sensor performance were studied. ${ }^{153,197}$ Guo et al. ${ }^{198}$ fabricated an enzymatic glucose biosensor using highly conductive N-doped
CVD-grown graphene (3D N-GR). The GOx enzyme was effectively immobilized on 3D N-GR using a chitosan (CS) biopolymer via molecular binding with $\mathrm{NH}_{2}$ groups. The fabricated 3D N-GR-CS-GOx biosensor showed a sensitivity of 226.24 $\mu \mathrm{A} \mathrm{mM}{ }^{-1} \mathrm{~m}^{-2}$ and a linear range of $0.1-1.3 \mathrm{mM}$ due to the high conductivity and porosity of the N-GR network, providing a large surface area for GOx enzyme loading and improving electron transport. Furthermore, Razmi et al. ${ }^{\mathbf{1 2 6}}$ demonstrated GQDs as an effective matrix for the immobilization of GOx on carbon ceramic electrode (CCE); the resulting GOx-GQD-CCE sensor showed high surface loading and strong affinity to enzymes while promoting DET, showing sensitivity of 0.085 $\mu \mathrm{A} \mu \mathrm{M}^{-1} \mathrm{~cm}^{-2}$ and a wide linear range of $5-1270 \mu \mathrm{M}$ with an LOD of $1.73 \mu \mathrm{M}$ for glucose detection. Manoj et al. ${ }^{199}$ demonstrated a biosensor using an aldehyde-functionalized ionic liquid (CHO-IL) and 3-(3-formyl-4-hydroxybenzyl)-3methylimidazolium hexafluorophosphate, as a platform for covalent immobilization (Fig. 6). Specifically, CHO-IL was immobilized on electrochemically reduced GO (EC-rGO) using the $\pi-\pi$ stacking of imidazolium and hydroxybenzyl groups with EC-rGO, which permitted the covalent bonding of Azure A mediator or GOx enzyme. The Azure A-immobilized biosensor showed a linear range of $0.03-1 \mathrm{mM}$, an LOD of $11.5 \mu \mathrm{M}$ and sensitivity of $133.2 \mu \mathrm{A} \mathrm{mM}^{-1} \mathrm{~cm}^{-2}$ for nonenzymatic detection of $\mathrm{H}_{2} \mathrm{O}_{2}$. The GOx-immobilized CHO-IL biosensor exhibited an improved sensing response with a sensitivity of $17.7 \mu \mathrm{A} \mathrm{mM}$ $\mathrm{cm}^{-2}$, a broad linear range of $0.05-2.4 \mathrm{mM}$ and an LOD of $17 \mu \mathrm{M}$ for glucose detection.

3.1.7 Graphene/polymer composite-based glucose biosensors. To enhance the catalytic function of immobilized GOx and the operational stability of the biosensor, GO is grafted with

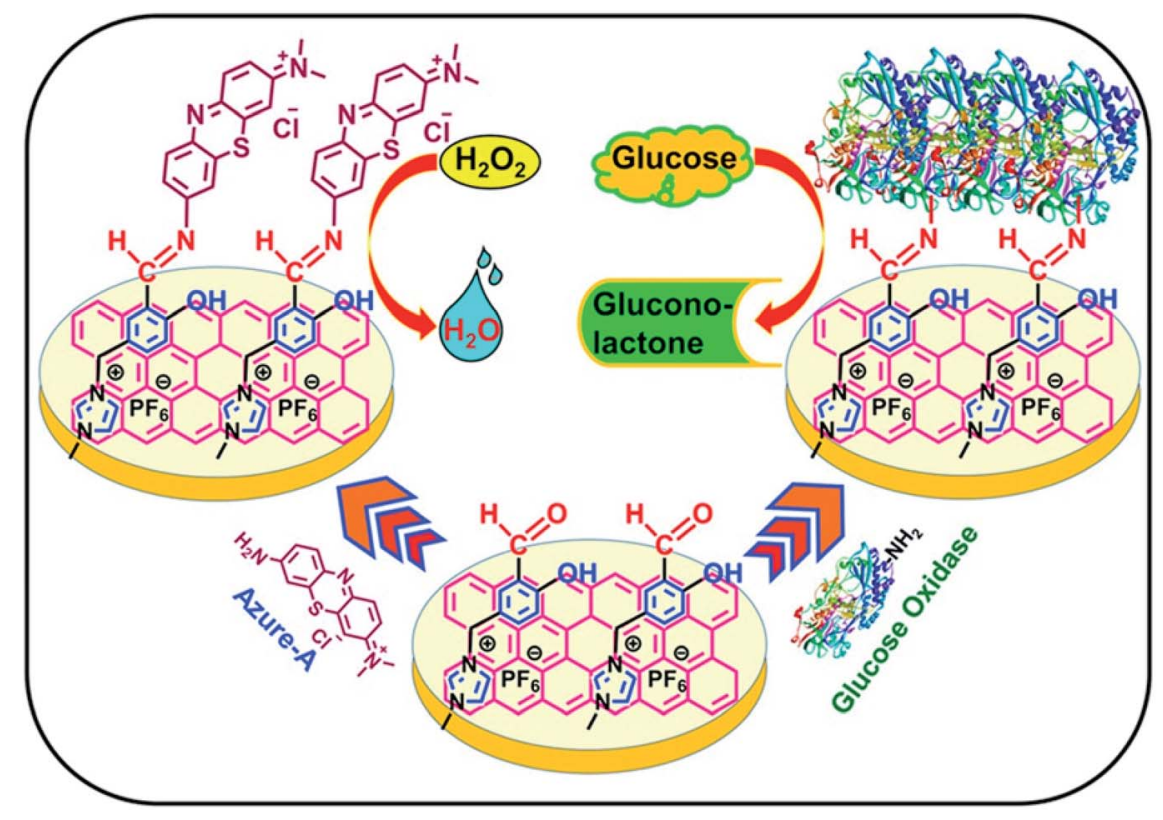

Fig. 6 Schematic illustration of the covalent immobilization of Azure A and GOx on the CHO-IL/EC-rGO/SPE platform. [Reprinted with permission from ref. 199, D. Manoj, K. Theyagarajan, D. Saravanakumar, S. Senthilkumar and K. Thenmozhi, Aldehyde Functionalized lonic Liquid on Electrochemically Reduced Graphene Oxide as a Versatile Platform for Covalent Immobilization of Biomolecules and Biosensing, Biosens. Bioelectron., 2018, 103, 104-112. Copyright@ Elsevier.] 
(A)

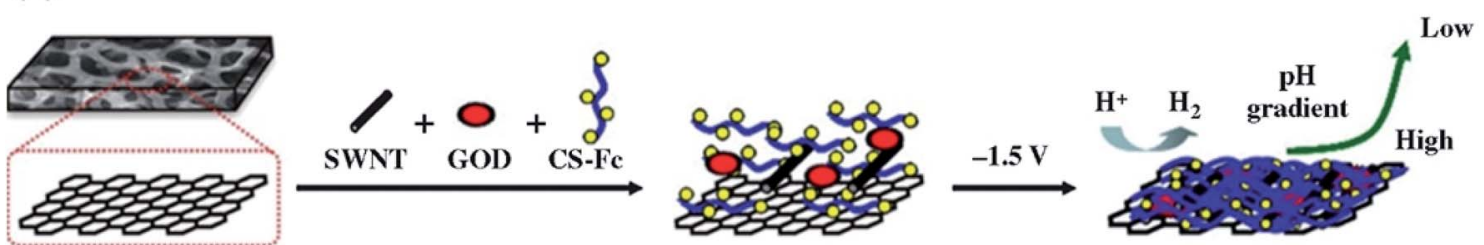

3D Graphene

\section{CS-Fc/SWNTs/GOD/3DG}
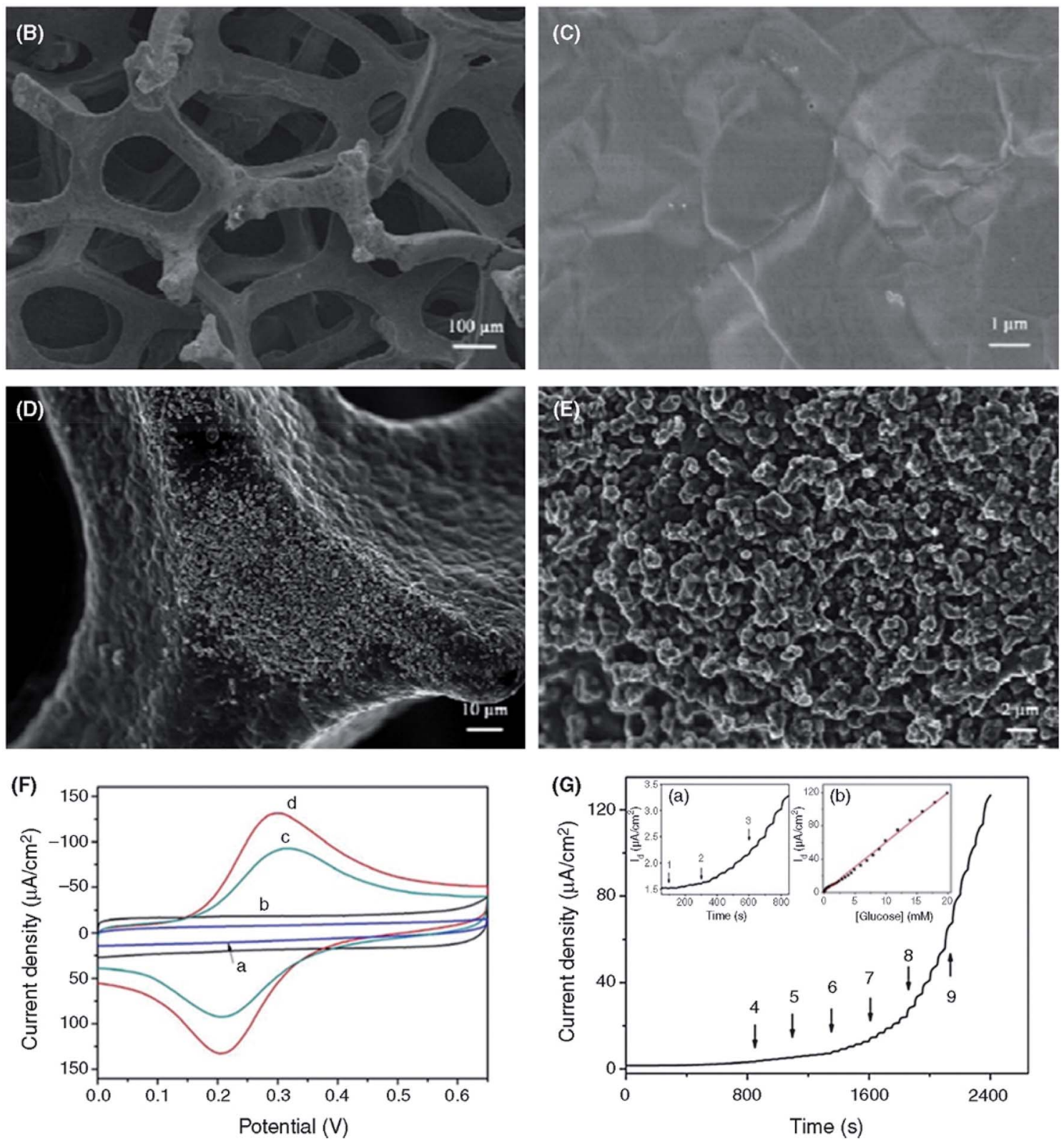

Fig. 7 (A) Schematic illustration of the preparation of 3D-GR-based enzymatic glucose biosensors using CS-mediated electrodeposition. (SWNT = SWCNT.). (B and C) SEM images of 3D-GR foam with low and high magnification. (D and E) SEM images of FC-CS/SWCNTs/GOx composite film electrodeposited on 3D graphene with low and high magnification. (F) CV curves of the (a) 3D-GR, (b) CS/GOx/3D-GR, (c) Fc-CS/GOx/3D-GR and (d) Fc-CS/SWCNTs/GOx/3D-GR electrodes in PBS (0.1 M, pH 7.0) at a scan rate of $100 \mathrm{mV} \mathrm{s}^{-1}$. (G) Amperometric response of the Fc-CS/SWCNTs/ GOx/3D-GR electrode upon the successively added glucose to stirred PBS (0.1 M, pH 7.0) at $0.4 \mathrm{~V}$. Inset (a) shows the magnified curve from 50 to $850 \mathrm{~s}$. Inset (b) shows the calibration plot of the current as a function of the glucose concentration. [Reprinted with permission from ref. 184, J. Liu, X. Wang, T. Wang, D. Li, F. Xi, J. Wang and E. Wang, Functionalization of Monolithic and Porous Three-Dimensional Graphene by One-Step Chitosan Electrodeposition for Enzymatic Biosensor, ACS Appl. Mater. Interfaces, 2014, 6, 19997-20002. Copyright@ American Chemical Society.] 
different polymers, such as polyethylene glycol (PEG), ${ }^{137}$ polyvinylpyrrolidone (PVP), ${ }^{192}$ or biopolymers, such as chitosan (CS). ${ }^{154}$ The polymer presents functional groups on the GO surface that enable the effective immobilization of redox enzymes without losing their biological functions and increase operational stability by suppressing leaching effects, while GO permits effective electron transport for enzymatic sensors. ${ }^{19,80}$ Kang et al. ${ }^{147}$ showed that a GR-chitosan (GR-CS) nanocomposite electrode is capable of high loading capacity $(1.12 \times$ $10^{-9} \mathrm{~mol} \mathrm{~cm}^{-2}$ ) for the immobilization of GOx enzyme, which exhibits DET with sensitivity of $37.9 \mu \mathrm{A} \mathrm{mM} \mathrm{m}^{-1} \mathrm{~cm}^{-2}$, a linear range of 0.08 to $12 \mathrm{mM}$ and an LOD of $0.02 \mathrm{mM}$. The CS biopolymer possesses amino $\left(-\mathrm{NH}_{3} / \mathrm{NH}_{2}\right)$ and hydroxyl $(-\mathrm{OH})$ functional groups, which facilitate the immobilizations of GOx while sustaining the biocatalytic functions and stabilizing the GOx enzymes. Similarly, Liu et al. ${ }^{141}$ reported the covalent immobilization of GOx enzymes on the carboxyl acid $(-\mathrm{COOH})$ groups of GO via amide bonds in the presence of EDC and NHS. Their GO/GOx composite glucose biosensor showed sensitivity of $8.045 \mathrm{~mA} \mathrm{M}^{-1} \mathrm{~cm}^{-2}$, a broad linear range and exhibited biocompatibility with human retinal pigment epithelium cells. Bharath et al. ${ }^{200}$ showed mesoporous 1D hydroxyapatite (HAp) nanorods on rGO for the efficient immobilization of GOx enzyme, displaying a highly enhanced electron transfer rate of rGO-HAp-GOx-GCE sensor with a superior sensitivity of $16.9 \mu \mathrm{A}$ $\mathrm{mM}^{-1} \mathrm{~cm}^{-2}$, a linear range of $0.1-11.5 \mathrm{mM}$ and an LOD of $0.03 \mathrm{mM}$. Liu et al. ${ }^{184}$ showed a facile, one-step chitosan (CS)based electrodeposition strategy for the fabrication of enzymatic glucose biosensors. The sensor was fabricated by electrodepositing a ferrocene (Fc)-functionalized CS hybrid (Fc-CS) and a single-walled carbon nanotube (SWCNT) solution to form a homogeneous film with GOx enzymes. Fc-CS/SWCNT/ GOx was immobilized via electrodeposition process on the surface of 3D graphene foam (GF) (Fig. 7A). The porous 3D structure is shown in Fig. 7B. Because of the superior properties of 3D GF, such as a large active surface area, high conductivity and fast mass transport dynamics, this sensor exhibited a wide linear range from $5.0 \mu \mathrm{M}$ to $19.8 \mathrm{mM}$, an LOD of $1.2 \mu \mathrm{M}$ and a fast response (95\% response within $8 \mathrm{~s}$ ) for the detection of glucose (Fig. 7C and D). Al-Sagur et al. ${ }^{201}$ demonstrated a highly sensitive enzymatic glucose biosensor based on lutetium phthalocyanine $\left(\mathrm{LuPc}_{2}\right)$ as a redox mediator in $\mathrm{rGO}$, integrated with polyacrylic acid (PAA) in vinyl-substituted polyaniline (VSPANI) multifunctional hydrogels. The fabricated PAA-rGO/VS$\mathrm{PANI} / \mathrm{LuPc}_{2} / \mathrm{GOx}$ biosensor showed a response time of $1 \mathrm{~s}$, sensitivity of $15.31 \mu \mathrm{AmM}^{-1} \mathrm{~cm}^{-2}$ in a linear range of 2-12 mM with an LOD of $25 \mu \mathrm{M}$ for glucose detection and 3 month long storage stability.

3.1.8 Graphene/nanoparticles (NPs) hybrid-based glucose sensors. The integration of graphene with inorganic nanoparticles has drawn significant interest in the development of hybrid nanostructures for electrochemical glucose sensors..$^{57,58,202}$ Integrating electrocatalytically active metal nanoparticles onto highly conductive GR surfaces is attractive for electrode fabrication because of the large accessible surface area, electrical conductivity and capacity for immobilizing enzymes. ${ }^{203}$ Furthermore, chemically derived graphene contains a large number of defects/vacancies and possesses functional groups such as carbonyls, epoxides, and hydroxyls, thus serving as a highly suitable matrix for the immobilization of inorganic nanoparticles as well as enzymes with enhanced stability and higher loading capacity. ${ }^{188}$ The hybrid GO/inorganic metal nanocomposites are primarily formed by decorating GO nanosheets with inorganic metal nanoparticles including AuNPs, ${ }^{149,159}$ AgNPs, ${ }^{158}$ PtNPs, ${ }^{172,204}$ PdNPs ${ }^{205} \mathrm{CuNPs}^{206}$ and RuNPs, metal oxide nanoparticles $\left(\mathrm{ZnO},{ }^{163,207,208} \mathrm{CuO},{ }^{209} \mathrm{ZrO}_{2},{ }^{156}\right.$ $\mathrm{SnO}_{2}, \mathrm{NiO},{ }^{210} \mathrm{MnO}_{2},{ }^{162} \mathrm{Fe}_{3} \mathrm{O}_{4}$ (ref. 211 and 212)), bimetallic nanoparticles (Pt/Pt NPs, ${ }^{157}$ ), and metal/metal oxide composites $\left(\mathrm{AuWO}_{3}\right.$ (ref. 213)). The surface functionalization of these supported NPs provides an effective approach for the site-specific conjugation of biomolecules without loss of activity. In particular, the integration of Au nanoparticles with the GOx enzyme can provide site-specific conjugation of the FAD site and improve the specific surface area, thus minimizing the electron tunneling distance to facilitate DET. ${ }^{214}$ Willner et al. ${ }^{215,216}$ reported the functionalization of AuNPs to create an FAD-active center of GOx for electrochemical enzymatic biosensors.

Graphene/nanoparticle composite-based electrochemical enzymatic glucose biosensors have been summarized in Table 1. Noble metal nanoparticles ( $\mathrm{Au}, \mathrm{Ag}, \mathrm{Pt}$, and $\mathrm{Pd}$ ) are widely used for the effective site-specific conjugation of enzymes. ${ }^{160,217}$ Palanisamy et al. ${ }^{158}$ developed a biosensor by immobilizing GOx on an EC-rGO/AgNP nanocomposite. The biosensor showed a pair of distinct redox peaks having a formal potential $\left(E^{\circ}\right)$ of $-0.422 \mathrm{~V}$ and a heterogeneous electron transfer rate constant $\left(K_{\mathrm{s}}\right)$ of $5.27 \mathrm{~s}^{-1}$, suggesting a high affinity between GOx and the EC-rGO/AgNP nanocomposite and fast DET. The biosensor exhibited high sensitivity toward glucose detection in the 0.5 to $12.5 \mathrm{mM}$ concentration range with an LOD of $0.16 \mathrm{mM}$. Claussen et al. ${ }^{218}$ demonstrated a biosensor with PtNPs decorated on multilayered graphene petal nanosheets (MGPNs). The performance of biosensor was optimized through electrodeposited PtNPs by changing their size and morphology. The designed GOx-PEDOT/PtNP/MGPN biosensor demonstrated a wide linear range of $0.01-50 \mathrm{mM}$ with an LOD of $0.3 \mu \mathrm{M}$ for glucose detection and a stable shelf-life by retaining $75 \%$ sensitivity over a time period of 5 week.

An enzymatic glucose biosensor based on the tannic acid (TA)mediated deposition of AuNPs onto rGO nanocomposites was demonstrated by Çakıroğlu and Özacar. ${ }^{186}$ The fabricated glucose biosensor exhibited a sensitivity of $18.73 \mathrm{~mA} \mathrm{mM}{ }^{-1} \mathrm{~cm}^{-2}$ and a linear range of 2-10 mM. Akkaya et al. ${ }^{187}$ also fabricated a glucose biosensor based on the direct electrochemistry of GOx on PtNPs supported on a TA-rGO nanocomposite, as shown in Fig. 8A. Specifically, after the electrochemical oxidation of tannic acid to quinone, the formation of $\pi-\pi$ interactions between GO and TA and of Schiff-base supported hydrogen bonding between GOx and TA that improved DET. The curves from CV measurements in PBS (0.05 M, pH 7.4) solution at $100 \mathrm{mV} \mathrm{s}^{-1}$ scan rate are shown in Fig. 8B. The GOx/PtNPs/rGO-GC-modified electrode exhibited redox peaks at a formal potential $\left(E^{\circ}\right)$ of $-0.462 \mathrm{~V}$ and the peak-to-peak separation $\left(\Delta E_{\mathrm{p}}\right)$ of $56 \mathrm{mV}$, indicating the fast electron transfer between PtNPs/rGO nanocomposite and GOx. The redox peak current linearly decreased as the concentration 
of glucose was increased (Fig. 8C). By adding glucose, more $\mathrm{GOx}(\mathrm{FAD})$ was transformed into $\mathrm{GOx}\left(\mathrm{FADH}_{2}\right)$ due to the biocatalytic reaction. The fabricated biosensor showed an enhanced sensitivity of $27.51 \mu \mathrm{A} \mathrm{mM}{ }^{-1} \mathrm{~cm}^{-2}$, a wide linear range of 2$10 \mathrm{mM}$ and an LOD of $1.21 \mu \mathrm{M}$. Notably, the sensitivity of the $\mathrm{rGO} / \mathrm{PtNP} / \mathrm{GOx}-\mathrm{GCE}$ electrode is higher than that of the $\mathrm{rGO} /$

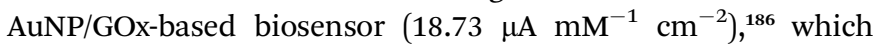
can be ascribed to the high catalytic activity of the PtNPs supported on the surface of rGO.
Bimetallic nanoparticles are well known to exhibit highly enhanced electrochemical activity compared to monometallic nanoparticles. ${ }^{219}$ Taking advantage of this property, Hossain and Park ${ }^{157}$ designed a glucose biosensor based on the immobilization of GOx on rGO modified with electrochemically deposited PtPdNPs. The resulting biosensor showed sensitivity of $24 \mu \mathrm{A} \mathrm{mM} \mathrm{mm}^{-1} \mathrm{~cm}^{-2}$, a linear range of $2-12 \mathrm{mM}$, an LOD of $0.001 \mathrm{mM}$, and a fast response time of $5 \mathrm{~s}$. In addition, metal oxide nanoparticles or magnetic nanoparticles supported on

(A)
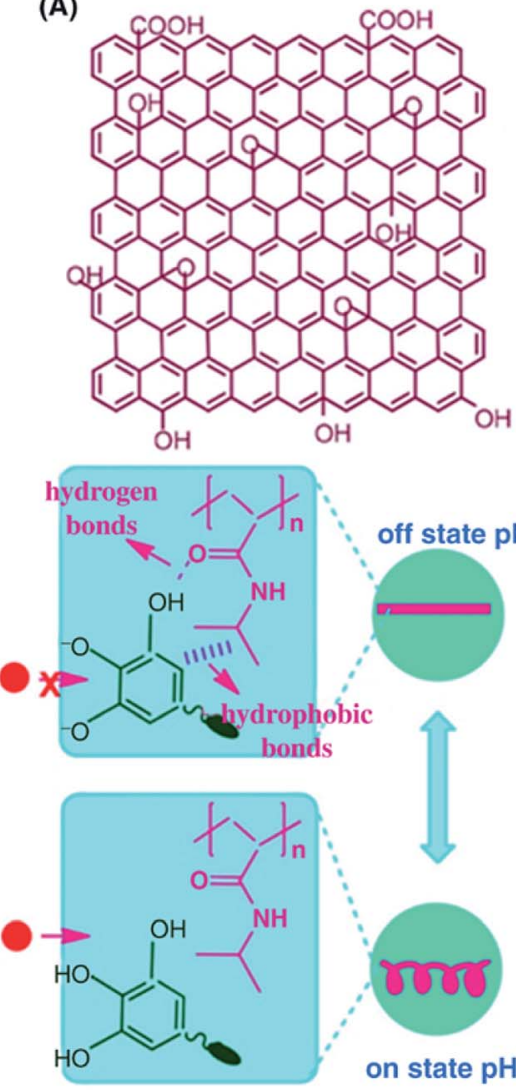

(B)

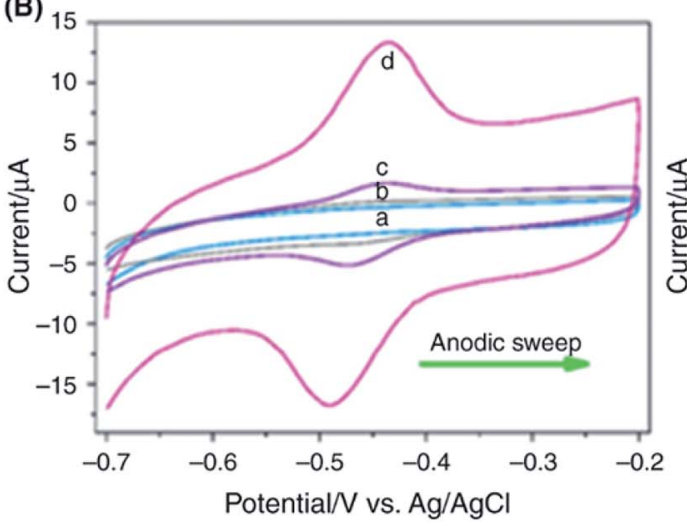

off state $\mathrm{pH} 7,20^{\circ} \mathrm{C}$
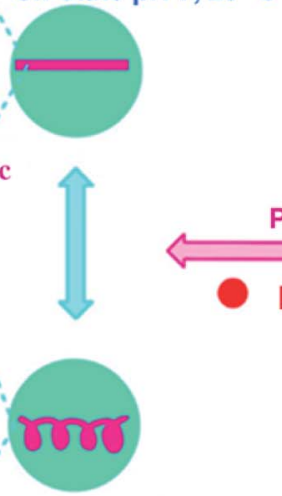

on state $\mathrm{pH} 4,40{ }^{\circ} \mathrm{C}$
Simultaneous reduction of $\mathrm{Pt}^{4+}$ and $\mathrm{GO}$

TA

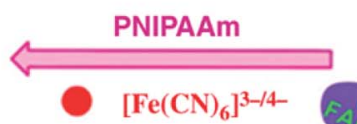

Hydrogen and Schiff base bond

-pre-oxidation of TA

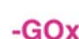

s
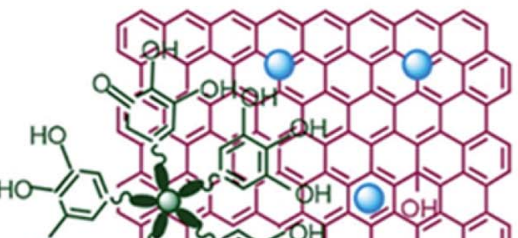

$\pi-\pi$ interaction
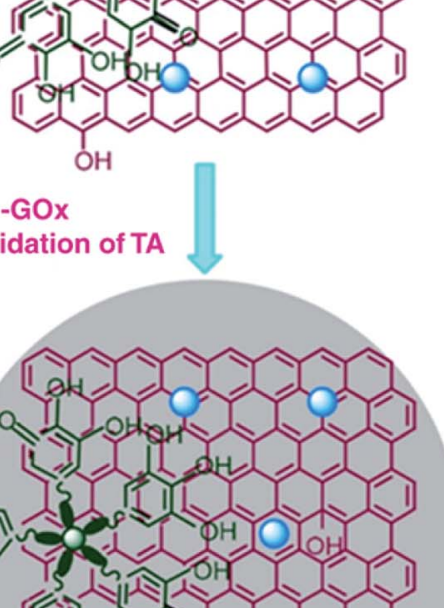

1)

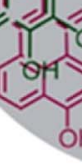

(C)

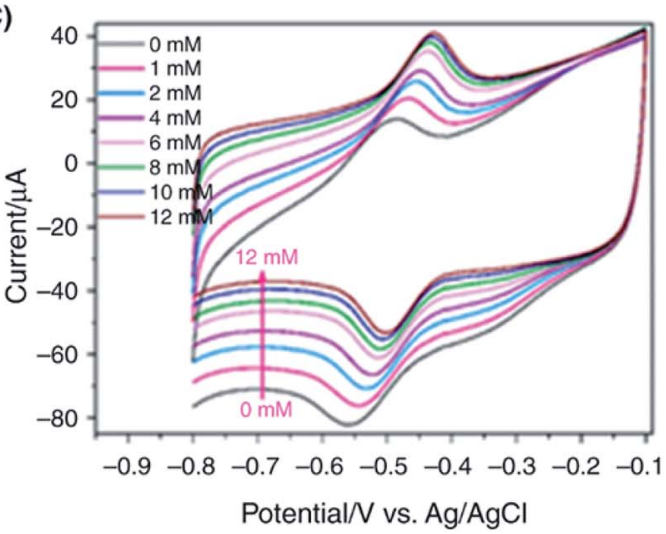

Fig. 8 (A) Schematic illustration of the tannic acid (TA)-assisted preparation of rGO/PtNPs/GOx-GCE-based biosensors for glucose sensing. (B) CV curves of different electrodes; (a) GOx/GCE, (b) GO-GOx/GCE, (c) rGO-GOx/GCE, and (d) rGO-PtNPs-GOx/GCE in deoxygenated PBS (0.1 M, $\mathrm{pH}$ 7.4) at a scan rate of $100 \mathrm{mV} \mathrm{s}^{-1}$. (C) CV curves of rGO-PtNPs-GOx/GCE recorded in $\mathrm{O}_{2}$-saturated PBS (0.1 M) at a scan rate of $100 \mathrm{mV} \mathrm{s}^{-1}$ as a function of different concentrations of glucose $(0-12 \mathrm{mM})$. [Reprinted with permission from ref. 187, B. Akkaya, B. Çakiroğlu and M. Özacar, Tannic Acid-Reduced Graphene Oxide Deposited with Pt Nanoparticles for Switchable Bioelectronics and Biosensors Based on Direct Electrochemistry, ACS Sustainable Chem. Eng., 2018, 6, 3805-3814. Copyright@ American Chemical Society.] 
Table 2 Graphene/nanoparticles (NPs) nanocomposite-based nonenzymatic glucose biosensors and their sensitivity, concentration detection range and $\mathrm{LOD}^{a}$

Graphene-based nanocomposites

Sensitivity $(\mu \mathrm{A}$

$\mathrm{NiCo}_{2} \mathrm{~N} / \mathrm{N}-\mathrm{GR}$ core-shell

GR/PtNiNPs

GR/CuO NPs nanocomposites

$3 \mathrm{D} \mathrm{GF} /$ cobalt oxide nanowires $\left(\mathrm{Co}_{3} \mathrm{O}_{4} \mathrm{NWs}\right)$ composite

GR-cobalt oxide nanoneedles

GR paper/PtAu- $\mathrm{MnO}_{2}$ nanocomposites

GO/NiONPs composite

$\mathrm{GO} / \mathrm{CuONPs}$ composite

$\mathrm{GR} / \mathrm{Cu}_{2} \mathrm{O}$ nanocubes

$\mathrm{Cu}-\mathrm{GR}$ nanoflowers

Pt nanoflowers/GO composite

$\mathrm{Mn}_{3} \mathrm{O}_{4} / 3 \mathrm{D}$ GR foam

$\mathrm{rGO} / \mathrm{CuO}$ nanoflowers/Pt nanocubes

$\mathrm{N}$-doped GR/CuNPs composite

$\mathrm{rGO} / \mathrm{Ni}(\mathrm{OH})_{2}$ nanostructures

PtPd-IL-rGO nanocomposite

$\mathrm{Ni}(\mathrm{OH})_{2} / 3 \mathrm{D} \mathrm{GF}$

GR/PtNi NPs

$\mathrm{Cu}_{2} \mathrm{O}$ NPs/graphene

PdCu NPs/3D GR hydrogel

GO nanoribbon/AuNPs hybrid

$\mathrm{Ni}(\mathrm{OH})_{2} / \mathrm{rGO} /$ MWCNTs

$\mathrm{SnO}_{2} / \mathrm{rGO}$ composite

PVP/GNs/NiNPs/CS composite

$\mathrm{rGO} / \mathrm{Au}-\mathrm{CuO}$ NPs

AuNPs/Ni-Al double hydroxide/SWCNTs/GR composite

S-GR/CuO NPs composite

$\mathrm{N}-\mathrm{rGO} / \mathrm{Mn}_{3} \mathrm{O}_{4} \mathrm{NPS}$

$\mathrm{CuNiO} / \mathrm{GO}$

GR/Pt nanoclusters/PVP composite

$\mathrm{GO} / \mathrm{Cu} \mathrm{NPs}$

$\mathrm{rGO} / \mathrm{Pt}-\mathrm{NiO}$ nanoplate arrays

Needlelike $\mathrm{NiCo}_{2} \mathrm{O}_{4} / 3 \mathrm{D} \mathrm{GF}$

rGO nanosheet/Cu nanowires composite

NiO NPs/polyaniline nanowire/GO composite

NiCo alloys/polypyrrole/rGO nanocomposites

$\mathrm{Cu}_{2} \mathrm{O} / \mathrm{AlOOH} / \mathrm{rGO}$ nanocomposite

$\mathrm{NiCo}_{2} \mathrm{O}_{4}$ nanowrinkles/rGO

$\mathrm{Cu} \mathrm{NPs} /$ pencil graphite

$\mathrm{CuCO}_{2} \mathrm{O}_{4}$ nanosheets/graphite paper

$\mathrm{AgPt} / \mathrm{rGO}$ composite

Hollow $\mathrm{CuCo}_{2} \mathrm{O}_{4} /$ porous rGO composite

GQD/CoNiAl-layered double hydroxide

GR/BiOCl nanohybrid

AuNPs/N-doped GR

CuS NPs/rGO

$\mathrm{NiCo}_{2} \mathrm{O}_{4} /$ nitrogen-doped $\mathrm{rGO} / \mathrm{IL}$ composite

CuNPs/EC-rGO composite

$\mathrm{N}$-doped GR/Cu nanostructures composite

Honeycomb-like CoNS/RGO/PPy nanocomposite

3D GR/Ni on ZnO nanorod arrays

Nanoneedle like $\mathrm{CuO} / \mathrm{N}$-doped rGO

Ni plasma-modified GR

rGO/CuS NF nanocomposite

3D $\mathrm{Cu}-\mathrm{Co} / \mathrm{rGO}$ nanocomposite

GNs/GR nanoribbons/NiNPs

CuNPs/rGO core-shell nanocomposite

$\mathrm{NiO} / \mathrm{PtNPs} / \mathrm{EC}-\mathrm{rGO}$ composite
$\left.\mathrm{mM}^{-1} \mathrm{~cm}^{-2}\right)$

Limit of detection

Ref.

$2.008 \mu \mathrm{M}$ to $7.15 \mathrm{mM}$

0.5-35 mM

$1 \mu \mathrm{M}$ to $8 \mathrm{mM}$

Up to $80 \mu \mathrm{M}$

$0.2-3 \mathrm{mM}$

0.1-30.0 mM

$3.13 \mu \mathrm{M}$ to $3.05 \mathrm{mM}$

$2.79 \mu \mathrm{M}$ to $2.03 \mathrm{mM}$

0.3-3.3 mM

5-900 $\mu \mathrm{M}$

2-20.3 mM

0.1-8 mM

Up to $12 \mathrm{mM}$

0.004-4.5 mM

$15 \mu \mathrm{M}$ to $30 \mathrm{mM}$

0.1-22 mM

$1 \mu \mathrm{M}$ to $1.17 \mathrm{mM}$

0.5-20 mM

$0.5 \mu \mathrm{M}$ to $2 \mathrm{mM}$

2-18 mM

$0.5 \mathrm{M}$ to $10 \mathrm{mM}$

10-1500 $\mu \mathrm{M}$

50-500 $\mu \mathrm{M}$

$0.1 \mu \mathrm{M}$ to $0.5 \mathrm{mM}$

$1 \mu \mathrm{M}$ to $12 \mathrm{mM}$

$10 \mu \mathrm{M}$ to $6.1 \mathrm{mM}$

$0.1-10.5 \mathrm{mM}$

$1.0-329.5 \mu \mathrm{M}$

0.05-6.9 mM

1-25 mM

Up to $12 \mathrm{mM}$

0.008-14.5 mM

$0.5 \mu \mathrm{M}$ to $0.59 \mathrm{mM}$

$1 \mu \mathrm{M}$ to $11 \mathrm{mM}$

$2 \mu \mathrm{M}$ to $5.560 \mathrm{mM}$

$0.5 \mu \mathrm{M}$ to $4.1 \mathrm{mM}$

$5.0 \mu \mathrm{M}$ to $14.77 \mathrm{mM}$

0.005-8.6 mM

1.0-100 mM

Up to $320 \mu \mathrm{M}$

0.003-7.72 mM

$0.5-3354 \mu \mathrm{M}$

$0.01-14.0 \mathrm{mM}$

$0.5-10 \mathrm{mM}$

$0.04-16.1 \mathrm{mM}$

$0.0001-3.88 \mathrm{mM}$

0.001-4.555 mM

$0.14-5091 \mathrm{mM}$

0.0005-5.0 mM

$0.5 \mu \mathrm{M}$ to $2.667 \mathrm{mM}$

$0.05 \mu \mathrm{M}$ to $1.11 \mathrm{mM}$

$0.5-639 \mu \mathrm{M}$

$1-1150 \mu \mathrm{M}$

1-2000 $\mu \mathrm{M}$

$1 \mu \mathrm{M}$ to $4 \mathrm{mM}$

$5 \mathrm{nM}$ to $5 \mathrm{mM}$

$0.001-2 \mathrm{mM}$

0.05-5.66 mM
1803

20.42

1065

3390

-

58.54

1087

262.52

-

11.3

0.64

360

3577

48.13

11.4

1.47

2.65

30.3

2939.24

48.0

59.1

2042

1930

103.8

2356

1989

1298.6

0.026

225.75

1.21

447.65

832.95

2524

1625

376.22

153.5

155.1

548.9

1467.5

3.625

129.32

2426

48.717

1.878

0.25

429.4

3760

445

1848

297.73

2030

0.0034

2213

53.5

240

2300

150

668.2

$50 \mathrm{nM}$

$10 \mu \mathrm{M} \quad 229$

$1 \mu \mathrm{M}$

$10 \mu \mathrm{M}$

$0.02 \mathrm{mM} 234$

$1 \mu \mathrm{M} \quad 210$

$0.69 \mu \mathrm{M} \quad 209$

$3.3 \mu \mathrm{M}$

$1 \mu \mathrm{M} \quad 236$

$2.0 \mu \mathrm{M}$

$10 \mu \mathrm{M} \quad 238$

$1.3 \mu \mathrm{M} \quad 226$

$15 \mu \mathrm{M} \quad 240$

$2 \mu \mathrm{M} \quad 241$

$0.34 \mu \mathrm{M} \quad 242$

$2 \mu \mathrm{M}$

$0.09 \mu \mathrm{M} \quad 244$

0-8 mM 245

$5 \mu \mathrm{M} \quad 246$

$2.7 \mu \mathrm{M} \quad 247$

$13.35 \mu \mathrm{M} \quad 248$

$30 \mathrm{nM} \quad 249$

$0.01 \mu \mathrm{M} \quad 250$

$1 \mu \mathrm{M} \quad 251$

$80 \mathrm{nM} 252$

$0.5 \mu \mathrm{M}$

$16 \mu \mathrm{M}$

$30 \mu \mathrm{M} \quad 255$

$3.4 \mu \mathrm{M} \quad 256$

$2.67 \mu \mathrm{M} \quad 257$

$0.38 \mu \mathrm{M} \quad 258$

$0.2 \mu \mathrm{M} \quad 227$

$0.5 \mu \mathrm{M} \quad 259$

$0.17 \mu \mathrm{M} \quad 230$

$2.6 \mu \mathrm{M} \quad 260$

$2 \mu \mathrm{M} \quad 261$

$0.44 \mu \mathrm{M} \quad 262$

$5 \mu \mathrm{M} \quad 263$

$1.8 \mu \mathrm{M} \quad 264$

$0.15 \mu \mathrm{M} \quad 265$

$6 \mu \mathrm{M} \quad 266$

$0.22 \mathrm{mM} \quad 267$

$12 \mu \mathrm{M} \quad 268$

$32 \mathrm{nM} \quad 269$

$0.18 \mu \mathrm{M} \quad 270$

$0.049 \mu \mathrm{M} \quad 271$

$0.014 \mu \mathrm{M} \quad 228$

$29 \mathrm{nM} \quad 272$

$0.15 \mu \mathrm{M} \quad 273$

$0.01 \mu \mathrm{M} \quad 274$

$1 \mu \mathrm{M} \quad 275$

$0.19 \mu \mathrm{M} \quad 276$

$0.15 \mu \mathrm{M} \quad 277$

$2.5 \mathrm{nM} \quad 278$

$0.34 \mu \mathrm{M} \quad 279$

$0.2 \mu \mathrm{M} \quad 280$
$25 \mathrm{nM} 232$

$0.01 \mu \mathrm{M} \quad 239$

${ }^{a}$ Abbreviations used: PVP: polyvinyl-pyrrolidone, EC-rGO: electrochemically reduced graphene oxide, GF: graphene foam, BiOCl: bismuth oxychloride, CuSNFs: copper sulfide nanoflake. 
graphene have been widely used to develop electrochemical enzymatic glucose biosensors (Table 1). Liu et al. ${ }^{\mathbf{1 8 0}}$ reported an enzymatic glucose sensor based on a composite of $\mathrm{MnO}_{2} \mathrm{NPs}$ supported on graphene. The GR-MnO${ }_{2}$ NPs-GOx-Nafion based sensor exhibited a high sensitivity of $3.30 \mu \mathrm{A} \mathrm{mM}{ }^{-1} \mathrm{~cm}^{-2}$, a wide linear range of 0.04 to $2 \mathrm{mM}$ with an LOD of $10 \mu \mathrm{M}$. The sensor displayed high selectivity for the determination of glucose level in the presence of interfering bioanalytes including AA and UA which may be associated with the use of Nafion in the modified electrode. Vilian et al. ${ }^{156}$ fabricated a glucose biosensor using GOx immobilized in a poly(L-lysine) (PLL) and rGO-zirconium oxide ( $\left.\mathrm{rGO}-\mathrm{ZrO}_{2}\right)$ nanocomposite. The sensor exhibited DET by displaying well-defined redox peaks at the formal potential $\left(E^{\circ}\right)$ of $-0.403 \mathrm{~V}$ with a small peak-to-peak separation $\left(\Delta E_{\mathrm{p}}=27 \mathrm{mV}\right)$. The GOx-PLL/rGO-ZrO $\mathrm{Zr}_{2}$ based sensor exhibited a sensitivity of $11.65 \mu \mathrm{AmM}^{-1} \mathrm{~cm}^{-2}$, a linear range of 0.29-14 $\mathrm{mM}$ and LOD of $0.13 \mathrm{mM}$. The biosensor showed recoveries of $97 \%$ to $102.5 \%$ in the spiked samples of human urine for glucose detection and $94.4 \%$ retention of original response after storing at $4{ }^{\circ} \mathrm{C}$ for a period of 1 month. Zhao et $a .^{208}$ reported a long-range electrical wiring of GOx using a $\mathrm{ZnO}$ nanorods/EC-reduced graphene heterostructure. The glucose biosensor fabricated by immobilizing GOx on ZnONRs/ EC-reduced graphene enabled contact with enzymes through the $\mathrm{ZnO}$ nanorods. The charge transfer was associated with $\mathrm{ZnO}$ nanorods between the redox center of FAD-GOx and the electrode. The fabricated GOx-ZnONRs/EC-reduced graphene/PTbased glucose biosensor showed DET characteristics with a sensitivity of $89.84 \mu \mathrm{A} \mathrm{mM} \mathrm{mm}^{-1} \mathrm{~cm}^{-2}$ and a linear response to glucose concentrations of $0.2-1.6 \mathrm{mM}$ with a correlation coefficient $(R)$ value of 0.998 . The $K_{\mathrm{s}}$ value of $0.92 \mathrm{~s}^{-1}$ indicated DET of GOx. Teymourian et al. ${ }^{\mathbf{1 6 1}}$ fabricated a glucose biosensor based on a $\mathrm{Fe}_{3} \mathrm{O}_{4} /$ rGO-modified GCE, which showed glucose sensing with a wide linear range of $0.5 \mathrm{mM}$ to $12 \mathrm{mM}$ and an LOD of $0.05 \mu \mathrm{M}$.

3.1.9 Graphene-based nonenzymatic glucose biosensors. The enzymatic glucose sensor exhibits high sensitivity and selectivity because of the superior catalytic functions of the enzymes. However, the catalytic function of enzymes such as GOx can be negatively influenced by many parameters, including $\mathrm{pH}$, temperature, pressure and humidity. ${ }^{220}$ In addition, the sensor performance and operational stability depend strongly on the immobilization method, biocatalytic functions of the enzyme, and DET characteristics, which greatly limit their practical applications. Although GOx is relatively stable compared to other enzymes, it quickly loses its activity below $\mathrm{pH}$ 2.0 and above $\mathrm{pH} 8 .^{220}$ Moreover, it has an apparent transition temperature of $55.8 \pm 1.28{ }^{\circ} \mathrm{C}$ and an activation energy of $280 \mathrm{~kJ} \mathrm{~mol}{ }^{-1} .^{221}$ Thus, GOx undergoes structural transformations and exhibits damage above $40{ }^{\circ} \mathrm{C}$. Therefore, the development of a low-cost, sensitive, enzyme-free approach to glucose detection is a desirable alternative to enzymatic methods. Nevertheless, given the sensitivity and selectivity of the commercially available glucose sensor strips, nonenzymatic methods have to meet stringent requirements for commercial viability. ${ }^{222}$
The unique electrochemical properties of graphene and its derivatives have also been applied to fabricate nonenzymatic glucose biosensors. Moreover, supporting inorganic nanoparticles on graphene allows significant enhancement of their electrocatalytic activity and sensing performance. ${ }^{223}$ Due to their strong electrocatalytic activity, noble metal nanoparticles have been decorated/supported on graphene to construct highly sensitive enzyme-less electrochemical glucose sensors. ${ }^{223}$ Table 2 summarizes the performance of nonenzymatic electrochemical glucose biosensing developed from graphene-based nanocomposites with a very wide variety of metal nanoparticles/nanostructures. ${ }^{\mathbf{2 1 0 , 2 2 4 - 2 8 0}}$ For example, Deepalakshmi et $a .^{224}$ used nitrogen-doped graphene (N-GR)-encapsulated nickel cobalt nitride $\left(\mathrm{NiCo}_{2} \mathrm{~N}\right)$ core-shell nanostructures for nonenzymatic detection of glucose and hydrogen peroxide. The synergistic effect of the $\mathrm{NiCo}_{2} \mathrm{~N} / \mathrm{N}-\mathrm{GR}$ core-shell nanostructures resulted in high sensitivity and selectivity. $\mathrm{NiCo}_{2} \mathrm{~N} / \mathrm{N}-\mathrm{GR} / \mathrm{GCE}$ sensor detected $1.09-\mathrm{mM}$ glucose concentration in human blood serum with $94.8 \%$ recovery, showed high selectivity for glucose detection against interfering biomolecules such as DA, $\mathrm{UA}, \mathrm{AA}$, lactic acid, fructose, lactose and $\mathrm{NaCl}$, as well as longterm environmental stability for glucose oxidation by retaining $92.31 \%$ of initial current response after 45 days at room temperature. Hoa et al. ${ }^{225}$ used Pt nanoparticles supported on GO hydrogel $(\mathrm{GOH})$ for nonenzymatic glucose detection. The PtNPs/GOH sensor showed a sensitivity of $137.4 \mu \mathrm{A} \mathrm{mM}^{-1} \mathrm{~cm}^{-2}$, which is 7-times greater than in the absence of PtNPs on GOH. In addition, the sensor showed selective detection in real blood samples and good anti-interference ability toward AA, UA, and DA interferents. Jiang et $a .^{226}$ prepared nitrogen-doped graphene (N-GR) decorated with copper nanoparticles (CuNPs) through simple thermal treatment for nonenzymatic glucose detection. The CuNPs/N-GR sensor showed greatly enhanced glucose-sensing performance with a sensitivity of $48.13 \mu \mathrm{A}$ $\mathrm{mM}^{-1}$, a linear range of $0.004-4.5 \mathrm{mM}$, an LOD of $1.3 \mu \mathrm{M}$, and the response time of $<5 \mathrm{~s}$. The peak current of glucose oxidation was found to be approximately 23 times greater compared with pristine CuNPs. Ju et al. ${ }^{227}$ demonstrated an enhanced nonenzymatic amperometric glucose biosensor based on rGO decorated with copper nanowires (CuNWs). The resulting sensor displayed enhanced sensitivity and LOD, which was attributed to the higher catalytic activity and electrical conductivity of the CuNWs. The sensitivity was found to be $1625 \mu \mathrm{A} \mathrm{cm}{ }^{-2} \mathrm{mM}^{-1}$ with a linear range up to $11 \mathrm{mM}$, an LOD of $0.02 \mu \mathrm{M}$ and a fast response time of $<2 \mathrm{~s}$ at a potential of $0.58 \mathrm{~V}$. Moreover, the sensor exhibited negligible interference from AA, DA, UA, acetamidophenol and other carbohydrates. Gowthaman et al. ${ }^{228}$ reported a nonenzymatic glucose biosensor based on a $\mathrm{Cu}$ nanostructure-deposited nitrogen-doped graphene (N-GR). Owing to the unique dendritic nanostructure of $\mathrm{Cu}$ on $\mathrm{N}-\mathrm{GR}$, the sensor exhibited 2-times greater glucose oxidation current than that of pristine N-GR, with a sensitivity of $1848 \mu \mathrm{AmM}^{-1}$ $\mathrm{cm}^{-2}$, a linear range of $0.0005-5.0 \mathrm{mM}$, and an LOD of 0.014 $\mu \mathrm{M}$. The biosensor showed high selectivity toward glucose determination against interfering bioanalytes including $\mathrm{Na}^{+}$, $\mathrm{K}^{+}, \mathrm{Mg}^{2+}, \mathrm{Ca}^{2+}, \mathrm{Cl}^{-}, \mathrm{SO}_{4}{ }^{2-}$, urea, oxalic acid, AA and UA. The recoveries of 97.34 to $99.52 \%$ and 97.19 to $98.01 \%$ in spiked 
(A) Graphene Oxide (GO)

(B)
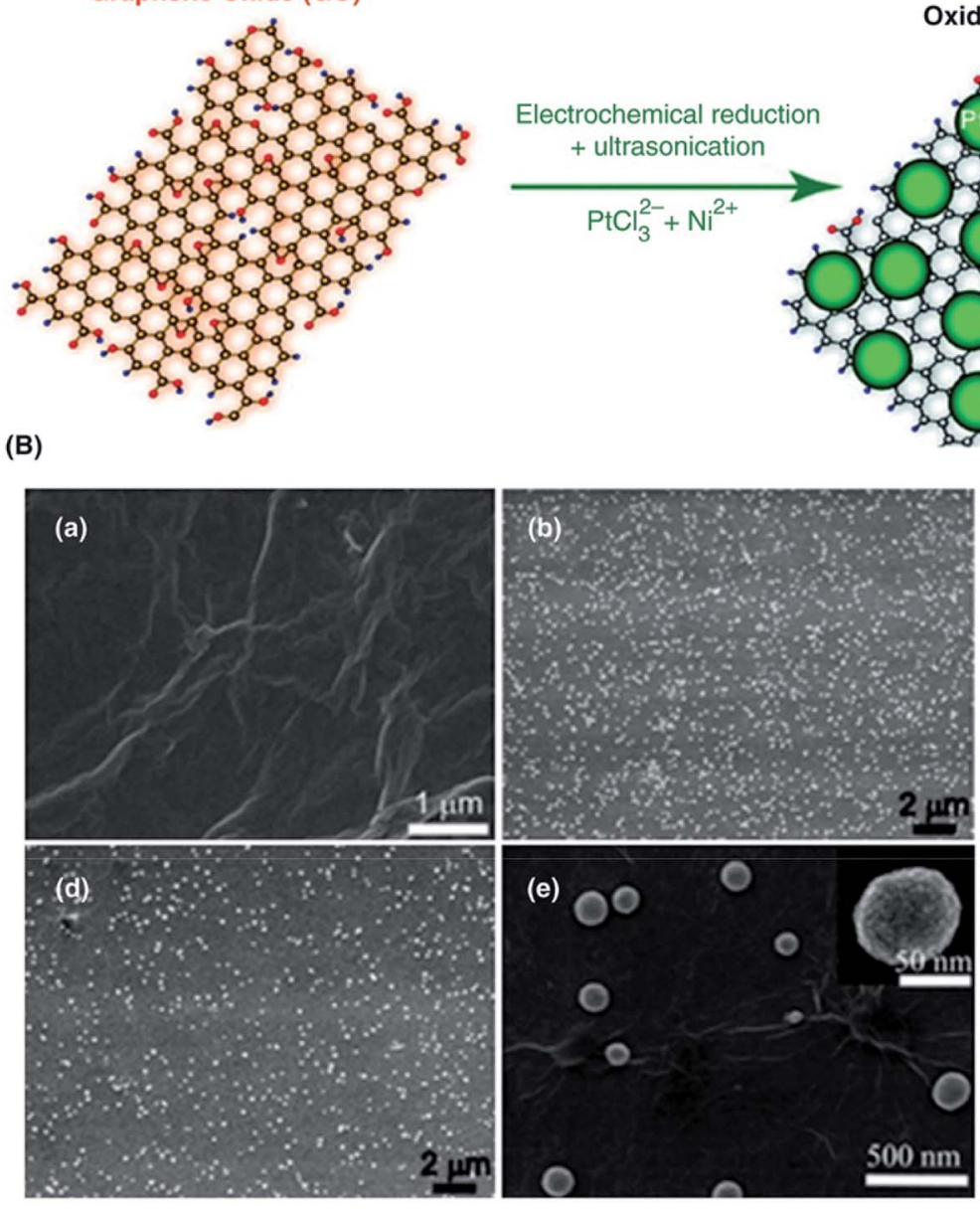

(C)

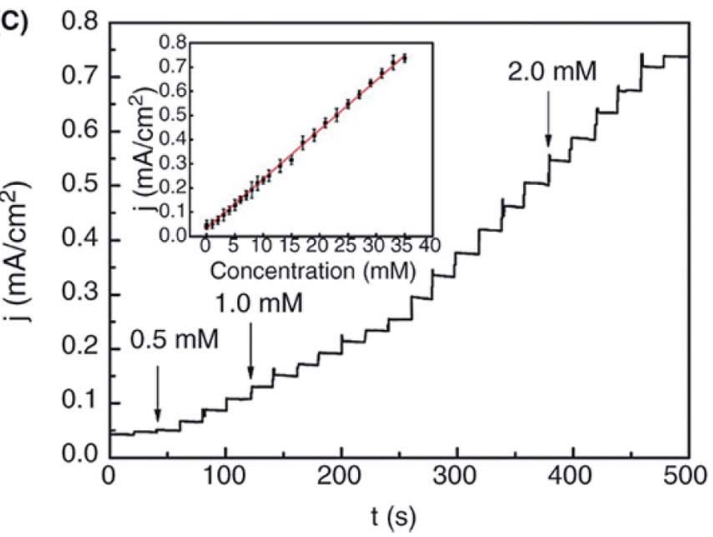

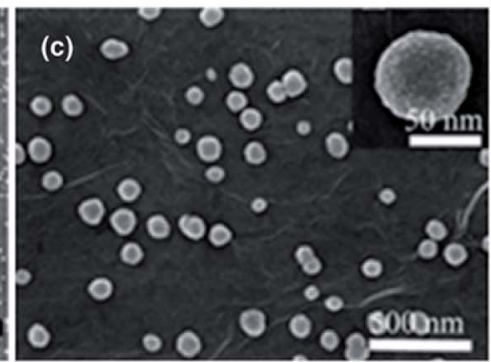

Reduced Graphene Oxide (RGO)
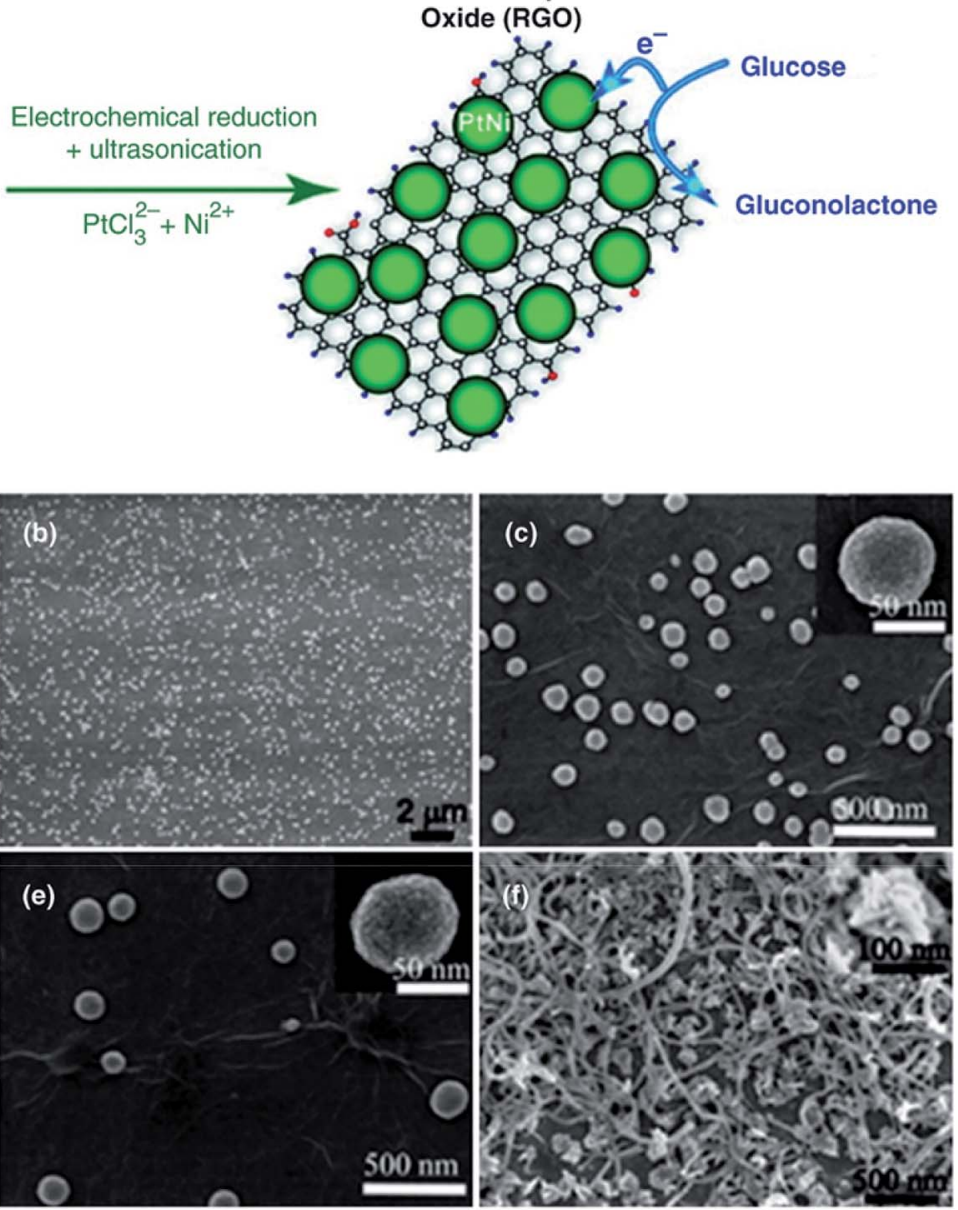

(D)

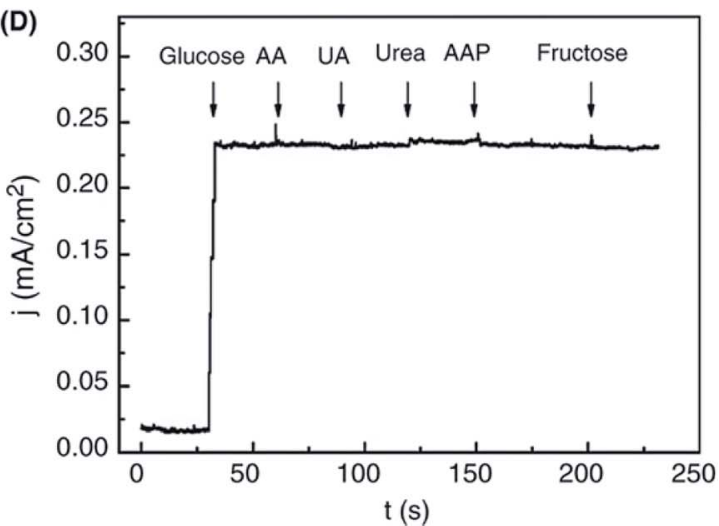

Fig. 9 (A) Schematic representation of the formation of PtNiNPs/rGO nanocomposites by electrochemical reduction method. (B) SEM images of the GO nanosheet, PtNiNPs/ERGO, PtNi NPs/CRGO nanocomposites and PtNiNPs/SWCNTs nanocomposites. (C) Amperometric response of PtNiNPs/ERGO/GCE after adding 0.5, 1.0, and $2.0 \mathrm{mM}$ glucose and the calibration curve (inset). (D) Influence of interfering bioanalytes including $0.5 \mathrm{mM}$ ascorbic acid (AA), $0.1 \mathrm{mM}$ uric acid (UA), $0.1 \mathrm{mM}$ urea, $0.5 \mathrm{mM} \mathrm{AAP,} \mathrm{and} 0.5 \mathrm{mM}$ fructose to $5.0 \mathrm{mM}$ glucose at the PtNiNPs/ERGO/GCE. Here, electrochemically and chemically reduced GO are ERGO and CRGO, respectively. [Reprinted with permission from ref. 229, H. Gao, F. Xiao, C. B. Ching and H. Duan, One-Step Electrochemical Synthesis of PtNi Nanoparticle-Graphene Nanocomposites for Nonenzymatic Amperometric Glucose Detection, ACS Appl. Mater. Interfaces, 2011, 3, 3049-3057. Copyright@ American Chemical Society.]

human blood serum and urine samples were achieved for the determination of glucose. Bimetallic nanoparticles supported on GO have also been widely employed to enhance the electrocatalytic activity and sensitivity of glucose biosensors. Gao et al. ${ }^{229}$ used sonication-assisted electrochemical synthesis to prepare bimetallic PtNiNPs/GR nanocomposite for nonenzymatic glucose detection (Fig. 9). The PtNiNPs are found to be uniformly distributed and supported on graphene surfaces 
compared with SWCNTs. The biosensor displayed a wide linear range of $0.5-35 \mathrm{mM}$, LOD of $10 \mu \mathrm{M}$, and a sensitivity of $20.42 \mu \mathrm{A} \mathrm{mM}^{-1} \mathrm{~cm}^{-2}$ with a negative potential of $-0.35 \mathrm{~V}$. The PtNiNPs/ERGO/GCE sensor also showed high selectivity for the determination of glucose against interfering bioanalytes including AA, UA, urea, AAP, and fructose, and recoveries of $91.3 \%$ to $108.2 \%$ for glucose detection in human urine samples. Sheng et al. ${ }^{230}$ reported a NiCoNPs/polypyrrole/rGO nanocomposite for nonenzymatic glucose sensing. The designed nonenzymatic glucose sensor displayed a sensitivity

\section{(A)}
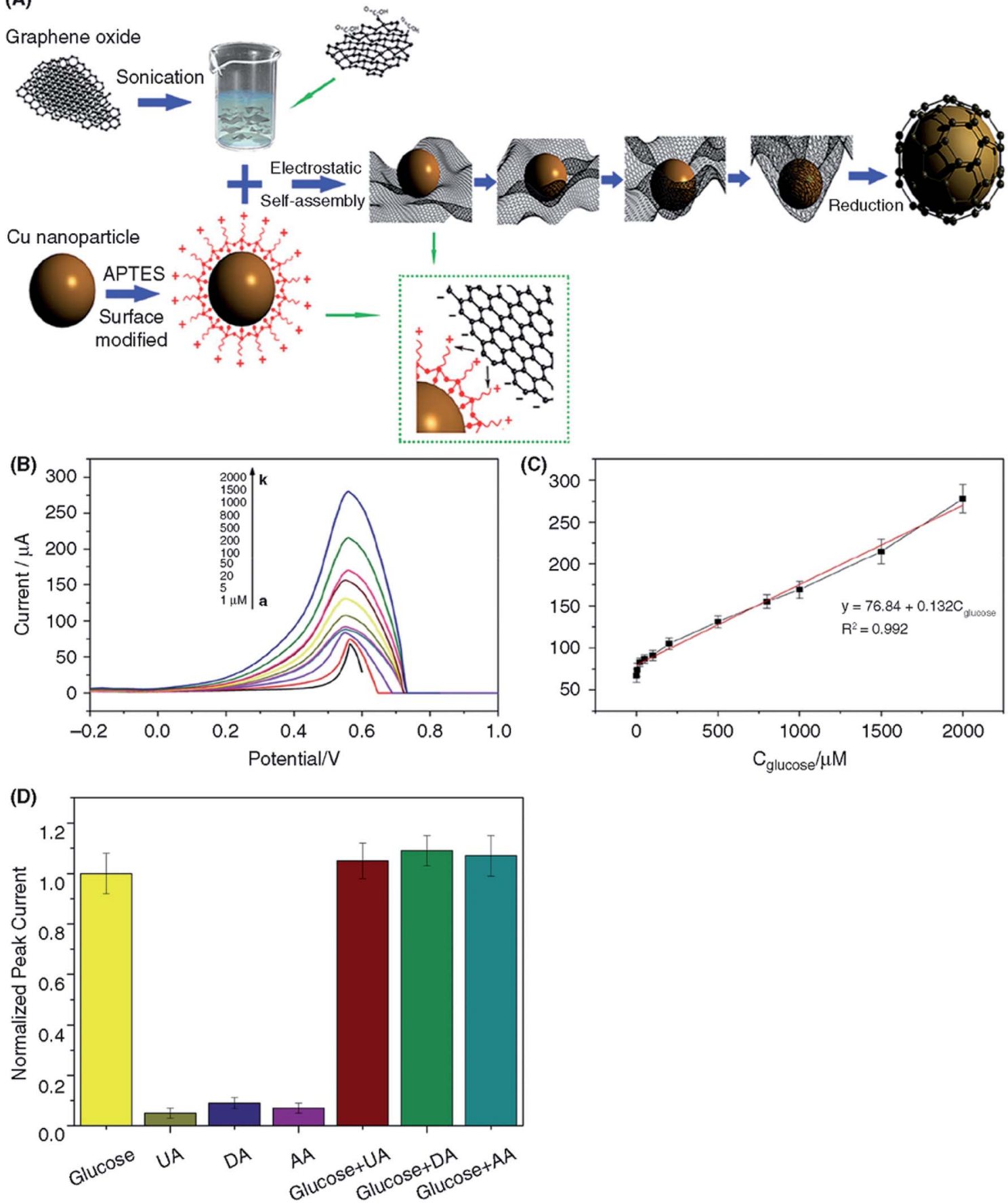

Fig. 10 (A) Schematic illustration of the preparation of graphene-encapsulated CuNPs. (B) DPV responses of graphene-encapsulated CuNPs composites at different concentrations (from a to k: $1-2000 \mu \mathrm{M}$ ) of glucose in $0.1 \mathrm{M} \mathrm{NaOH}$. (C) Linear relationship between the peak current and the glucose concentration. (D) Normalized DPV peak current of the CuNPs(arGO composites with the addition of $1.0 \mathrm{mM}$ glucose as the control and in the presence of $2.0 \mathrm{mM}$ uric acid (UA), $2.0 \mathrm{mM}$ dopamine (DA), and $2.0 \mathrm{mM}$ ascorbic acid (AA) as interferents and their corresponding mixtures. The CuNPs@rGO composite-based sensor shows high selectivity for detecting glucose. [Reprinted with permission from ref. 279, $Q$. Zhang, Q. Luo, Z. Qin, L. Liu, Z. Wu, B. Shen and W. Hu, Self-Assembly of Graphene-Encapsulated Cu Composites for Nonenzymatic Glucose Sensing, ACS Omega, 2018, 3, 3420-3428. Copyright@ American Chemical Society.] 
of $153.5 \mu \mathrm{A} \mathrm{mM} \mathrm{m}^{-1} \mathrm{~cm}^{-2}$, a wide linear range of $0.5 \mu \mathrm{M}$ to 4.1 $\mathrm{mM}$, and an LOD of $0.17 \mu \mathrm{M}$ for the glucose detection.

In addition to metal/graphene hybrid nanostructures, copper $(\mathrm{Cu})$, and nickel $(\mathrm{Ni})$ oxide nanostructures have been widely studied in the development of nonenzymatic electrochemical glucose sensors due to their high electrochemical activity and stability. Song et $a .^{209}$ demonstrated a nonenzymatic glucose biosensor based on $\mathrm{CuO}$ nanoparticles (CuONPs) supported on GO nanosheet with a high sensitivity of $262.52 \mu \mathrm{A} \mathrm{mM}^{-1} \mathrm{~cm}^{-2}$ and a wide linear range between $2.79 \mu \mathrm{M}$ and $2.03 \mathrm{mM}$ with an LOD of $0.69 \mu \mathrm{M}$ under $0.7 \mathrm{~V}$ detection potential. In the real human serum, the CuONPs/GO sensor also showed a similar broad linear range of $2.55 \mu \mathrm{M}$ to $0.5 \mathrm{mM}$ with a sensitivity of $285.38 \mu \mathrm{A} \mathrm{mM} M^{-1} \mathrm{~cm}^{-2}$. The sensor also displayed selectivity toward glucose against AA and UA interferents and good stability at room temperature as the sensitivity was found to decrease by $12.5 \%$ after 4 weeks. Wang et al. ${ }^{257}$ prepared a Pt$\mathrm{NiO}$ array/rGO nanocomposite-based biosensor with a sensitivity of $832.95 \mu \mathrm{A} \mathrm{mM}^{-1} \mathrm{~cm}^{-2}$, a linear range of $0.008-14.5 \mathrm{mM}$, and an LOD of $2.67 \mu \mathrm{M}$. The enhanced performance was ascribed to the strong catalytic activity of the metal-metal oxide (Pt-NiO) combined with rGO, as well as the high porosity of the nanocomposite material. Moreover, graphene functionalized with metal oxide nanoparticles such as nitrogen-doped $\mathrm{rGO} /$ $\mathrm{Mn}_{3} \mathrm{O}_{4} \mathrm{NPs}^{253}$ or GO hydrogels $/ \mathrm{Co}_{3} \mathrm{O}_{4}$ nanoflowers ${ }^{281}$ has been applied to the fabrication of biosensors with improved

(A)
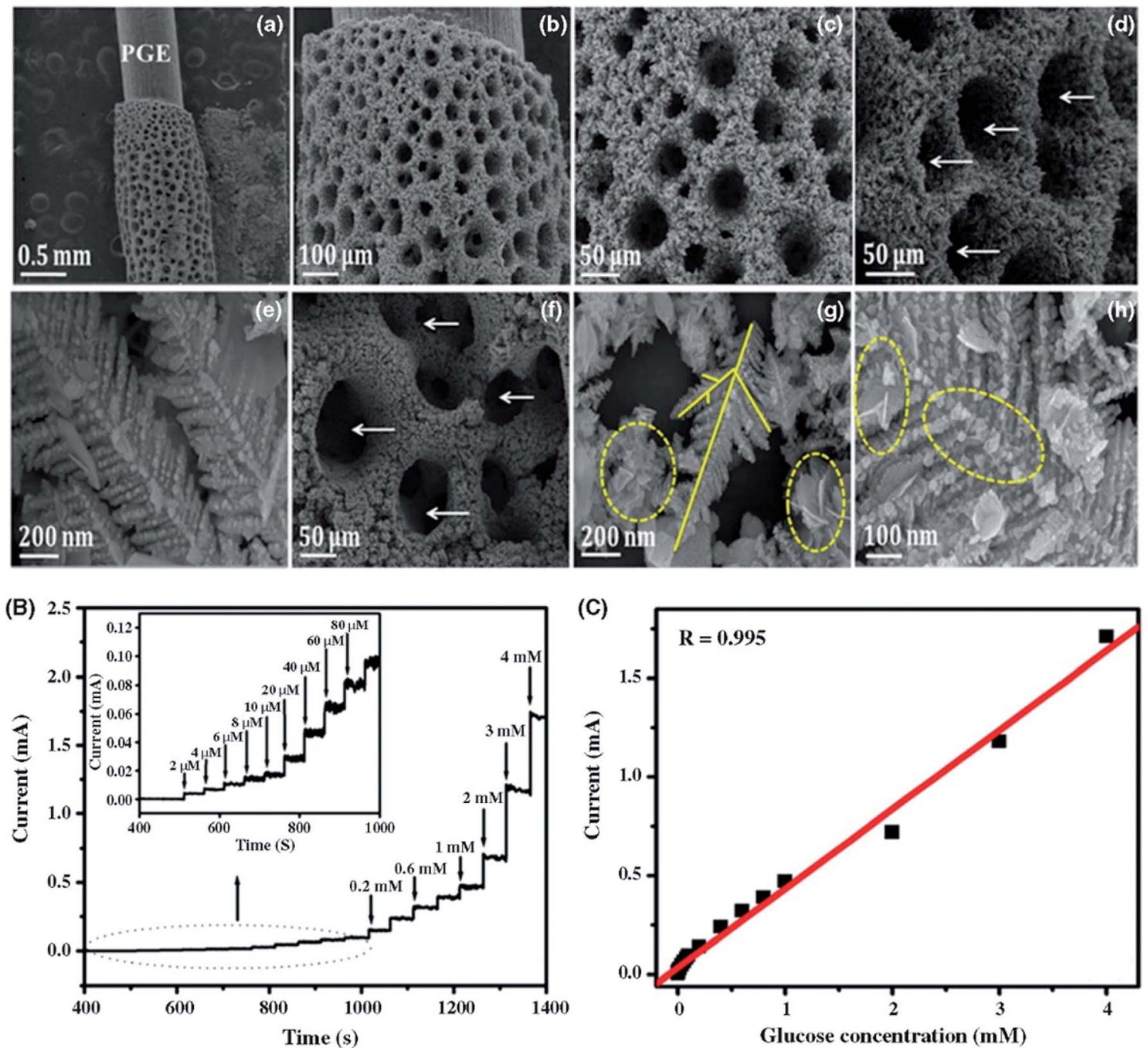

Fig. 11 Structural characterization of $\mathrm{Cu}-\mathrm{Co} / \mathrm{rGO}$ nanostructures on a pencil graphite electrode (PGE). (A) SEM images of Cu/PGE (a-C), Cu$\mathrm{Co} / \mathrm{PGE}(\mathrm{d}$ and $\mathrm{e})$, and $\mathrm{Cu}-\mathrm{Co} / \mathrm{rGO} / \mathrm{PGE}(\mathrm{f}-\mathrm{h})$. White arrows indicate interspaces with voids, and yellow solid and dotted lines indicate the primary and secondary trunks of dendrites and rGO, respectively. (B) Glucose-sensing performance of the Cu-Co/rGO-PGE-modified electrode. (a) Amperometric response after the successive addition of glucose at $0.4 \mathrm{~V} \mathrm{vs}$. Ag/AgCl. (Inset) Amperometric response of Cu-Co/rGO/ PGE toward low glucose concentration ranges between 1-100 $\mu \mathrm{M}$. (C) Calibration plot of $\mathrm{Cu}-\mathrm{Co} / \mathrm{rGO} / \mathrm{PGE}$ amperometric responses with respect to glucose concentration. [Reprinted with permission from ref. 277, K. J. Babu, S. Sheet, Y. S. Lee and G. G. Kumar, Three-Dimensional Dendrite $\mathrm{Cu}-\mathrm{Co} /$ Reduced Graphene Oxide Architectures on a Disposable Pencil Graphite Electrode as an Electrochemical Sensor for Nonenzymatic Glucose Detection, ACS Sustainable Chem. Eng., 2018, 6, 1909-1918. Copyright@ American Chemical Society.] 
sensitivity and selectivity for the electrochemical detection of glucose. Dong et al. ${ }^{232}$ designed cobalt oxide nanowires $\left(\mathrm{Co}_{3} \mathrm{O}_{4}\right.$ NWs)/3D-graphene foam with high porosity for nonenzymatic electrochemical glucose detection. The $3 \mathrm{D}$ graphene $/ \mathrm{Co}_{3} \mathrm{O}_{4}$ nanocomposite sensor exhibited a sensitivity of $3.39 \mathrm{~mA} \mathrm{mM}^{-1}$ $\mathrm{cm}^{-2}$, an LOD of $<25 \mathrm{nM}(\mathrm{S} / \mathrm{N}=8.5)$ and anti-interference ability against AA, UA and proteins, which was attributed to the unique $3 \mathrm{D}$ porous nanostructures. Rao et al. ${ }^{270}$ reported an enzyme-free electrochemical glucose sensor based on a $\mathrm{NiCo}_{2} \mathrm{O}_{4} /$ nitrogen-doped $\mathrm{rGO} /$ ionic liquid ternary composite. The resulting sensor showed high electrocatalytic activity toward glucose with a sensitivity of $3.76 \mathrm{~mA} \mathrm{mM}^{-1} \mathrm{~cm}^{-2}$, a wide linear response from $0.001 \mathrm{mM}$ to $4.555 \mathrm{mM}$ and an LOD of 0.18 $\mu \mathrm{M}$ under a potential of $0.5 \mathrm{~V}$. The enhanced sensitivity was attributed to the synergistic effect of the $\mathrm{NiCo}_{2} \mathrm{O}_{4}, \mathrm{GO}$ and ionic liquid. Moreover, the N-doping of rGO further expedited the electron transfer of the ionic liquid.

An approach to synthesize of rGO-encapsulated CuNPs using an electrostatic self-assembly method was reported by Zhang et al., ${ }^{279}$ as shown in Fig. 10A. The obtained $\mathrm{Cu}$ core and rGO shell nanocomposite exhibited high stability compared to bare CuNPs because CuNPs in the composite were protected from oxidation by the rGO shell. The differential pulse voltammetry (DPV) response of glucose in 0.1-M NaOH was measured in the 1-2000 $\mu \mathrm{M}$ concentration range and showed a linear relationship between the peak current and the glucose concentration. Moreover, the resulting CuNPs/rGO nanocomposite exhibited high electrocatalytic activity with a wide linear range from $1 \mu \mathrm{M}$ to $2 \mathrm{mM}$, a sensitivity of $150 \mu \mathrm{AmM}^{-1}$ $\mathrm{cm}^{-2}$, and an LOD of $0.34 \mu \mathrm{M}$ in addition to good reproducibility and selectivity for the oxidation of glucose (Fig. 10B). The selectivity of the CuNPs/rGO core-shell nanocomposite-based sensor for glucose detection was examined by measuring the normalized DPV peak current with addition of 1.0-mM glucose alone as the control and then in the presence of 2.0-mM UA, 2.0$\mathrm{mM}$ DA and 2.0-mM AA as interfering analytes and the corresponding mixtures with glucose (Fig. 10C bottom). The resulting DPV peak current was 5.4, 8.9 and $6.3 \%$ for UA, DA, and AA compared with $1.0-\mathrm{mM}$ glucose in $0.1-\mathrm{M} \mathrm{NaOH}$, respectively, showing high selectivity for glucose detection in the presence of each interferent. Babu et al. ${ }^{277}$ fabricated nonenzymatic glucose sensors using 3D copper-cobalt/rGO (Cu-Co/rGO) nanostructures which were electrochemically deposited over a pencil graphite electrode (PGE) (Fig. 11A). The Cu-Co/rGO/PGE sensor displayed high glucose detection with enhanced sensitivity (240

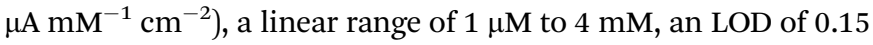
$\mu \mathrm{M}$ (Fig. 11B) as well as anti-interfering property against AA, UA, DA, citric acid (CA), NaCl, urea, acetaminophen (AP) and chloride poisoning resistance. The high analytical performance of the biosensor was associated with the 3D hierarchical dendrite-like

Table 3 Cholesterol biosensors developed from graphene nanocomposite-based modified electrodes and their linear detection range, sensitivity and limit of detection ${ }^{a}$

\begin{tabular}{|c|c|c|c|c|c|}
\hline Graphene-based nanocomposites & Linear range & 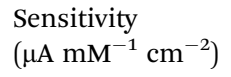 & Limit of detection (LOD) & $K_{\mathrm{m}}(\mathrm{mM})$ & Ref. \\
\hline GR-PtNPs hybrid & Up to $12 \mathrm{mM}$ & 2.07 & $0.2 \mu \mathrm{M}$ & 5.0 & 287 \\
\hline Cholesterol esterase-functionalized GR & $50-300 \mu \mathrm{M}$ & 443.25 & $15 \mu \mathrm{M}$ & - & 288 \\
\hline ChOx/hemin-GR nanosheets & $0.17-1120 \mu \mathrm{M}$ & - & $0.06 \mu \mathrm{M}$ & - & 289 \\
\hline $\mathrm{CeO}_{2} / \mathrm{GR}$ composites & $12 \mu \mathrm{M}$ to $7.2 \mathrm{mM}$ & - & $4 \mu \mathrm{M}$ & - & 290 \\
\hline $\mathrm{TiO}_{2}-\mathrm{GR}-\mathrm{Pt}-\mathrm{Pd}$ hybrid & $0.05 \mu \mathrm{M}$ to $5.9 \mathrm{mM}$ & - & $0.017 \mu \mathrm{M}$ & 0.21 & 291 \\
\hline Pd-Pt NPs/GR nanocomposite & $2.2 \mu \mathrm{M}$ to $0.52 \mathrm{mM}$ & - & $0.75 \mu \mathrm{M}$ & 0.11 & 292 \\
\hline MWCNTs/GO/thionine-AuNPs & $0.15-828 \mu \mathrm{M}$ & - & $50 \mathrm{nM}$ & - & 293 \\
\hline GR/PVP/PANI nanocomposite & $50 \mu \mathrm{M}$ to $10 \mathrm{mM}$ & - & $1 \mu \mathrm{M}$ & - & 294 \\
\hline Self-assembled GR & $0.05-0.35 \mathrm{mM}$ & 124.57 & $0.05 \mu \mathrm{M}$ & 1.22 & 295 \\
\hline rGO/dendritic Pd nanostructure & $0.005-0.014 \mathrm{mM}$ & - & $0.05 \mu \mathrm{M}$ & - & 296 \\
\hline $\mathrm{GR} / \mathrm{IL}$ & $0.25-215 \mu \mathrm{M}$ & 4163 & $0.5 \mathrm{mM}$ & 2.32 & 297 \\
\hline CS/GR nanocomposites & $0.005-1.0 \mathrm{mM}$ & - & $0.715 \mu \mathrm{M}$ & 17.39 & 298 \\
\hline MWCNTs/GO/AuNPs & $0.15-828 \mu \mathrm{M}$ & - & $0.05 \mu \mathrm{M}$ & - & 293 \\
\hline ZnO/AgNWs/GR-CS nanocomposites & $6.5 \mu \mathrm{M}$ to $10 \mathrm{mM}$ & 9.2 & $0.287 \mu \mathrm{M}$ & 0.295 & 299 \\
\hline $\mathrm{MB} /$ calix[6]arene-functionalized GR & $0.50-50.00 \mu \mathrm{M}$ & - & $0.20 \mu \mathrm{M}$ & - & 300 \\
\hline Ag nanowires/CS/GO nanocomposite & $\mathrm{Up}$ to $400 \mathrm{mg} \mathrm{dL}^{-1}$ & 13.628 & $0.427 \mathrm{mg} \mathrm{dL}^{-1}$ & 2.813 & 301 \\
\hline CdTe QD/MWCNTs/rGO nanoribbons & $1 \mu \mathrm{M}$ to $1 \mathrm{mM}$ & - & $0.33 \mu \mathrm{M}$ & - & 302 \\
\hline $\mathrm{SiO}_{2} \mathrm{NPs} / \mathrm{rGO}$ hybrid composite & $2.6-15.5 \mathrm{mM}$ & 11.1 & $1.3 \mathrm{mM}$ & 0.49 & 303 \\
\hline $\mathrm{NiO}$ flowers/CVD-grown GR & $2-40 \mu \mathrm{M}$ & - & $0.13 \mu \mathrm{M}$ & - & 304 \\
\hline PSS/polymeric IL/GR nanocomposite & $0.01-10.4 \mathrm{mM}$ & - & $3.5 \mu \mathrm{M}$ & - & 305 \\
\hline Polypyrrole/rGO/ChOx composite & $0.01-6 \mathrm{mM}$ & 1095.3 & $3.78 \mu \mathrm{M}$ & - & 306 \\
\hline GO-SH/AuNPs composite & $0.05-11.45 \mathrm{mM}$ & 273 & $0.2 \mathrm{nM}$ & 0.18 & 26 \\
\hline $\mathrm{TiO}_{2}$ nanowires/3D GR nanostacks & $0.05-8.0 \mathrm{mM}$ & 3.82 & $6 \mu \mathrm{M}$ & - & 307 \\
\hline rGO nanosheet/PEI hybrid & $2.5-25 \mu \mathrm{M}$ & - & $0.5 \mu \mathrm{M}$ & - & 181 \\
\hline $\mathrm{CeO}_{2} / \mathrm{N}$-doped GR composite & $4.0 \mu \mathrm{M}$ to $5 \mathrm{mM}$ & - & $1.33 \mu \mathrm{M}$ & - & 308 \\
\hline $\mathrm{Ag} /$ AuNPs-GO & $0.01-5000 \mu \mathrm{g} \mathrm{mL}^{-1}$ & 0.084 & $0.001 \mu \mathrm{g} \mathrm{mL}^{-1}$ & - & 309 \\
\hline
\end{tabular}

${ }^{a}$ Abbreviations used: CS: chitosan, ILs: ionic liquids, SPE: screen-printed electrode, MB: methylene blue, GO-SH: thiol-functionalized graphene oxide. 
architecture having controlled pore diameter, large surface area and high conductivity for rapid electron transfer.

\subsection{Cholesterol biosensors}

Cholesterol and its fatty acid esters are among the main constituents of mammalian cell membranes and are precursors of extra biological materials including steroid hormones and bile acid. Accurate determination of the cholesterol content in blood is very important for the diagnosis and prevention of various heart diseases. Excessive blood cholesterol is considered to be a severe threat for cardiovascular diseases such as atherosclerosis, hypertension, myocardial infarction, and cardiopathy. ${ }^{282}$ The accurate estimation of blood cholesterol is essential for the assessment of atherosclerosis and other lipidrelated disorders and to determine the risk of thrombosis and myocardial infarction. ${ }^{283}$ A normal level of total cholesterol in the human blood is less than $5.17 \mathrm{mM}$, but this value can differ based on individuals and their age, body-weight, and gender. The higher limit of total cholesterol in human blood is in the range of 5.17-6.18 $\mathrm{mM}$ and cholesterol in values above $6.21 \mathrm{mM}$ are considered high. ${ }^{283}$ Thus, biosensors for the precise detection of cholesterol in the human blood samples are critical for healthcare. Multiple analytical methods including electrochemical sensing have been developed for sensitive and selective monitoring of cholesterol.
(A)

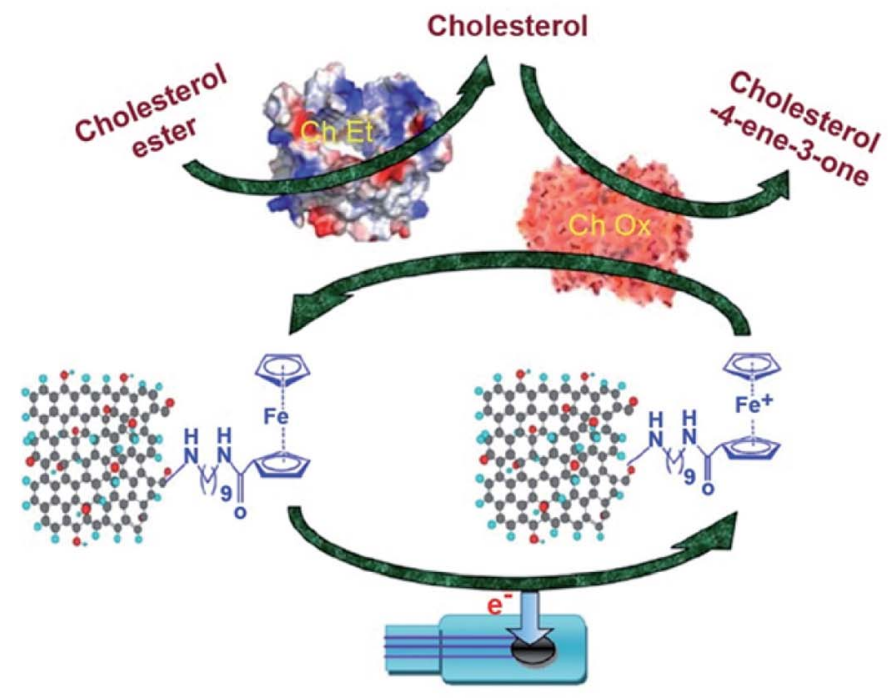

(B)

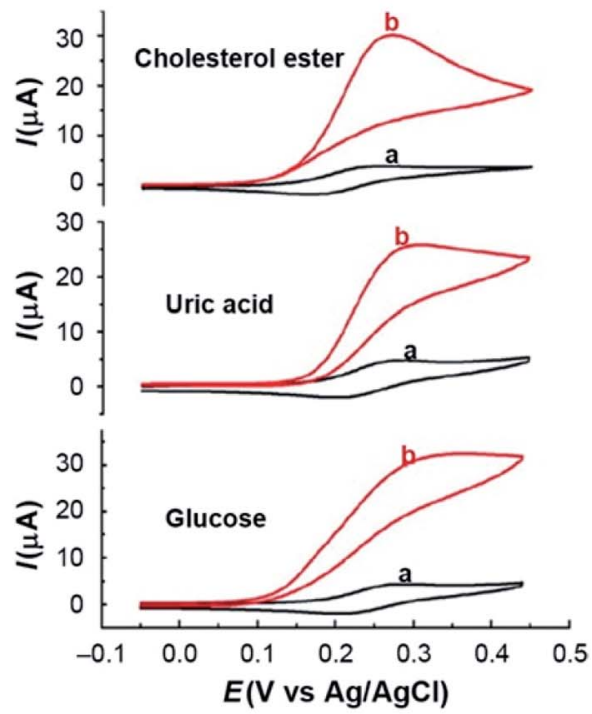

(C)
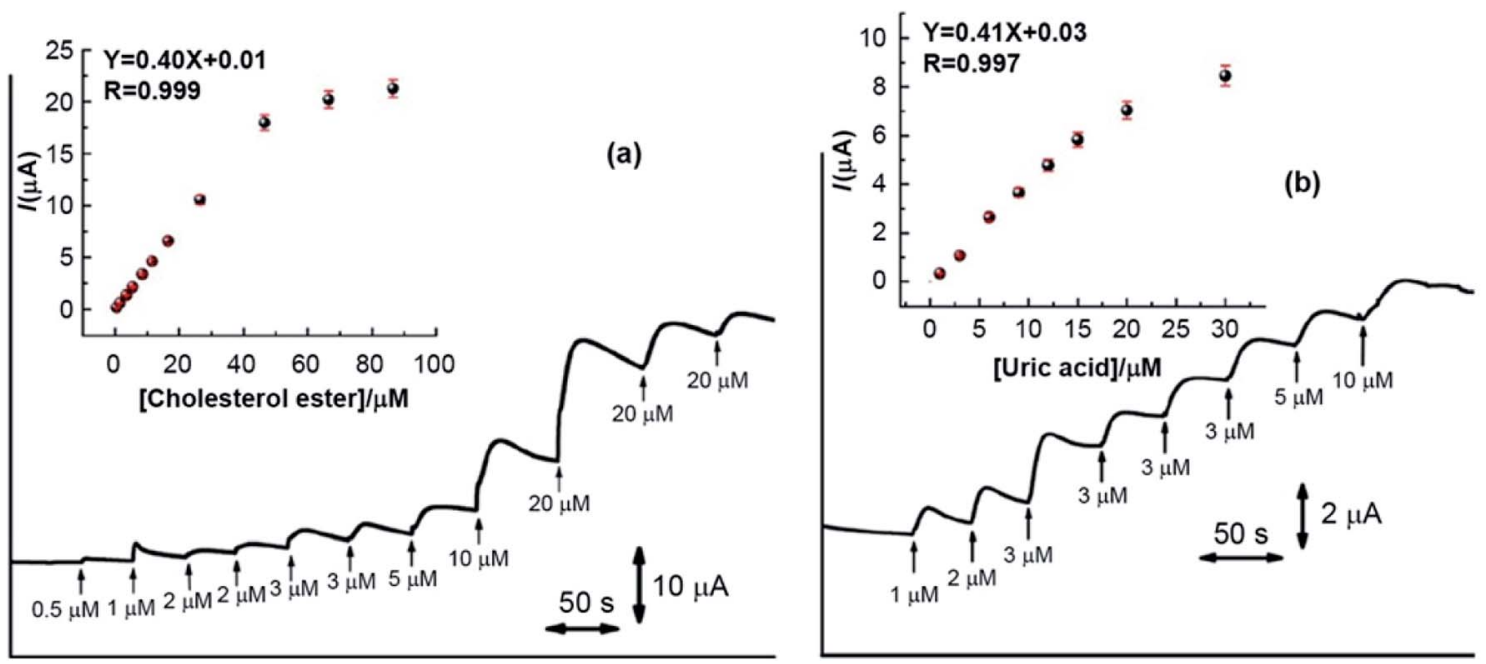

Fig. 12 (A) Scheme illustrating the biosensor based on Fc-GO on SPE for the detection of cholesterol. (B) CV response of the Fc-GO based biosensors in the (a) absence and (b) presence of cholesterol ester, uric acid and glucose. (C) Amperometric response of the Fc-GO based biosensor for detecting cholesterol and uric acid. Arrows indicate the aliquots of (a) cholesterol ester and (b) uric acids, added at regular intervals into PBS ( $\mathrm{pH}$ 7.2). Insets show the corresponding calibration plots. [Reprinted with permission from ref. 284, R. S. Dey and C. R. Raj, RedoxFunctionalized Graphene Oxide Architecture for the Development of Amperometric Biosensing Platform, ACS Appl. Mater. Interfaces, 2013, 5, 4791-4798. Copyright@ American Chemical Society.] 
Similar to electrochemical glucose biosensing, the sensing of cholesterol can be accomplished by two approaches. (i) The enzymatic reaction of cholesterol with cholesterol oxidase (ChOx), i.e., the concentration of cholesterol can be estimated by monitoring the concentration of hydrogen peroxide $\left(\mathrm{H}_{2} \mathrm{O}_{2}\right)$ generated through the enzymatic reaction. The ChOx enzyme is most commonly used for the detection of cholesterol because it easily catalyzes the oxidation of cholesterol to cholest-4-3-one and $\mathrm{H}_{2} \mathrm{O}_{2} \cdot{ }^{284}$ (ii) Enzyme-free direct biocatalytic oxidation of cholesterol. ${ }^{285}$ Various nanomaterials, such as metal and metal oxide nanoparticles, CNTs, GR, and polymer nanocomposites, have been successfully applied to develop cholesterol biosensors. ${ }^{286}$ Among the nanomaterials developed, graphene holds a great promise for the detection of cholesterol with highly enhanced sensitivity and selectivity due to its fast electron transport process, good mechanical properties and biocompatibility. ${ }^{286}$ Graphene-based cholesterol biosensors are summarized in Table 3.

3.2.1 Enzymatic detection of cholesterol. Graphene-based nanocomposites have been extensively investigated for the fabrication of enzyme-based cholesterol sensors. For example, Dey et al. ${ }^{284}$ fabricated a redox enzyme-functionalized GO based biosensor by covalently conjugated the ferrocene (Fc) redox units to the GO backbone through diamine in order to facilitate the enhanced electron transfer rate. Fig. 12 shows the schematic of Fc-GO based biosensor the detection of cholesterol and mechanism of the enzymatic reaction, $\mathrm{CV}$ response of the biosensors in the absence and presence of cholesterol ester, UA and glucose and amperometric response of the cholesterol biosensor with subsequent addition of cholesterol at a fixed potential of $0.3 \mathrm{~V}$. The biosensor showed a linear response ranging from 0.5 to $46.5 \mu \mathrm{M}$ with a sensitivity of $5.71 \mu \mathrm{A} \mu \mathrm{M}^{-1}$ $\mathrm{cm}^{-2}$ and an LOD of $0.1 \mu \mathrm{M}$ with a response time of $4 \mathrm{~s}$ for the detection of cholesterol. The Fc-GO based biosensors also exhibited a linear range of 0.001 to $0.02 \mathrm{mM}$ and 0.2 to $19.7 \mathrm{mM}$, sensitivities of $5.85 \mu \mathrm{A} \mu \mathrm{M}^{-1} \mathrm{~cm}^{-2}$ and $20.71 \mu \mathrm{A}$ $\mathrm{mM}^{-1} \mathrm{~cm}^{-2}$ with LODs of $0.1 \mu \mathrm{M}$ and $1 \mu \mathrm{M}$ for detecting UA and glucose, respectively. The clinical application of the Fc-GO biosensors was demonstrated by detecting cholesterol ester, UA and glucose in human serum samples. Li et al. ${ }^{298}$ showed a cholesterol biosensor based on a CS biopolymer/GR nanocomposite. The availability of amine $\left(\mathrm{NH}_{3} / \mathrm{NH}_{2}\right)$ and hydroxyl $(\mathrm{OH})$ side groups in $\mathrm{CS}$ biopolymer not only facilitates the stable conjugation of ChOx enzyme but also increased the DET between the enzyme and the electrode surface. The ChOx/CS-GR based biosensor exhibited a linear range of 0.005 to $1.0 \mathrm{mM}$ with an LOD of $0.715 \mu \mathrm{M}$ for cholesterol detection and selectivity against interfering bionalytes such as DA, AA, UA, and glucose. Ruecha et al. ${ }^{294}$ used graphene/poly(vinylpyrrolidone)/ poly(aniline) (GR/PVP/PANI) nanocomposites to fabricate cholesterol biosensors. The sensor showed a linear range from $50 \mu \mathrm{M}$ to $10 \mathrm{mM}$ with an LOD of $1 \mu \mathrm{M}$ and clinical use for cholesterol detection in human serum samples.

Combining the noble metal nanoparticles with graphene has been shown to significantly enhance the electrocatalytic properties and subsequent detection of cholesterol. ${ }^{161,296,310} \mathrm{Pt}$ and Pd nanoparticles exhibit superior electrocatalytic activity to other noble metal nanoparticles. The fabrication of biosensor using PtNPs/GR ${ }^{287}$ and PdNPs/rGO ${ }^{296}$ hybrid nanostructures has been reported for the electrochemical detection of cholesterol. The PdNPs/rGO hybrid nanostructure-based biosensor displayed an increased sensitivity of $5.12 \mu \mathrm{A} \mu \mathrm{M}^{-1} \mathrm{~cm}^{-2}$ and an LOD of $0.05 \mu \mathrm{M}$, due to the high electrocatalytic activity of dendritic Pd nanostructures supported on rGO surface. Cao et $a l .{ }^{292}$ developed a cholesterol biosensor based on bimetallic PtPd nanoparticles supported on CS-GR-functionalized GCE. Owing to the synergetic catalytic activity of $\mathrm{Pt}$ and $\mathrm{Pd}$ nanoparticles, the fabricated PtPdNPs-CS-GR biosensor showed improvement in the detection range from $2.2 \times 10^{-6}$ to $5.2 \times 10^{-4} \mathrm{M}$, and the LOD was $0.75 \mu \mathrm{M}$ because of accelerated DET between the redox enzyme and the electrode surface. Metal oxide nanostructures supported on GR have also been used to develop cholesterol sensors. Komathi et $a l^{307}$ used titanium dioxide $\left(\mathrm{TiO}_{2}\right)$ nanowires supported on $3 \mathrm{D}$ graphene nanostacks for the fabrication of dual-mode cholesterol biosensors. The biosensor exhibited a sensitivity of $3.82 \mu \mathrm{A} \mathrm{mM} \mathrm{mm}^{-1} \mathrm{~cm}^{-2}$ and an LOD of $6 \mu \mathrm{M}$. The sensing performance is slightly lower than that of previous metal nanoparticles supported on GO due to the lower electrocatalytic activity and conductivity of $\mathrm{TiO}_{2}$ nanostructures.

3.2.2 Nonenzymatic detection of cholesterol. The stability of enzymes such as GOx and ChOx is easily affected by factors such as temperature, humidity, $\mathrm{pH}$, etc. Nonenzymatic detection has numerous advantages over the enzyme-based approach including simple fabrication process, reproducibility, stability, low cost and freedom from oxygen limitation. ${ }^{285}$ Therefore, significant research efforts have been focused on developing biosensors for nonenzymatic cholesterol detection. Moreover, owing to the strong catalytic ability and facile attachment of metal NPs to the electrode surface, GR-based nanocomposites have been extensively investigated as electrode materials. ${ }^{75}$

A nonenzymatic cholesterol sensor based on $\beta$-cyclodextrin $(\beta-C D)$-modified chemically converted graphene was reported by Agnihotri et al. ${ }^{311}$ Methylene blue (MB) was used as a redox indicator in complex to form a cholesterol-sensing matrix. The resulting sensor showed an LOD as low as $1 \mu \mathrm{M}$. Lakshmi et $a{ }^{3 \mathbf{3 1 2}}$ developed a biosensor using polyaniline nanofiber/ graphene microflowers for the detection of cholesterol, achieving an enhanced sensitivity of $0.101 \mu \mathrm{A} \mathrm{mg}^{-1} \mathrm{dL} \mathrm{cm}^{-2}$

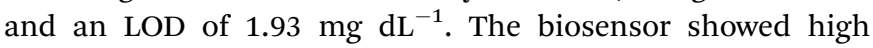
selectivity toward cholesterol detection against interfering bioanalytes including AA, citric acid, urea, glucose, and cysteine. Alexander et $a .^{313}$ reported a nonenzymatic cholesterol sensor fabricated from a modified GO-based molecularly imprinted polymer (MIP). The GO-MIP based sensor showed high sensitivity with an LOD of $0.1 \mathrm{nM}$ and a fast response time of $2 \mathrm{~min}$. Rengaraj et al. ${ }^{\mathbf{3 0 4}}$ developed a nonenzymatic cholesterol biosensor using nickel oxide (NiO)/graphene composite. The developed sensor displayed a sensitivity of $40.6 \mathrm{~mA} \mu \mathrm{M}^{-1} \mathrm{~cm}^{-2}$, an LOD of $0.13 \mathrm{mM}$, and a fast response time of $5 \mathrm{~s}$. Moreover, the biosensor showed the ability to detect the cholesterol content in a milk sample, reproducibility and long-term stability. 
Table 4 Hydrogen peroxide $\left(\mathrm{H}_{2} \mathrm{O}_{2}\right)$ biosensors fabricated using graphene nanocomposite-based modified electrodes ${ }^{a}$

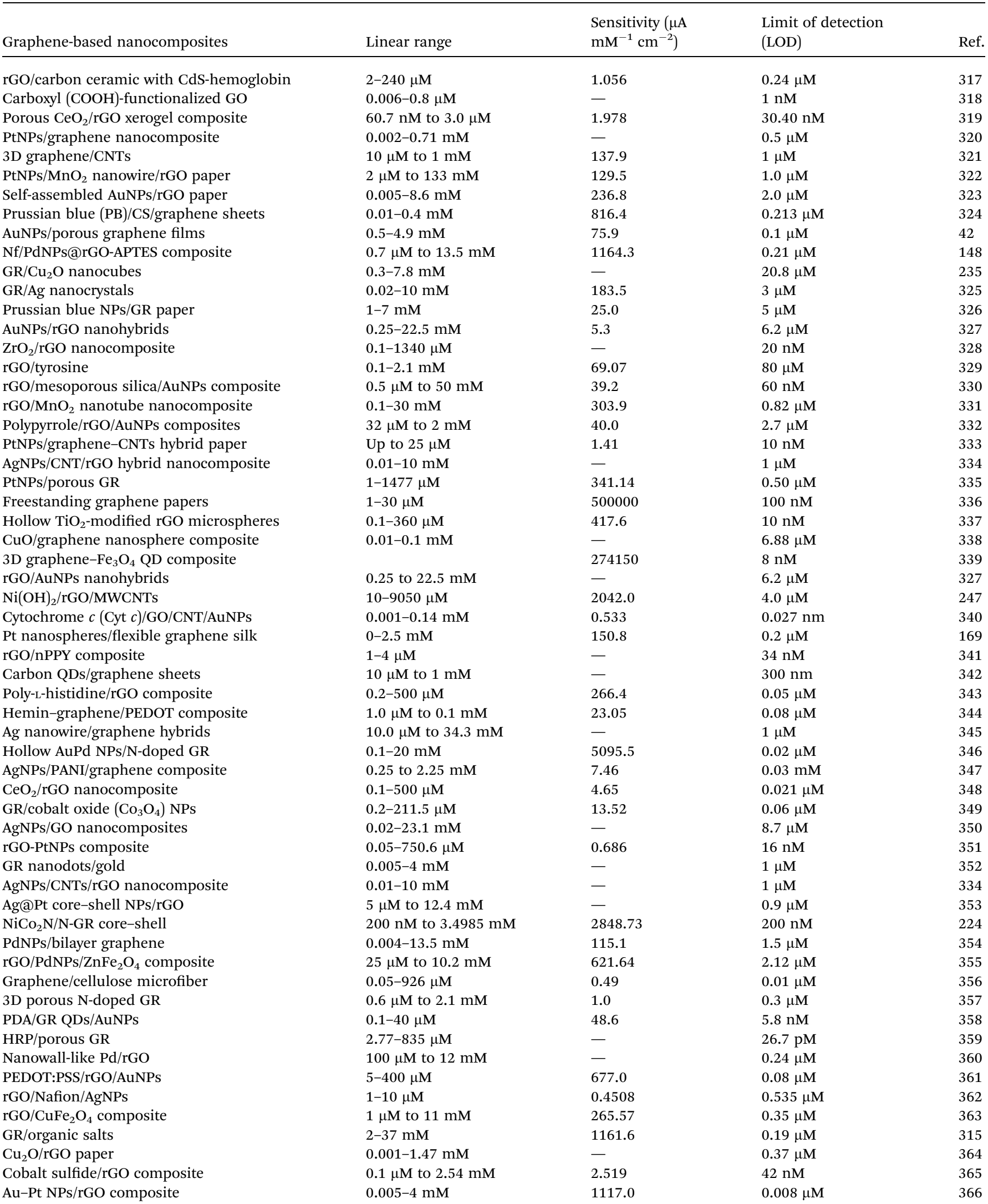

${ }^{a}$ Abbreviations used: ChOx: cholesterol oxidase, PVP: polyvinylpyrrolidone, PANI: polyaniline, MWCNTs: multiwalled carbon nanotubes, PEI: polyethyleneimine, PEDOT: poly(3,4-ethylenedioxythiophene), HRP: horseradish peroxidase. 


\subsection{Hydrogen peroxide $\left(\mathrm{H}_{2} \mathrm{O}_{2}\right)$ biosensors}

Hydrogen peroxide $\left(\mathrm{H}_{2} \mathrm{O}_{2}\right)$ sensors have attracted much research interest because $\mathrm{H}_{2} \mathrm{O}_{2}$ is not only a byproduct of numerous enzymatic reactions but also plays an important role as a mediator in clinical, food, pharmaceutical and environmental monitoring applications due to its superior oxidizing and reducing properties. ${ }^{66}$ In living organisms, $\mathrm{H}_{2} \mathrm{O}_{2}$ is a byproduct of enzymatic reactions involving different oxidases and plays an essential role in regulating various biological signaling transduction processes, and enzymatic biosensing appears feasible. Unfortunately, the overpotentials (300-600 mV against a reference electrode) applied to the working electrode usually generate a significant amount of interference. ${ }^{314}$ Thus, intensive investigations have focused on the development of $\mathrm{H}_{2} \mathrm{O}_{2}$ sensors with reduced oxidation/reduction overpotentials. ${ }^{66}$

Graphene-based nanomaterials have attracted extensive interest for sensing $\mathrm{H}_{2} \mathrm{O}_{2}$ because of their high specific surface area, good mechanical strength and superior electric conductivity ${ }^{66}$ Furthermore, the integration of metal NPs onto the surface of graphene could significantly improve the active surface area to enable adsorption and speed up electron transfer processes of analyte between the electrode and detection molecules. Table 4 lists the $\mathrm{H}_{2} \mathrm{O}_{2}$ biosensors developed from graphene-based nanocomposites. Initially, Zhou et al. ${ }^{\mathbf{1 3 1}}$ demonstrated a modified electrode with chemically reduced GO, showing a much lower onset potential of $\mathrm{H}_{2} \mathrm{O}_{2}$ oxidation/ reduction at $0.2 / 0.1 \mathrm{~V}(v s$. $\mathrm{Ag} / \mathrm{AgCl})$ than with a graphitemodified electrode. The enhanced performance is ascribed to the high electrocatalytic activity and surface defects. Hou et $a l^{315}$ performed the organic salt-mediated preparation of pristine graphene and used it for the nonenzymatic electrochemical detection of $\mathrm{H}_{2} \mathrm{O}_{2}$. The biosensor exhibited improved electrocatalytic activity for $\mathrm{H}_{2} \mathrm{O}_{2}$ detection with a wide linear range from 2.0 to $437.0 \mu \mathrm{M}$ with LOD of $0.19 \mu \mathrm{M}$. Yu et al. ${ }^{316}$ prepared multilayer composite films of (phthalocyaninato)(porphyrinato)europium(III) triple-decker compound ( $\mathrm{Pc}) \mathrm{Eu}(\mathrm{Pc})$ $\left.\mathrm{Eu}[\text { trans-T(COOCH})_{2} \mathrm{PP}\right](1)$ and GO for the detection of $\mathrm{H}_{2} \mathrm{O}_{2}$. The combination of the high electrical conductivity and a large surface area of GO with the semiconducting triple-decker porphyrin compound in the indium tin oxide electrode enabled high $\mathrm{H}_{2} \mathrm{O}_{2}$-sensing activity because of the optimized triple-decker molecular packing on the GO surface. The tripledecker/GO sensor showed a sensitivity of $7.4 \mu \mathrm{A} \mathrm{mM^{-1 }}$ with a wide linear range of $0.05-1800 \mu \mathrm{M}$, an LOD of $0.017 \mu \mathrm{M}$, and a response time of $0.03 \mathrm{~s} \mu \mathrm{M}^{-1}$.

Noble metal nanoparticles such as $\mathrm{Ag}, \mathrm{Au}, \mathrm{Pt}, \mathrm{Pd}$, and $\mathrm{Cu}$ decorated on GO surface have attracted significant interest for developing electrochemical $\mathrm{H}_{2} \mathrm{O}_{2}$ sensors due to their unique catalytic properties. ${ }^{66,367,368}$ Guo et al. ${ }^{369}$ prepared $\mathrm{Pt}$ nanoparticle/graphene hybrid nanosheets through a microwave-assisted heating method and used it for the electrochemical sensing of $\mathrm{H}_{2} \mathrm{O}_{2}$. The modified electrode showed increased in electrochemical performance for the detection of $\mathrm{H}_{2} \mathrm{O}_{2}$ in the linear range of 1 to $500 \mu \mathrm{M}$ with a detection limit of $80 \mathrm{nM}$. Moreover, the sensor exhibited high selectivity for the detection of $\mathrm{H}_{2} \mathrm{O}_{2}$ in the presence of UA and AA interferents. $\mathrm{Ag}$ and $\mathrm{Au}$ nanoparticles exhibit high catalytic ability toward $\mathrm{H}_{2} \mathrm{O}_{2}$. Zhan et $a l .{ }^{370}$ demonstrated the hydrothermal synthesis of AgNPs and used them to decorate $3 \mathrm{D}$ graphene for a $\mathrm{H}_{2} \mathrm{O}_{2}$ sensor. Owing to the higher catalytic activity of AgNPs, higher conductivity and reactivity of the $3 \mathrm{D}$ graphene porous structure, the fabricated sensor showed a wide linear range between 0.03$16.21 \mathrm{mM}$ and an LOD of $14.9 \mu \mathrm{M}$. One-dimensional Ag nanostructures such as $\mathrm{Ag}$ nanorods $\mathrm{s}^{371}$ and nanowires ${ }^{345}$ were also decorated on graphene surface to design sensors with improved sensitivity for the detection of $\mathrm{H}_{2} \mathrm{O}_{2}$. In addition to metal nanoparticles, metal oxide nanostructures were also supported on graphene for fabricating $\mathrm{H}_{2} \mathrm{O}_{2}$ biosensors. Karthikeyan et al. ${ }^{363}$ developed copper ferrite $\left(\mathrm{CuFe}_{2} \mathrm{O}_{4}\right)$ supported rGO nanocomposite-based sensor which showed a high sensitivity of $265.57 \mu \mathrm{A} \mathrm{mM}^{-1} \mathrm{~cm}^{-2}$, a linear range of $1 \mu \mathrm{M}$ to $11 \mathrm{mM}$, and an LOD of $0.35 \mu \mathrm{M}$. Yusoff et al. ${ }^{362}$ developed a biosensor using rGO-Nafion@silver6 (rGO-Nf@Ag6) hybrid-modified GCEs for the detection of $\mathrm{H}_{2} \mathrm{O}_{2}$. The sensor exhibited high sensitivity $0.4508 \mu \mathrm{A} \mathrm{mM}^{-1}$, with an LOD of $0.535 \mu \mathrm{M}$ due to the coupling of rGO-Nf with AgNPs, which enhanced the performance due to more active surface area that enabling the interaction of analyte and the fast electron transfer. Chen et $a l^{372}$ developed a biosensor based on a PtNPs-carbon quantum dot (CQD)/ionic liquid-functionalized $\mathrm{GO}$ nanocomposite for detecting $\mathrm{H}_{2} \mathrm{O}_{2}$. The unique PtNPs-CQDs/IL-GO structure improved the electrocatalytic reduction of $\mathrm{H}_{2} \mathrm{O}_{2}$ and offered abundant active sites for involved electrochemical redox reactions. The fabricated sensor showed a linear range of 1 to $900 \mu \mathrm{M}$ and an LOD of $0.1 \mu \mathrm{M}$ for detecting $\mathrm{H}_{2} \mathrm{O}_{2}$. Sun et al. ${ }^{373}$ used PtNi nanowires on rGO for developing $\mathrm{H}_{2} \mathrm{O}_{2}$ sensor. The sensor showed with a wide linear range between $1 \mathrm{nM}$ to $5.3 \mathrm{mM}$ and an LOD of $0.3 \mathrm{nM}$ for the sensing of $\mathrm{H}_{2} \mathrm{O}_{2}$ due to the plentiful active sites available through PtNi nanowires. Bai et al. ${ }^{366}$ developed nonenzymatic $\mathrm{H}_{2} \mathrm{O}_{2}$ sensors using $\mathrm{Au}$ and $\mathrm{Pt}$ alloy microspheres decorated on rGO. The resulting biosensor exhibited a sensitivity of $1117.0 \mu \mathrm{A}$ $\mathrm{mM}^{-1} \mathrm{~cm}^{-2}$, a linear range of $0.005-4.0 \mathrm{mM}$ and an LOD of $0.008 \mu \mathrm{M}$ for the determination of $\mathrm{H}_{2} \mathrm{O}_{2}$. Deepalakshmi et al. ${ }^{224}$ used $\mathrm{NiCo}_{2} \mathrm{~N} / \mathrm{N}-\mathrm{GR} / \mathrm{GCE}$ sensor for nonenzymatic detection of $\mathrm{H}_{2} \mathrm{O}_{2}$. NiCo $\mathrm{Ni}_{2} \mathrm{~N} / \mathrm{N}-\mathrm{GR}$ core-shell nanostructure-based sensor detected $0.463 \mathrm{mM} \mathrm{H}_{2} \mathrm{O}_{2}$ concentration in human blood serum with $91.7 \%$ recovery, showed high selectivity toward $\mathrm{H}_{2} \mathrm{O}_{2}$ against interferents including DA, UA, AA, glucose, urea, and $\mathrm{NaCl}$, and retained $91.05 \%$ and $98.52 \%$ of initial current response after 45 days at $<5{ }^{\circ} \mathrm{C}$ and $3000 \mathrm{~s}$, respectively.

The assembly of flexible electrodes using few-layer graphene nanosheets into different freestanding structures with high electrical conductivity, mechanical strength, and structural uniformity has shown great promise for the design of flexible biosensors. ${ }^{40}$ The biosensor performance can be optimized by controlling the particle size and morphology of inorganic NPs, and their degree of loading and uniform distribution on the freestanding graphene films. For instance, the homogeneous dispersion or self-assembly of the different nanoparticles over graphene nanosheets offers a large specific surface area and improved electron transport through conducting networks. ${ }^{374}$ $\mathrm{Xi}$ et $a .^{\mathbf{4 2}}$ prepared AuNPs containing porous graphene 
(A)

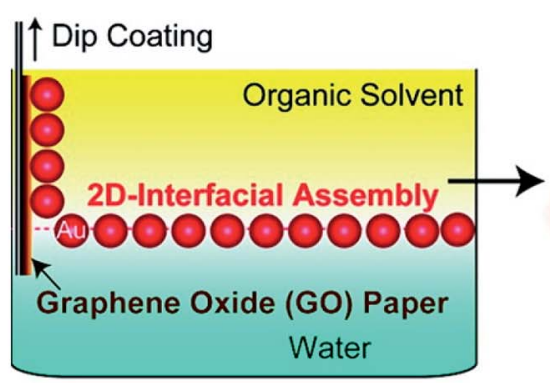

(B)
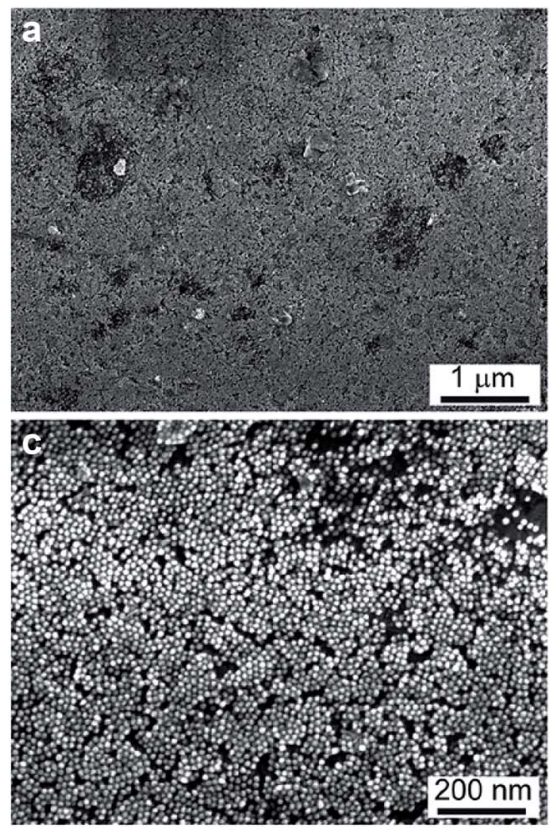
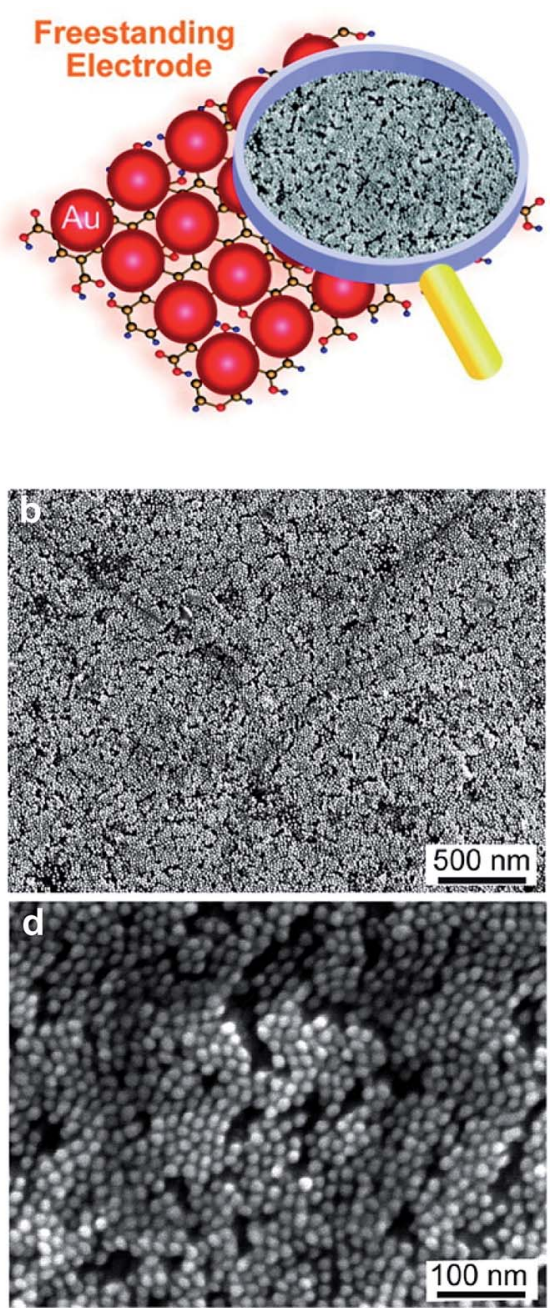

Fig. 13 (A) Schematic illustration of the preparation of 2D-assembly of AuNPs on GO paper to develop hybrid electrodes. (B) Different magnifications of the SEM images of 2D-assembly of AuNPs transferred on the GO paper where AuNPs formed a highly packed monolayer on the GO paper. [Reprinted with permission from ref. 323, F. Xiao, J. Song, H. Gao, X. Zan, R. Xu and H. Duan, Coating Graphene Paper with 2DAssembly of Electrocatalytic Nanoparticles: A Modular Approach toward High-Performance Flexible Electrodes, ACS Nano, $2012,6,100-110$. Copyright@ American Chemical Society.]

nanosheets by the electrostatic layer-by-layer (LbL) assembly of AuNPs with BSA to fabricate the electrode material for the detection of $\mathrm{H}_{2} \mathrm{O}_{2}$. The AuNPs/porous GR-based sensor exhibited enhanced electrochemical detection of $\mathrm{H}_{2} \mathrm{O}_{2}$ with a sensitivity of $75.9 \mu \mathrm{A} \mathrm{cm}^{-2} \mathrm{mM}^{-1}$, a linear range from $0.5 \mu \mathrm{M}$ to $4.9 \mathrm{mM}$, and an LOD of $0.1 \mu \mathrm{M}$ compared with AuNPs/ nonporous graphene composite. Xiao et al. ${ }^{323}$ developed flexible electrodes by assembling AuNPs on the freestanding GO paper. Specifically, the 2D array of AuNPs at oil-water interfaces were transferred on a freestanding GO paper, enabling a densely assembled monolayer of AuNPs on the surface of GO paper (Fig. 13). The fabricated sensor showed improved electrochemical properties, with a sensitivity of $236.8 \mu \mathrm{A} \mathrm{mM}{ }^{-1}$ $\mathrm{cm}^{-2}$, a linear range of $0.005-8.6 \mathrm{mM}$ and an LOD value of 2.0 $\mu \mathrm{M}(\mathrm{S} / \mathrm{N}=3)$ for the detection of $\mathrm{H}_{2} \mathrm{O}_{2}$. The AuNPs/rGO paperbased sensor showed a linear range of 0.01 to $46 \mathrm{mM}$, sensitivity of $52.36 \mu \mathrm{A} \mathrm{mM} \mathrm{mm}^{-1} \mathrm{~cm}^{-2}$, and the LOD of $5.0 \mu \mathrm{M}$ for glucose detection. The AuNPs/GO paper hybrid electrode also showed long-term stability by retaining $96.8 \%$ of its initial peak current value for glucose oxidation after 200 cycles and over $90 \%$ initial catalytic current value up to 60 days after the electrode fabrication. Liang et al. ${ }^{169}$ prepared a freestanding graphene oxide-silk fiber nanocomposite film by then supported spiky structured PtNPs on the film substrate for fabrication of the $\mathrm{H}_{2} \mathrm{O}_{2}$ sensor. The resultant sensor based on spiky PtNP-decorated nanocomposite films showed a linear range of $0-2.5 \mathrm{mM}$, an LOD of $0.2 \mu \mathrm{M}$ and the improved sensitivity of $0.56 \mathrm{~mA} \mathrm{mM}^{-1}$ $\mathrm{cm}^{-2}$ for detecting $\mathrm{H}_{2} \mathrm{O}_{2}$ and the LOD of $1 \mu \mathrm{M}$ with a sensitivity of $150.8 \mu \mathrm{A} \mathrm{mM}{ }^{-1} \mathrm{~cm}^{-2}$ for the glucose detection.

Hybrid nanocomposites of 3D graphene with NPs have attracted tremendous attention because of their unique physical and chemical properties, such as high electron mobility, exceptional impermeability and a large specific surface area for the development of $\mathrm{H}_{2} \mathrm{O}_{2}$ sensors. ${ }^{132,375,376}$ Cheng et al. ${ }^{364}$ reported $\mathrm{Cu}_{2} \mathrm{O}$ microcrystals decorating highly porous $3 \mathrm{D}$ graphene aerogel (GA) for the electrochemical detection of $\mathrm{H}_{2} \mathrm{O}_{2}$. 


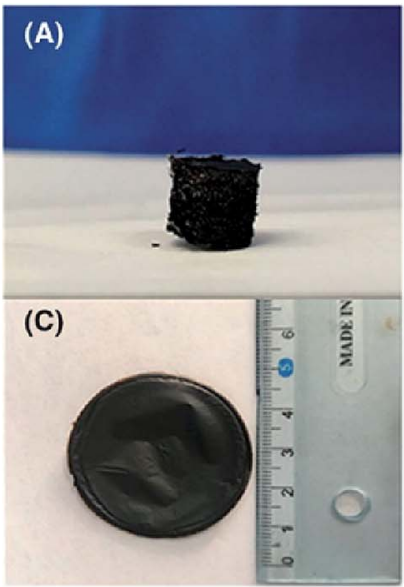

Graphene oxide sheet 3D $\mathrm{GA}-\mathrm{Cu}_{2} \mathrm{O}$
(B)

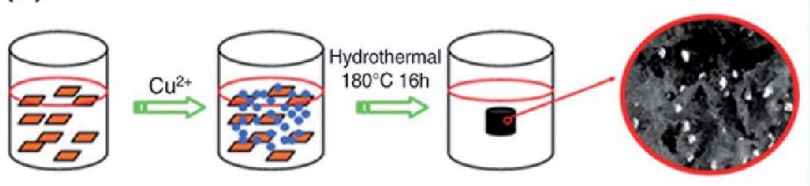

(D)

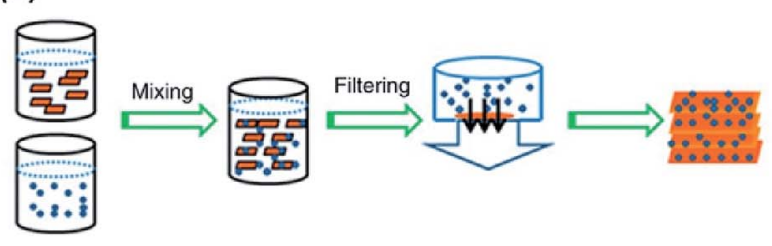

- $\mathrm{Cu}^{2+}$

- $\mathrm{Cu}_{2} \mathrm{O}$ nanocube

\section{D $\mathrm{Cu}_{2} \mathrm{O}-\mathrm{rGO}-\mathrm{P}$}

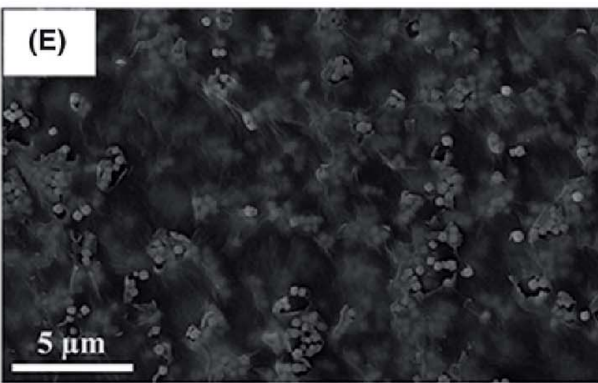

\section{(G)}

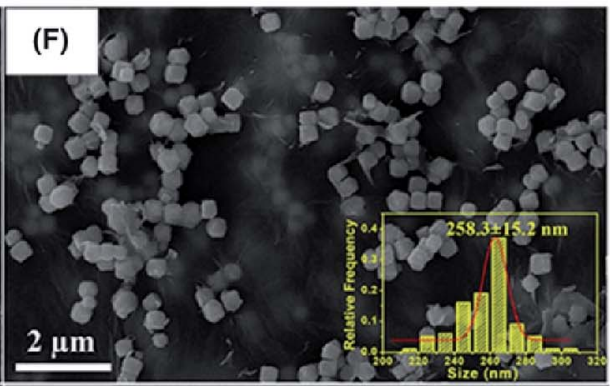

(H)
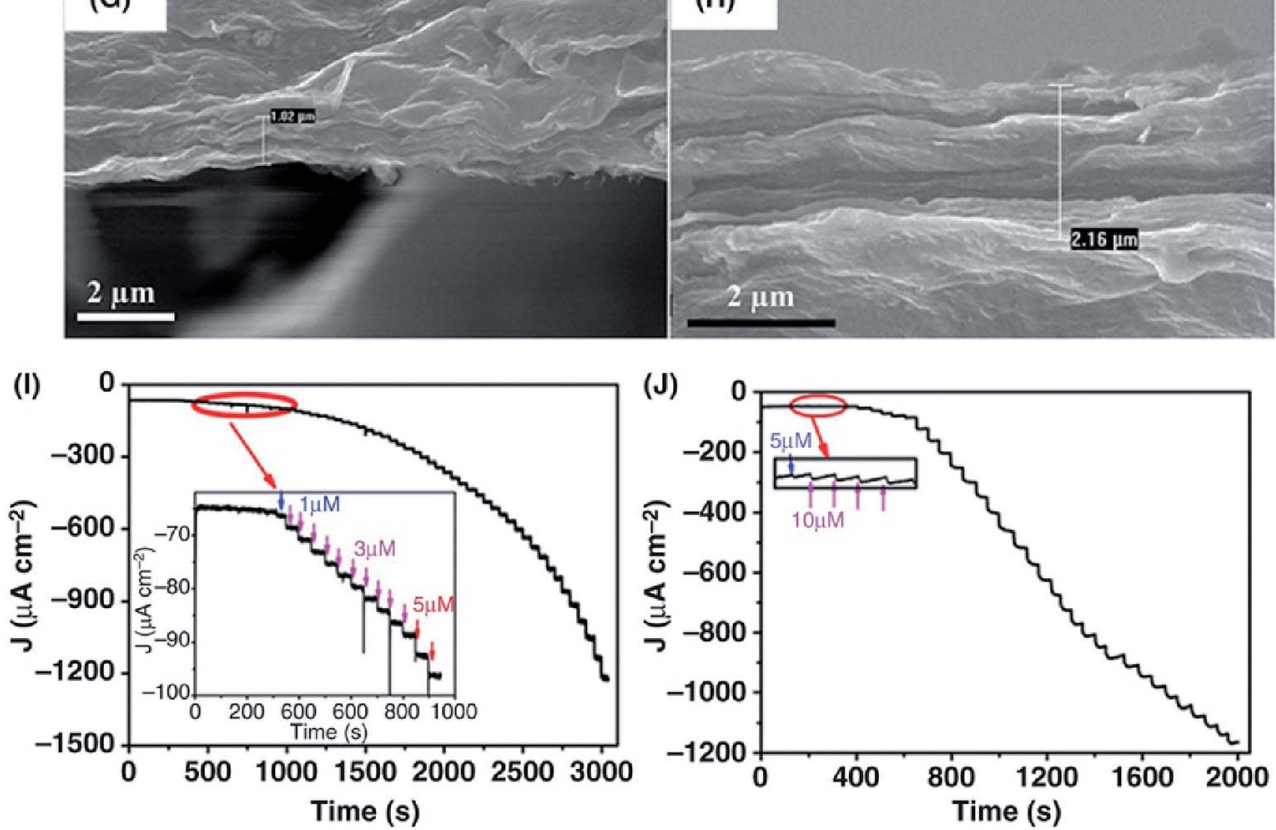

Fig. 14 (A) Photographs of 3D- $\mathrm{Cu}_{2} \mathrm{O}-\mathrm{GA}$ fabricated through a freeze-drying process and paper-like 2D Cu $2 \mathrm{O}-\mathrm{rGO}-\mathrm{P}$ nanostructure obtained by filtration (C). Schematic illustration of the synthesis of 3D $\mathrm{Cu}_{2} \mathrm{O}-\mathrm{GA}$ composite $(\mathrm{B})$ and paper-like 2D $\mathrm{Cu}_{2} \mathrm{O}-\mathrm{rGO}-\mathrm{P}$ nanostructure (D). (E and F) SEM images obtained at different magnifications of the $2 \mathrm{D} \mathrm{Cu}_{2} \mathrm{O}-\mathrm{rGO}$ paper without thermal annealing. Inset in (F) shows the dispersion of $\mathrm{Cu}_{2} \mathrm{O}$ nanocubes in the $\mathrm{rGO}$ paper in terms of the size distribution histogram. SEM images of the 2D $\mathrm{Cu}_{2} \mathrm{O}-\mathrm{rGO}$ paper before $(\mathrm{G})$ and after $(\mathrm{H})$ thermal annealing. Amperometric curves of 3D Cu${ }_{2} \mathrm{O}-\mathrm{GA} / \mathrm{GC}$ composite electrode (I) and paper-like 2D Cu $\mathrm{Cu}_{2} \mathrm{O}-\mathrm{rGO}-\mathrm{P} / \mathrm{GC}$ composite electrode (J) with $\mathrm{H}_{2} \mathrm{O}_{2}$ addition in $\mathrm{N}_{2}$-saturated $0.1 \mathrm{M}$ PBS solution $(\mathrm{pH}=7.0)$ at the applied potential of $-0.4 \mathrm{~V}$. The insets in (I) and (J) show the magnified cathodic current response measured at the low $\mathrm{H}_{2} \mathrm{O}_{2}$ concentrations. [Reprinted with permission from ref. 364, C. Cheng, C. Zhang, X. Gao, Z. Zhuang, C. Du and W. Chen, 3D Network and 2D Paper of Reduced Graphene Oxide/ $\mathrm{Cu}_{2} \mathrm{O}$ Composite for Electrochemical Sensing of Hydrogen Peroxide, Anal. Chem., 2018, 90, 1983-1991. Copyright@ American Chemical Society.] 
(A)
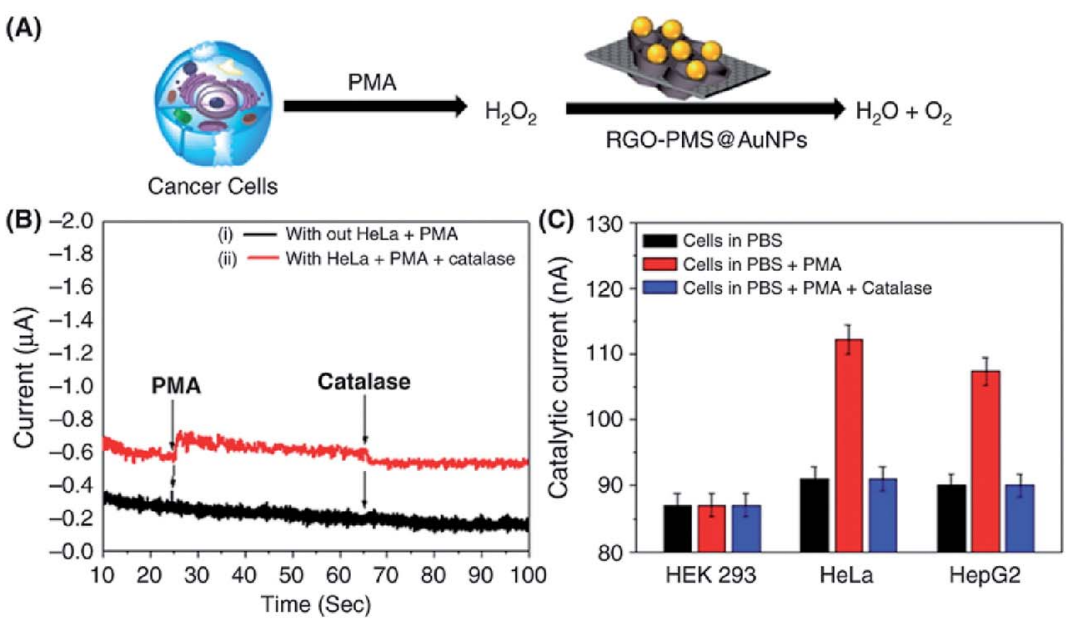

(D)
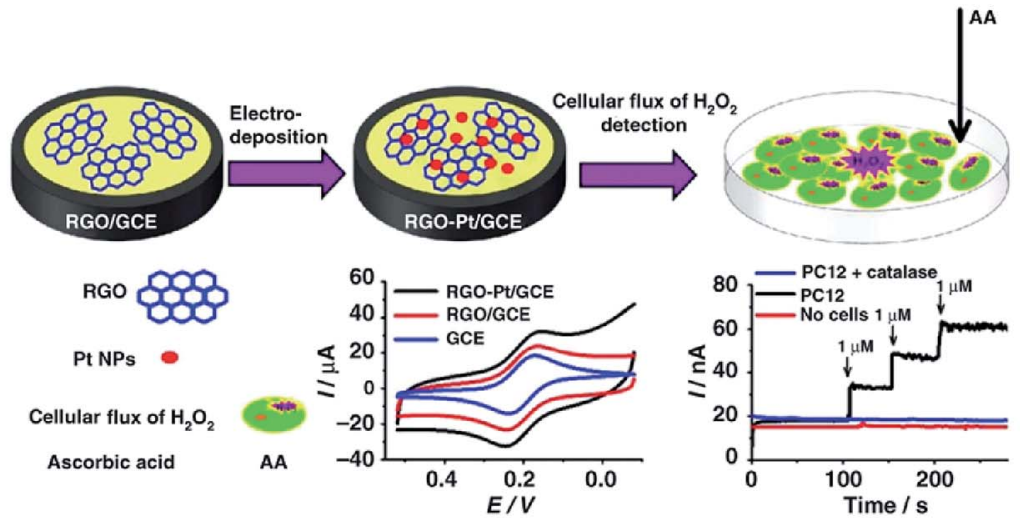

(E)
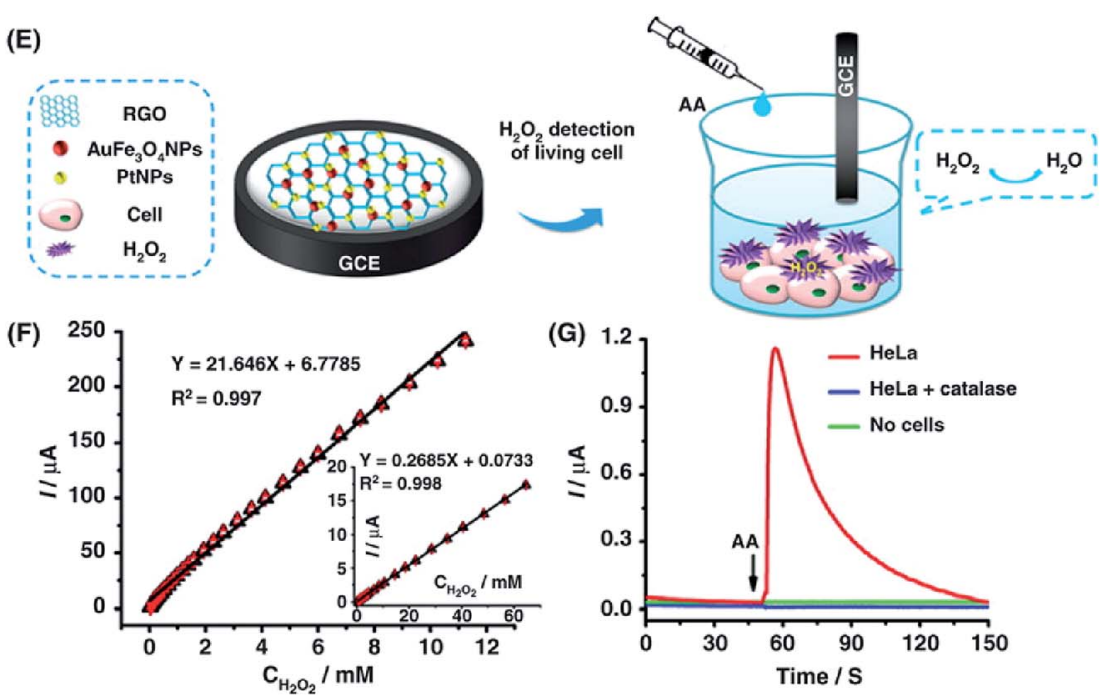

Fig. 15 Different graphene nanocomposite-based electrode materials for the detection of $\mathrm{H}_{2} \mathrm{O}_{2}$ in living cells. (A) Schematic representation of the in vitro detection of $\mathrm{H}_{2} \mathrm{O}_{2}$ using the rGO-PMS@AuNP/GCE. (B) Amperometric response of the rGO-PMS@AuNPs/GC electrode (i) with and (ii) without of HeLa cells after sequential addition of PMA and catalase to $0.1 \mathrm{M} \mathrm{PBS}$ at $-0.75 \mathrm{~V}$. (C) Cellular assay comparison of $\mathrm{H}_{2} \mathrm{O}_{2}$ detection for HEK 293, HeLa, and HepG2 cells. [Reprinted with permission from ref. 330, S. K. Maji, S. Sreejith, A. K. Mandal, X. Ma and Y. Zhao, Immobilizing Gold Nanoparticles in Mesoporous Silica Covered Reduced Graphene Oxide: A Hybrid Material for Cancer Cell Detection through Hydrogen Peroxide Sensing, ACS Appl. Mater. Interfaces, 2014, 6, 13648-13656. Copyright@ American Chemical Society.] (D) Schematic of the rGOPtNPs-modified GCE for detecting $\mathrm{H}_{2} \mathrm{O}_{2}$ efflux from cells stimulated with ascorbic acid (AA). [Reprinted with permission from ref. 381, Y. Zhang, X. Bai, X. Wang, K.-K. Shiu, Y. Zhu and H. Jiang, Highly Sensitive Graphene-Pt Nanocomposites Amperometric Biosensor and Its Application in Living Cell $\mathrm{H}_{2} \mathrm{O}_{2}$ Detection, Anal. Chem., 2014, 86, 9459-9465. Copyright $\odot$ American Chemical Society.] (E) Schematic of the rGO/AuFe $\mathrm{O}_{4} /$ PtNPs-modified GCE for detecting $\mathrm{H}_{2} \mathrm{O}_{2}$ efflux from cells stimulated with ascorbic acid (AA). (F) Amperometric response upon adding AA in PBS containing HeLa cells at $\mathrm{OV}$ (red), containing HeLa cells and catalases (blue), or without cells (green). (G) $\mathrm{Amount}$ of $\mathrm{H}_{2} \mathrm{O}_{2}$ released by $\mathrm{LO}_{2}$, $\mathrm{HeLa}$, HepG2, and U87 cells stimulated by 1 MM AA. [Reprinted with permission from ref. 382, L. Wang, Y. Zhang, C. Cheng, X. Liu, H. Jiang and X. Wang, Highly Sensitive Electrochemical Biosensor for Evaluation of Oxidative Stress Based on the Nanointerface of Graphene Nanocomposites Blended with Gold, $\mathrm{Fe}_{3} \mathrm{O}_{4}$, and Platinum Nanoparticles, ACS Appl. Mater. Interfaces, 2015, 7, 18441-18449. Copyright@ American Chemical Society.] 
Fig. 14 shows the synthesis and photographs of $3 \mathrm{D}-\mathrm{Cu}_{2} \mathrm{O}-\mathrm{GA}$ and paper-like 2D $\mathrm{Cu}_{2} \mathrm{O}-\mathrm{rGO}-\mathrm{P}$ nanostructures, their SEM images and amperometric curves of $3 \mathrm{D} \mathrm{Cu}_{2} \mathrm{O}-\mathrm{GA} / \mathrm{GC}$ and paperlike $2 \mathrm{D} \mathrm{Cu} \mathrm{Cu}_{2} \mathrm{O}$-rGO-P/GC composite electrodes after adding $\mathrm{H}_{2} \mathrm{O}_{2}$ in $\mathrm{N}_{2}$-saturated PBS solution at $-0.4 \mathrm{~V}$. The developed $3 \mathrm{D} \mathrm{Cu}_{2} \mathrm{O}$ GA-based biosensor exhibited high electrocatalytic activity for the reduction of $\mathrm{H}_{2} \mathrm{O}_{2}$ and improved sensing performance with a linear range from $1.0 \mu \mathrm{M}$ to $1.47 \mathrm{mM}$ and an LOD of $0.37 \mu \mathrm{M}$. The paper-like 2D $\mathrm{Cu}_{2} \mathrm{O}$-rGO-P nanostructure showed further improved $\mathrm{H}_{2} \mathrm{O}_{2}$ sensing with a wide linear range of $5.0 \mu \mathrm{M}$ to $10.56 \mathrm{mM}$ and the LOD of $3.78 \mu \mathrm{M}$. Both sensors based on 3D $\mathrm{Cu}_{2} \mathrm{O}-\mathrm{GA}$ and $2 \mathrm{D} \mathrm{Cu}_{2} \mathrm{O}-\mathrm{rGO}-\mathrm{P}$ nanocomposites showed high selectivity toward the detection of $\mathrm{H}_{2} \mathrm{O}_{2}$ as no interference was observed from the trace amounts of DA, AA, UA and $\mathrm{NaCl}$ interferents. Lin et al. ${ }^{377}$ developed an electrochemical $\mathrm{H}_{2} \mathrm{O}_{2}$ sensor using 3D rGO structure decorated with molybdenum disulfide $\left(\mathrm{MoS}_{2}\right)$ QDs. The developed rGO-MoS 2 QD compositebased sensor exhibited a broad linear range of 0.01 to $5.57 \mathrm{mM}$, an LOD of $1.90 \mu \mathrm{M}$, and stability up to 18 days.

3.3.1 Detection of $\mathrm{H}_{2} \mathrm{O}_{2}$ in living cells. The development of biosensors to detect $\mathrm{H}_{2} \mathrm{O}_{2}$ from biological cells such as cancer cells is gaining a lot of interest. Zhang et al. ${ }^{378}$ reported a $\mathrm{H}_{2} \mathrm{O}_{2}$ sensor based on nitrogen- and sulfur-codoped GQDs/graphene nanosheets. The sensor displayed a wide linear range from 0.4 $\mu \mathrm{M}$ to $33 \mathrm{mM}$ with an LOD of $26 \mathrm{nM}$, in addition to high selectivity, reproducibility and long-term stability for $\mathrm{H}_{2} \mathrm{O}_{2}$ detection in human serum samples and in the secretions released by Raw 264.7 cells. The NS-GQDs/GR hybrid nanocomposite exhibited high performance for the detection of $\mathrm{H}_{2} \mathrm{O}_{2}$ due to the high electrical conductivity, specific surface area and the availability of plentiful doping sites and edges. Sun et al. ${ }^{333}$ demonstrated the real-time electrochemical detection of $\mathrm{H}_{2} \mathrm{O}_{2}$ in live cells using a PtNP-decorated GR/CNT hybrid paper electrode. The flexible PtNP/GR-CNT hybrid paper-based sensor showed a linear range as low as $25 \mathrm{mM}$ and an LOD of $10 \mathrm{nM}$ and the sensitivity of $1.41 \mu \mathrm{A} \mu \mathrm{M}^{-1} \mathrm{~cm}^{-2}$. Zhang et al. ${ }^{379}$ developed a freestanding nanohybrid paper electrode using 3D ionic liquid-functionalized graphene supported by gold nanoflowers for the in situ electrochemical detection and real-time monitoring of $\mathrm{H}_{2} \mathrm{O}_{2}$ released from live breast cancer cells. The composite-based nonenzymatic electrochemical sensor showed sensitivity toward $\mathrm{H}_{2} \mathrm{O}_{2}$ with a linear range of $0.5 \mu \mathrm{M}$ to $2.3 \mathrm{mM}$ and an LOD of $100 \mathrm{nM}$ due to the unique 3D structure and high electrocatalytic activity originating from the Au nanoflowers. Moreover, the developed sensor was able to discriminate normal breast cells (HBL-100) from the cancer breast cells (MDA-MB-231 and MCF-7 cells) and monitored $\mathrm{H}_{2} \mathrm{O}_{2}$ released from the breast cancer cells. Liu et al. ${ }^{335}$ reported PtNP-decorated porous graphene for detecting extracellular $\mathrm{H}_{2} \mathrm{O}_{2}$ released from the living cells. The biosensor showed good electrocatalytic reduction of $\mathrm{H}_{2} \mathrm{O}_{2}$ with a sensitivity of $341.14 \mu \mathrm{AmM}^{-1} \mathrm{~cm}^{-2}$, a linear range of 1.0-1477 $\mu \mathrm{M}$ and an LOD as low as $0.5 \mu \mathrm{M}$. The improved sensor performance can be attributed to the uniform dispersion of PtNPs on the larger surface area and abundant active sites provided by the porous graphene nanostructure.

Maji et $a .^{330}$ developed a sensor based on sandwich-like periodic silica-coated rGO-AuNPs for the detection of $\mathrm{H}_{2} \mathrm{O}_{2}$ released from living tumor cells (Fig. 15A). The sensor showed a sensitivity of $39.2 \mu \mathrm{AmM}^{-1} \mathrm{~cm}^{-2}$, a wide linear range from 0.5 $\mu \mathrm{M}$ to $50 \mathrm{mM}$ and an LOD of $60 \mathrm{nM}$. The sensor also detected glucose and $\mathrm{H}_{2} \mathrm{O}_{2}$ in human urine and nanomolar level sensitivity for $\mathrm{H}_{2} \mathrm{O}_{2}$ released from living HeLa and HepG2 tumor cells. Similarly, the detection of $\mathrm{H}_{2} \mathrm{O}_{2}$ in a biological system was accomplished Ju et al. ${ }^{380}$ using an electrochemical sensor based on AuNPs supported on nitrogen-doped GQDs (AuNPs/NGQDs). The fabricated sensor exhibited high sensitivity and selectivity for the detection of $\mathrm{H}_{2} \mathrm{O}_{2}$ released from human cervical cancer cells with a sensitivity of $186.22 \mu \mathrm{A} \mathrm{mM} \mathrm{m}^{-1} \mathrm{~cm}^{2}$, a linear range of $0.25-13327 \mu \mathrm{M}$ and an LOD of $0.12 \mu \mathrm{M}$. The performance of the biosensor is attributed to the catalytic activity of the exposed (111) facets of the AuNPs and the dimension of the GQDs, which facilitate electron transfer between the AuNPs/N-GQDs and the GCE surface. Zhang et al. ${ }^{381}$ demonstrated an $\mathrm{H}_{2} \mathrm{O}_{2}$ sensor based on PtNPs/rGO nanocomposites to detect $\mathrm{H}_{2} \mathrm{O}_{2}$ from living cells (Fig. 15B). The sensor showed high sensitivity with a linear range from $0.5 \mu \mathrm{M}$ to $3.475 \mathrm{mM}$ and an LOD of $\sim 0.2 \mu \mathrm{M}$. Wang et al. ${ }^{382}$ prepared a modified electrode using rGO blended with dumbbell-like $\mathrm{AuFe}_{3} \mathrm{O}_{4}$ and Pt NPs ( $\left.\mathrm{rGO} / \mathrm{AuFe}_{3} \mathrm{O}_{4} \mathrm{NP} / \mathrm{PtNP}\right)$ for the detection of $\mathrm{H}_{2} \mathrm{O}_{2}$ released from living cancer cells (Fig. 15C). The fabricated $\mathrm{rGO} / \mathrm{AuFe}_{3} \mathrm{O}_{4} / \mathrm{PtNPs}$ modified electrode-based sensor shows high catalytic activity and low overpotential for $\mathrm{H}_{2} \mathrm{O}_{2}$ detection. Moreover, the biosensor displayed improved selectivity and sensitivity with a linear range from $0.5 \mu \mathrm{M}$ to $11.5 \mathrm{mM}$ and an LOD of $\sim 0.1 \mu \mathrm{M}$ as well as no interference from competing UA, AA and glucose bioanalytes. The sensor was used to measure the release of $\mathrm{H}_{2} \mathrm{O}_{2}$ from living cells, including both tumor cells and healthy normal cells using external stimulation where more $\mathrm{H}_{2} \mathrm{O}_{2}$ was released from tumor cells compared with normal cells. The quantity of $\mathrm{H}_{2} \mathrm{O}_{2}$ released from human cervical cancer cell HeLa was found to be to almost twice than that of normal hepatic cells $\mathrm{LO}_{2}$. The results showed nanocomposite-based biosensor as a promising platform for nonenzymatic $\mathrm{H}_{2} \mathrm{O}_{2}$ detection in clinical diagnostics to determine oxidative stress in different types of normal and diseases cells. Sun et al. ${ }^{383}$ reported a graphene-decorated intermetallic $\mathrm{PtPb}$ nanoplate (PtPb-NPs/GR) nanocomposite-based electrochemical sensor for the detection of $\mathrm{H}_{2} \mathrm{O}_{2}$. This sensor showed high electrocatalytic activity for the reduction of $\mathrm{H}_{2} \mathrm{O}_{2}$ with a linear range from $2 \mathrm{nM}$ to $2.5 \mathrm{mM}$ and an LOD of $2 \mathrm{nM}$. Moreover, the sensor also detected $\mathrm{H}_{2} \mathrm{O}_{2}$ released from Raw 264.7 cells. The high performance of this biosensor was attributed to the synergy between the $\mathrm{PtPb}$ nanoplates and graphene as well as abundance of electrocatalytic active sites available on the PtPb nanoplates. The same research group also fabricated $\mathrm{H}_{2} \mathrm{O}_{2}$ biosensor by assembling atomic-thick PtNi nanowires on rGO employing an ultra-sonic self-assembly technique. ${ }^{373}$ The resulting PtNiNWs/rGO-based biosensor exhibited electrocatalytic reduction of $\mathrm{H}_{2} \mathrm{O}_{2}$ in a wide linear range from $1.0 \mathrm{nM}$ to $5.3 \mathrm{mM}$ with an LOD of $0.3 \mathrm{nM}$ at a potential of $-0.6 \mathrm{~V}$ ( $v s$. Ag/ $\mathrm{AgCl}$ ) and also detected real-time $\mathrm{H}_{2} \mathrm{O}_{2}$ release from a murine macrophage cell line (Raw 264.7 cell). 


\subsection{Nucleic acid (DNA/RNA) biosensors}

The detection of nucleic acids has attracted attention due to the variability and specificity of the sequence. For example, NA biosensors can be used to detect single-stranded DNA (ssDNA) that can hybridize with the complementary strand. The high sensitivity and selectivity of these biosensors is important in gene engineering and in the diagnosis of genetic diseases since any damage to DNA molecules strongly influences genetic and chronic diseases. ${ }^{3 \mathbf{8 4}}$ Several signal transduction methods have been employed for the detection of DNA, including fluorescence, electrochemistry, electrochemiluminescence (ECL), chemiluminescence (CL), colorimetry, and surface plasmon resonance (SPR). ${ }^{\mathbf{1 1}}$ The electrochemical detection method is

Table 5 Graphene nanocomposite-based biosensors for detecting nucleic acids and NADH ${ }^{a}$

Graphene-based

nanocomposites

Nucleic acids

3D GR/AuNP nanocomposite

Poly(xanthurenic acid)/rGO composite

SPAN/GO composite

rGO modified GCE

AuNPs/toluidine blue-GO composite

GR/Au nanorods/polythionine

Flower-like $\mathrm{VS}_{2} / \mathrm{GR} / \mathrm{AuNPS}$

EC-rGO/GR

GR/Au nanoclusters composite

rGO/AuNPs

AuNPs/rGO/PANI nanocomposite

rGO

N-doped GR/AuNPs

$\mathrm{rGO} / \mathrm{ZrO}_{2}$ nanocomposite

GO nanosheet/AgNPs nanocomposite

GR/Nafion composite

EC-rGO

$p$-rGO/AuNPs

GR/flavin mononucleotides

dsDNA/poly(L-cysteine) $/ \mathrm{Fe}_{3} \mathrm{O}_{4} \mathrm{NPs} / \mathrm{GO}$ nanocomposite

Au-transferred CVD-grown GR

rGO

Thionine functionalized rGO

AQMS-rGO composite

Au nanocages/GR nanoribbons

GR QDs

GO/Au nanorod nanocomposite

$\mathrm{MgO}$ nanoflowers/GO-AuNPs nanocomposite

N-doped GR/AuNPs/hemin/G-quadruplexes
AuNPs/rGO/GCE

Au nanoclusters/GR

$\mathrm{CS} / \mathrm{N}$-doped rGO

Target

DNA

DNA

DNA

Amelogenin gene

DNA

HPV

AIV H5N1 gene

ssDNA

HIV gene

DNA

DNA

DNA

DNA

(Mycobacterium

tuberculosis)

DNA

DNA

miRNA-21

DNA

HIV gene

HIV gene

ssDNA

(Listeria

monocytogenes)

DNA

DNA

DNA

DNA

DNA

DNA

DNA

miRNA-155

miRNA-155

miRNA

miRNA

miRNA
Detection

technique

Linear range

CV, EIS

EIS

EIS

EIS, CV

DPV

-

EIS, CV

DPV

CV, DPV

LSV

DPV

$50 \mathrm{fM}$ to $5.0 \mathrm{pM}$
$0.1 \mathrm{fM}$ to $0.1 \mathrm{nM}$
$0.1 \mathrm{fM}$ to $0.1 \mathrm{nM}$
$1 \times 10^{-20}$ to $1 \times$
$10 \mathrm{pM}$ to $1 \mathrm{nM}$
$0.1 \mathrm{pM}$ to $0.1 \mathrm{nM}$
$0.5 \mathrm{pM}$ to $0.5 \mathrm{nM}$
$3 \times 10^{-20}$ to $10^{-1}$
$0.1 \mathrm{fM}$ to $100 \mathrm{nM}$
$10 \mathrm{fM}$ to $1 \mu \mathrm{M}$
$0.2 \mathrm{fM}$ to $20 \mathrm{pM}$
$0.1 \mathrm{pM}$ to $10 \mathrm{nM}$
$1.0 \mathrm{fM}$ to $1.0 \mathrm{nM}$

$1.0 \mathrm{fM}$ to $1.0 \mathrm{nM}$
$10 \mathrm{fM}$ to $0.1 \mu \mathrm{M}$
$10 \mathrm{fM}$ to $0.1 \mathrm{nM}$
$10 \mathrm{fM}$ to $10 \mathrm{nM}$
$0.1-100 \mathrm{pM}$
$1 \mathrm{pM}$ to $1 \mathrm{nM}$
$0.1 \mathrm{fM}$ to $1 \mu \mathrm{M}$

EIS

-

DPV

EIS

EIS

DPV

$0.1 \mathrm{fM}$ to $1 \mu \mathrm{M}$

DPV

$\mathrm{CV}$, EIS

$0.1 \mathrm{fM}$ to $1.0 \mu \mathrm{M}$

Hall

0.01-30.0 $\mu \mathrm{M}$

$1 \mathrm{pM}$ to $100 \mathrm{nM}$

$1 \mathrm{fM}$ to $1 \mathrm{nM}$

DPV

DPV

CV, EIS

CV

CV

$\mathrm{CV}$

EIS

CV, DPV

$0.01 \mathrm{fM}$ to $1 \mathrm{pM}$

$1 \mathrm{fM}$ to $0.01 \mu \mathrm{M}$

$1 \mathrm{fM}$ to $100 \mathrm{pM}$

$1 \mathrm{fM}$ to $100 \mathrm{pM}$

$2.0 \mathrm{fM}$ to $8.0 \mathrm{pM}$

$0.1-100 \mathrm{fM}$

5-20 $\mu \mathrm{g} \mathrm{mL}^{-1}$

$10 \mathrm{fM}$ to $1 \mathrm{nM}$

$\begin{array}{lll}\text { NADH } & \text { CV } & 1-100 \mu \mathrm{M} \\ \text { NADH } & \text { CV } & 0-500 \mu \mathrm{M} \\ \text { NADH } & \text { CV } & 1.0 \mathrm{fM} \text { to } 10 \mathrm{pM} \\ \text { NADH } & \text { CV } & 50 \mathrm{nM} \text { to } 500 \mu \mathrm{M} \\ \text { NADH } & \text { CV } & 0.166-1.772 \mu \mathrm{M} \\ \text { NADH } & \text { CV } & 0.5-12 \mu \mathrm{M} \\ \text { NADH } & \text { CV } & 0.01-0.2 \mathrm{mM} \\ \text { NADH } & & 0.4-718 \mu \mathrm{M} \\ \text { NADH } & \text { DPV } & 0.32-220 \mu \mathrm{M} \\ \text { NADH } & \text { CV } & 0.083 \mu \mathrm{M} \text { to } 1.05 \mathrm{mM}\end{array}$

Limit of

detection (LOD) Ref.

\section{NADH}

N-doped GR/AuNPs nanocomposite rGO/AuNPs

$\begin{array}{ll}300 \mathrm{nM} & 423 \\ 0.6 \mu \mathrm{M} & 160 \\ 1.0 \mathrm{fM} & 424 \\ 1.13 \mathrm{nM} & 425 \\ 1.3 \mu \mathrm{M} & 426 \\ 0.37 \mu \mathrm{M} & 427 \\ 3.5 \mu \mathrm{M} & 428 \\ 25 \mathrm{nM} & 429 \\ 0.16 \mu \mathrm{M} & 430 \\ 9.0 \mathrm{nM} & 431\end{array}$

${ }^{a}$ Abbreviations used: NADH: nicotinamide adenine dinucleotide, SWCNTs: single-walled carbon nanotubes, LSV: linear sweep voltammetry, MDR: multidrug resistance, HPV: human papillomavirus, PAH: poly(allylamine hydrochloride), AQMS: anthraquinone-2-sulfonic acid. AIV: avian influenza virus, CILE: carbon ionic liquid electrode, SPAN: sulfonated polyaniline. 
considered an efficient approach for NA detection because of its low cost, simple operation, high sensitivity, fast response, miniaturization, and easy use in molecular diagnosis. The electrochemical NA detection mechanisms involve binding to a probe immobilized on the sensing surface and thereafter monitoring the change in a generated signal. The sensitivity and specificity of NA detection depend strongly on the immobilization of specific probes and also on shunning nonspecific binding to the sensing surface. ${ }^{385}$

Graphene and its derivatives have attracted much interest for achieving fast, label-free, highly sensitive and selective DNA/ RNA detection and sequencing. ${ }^{386}$ Owing to their unique molecular structure and electronic properties, DNA and RNA can easily form $\pi-\pi$ bonds between their conjugated systems and the nucleobases. ${ }^{387}$ Theoretical studies have also provided evidence that the molecular interaction between DNA/RNA and graphene is highly dominated by weak non-covalent $\pi-\pi$ interactions. ${ }^{\mathbf{3 8 8 , 3 8 9}}$ Hence, the electronic structure of the graphene-nucleobase system can be easily tuned by altering the substrate surface and the adsorbate. ${ }^{390}$ Additionally, the inherent electrical conductivity of graphene and graphenebased derivatives support effective signal amplification. Table 5 summarizes the DNA/RNA biosensors developed from graphene-based nanocomposites and their sensing capabilities using different electrochemical methods.

Loan et al. ${ }^{\mathbf{4 1 3}}$ demonstrated a Hall effect device consisting of gold films deposited on graphene layers for improved sensitivity in hybridization detection, with a wide linear range from 1 pM to $100 \mathrm{nM}$ for the detection of DNA targets. AuNPs have been widely employed with graphene for the detection of DNA because covalent bonding between AuNPs and multiple thiolated probe ssDNAs supports both the loading efficiency and capacity. Wang et al. ${ }^{\mathbf{4 3 2}}$ described an electrochemical DNA biosensor based on AuNP-decorated rGO. The biosensor showed good selectivity toward single-base mismatched target DNA with a linear detection range of $0.1 \mu \mathrm{M}$ to $0.1 \mathrm{fM}$ and an LOD of 35 aM. Huang et al. ${ }^{396}$ developed a DNA biosensor for electrochemical detection of human papillomavirus (HPV) using graphene/Au nanorods/polythionine (GR/AuNRs/PT). The fabricated DNA biosensor exhibited high performance for the detection of HPV DNA in a range from $1 \times 10^{-13}$ to $1 \times$ $10^{-10} \mathrm{~mol} \mathrm{~L}^{-1}$ with an LOD of $4.03 \times 10^{-14} \mathrm{~mol} \mathrm{~L}^{-1}$. Wang et al. $^{\mathbf{4 3 3}}$ fabricated a peroxide mimic by loading iron(III) mesotetrakis( $N$-methylpyridinum-4-yl)porphyrin (FeTMPyP) and streptavidin onto graphene oxide. The designed biosensor was used to detect a biotinylated molecular beacon for the electrochemical detection of DNA (Fig. 16A). The sensor showed a high electrochemical response for detecting DNA with an LOD reaching to $22 \mathrm{aM}$ and a broad linear relationship from $10 \mathrm{pM}$ to $100 \mathrm{aM}$, ranging five orders of magnitude (Fig. 16B). Yang et $a .^{411}$ fabricated an electrochemical DNA sensor using bifunctionalized graphene with riboflavin $5^{\prime}$-monophosphate sodium salt (FMNS). The GR/FMNS sensor performance was compared with that of the conventional electrochemical indicator $\left(\left[\mathrm{Fe}(\mathrm{CN})_{6}\right]^{3-/ 4-}\right)$. The designed DNA sensor exhibited high sensitivity, selectivity, and stability with an LOD of $8.3 \times$ $10^{-17} \mathrm{M}$, which is slightly higher $\left(7.4 \times 10^{-17} \mathrm{M}\right)$ than that of
$\left(\left[\mathrm{Fe}(\mathrm{CN})_{6}\right]^{3-/ 4-}\right)$ indicator. Singh et al. $^{\mathbf{4 3 4}}$ developed a GO/CS nanocomposite-based DNA biosensor for the identification of typhoid. The biosensor was constructed by the covalent immobilization of a Salmonella typhi-specific $5^{\prime}$-amine-labeled sSDNA probe on GO-CS/ITO with GA as cross-linkers. The ssDNA/GO-CS-based biosensor can effectively differentiate between complementary, non-complementary and one-base mismatch sequences with a detection range of $10 \mathrm{fM}$ to $50 \mathrm{nM}$ and an LOD of $10 \mathrm{fM}$ within hybridization times of $60 \mathrm{~s}$ for the complementary sequence. Moreover, the resulting sSDNA/GO-CS biosensor enables the detection of complementary targets in serum samples with a low LOD of $100 \mathrm{fM}$. The high performance of the sensor originated from the electrochemical activity of GO and to the capability of CS biopolymer to enhance the DNA immobilization and enable the electron transfer between DNA and the ITO electrode surface.

The integration of AuNPs with GO or rGO is another valuable approach for developing DNA biosensors. Peng et al. ${ }^{395}$ reported

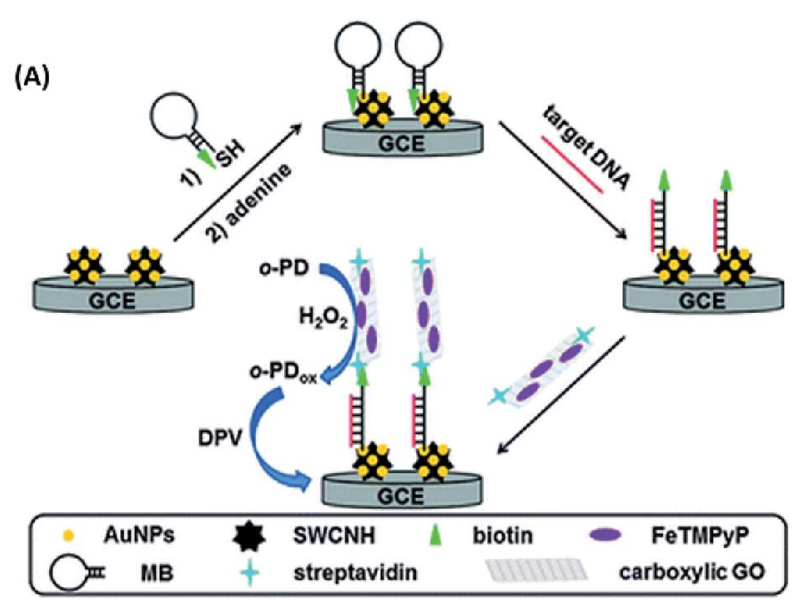

(B)
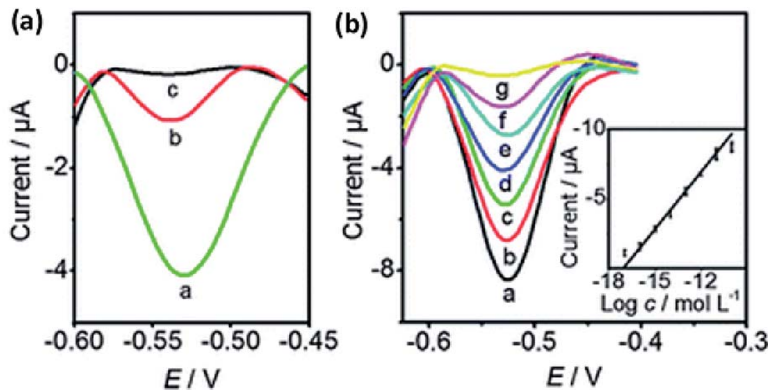

Fig. 16 (A) Schematic illustration of graphene/ferric porphyrin (FeTMPyP) based electrochemical sensor for DNA detection as a horseradish peroxidase (HRP)-mimicking trace label. (B) DPV responses at target DNA concentration of $10 \mathrm{fM}$ with (a) FeTMPyPstreptavidin-GO bioconjugate, (b) HRP-streptavidin-GO as trace label, and (c) in the absence of trace label. DPV curves at different target DNA concentrations of (a) $10 \mathrm{pM}$, (b) $1 \mathrm{pM}$, (c) $100 \mathrm{fM}$, (d) $10 \mathrm{fM}$, (e) $1.0 \mathrm{fM}$, (f) $100 \mathrm{aM}$ and (g) $0 \mathrm{aM}$. Inset is a plot of peak versus the logarithm of target DNA concentration. [Reprinted with permission from ref. 433, Q. Wang, J. Lei, S. Deng, L. Zhang and H. Ju, GrapheneSupported Ferric Porphyrin as a Peroxidase Mimic for Electrochemical DNA Biosensing, Chem. Commun., 2013, 49, 916-918. Copyright $(\subseteq)$ Royal Society of Chemistry.] 


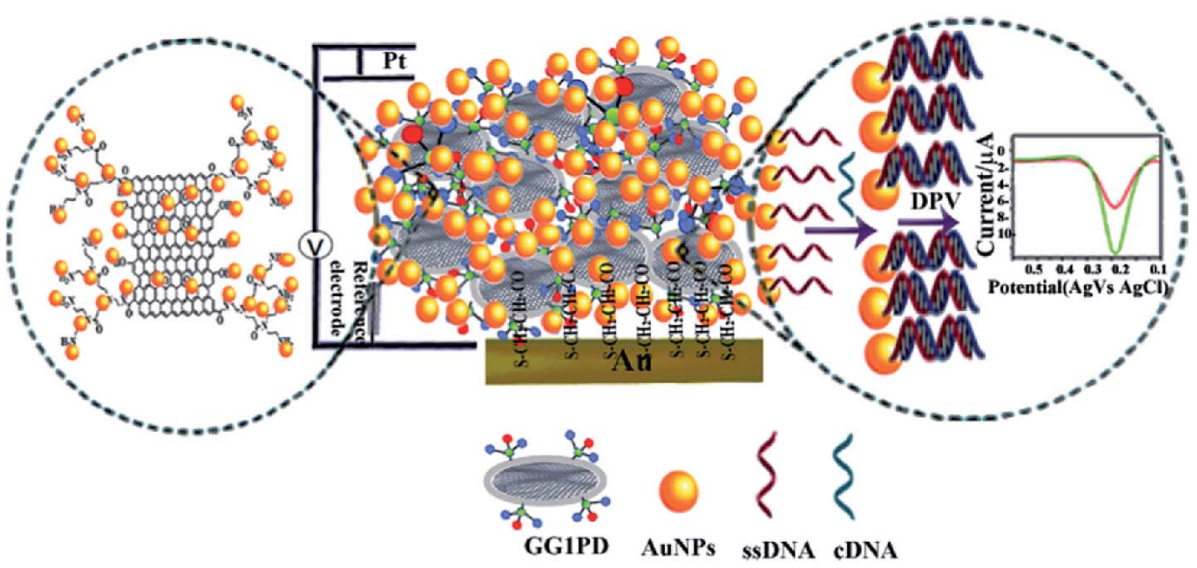

Fig. 17 Schematic illustration of the LbL-assembled AuNP-decorated first-generation (G1) PD with rGO core as a label-free biosensor with controllable 3D nanoarchitecture for the rapid detection of DNA hybridization. [Reprinted with permission from ref. 436, K. Jayakumar, M. B. Camarada, V. Dharuman, R. Rajesh, R. Venkatesan, H. Ju, M. Maniraj, A. Rai, S. R. Barman and Y. Wen, Layer-by-Layer-Assembled AuNPsDecorated First-Generation Poly(amidoamine) Dendrimer with Reduced Graphene Oxide Core as Highly Sensitive Biosensing Platform with Controllable 3D Nanoarchitecture for Rapid Voltammetric Analysis of Ultratrace DNA Hybridization, ACS Appl. Mater. Interfaces, 2018, 10, 2154121555. Copyright@ American Chemical Society.]

a label-free electrochemical DNA biosensor for the detection of multidrug resistance (MDR) gene using AuNPs/toluidine blue (TB)-GO-modified electrode. The AuNPs/TB-GO based sensor showed an LOD of $2.95 \mathrm{pM}$ with good selectivity. Han et al. ${ }^{\mathbf{4 3 5}}$ fabricated sensor from gold nanorods (AuNRs) supported on GO nanosheets. The constructed DNA sensor detected complementary DNA from $1.0 \mathrm{nM}$ to $10 \mathrm{fM}$ with an LOD of 3.5 fM. Moreover, the sensor showed high selectivity as it can effectively discriminate complementary DNA sequences from a large quantity of single-base mismatched DNA from a ratio of 1000 : 1. Fang et al. $^{397}$ developed a label-free electrochemical aptasensor using the self-assembly of DNA aptamers on flowerstructured $\mathrm{VS}_{2}$ and AuNPs supported on graphene for the detection of DNA. Specifically, the gene sequence of avian influenza virus $\mathrm{H} 5 \mathrm{~N} 1$ was selected as the target DNA, and the $\left[\mathrm{Fe}(\mathrm{CN})_{6}\right]^{3-/ 4-}$ solution was employed as the electrochemical indicator. This $\mathrm{VS}_{2} / \mathrm{AuNPs} / \mathrm{GR}$ composite-based sensor displayed a wide linear detection range from $5.0 \times 10^{-13}$ to $5.0 \times$ $10^{-10} \mathrm{M}$ with an LOD of $52 \mathrm{fM}$ for the detection of the H5N1specific genetic sequence. Jayakumar et $a l^{436}$ synthesized a 3D nanoarchitecture based on the layer-by-layer (LbL) assembly of AuNPs, which was supported by poly(amido-amine) dendrimer using rGO as a core for the ultra-low level detection of DNA hybridization, as shown in Fig. 17. Specifically, the biosensor was designed using $[\mathrm{Fe}(\mathrm{CN}) 6]^{3-/ 4-}$ for covalent immobilization of thiol-functionalized ssDNA. The DNA biosensor was fast, highly sensitive and selective for DNA hybridization with a linear range of $1 \times 10^{-6}$ to $1 \times 10^{-13} \mathrm{~g} \mathrm{~m}^{-1}$ and achieved an LOD of $9.07 \times 10^{-14} \mathrm{~g} \mathrm{~m}^{-1}$.

Several signal enhancement strategies to amplify DNA detection have been demonstrated. For example, Wang et al. ${ }^{399}$ reported an electrochemical biosensor for human immunodeficiency virus (HIV) gene detection using graphene/Au nanoclusters (GR/AuNCs) with an exonuclease III (Exo III) amplification strategy (Fig. 18). The biosensor platform having extremely high specific area offered more fixed active sites for the cyctosine (C)-rich capture probes. The biosensor exhibited high sensitivity and selectivity toward HIV target DNA with an LOD of 30 aM. The GR/AuNCs biosensor showed high selectivity toward detecting HIV target DNA (10 nM concentration), compared with 10 times larger concentrations of three other interfering DNA sequences; single-base mismatched DNA (100 $\mathrm{nM}$ ), four-base mismatched DNA (100 nM), and unmatched DNA (100 nM) as well as high reproducibility for detecting HIV target DNA with five different electrodes. Moreover, the resulting GR/AuNCs biosensor showed good performance in HIVinfected human blood DNA. Wang et al. ${ }^{\mathbf{4 0 1}}$ developed an electrochemical DNA biosensor based on functionalized $\mathrm{Au}$ nanoclusters/graphene hybrids and Exo III-supported cascade target recycling (Fig. 19). In this designed biosensor, Exo IIIsupported cascade recycling that was induced by the target DNA, resulted in the final cleavage product, which acted as a linker between the capture probe and the functionalized $\mathrm{Au}$ nanoclusters/GR nanohybrid acting as interface. Using this signal enhancement strategy, the fabricated biosensor showed DNA detection range from $0.02 \mathrm{fM}$ to $20 \mathrm{pM}$ and an LOD of $0.057 \mathrm{fM}$. Functionalized Pt nanoclusters/graphene hydridbased biosensor also detected DNA using the catalysis of $3^{\prime}, 5,5^{\prime}$-tetramethylbenzidine-hydrogen peroxide $\left(\mathrm{TMB}-\mathrm{H}_{2} \mathrm{O}_{2}\right)$ an electrochemical signal.

3.4.1 Graphene nanopore-mediated DNA detection. Graphene nanopores have emerged as a promising route to fabricate devices for simultaneously measuring the ionic current and changes in local voltage in the transistor. ${ }^{\mathbf{4 3 7 - 4 3 9}}$ Graphene atomic layers serve as an efficient membrane that allows single-base resolution in an ionic current because of its single atomic-layer thickness. $^{\mathbf{4 4 0}}$ Sadeghi et al. ${ }^{\mathbf{4 4 1}}$ demonstrated DNA sequencing using bilayer graphene with nanopores by observing the currentvoltage characteristics of a bilayer graphene junction. Liu et al. ${ }^{\mathbf{4 0 3}}$ reported an electrochemical DNA sensor based on the signal 
(A)

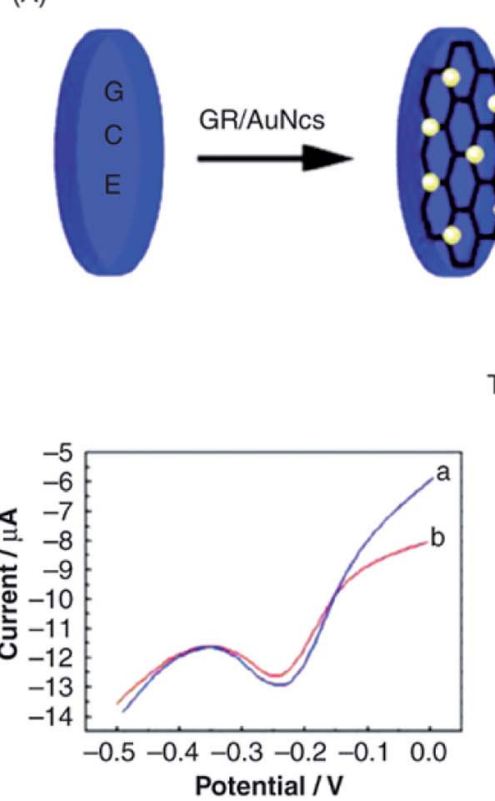

(B)

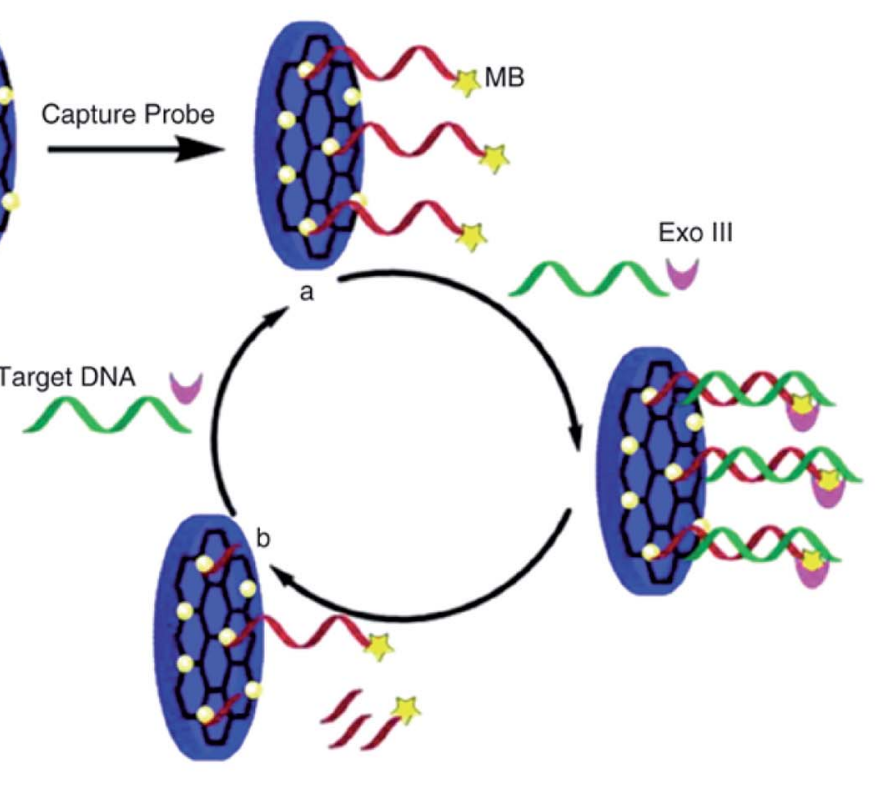

(C)
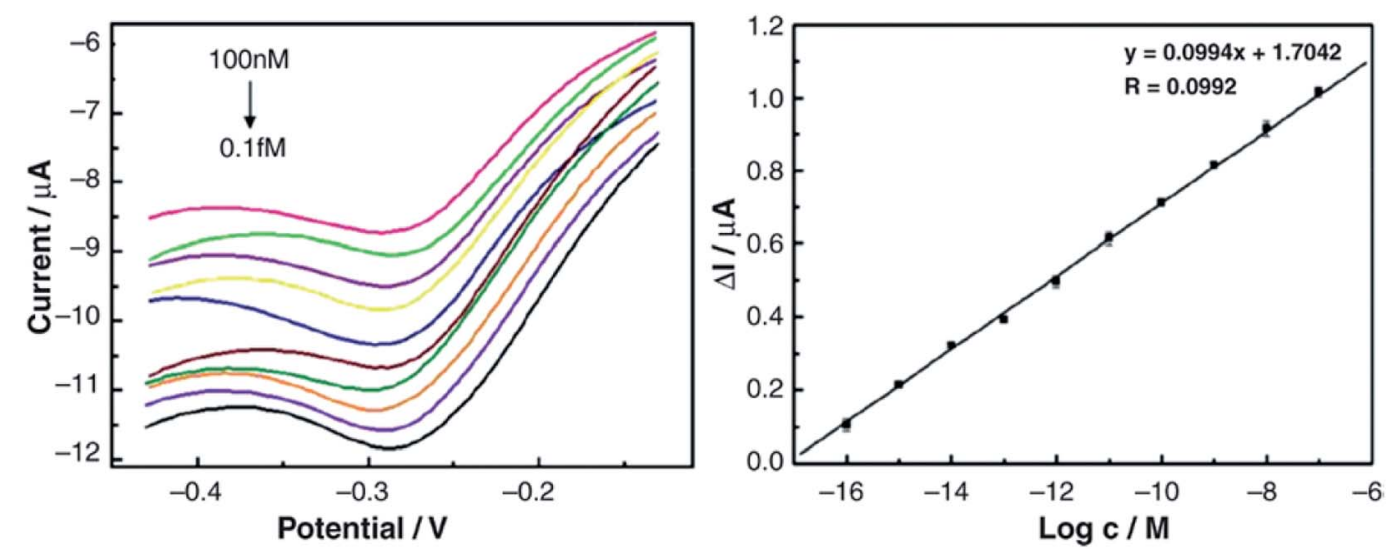

Fig. 18 (A) Schematic illustration for the biosensor fabrication process using graphene/gold nanoclusters (GR/AuNCs) modified GCE with exonuclease III (Exo III) supported target DNA recycling for detecting HIV DNA. (A) The initial signal obtained from the capture probe. (B) DPV of AuNCs/GR/GCE biosensor after incubation with $100 \mathrm{nM}, 10 \mathrm{nM}, 1 \mathrm{nM}, 100$ pM, 10 pM, 1 pM, $100 \mathrm{fM}, 10 \mathrm{fM}, 1 \mathrm{fM}$, and $0.1 \mathrm{fM}$ concentrations of target HIV gene and Exo III. (C) The linear relationship showing current change as a function of the logarithmic value of the target DNA concentration within a $0.1 \mathrm{fM}$ to $100 \mathrm{nM}$ range. [Reprinted with permission from ref. 399, Y. Wang, X. Bai, W. Wen, X. Zhang and S. Wang, Ultrasensitive Electrochemical Biosensor for HIV Gene Detection Based on Graphene Stabilized Gold Nanoclusters with Exonuclease Amplification, ACS Appl. Mater. Interfaces, 2015, 7, 18872-18879. Copyright@ American Chemical Society.]

amplification of rGO and gold nanoparticles/polyaniline (AuNPs/ PANI) hybrid composite for detecting specific DNA insertion sequence IS6110 of pathogenic bacteria Mycobacterium tuberculosis. The sensor showed specificity in the wide linear range from $1.0 \times 10^{-15}$ and $1.0 \times 10^{-9} \mathrm{M}$.

3.4.2 Graphene-based miRNA detection. Similar to DNA (nitrogenous bases of DNA are guanine (G), adenine (A), cytosine (C), thymine (T)), RNA is also a chain of nucleotides having four nitrogenous bases guanine (G), adenine (A), cytosine (C) and uracil (U); however, it is usually single stranded. MicroRNAs (miRNAs) are small, endogenous and single-stranded noncoding RNAs (approximately 18-25 nucleotides) that play vital roles in regulating gene expression through messenger RNA
(mRNA) degradation or translational repression. ${ }^{\mathbf{4 4 2}}$ MiRNAs are involved in many physiological processes of the human body, such as cell proliferation, differentiation and apoptosis, and their abnormal regulation will result in various diseases, including different types of cancer, kidney disease, central nervous system disease, cardiovascular disease, diabetes and viral infections. ${ }^{\mathbf{4 4 3}}$ Therefore, the precise and rapid detection of multiple miRNAs is of great importance to control and prevent various types of diseases. The isolation of miRNA is commonly carried out through commercial kits; however, there are several limitations since the kits contain numerous interfering RNAs, and the concentration of miRNA is not sufficient for detection. ${ }^{443}$ Several strategies have been explored for the sensitive 
(A)
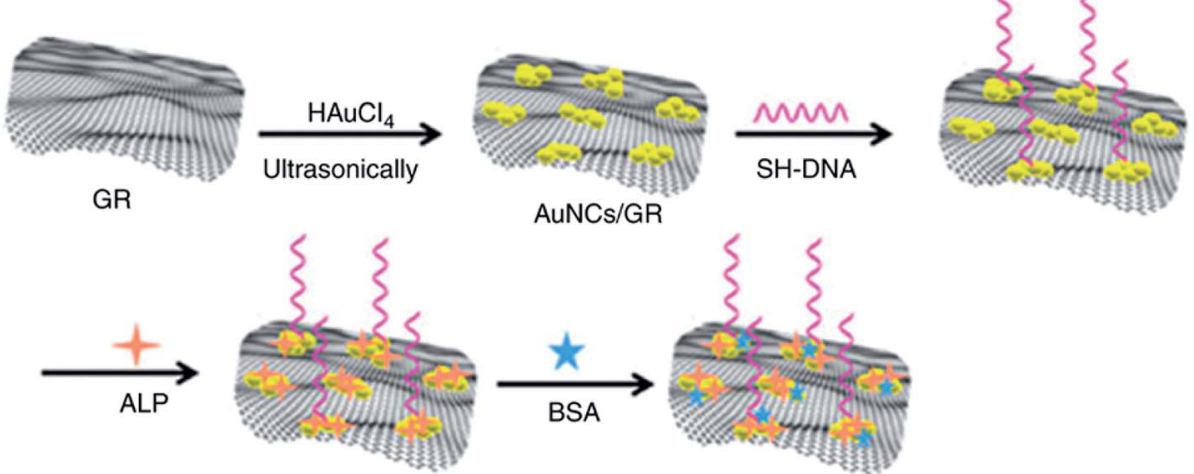

AuNCs/GR-DNA-ALP Conjugates

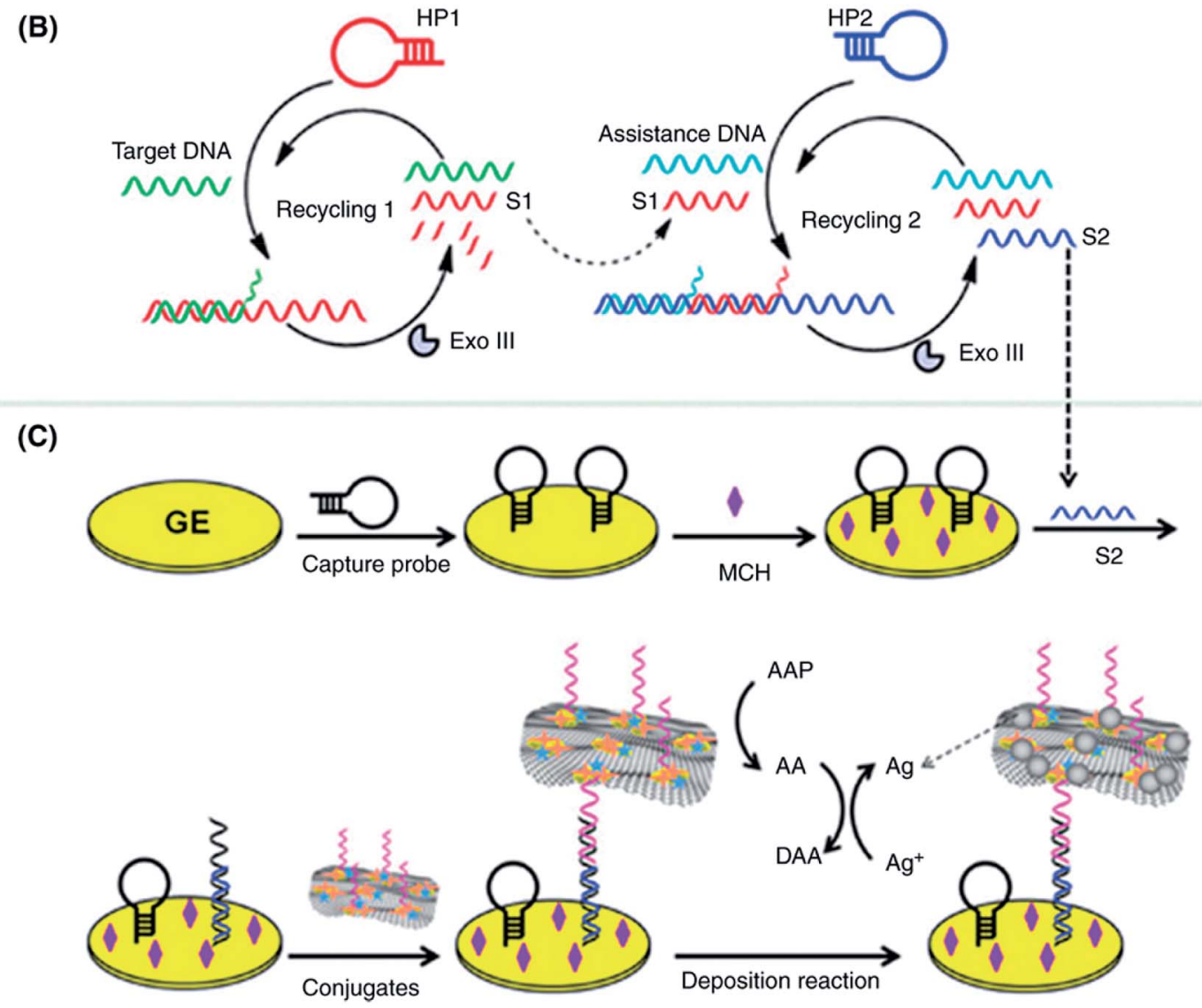

Fig. 19 Schematic illustration of the multiply amplified electrochemical biosensor for target DNA detection. (A) Fabrication steps of the functionalized AuNCs/GR nanohybrids; (B) principle of the target-triggered Exo III-assisted cascade target recycling; (C) construction of the biosensor using functionalized AuNC/GR nanohybrids as the interfaces of the enzyme-catalyzed silver deposition reaction. [Reprinted with permission from ref. 401, W. Wang, T. Bao, X. Zeng, H. Xiong, W. Wen, X. Zhang and S. Wang, Ultrasensitive Electrochemical DNA Biosensor Based on Functionalized Gold Clusters/Graphene Nanohybrids Coupling with Exonuclease III-Aided Cascade Target Recycling, Biosens. Bioelectron., 2017, 91, 183-189. Copyright@ Elsevier.]

detection of miRNA through significant challenges to improve the sensitivity, detection limit and sensitivity still remain. Several signal amplification strategies have been studied to enhance the sensitivity. ${ }^{43}$ Different approaches for the signal amplification are included in Table 5 .

GO and rGO exhibit strong affinity for single-stranded nucleic acids (ssNAs) via hydrogen bonding or $\pi-\pi$ interactions ${ }^{444}$ Hence, GO and rGO have been extensively used for sensing miRNAs. Hu et al. ${ }^{418}$ developed a miRNA electrochemical biosensor based on a GQDs and horseradish peroxidase (HRP). The GQDs/HRP can efficiently catalyze the $\mathrm{H}_{2} \mathrm{O}_{2}$ supported oxidation of 3,3,5,5-tetramethylbenzidine (TMB), resulting in an enhanced electrochemical signal and improved performance of the biosensor with a linear range from $1 \mathrm{fM}$ to 
$100 \mathrm{pM}$ and an LOD of $0.14 \mathrm{fM}$. Azimzadeh et al. ${ }^{\mathbf{4 1 9}}$ reported an electrochemical nanobiosensor for detecting plasma miRNA155 utilizing GO sheets decorated with thiol-functionalized gold nanorods (AuNRs), which were deposited on a GCE surface. The biosensor showed a linear range from $2.0 \mathrm{fM}$ to 8.0 pM, with an LOD of $0.6 \mathrm{fM}$ for detecting the target miRNA and was able to differentiate between complementary target miRNA, non-complementary, single- and three-base mismatch miRNA; therefore, this sensor was also used in clinical detection of the breast cancer cells. Cardoso et al. ${ }^{\mathbf{4 4 5}}$ fabricated an electrochemical biosensor for monitoring miRNA-155 down to the attomolar concentration level in breast cancer cells. The sensor was fabricated in two steps: first the immobilization of antimiRNA-155 onto the thiol-modified gold-screen-printed electrode and then followed by protecting the areas of nonspecific binding using mercaptosuccinic acid. The sensor detected miRNA-155 in a linear range from $10 \mathrm{aM}$ to $1.0 \mathrm{nM}$ with an LOD of $5.7 \mathrm{aM}$ in human serum. The biosensor also showed selectivity for the breast cancer antigen (CA-15.3) and BSA without any interference from cell lines of melanoma. The graphenebased biosensors can be used for clinical diagnosis of various diseases.

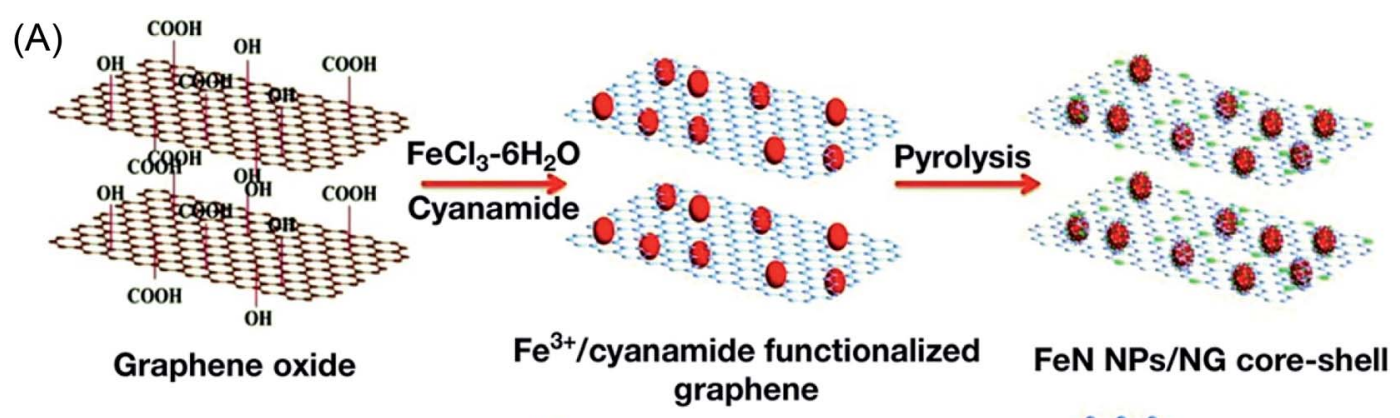

FeN NPs

FeN NPs (core) and NG (shell)

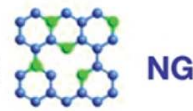

(B)

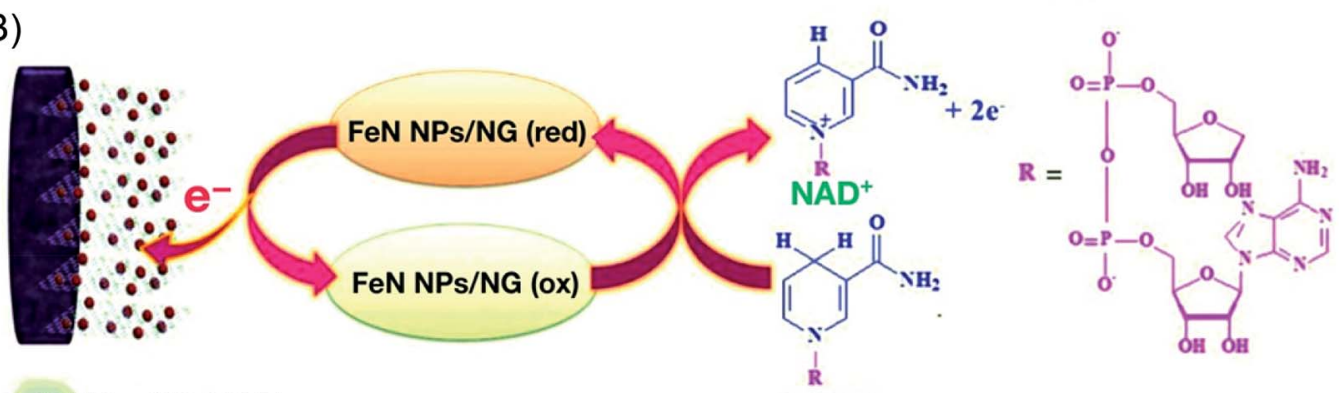

FeN NPs-NG/GCE

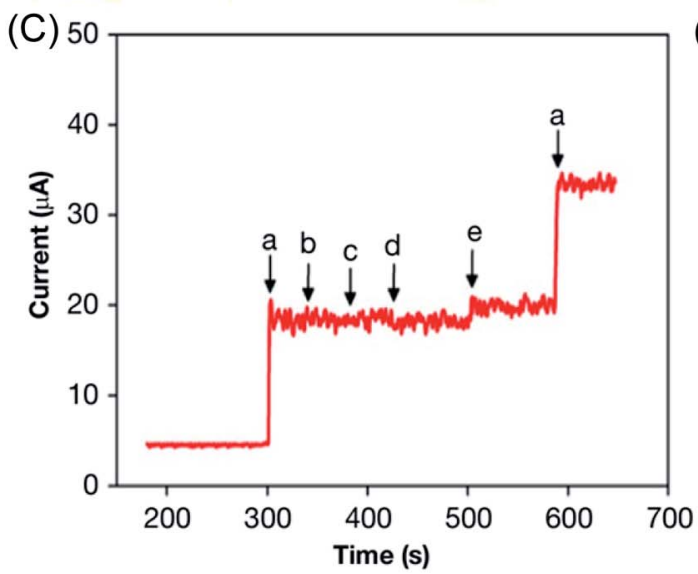

(D)

NADH

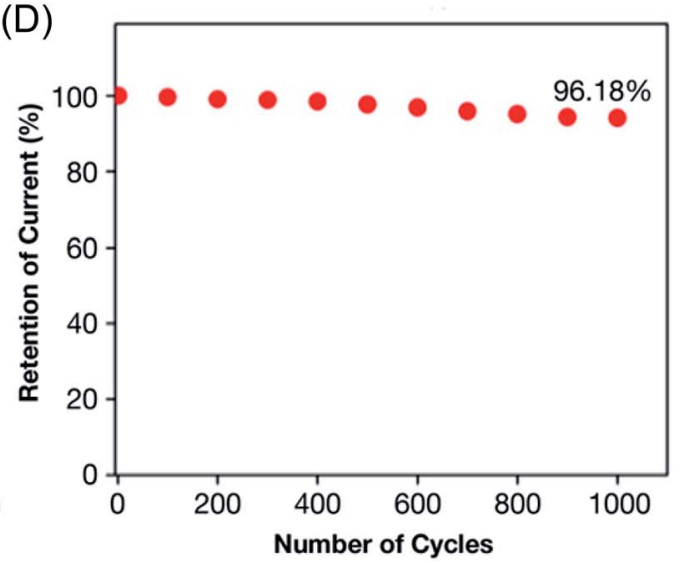

Fig. 20 (A) Fabrication of the iron nitride (FeN) NPs/NG core-shell hybrid. (B) NADH measurement mechanism involving a FeN NPs/NG coreshell hybrid-based electrode. (C) Selective amperometric response of FeN NPs/NG/GCE after adding (a) $1 \mathrm{mM} \mathrm{NADH,} \mathrm{(b)} 1 \mathrm{mM}$ glucose, (c) UA, (d) dopamine, and (e) AA at an applied potential of +0.35 V. (D) Stability of FeN NPs/NG/GCE based sensor in $0.5 \mathrm{mM} \mathrm{NADH}$ at an applied potential of $+0.35 \mathrm{~V}$ showing $96.18 \%$ of initial current retention after 1000 cycles. [Reprinted with permission from ref. 429, J. Balamurugan, T. D. Thanh, N. H. Kim and J. H. Lee, Facile Fabrication of FeN Nanoparticles/Nitrogen-Doped Graphene Core-Shell Hybrid and Its Use as a Platform for NADH Detection in Human Blood Serum, Biosens. Bioelectron., 2016, 83, 68-76. Copyright@ Elsevier.] 


\subsection{Detection of enzymes}

Nicotinamide adenine dinucleotide $(\mathrm{NADH})$ is a coenzyme involved in metabolic processes, and the $\mathrm{NAD}^{+} / \mathrm{NADH}$ redox couple (standard redox potential for half-reaction $=-0.32 \mathrm{~V}$ ) is involved in numerous enzymatic reactions in biological systems. A high overpotential (e.g., $0.6 \mathrm{~V} v s$. SCE) is needed for the direct oxidation of NADH with traditional electrodes. ${ }^{\mathbf{4 4 6}}$ Moreover, the electrooxidation of NADH is mostly an irreversible reaction, which causes significant polluting of electrodes. ${ }^{74}$ Graphene-based electrode materials have shown a great promise for the electrochemical detection of $\mathrm{NADH}$ with reduced overpotential and such results are summarized in Table 5. Tabrizi and Zand ${ }^{\mathbf{4 4 7}}$ reported synthesis of rGO using $\mathrm{NADH}$ as a mild reducing agent. The GO-modified electrode showed high electrocatalytic activity for the oxidation of $\mathrm{NADH}$, the detection of NADH at a reduced potential of $1.35 \mathrm{~V}$ with an LOD of $0.6 \mu \mathrm{M}$. Govindhan et al. ${ }^{\mathbf{4 2 5}}$ developed an electrochemical NADH sensor using a AuNPs/rGO nanocomposite without any redox mediators or enzymes. The fabricated sensor showed a sensitivity of $0.916 \mu \mathrm{A} \mu \mathrm{M}^{-1} \mathrm{~cm}^{-2}$, a linear range of $50 \mathrm{nM}$ to $500 \mathrm{mM}$ and an LOD of $1.13 \mathrm{nM}$, with no interference from AA, glucose, glutathione, or quinine bioanalytes. Mutyala et $a{ }^{427}$ developed a NADH biosensor using nitrogen-doped graphene-modified electrodes. The NADH biosensor displayed an enhanced sensitivity of $0.16 \mu \mathrm{A} \mu \mathrm{M}^{-1}$ with a linear range of 0.5-12 $\mu \mathrm{M}$ and an LOD of $0.37 \mu \mathrm{M}$. Moreover, the sensor exhibited stability after a period of 15 days with low surface contamination. Balamurugan et al. ${ }^{\mathbf{4 2 9}}$ reported an iron nitride (FeN) nanoparticle-encapsulated nitrogen-doped graphene for the electrochemical detection of NADH. Fig. 20 shows the fabrication and NADH measurement mechanism for the FeN NPs/NG core-shell hybrid electrode based biosensor, high selectivity of FeN NPs/NG/GCE toward NADH against interfering bioanalytes including glucose, UA, DA, and AA at an applied potential of $+0.35 \mathrm{~V}$, and also high stability of FeN NPs/NG/GCE sensor with $98.18 \%$ retention of initial current after 1000 cycles. The sensor exhibited sensitivity of $0.028 \mu \mathrm{A} \mu \mathrm{M}^{-1} \mathrm{~cm}^{-2}$, a wide linear range of 0.4 to $718 \mu \mathrm{M}$, and an LOD of $25 \mathrm{nM}$. In addition, the FeN NPs/NG/GCE biosensor showed negligible interference effects in the presence of common bioanalytes existing in human serum samples. Li et $a$ al $^{\mathbf{4 2 4}}$ fabricated a graphene-DNA tetrahedron-gold nanoparticle (AuNP)-modified gold disk electrode for the detection of NADH which showed a very low of LOD of $1 \mathrm{fM}$ with a significantly decreased potential of $0.28 \mathrm{~V}$ (vs. $\mathrm{Ag} / \mathrm{AgCl}$ ). The graphene biosensors can be used for the clinical diagnosis of NADH-related diseases.

\subsection{Detection of dopamine (DA), ascorbic acid (AA) and uric acid (UA)}

Dopamine is known to be a neurotransmitter present in the brain, and its level impacts the physiological functions in human body and also helps effect the treatment of disorders related to the central nervous system including Parkinson disease. ${ }^{\mathbf{4 4 8}}$ The electrochemical biosensors have been widely employed to detect a variety of biochemicals such as neurotransmitters in the brain. ${ }^{449-451}$ DA, AA and UA exhibit electrooxidation peaks at nearly the same potentials; therefore, many studies have focused on separating their signal potential with enhanced sensitivity and selectivity. ${ }^{\mathbf{4 5 2 , 4 5 3}}$ Ascorbic acid is an essential component of the human diet that helps prevent scurvy. It is present in the mammalian brain together with several neurotransmitter amines such as dopamine, epinephrine and norepinephrine. Moreover, UA and other oxypurines are the main products of human's purine metabolism. High levels of UA can lead to several diseases, including hyperuricemia, gout and Lesch-Nyhan disease. Therefore, accurate detection of DA, AA and UA is highly desirable in clinical monitoring and diagnosis of such diseases.

Carbon-based nanomaterials such as CNTs and GBMs have been studied for the electrochemical detection of DA, AA, and UA due to their unique electronic structure, high surface area, and electrical conductivity. ${ }^{\mathbf{1 9 , 4 5 4}}$ Graphene and GO have emerged as the most promising electrode materials for the detection of a wide range of analytes because of their enhanced electron transfer rate and structural orientation. ${ }^{\mathbf{4 5 5 , 4 5 6}}$ The simultaneous detection of DA, AA, and UA using graphene-based electrodes has been accomplished and such data are summarized in Table 6. Gao et $a l^{457}$ reported a GO-modified GCE for the selective detection of DA in the presence of interfering AA bioanalyte. The designed biosensor showed an LOD of $0.27 \mu \mathrm{M}$ for detecting DA, while no interference was observed by the addition of AA. The electrostatic repulsion between the GO and AA biomolecules makes the oxidation of AA difficult at the electrode surface. In comparison, the $\pi-\pi$ stacking as well as the electrostatic attraction between dopamine aromatic ring and delocalized $\pi$-electrons of GO promotes the oxidation. Li et al. ${ }^{458}$ used 3D nanocomposite based on $\mathrm{MoS}_{2}$ nanospheres, PANI loaded on rGO for simultaneous electrochemical detection of AA, DA and UA. Fig. 21 shows the differential pulse voltammetry (DPV) curves of $\mathrm{MoS}_{2}-\mathrm{PANI} / \mathrm{rGO} / \mathrm{GCE}$ electrode-based sensor and the plot of peak currents as a function of AA, DA and UA concentrations. The oxidation peak currents showed a linear increase with increasing AA, DA and UA concentrations. The $\mathrm{MoS}_{2}-\mathrm{PANI} / \mathrm{rGO}$ nanocomposite-based sensors displayed sensitivity over wide concentration ranges and low LODs for AA, DA and UA. DPV curves measured with $\mathrm{MoS}_{2}-\mathrm{PANI} / \mathrm{rGO} / \mathrm{GCE}$ electrode for a ternary nanocomposite of AA, DA, and UA biomolecules with their different concentrations showed three well-defined anodic peaks at different potentials; $0.052 \mathrm{mV}$ for AA, $0.196 \mathrm{mV}$ for DA, and $0.304 \mathrm{mV}$ for UA and simultaneously detected these three analytes with LODs of $2.49,0.65$ and $0.40 \mu \mathrm{M}$ for AA, DA and UA, respectively. The electrochemical sensor also showed high selectivity toward AA, DA and UA in the presence of interfering analytes including $\mathrm{ZnSO}_{4}, \mathrm{NaNO}_{3}$, glucose, citric acid, and glycine. Devaramani et al. $^{\mathbf{4 5 6}}$ used a covalently linked $p$-aminobenzene sulfonate multilayer on the surface of a graphite pencil core electrode for selectively detecting dopamine. The sensor exhibited a linear range of $0.5-10 \mu \mathrm{mol} \mathrm{L}^{-1}$ and an LOD of 0.095 $\mu \mathrm{mol} \mathrm{L}{ }^{-1}(\mathrm{~S} / \mathrm{N}=3)$.

To enhance the high electrocatalytic activity and sensitivity, graphene and GO can be doped with nitrogen, ${ }^{\mathbf{4 6 6 , 4 9 2}}$ modified with polymers, ${ }^{\mathbf{4 6 7 , 4 6 8 , 4 7 0}}$ and decorated with different inorganic metal and metal oxide nanoparticles ${ }^{\mathbf{1 9}}$ for the 
Table 6 Graphene-based biosensors for detecting dopamine (DA), ascorbic acid (AA), and uric acid (UA) ${ }^{a}$

\begin{tabular}{|c|c|c|c|c|c|c|c|c|}
\hline \multirow[b]{2}{*}{ Graphene-based nanocomposites } & \multicolumn{3}{|c|}{ Linear range $(\mu \mathrm{M})$} & \multicolumn{3}{|c|}{ Limit of detection $(\mu \mathrm{M})$} & \multirow[b]{2}{*}{ Sensitivity } & \multirow[b]{2}{*}{ Ref } \\
\hline & AA & DA & UA & $\mathrm{AA}$ & DA & $\mathrm{UA}$ & & \\
\hline GR/PtNPs & $0.15-34.4$ & $0.03-8.13$ & $0.05-11.85$ & 0.15 & 0.03 & 0.05 & 0.969 & 460 \\
\hline MWCNTs/GO nanoribbons & $0.1-8.5$ & $0.15-12.15$ & $0.15-11.4$ & 0.06 & 0.08 & 0.07 & - & 461 \\
\hline $\mathrm{MoS}_{2}$-PANI/rGO nanocomposite & $50-8000$ & $5.0-500$ & $1.0-500$ & 22.20 & 0.70 & 0.36 & - & 458 \\
\hline AuNPs/ $\beta$-cyclodextrin/GR & $300-2000$ & $0.50-150$ & $0.5-50$ & 10 & 0.15 & 0.21 & - & 464 \\
\hline Pd NPs/GR/CS composite & $100-4000$ & $20-200$ & $0.5-200$ & 20 & 0.1 & 0.17 & - & 465 \\
\hline N-doped carbon spheres/rGO & $50-1200$ & $0.5-90$ & $1-70$ & 0.65 & 0.012 & 0.018 & - & 466 \\
\hline GR/CS nanocomposite & - & $0.1-140$ & $1-125$ & - & 0.05 & 0.1 & - & 467 \\
\hline Polyaniline/GO fibrous nanocomposite & $25-200$ & $2-18$ & $2-18$ & 20 & 0.5 & 0.2 & 2.0 & 468 \\
\hline Tryptophan/GR & $0.2-3.4$ & $0.5-10$ & $10-1000$ & 10.09 & 0.29 & 1.24 & - & 469 \\
\hline Ni-Co hexacyanoferrate/GR & $0.5-2000$ & $0.2-500$ & - & 0.15 & 0.06 & - & & 474 \\
\hline GR/CNTs/sulfonated CS & $1.25-442$ & $1.25-357$ & $1.25-533$ & 0.06 & 0.06 & 0.06 & 1.68 & 475 \\
\hline AgNWs/rGO & $45-1550$ & $40-450$ & $35-300$ & 0.81 & 0.26 & 0.30 & - & 476 \\
\hline rGO-CNTs/ITO & $10-200$ & $0.2-8$ & $0.2-16$ & 5.31 & 0.04 & 0.17 & - & 477 \\
\hline Au@PdNPs/rGO composite & $1-800$ & $0.1-100$ & $0.1-350$ & 0.28 & 0.024 & 0.02 & - & 478 \\
\hline N-doped GR flakes & $80-2000$ & $0.5-150$ & $3-60$ & 0.58 & 0.015 & 0.015 & - & 479 \\
\hline Hemin-GO sheets & $1-100$ & $0.5-40$ & $0.5-50$ & 0.3 & 0.17 & 0.17 & - & 480 \\
\hline Pd@Ag nanoflowers/rGO & $1-4100$ & $0.05-112$ & $3-186$ & 0.185 & 0.017 & 0.654 & - & 481 \\
\hline GR fibers $/ \mathrm{NiCo}_{2} \mathrm{O}_{4}$ & $200-750$ & $1-13$ & $10-26$ & 50 & 0.1 & 0.2 & - & 482 \\
\hline PtNPs/GR paper & $0.087-100$ & - & - & 0.005 & - & 2.00 & - & 483 \\
\hline GR/ZnO composite & $50-2350$ & $1-70$ & $3-330$ & 3.71 & 0.33 & 1.08 & - & 484 \\
\hline PAA/polypyrrole/GO & $300-440$ & $32-60$ & - & 1.962 & 1.025 & - & - & 485 \\
\hline
\end{tabular}

${ }^{a}$ Abbreviations used: HP: hydrophilic polymer, CNT: carbon nanotubes, CTAB: cetyl trimethylammonium bromide, PPy: polypyrrole, CVD: chemical vapor deposition, GRHG: graphene hydrogel, PAA: polyacrylic acid, PAM: polyacrylamide.

amplified detection of DA, AA, and UA. Yue et al. ${ }^{493}$ reported a vertically aligned $\mathrm{ZnO}$ nanowire array (NWA) assembled on 3D GF and applied to the detection of UA, DA and AA employing DPV technique, as shown in Fig. 22. The fabricated ZnO NWA/GF sensor showed high selectivity for both UA and DA with an LOD of 1 nM. DA and UA are considered biomarkers for the diagnosis of Parkinson disease. The sensors exhibited high performance in real samples for the detection of UA levels at $265 \pm 20 \mu \mathrm{M}$ in the serum of patients suffering from Parkinson disease compared to UA levels of $355 \pm 30 \mu \mathrm{M}$ in healthy individuals. Srivastava et al. ${ }^{494}$ prepared functionalized multilayer graphene for developing an amperometric urea biosensor that showed a linear range from 10 to $100 \mathrm{mg} \mathrm{dL}^{-1}$ and a high sensitivity of $5.43 \mu \mathrm{A} \mathrm{mg}^{-1}$ $\mathrm{dL} \mathrm{cm}{ }^{-2}$ with an LOD of $3.9 \mathrm{mg} \mathrm{dL}^{-1}$. Dutta et al. ${ }^{495}$ fabricated an enzyme-free urea sensor employing a nanocomposite of $\mathrm{SnO}_{2}$ QDs/rGO. Due to the synergistic effect between these components, the developed sensor displayed high electrochemical activity for the detection of urea in a linear range of $1.6 \times 10^{-14}$ to $3.9 \times 10^{-12} \mathrm{M}$ and a very low LOD of $11.7 \mathrm{fM}$.

\subsection{Detection of cancer biomarkers}

The foremost cause of human death worldwide is cancer. The accurate detection and diagnosis of cancer during its early stage is significantly important for its successful treatment. ${ }^{496}$ Cancer diagnostic techniques such as blood analysis, clinical bioimaging and morphological changes in cancer cells have been widely employed for cancer diagnosis. However, these techniques still lack the required sensitivity, selectivity and reproducibility; therefore, these are not so accurate in the precise early stage detection of cancer cells. ${ }^{497}$ The use of cancer biomarkers seems more promising for early stage cancer detection, monitoring, diagnosis and subsequent treatment. ${ }^{498,499} \mathrm{~A}$ biomarker is defined as a biological feature that 
(a)

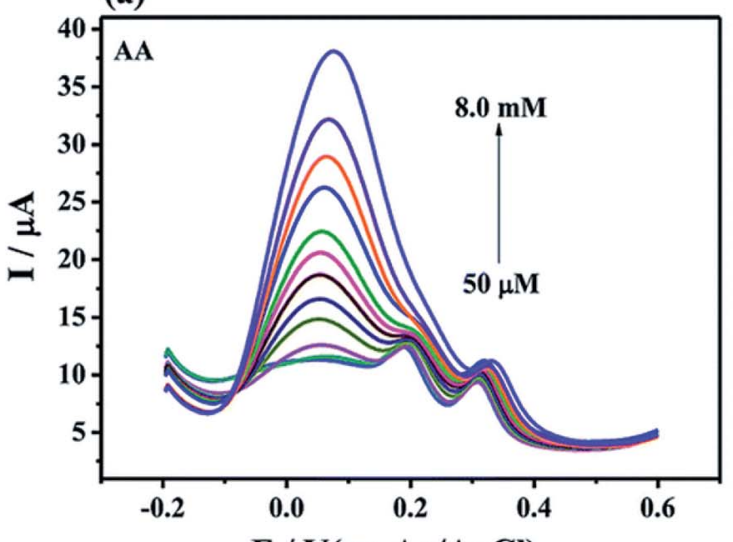

(c)

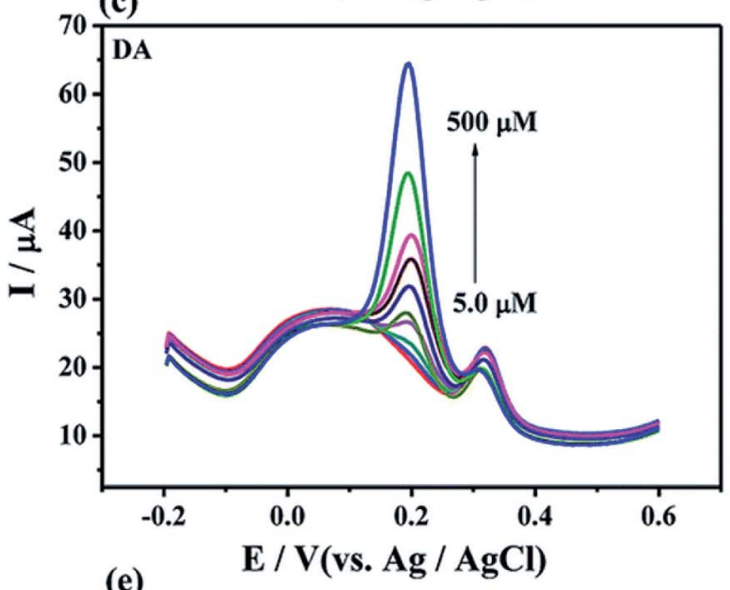

(e)

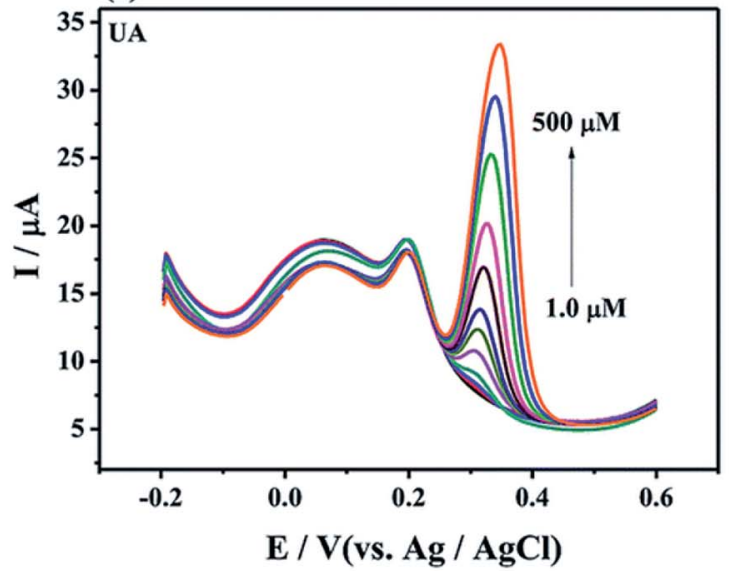

(b)

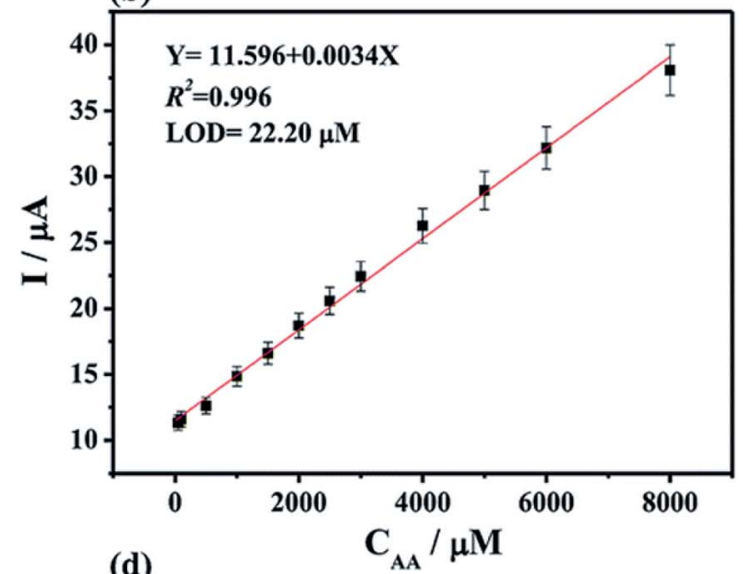

(d)

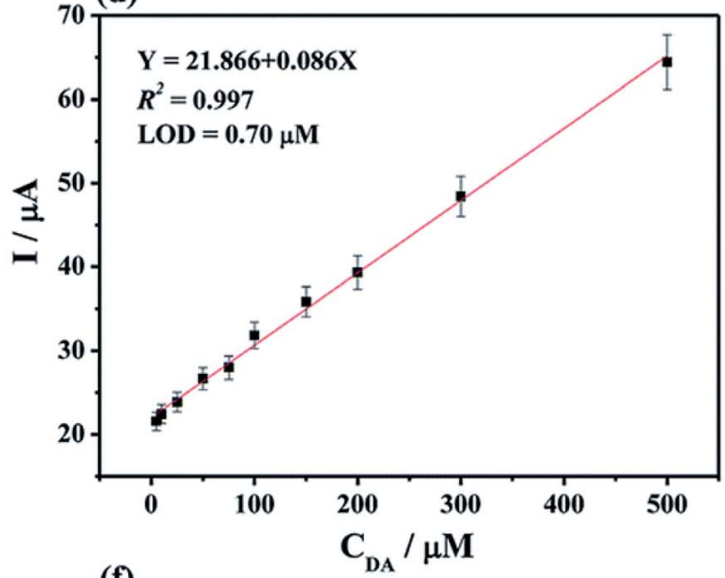

(f)

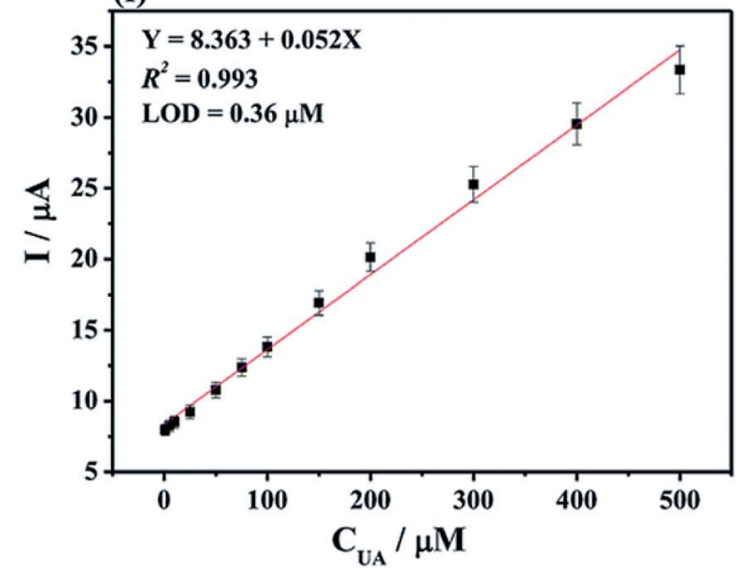

Fig. 21 (a) DPV curves of $\mathrm{MOS}_{2}-\mathrm{PANI} / \mathrm{rGO} / \mathrm{GCE}$ electrode and (b) the plot of peak currents as a function of $A A$ concentration from $50 \mu M$ to $8.0 \mathrm{mM}$ containing $75 \mu \mathrm{M}$ DA and $75 \mu \mathrm{M}$ UA. (c) DPV curves of $\mathrm{MoS}_{2}-\mathrm{PANI} / \mathrm{rGO} / \mathrm{GCE}$ and (d) the plot of peak currents as a function of DA concentration from 5.0 to $500 \mu \mathrm{M}$ containing $1.0 \mathrm{mM} \mathrm{AA}$ and $75 \mu \mathrm{M} \cup \mathrm{A}$. (e) DPV curves of $\mathrm{MoS}_{2}-\mathrm{PANI} / \mathrm{rGO} / \mathrm{GCE}$ and (f) the plot of peak currents as a function of UA concentration from $1.0 \mu \mathrm{M}$ to $500 \mu \mathrm{M}$ containing $1.0 \mathrm{mM}$ AA and $75 \mu \mathrm{M}$ DA. The solution used in the measurements was $0.1 \mathrm{M}$ PBS (pH 7.0) containing 0.1 M KCl. [Reprinted with permission from ref. 458, S. Li, Y. Ma, Y. Liu, G. Xin, M. Wang, Z. Zhang and Z. Liu, Electrochemical sensor based on a three dimensional nanostructured $\mathrm{MoS}_{2}$ nanosphere-PANI/reduced graphene oxide composite for simultaneous detection of ascorbic acid, dopamine, and uric acid, RSC Adv., 2019, 9, 2997-3003. Copyright@ Royal Society of Chemistry.]

can be an indicator of both normal and abnormal biological processes, pathogenic conditions, or pharmacological responses to applied therapeutic interventions. ${ }^{500}$ There is an increasing demand for disease diagnostic tools which require highly sensitive and accurate methods for detecting cancerrelated biomarkers. ${ }^{69,499}$ In particular, the clinical detection of cancer biomarkers is critically important for early-stage diagnosis of cancer-related diseases and proteomics research to 
(A)
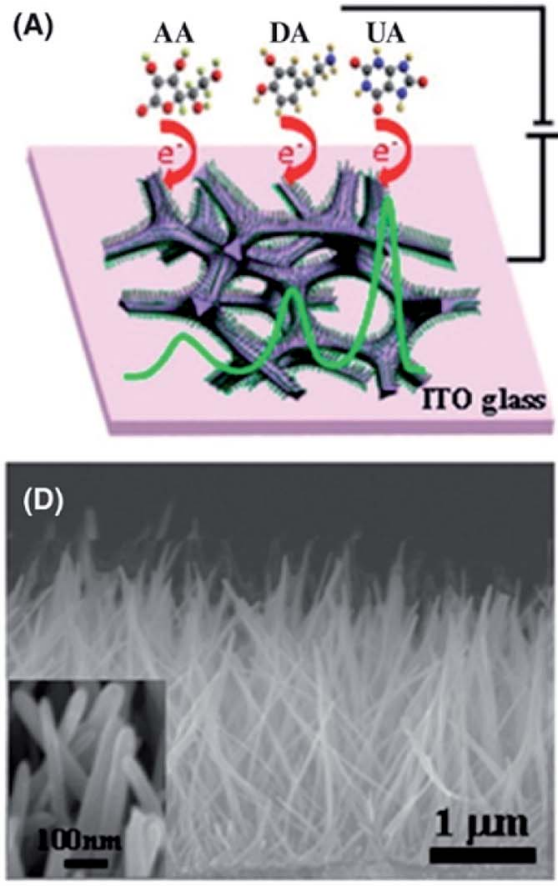

(G)

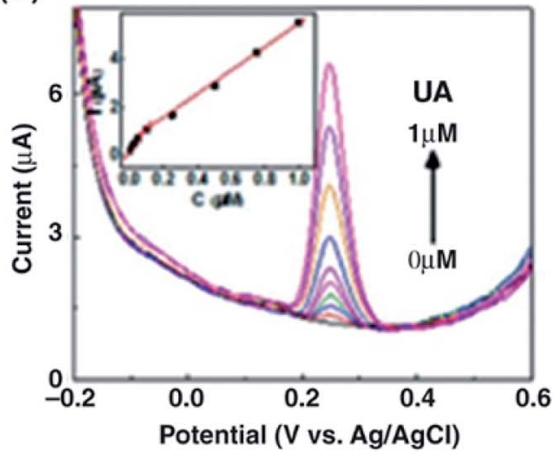

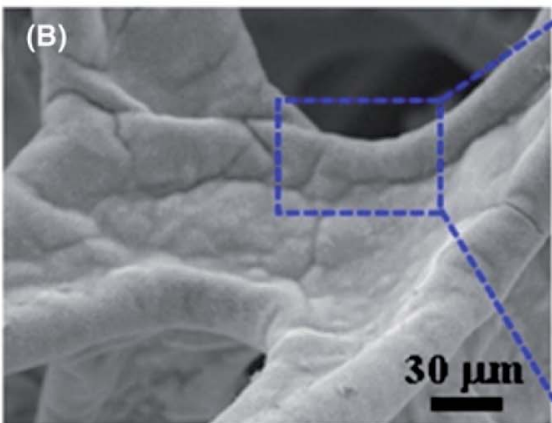
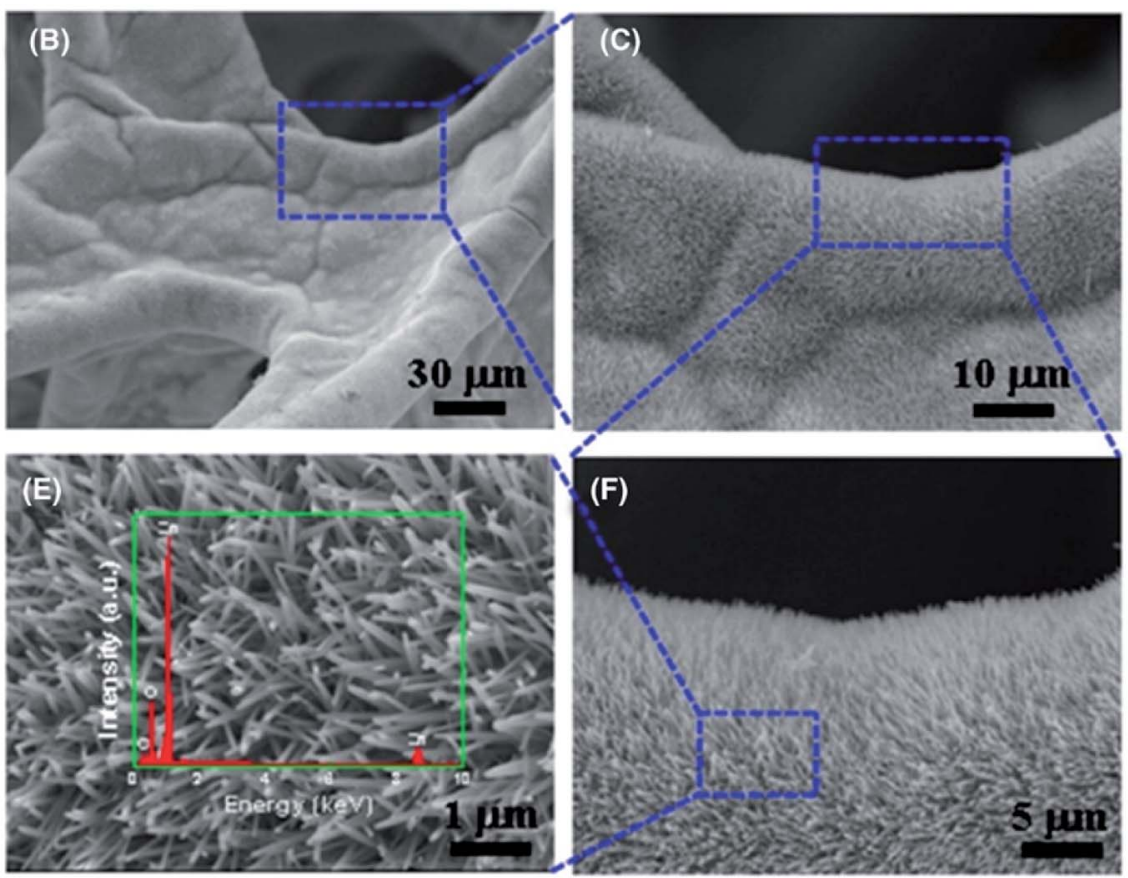

(H)

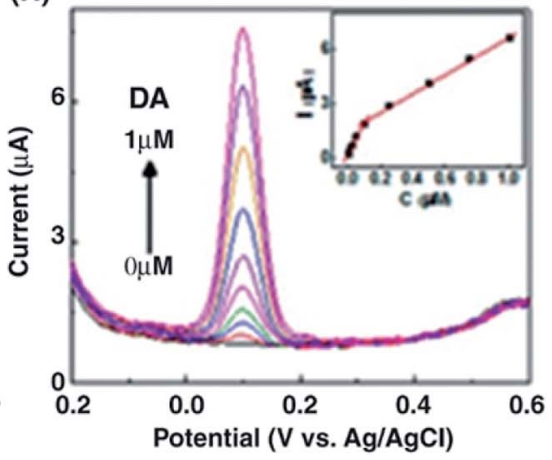

(I)

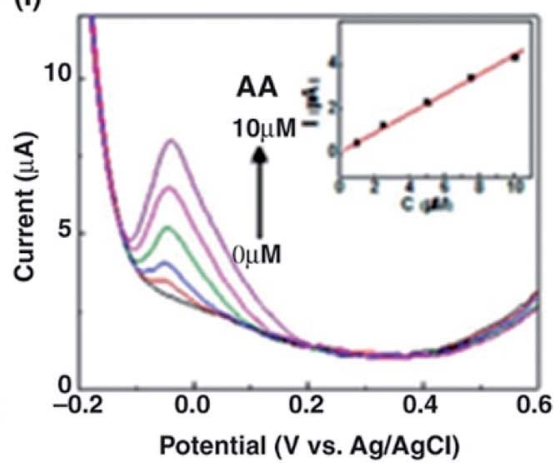

Fig. 22 (A) Schematic illustration of the ZnO NWAs/GF electrode for the simultaneous detection of UA, DA, and AA. (B-E) SEM images of the ZnO NWAs assembled on the 3D GF recorded at different magnifications. Inset shows the EDX of the ZnO NWAs. (F) SEM images showing the height of ZnO NWAs ( 2 $\mu \mathrm{m})$. Inset shows the diameter of the ZnO NWAs ( 40 nm). (G-l) DPV curves for UA, DA, and AA measured using a ZnO NWA/ GF electrode at different concentrations. The UA concentrations from the bottom are $0-1 \mu \mathrm{M}$. The DA concentrations from the bottom are $0-1$ $\mu \mathrm{M}$. The AA concentrations from the bottom are 0-10 $\mu \mathrm{M}$. Insets show plots of the oxidation peak current as a function of concentration of each biomolecule, showing two slopes for UA and DA. [Reprinted with permission from ref. 493, H. Y. Yue, S. Huang, J. Chang, C. Heo, F. Yao, S. Adhikari, F. Gunes, L. C. Liu, T. H. Lee, E. S. Oh, B. Li, J. J. Zhang, T. Q. Huy, N. V. Luan and Y. H. Lee, ZnO Nanowire Arrays on 3D Hierarchical Graphene Foam: Biomarker Detection of Parkinson's Disease, ACS Nano, 2014, 8, 1639-1646. Copyright@ American Chemical Society.]

understand of the biological processes involved in causing cancer. ${ }^{501}$ To discard false positives in the diagnosis of cancer that may result from the population inequality in the genetic expression of a single biomarker, concurrent detection of a series of protein biomarkers are required. ${ }^{498}$ In recent years, electrochemical techniques have become attractive for the immunoassay of protein biomarkers, owing to their low cost, simplicity, high sensitivity, and miniaturization. However, their sensing performance still needs to be improved for rapid and sensitive detection.

Nanomaterials offer potential platforms to diagnosis and treatment of cancer; therefore, research efforts have moved from the preclinical stage to practical point-of-care applications. ${ }^{502}$ Nanomaterials such as metal NPs, magnetic NPs, QDs, and CNTs have been studied in the design of biosensors to increase the sensitivity of electrochemical detection techniques. ${ }^{503-505}$ Graphene and GO are particularly attractive materials for use in biomarker detection owing to their easy surface modification chemistry and unique electrocatalytic, optical, chemical, and electrical properties. ${ }^{\mathbf{5 0 6 , 5 0 7}}$ Furthermore, metal nanoparticles such as AuNPs have been used as immobilization matrices in developing electrochemical biosensors due to their strong adsorption abilities, easy surface modification, high stability and electrical conductivity. ${ }^{508}$ Table 7 
summarizes graphene-based nanocomposites using cancer biomarkers for various disease diagnosis. Zhu et al. ${ }^{509}$ developed an electrochemiluminescence immunosensor using CdSe QD-decorated poly(diallyldimethylammonium chloride)graphene and gold nanoparticles for the detection of ractopamine. PDDA-graphene and AuNPs were used in order to improve electron conduction, efficient loading of antibodies, and to create a large surface area for antigen binding. The sensor showed a linear range of 0.01 to $1000 \mathrm{ng} \mathrm{mL}^{-1}$ and a low LOD of $2.6 \mathrm{pg} \mathrm{mL} \mathrm{m}^{-1}(\mathrm{~S} / \mathrm{N}=3)$ and displayed high stability as well as sensitivity for determining biomarkers. Kumar et al. ${ }^{\mathbf{5 1 0}}$ developed PdNP-decorated rGO-based electrochemical sensor for the label-free detection of prostate-specific antigen (PSA), a prostate cancer biomarker. The fabricated biosensor exhibited

Table 7 Graphene-based biosensors for detecting different cancer biomarkers ${ }^{a}$

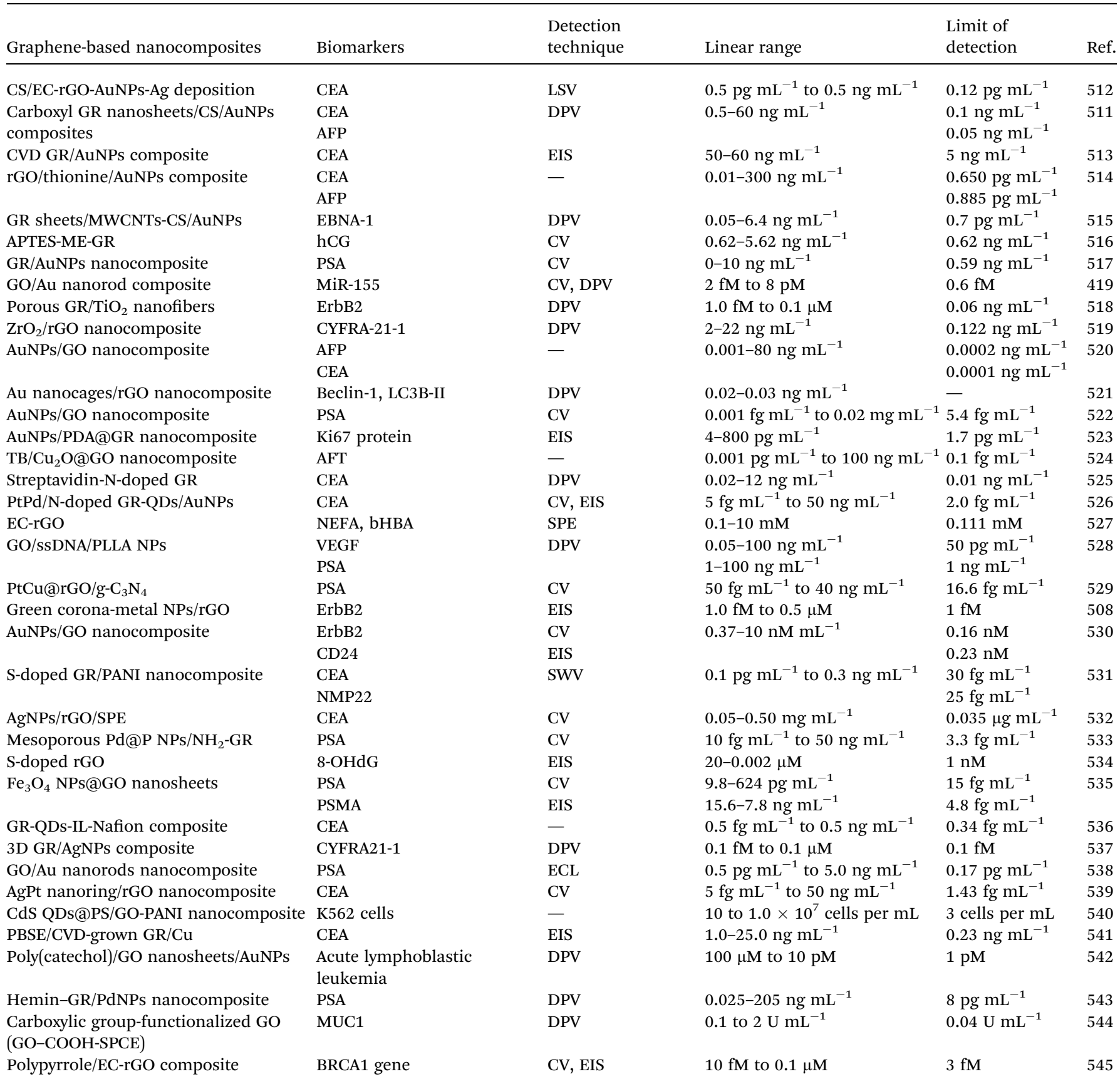

${ }^{a}$ Abbreviations used: CEA: carcinoembryonic antigen, AFP: $\alpha$-fetoprotein, DPV: differential pulse voltammetry, NMP22: nuclear matrix protein 22, SWV: square wave voltammetry, NEFA: nonesterified fatty acids, bHBA: beta-hydroxybutyrate, ErbB2: epidermal growth factor receptor, MUC1: mucin1, AFT: a-fetoprotein, MPS: mesoporous silica, hCG: human chorionic gonadotropin, APTES: 3-aminopropyl-triethoxysilane, ME-GR: multilayer epitaxial graphene, SPE: screen-printed carbon electrode, 8-OHdG: 8-hydroxy-2'-deoxyguanosine, EBNA-1: Epstein-Barr virus nuclear antigen 1, TB: toluidine blue, SPE: screen-printed electrode, SPCE: screen-printed carbon electrode, VEGF: vascular endothelial growth factor, PSA: prostate-specific antigen, PSMA: prostate-specific membrane antigen, PDA: polydopamine, PS: polystyrene, PANI: polyaniline, BRCA: breast cancer gene, CYFRA21-1: cytokeratin fragment antigen 21-1, PLLA: poly(L-lactide), IL: ionic liquid, PBSE: 1-pyrenebutanoic acid succinimidyl ester. 
a high sensitivity of $28.96 \mathrm{~mA} \mathrm{~mL} \mathrm{ng} \mathrm{m}^{-1} \mathrm{~cm}^{-2}$ with an LOD of 10 $\mathrm{pg} \mathrm{mL}^{-1}$. Ali et al. ${ }^{508}$ reported the green synthesis of metal (Pd, $\mathrm{Pt}, \mathrm{Ag}$ and $\mathrm{Au}$ ) nanoparticles on rGO sheets using a black pepper extract and applied the nanocomposite-based sensor to the detection of epidermal growth factor receptor in breast cancer diagnosis. The designed biosensor can be employed for detecting ErbB2 concentrations ranging from $1.0 \mathrm{fM}$ to $0.5 \mathrm{mM}$. Chen et al. ${ }^{511}$ reported an immunosensor using graphene-based nanocomposites for simultaneously detecting multiple cancer biomarkers, such as carcinoembryonic antigen (CEA) and $\alpha$ fetoprotein (AFP), as shown in Fig. 22A. In this study, the carboxyl-functionalized graphene nanosheet was first immobilized with toluidine blue and labeled with anti-CEA $(\mathrm{Ab} 2,1)$ or immobilized with Prussian blue and labeled with anti-AFP $(\mathrm{Ab} 2,2)$. Thereafter, the captured anti-CEA $(\mathrm{Ab} 1,1)$ and antiAFP (Ab1,2) were immobilized on a CS-AuNP-modified electrode via EDC/NHS. The developed biosensors showed a linear

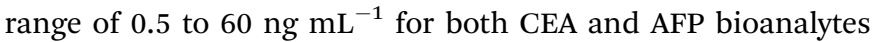
and an LOD of $0.1 \mathrm{ng} \mathrm{mL} \mathrm{md}^{-1}$ and $0.05 \mathrm{ng} \mathrm{mL}^{-1}$ for CEA and AFP, respectively (Fig. 23B).

Cancer biomarkers have been developed using graphenebased nanocomposites. Ren et al. ${ }^{531}$ used sulfur-doped GO/ polyaniline (PANI) composite as a matrix and mesoporous NKF-5-3 as a label for developing tri-antibody dual-channel immunological biosensors for the detection of cancer biomarkers, such as carcino-embryonic antigen (CEA) and nuclear matrix protein 22 (NMP22) as shown in Fig. 24A. In this approach, two types of labels were prepared on the biosensor using two antibodies (anti-CEA anti-NMP22) and two antigens (CEA and NMP22). Toluidine blue (TB) and neutral red were employed as the signals to detect the CEA and NMP22 antigens. The immunosensor biosensor was incubated with two types of labels; AuNP-NKF-5-3|anti-NMP22|neutral red|BSA and AuNPNKF-5-3|anti-CEA|TB|BSA which were NMP22 and CEA labels, respectively. The tri-antibody dual-channel immunosensor showed improved sensing performance having linear ranges of $0.1 \mathrm{pg} \mathrm{mL}^{-1}$ to $0.3 \mathrm{ng} \mathrm{mL} \mathrm{m}^{-1}$ for both NMP22 and CEA, and the low LODs of $25 \mathrm{fg} \mathrm{mL}^{-1}$ for NMP22 antigen and $30 \mathrm{fg} \mathrm{mL}^{-1}$ for CEA antigen. The immunosensor biosensor also exhibited high selectivity toward NMP22 and CEA in the presence of interfering bioanalytes including BSA, $\alpha$-fetoprotein, human immunoglobulin G (HIgG), PSA, and breast cancer gene BRCA1 and can be used for the analysis of bladder carcinoma biomarkers. Ali Saeed et al. ${ }^{530}$ fabricated an electrochemical DNA biosensor for detecting the ErbB2 and CD24 biomarkers in order to evaluate breast cancer. Their strategy was based on a sandwich hybridization assay by immobilizing the capture DNA probe of the target DNA on GO-modified AuNPs (Fig. 24B). An HRP-

(A)
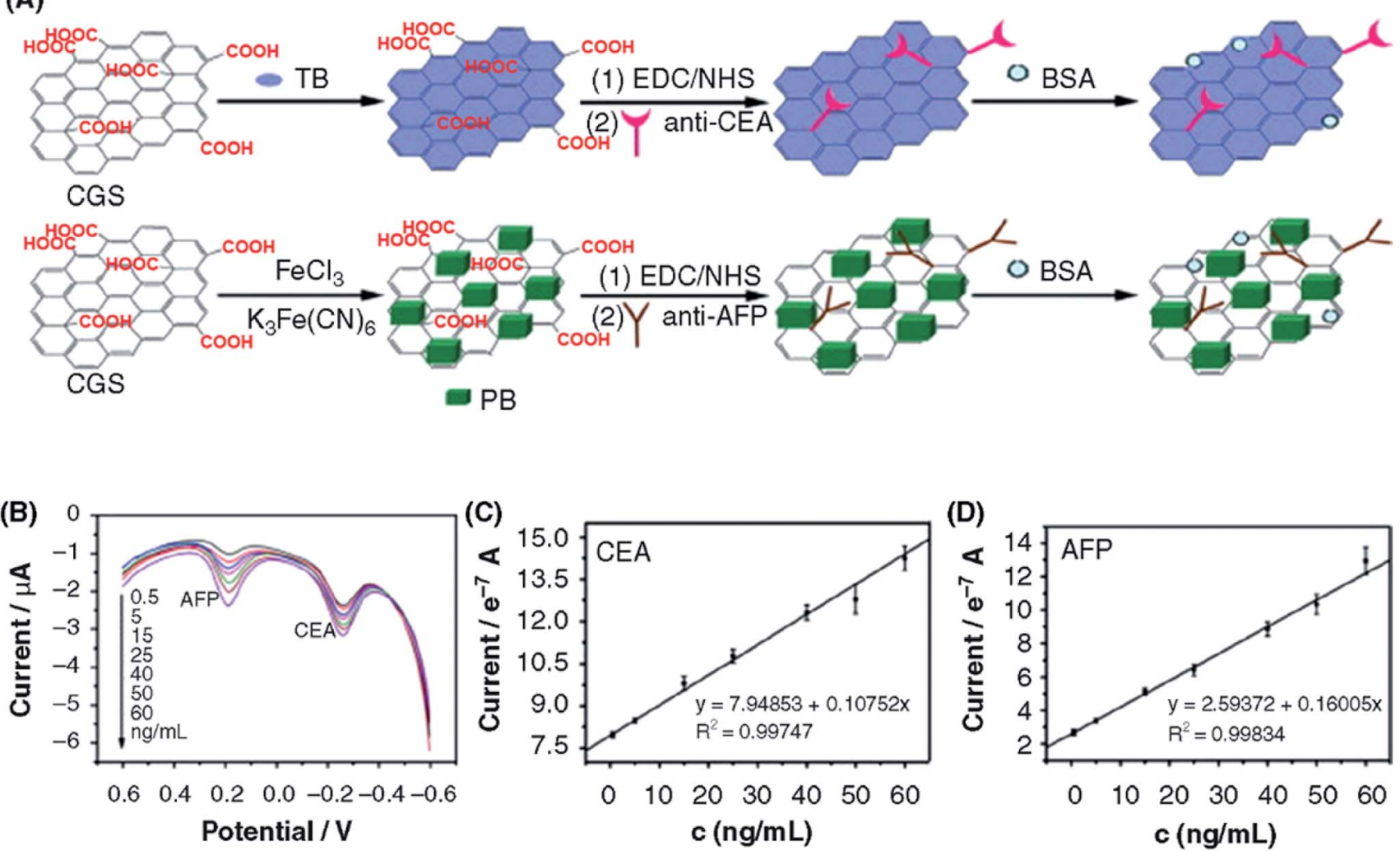

Fig. 23 (A) Schematic illustration of the preparation of biofunctional CGS nanocomposites. (B) DPV responses of the proposed immunosensor after incubation with different concentrations of CEA and AFP. (C) and (D) Calibration curves of the multiplex immunoassay toward CEA and AFP in 0.1 M PBS, pH 6.5. [Reprinted with permission from ref. 511, X. Chen, X. Jia, J. Han, J. Ma, Z. Ma, Electrochemical immunosensor for simultaneous detection of multiplex cancer biomarkers based on graphene nanocomposites, Biosens. Bioelectron., 2013, 50, 356-361. Copyright $\odot$ Elsevier.] 
(A)

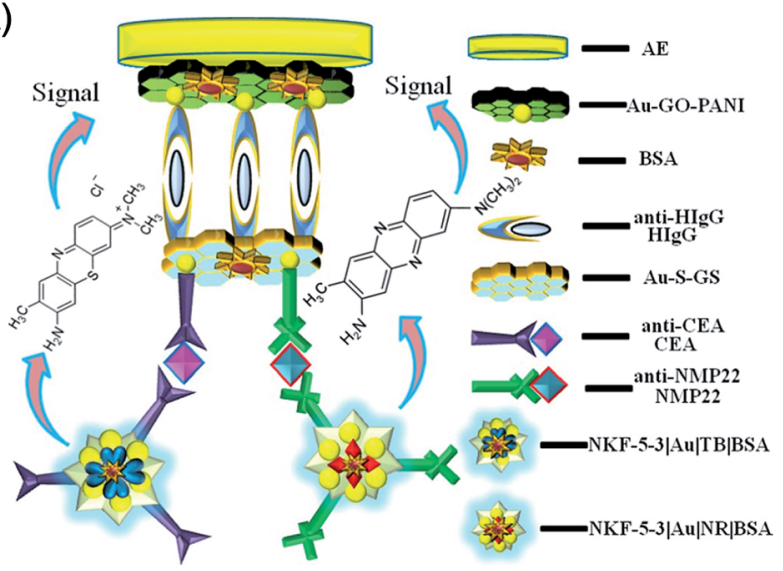

(B)

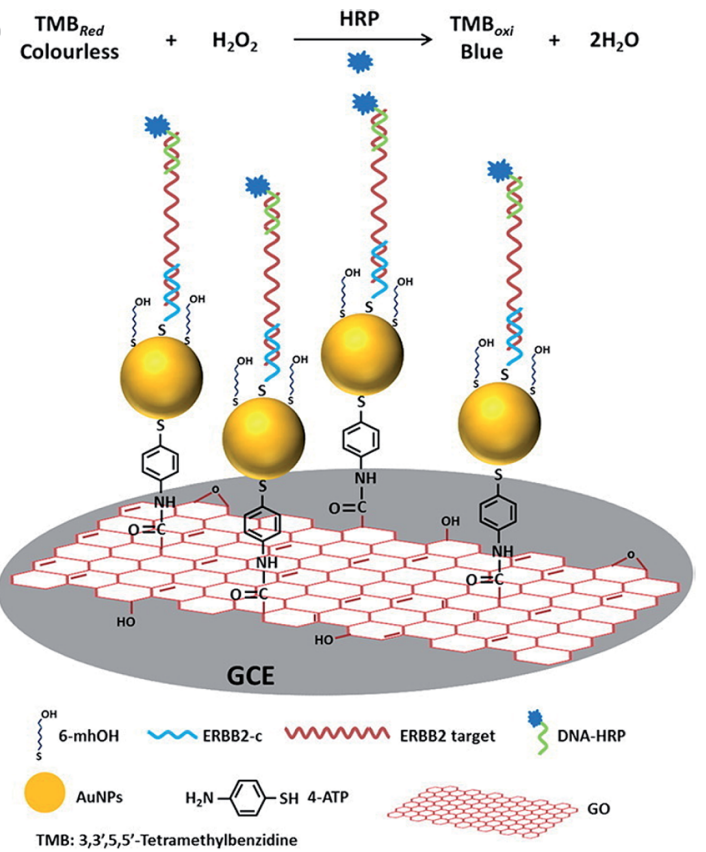

Fig. 24 (A) Schematic representation of the fabrication of the triantibody dual-channel immunological biosensor for detecting cancer biomarkers; nuclear matrix protein 22 (NMP22) and carcino-embryonic antigen (CEA). [Reprinted with permission from ref. 531, X. Ren, $\mathrm{H}$. Ma, T. Zhang, Y. Zhang, T. Yan, B. Du and Q. Wei, Sulfur-Doped Graphene-Based Immunological Biosensing Platform for Multianalysis of Cancer Biomarkers, ACS Appl. Mater. Interfaces, 2017, 9, 3763737644. Copyright@ American Chemical Society]. (B) Schematic presentation of the proposed sandwich-type DNA sensor showing the prepared AuNPs-GO/GCE and the hybridization of target DNA with the specific capture probe and HRP-labeled probe. [Reprinted with permission from ref. 530, A. A. Saeed, J. L. A. Sánchez, C. K. O'Sullivan, M. N. Abbas, DNA Biosensors Based on Gold NanoparticlesModified Graphene Oxide for the Detection of Breast Cancer Biomarkers for Early Diagnosis, Bioelectrochemistry, 2017, 118, 91-99. Copyright@ Elsevier.]

labeled probe was used to detect the concentration of the DNA biomarker. The biosensor achieved the sensitivities of $378 \mathrm{nA}$ $\mathrm{nM}^{-1}$ with an LOD of $0.16 \mathrm{nM}$ for ErbB2 breast cancer biomarker and $219 \mathrm{nA} \mathrm{nM}^{-1}$ with LOD of $0.23 \mathrm{nM}$ for CD24 control marker. Song et al. ${ }^{515}$ reported a sandwich-type electrochemical biosensor using an AuNPs/MWCNTs/GR/CS nanocomposite with a signal amplification strategy involving a hybridization chain reaction (HCR) for the detection of Epstein-Barr virus nuclear antigen 1 (EBNA-1). Similar to the sandwich immunoassay approach, the sandwich immunereactions were initially formed by functionalization with the captured antibody, EBNA-1 and a secondary antibody in DNAconjugated carboxyl MWCNTs. The double-stranded DNA (dsDNA) was formed from the HCR. This HCR-assisted amperometric signal amplification strategy significantly increased the detection range of target concentrations (0.05$6.4 \mathrm{ng} \mathrm{mL}{ }^{-1}$ ) with an LOD as low as $0.7 \mathrm{pg} \mathrm{mL}^{-1}$. Jang et al. ${ }^{517}$ showed a 3D electrochemical immunosensor based on a GR/ AuNP composite for the label-free detection of PSA over a linear range of $0-10 \mathrm{ng} \mathrm{mL}^{-1}$ with an LOD of $0.59 \mathrm{ng} \mathrm{mL}^{-1}$. Feng et al. ${ }^{529}$ reported a sandwich-type immunosensor for detecting PSA using AuNPs supported on thionine functionalized GO. In addition, bimetallic PtCu NPs were loaded on $\mathrm{rGO} / \mathrm{graphitic}$ carbon nitride $\left(\mathrm{g}-\mathrm{C}_{3} \mathrm{~N}_{4}\right)$ and used as labels for combining secondary antibodies $\left(\mathrm{Ab}_{2}\right)$ and amplification of signals. The designed immunosensor displayed a linear range from $50 \mathrm{fg} \mathrm{mL}^{-1}$ to $40 \mathrm{ng} \mathrm{mL}{ }^{-1}$ with an LOD of $16.6 \mathrm{fg} \mathrm{mL} \mathrm{mL}^{-1}$ for the detection of PSA biomarker.

A chemical nose/tongue was developed by Wu et al. ${ }^{546}$ using a functionalized graphene array-based electrochemical biosensor for differentiating the cancerous, metastatic human breast cells, multidrug-resistant cancerous, and artificial circulating tumor cells (CTCs). This approach enables the biosensor to analyze different cell types at a very low level (100 cells). Fig. 25 shows the schematic of the cell detecting system, seven graphene derivatives including P1: BSA/chemically converted graphene (CCG), P2: CCG, P3: chitosan (Chit)/CCG, P4: polydopamine (DA)/CCG, P5: calf thymus DNA/CCG, P6: gelatin (Gel)/CCG, and P7: polyethylene glycol (PEG)/CCG, discrimination of 5 different human cancerous cell lines including lung (A549), cervical (HeLa), liver (HepG2), leukemia (K562), and breast (MCF-7) with P1-P7 graphene probes at a low cancer cell density of 100 cells, jackknifed classification using linear discriminant analysis (LDA) and canonical score plot for the graphene derivatives array-based biosensor. A single graphene array electrochemical sensor can detect 5 different human cancerous cell types for diagnostic purposes where two combined graphene derivative probes successfully obtained $100 \%$ classification accuracy. The LDA plots showed certain patterns and differentiated human cancerous cells from each other, having two canonical factors variance of 70.06 and $29.94 \%$ for $\mathrm{P} 1+\mathrm{P} 4,68.66$ and $31.34 \%$ for $\mathrm{P} 1+\mathrm{P} 5,51.91$ and $48.09 \%$ for $\mathrm{P} 2+\mathrm{P} 4,82.95$ and $17.05 \%$ for P2 + P5 for the A549, HeLa, HepG2, K562 and MCF-7 human cancerous cell lines. The LDA separated human cancerous cell lines into five discrete clusters. The functionalized graphene derivatives can be used for the application in the field of clinical cancer diagnostics.

3.7.1 Microfluidic electrochemical immunoassays. The microfluidic devices have attracted significant interest for point-of-care diagnostic systems. ${ }^{547} \mathrm{~A}$ microfluidics-based detection device is a promising approach for the detection of clinical biomarkers because of its simplicity, low cost, 
(A)

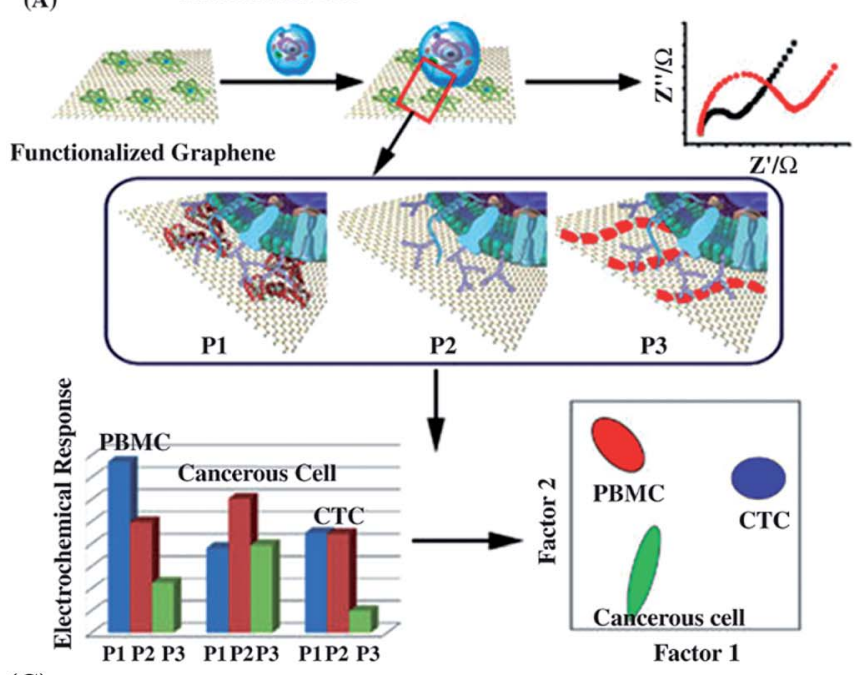

(B)

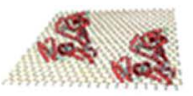

P1: BSA/CCG

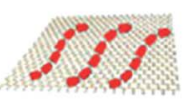

P3: Chit/CCG

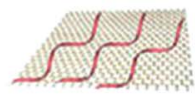

P5: DNA/CCG

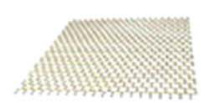

P2: CCG

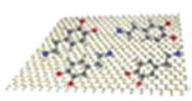

P4: DA/CCG

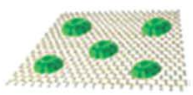

P6: GeI/CCG

(C)
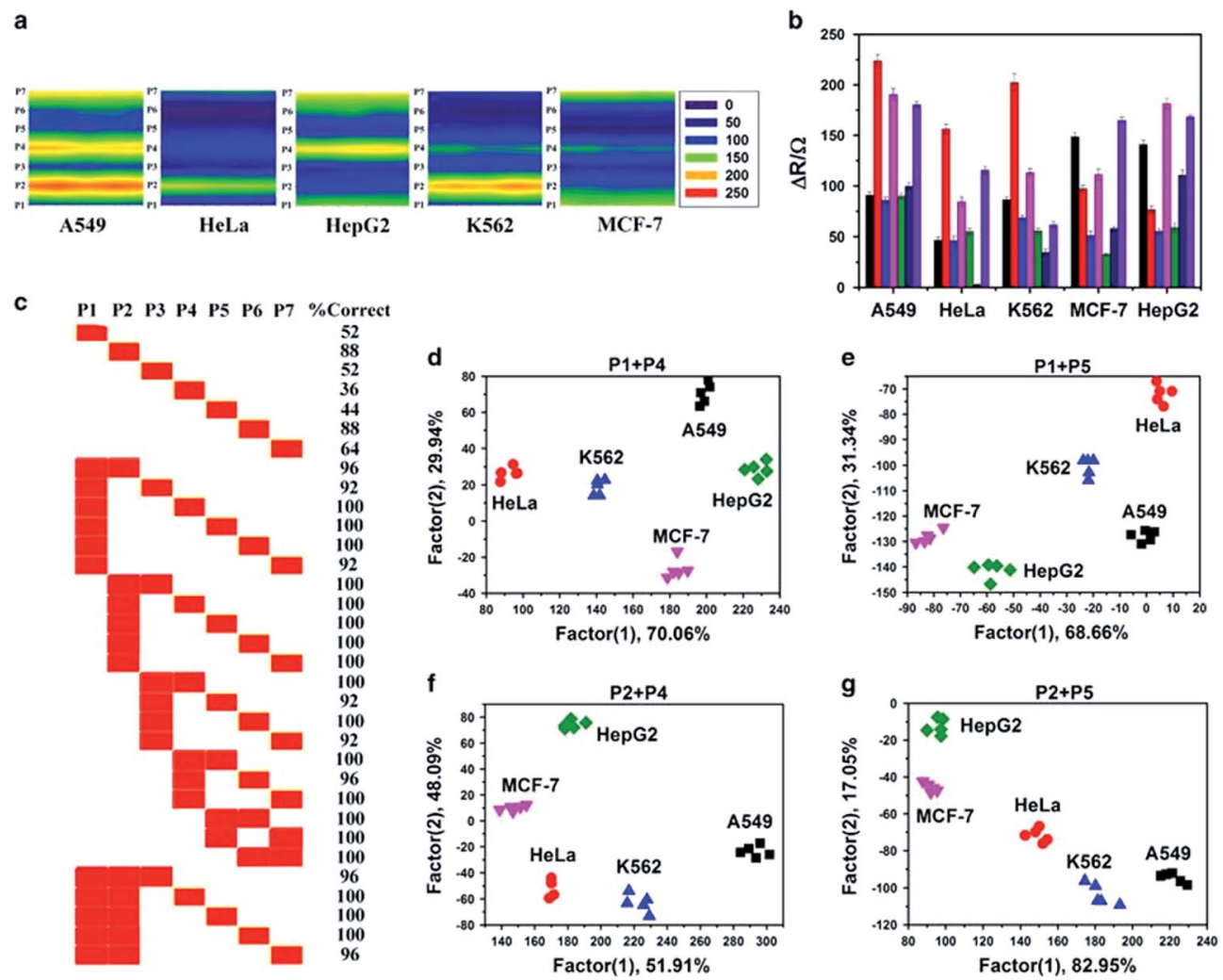

Fig. 25 (A) Schematic illustration of the cell detecting system and the molecular interactions between the functionalized graphene (P1, P2, P3) and the different cell types including human peripheral blood mononuclear cells (PBMCs), cancerous cells and circulating tumor cells (CTCS). (B) Illustration of the seven functionalized graphene (P1-P7) derivatives used for the identification of normal, cancerous cells and CTCs. The seven graphene (P1-P7) derivatives include P1: BSA/chemically converted graphene (CCG), P2: CCG, P3: chitosan (Chit)/CCG, P4: polydopamine (DA)/ CCG, P5: calf thymus DNA/CCG, P6: gelatin (Gel)/CCG, and P7: polyethylene glycol (PEG)/CCG. (C) Discrimination of different cancerous cell types at a cancer cell density of 100 cells: (a) 2D electrochemistry contour plots of 5 different cancer cell lines including lung (A549), cervical (HeLa), liver (HepG2), leukemia (K562), and breast (MCF-7) to P1-P7 graphene probes; (b) changes in the electron-transfer resistance at the electrolyte/graphene interface measured by the electrochemical impedance spectra for five different cancerous cell lines; A549, HeLa, HepG2, K562 and MCF-7 using P1-P7 graphene derivatives; (c) jackknifed classification recorded using linear discriminant analysis (LDA) for P1-P7 graphene derivatives for A549, HeLa, HepG2, K562 and MCF-7 human cancerous cells; and canonical score plots for the functionalized graphene array-based electrochemical sensor containing P1 + P4 (d), P1 +P5 (e), P2 + P4 (f), and P2 + P5 (g). [Reprinted with permission from ref. 546 , L. Wu, H. Ji, Y. Guan, X. Ran, J. Ren and X. Qu, A Graphene-Based Chemical Nose/Tongue Approach for the Identification of Normal, Cancerous and Circulating Tumor Cells, NPG Asia Mater., 2017, 9, e356. Copyright@ Nature Publishing Group.] 
(A)

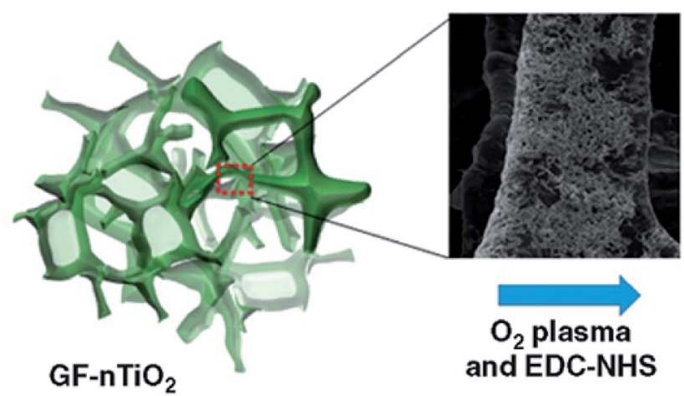

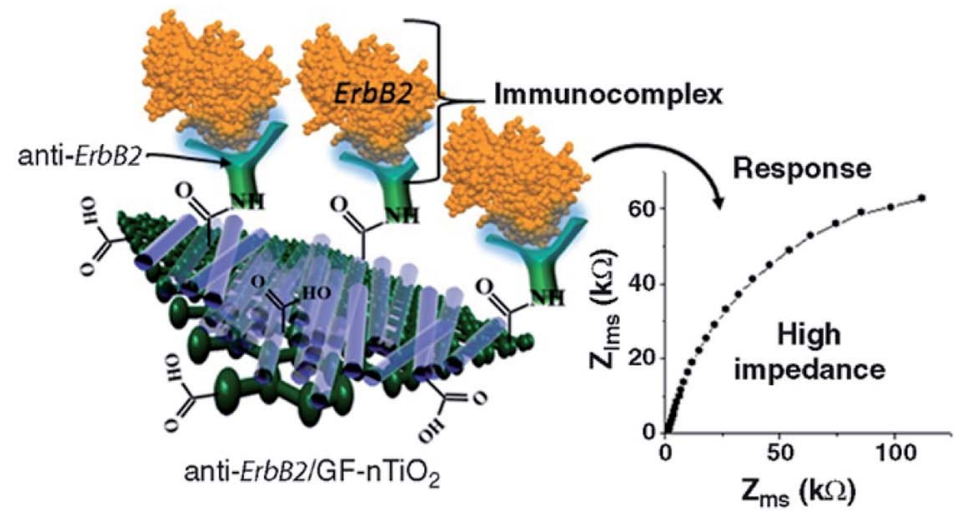

(B)

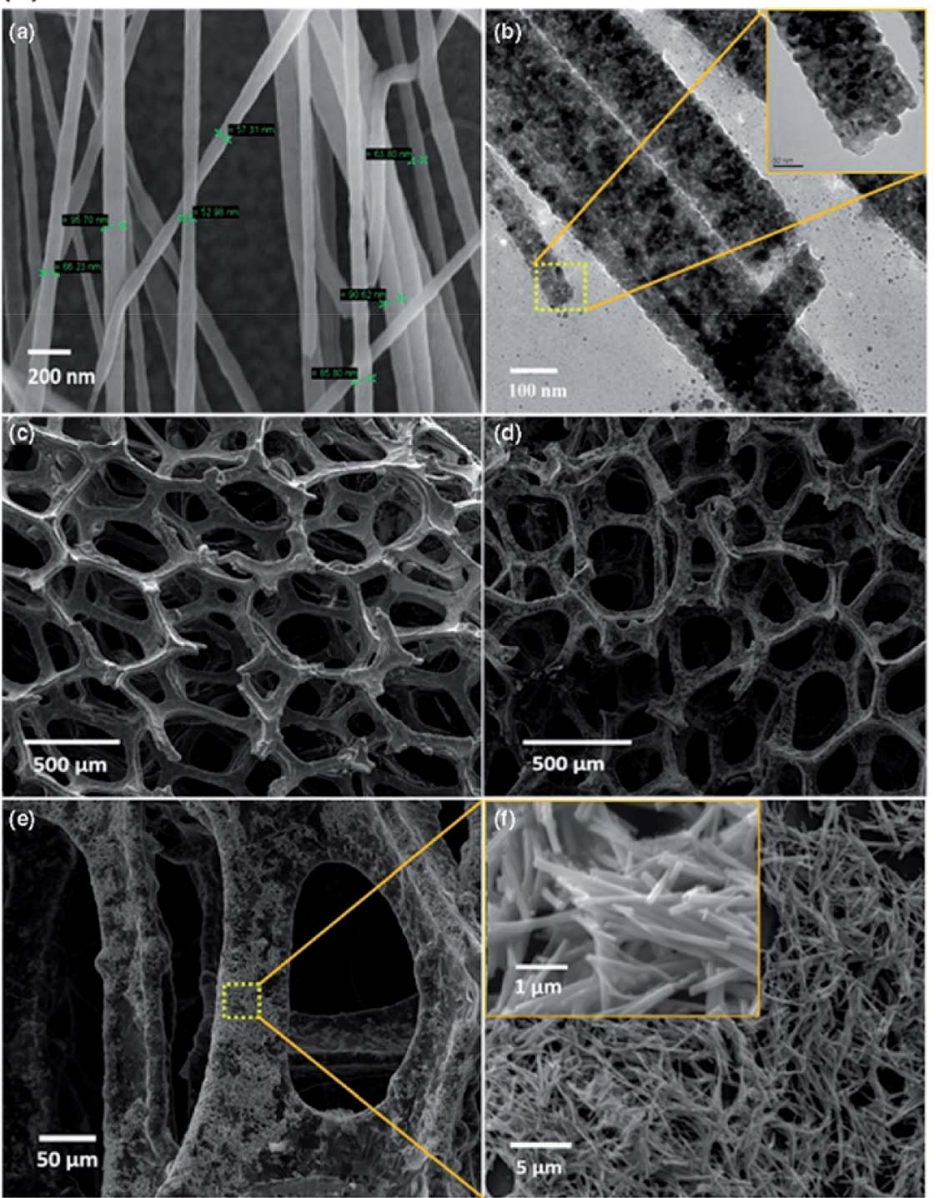

(C) 32
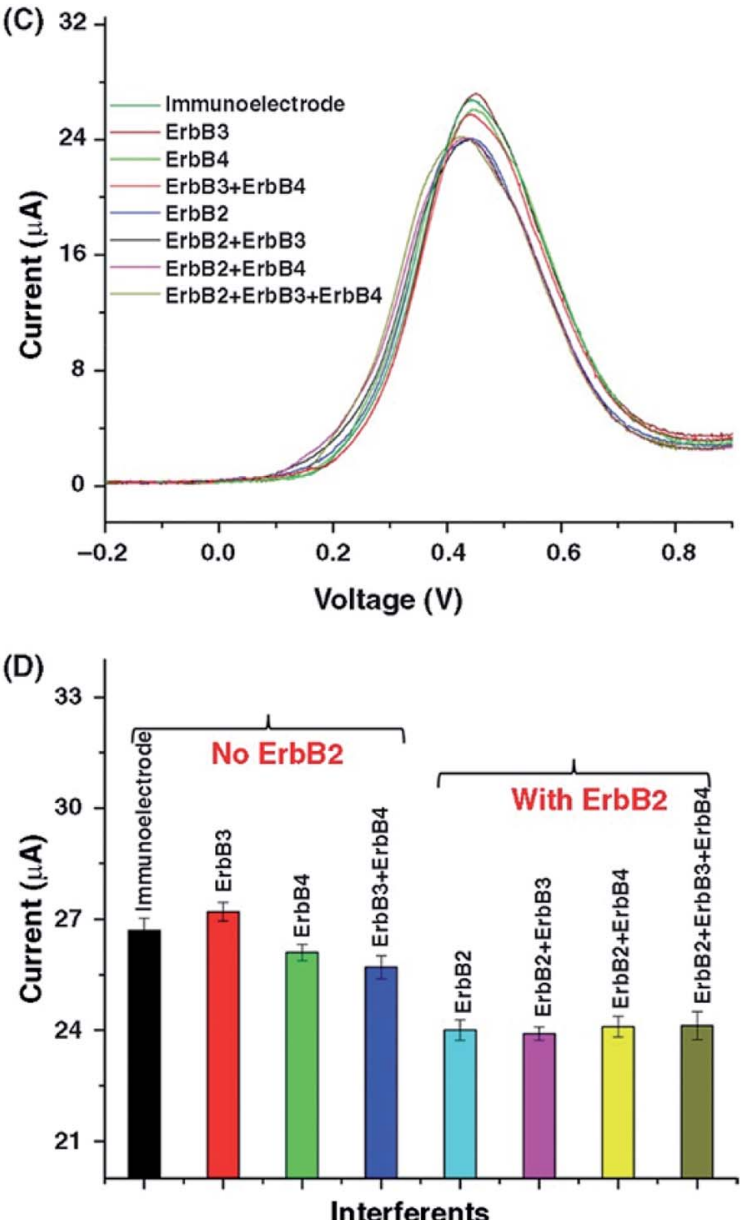

Fig. 26 (A) Functionalization of anti-ErbB2 molecules on the outer surfaces of GF and GF- nTiO, electrodes. (B) FESEM (a) and TEM (b) images of carbon-doped $n \mathrm{TiO}_{2}$. The inset shows a magnified image of a single $n \mathrm{TiO}_{2}$. (c) SEM image of $3 \mathrm{D}$ GF. (d-f) $\mathrm{SEM}$ images of the GF- $n \mathrm{TiO} 2$ nanocomposite. (C) DPV curves of biosensor in the presence of ErbB3 and ErbB4 antigens. (D) Histogram displaying the peak current of the biosensor in the presence of different interfering bionanalytes. [Reprinted with permission from ref. 518, M. A. Ali, K. Mondal, Y. Jiao, S. Oren, Z. Xu, A. Sharma and L. Dong, Microfluidic Immuno-Biochip for Detection of Breast Cancer Biomarkers Using Hierarchical Composite of Porous Graphene and Titanium Dioxide Nanofibers, ACS Appl. Mater. Interfaces, 2016, 8, 20570-20582. Copyright@ American Chemical Society.]

portability, low reagent consumption and capability of multiplex analysis using a single chip. ${ }^{548}$ Likely, a paper-based microfluidic device is also considered a simple approach for biosensing applications due to its low cost. ${ }^{549}$ Graphene-based materials have been used for fabricating microfluidic devices to detect cancer biomarkers at ultra-low concentrations. Wu et al. ${ }^{550}$ developed a paper-based microfluidic electrochemical immunodevice using graphene thin film for the multiplexed detection of four types of cancer biomarkers including AFP, CEA, cancer antigen 125 (CA125) and carbohydrate antigen 153 (CA153). They used horseradish peroxidase (HRP), antibody coimmobilized silica NPs and graphene to enable dual signal 
amplification, which enabled LODs of 0.001, 0.005, 0.001, and $0.005 \mathrm{ng} \mathrm{mL}^{-1}$ for AFP, CEA, CA125, and CA153 biomarkers, respectively. Ali et al. $^{518}$ demonstrated a label-free microfluidic immunosensor fabricated using highly porous graphene foam (GF) integrated with carbon-doped $\mathrm{TiO}_{2}$ nanofibers for the detection of epidermal growth factor receptor 2 (ErbB2) proteins. Fig. 26 shows the functionalization of anti-ErbB2 molecules on the outer surfaces of GF and $\mathrm{GF}-n \mathrm{TiO}_{2}$ electrodes, microscopic images of carbon-doped $n \mathrm{TiO}_{2}, 3 \mathrm{D}$ GF and $\mathrm{GF}-n \mathrm{TiO}_{2}$ nanocomposite, DPV curves in the presence of ErbB3 and ErbB4 antigens and histogram displaying the peak current in the presence of different interfering bionanalytes. The rodlike $\mathrm{TiO}_{2}$ nanofibers consisted of $\mathrm{TiO}_{2}$ grains. The GF has 500-600 $\mu \mathrm{m}$ size pores which are sufficient for the penetration of $\mathrm{TiO}_{2}$ nanofibers. The large surface area and porous structure of $\mathrm{GF}-n \mathrm{TiO}_{2}$ composite allows easy adsorption of analytes. The sensor detected breast cancer biomarkers with a high sensitivity of $0.585 \mu \mathrm{A} \mu \mathrm{M}^{-1} \mathrm{~cm}^{-2}$, a very wide linear range of $1.0 \mathrm{fM}$ to 0.1 $\mu \mathrm{M}$ concentrations of the target ErbB2 antigen and an LOD of 1 fM ErbB2. The peak current of the microfluidic sensor showed no significant change after adding $1 \mathrm{nM}$ ErbB2 antigen to the mixed solution of interfering ErbB3, ErbB4, and ErbB3 + ErbB4 antigens, which indicated high selectivity toward ErbB2 in the breast cancer cells. The relative standard deviation (RSD) of $\pm 1.0 \%$ was observed for the microfluidic sensor for 42 days storage at $4{ }^{\circ} \mathrm{C}$, showing high stability due to strong molecular interactions between $\mathrm{GF}-n \mathrm{TiO}_{2}$ composite and anti-ErbB2. Singh et al. ${ }^{551}$ demonstrated a microfluidic biochip fabricated using a microporous manganese oxide $\left(\mathrm{Mn}_{3} \mathrm{O}_{4}\right) / \mathrm{rGO}$ nanocomposite for human cardiac troponin I (cTnI) detection. The designed microfluidic chip showed high sensitivity, stability and reproducibility for the analysis of human cardiac troponin I molecules in a wide concentration range of 0.008 to $20 \mathrm{ng} \mathrm{mL}$ and achieved an LOD of $8.0 \mathrm{pg} \mathrm{mL}^{-1}$ and negligible interference from other competing biomarkers including cardiac troponin $\mathrm{C}$ (cTnC), cardiac troponin $\mathrm{T}$ (cTnT), B-type natriuretic peptide (BNP), and myoglobin.

\subsection{Detection of pathogens}

Pathogens are key contaminants in water, including viruses, fungi, bacteria and protozoan parasites, and are responsible for various kinds of water-borne diseases. The presence of pathogens in water and food products is a major concern due to their rapid growth and harmful health effects to human. ${ }^{\mathbf{5 2 - 5 5 4}}$ Furthermore, pathogenic microorganisms such as fungi, bacteria, viruses, viroids, phytoplasma and nematodes cause infectious diseases that lead to a decrease in agricultural productivity worldwide. The early detection of plant pathogens is an important first step to prevent plant disease in greenhouses, agricultural fields and at the national borders. ${ }^{555} \mathrm{~A}$ report from the World Health Organization (WHO) in 2007 indicated that the water- and food-borne infections account for approximately 4 billion episodes of diarrhea per year worldwide, causing approximately 1 to 2 million deaths. ${ }^{73}$ The real-time and sensitive detection of pathogens is of great importance to monitor and control such pathogen-related fatalities. Among the various pathogens, enterohemorrhagic Escherichia coli bacterial strains is the most life-threatening and serious foodborne outbreaks. ${ }^{556}$ Therefore, the sensitive detection and control of foodborne bacterial pathogens, such as Escherichia coli serotype O157:H7, remains a major scientific challenge in food safety in order to prevent severe diseases in humans. ${ }^{557}$ Nanostructured materials, including graphene and CNTs, have been exploited to fabricate electrochemical biosensors for the sensitive and selective detection of pathogens. ${ }^{73}$ Progress in the nanomaterial-based detection of pathogens through electrochemical methodologies has been reported previously. ${ }^{73,558}$ The electrochemical sensors for the detection of pathogenic bacteria using graphene-based nanocomposites are summarized in Table 8 .

Tiwari et $a l .{ }^{559}$ demonstrated an electrochemical sensor based on a GO-modified iron oxide-CS nanocomposite for pathogen detection. The sensor was fabricated through the covalent immobilization of $E$. coli $\mathrm{O} 157: \mathrm{H} 7$ as a specific probe oligonucleotide sequence on the nanocomposite films. The sensor showed a linear response to complementary DNA in $10^{-6} \mathrm{M}$ to $10^{-14} \mathrm{M}$ concentration range with an LOD of $1 \times$ $10^{-14} \mathrm{M}$. Pandey et al. ${ }^{564}$ reported a graphene-based label-free biosensor for detecting pathogenic bacteria. Graphene was interfaced with interdigitated microelectrodes for designing a biosensor that was biofunctionalized using E. coli O157:H7specific antibodies (Fig. 27). The capacitance change of the sensor showed the sensitivity of 10-100 cells per $\mathrm{mL}$ of the $E$. coli O157:H7 strain.

\subsection{Detection of food toxins}

Environmental conditions such as insect damage, temperature, and humidity may lead to increased infection and toxin accumulation. Mycotoxins are toxic secondary metabolites generated by fungi including Aspergillus flavus, Aspergillus parasiticus, Aspergillus ochraceus, Penicillium verrucosum that readily grow on crops such as cereals, nuts, dried fruits, and beans during plant growth, harvesting, drying, processing and storage. Hence, the efficient monitoring and detection of foodborne pathogens and toxins (sterigmatocystin, aflatoxin, ochratoxin, etc.) are of paramount significance. ${ }^{599}$ Although several methods have been developed to maintain the quality of food nutrients and to prevent contamination of food, still diseases due to foodborne pathogens have increased considerably. Food and water contamination by pathogenic microorganisms causes 1.8 million deaths per year worldwide. ${ }^{600}$ Aflatoxin-B1 (AFB1) is a highly toxic and carcinogenic compound generated by Aspergillus species of fungi. Among more than 20 aflatoxins, only four compounds, aflatoxin-B1 (AFB1), AFB2, AFG1, and AFG2, are classified as human carcinogens. AFB1 is more toxic relative to the other types of aflatoxins that cause human liver cancer. ${ }^{575}$ Various analytical techniques have been developed and applied for the fast, sensitive and selective detection of diverse toxins in foods and food products, including fluorescence and electrochemical sensing, colorimetry, immunoassays, and chromatographic separation. ${ }^{599}$ However, electrochemical methods are highly sensitive and easy for the detection of food toxins. ${ }^{\mathbf{6 0 1}}$ 
Table 8 Graphene-based biosensors for the detection of pathogens, food toxins, and metal ions ${ }^{a}$

Graphene-based nanocomposites

Pathogenic bacteria

GO/CS nanocomposite

GO/iron oxide/CS composite

$\mathrm{GO} /$ nickel ferrite/CS composite

$\mathrm{GO} /$ thionine assembly

rGO/AuNPs nanocomposite

GR/copper oxide-cysteine

GR

Nanoporous alumina

membrane/GR-QDs

Functionalized bridged rebar GR

EC-rGO/CS nanocomposite

rGO/AuNPs/Ab $/$ /IMB/SPCE

rGO-azophloxine nanocomposite

ssDNA/GO/CS nanocomposite

AuNPs-PdNPs/EC-rGO

3D GR nanosheet/AuNPs

GO/AuNPs

rGO/polyethylenimine

\section{Food toxins}

ss-HSDNA/rGO aerogel

Anti-AFB ${ }_{1} / \mathrm{rGO} / \mathrm{ITO}$

AuNPs/carboxylated rGO

rGO/PPy/PPa nanocomposite

NiNPs/rGO nanosheets

GR-thionine-aptamer

DNA aptamer/GR

\section{Metal ions}

AuNPs/GR/cysteine composite

GO modified Au electrode

GR nanodots/porous Au electrode

GO/AgNPs composite

Hollow AuPd-flower-like

$\mathrm{MnO}_{2}$-hemin@rGO composite

DNA-modified 3D rGO/CS composite

AuNPs/rGO nanocomposite

Heparin modified CS/GR composite

rGO/AuNPs composite

$\mathrm{Pi}-\mathrm{A} / \mathrm{rGO}$ nanocomposite

Nile red-GR composite

rGO-CS/poly-L-lysine nanocomposite

GR-Au modified electrode Ruthenium(II) bipyridine $\left[\mathrm{Ru}(\mathrm{bpy})_{3}\right]^{2+}$ complex/GO composite

L-Cysteine/AuNPs/rGO

$\mathrm{MnFe}_{2} \mathrm{O}_{4} / \mathrm{GO}$ nanocomposite

Pathogen/food toxin

Detection

method

Detection range

Limit of detection

(LOD)

Ref.

S. Typhi-specific DNA

E. coli $\mathrm{O} 157: \mathrm{H7}$

E. coli $\mathrm{O} 157: \mathrm{H} 7$

Cry1C

S. aureus

E. coli $\mathrm{O} 157: \mathrm{H} 7$

E. coli $\mathrm{O} 157: \mathrm{H7}$

Salmonella

typhimurium

E. coli $\mathrm{O} 78: \mathrm{K} 80: \mathrm{H} 11$

Salmonella enterica

Salmonella pullorum

S. typhimurium

E. coli $\mathrm{O} 157: \mathrm{H} 7$

Lomefloxacin

Amoxicillin

Sulfate-reducing

bacteria (SRB)

Cronobacter sakazakii

E. coli UTI89 bacteria

$\begin{array}{ll}\text { Aflatoxin B1 (AFB1) } & \text { EIS } \\ \text { AFB1 } & \text { CV } \\ \text { AFB1 } & \text { EIS } \\ \text { AFB1 } & \text { EIS } \\ \text { Mycotoxin (AFB1) } & \text { CV } \\ \text { Ochratoxin A } & \text { DPV } \\ \beta \text {-Lactoglobulin } & \text { SWV } \\ \text { milk protein } & \end{array}$

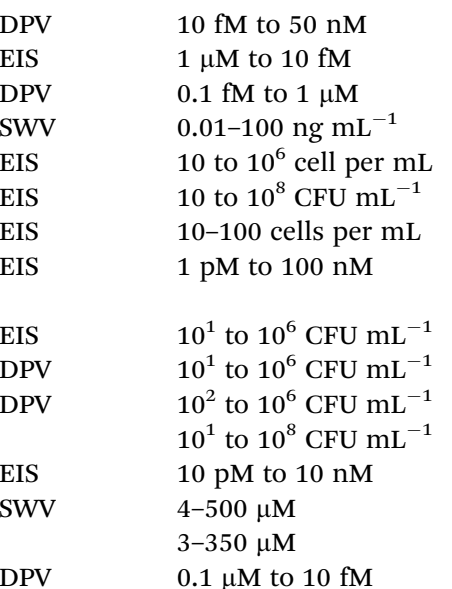

DPV

DPV

EIS
CV
EIS
EIS
CV
DPV
SWV

$\mathrm{Cd}^{2}$

$\mathrm{Pb}^{2}$

$\mathrm{Pb}^{2+}$

$\mathrm{Hg}^{2+}$

$\mathrm{Cu}^{2+}$

$\mathrm{Cu}^{2+}$

$\mathrm{Pb}^{2+}$

$\mathrm{Pb}^{2+}$

$\mathrm{Hg}^{2+}$

$\mathrm{Pb}^{2+}$

$\mathrm{Fe}^{3+}$

$\mathrm{Cu}^{2+}$

$\mathrm{Fe}^{3+}$

$\mathrm{Cd}^{2+}$

$\mathrm{Pb}^{2}$

$\mathrm{Cu}^{2+}$

$\mathrm{Hg}^{2+}$

$\mathrm{Cd}^{2+}$

$\mathrm{Pb}^{2+}$

$\mathrm{Hg}^{2+}$

$\mathrm{Cu}^{2+}$

$\begin{array}{ll}\text { SWASV } & 0.5-40 \mu \mathrm{g} \mathrm{L}^{-1} \\ \text { SWV } & 0-50 \mathrm{ppb} \\ & 0-15 \mathrm{ppb} \\ & 0-200 \mathrm{ppb} \\ \text { SWV } & 0.006-2.5 \mu \mathrm{M} \\ & 0.009-4 \mu \mathrm{M} \\ \text { SWV } & 0.1 \mathrm{nM} \text { to } 10 \mu \mathrm{M} \\ \text { DPV } & 0.1 \mathrm{pM} \text { to } 200 \mathrm{nM} \\ & \\ \text { EIS } & 0.1 \mathrm{nM} \text { to } 10 \mathrm{nM} \\ \text { CV, EIS } & 10 \mathrm{ng} \mathrm{L} \text { to } 1.0 \mu \mathrm{g} \mathrm{L} \mathrm{L}^{-1} \\ \text { SWASV } & 1.125-8.25 \mu \mathrm{g} \mathrm{L} \\ \text { DPV } & 30-3000 \mathrm{nM} \\ \text { SWASV } & 5-300 \mu \mathrm{g} \mathrm{L}^{-1} \\ \text { CV, DPV } & 30-1000 \mu \mathrm{M} \\ \text { DPASV } & 0.05-10 \mu \mathrm{g} \mathrm{mL} \mathrm{mL}^{-1} \\ & \\ \text { CV, SWV } & 1 \mathrm{aM} \text { to } 100 \mathrm{nM}^{-1} \\ \text { CV, EIS } & 0.5-0.3 \mu \mathrm{M} \\ & 0.05-0.25 \mu \mathrm{M} \\ & 0.05-1.8 \mu \mathrm{M} \\ & 0.1-1.2 \mu \mathrm{M} \\ \text { DPSV } & 2-60 \mu \mathrm{mL} \mathrm{m}^{-1} \\ \text { SWASV } & 0.2-1.1 \mu \mathrm{M}\end{array}$

$\begin{array}{ll}10 \mathrm{fM} & 434 \\ 10 \mathrm{fM} & 559 \\ 0.1 \mathrm{fM} & 560 \\ 3.2 \mathrm{pg} \mathrm{mL}^{-1} & 561 \\ 10 \mathrm{CFU} \mathrm{mL} L^{-1} & 562 \\ 3.8 \mathrm{CFU} \mathrm{mL} & 563 \\ - & 564 \\ 1 \mathrm{pM} & 565 \\ & \\ 10 \mathrm{CFU} \mathrm{mL} & \\ 10^{1} \mathrm{CFU} \mathrm{mL} & 566 \\ 89 \mathrm{CFU} \mathrm{mL} & 567 \\ 10^{1} \mathrm{CFU} \mathrm{mL} & 568 \\ 3.584 \mathrm{pM}^{-1} & 569 \\ 81 \mathrm{nM} & 570 \\ 9 \mu \mathrm{M} & 571 \\ 9.41 \mathrm{fM}^{-1} & \\ 2 \times 10^{1} \mathrm{CFU} \mathrm{mL}^{-1} & 573 \\ 10 \mathrm{CFU} \mathrm{mL} & \end{array}$

$0.04 \mathrm{ng} \mathrm{mL}^{-1} \quad 575$ $0.12 \mathrm{ng} \mathrm{mL}^{-1} \quad 576$ $0.1 \mathrm{ng} \mathrm{mL}^{-1}$ $10 \mathrm{fg} \mathrm{mL}^{-1} \quad 578$ $0.16 \mathrm{ng} \mathrm{mL}^{-1} \quad 579$ $5.6 \mathrm{pg} \mathrm{mL}^{-1} \quad 580$ $20 \mathrm{pg} \mathrm{mL}^{-1} \quad 581$

$\begin{array}{ll}0.1 \mu \mathrm{g} \mathrm{L}^{-1} & 582 \\ 0.05 \mu \mathrm{g} \mathrm{L}^{-1} & \\ 0.4 \mathrm{ppb} & 583 \\ 0.8 \mathrm{ppb} & \\ 1.2 \mathrm{ppb} & \\ 0.8 \mathrm{nM} & 584 \\ 1 \mathrm{nM} & \\ 80 \mathrm{pM} & 585 \\ 0.034 \mathrm{pM} & 586 \\ & \\ 0.016 \mathrm{mM}^{-1} & 587 \\ 1.5 \mathrm{ng} \mathrm{L} & 588 \\ 0.03 \mu \mathrm{g} \mathrm{L} & 589 \\ 3.5 \mathrm{nM} & 590 \\ 0.67 \mu \mathrm{g} \mathrm{L} & 591 \\ 24.9 \mu \mathrm{M} & 592 \\ 0.01 \mu \mathrm{g} \mathrm{mL} & \\ 0.02 \mu \mathrm{g} \mathrm{mL} \mathrm{mL}^{-1} & 593 \\ 0.02 \mu \mathrm{g} \mathrm{mL} & \\ 0.001 \mathrm{aM} & \\ 2.8 \mathrm{nM} & 594 \\ 1.41 \mathrm{nM} & 595 \\ 2.3 \mathrm{nM} & \\ 1.6 \mathrm{nM} & \\ 0.037 \mu \mathrm{g} \mathrm{mL}^{-1} & 596 \\ 0.0883 \mu \mathrm{M} & 597\end{array}$


Table 8 (Contd.)

\begin{tabular}{|c|c|c|c|c|c|}
\hline \multirow[t]{2}{*}{ GQDs@VMSF } & $\mathrm{Hg}^{2+}$ & DPV & $10 \mathrm{pM}$ to $1 \mathrm{nM}$ & $9.8 \mathrm{pM}$ & 598 \\
\hline & $\mathrm{Cd}^{2+}$ & & $20 \mathrm{nM}$ to $1 \mu \mathrm{M}$ & $4.3 \mathrm{nM}$ & \\
\hline
\end{tabular}

${ }^{a}$ Abbreviations used: $\mathrm{MoS}_{2}$ : molybdenum disulfide, PANI: polyaniline, PPy: polypyrrole, PPa: pyrrolepropylic acid, GQDs: graphene quantum dots, LMF: lomefloxacin, AMX: amoxicillin, EIS: electrochemical impedance spectroscopy, SWV: square wave voltammetry, SWASV: square wave anodic stripping voltammetry, Ab2: secondary antibody, IMB: immunomagnetic beads, Pi-A: $N$-(2-(1-(p-tolyl)-1H-phenanthro[9,10- $d]$ imidazol-2-yl)phenyl) picolinamide, DPASV: differential pulse anodic stripping voltammetry, ochratoxin A (OTA): (2S)-2-[[(3R)-5-chloro-8-hydroxy-3-methyl-1-oxo-3,4dihydroisochromene-7-carbonyl]amino]-phenylpropanoic acid, VMSF: vertically ordered mesoporous silica-nanochannel film.

(A)
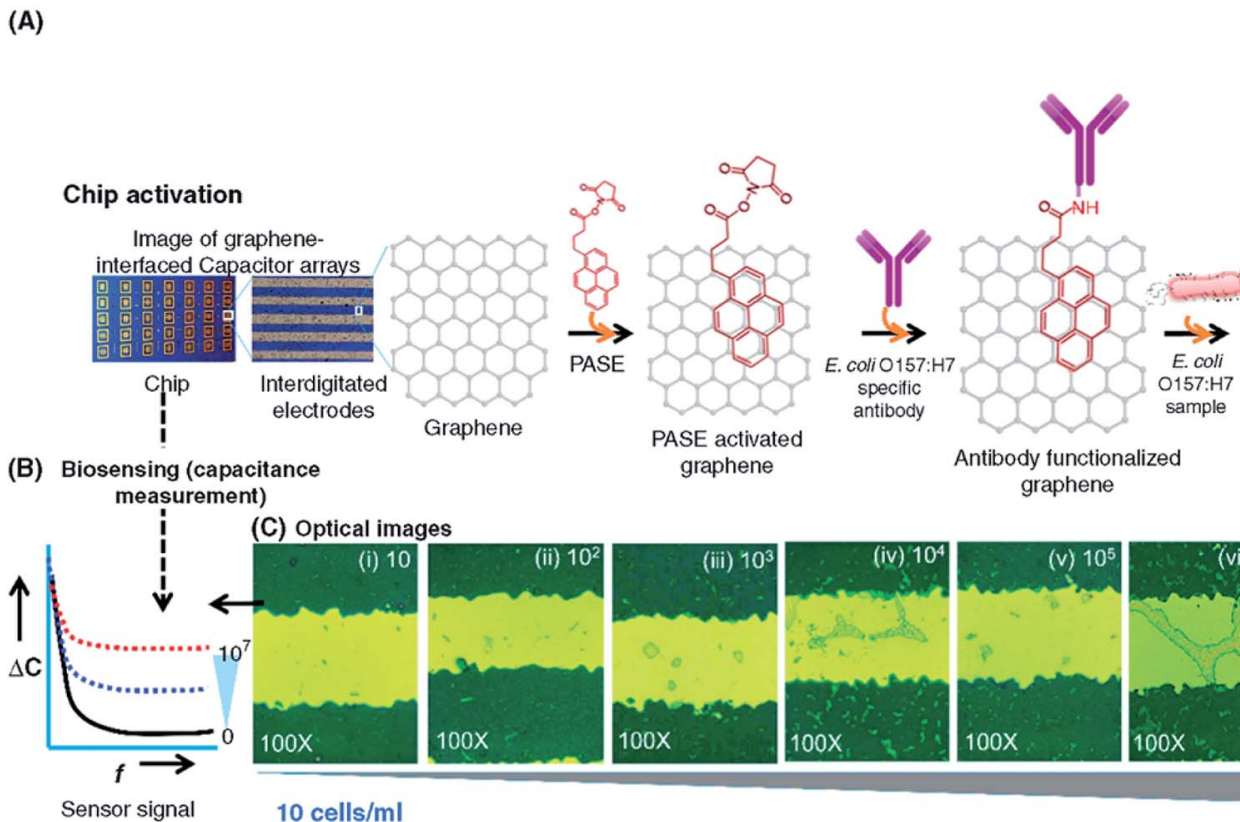

(C) Optical images
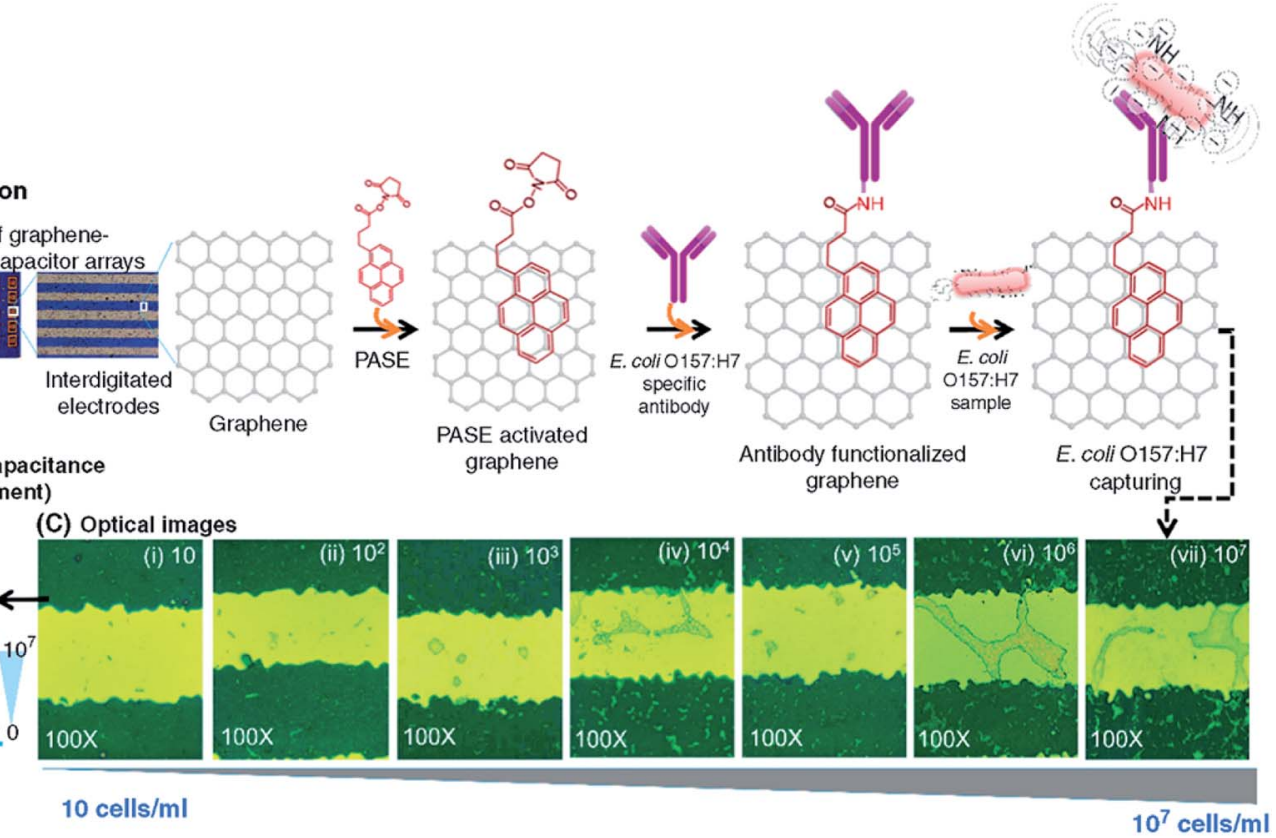

Fig. 27 (A) (Left) Photographs of a real graphene-interfaced chip. (Right) The PASE activation and antibody immobilization processes. (B) Onchip biosensing of $E$. coli O157: $\mathrm{H} 7$ through capacitance change measured between Au microelectrodes. (C) Optical micrographs of captured $E$. coli $0157: \mathrm{H} 7$ cells on graphene-interface chips through targeted antibodies that are covalently attached to the surfaces of the chips. [Reprinted with permission from ref. 564, A. Pandey, Y. Gurbuz, V. Ozguz, J. H. Niazi and A. Qureshi, Graphene-Interfaced Electrical Biosensor for Label-Free and Sensitive Detection of Foodborne Pathogenic E. coli O157:H7, Biosens. Bioelectron., 2017, 91, 225-231. Copyright $\odot$ Elsevier.]

Nanomaterials such as inorganic nanoparticles and carbonbased nanomaterials (CNTs, graphene) have been effectively used for the detection of food contaminants. ${ }^{\mathbf{6 0 2 , 6 0 3}}$ Table 8 summarizes the graphene-based biosensors for the detection of pathogens, food toxins, and metal ions.

Srivastava et al. ${ }^{576}$ demonstrated an electrochemical sensor by covalently binding monoclonal antibodies to AFB1 (antiAFB1) onto the rGO surface for detecting food toxin (AFB1). The rGO/anti-AFB1 based sensor showed high sensitivity of $68 \mu \mathrm{A}$ $\mathrm{ng}^{-1} \mathrm{~mL} \mathrm{~cm}^{-2}$ and an LOD of $0.12 \mathrm{ng} \mathrm{mL}^{-1}$. The same research group also reported a label-free sensor using a AuNP-supported rGO nanosheet, and the surface was bioconjugated with monoclonal antibodies against AFB1. The BSA/anti-AFB1/
AuNPs/rGO nanocomposite-based immunosensor showed good stability up to 56 days and the detection of AFB1 with a sensitivity of $182.4 \mu \mathrm{Ag}^{-1} \mathrm{~mL} \mathrm{~cm}^{-2}$ and a linear range of $0.1-$ $12 \mathrm{ng} \mathrm{mL}{ }^{-1}$ with an LOD of $0.1 \mathrm{ng} \mathrm{mL}^{-1} .{ }^{.77}$ Moreover, this group also demonstrated the fabrication of label-free immunosensors that used rGO sheets decorated with antibody-conjugated nickel nanoparticles for the electrochemical detection of AFB1 mycotoxin. ${ }^{579}$ Owing to the high electrocatalytic activity of NiNPs, the fabricated BSA/anti-AFB1/rGO-NiNPs immunosensor exhibited an enhanced electron transfer rate and sensitivity of $129.6 \mu \mathrm{A} \mathrm{ng} \mathrm{mL} \mathrm{L}^{-1} \mathrm{~cm}^{-2}$, a linear detection range of 1.0-8.0 ng mL ${ }^{-1}$ with an LOD of $0.16 \mathrm{ng} \mathrm{mL}^{-1}$ for $\mathrm{AFB}_{1}$ and stability up to 42 days. Krittayavathananon et al. ${ }^{575}$ showed an 
impedimetric biosensor for detection of the AFB1 level employing rGO aerogel labeled with ssDNA on a rotating disk electrode. The sensor detected AFB1 in a linear concentration range of $7 \times 10^{-8}$ to $1 \times 10^{-10} \mathrm{~g} \mathrm{~mL}^{-1}$ with an LOD of $0.04 \mathrm{ng}$ $\mathrm{mL}^{-1}$. Wang et al. ${ }^{578}$ reported the detection of AFB1 using an rGO/polypyrrole/pyrrolepropylic acid nanocomposite-based impedimetric immunosensor. The sensor showed improved sensitivity for the detection of AFB1 in a linear range of $10 \mathrm{fg}$ $\mathrm{mL}^{-1}$ to $10 \mathrm{pg} \mathrm{mL}^{-1}$. Kaur et al. ${ }^{604}$ developed an electrochemical aptasensor using functionalized GR (f-GR)-doped chitosan composite for detecting mycotoxin ochratoxin A (OTA). The fGR played a role in increasing the surface area of the electrode whereas CS hindered the leaching of aptamer (APT). The streptavidin (SA) attached in f-GR was immobilized by ATP molecules through SA-biotin coupling process. The APT/SA/CS-fGR composite-based biosensor showed a linear range of $1 \mu \mathrm{g}$ $\mathrm{mL}^{-1}$ to $1 \mathrm{fg} \mathrm{mL} \mathrm{m}^{-1}$, and an LOD of $1 \mathrm{fg} \mathrm{mL}^{-1}$ for standard sample and $0.01 \mathrm{ng} \mathrm{mL}^{-1}$ for spiked grape juice samples for the OTA detection with a response time of $8 \mathrm{~min}$ and retained $91.4 \%$, $70.7 \%$ and $53.8 \%$ of initial responses after 7,21 , and 28 days, respectively. The OTA recoveries from the grape juice were found to be between $90 \%$ and $101 \%$. Jiang et al. ${ }^{605}$ used AuNPs/rGO nanocomposite to amplify the electrochemical impedimetric signal of an aptasensor for mycotoxin ochratoxin A (OTA) detection within picomolar level sensitivity. The negative assembled reporter DNA covered almost the entire surface of the AuNPs/rGO nanocomposite and effectively repelled the $\left[\mathrm{Fe}(\mathrm{CN})_{6}\right]^{4-/ 3-}$ anions, which enabled considerable amplification for the detection of OTA. The designed sensor displayed a good response for OTA concentrations ranging from $1.0 \mathrm{pg} \mathrm{mL} \mathrm{mL}^{-1}$ to $50 \mathrm{ng} \mathrm{mL}^{-1}$ with an LOD of $0.3 \mathrm{pg} \mathrm{mL}$.

\subsection{Detection of toxic heavy metal ions}

Toxic heavy metal ions including of arsenic $\left(\mathrm{As}^{3+}\right)$, lead $\left(\mathrm{Pb}^{2+}\right)$, chromium $\left(\mathrm{Cr}^{3+}\right.$ or $\left.\mathrm{Cr}^{6+}\right)$, cadmium $\left(\mathrm{Cd}^{2+}\right)$, and mercury $\left(\mathrm{Hg}^{2+}\right)$ may get accumulated in the human body through food chains. These highly toxic pollutants are non-biodegradable and may have adverse effects on the central nervous, immune and reproductive systems even at very low trace amounts. ${ }^{606}$ It is critical to develop new analytical techniques for monitoring toxic heavy metal ions in water, food and the environment. ${ }^{607}$ The electrochemical approach is a simple low-cost method and facilitates real-time monitoring of pollutants compared to other spectroscopic and optical techniques. ${ }^{608}$ Different types of nanostructured materials have been employed for effectively monitoring environmental pollutants. Graphene and its nanocomposites have shown great promise for removing toxic heavy metals and organic dyes from aqueous solutions. ${ }^{609,610}$ The graphene-based electrochemical sensors used for detecting various metal ions are summarized in Table 8 .

Muralikrishna et al. ${ }^{\mathbf{6 1 1}}$ utilized L-cysteine-functionalized GO for the simultaneous detection of $\mathrm{Cd}$ (II), $\mathrm{Cu}$ (II), $\mathrm{Pb}$ (II), and $\mathrm{Hg}$ (II) in the linear detection range of 0.4 to $2.0 \mu \mathrm{M}$ for $\mathrm{Cd}^{2+}, \mathrm{Cu}^{2+}$ and $\mathrm{Hg}^{2+}$ and 0.4 to $1.2 \mu \mathrm{M}$ for $\mathrm{Pb}^{2+}$. The L-cysteine/GO compositebased sensor showed LODs of $0.366,0.261,0.416$ and 1.113 $\mu \mathrm{g} \mathrm{\textrm {L } ^ { - 1 }}$ for $\mathrm{Cd}^{2+}, \mathrm{Cu}^{2+}, \mathrm{Pb}^{2+}$, and $\mathrm{Hg}^{2+}$, respectively. The limits of metal ion detection were found to be lower than the WHO standards. Gumpu et al. ${ }^{595}$ used a ruthenium(II) bipyridine complex $\left(\left[\mathrm{Ru}(\mathrm{bpy})_{3}\right]^{2+}\right)$ and GO nanocomposite to detect $\mathrm{Cd}(\mathrm{II})$, $\mathrm{Pb}$ (II), $\mathrm{As}$ (III) and $\mathrm{Hg}$ (II) metal ions. The sensor showed the lowest detection limits of $2.8 \mathrm{nM}$ for $\mathrm{Cd}(\mathrm{II}), 1.41 \mathrm{nM}$ for $\mathrm{Pb}(\mathrm{II}), 2.3 \mathrm{nM}$ for As(III) and $1.6 \mathrm{nM}$ for $\mathrm{Hg}$ (II) metal ions. Zhang et al. ${ }^{612}$ reported the use of $\mathrm{TiO}_{2}$-graphene hybrid nanostructures for $\mathrm{Pb}(\mathrm{II})$ and $\mathrm{Cd}(\mathrm{II})$ detection. The sensor showed linear ranges from $1.0 \times 10^{-8} \mathrm{M}$ to $3.2 \times 10^{-5} \mathrm{M}$ and $6.0 \times 10^{-7} \mathrm{M}$ to $3.2 \times$ $10^{-5} \mathrm{M}$ and LODs of $1.0 \times 10^{-10} \mathrm{M}$ and $2.0 \times 10^{-9} \mathrm{M}$ for $\mathrm{Pb}^{2+}$ and $\mathrm{Cd}^{2+}$ respectively. Palanisamy et al. ${ }^{613}$ reported the electrochemical determination of $\mathrm{Hg}$ (II) based on activated graphitemodified screen-printed carbon electrodes. The sensor exhibited a linear range of 0.05-14.77 ppm and an LOD of $4.6 \mathrm{ppb}$ with a sensitivity of $81.5 \mathrm{~mA} \mathrm{ppm} \mathrm{cm}^{-2}$. The LOD value is considerably below the guideline values for $\mathrm{Hg}$ (II) in drinking water. Seenivasan et al. ${ }^{\mathbf{6 1 4}}$ synthesized cysteine-functionalized GO that used carbonyldiimidazole as a cross-linker for the electrochemical detection of $\mathrm{Pb}^{2+}$ ions in water using DPV. The resulting sensor showed a linear range of 1.4-28 ppb with an LOD of $0.07 \mathrm{ppb}(\mathrm{S} / \mathrm{N}=3)$ for the detection of $\mathrm{Pb}^{2+}$ ions. This value is 2 -fold lower than the $10 \mathrm{ppb}$ threshold for drinking water set by WHO. Zhang et al. ${ }^{594}$ reported the activation of thymine- $\mathrm{Hg}^{2+}$-thymine $\left(\mathrm{T}-\mathrm{Hg}^{2+}-\mathrm{T}\right)$ coordination chemistry for the detection of $\mathrm{Hg}^{2+}$ with an LOD of $0.001 \mathrm{aM}$ using an electrodeposited graphene-Au-modified electrode for signal amplification. Fig. 28 shows a schematic illustration of the sensing approach for the detection of $\mathrm{Hg}^{2+}$, square wave voltammograms (SWV) of the biosensor as a function of $\mathrm{Hg}^{2+}$ ion concentration and its selectivity toward $\mathrm{Hg}^{2+}$ ions among various interfering metal ions and their mixture containing $\mathrm{Hg}^{2+}$ ions. In this sensor design, the electrodeposited GR-Au on a GCE surface is functionalized with a 10-mer thymine-rich DNA probe (P1), and the Au nanocarrier is functionalized with a 29mer guanine-rich DNA probe (P3) labeled with MB to further improve the signal response. With the addition of $\mathrm{Hg}^{2+}$, the T-T mismatched dsDNA between P1 and a 22-mer thymine-rich DNA probe (P2) forms on the surface of the electrode because of $\mathrm{T}-\mathrm{Hg}^{2+}-\mathrm{T}$ coordination chemistry and the introduction of the MB-nano-Au-P 3s for P2 hybridization. The SWV of the mercuric sensor showed a very broad range of $\mathrm{Hg}^{2+}$ ions concentration from $10 \mu \mathrm{m}$ to $0.001 \mathrm{aM}$ where the detection of $\mathrm{Hg}^{2+}$ ions ranged from $1.0 \mathrm{aM}$ to $100 \mathrm{nM}$ with an LOD of $0.001 \mathrm{aM}$. Furthermore, biosensor also showed very high selectivity toward $\mathrm{Hg}^{2+}$ ions among various interfering metal ions when measurements were performed by the square wave voltammetry using $10 \mathrm{nM}$ of $\mathrm{Hg}^{2+}$ and $500 \mathrm{nM}$ of $\mathrm{K}^{+}, \mathrm{Ca}^{2+}, \mathrm{Ba}^{2+}, \mathrm{Cd}^{2+}, \mathrm{Cr}^{2+}, \mathrm{Co}^{2+}, \mathrm{Cu}^{2+}, \mathrm{Mg}^{2+}, \mathrm{Mn}^{2+}$, $\mathrm{Pb}^{2+}, \mathrm{Ni}^{2+}, \mathrm{Zn}^{2+}, \mathrm{Fe}^{3+}, \mathrm{Al}^{3+}$, and their mixture with $10 \mathrm{nM}$ of $\mathrm{Hg}^{2+}$ respectively, where the sensitivity of interfering metal ions were found be less than $5 \%$ compared with $\mathrm{Hg}^{2+}$ ions. Yu et al. ${ }^{615}$ reported a noncovalently modified rGO sensor for the detection of $\mathrm{Hg}^{2+}$ down to the picomolar concentration range. The resulting sensor could detect $\mathrm{Hg}^{2+}$ ions at low concentrations up to $0.1 \mathrm{nM}$, which is lower than the limits of the WHO and the United States Environmental Protection Agency (US EPA). Lu et $a l .{ }^{598}$ developed electrochemical sensors for detecting $\mathrm{Hg}^{2+}$, $\mathrm{Cu}^{2+}, \mathrm{Cd}^{2+}$ ions and dopamine using silica nanochannel- 
(A)

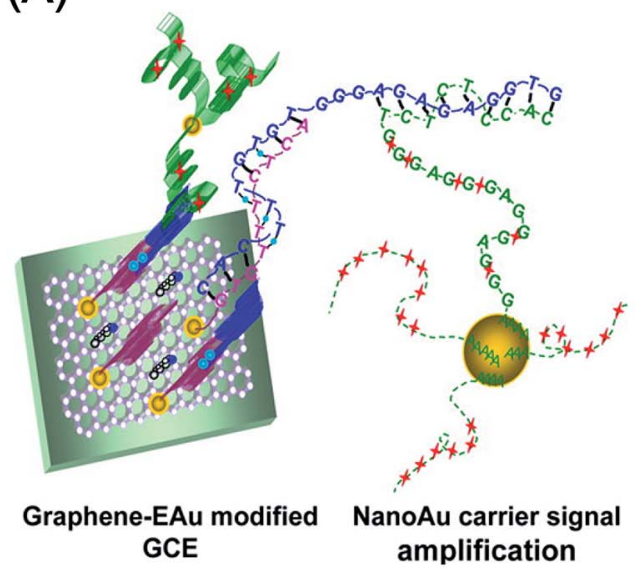

(B)

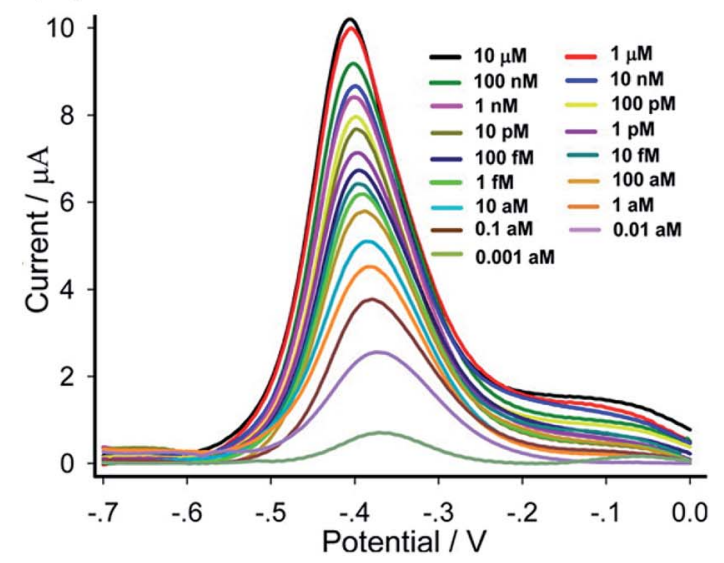

(C)

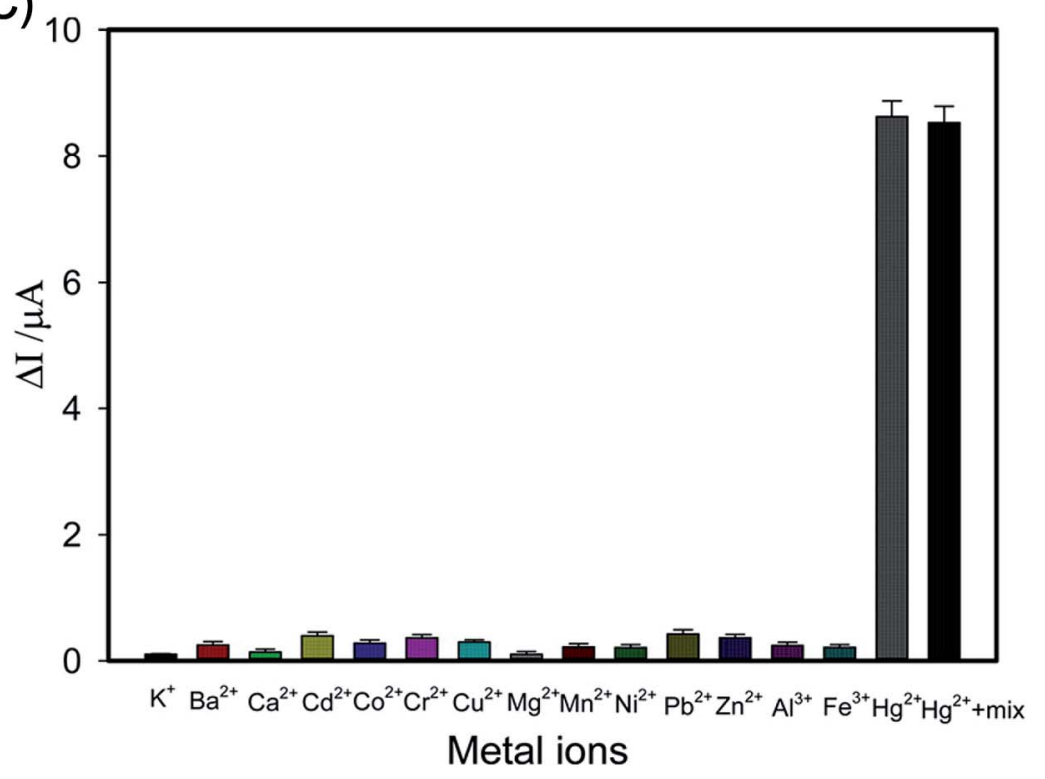

Fig. 28 (A) Schematic illustration of the sensing strategy for the detection of $\mathrm{Hg}^{2+}$ using a graphene/nano-Au composite for signal amplification. (B) Square wave voltammograms (SWV) of mercuric biosensor as a function of $\mathrm{Hg}^{2+}$ ions concentration in the $10 \mu \mathrm{m}$ to $0.001 \mathrm{aM} \mathrm{range} \mathrm{in} 20 \mathrm{~mL}$ Tris having $10 \mathrm{mM} \mathrm{KCl}$. (C) Mercuric biosensor showing the selectivity of $\mathrm{Hg}^{2+}$ ions among various interfering metal ions measured using $10 \mathrm{nM}$ of $\mathrm{Hg}^{2+}, 500 \mathrm{nM}$ of interfering metal ions $\mathrm{K}^{+}, \mathrm{Ba}^{2+}, \mathrm{Ca}^{2+}, \mathrm{Cd}^{2+}, \mathrm{Co}^{2+}, \mathrm{Cr}^{2+}, \mathrm{Cu}^{2+}, \mathrm{Mg}^{2+}, \mathrm{Mn}^{2+}, \mathrm{Ni}^{2+}, \mathrm{Pb}^{2+}, \mathrm{Zn}^{2+}, \mathrm{Al}^{3+}, \mathrm{Fe}^{3+}$, and their $\mathrm{Corresponding}^{2}$ mixture having $10 \mathrm{nM}$ of $\mathrm{Hg}^{2+}$ respectively. [Reprinted with permission from ref. 594, Y. Zhang, G. M. Zeng, L. Tang, J. Chen, Y. Zhu, X. X. He and Y. He, Electrochemical Sensor Based on Electrodeposited Graphene-Au Modified Electrode and NanoAu Carrier Amplified Signal Strategy for Attomolar Mercury Detection, Anal. Chem., 2015, 87, 989-996. Copyright $\odot$ American Chemical Society.]

confined GQDs. Fig. 29 shows DPV curves recorded with vertically ordered mesoporous silica-nanochannel film (VMSF)/ITO electrode, OH-GQD@VMSF/ITO and $\mathrm{NH}_{2}$-GQD@VMSF/ITO electrodes for the detection of $\mathrm{Hg}^{2+}, \mathrm{Cu}^{2+}$, and $\mathrm{Cd}^{2+}$. Both $\mathrm{OH}^{-}$ GQD@VMSF/ITO and $\mathrm{NH}_{2}$-GQD@VMSF/ITO electrodes exhibited significantly amplified current signals for detecting $\mathrm{Hg}^{2+}$, $\mathrm{Cu}^{2+}$, and $\mathrm{Cd}^{2+}$ ions than that of VMSF/ITO electrode having no GQDs. This indicates that the significant increase in current signals is associated with the GQDs due to their molecular interactions with $\mathrm{Hg}^{2+}, \mathrm{Cu}^{2+}$, and $\mathrm{Cd}^{2+}$ ions, efficient electronic transfer between GQDs and metal ions as well as high specific surface area inherited by GQDs. DPV curves were also recorded as a function of different concentrations of $\mathrm{Hg}^{2+}$ and $\mathrm{Cu}^{2+}$ using OH-GQD@VMSF/ITO electrode and as a function of different concentrations of $\mathrm{Cd}^{2+}$ using $\mathrm{NH}_{2}$-GQD@VMSF/ITO electrode. The OH-GQD@VMSF/ITO electrode-based sensing platform could detect $\mathrm{Hg}^{2+}$ and $\mathrm{Cu}^{2+}$ ions at concentrations as low as 9.8 $\mathrm{pM}$ and $8.3 \mathrm{pM}$, respectively, compared with the LOD of $0.3 \mu \mathrm{M}$ for $\mathrm{Hg}^{2+}$ and $32 \mathrm{nM}$ for $\mathrm{Cu}^{2+}$ using VMSF/ITO electrode. Likely, the $\mathrm{NH}_{2}$-GQD@VMSF/ITO electrode showed an LOD of $4.3 \mathrm{nM}$ for $\mathrm{Cd}^{2+}$ compared with $120 \mathrm{nM}$ using VMSF/ITO electrode. The LOD for detecting $\mathrm{Hg}^{2+}$ was reduced by 5 orders of magnitude by incorporating OH-GQDs into the electrode. The ITO electrode was replaced by Au electrode, the resulting OH-GQD@VMSF/Au 

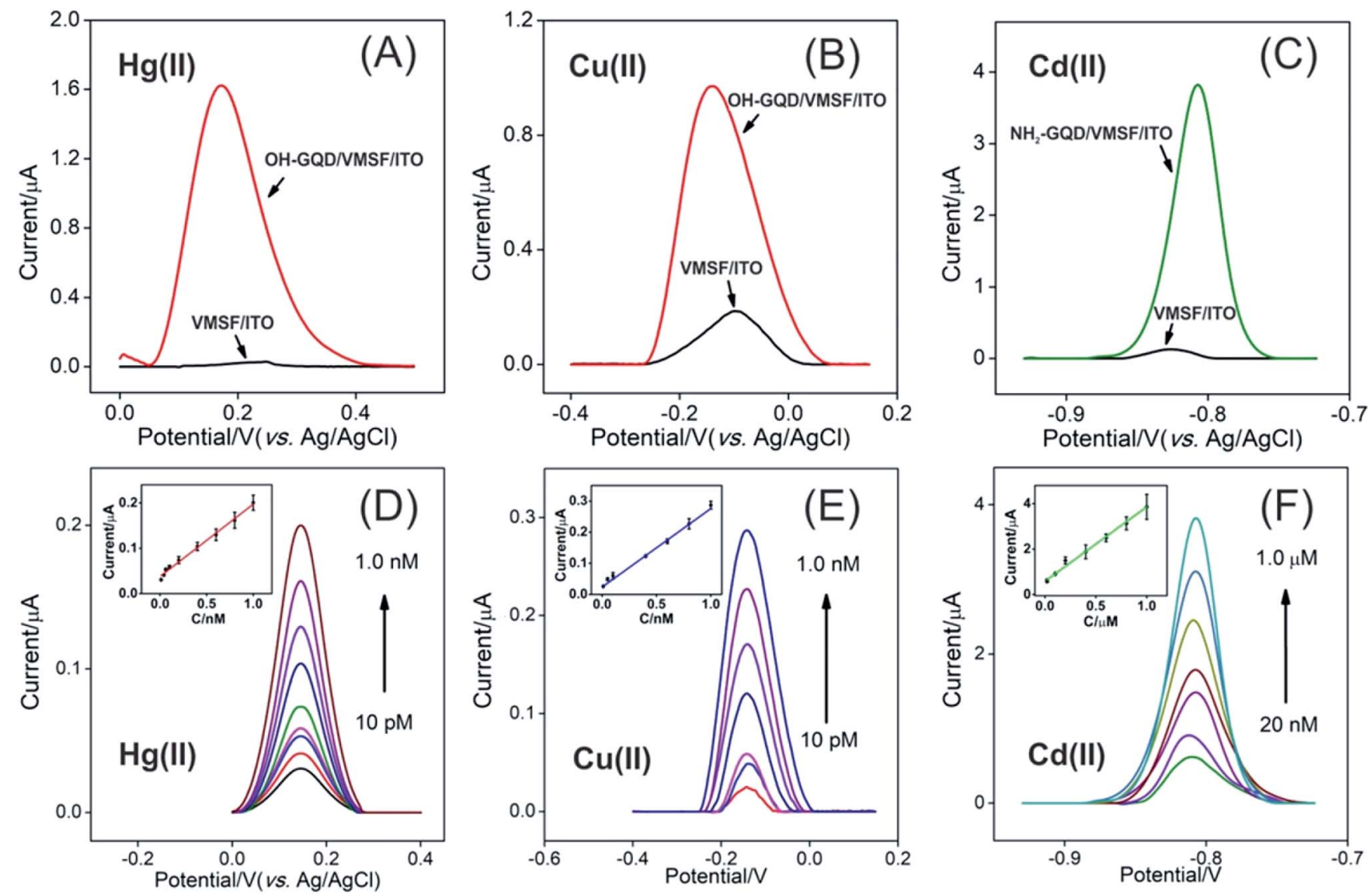

Fig. 29 (A-C) A comparison of DPV curves recorded with different electrodes including vertically ordered mesoporous silica-nanochannel film (VMSF)/ITO electrode, OH-GQDaVMSF/ITO and NH $\mathrm{H}_{2}$-GQD @VMSF/ITO electrodes for detecting $\mathrm{Hg}^{2+}(0.5 \mu \mathrm{M}), \mathrm{Cu}^{2+}(1.0 \mu \mathrm{M})$, and Cd $\mathrm{Cd}^{2+}(1.0 \mu \mathrm{M})$, respectively. ( $D$ and $E$ ) DPV curves recorded as a function of different concentrations of $\mathrm{Hg}^{2+}$ and $\mathrm{Cu}^{2+}$ using OH-GQDQVMSF/ITO electrode. (F) DPV curves obtained as a function of different concentrations of $\mathrm{Cd}^{2+}$ using $\mathrm{NH}_{2}-\mathrm{GQD}(\mathrm{VVMSF} / \mathrm{ITO}$ electrode. The (D)-(F) insets show linear curves obtained in a very low concentration range. [Reprinted with permission from ref. 598, L. Lu, L. Zhou, J. Chen, F. Yan, J. Liu, X. Dong, F. Xi and P. Chen, Nanochannel-Confined Graphene Quantum Dots for Ultrasensitive Electrochemical Analysis of Complex Samples, ACS Nano, 2018, 12, 12673-12681. Copyright@ American Chemical Society.]

electrode showed a linear range of $200 \mathrm{~nm}$ to $100 \mu \mathrm{M}$ and an LOD of $120 \mathrm{~nm}$ for the detection of dopamine. These GQD@VMSF/ITO electrode-based sensors also showed high sensitivity and selectivity toward $\mathrm{Hg}^{2+}, \mathrm{Cu}^{2+}$, and $\mathrm{Cd}^{2+}$ ions in presence of other interfering chemical analytes including $\mathrm{Ca}^{2+}, \mathrm{Mg}^{2+}, \mathrm{Co}^{2+}, \mathrm{Fe}^{3+}, \mathrm{Cr}^{3}$, BSA and hemoglobin. Similarly, the OH-GQD@VMSF/Au electrode-based sensor showed high selectivity toward dopamine in the presence of other interfering bioanalytes including common ions, amino acids, protein (concanavalin A), ascorbic acid and uric acid.

\subsection{Detection of pesticides}

Pesticides are widely employed in agriculture to control weeds and pests and to increase food production around the world. Herbicides and insecticides used to protect plants can be potentially toxic to human. Their overuse and improper disposal can cause significant food and environmental contamination as pesticides easily dissolve in water and do not decompose easily so they may have harmful health effects. ${ }^{616}$ Therefore, it is important to develop biosensors for the sensitive detection of agro-hazardous pesticide residues in food and water to avoid toxic effects to humans. Organophosphorus pesticides (OPs) are used as insecticides to protect crops worldwide, but they are equally unsafe to public health due to their high neurotoxicity. ${ }^{617}$ Acetylcholinesterase (AChE), a kind of acetylhydrolase, is a catalytic enzyme present in the central nervous system that catalyzes the hydrolysis of neurotransmitters such as acetylcholine and choline esters. AChE inhibitorbased drugs have been approved by the FDA for clinical trials and the management of Alzheimer disease, which is a neurodegenerative disorder. ${ }^{618-620}$ The catalytic activity of AChE is significantly inhibited by exposure to trace amounts of environmentally toxic OPs and OP nerve agents. ${ }^{621-623}$ This catalytic inhibition concept has been used to develop various types of AChE-based biosensors for detecting OPs. ${ }^{624-627}$ AChE can be easily immobilized on the surface of nanostructured materials to improve the sensitivity of biosensors for detecting pesticide residues. ${ }^{628-634}$ Graphene-based materials also offer high surface area and plentiful active sites; therefore, they have been used for developing AChE inhibition biosensors.

Two-dimensional layered nanomaterials analogous to graphene $^{635}$ and transition metal dichalcogenides (TMDs) ${ }^{636}$ have attracted significant research interest for developing biosensors 
for the electrochemical detection of pesticides. Owing to its high surface area and unique electrocatalytic properties, graphene can markedly enhance biosensing performance (Table 9). AChE electrochemical biosensors with graphene nanocompositemodified GCEs have been studied for the detection of different types of pesticides: methyl parathion, carbofuran, ${ }^{637}$ chlorpyrifos, ${ }^{\mathbf{6 3 8 - 6 4 0}}$ imidacloprid, ${ }^{\mathbf{6 4 1}}$ methyl parathion, ${ }^{\mathbf{6 4 2}}$ phoxim with graphene/GCE, ${ }^{643}$ poly(3-methylthiophene)/nitrogen-doped graphene (P3MT/N-GR), ${ }^{644}$ and carboxylic chitosan (CChit)/silver nanoclusters-rGO (AgNCs-rGO), ${ }^{645}$ paraoxon and chlorpyrifos with $\mathrm{TiO}_{2}$-GO/UiO-66 composite, ${ }^{646}$ carbaryl with MWCNTs/GO nanoribbons, ${ }^{647}$ carbaryl and chlorpyrifos with AgNPs-CGR/NF composite, ${ }^{648}$ chlorpyrifos and carbofuran with ZnONPs-CGR/ $\mathrm{NF}$ composite, ${ }^{649}$ carbaryl and monocrotophos with ionic liquid-functionalized graphene (IL-GR)/gelatin, ${ }^{650}$ monocrotophos with Prussian blue nanocubes (PBNCs)/rGO, ${ }^{651}$ malathion and carbaryl with rGO-AuNP/ $\beta$-cyclodextrin/Prussian blue-CS nanocomposites (rGO-AuNPs/ $\beta$-CD/PB-Chit), ${ }^{652}$ dichlorvos with multilayered Chit@ $\mathrm{TiO}_{2}-\mathrm{Chit} / \mathrm{rGO},{ }^{653}$ fenitrothion with cerium oxide nanoparticle-decorated $\mathrm{rGO}\left(\mathrm{CeO}_{2}\right.$ rGO) ${ }^{654}$ diuron with rGO-AuNPs, ${ }^{655}$ paraoxon-ethyl with rGOAuNPs/polypyrrole (PPy), ${ }^{656}$ carbaryl with GR/PANI, ${ }^{657}$ carbaryl with an electrochemically induced porous GO network, ${ }^{658}$ methyl parathion and malathion with plant esterase (PLaE)Chit/AuNPs-graphene nanosheets (PLaE-Chit/AuNPs-GNs) ${ }^{659}$

An electrochemical AChE biosensor using rGO/silver nanoclusters (AgNCs)/chitosan composite was fabricated by Zheng et $a l .{ }^{645}$ The immobilization of AChE on a GCE modified with rGO and AgNCs was accomplished using carboxylic chitosan as a cross-linking agent. The biosensor exhibited a wide linear range of $0.2-250 \mathrm{nM}$ and an LOD of $81 \mathrm{pM}$ for phoxim with $\mathrm{S} / \mathrm{N}$ $=3$. Bao et al. ${ }^{659}$ developed a sensor using plant esterase-

Table 9 Graphene nanocomposite-modified glassy carbon electrode (GCE)-based acetylcholinesterase (AChE) electrochemical biosensors for detecting pesticides ${ }^{a}$

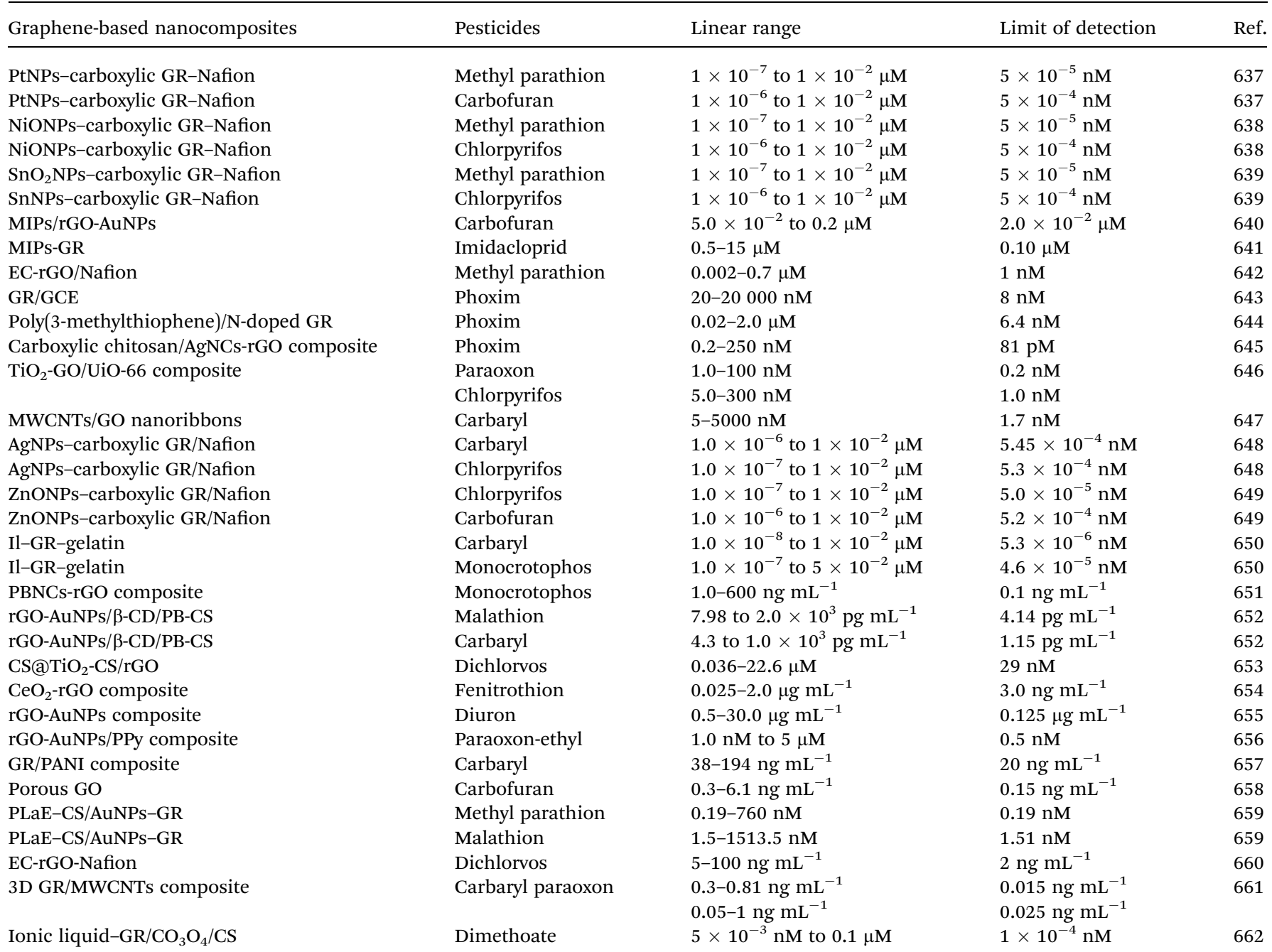

${ }^{a}$ Abbreviations used AChE: acetylcholinesterase, GCE: glassy carbon electrode, PLaE: plant esterase, GNs: graphene nanosheets, AuNPs: gold nanoparticles, carboxylic graphene (C-GR), Nafion (NF), PtNPs: platinum nanoparticles, NiONPs: nickel oxide nanoparticles, SnO ${ }_{2} \mathrm{NPs}$ : tin oxide nanoparticles, MIPs: molecularly imprinted polymers, C-CS: carboxylic chitosan, MWCNTs: multiwalled carbon nanotubes, Il-GR: ionic liquidfunctionalized graphene, rGO: reduced graphene oxide, $\beta$-CD: $\beta$-cyclodextrin, PBNCs: Prussian blue nanocubes, CeO ${ }_{2}$ : cerium oxide, PPy: polypyrrole, PANI: polyaniline, methyl parathion: $O, O$-dimethyl-O-(4-nitrophenyl) phosphorothioate. 


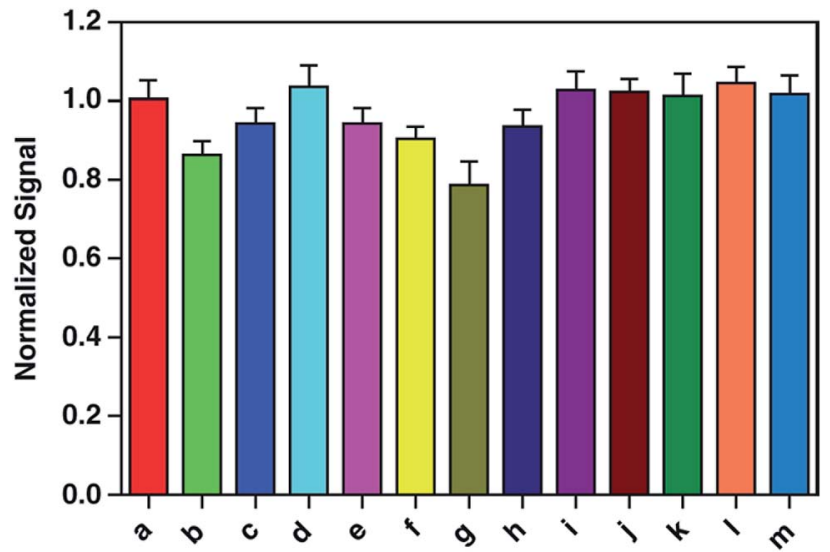

Fig. 30 DPVs at the glassy carbon electrode GCE on the PLaE-Chit/ AuNPs-GN nanocomposite for (a) $10 \mathrm{ppb}$ of methyl parathion and then $10 \mathrm{ppb}$ of methyl parathion mixed with (b) $10 \mathrm{ppb}$ of carbendazim, (c) $10 \mathrm{ppb}$ of lindane, (d) $1 \mathrm{ppm}$ of $\mathrm{Fe}^{3+}$, (e) $1 \mathrm{ppm}$ of $\mathrm{Zn}^{2+}$, (f) $1 \mathrm{ppm}$ of $\mathrm{Cu}^{2+}$, (g) $1 \mathrm{ppm}$ of $\mathrm{Pb}^{2+}$, (h) $1 \mathrm{ppm}$ of $\mathrm{K}^{+}$, (i) $1 \mathrm{ppm}$ of $\mathrm{NO}^{3-}$, (j) $1 \mathrm{ppm}$ of $\mathrm{PO}_{4}{ }^{3-}$, (k) 1 ppm of $\mathrm{SO}_{4}{ }^{2-}$, (l) $0.5 \mathrm{mM}$ glucose, or (m) $0.5 \mathrm{mM}$ citric acid. [Reprinted with permission from ref. 659, J. Bao, C. Hou, M. Chen, J. Li, D. Huo, M. Yang, X. Luo and Y. Lei, Plant Esterase-Chitosan/Gold Nanoparticles-Graphene Nanosheet Composite-Based Biosensor for the Ultrasensitive Detection of Organophosphate Pesticides, J. Agric. Food Chem., 2015, 63, 10319-10326. Copyright@ 2018 American Chemical Society.]

chitosan/AuNPs-graphene nanosheets for the detection of methyl parathion and malathion. Esterase is a plant enzyme that dissociates esters into acid and alcohol components and catalyzes OPs with a similar mechanism to that of the AChE enzyme. The nanostructures of graphene nanosheets, AuNPGNs, and PLaE-Chit/AuNP-GN nanocomposites were characterized using SEM and TEM. AuNPs approximately $13 \mathrm{~nm}$ in diameter were uniformly distributed on the crumpled GN surfaces. The particles of PLaE and CS nanocomposite were clearly visible on the AuNP-GN composites, where TEM images confirmed the formation of hybrid composites suitable for detecting OPs. The nanocomposite-based biosensor showed detection limits of $0.19 \mathrm{nM}(50 \mathrm{ppt})$ for methyl parathion and $1.51 \mathrm{nM}(0.5 \mathrm{ppb})$ for malathion $(\mathrm{S} / \mathrm{N}=3)$. The biosensor also exhibited high selectivity because no interference from other analytes, such as glucose, citric acid, metal ions and oxygencontaining inorganic ions, was observed. The specificity of the sensor was evaluated for the detection of OPs by comparing the normalized signal of 0.1 M PBS buffer solution ( $\mathrm{pH}$ 6.5) containing $0.5 \mathrm{mM}$ 1-naphthyl acetate in $10 \mathrm{ppb}$ of methyl parathion $(100 \%)$ with the normalized signals recorded in the presence of methyl parathion in combination with different interfering analytes (Fig. 30). The DPV data did not show any significant changes in the normalized signals for the presence of (c) $10 \mathrm{ppb}$ lindane, (d) $1 \mathrm{ppm} \mathrm{Fe}^{3+}$, (e) $1 \mathrm{ppm} \mathrm{Zn}^{2+}$, (f) $1 \mathrm{ppm}$ $\mathrm{Cu}^{2+}$, (h) $1 \mathrm{ppm} \mathrm{K}$, (i) $1 \mathrm{ppm} \mathrm{NO}^{3-}$, (j) $1 \mathrm{ppm} \mathrm{PO}_{4}{ }^{3-}$, (k) $1 \mathrm{ppm}$ $\mathrm{SO}_{4}{ }^{2-}$, (l) $0.5 \mathrm{mM}$ glucose, and (m) $0.5 \mathrm{mM}$ citric acid after mixing with $10 \mathrm{ppb}$ of methyl parathion. Interference with the $10 \mathrm{ppb}$ methyl parathion was observed only with (b) $10 \mathrm{ppb}$ of carbendazim and $(\mathrm{g}) 1 \mathrm{ppm} \mathrm{Pb}^{2+}$ with detection of 85.97 and $78.37 \%$, respectively. The biosensor also showed storage
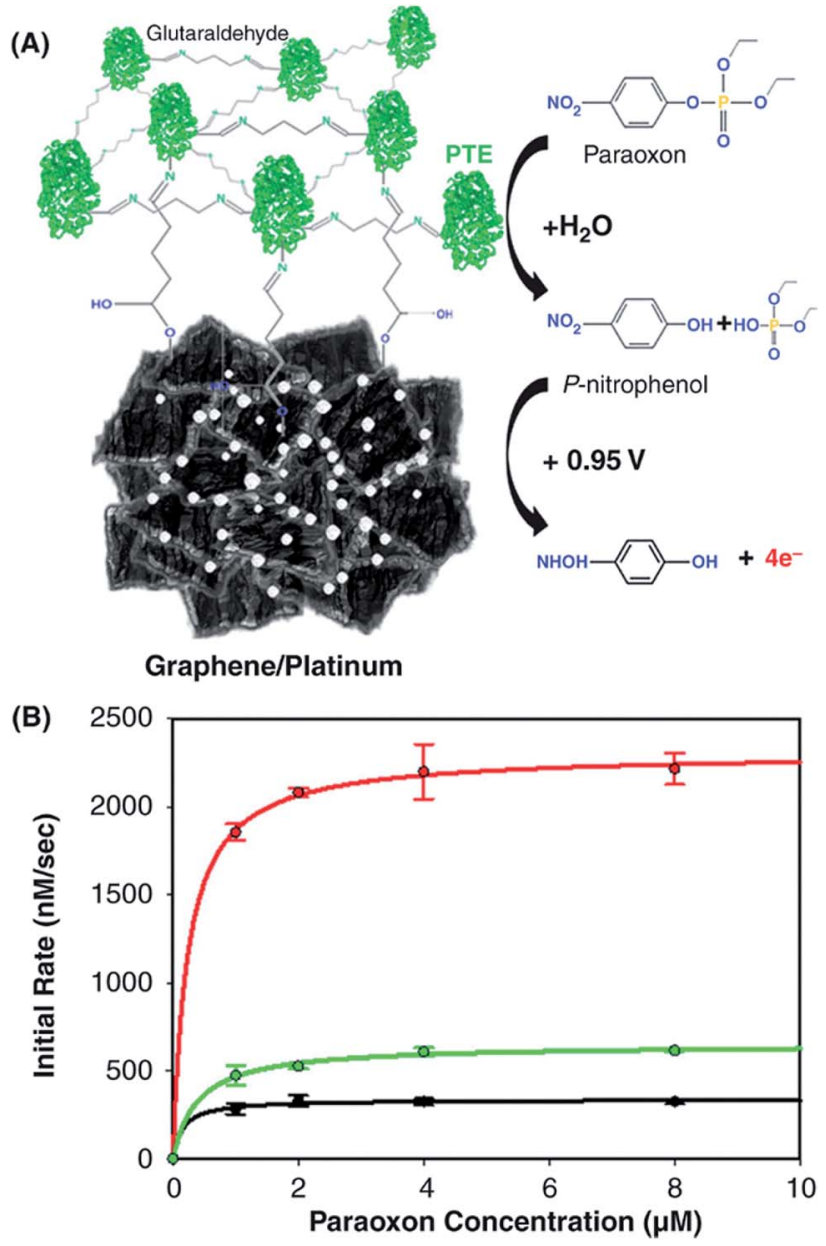

Fig. 31 (A) Schematic illustration of phosphotriesterase (PTE) enzyme functionalization on the PtNPs-inkjet maskless lithography-PGE (PtNP-IML-PGE) surface using glutaraldehyde (GA). Hydrolysis of paraoxon into $p$-nitrophenol by the immobilized PTE enzyme and thereafter, successive oxidation of $p$-nitrophenol at the surface of graphene electrode at a working potential of $+0.95 \mathrm{~V} v$ s. $\mathrm{Ag} / \mathrm{AgCl}$. (B) Enzyme progress of $p$-nitrophenol production rate for varying paraoxon concentrations using enzyme inks created using 2 nM (black), $4 \mathrm{nM}$ (green), and $20 \mathrm{nM}$ (red) concentrations of PTE. [Reprinted with permission from ref. 663, J. A. Hondred, J. C. Breger, N. J. Alves, S. A. Trammell, S. A. Walper, I. L. Medintz and J. C. Claussen, Printed Graphene Electrochemical Biosensors Fabricated by Inkjet Maskless Lithography for Rapid and Sensitive Detection of Organophosphates, ACS Appl. Mater. Interfaces, 2018, 10, 11125-11134. Copyright(C 2018 American Chemical Society.]

stability, as no decrease in the normalized signal was observed after 9 days, and $73 \%$ of the initial signal was retained after 15 days.

Hondred et al. ${ }^{663}$ developed an electrochemical sensor with Pt nanoparticles electrodeposited on patterned graphene electrodes (PGEs) for detecting OPs. The electrode was first fabricated via inkjet maskless lithography (IML) followed by the electrodeposition of Pt nanoparticles to enhance its surface area, electrical conductivity, and electrocatalytic activity. Next, the enzyme phosphotriesterase was conjugated using the bifunctional cross-linking molecule glutaraldehyde (GA), as shown in Fig. 31. The fabricated PtNP-IML-PGE/GA biosensor 
showed rapid detection of paraoxon pesticide with $5 \mathrm{~s}$ response time, a sensitivity of $370 \mathrm{nA} \mu \mathrm{M}$, a linear range of $0.1-1 \mu \mathrm{M}$, and an LOD of $3 \mathrm{nM}$. In comparison, the biosensor based on IMLPGE/GA (without PtNPs) showed a sensitivity of $270 \mathrm{nA} \mu \mathrm{M}$, a linear range of $0.1-1 \mu \mathrm{M}$, and an LOD of $12 \mathrm{nM}$. The PtNPIML-PGE/GA biosensor showed reusability over 12 repeated operations by retaining $95 \%$ sensitivity and $70 \%$ stability of anodic current signal to paraoxon after 8 weeks. The biosensor exhibited high selectivity toward paraoxon detection against interfering chemical nerve agents including $p$-nitrophenol, chlorpyrifos methyl, parathion, dichlofenthion, fenitrothion, phoxim, and dimethoate. These PtNP-IML-PGEs biosensors can be manufactured in large quantities at a low-cost and used in-field for detecting pesticides and other biochemical agents in river water, tap water and soil samples.

\section{Graphene-based fluorescent biosensors}

Fluorescence-based biosensing is a well-established optical tool with many advantages, such as high sensitivity, rapid detection time and easy operation, making it an ideal platform for biological, biochemical and medical research. ${ }^{664}$ The development of fluorescent biosensors to detect different biological molecules, such as glucose, $\mathrm{H}_{2} \mathrm{O}_{2}$, dopamine, NAs, food toxins and metal ions, is of great interest to medicine since this technique can provide direct readouts of molecular localization in different biological organisms and living cells. Since the discovery of green fluorescent protein (GFP), which enabled the observation of molecular interactions within a $10 \mathrm{nM}$ range through the Förster resonance energy transfer (FRET) mechanism, a wide variety of fluorescent biosensors have been developed utilizing different types of nanomaterials with high sensitivity and selectivity, and low detection limits. ${ }^{665}$ Specifically, carbon-based nanomaterials such as GR or GO have been found to quench fluorescence very effectively, which makes them strong acceptors in the FRET process over the entire visible region. In addition, further functionalization with carboxyl $(-\mathrm{COOH})$ groups has been applied to fluorescent sensors in order to attain high fluorescence quenching and ssDNA interaction through $\pi-\pi$ stacking. ${ }^{21} \mathrm{GO}$ has been found to exhibit high florescence quenching up to a distance of $300 \AA{ }^{666}$

Graphene-based fluorescence quenching has been predominantly accredited to FRET due to the linear dispersion of Dirac electrons and the relatively distant effective gap between fluorescent molecules and graphene. ${ }^{67}$ The linear dispersion of Dirac fermions allows electron-hole pairs in graphene to resonate with photons of various frequencies that are emitted from fluorescent molecules. This kind of FRET mechanism and the high-efficiency quenching ability of graphene for all types of fluorophores makes GO promising for the development of new fluorescent-based biological sensors and other devices for their use in vivo and in vitro. ${ }^{21,462,668}$ Moreover, the amphiphilicity of GO facilitates the adsorption of diverse biomolecules on its surface due to its high binding affinity with biomolecules via $\pi-$ $\pi$ stacking. ${ }^{669}$ GO nanosheet shows the capability of efficient energy transfer when the adsorbed biomolecules are conjugated with a fluorescent dye, yielding fluorescence quenching with minimal background signal because of the absence of graphene's energy bandgap and the existing close-distance $\pi-\pi$ stacking. The progress made in fluorescence-based biosensors have been discussed. ${ }^{17,21,128}$ The graphene- and graphenenanocomposite-based fluorescent biosensors for the detection of various biomolecules are summarized in Table 10 .

\subsection{Fluorescent detection of glucose, cholesterol, $\mathrm{H}_{2} \mathrm{O}_{2}$, and dopamine}

Graphene-based nanocomposites have been widely applied to the fluorescence-based detection of glucose, $\mathrm{H}_{2} \mathrm{O}_{2}$, DA, and so forth, with enhanced sensitivity and selectivity (Table 10). Li et $a l .{ }^{670}$ developed tyramine (TYR)-functionalized GQDs (TYRGQDs) for fluorescent biosensing of $\mathrm{H}_{2} \mathrm{O}_{2}$ and four metabolites including glucose, cholesterol, L-lactate, and xanthine in the blood. In the TYR-GQDs conjugate, the tyramine is covalently attached on the surface of GQDs through the reaction between the amino $\left(-\mathrm{NH}_{2}\right)$ groups of TYR and carboxyl $(-\mathrm{COOH})$ groups of GQDs. In order to detect these four metabolites, their corresponding oxidases such as glucose oxidase, cholesterol oxidase, $\mathrm{L}$-lactate oxidase, and xanthine oxidase were added into the $10 \mathrm{mM}$ PBS (pH 7.0) containing $0.1 \mathrm{mg} \mathrm{mL}{ }^{-1}$ TYR-GQDs conjugate to trigger photoluminescence (PL) quenching. Fig. 32 shows a schematic of fluorescence biosensing using TYR-GQDs, and PL spectra of $0.1 \mathrm{mg} \mathrm{mL}^{-1}$ TYR-GQDs conjugate in PBS containing $2.5 \mu \mathrm{M}$ glucose oxidase, $10 \mu \mathrm{M}$ cholesterol oxidase and $5 \mu \mathrm{M}$ L-lactate oxidase at different concentrations of glucose, cholesterol and L-lactate, respectively. The TYR-GQDbased fluorescence biosensors exhibited LODs of $7.2 \times$ $10^{-9} \mathrm{M}, 3.2 \times 10^{-10} \mathrm{M}$, and $1.2 \times 10^{-9} \mathrm{M}$ with linear ranges of 5 $\times 10^{-8}$ to $2 \times 10^{-6} \mathrm{M}, 1 \times 10^{-9}$ to $1.5 \times 10^{-7} \mathrm{M}$, and $8 \times 10^{-8}$ to $1 \times 10^{-5} \mathrm{M}$ for glucose, $\mathrm{H}_{2} \mathrm{O}_{2}$ and cholesterol detection, respectively. The biosensor showed a linear range of $2 \times 10^{-7}$ to $2.5 \times 10^{-5} \mathrm{M}$ and $1 \times 10^{-7}$ to $1.8 \times 10^{-5} \mathrm{M}$ with LOD of $4.7 \times$ $10^{-8} \mathrm{M}$ and $3.2 \times 10^{-8} \mathrm{M}$ for L-lactate and xanthine detection, respectively. The evident PL quenching was not observed for TYR-GQDs after adding either glucose oxidase or glucose and other interferents such as fructose, lactose and maltose mixed with glucose oxidase, indicating high selectivity of the biosensor toward glucose detection. These TYR-GQD-based fluorescence biosensors showing high sensitivity and selectivity, can be used for healthcare for diagnosis of metabolic and other related diseases.

Chen et $a .^{708}$ demonstrated a GO-based label-free near infrared (NIR) fluorescent sensor for detecting dopamine. The good affinity of DA molecules with GO allows multiple noncovalent interactions. The effective quenching of GO when DA molecules are adsorbed onto the GO nanosheet enables biosensing by way of direct readout of the NIR fluorescence of the GO in the NIR for the effective detection of DA. The sensor showed an LOD of $94 \mathrm{nM}$ and a $2.0 \%$ relative standard deviation; it was used to DA determination in biological fluids with 98 to $115 \%$ quantitative recovery. Cheng et al. ${ }^{709}$ demonstrated a label-free FRET-based biosensor using doxorubicin (DOX)- 
Table 10 Graphene-based fluorescent biosensors for detecting biomolecules, nucleic acids, biomarkers, pathogens, food toxins, metal ions and pesticides $^{a}$

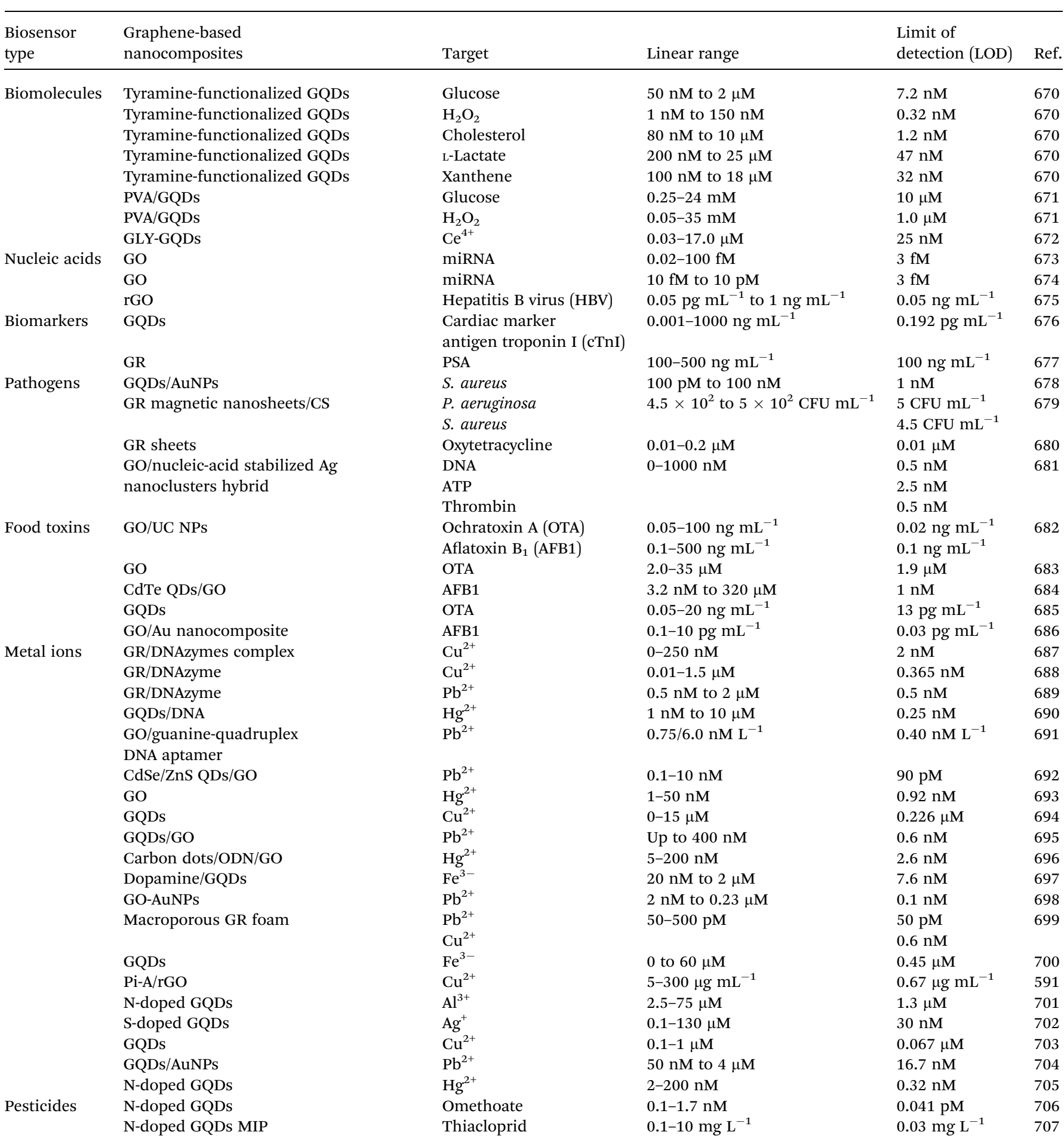

${ }^{a}$ Abbreviations used PVA: poly(vinyl alcohol), PSA: (prostate specific antigen, a cancer marker), GLY: glycine, AFB1: aflatoxin B1, cTnI: cardiac marker antigen troponin I in blood, ATP: adenosine triphosphate, Pi-A: $N$-(2-(1-( $p$-tolyl)- $1 H$-phenanthro[9,10- $d]$ imidazol-2-yl)phenyl)picolinamide, CDs: carbon dots, ODN: oligodeoxyribonucleotide, MIP: molecularly imprinted polymer. Upconversion fluorescent $\mathrm{NPs} \mathrm{BaY}_{0.78} \mathrm{~F}_{5}: \mathrm{Yb}_{0.2}, \mathrm{Er}_{0.02}$ and $\mathrm{BaY}_{0.78} \mathrm{~F}_{5}: \mathrm{Yb}_{0.7}, \mathrm{Tm}_{0.02}$ nanoparticles.

functionalized GO for detecting DA in human serum, aqueous solutions and cells. The fluorescence is quenched upon adsorption onto GO. Upon the addition of DA, the preadsorbed DOX is released by the specific interaction occurring between
DA and GO due to the significantly higher binding affinity of DA molecules, which recovers the fluorescence of DOX and enables selective sensing of DA. The fluorescent DOX-GO sensor showed a high response for the detection of DA in the $8.3 \times 10^{-7} \mathrm{M}$ to 3.3 
$\times 10^{-5} \mathrm{M}$ concentration range an aqueous solution and 1.44-11.48 $\mu \mathrm{mol} \mathrm{L}{ }^{-1}$ in a human serum sample. A linear relationship was observed between the ratio of fluorescence intensity $\left(F_{0} / F\right)$ of DOX before and after adding GO as a function of the GO concentration over 0.5 to $20 \mu \mathrm{g} \mathrm{mL}{ }^{-1}$ range at $598 \mathrm{~nm}$.

\subsection{Fluorescence detection of nucleic acids}

Fluorescence-based detection has become an attractive research area for a wide range of applications. It is suitable for NA sensors where the target-triggered conformational change of an oligonucleotide affects the fluorescence of DNA-binding dyes such as OliGreen, TOTO, and ethidium bromide. ${ }^{11}$ Carbon nanomaterials functionalized with carboxyl $(-\mathrm{COOH})$ groups have been commonly used in NA sensors, which offer great fluorescence quenching and interaction with ssDNA via $\pi-\pi$ stacking. Specifically, graphene and GO-based fluorescent sensors have been widely employed for the detection of biologically active molecules such as DNA, RNA, and proteins..$^{\mathbf{2 1 , 6 0 2}}$ Most of these biosensor designs involve the use of DNA as the basis for templates for targeted molecule recognition and reporting. These DNA segments including aptamers have been (a)
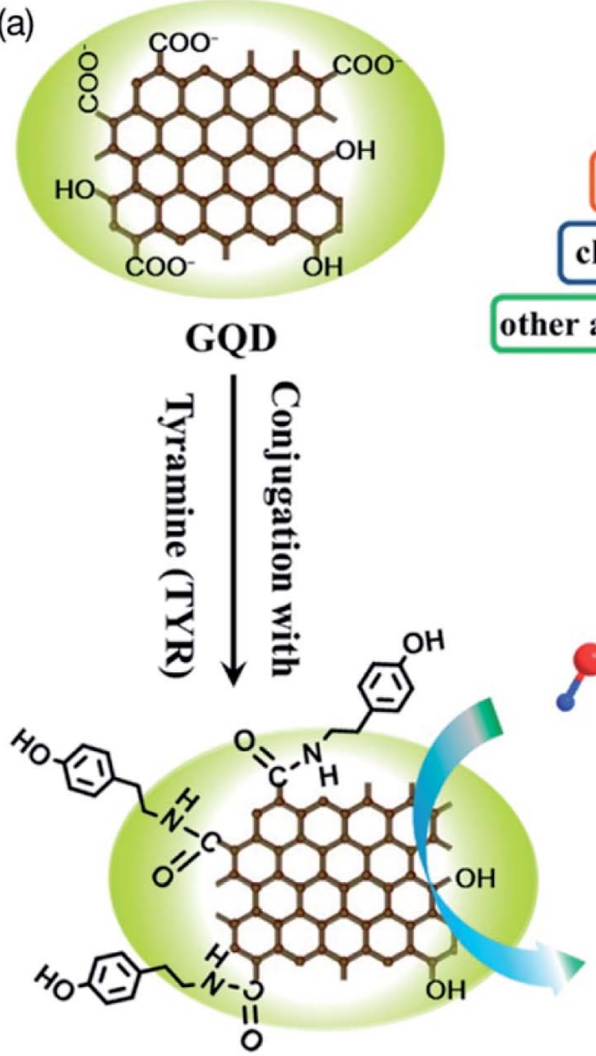

TYR-GQD

(b)

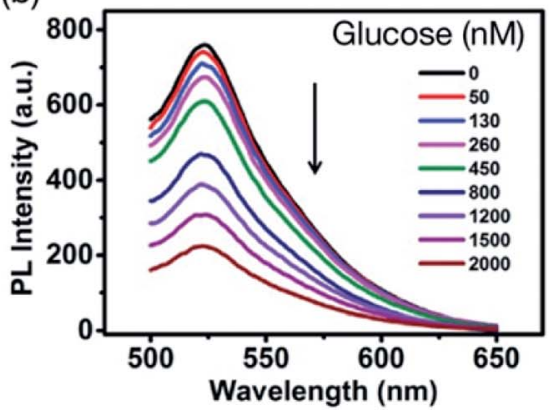

(c)

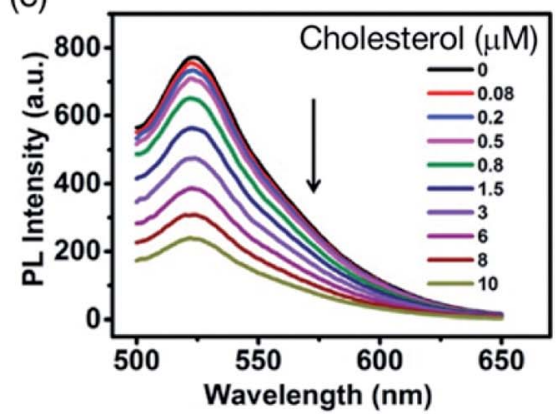

Product

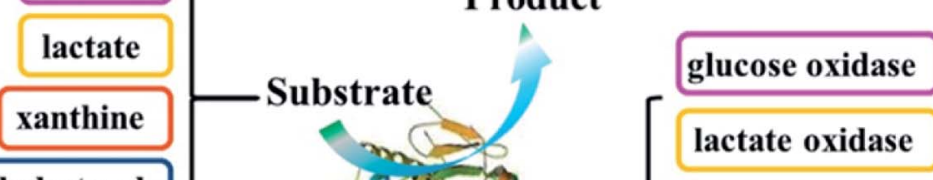

cholesterol other analytes...

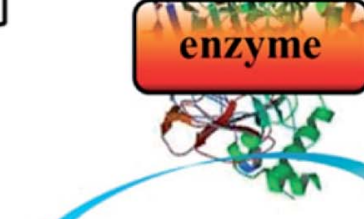

$\mathrm{C}\left(\mathbf{O}_{2}\right)$ $\left(\mathrm{H}_{2} \mathrm{O}_{2}\right)$
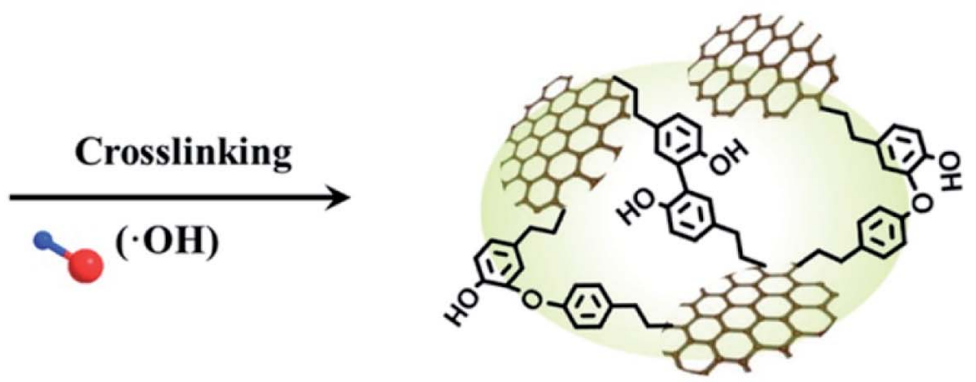

Fluorescence quenching

(d)

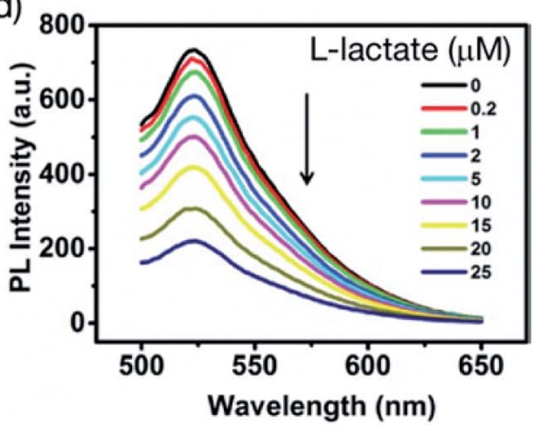

Fig. 32 (a) Schematic illustration of fluorescence detection scheme using tyramine-functionalized GQDs (TYR-GQDs). (b) Photoluminescence $(\mathrm{PL})$ spectra of $0.1 \mathrm{mg} \mathrm{mL}^{-1}$ TYR-GQD in PBS ( $\mathrm{pH} 7.0$ ) containing $2.5 \mu \mathrm{M}$ glucose oxidase and at different concentrations of glucose. (c) PL spectra of spectra of $0.1 \mathrm{mg} \mathrm{mL}^{-1}$ TYR-GQD in PBS ( $\mathrm{pH}$ 7.0) containing $10 \mu \mathrm{M}$ cholesterol oxidase and at different concentrations of cholesterol. (d) PL spectra of $0.1 \mathrm{mg} \mathrm{mL}^{-1}$ TYR-GQD in PBS (pH 7.0) containing $5 \mu \mathrm{M}$ lactate oxidase and at different concentrations of L-lactate. [Reprinted with permission from ref. 670, N. Li, A. Than, X. Wang, S. Xu, L. Sun, H. Duan, C. Xu and P. Chen, Ultrasensitive Profiling of Metabolites Using TyramineFunctionalized Graphene Quantum Dots, ACS Nano, 2016, 10, 3622-3629. Copyright@ American Chemical Society.] 
used for the selective binding of target analytes or as enzyme substrates and the signal transducer is graphene or GO. Moreover, many fluorescent nanomaterials such as QDs, nanoclusters (NCs), and upconversion nanoparticles (UCNPs) have been developed for their use in biosensing.

Xing et al. ${ }^{710}$ demonstrated a fluorescence aptasensor using a dsDNA/GO complex through dsDNA and GO binding in the presence of certain salts to study exonuclease I (Exo I) activities. In this sensing probe, the competitor gets hybridized with the aptamer in the absence of the target, which hinders the absorption of the competitor by Exo I, therefore, the formed dsDNA is adsorbed onto the surface of GO, giving rise to fluorescence quenching. On the other hand, the aptamer prefers binding with its target after the target is introduced into the system; this results in a nuclease reaction, and a minor change in fluorescence is observed when GO is introduced because of the weak affinity between generated mononucleotides and GO.
A smaller dissociation constant $\left(K_{\mathrm{d}}\right)$ value of $311.0 \mu \mathrm{M}$ and an LOD of $3.1 \mu \mathrm{M}$ were obtained for dsDNA and GO-based fluorescence aptasensor, compared with the conventional dyelabeled aptamer and GO complex-based sensing platform $\left(K_{\mathrm{d}}\right.$ $=688.8 \mu \mathrm{M}$ and LOD $=21.2 \mu \mathrm{M})$. Chen et al. ${ }^{711}$ demonstrated a nitrogen- and sulfur-codoped rGO (N,S-rGO) as a sensing probe for the fluorescent detection of various biological species through simple fluorescence quenching and recovery (Fig. 33A). Doping with $\mathrm{N}$ and $\mathrm{S}$ heteroatoms can effectively activate the $\mathrm{sp}^{2}$-hybridized carbon lattice, which greatly improves the electron/energy acceptor. The QD fluorescence was found to be quenched after mixing QD-labeled with human disease-related genes such as hepatitis B virus (HBV) DNA and HIV DNA molecular beacon probes with $\mathrm{N}, \mathrm{S}-\mathrm{rGO}$; the recovery of QD fluorescence was observed after adding target HBV and HIV DNA. The biosensor showed the target virus DNA with an LOD of $2.4 \mathrm{nM}$ with a linear range of $5-100 \mathrm{nM}$ for $\mathrm{HBV}$ and $3.0 \mathrm{nM}$
(A)

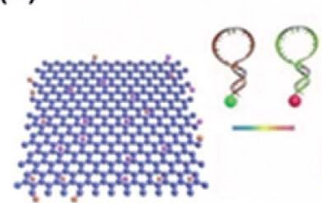
$=$ N,S-rGO

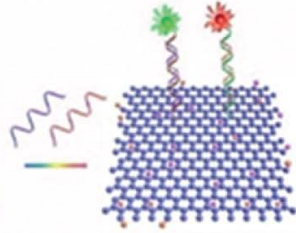

= HIV Probe

$=$ HIV DNA
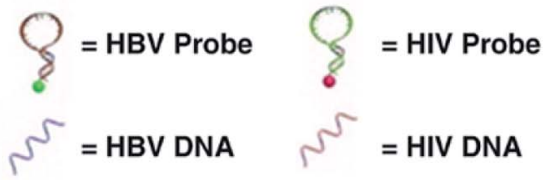

(B)

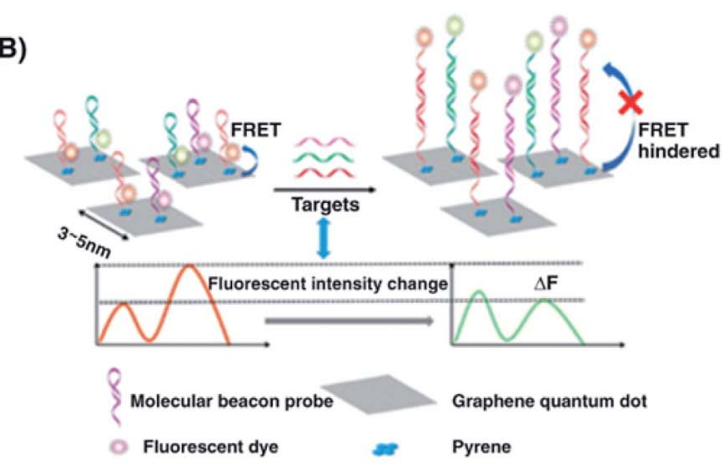

(D)

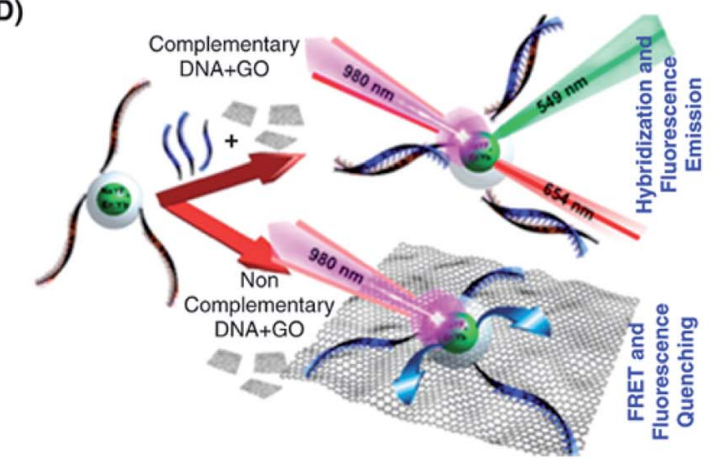

(C)

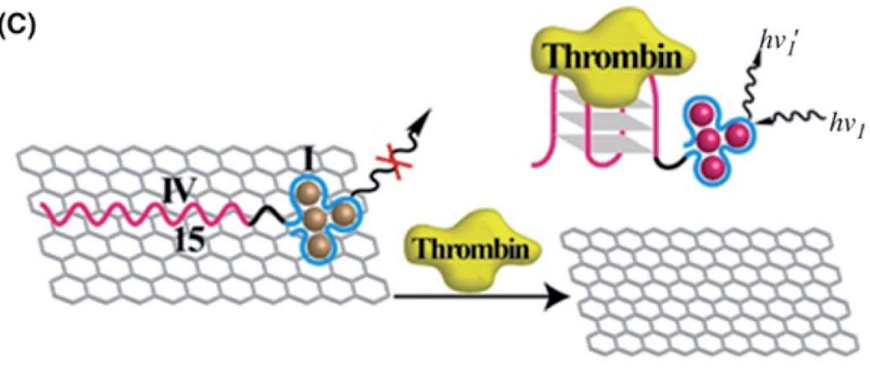

Fig. 33 (A) Schematic illustration showing the multicolored P-QD- and N, S-codoped rGO-(N, S-rGO) based DNA biosensor for the sensitive detection of HBV DNA and HIV DNA. [Reprinted with permission from ref. 711, L. Chen, L. Song, Y. Zhang, P. Wang, Z. Xiao, Y. Guo and F. Cao, Nitrogen and Sulfur Codoped Reduced Graphene Oxide as a General Platform for Rapid and Sensitive Fluorescent Detection of Biological Species, ACS Appl. Mater. Interfaces, 2016, 8, 11255-11261. Copyright@ American Chemical Society.] (B) Schematic illustration of the FRETbased biosensing platform using GQDs and pyrene-functionalized molecular beacon probes for miRNA detection. [Reprinted with permission from ref. 712, H. Zhang, Y. Wang, D. Zhao, D. Zeng, J. Xia, A. Aldalbahi, C. Wang, L. San, C. Fan, X. Zuo and X. Mi, Universal Fluorescence Biosensor Platform Based on Graphene Quantum Dots and Pyrene-Functionalized Molecular Beacons for Detection of MicroRNAs, ACS Appl. Mater. Interfaces, 2015, 7, 16152-16156. Copyright@ American Chemical Society.] (C) Aptamer biosensor based on a NA/AgNC/GO hybrid system for the detection of thrombin. [Reprinted with permission from ref. 681, X. Liu, F. Wang, R. Aizen, O. Yehezkeli and I. Willner, Graphene Oxide/ Nucleic-Acid-Stabilized Silver Nanoclusters: Functional Hybrid Materials for Optical Aptamer Sensing and Multiplexed Analysis of Pathogenic DNAs, J. Am. Chem. Soc., 2013, 135, 11832-11839. Copyright $\odot$ American Chemical Society.] (D) Schematic representation showing the mechanism of UC NPs/GO in the presence and absence of complementary DNA. [Reprinted with permission from ref. 713, P. Alonso-Cristobal, P. Vilela, A. El-Sagheer, E. Lopez-Cabarcos, T. Brown, O. L. Muskens, J. Rubio-Retama, A. G. Kanaras, Highly Sensitive DNA Sensor Based on Upconversion Nanoparticles and Graphene Oxide, ACS Appl. Mater. Interfaces, 2015, 7, 12422-12429. Copyright@ American Chemical Society.] 
with a linear range of 7-80 nM for HIV. The molecular beacon probes with thrombin and immunoglobulin (IgE) aptamers showed LODs of $0.17 \mathrm{ng} \mathrm{mL}{ }^{-1}$ for thrombin and $0.19 \mathrm{ng} \mathrm{mL} \mathrm{m}^{-1}$ for IgE proteins. The N,S-rGO-based QD sensor showed 99 to $100.6 \%$ quantitative recovery. Zhang et $a .^{712}$ showed a fluorescence biosensor using GQDs and pyrene-functionalized molecular beacons (py-MBs) for detecting miRNAs (Fig. 33B). Pyrene was used to functionalize and trigger specific FRET between GQDs and py-MBs containing fluorescent dyes and the target detection was realized by the change in fluorescence intensity. The biosensor showed a linear range of $0.1 \mathrm{nM}$ to $200 \mathrm{nM}$ with LOD of $100 \mathrm{pM}$ and high selectivity for the detection of target miRNA miR-155 in the presence three other interfering miRNAs including miR-21, miR-210, and miR-196a.

Metal nanoclusters such as Ag nanoclusters (NCs) also show promise for greatly enhanced detection limits and sensitivity of fluorescence-based biosensors. Metal NCs can either increase or quench fluorophore emission, depending on the distance between a metal surface and the resulting molecular orientation. Liu et al. ${ }^{681}$ developed a fluorescent DNA sensor and aptasensor for the detection of infectious pathogens based on a silver nanocluster/GO hybrid system (Fig. 33C). By conjugating oligonucleotide sequences to NA-protected AgNCs, the fluorescence was generated by the desorption of AgNCs from GO surface by forming duplex DNA structures or complexes with aptamersubstrate. This approach enabled thrombin detection with an LOD of $0.5 \mathrm{nM}$. The hybrid biosensor was also used for the detection of HBV, HIV, and Treponema pallidum (syphilis) genes. The upconversion nanoparticle-based FRET detection approach has attracted significant research interest. Alonso-Cristobal et $a l^{713}$ reported a highly sensitive DNA biosensor based on FRET pairing between erbium- and ytterbium-doped $\beta-\mathrm{NaYF}_{4}$
$\left(\mathrm{NaYF}_{4}: \mathrm{Yb}, \mathrm{Er}\right)$ UCNP fluorescent donors and GO quencher (Fig. 33D). In this sensing probe, the DNA-functionalized $\mathrm{NaYF}_{4}: \mathrm{Yb}, \mathrm{Er} @ \mathrm{SiO}_{2}$ nanoparticles were near the GO surface, and FRET fluorescence quenching was induced by the $\pi-\pi$ stacking interactions occuring between the GO and nucleobases of the DNA because of the fluorescence emission overlapping of NaYF4:Yb,Er@SiO ${ }_{2}$ NPs and the GO absorption spectrum. This nanoparticle-mediated upconversion approach showed high sensitivity with an LOD of 5 pM. Huang et al. $^{714}$ designed a FRETbased biosensor from DNA-functionalized $\mathrm{NaYF}_{4}: \mathrm{Yb}, \mathrm{Tm} @ \mathrm{NaYF}_{4}$ UCNPs utilizing liquid-liquid interface-based ligand exchange. The resulting biosensor exhibited a high sensitivity with an LOD of $1 \times 10^{-4} \mathrm{U} \mathrm{mL}^{-1}$ for S1 nuclease.

4.2.1 Amplified detection of nucleic acids. There has been significant effort to enhance the signal intensity in order to detect the trace amounts of bioanalytes. Numerous strategies have been developed for signal enhancement, including ligation chain reaction (LCR), polymerase chain reaction (PCR), and rolling circle amplification (RCA).${ }^{128,715}$ RCA offers the capability of creating DNA products through a replication process that may contain thousands of tandem sequence repeats; therefore, RCA is a powerful tool for signal amplification. Numerous reports utilizing this unique strategy have appeared (see Table 11) to enhance the sensitivity of the detection of biomolecules. ${ }^{\mathbf{4 3}}$

Liu et $a .^{716}$ developed a sensor using graphene for the detection of DNA probes, through an RCA signal-amplified technique. The working mechanism of this rGO-aptamer RCA strategy is displayed in Fig. 34A. The sensing design consists of an ssDNA probe and an rGO transducing mediator in order to amplify the molecular detection. Specifically, the sensing probe is designed with 2 sequence domains: a $3^{\prime}$ primer domain that initiates RCA and a $5^{\prime}$ domain consisting of an aptamer for

Table 11 Graphene-based signal enhancement strategies

\begin{tabular}{|c|c|c|c|c|c|c|}
\hline $\begin{array}{l}\text { Graphene- } \\
\text { based platform }\end{array}$ & Amplification method & Target & & Linear range & Limit of detection & Ref. \\
\hline GR & Rolling circle amplification & Nucleic acid & DNA & $10 \mathrm{pM}$ & $10-200 \mathrm{pM}$ & 716 \\
\hline GR & Hybridization chain reaction & Nucleic acid & miRNA & $10-2000 \mathrm{fM}$ & $4.2 \mathrm{fM}$ & 718 \\
\hline $\begin{array}{l}\text { Au nanoclusters/ } \\
\text { GR hybrid }\end{array}$ & $\begin{array}{l}\text { Exo III-aided cascade } \\
\text { target recycling }\end{array}$ & Nucleic acid & DNA & $0.02 \mathrm{fm}$ to $20 \mathrm{pM}$ & $0.057 \mathrm{fM}$ & 401 \\
\hline GO & Hybridization chain reaction & Nucleic acid & RNA & $5 \mathrm{pM}$ to $12 \mathrm{nM}$ & $2.7 \mathrm{pM}$ & 719 \\
\hline GO & $\begin{array}{l}\text { Isothermal exponential } \\
\text { amplification }\end{array}$ & Nucleic acid & miRNA & $10 \mathrm{fM}$ to $10 \mathrm{pM}$ & $3 \mathrm{fM}$ & 674 \\
\hline rGO & In situ reverse transcription & Nucleic acid & miRNA & $10 \mathrm{fM}$ to $100 \mathrm{pM}$ & $1.318 \mathrm{pM}$ & 721 \\
\hline GO & $\begin{array}{l}\text { Nicking enzyme signal } \\
\text { amplification }\end{array}$ & Protein & Thrombin & $2 \mathrm{fM}$ to $200 \mathrm{nM}$ & $1.0 \mathrm{fm}$ & 722 \\
\hline GO & $\begin{array}{l}\text { DNase I-mediated } \\
\text { cyclic signal } \\
\text { amplification }\end{array}$ & Bacteria & $S$. Paratyphi A & $\begin{array}{l}1 \times 10^{2} \text { to } 1 \times 10^{11} \\
\text { cells per } \mathrm{mL}\end{array}$ & $\begin{array}{l}1 \times 10^{2} \\
\text { cells per } \mathrm{mL}\end{array}$ & 723 \\
\hline GO-AuNCs & HCR & Food toxin & AFB1 & $0.1-10 \mathrm{pg} \mathrm{mL}^{-1}$ & $0.03 \mathrm{pg} \mathrm{mL}^{-1}$ & 686 \\
\hline GO & Hybridization chain reaction & Metal ion & $\mathrm{Hg}^{2+}$ & $0-1 \mathrm{nM}$ & $0.3 \mathrm{nM}$ & 602 \\
\hline GR & GR/DNAzyme & Metal ion & $\mathrm{Pb}^{2+}$ & Up to $100 \mathrm{nM}$ & $300 \mathrm{pM}$ & 726 \\
\hline
\end{tabular}


(A)

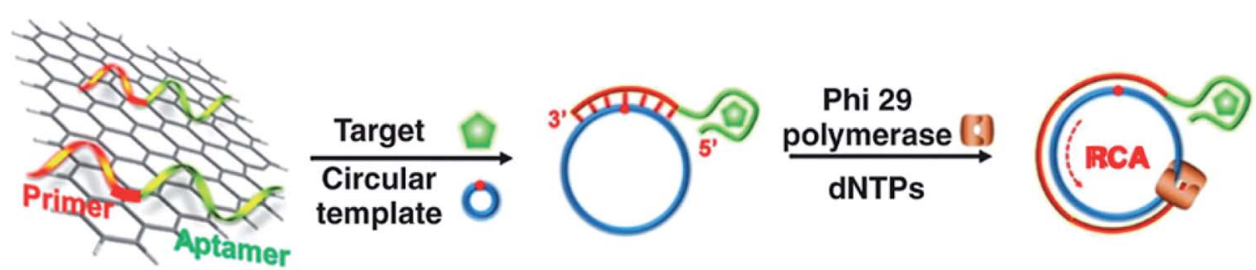

(B)

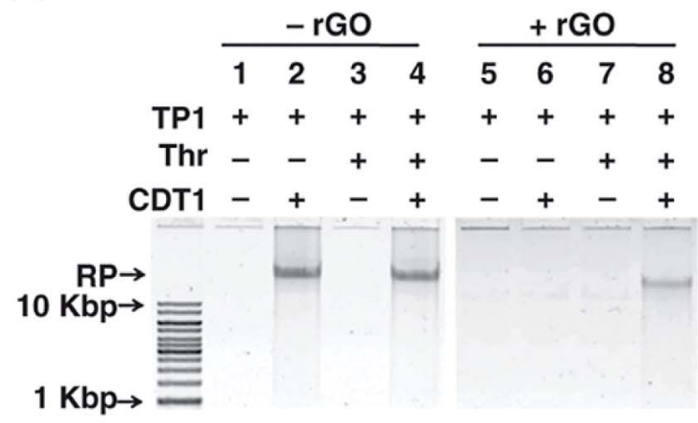

(C)

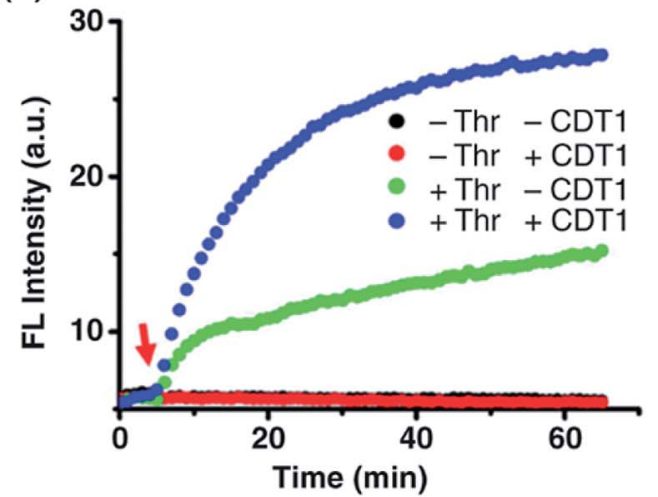

(D)

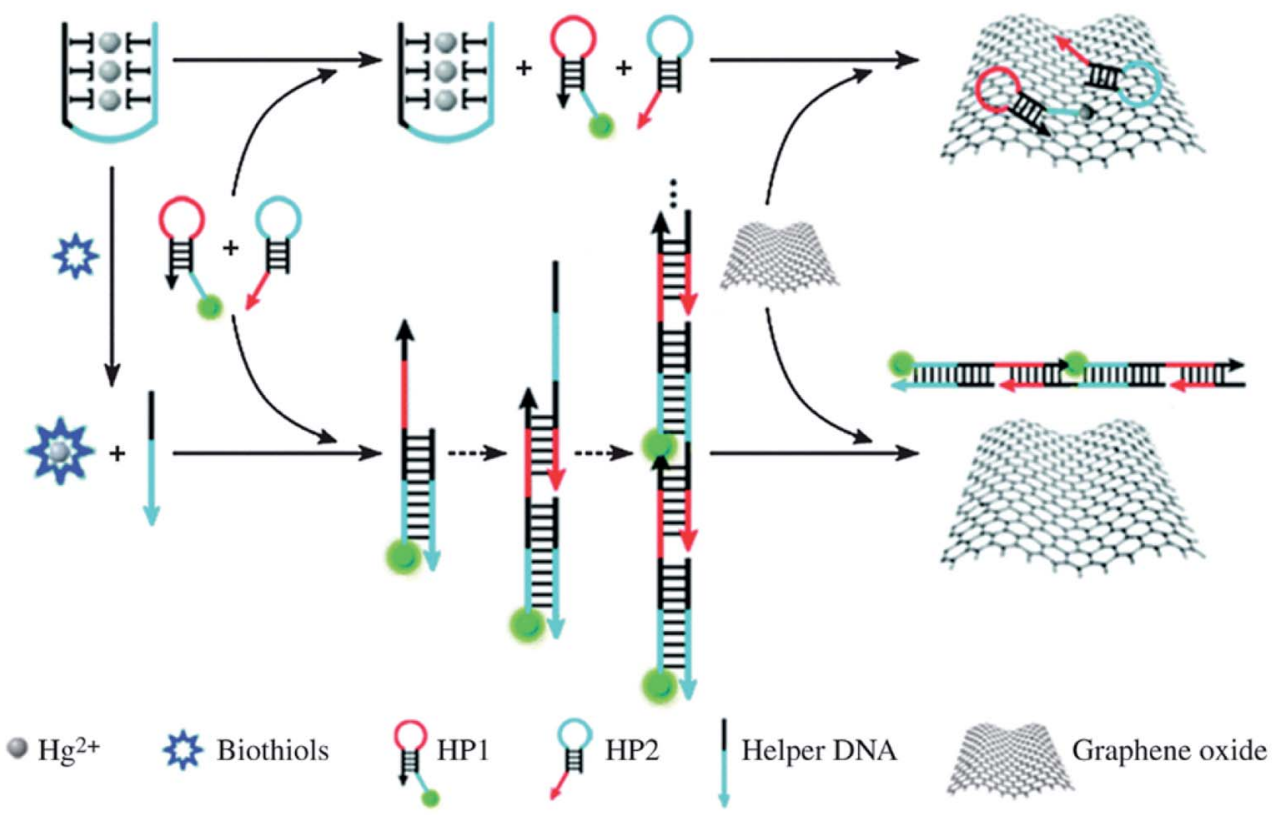

Fig. 34 (A) Schematic illustration of the rGO-aptamer-RCA based sensing probe. (B) Analysis of RCA products using $0.6 \%$ agarose gel electrophoresis. Each reaction was conducted at $30^{\circ} \mathrm{C}$ for $1 \mathrm{~h}$ in $60 \mu \mathrm{L}$ of target binding buffer (4,5,6,7-tetrabromobenzotriazole (TBB), $20 \mathrm{mM}$ PBS, $150 \mathrm{mM} \mathrm{NaCl}, 20 \mathrm{mM} \mathrm{KCl}$, and $5 \mathrm{mM} \mathrm{MgCl} 2$ at $\mathrm{pH}$ 7.5) having components of rGO-adsorbed functional thrombin probe TP1 (250 nM), circular DNA template (CDT1) $(8 \mathrm{nM})$, and thrombin (Thr; $200 \mathrm{nM})$. (C) Time-dependent fluorescence response of rGO-adsorbed FAM-labeled TP1 (250 $\mathrm{nM})$ in the presence of $\operatorname{Thr}(200 \mathrm{nM}), \mathrm{CDT1}(8 \mathrm{nM})$, or both. Excitation wavelength $\left(\lambda_{\text {ex }}\right) /$ emission wavelength $\left(\lambda_{\text {em }}\right)=494 \mathrm{~nm} / 518 \mathrm{~nm}$. $[$ Reprinted with permission from ref. 716, M. Liu, J. Song, S. Shuang, C. Dong, J. D. Brennan and Y. A. Li, Graphene-Based Biosensing Platform Based on the Release of DNA Probes and Rolling Circle Amplification, ACS Nano, 2014, 8, 5564-5573. Copyright@ American Chemical Society.] (D) Illustration of the GO-based platform coupled with hybridization chain reactions (HCR) for biothiol analysis. [Reprinted with permission from ref. $724, \mathrm{~J}$. Ge, Z.-M. Huang, Q. Xi, R.-Q. Yu, J.-H. Jiang and X. Chu, A Novel Graphene Oxide Based Fluorescent Nanosensing Strategy with Hybridization Chain Reaction Signal Amplification for Highly Sensitive Biothiol Detection, Chem. Commun., 2014, 50, 11879-11882. Copyright@ Royal Society of Chemistry.]

molecular recognition (Fig. 34B). The rGO surface adsorbs the ssDNA probe via $\pi-\pi$ stacking, preventing the probe's primer region from being used for RCA initiation. The adsorbed probes undergo conformational change in the presence of any targeted molecule, releasing the probes from the surface of the rGO, exposing the primer domain for RCA. The detection of the target was therefore, in effect, used for the detection of the products of the RCA. This RCA approach showed the detection of ATP and DNA with LODs as low as $60 \mathrm{nM}$ and $0.8 \mathrm{pM}$, respectively. Hong et $a l^{717}$ developed a GO-based biosensor combined with the 
target-specific RCA technique for the fluorometric detection of miRNA. By utilizing this approach, the resulting sensor exhibited a highly enhanced quantitative analysis of miRNA with an LOD as low as $0.4 \mathrm{pM}$. Wen et $a .^{725}$ demonstrated a GOassisted RCA probe for the sensitive detection of Ebola virus (EBOV). No RCA products were obtained in the absence of the EBOV gene; however, the fluorescence quenching of fluorescein amidate (FAM) was observed when FAM-labeled detection probe was adsorbed on the GO surface. The sensor showed high performance both in aqueous solution and in 1\% serum for determination of the EBOV gene with an LOD of $1.4 \mathrm{pM}$.

Special types of enzymes consisting of DNA, also known as DNAzymes, have been developed to perform various catalytic functions as an alternative to traditional protein enzymes. ${ }^{727}$ Since the first report on DNAzyme by Breaker and Joyce ${ }^{727}$ in 1994, numerous DNAzyme sequences have been discovered, showing high catalytic activities in various chemical reactions. DNAzymes can readily interact with various fluorescent tags, functional molecules and solid surfaces, which facilitate the highly sensitive detection of target analytes. Furthermore, the development of different DNA amplification techniques has enabled the enhancement of the signal to improve the sensitivity of assays. ${ }^{72,727}$ Enzymatic signal amplification approaches suffer from several limitations due to the influence of parameters such as temperature and $\mathrm{pH}$. Therefore, signal amplification approaches without using an enzymatic process are highly desirable to overcome such limitations. HCR amplification is one of the methods that have been widely employed to enhance the signal. Ge et al. ${ }^{724}$ demonstrated a GO-based fluorescentbased biosensor for biothiol detection with hairpin DNAselective fluorescence quenching and signal amplification via thymine- $\mathrm{Hg}$ (II)-thymine $\left(\mathrm{T}-\mathrm{Hg}^{2+}-\mathrm{T}\right)$ and $\mathrm{HCR}$ coordination control. The sensing mechanism is shown in Fig. 34D. The target biothiol molecules initiate the formation of long duplex chains utilizing $\mathrm{T}-\mathrm{Hg}^{2+}-\mathrm{T}$ coordination chemistry, giving rise to an amplified fluorescence emission. The fluorescence quenching ability of GO and HCR reduces the background signal and enhances the detection sensitivity. The biosensor showed an LOD of $0.08 \mathrm{nM}$ for biothiols. Xi et al. ${ }^{728}$ also developed a fluorescence biosensor by combining fluorescence quenching ability of GO with HCR amplification. The biosensor showed a dynamic range of 0.0001 to $100 \mathrm{U} \mathrm{mL}^{-1}$ and an LOD of $0.00006 \mathrm{U} \mathrm{mL}^{-1}$.

\subsection{Fluorescent detection of pathogens and food toxins}

The strong fluorescence quenching properties of graphenebased nanomaterials have attracted tremendous attention for developing aptamer-based fluorescence quenching sensing probes for detecting pathogens and food toxins. ${ }^{729}$ Shi et al. ${ }^{678}$ developed a FRET biosensor using GQDs and AuNPs for Staphylococcus aureus, achieving a fluorescence quenching efficiency of approximately $87 \%$ in a $100 \mathrm{nM}$ target oligo and an LOD of $1 \mathrm{nM}$. The biosensor was designed by immobilizing capture probes on GQDs and conjugating the reporter probes to AuNPs. The sandwich-type structure was formed by cohybridizing the target oligos with the capture probes to trigger the FRET effect. Lu et al. ${ }^{684}$ developed an aptamer-based fluorescence recovery assay for detecting AFB1 mycotoxin, a secondary fungal metabolite of A. flavus, using a FRET-based quenching system composed of CdTe QDs and GO. In this system, a thiolated aptamer specific for AFB1 was linked to the surface of CdTe QDs; thus, the fluorescence of the aptamermodified CdTe QDs was quenched by the GO. The fluorescence is restored after adding AFB1 which also depends on the quantity of AFB1. The developed CdTe QDs/GO showed a wide linear range of $3.2 \mathrm{nM}$ to $320 \mu \mathrm{M}$ and an LOD of $1.0 \mathrm{nM}$. Moreover, sensor showed a linear range of $1.6 \mathrm{nM}$ to $160 \mu \mathrm{M}$ and an LOD of $1.4 \mathrm{nM}$ in peanut oil solution.

$\mathrm{Wu}$ et al. $^{\mathbf{6 8 2}}$ developed an aptasensor for detecting mycotoxins based on multicolor UCNPs as donors and GO as an acceptor. Two mycotoxins, ochratoxin A (OTA) and fumonisin B1 (FB1) were used in this study. The UCNPs $\mathrm{BaY}_{0.78} \mathrm{~F}_{5}: \mathrm{Yb}_{0.2}$,$\mathrm{Er}_{0.02}$ and $\mathrm{BaY}_{0.78} \mathrm{~F}_{5}: \mathrm{Yb}_{0.7}, \mathrm{Tm}_{0.02}$ were prepared and functionalized via OTA-aptamer and FB1-aptamer immobilization (Fig. 35A). Both tetraethyl orthosilicate (TEOS) and APTES were used to prepare amino-modified $\mathrm{BaYF}_{5}: \mathrm{Yb}$ Er/Tm UCNPs, which were then be conjugated with avidin employing GA method. The avidin-conjugated $\mathrm{BaYF}_{5}: \mathrm{Yb}$ Er/Tm UCNPs were linked with biotin-functionalized OTA- and FB1-aptamers, respectively. Strong upconversion fluorescence was displayed by both the OTA aptamer-modified $\mathrm{BaYF}_{5}: \mathrm{YbEr}$ UCNPs and the FB1 aptamer-modified $\mathrm{BaYF}_{5}: \mathrm{Yb}$ Tm UCNPs at a $980 \mathrm{~nm}$ excitation wavelength. GO completely quenches the upconversion fluorescence of UCNPs owing to the strong overlap between the GO absorption and multicolor UCNP fluorescence spectra. Due to changes in aptamer formation when they bind OTA and FB1, aptamer-modified UCNPs form further away from the GO surface. This aptasensor exhibited a linear range of 0.05-100 ng $\mathrm{mL}^{-1}$ and $0.1-500 \mathrm{ng} \mathrm{mL}^{-1}$ for detection of OTA and FB1 with LODs of 0.02 and $0.1 \mathrm{ng} \mathrm{mL}{ }^{-1}$, respectively. Fig. 35B shows the selectivity for OTA and FB1 against other interfering mycotoxins, including aflatoxins AFB1, AFB2, AFG1, and AFG2, and $\mathrm{AFG}_{2}$, fumonisin B2 (FB2) and zearalenone (ZEN). A dramatic enhancement in fluorescent intensity was observed for OTA and FB1 compared with other mycotoxins at a concentration of $10 \mathrm{ng}$ $\mathrm{mL}^{-1}$. The fluorescent intensity showed no change with other mycotoxins because UCNPs/GO form specific interactions with OTA and FB1 aptamers. Yan et al. ${ }^{723}$ developed a sensitive and highly selective DNA aptasensor to detect $S$. Paratyphi A by using GO as an efficient fluorescence quencher and two short carboxyfluorescein-modified sequences in a DNase I-mediated target recycling amplification (TRA) approach. When the target is absent, the aptasensor selectively bound with GO, causing fluorescence quenching of FAM. In the presence of the $S$. Paratyphi A target, the aptasensor disconnects from the GO surface due to aptasensor-to-target binding, resulting into a strong fluorescence that could be further amplified and detected by a DNase Imediated TRA strategy. The resulting aptasensor displayed high sensitivity and selectivity with an LOD of $1 \times 10^{2}$ cells per $\mathrm{mL}$. Li et al. ${ }^{686}$ reported a fluorescence quenching based sensing probe for aflatoxin B1 (AFB1) combined with an HCR amplification approach using a GO/AuNC nanocomposite. The HCR strategy displayed $94 \%$ quenching efficiency for the fluorescent probes 
(A)

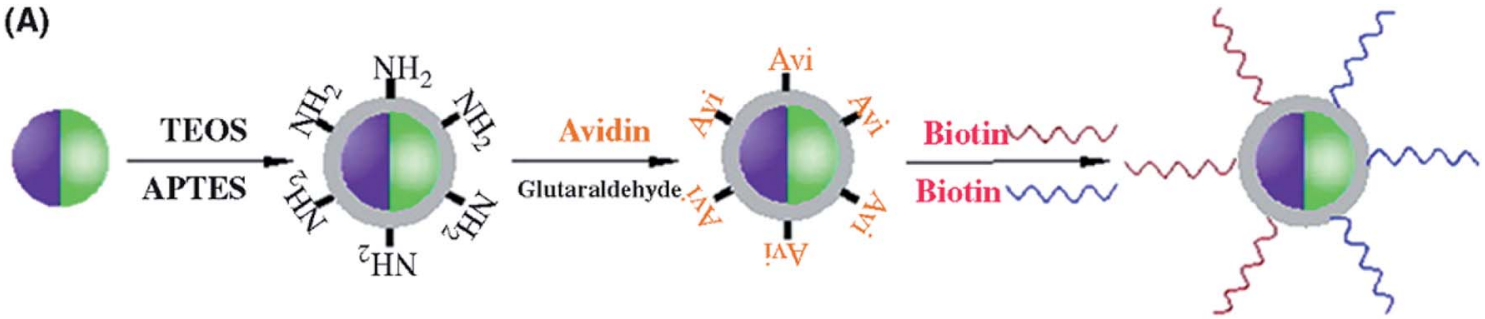
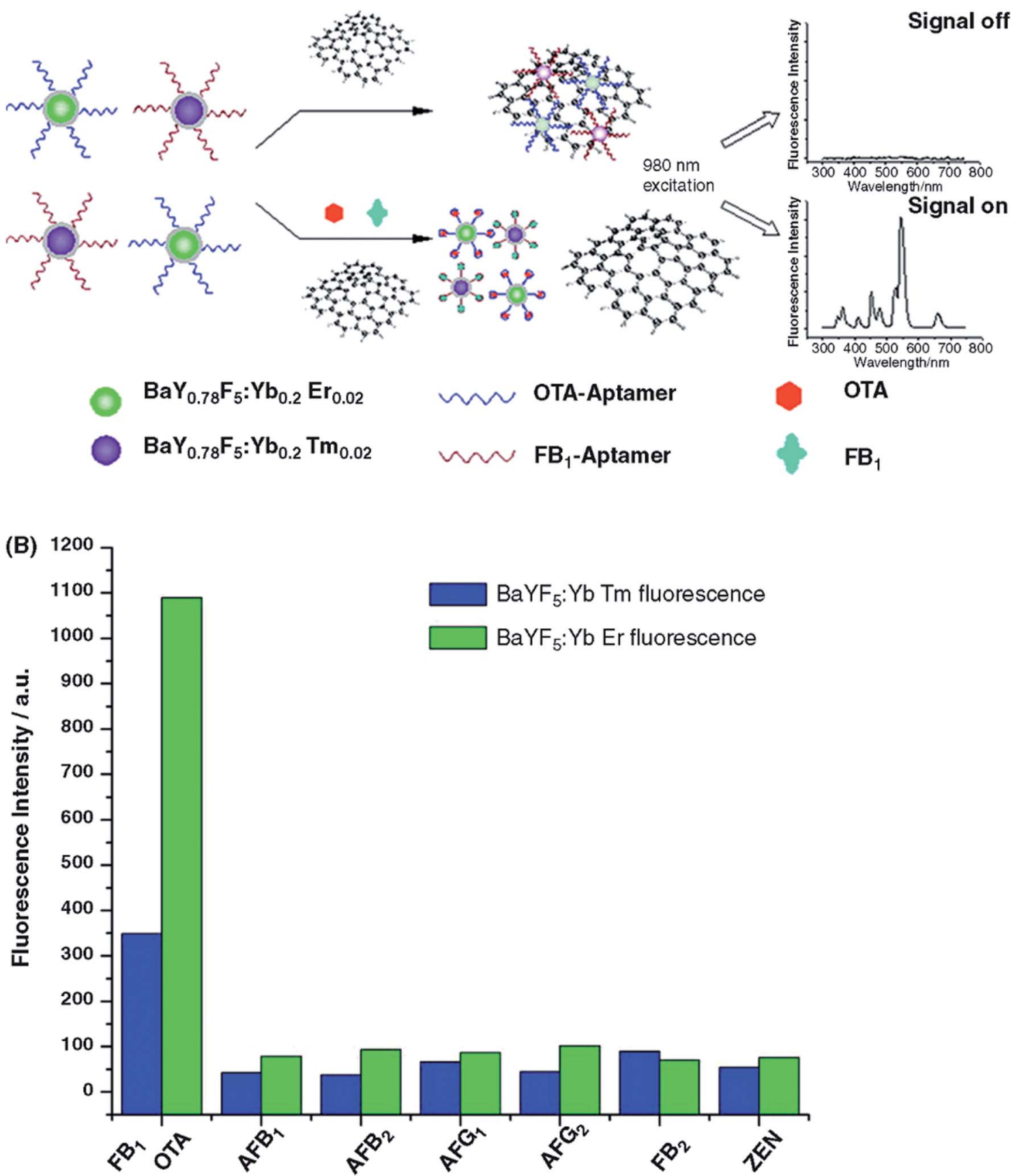

Fig. 35 (A) Schematic representation of the immobilization of ochratoxin A (OTA) aptamers and fumonisin B1 (FB1) aptamers and their multiplex upconversion FRET between aptamer-UCNPs and GO for detecting OTA and FB1. (B) Fluorescence signal change for different mycotoxins at a concentration of $10 \mathrm{ng} \mathrm{mL}^{-1}$. FB1 and OTA show a significant increase in fluorescence intensity compared with other mycotoxin homologs, including AFB1, AFB2, AFG1, AFG2, fumonisin B2 (FB2) and zearalenone (ZEN), which are commonly found in foods. [Reprinted with permission from ref. 682, S. Wu, N. Duan, X. Ma, Y. Xia, H. Wang, Z. Wang and Q. Zhang, Multiplexed Fluorescence Resonance Energy Transfer Aptasensor between Upconversion Nanoparticles and Graphene Oxide for the Simultaneous Determination of Mycotoxins, Anal. Chem., 2012, 84, 62636270. Copyright@ American Chemical Society.] 
and considerable enhancement of the LOD, reaching $0.03 \mathrm{pg}$ $\mathrm{mL}^{-1}$ for AFB1 detection.

\subsection{Fluorescent detection of toxic heavy metal ions}

Graphene-based nanocomposites have been widely used as fluorescent probes to detect metal ions such as $\mathrm{Ag}^{+}, \mathrm{Pb}^{2+}, \mathrm{Hg}^{2+}$, $\mathrm{Cu}^{2+}$, and $\mathrm{Fe}^{3+}$ with high levels of sensitivity and selectivity. ${ }^{21}$ Metal ions can bind selectively to specific DNA nitrogenous bases. Therefore, DNAzyme-based sensors with different signal transduction mechanisms have been developed using graphene. ${ }^{72}$ However, the diverse chemical structures of graphene, GO and rGO lead to complexities in the selective detection of multiple metal ions. Wen et al. ${ }^{330}$ demonstrated a fluorescent nanoprobe using GO and an Ag-specific oligonucleotide for the detection of $\mathrm{Ag}(\mathrm{I})$ ions. The sensor exhibited an LOD of $20 \mathrm{nM}$, and the Stern-Volmer constant $\left(K_{\mathrm{sv}}\right)$ of $\mathrm{Ag}^{+}$to silver-specific cytosine-rich oligonucleotide (DNA oligonucleotide SSO $=5^{\prime}$ FAM-CTCTCTTCTCTTCATTTTTCAACA CAACACAC-3') was calculated to be $12 \mu \mathrm{M}$. The fluorescence response satisfies the sensitivity requirement $\left(4.610^{-7} \mathrm{M}\right)$ of the US EPA for drinking water. Wang et al. ${ }^{731}$ reported the self-assembly of fluorescent chemodosimeter (FC) molecules onto a GO surface for the detection of fluoride ions $\left(\mathrm{F}^{-}\right)$. The fluorescent sensing of $\mathrm{F}^{-}$ions using $\mathrm{GO} / \mathrm{FC}$ was approximately 5 -fold larger than that without $\mathrm{GO}$, the response time was shortened from $4 \mathrm{~h}$ to $30 \mathrm{~min}$, and the sensitivity was 2-fold greater than that without GO.

GQDs have been investigated as a new class of fluorescent sensors for detecting a wide range of metal ions due to their improved optical properties and fluorescence quenching capability compared to GR and GO. ${ }^{102}$ In addition, the small size, improved photostability, good solubility, chemical inertness and high biocompatibility of the GQDs are advantageous for the fabrication of fluorescent biosensors with high sensitivity and selectivity in the presence of interfering analytes. ${ }^{102}$ Surfacefunctionalized GQDs have been applied for the fluorescent detection of metal ions. Zhu et al. ${ }^{700}$ demonstrated a fluorescent approach using GQDs for the detection of $\mathrm{Fe}^{3+}$ ions (Fig. 36). The strong coordination between the hydroxyl $(-\mathrm{OH})$ groups on the GQDs and $\mathrm{Fe}^{3+}$ ions forms aggregates of iron hydroxide, which gives rise to fluorescence quenching. The GQD sensor displayed a very low solubility product constant $\left(K_{\mathrm{sp}}\right) 2.8 \times$ $10^{-39}$ for ferric hydroxide $\left[\mathrm{Fe}(\mathrm{OH})_{3}\right]$ compared with $\mathrm{Cu}(\mathrm{OH})_{2}$ and $\mathrm{Ni}(\mathrm{OH})_{2}$, leading to superior sensitivity and an LOD as low as $0.45 \mu \mathrm{M}$. The GQDs sensor showed high selectivity toward $\mathrm{Fe}^{3+}$ ions over 10 other interfering metal ions including $\mathrm{Ni}^{2+}$, $\mathrm{Co}^{2+}, \mathrm{Cu}^{2+}, \mathrm{Pb}^{2+}, \mathrm{Ca}^{2+}, \mathrm{Al}^{3+}, \mathrm{K}^{+}, \mathrm{Mg}^{2+}, \mathrm{Na}^{+}$, and $\mathrm{Zn}^{2+}$ at the same concentrations of $5 \mu \mathrm{M}$ for all metal ions. Ananthanarayanan et al. ${ }^{732}$ demonstrated an IL (1-butyl-3-methylimidazolium hexafluorophosphate $\left(\mathrm{BMIMPF}_{6}\right)$ in acetonitrile)-functionalized GQD derived from CVD-grown 3D graphene for the sensitive detection of $\mathrm{Fe}^{3+}$ ions. The $\mathrm{BMIM}^{+}$functionalization of GQDs due to the high binding affinity of ferric ions with the imidazole ring of $\mathrm{BMIM}^{+}$enabled enhanced fluorescence quenching for a wide range of $\mathrm{Fe}^{3+}$ ions and achieved an LOD of $7.2 \mu \mathrm{M}$. Guo et al. ${ }^{733}$ reported a fluorescent biosensor based on $\mathrm{N}$-(rhodamine B) lactam-ethylenediamine-functionalized GQDs (RBD-GQDs) for the sensitive detection of $\mathrm{Fe}^{3+}$ in $0-1 \mu \mathrm{M}$ concentration range with an LOD of $0.02 \mu \mathrm{M}$ in pancreatic cancer stem cells. The $\mathrm{Fe}^{3+}$ binding with RBD-GQDs, turns on the biosensor in cancer stem cells after forming RBD-GQDs-Fe ${ }^{3+}$ complex of orange-red fluorescence. RBD-GQDs showed high selectivity toward $\mathrm{Fe}^{3+}$ ions in the presence other interfering cations including $\mathrm{Na}^{+}, \mathrm{K}^{+}, \mathrm{NH}_{4}{ }^{+}, \mathrm{Ca}^{2+}, \mathrm{Cu}^{2+}, \mathrm{Cd}^{2+}, \mathrm{Co}^{2+}, \mathrm{Hg}^{2+}, \mathrm{Mg}^{2+}$, $\mathrm{Mn}^{2+}, \mathrm{Pb}^{2+}, \mathrm{Ni}^{2+}, \mathrm{Zn}^{2+}$, and $\mathrm{Al}^{3+}$. RBD-GQDs-Fe ${ }^{3+}$ also showed high photostability in living cells as the fluorescence intensity did not decrease after $12 \mathrm{~h}$ continuous excitation. Chowdhury and Doong ${ }^{697}$ demonstrated dopamine-functionalized GQDs with bright blue fluorescence for the detection of low levels of $\mathrm{Fe}^{3+}$ ions. The complexation and oxidation of DA were associated with detection of $\mathrm{Fe}^{3+}$ with a linear range of $20 \mathrm{nM}$ to $2 \mu \mathrm{M}$ and an LOD of $7.6 \mathrm{nM}$. Moreover, the resulting DA-GQD fluorescent biosensor was highly selective toward $\mathrm{Fe}^{3+}$ ions in the presence of other interfering metal ions including $\mathrm{Al}^{3+}, \mathrm{Au}^{3+}$, $\mathrm{Fe}^{2+}, \mathrm{Hg}^{2+}, \mathrm{Ca}^{2+}, \mathrm{Cd}^{2+}, \mathrm{Cu}^{2+}, \mathrm{Co}^{2+}, \mathrm{Cr}^{3+}, \mathrm{Ni}^{2+}, \mathrm{Pb}^{2+}, \mathrm{Mg}^{2+}$, and $\mathrm{Mn}^{2+}$, and $\mathrm{Zn}^{2+}$; however, small quenching was observed for the competitive $\mathrm{Fe}^{2+}$ and $\mathrm{Cu}^{2+}$ ions which may be associated with the adsorption of these metal ions onto the surface of GQDs. The DA-GQDs sensor shows higher sensitivity with a lower LOD compared with RBD-GQDs sensor for detecting $\mathrm{Fe}^{3+}$ ions.

The heteroatom doping of GQDs drastically enhances their electrical properties, leading to unusual fluorescent properties and surface reactivity ${ }^{88}$ Li et al. ${ }^{734}$ prepared sulfur-doped GQDs with stable blue-green fluorescence for the enhanced detection of $\mathrm{Fe}^{3+}$. S-doping effectively alters the local electronic density of the GQDs and thus the coordination interaction between $\mathrm{Fe}^{3+}$ and phenolic hydroxyl groups on the surface. This coordination interaction induces efficient fluorescence quenching, enabling improved sensitivity in a linear range of $0-0.7 \mathrm{mM}$ and an LOD of $4.2 \mathrm{nM}$. This biosensor was also useful for the direct analysis of $\mathrm{Fe}^{3+}$ in human serum samples, indicating its potential for future clinical diagnosis. Peng et al..$^{705}$ developed a facile approach for the rapid detection of $\mathrm{Hg}^{2+}$ using N-GQDs, which increase the complexation rate between 5,10,15,20-tetrakis(1methyl-4-pyridinio)porphyrin

tetra( $p$-toluenesulfonate) (TMPyP) and manganese ions $\left(\mathrm{Mn}^{2+}\right)$. In this strategy, the substitution reaction between the deformed TMPyP nucleus and the $\mathrm{Hg}^{2+}$ ions facilitates the effective detection of small divalent metal ions by utilizing N-GQDs. At the same time, the effective formation of metalloporphyrin coupled with the fluorescence quenching of porphyrins improves the fluorescence of $\mathrm{N}$-GQDs due to the inner filter effect between porphyrins and NGQDs from the back. This sensor detected $\mathrm{Hg}^{2+}$ with an LOD of $0.2 \mathrm{nM}$. Fig. 37 shows the fluorescence spectra of (TMPyP) after the addition of N-GQDs, $\mathrm{Mn}^{\mathrm{II}}$, and different concentrations of $\mathrm{Hg}^{\mathrm{III}}$ ranging from 0 to $200 \mathrm{nM}$, a plot of the fluorescence ratio $\left(I_{490} / I_{658}\right) v s . \mathrm{Hg}^{\mathrm{II}}$ concentrations, the absorption spectra and the selectivity of $\mathrm{Hg}^{\mathrm{II}}$ among different interfering analytes including $\mathrm{Ba}^{\mathrm{II}}, \mathrm{Cd}^{\mathrm{II}}, \mathrm{Co}^{\mathrm{II}}, \mathrm{Cu}^{\mathrm{II}}, \mathrm{Fe}^{\mathrm{II}}, \mathrm{Mg}^{\mathrm{II}}, \mathrm{Ni}^{\mathrm{II}}, \mathrm{Pb}^{\mathrm{II}}, \mathrm{Zn}^{\mathrm{II}}, \mathrm{HCO}_{3}{ }^{-}, \mathrm{NO}_{3}{ }^{-}, \mathrm{Cl}^{-}$, and $\mathrm{SO}_{4}{ }^{2-}$, where the system of $\mathrm{N}$-GQDs and $\mathrm{Mn}^{\mathrm{II}}$ is highly selective in detecting $\mathrm{Hg}^{\mathrm{II}}$ over a very wide range of concentrations.

4.4.1 Amplified detection of heavy metal ions. Numerous strategies have been pursued to enhance the fluorescent signal 
(A)

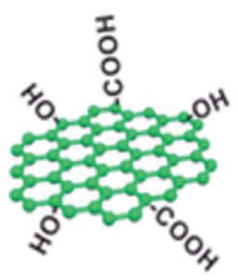

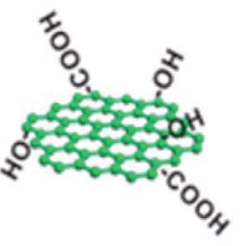
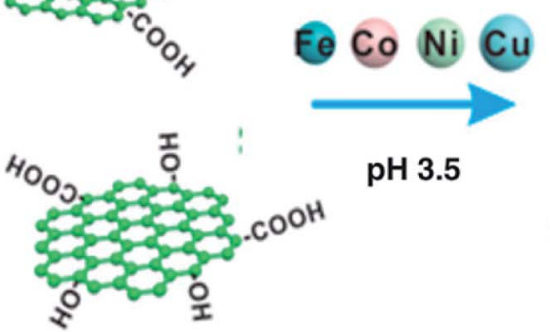

pH 3.5

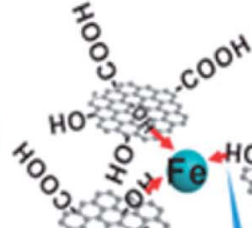<smiles>[GeH3]</smiles><smiles>C[14CH2]O[14C@H](O)[14CH2]O</smiles>
$2 \mathrm{CO}_{/} \mathrm{Ni}$

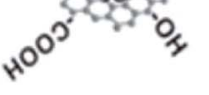

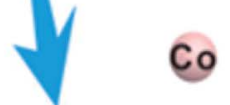

Co
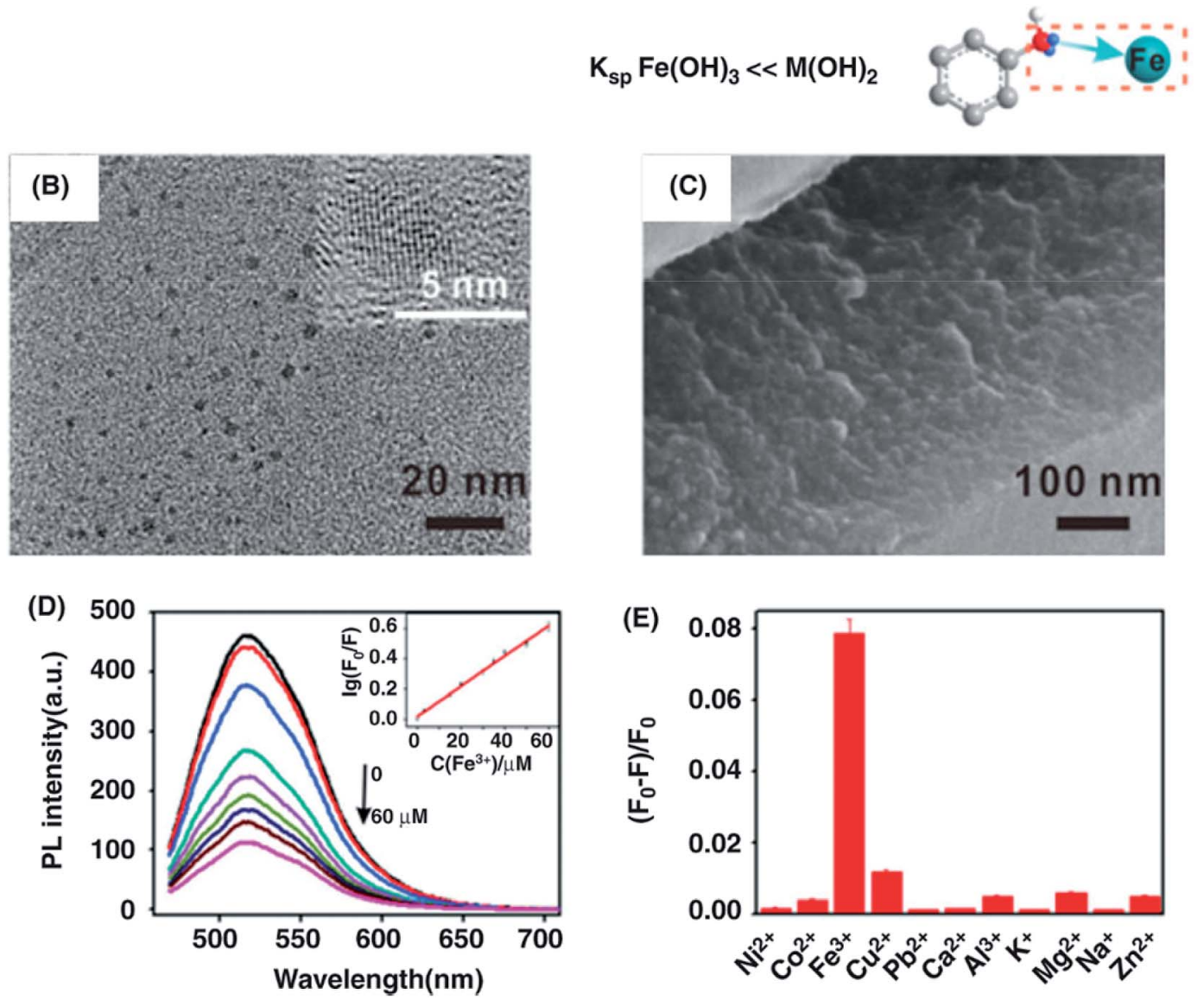

Fig. 36 (A) Fe ${ }^{3+}$ ions sensing platform showing GQD fluorescence quenching by $\mathrm{Fe}^{3+}$ ions and the selectivity of Fe ${ }^{3+}$ ions by GQDs among other interfering metal ions. (B) TEM and HETEM images of the GQDs. Inset shows the lattice fringes of GQDs. (C) SEM image of GQD aggregation induced after adding $\mathrm{Fe}^{3+}$ ions. (D) PL intensity of GQDs in different concentrations of $\mathrm{Fe}^{3+}$ ranging from 0 to $60 \mu \mathrm{M}$. Inset shows a linear calibration plot for detecting $\mathrm{Fe}^{3+}$ ions. (E) Selectivity of GQDs toward $\mathrm{Fe}^{3+}$ ions over 10 other common interfering metal ions at identical concentrations of $5 \mu \mathrm{M}$ for all metal ions. [Reprinted with permission from ref. 700, X. Zhu, Z. Zhang, Z. Xue, C. Huang, Y. Shan, C. Liu, X. Qin, W. Yang, X. Chen and T. Wang, Understanding the Selective Detection of Fe ${ }^{3+}$ Based on Graphene Quantum Dots as Fluorescent Probes: The $K_{\text {sp }}$ of a Metal Hydroxide-Assisted Mechanism, Anal. Chem., 2017, 89, 12054-12058. Copyright@ American Chemical Society.]

for the amplified detection of various metal ions, as shown in Table 10. Effective DNAzyme-based sensors have been designed to detect a broad range of metal ions using various signal transduction mechanisms. ${ }^{21}$ Wen et al. ${ }^{689}$ developed a GO-based DNAzyme for selectively detecting $\mathrm{Pb}^{2+}$. Their biosensor was fabricated by employing a $\mathrm{Pb}^{2+}$-dependent DNAzyme consisting of 2 strands, an enzyme strand, and a Cy3 fluorescent dyetagged ribonucleotide-containing substrate strand; the ribonucleotide was the site of cleavage. Cy3 fluorescent dye namely 1,1'-bis(3-hydroxypropyl)-3,3,3', $3^{\prime}$-tetramethylindocarbocyanine, consists of a conjugated chain of three methine $(=\mathrm{CH}-)$ groups. This modification of the DNAzyme enabled an extension of 5 bases at the $3^{\prime}$ end of the substrate and 5 complementary bases at the $5^{\prime}$ end of the enzyme strand. The rigid DNAzyme double strand did not bind GO well, and there was strong emission from Cy3 dye, without $\mathrm{Pb}^{2+}$. When $\mathrm{Pb}^{2+}$ is 
introduced, the substrate strand is irreversibly cleaved at this site, leading to the duplex DNAzyme being disassembled into the $3^{\prime}$ - and $5^{\prime}$ - fragments of the substrate strand and the enzyme strand. The GO surface adsorbed the short Cy3 dye-containing segment of DNA, resulting in significant fluorescence quenching. The resulting fluorescent sensor exhibited an LOD of $0.5 \mathrm{nM}$ for the detection of $\mathrm{Pb}^{2+}$, which is significantly lower than the limit set by the US EPA for drinking water (72 nM). Zhao et $a .^{726}$ reported a GO-based DNAzyme containing different numbers of bases for the amplified fluorescence "turnon" detection of $\mathrm{Pb}^{2+}$. The hybrid DNAzyme-substrate was used for molecular recognition and as signal reporter. GO acted as a highly effective quencher. The resulting biosensor exhibited an amplified fluorescence signal with detection of $\mathrm{Pb}^{2+}$ with an LOD of $300 \mathrm{pM}$. The sensor also was effectively employed in $\mathrm{Pb}^{2+}$ analysis in samples of river water. The DNAzyme-GO-based fluorescence sensor also showed high selectivity toward $\mathrm{Pb}^{2+}$ ions among nine other interfering metal ions, including $\mathrm{Hg}^{2+}$, $\mathrm{Zn}^{2+}, \mathrm{Mn}^{2+}, \mathrm{Co}^{2+}, \mathrm{Cd}^{2+}, \mathrm{Mn}^{2+}, \mathrm{Ca}^{2+}, \mathrm{Ni}^{2+}, \mathrm{Cu}^{2+}$, and $\mathrm{Fe}^{3+}$, and the fluorescence response toward $\mathrm{Pb}^{2+}$ was found to be remarkably high. Liu et $a l .{ }^{688}$ demonstrated a "turn-on" fluorescent biosensor based on a graphene-DNAzyme catalytic beacon for the sensitive detection of $\mathrm{Cu}^{2+}$. The graphene acts as substrate and quencher for the $\mathrm{Cu}^{2+}$-dependent DNAzyme, promoting graphene-quenched DNAzyme complex formation that through a process of self-assembly. The quenched fluorescence was recovered in $15 \mathrm{~min}$. The sensors exhibited improved sensitivity with an LOD of $0.365 \mathrm{nM}$. Liu et al. ${ }^{687}$ also reported label-free fluorescent detection of $\mathrm{Cu}^{2+}$, using an externally introduced fluorophore and cleavage of DNA internally in a graphene/ DNAzyme complex. This sensor showed an LOD as low as 2 nM. Fang et al. ${ }^{699}$ showed a macroporous GF-based DNAzyme for the detection of $\mathrm{Pb}^{2+}$ and $\mathrm{Cu}^{2+}$. This biosensor achieved LOD of $50 \mathrm{pM}$ for $\mathrm{Pb}^{2+}$ and $0.6 \mathrm{nM}$ for $\mathrm{Cu}^{2+}$, and the sensor also showed detection of these ions in river water samples with high recovery.

Enzymeless signal amplification strategies have also been widely applied for the sensitive and selective detection of metal ions. Huang et $a .^{602}$ developed a GO-based fluorescent biosensor using HCR for detecting $\mathrm{Hg}^{2+}$ in aqueous solution using GO to adsorb ssDNA. The biosensor showed an LOD of $0.3 \mathrm{nM}$ for $\mathrm{Hg}^{2+}$ detection. They constructed a thymine-rich sSDNA initiator and two fluorescently labeled hairpin probes (Fig. 38). The GO surface adsorbed the hairpins in the absence of $\mathrm{Hg}^{2+}$; one of the hairpin probes quenched the fluorescence. However, the presence of $\mathrm{Hg}^{2+}$ initiates the HCR process between the two hairpin probes using the coordination chemistry of the thymine- $\mathrm{Hg}^{2+}$-thymine coordination compound.
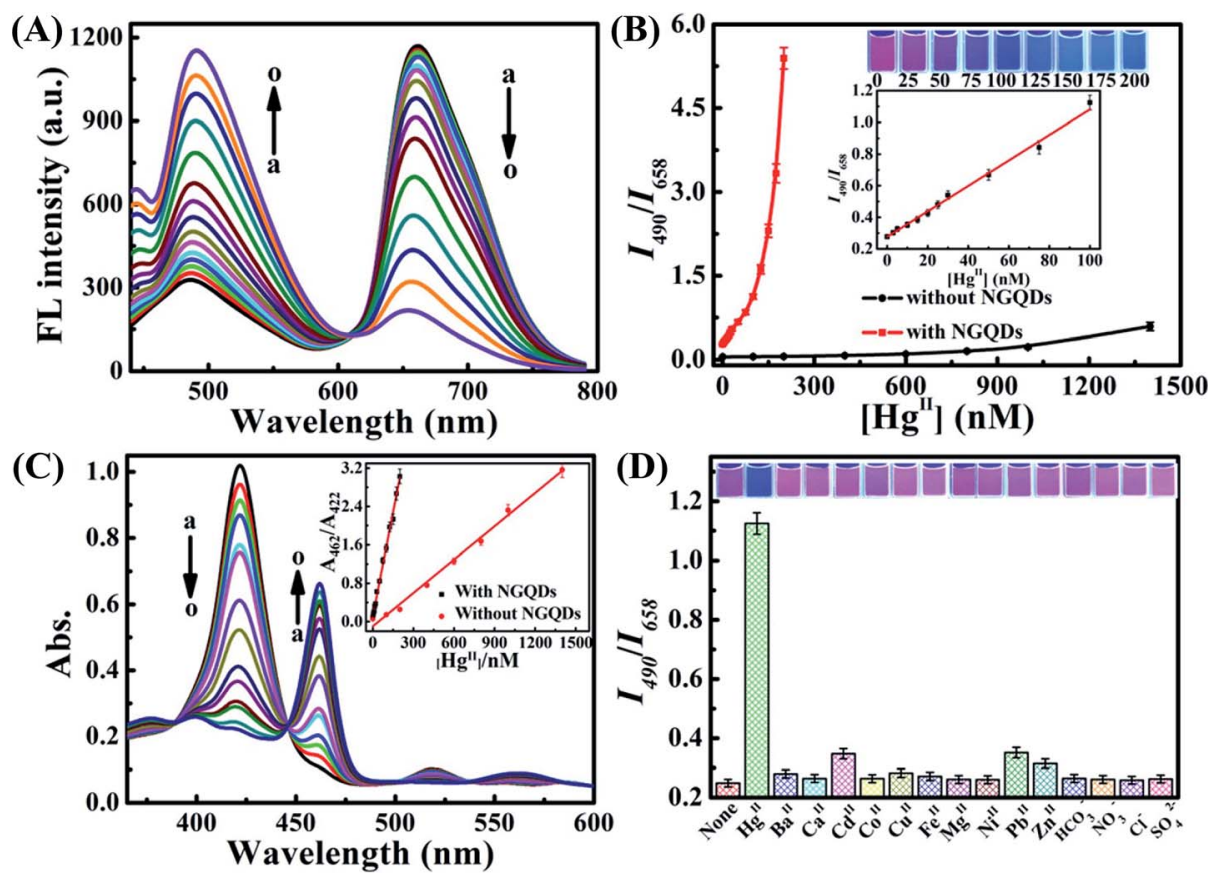

Fig. 37 (A) Fluorescence spectra of $5.0 \mu \mathrm{M}$ 5,10,15,20-tetrakis(1-methyl-4-pyridinio)porphyrin tetra( $p$-toluenesulfonate) (TMPyP) after the addition of $20 \mu \mathrm{g} \mathrm{L}^{-1} \mathrm{~N}-\mathrm{GQDs}, 40 \mu \mathrm{M} \mathrm{Mn}$ ", and different concentrations of $\mathrm{Hg}^{\prime \prime}$ ranging from 0 to $200 \mathrm{nM}$. (B) Plot of the fluorescence ratio (/490/ $\left.I_{658}\right)$ vs. $\mathrm{Hg}^{\prime \prime}$ concentrations $(0-200 \mathrm{nM})$ in the presence or absence $\left(\mathrm{Hg}^{\prime \prime}\right.$ concentrations of 0 to $\left.1400 \mathrm{nM}\right)$ of $\mathrm{N}$-GQDs. Inset shows photographs corresponding to the $\mathrm{Hg}^{\prime \prime}$ concentrations and the plot of $\left(/_{490} / I_{658}\right) \mathrm{vs}$. $\mathrm{Hg}^{\prime \prime}$ concentration $(0-100 \mathrm{nM})$ in the presence of $\mathrm{N}$-GQDs. (C) Absorption spectra of $5.0 \mu \mathrm{M}$ TMPyP after the addition of $40 \mu \mathrm{M} \mathrm{Mn}$ "I, $20 \mu \mathrm{g} \mathrm{L}^{-1} \mathrm{~N}-\mathrm{GQDs}$, and different concentrations of Hg"; inset shows plots of $A_{462} / A_{422}$ vs. Hg" concentration with and without N-GQDs. (D) The selectivity for $\mathrm{Hg}^{\prime \prime}$ among different interfering analytes. The fluorescence quenching efficiency $\left(/ 490 / I_{658}\right)$ of $5.0 \mu \mathrm{M}$ TMPyP after the addition of $40 \mu \mathrm{M} \mathrm{Mn} ", 100 \mathrm{nM} \mathrm{Hg}$ " and $20 \mu \mathrm{g} \mathrm{L}^{-1} \mathrm{~N}$-GQDs and other interfering ions, including $\mathrm{Ba}^{\prime \prime}, \mathrm{Cd}^{\prime \prime}, \mathrm{Co}^{\prime \prime}, \mathrm{Cu}^{\prime \prime}, \mathrm{Fe}^{\prime \prime}, \mathrm{Ni}^{\prime \prime}, \mathrm{Pb}^{\prime \prime}, \mathrm{Zn}^{\prime \prime}, \mathrm{HCO}_{3}{ }^{-}, \mathrm{Cl}^{-}$at $500 \mathrm{nM}$ concentration; $\mathrm{Mg}^{\prime \prime}, \mathrm{SO}_{4}{ }^{2-}, \mathrm{NO}_{3}{ }^{-}$at $100 \mu \mathrm{M}$ concentration; and $\mathrm{Ca}^{\prime \prime}$ at $200 \mu \mathrm{M}$ concentration; inset shows photographs of the fluorescence changes under $365 \mathrm{~nm}$ UV light. [Reprinted with permission from ref. 705 , D. Peng, L. Zhang, R.-P. Liang and J.-D. Qiu, Rapid Detection of Mercury lons Based on Nitrogen-Doped Graphene Quantum Dots Accelerating Formation of Manganese Porphyrin, ACS Sens., 2018, 3, 1040-1047. Copyright@ American Chemical Society.] 
The GO surface cannot adsorb the final HCR double-stranded DNA (dsDNA); therefore, the fluorescence of the system is released by the GO and recovered.

\subsection{Fluorescence detection of pesticides}

Nanomaterials offer unique possibilities in optical biosensing platforms for the detection of food and chemical contaminants. ${ }^{603}$ In particular, GQDs have been studied for the detection of pesticides through FRET mechanisms because of their unique fluorescence quenching properties. GQDs have also been used as fluorophores for the fabrication of highly efficient fluorescence-based biosensors for pesticide detection. ${ }^{\mathbf{1 0 3}}$ Zor et al. ${ }^{735}$ developed a GQD-based multifunctional nanocomposite as a photoluminescent sensor probe in which molecularly imprinted polypyrrole captures the chemical species, magnetic silica beads support the separation mechanism and GQDs optically recognize the target chemical species. This sensor showed an LOD of $12.78 \mathrm{ppb}$ in water and $42.56 \mathrm{ppb}$ in seawater for the tributyltin (TBT) pesticide. Lin et al. ${ }^{706}$ reported an $\mathrm{N}$ GQD-based aptamer biosensor for the detecting pesticide omethoate with an LOD of $0.041 \mathrm{nM}$. In this study, the GQDs were first doped with nitrogen to enhance the fluorescence quantum efficiency to $30 \%$, followed by coupling with an omethoate aptasensor. The fluorescence polarization technique based on the polarization signal of GQDs lowered the LOD from $0.041 \mathrm{nM}$ to $0.029 \mathrm{fM}$. Gao et al. ${ }^{736}$ developed a pesticide biosensor array for the detection of multiple pesticides using $N$ (aminobutyl)- $N$-(ethylisoluminol) (ABEI)-functionalized GQDs. The biosensor array can be used to effectively differentiate different pesticides, such as flubendiamide, thiamethoxam, dimethoate, chlorpyrifos and dipterex, and the ABEI-GQDbased sensor detected pesticides with LOD of $10 \mathrm{ng} \mathrm{mL}^{-1}$. Yan et al. ${ }^{737}$ used a GQD-manganese dioxide nanosheet-based fluorescent sensor for detecting glutathione in living cells. The sensor showed a linear range of $0.5-10 \mu \mathrm{mol} \mathrm{L} \mathrm{L}^{-1}$ and an LOD of $150 \mathrm{nmol} \mathrm{L}^{-1}$ for glutathione. The $\mathrm{MnO}_{2}$ nanosheets act as a quencher for the GQDs through FRET and recognize glutathione molecules. The fluorescence sensor also showed high selectivity toward glutathione among common interfering analytes, including $\mathrm{Na}^{+}, \mathrm{K}^{+}, \mathrm{Ca}^{2+}, \mathrm{Mg}^{2+}, \mathrm{Mn}^{2+}$, aspartic acid, glycine, tyrosine, glucose, fructose, BSA, acetylcholinesterase (AChE), tyrosinase, glucose oxidase (GOx), and trypsin. The

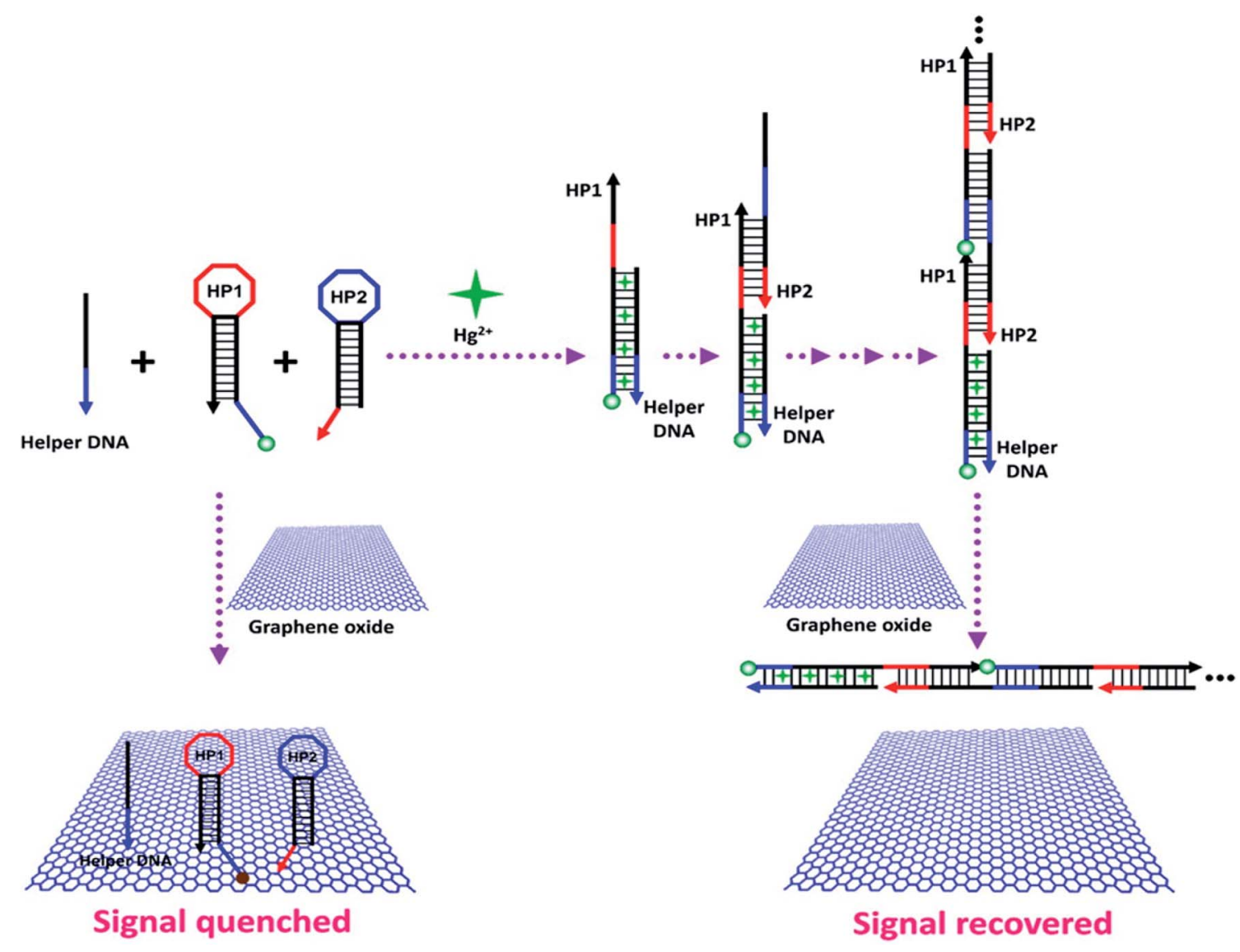

Fig. 38 Amplified fluorescent sensing mechanism of detecting $\mathrm{Hg}^{2+}$ through $\mathrm{HCR}$. In the absence of $\mathrm{Hg}^{2+}$, GO absorbs the DNA probes (helper DNA, HP1, and HP2) via noncovalent interactions and quenches the fluorescence of HP1. However, in the presence of Hg ${ }^{2+}$, the helper DNA opens HP1 because of the formation of stable $\mathrm{T}-\mathrm{Hg}^{2+}-\mathrm{T}$ structures and consequently induces continuous HP1-HP2 hybridizations, which cannot adsorb on GO, leading to the generation of amplified fluorescence. [Reprinted with permission from ref. 602, J. Huang, X. Gao, J. Jia, J.-K. Kim and Z. Li, Graphene Oxide-Based Amplified Fluorescent Biosensor for $\mathrm{Hg}^{2+}$ Detection through Hybridization Chain Reactions, Anal. Chem., 2014, 86, 3209-3215. Copyright@ American Chemical Society.] 
fluorescence intensity remained almost constant, while the fluorescence intensity of the GQD- $\mathrm{MnO}_{2}-\mathrm{GSH}$ sensor was found to increase only after adding glutathione (Fig. 39). Liu et ll. $^{707}$ used a GQD- $\mathrm{MnO}_{2}$ nanosheet MIP to fabricate a fluorescent sensor for thiacloprid detection. The sensor showed a linear range of 0.1 to $10 \mathrm{mg} \mathrm{L}^{-1}$ and a low LOD of $0.03 \mathrm{mg} \mathrm{L}^{-1}$ in the thiacloprid pesticide detection. Nemati et al. ${ }^{738}$ detected ethion using $\mathrm{N}$ - and $\mathrm{S}$ - codoped GQD-based fluorescent biosensors. The N/S-GQD-based sensors showed a linear concentration range of 19.2-961.2 $\mu \mathrm{g} \mathrm{L}^{-1}$ and an LOD of $8 \mu \mathrm{g}$ $\mathrm{L}^{-1}$ for detecting ethion in food samples in the presence of mercury $\left(\mathrm{Hg}^{2+}\right)$ ions.

\section{Selectivity of graphene-based biosensors}

The performance of a biosensor is evaluated based on its high sensitivity and selectivity toward a target biochemical analyte, achieved by excluding other interfering chemical species. Graphene-based sensor arrays have been developed for the simultaneous detection, differentiation and categorization of various analytes within a mixture by using linear discriminant analysis (LDA) and principal component analysis (PCA) to identify patterns. The high selectivity of graphene-based sensor
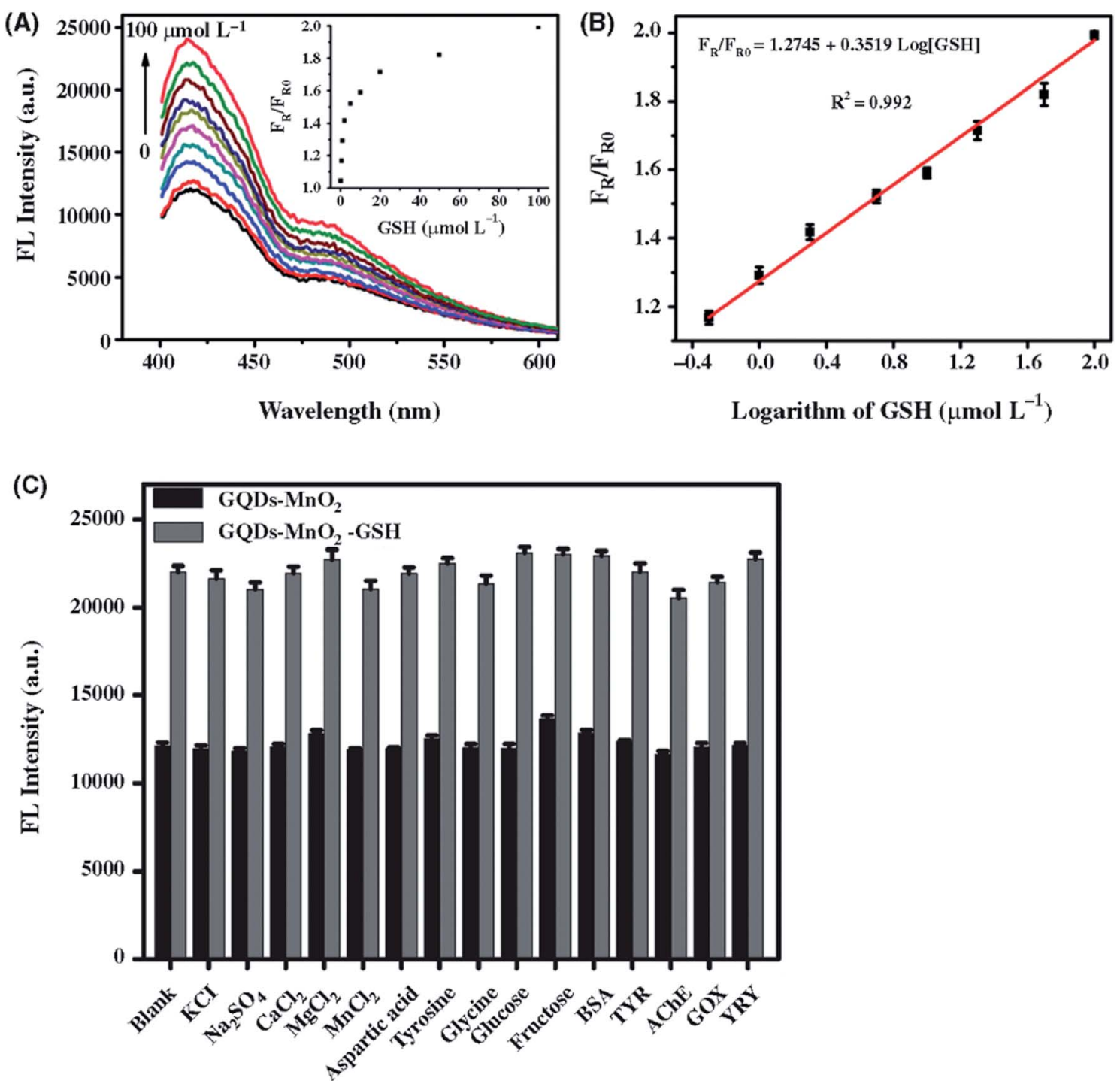

Fig. 39 (A) Fluorescence (FL) intensity of $\mathrm{GQDs}-\mathrm{MnO}_{2}$ sensor with varying concentrations of glutathione (GSH) from 0 to $100 \mu \mathrm{mol} \mathrm{L}^{-1}$. Inset shows the $\mathrm{FL}$ intensity ratio trend varying concentrations of GSH. (B) Plot of $\mathrm{FL}$ intensity ratio $\left(F_{\mathrm{R}} / F_{\mathrm{RO}}\right)$ against the logarithm of the concentration of GSH. (C) Selectivity of the GQD- $\mathrm{MnO}_{2}$-based fluorescence sensor toward glutathione pesticide. Fluorescence (FL) intensities of the GQD$\mathrm{MnO}_{2}$ and GQD- $\mathrm{MnO}_{2}$-glutathione (GSH) sensors measured in the presence of 21 different interfering analytes. The concentration was $100 \mu \mathrm{g} \mathrm{mL}{ }^{-1}$ for protein analytes, including bovine serum albumin (BSA), tyrosinase (TYR), glucose oxidase (GOx), acetyl cholinesterase (AChE), and trypsin (TRY), and $500 \mu \mathrm{mol} \mathrm{L}{ }^{-1}$ for nonprotein analytes, including the inorganic salts $\mathrm{KCl}, \mathrm{Na}_{2} \mathrm{SO}_{4}, \mathrm{CaCl}_{2}, \mathrm{MgCl}_{2}$, and $\mathrm{MnCl}_{2}$ as well as aspartic acid, tyrosine, glycine, glucose and fructose. The fluorescence intensities increased only after the addition of GSH to the GQD-MnO 2 system and also showed recovery (blank column). [Reprinted with permission from ref. 737, X. Yan, Y. Song, C. Zhu, J. Song, D. Du, X. Su and Y. Lin, Graphene Quantum Dot- $\mathrm{MnO}_{2}$ Nanosheet Based Optical Sensing Platform: A Sensitive Fluorescence "Turn off-on" Nanosensor for Glutathione Detection and Intracellular Imaging, ACS Appl. Mater. Interfaces, 2016, 8, 21990-21996. Copyright@ American Chemical Society.] 

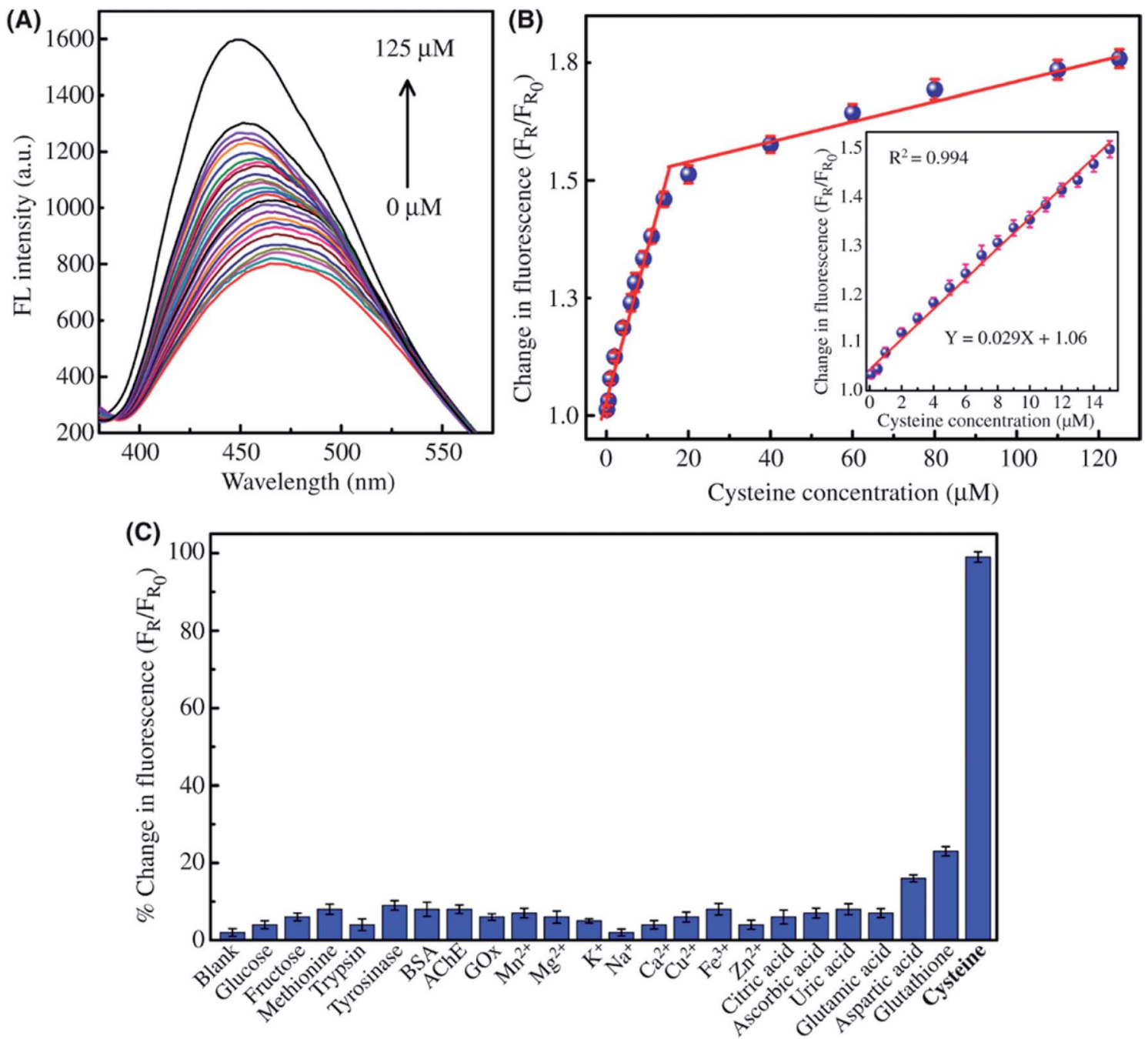

Fig. 40 (A) Fluorescence spectra of $\mathrm{N}$-doped GQDs decorated with $\mathrm{V}_{2} \mathrm{O}_{5}$ nanosheets $\left(\mathrm{N}-\mathrm{GQD}\left(\mathrm{V}_{2} \mathrm{O}_{5}\right)\right.$ of cysteine in the concentration range of $0-125 \mu \mathrm{M}$. (B) The change of fluorescence intensity as a function of cysteine concentration in the range of $0-125 \mu \mathrm{M}$. The inset shows a linear relationship in the $0-15 \mu \mathrm{M}$ concentration range. (C) Selectivity of the $\mathrm{N}-\mathrm{GQD}\left(\mathrm{V}_{2} \mathrm{O}_{5}\right.$ biosensors in the presence of 22 common interfering electrolytes and biological analytes, including metal ions, inorganic salts, amino acids, sugar, reducing agents, proteins, and glucose oxidase (GOx). Reprinted with permission from ref. 750, A. B. Ganganboina, A. D. Chowdhury and R.-A. Doong, N-Doped Graphene Quantum DotsDecorated $\mathrm{V}_{2} \mathrm{O}_{5}$ Nanosheet for Fluorescence Turn Off-On Detection of Cysteine, ACS Appl. Mater. Interfaces, 2017, 10, 614-624. Copyright@ 2018 American Chemical Society.

arrays was reported for the detection of volatile organic compounds (VOCs), ${ }^{739}$ gases, ${ }^{740}$ organophosphate pesticides,${ }^{741}$ bisphenol A, ${ }^{742,743}$ cancer biomarkers,${ }^{744}$ proteins, ${ }^{745,746}$ dopamine (DA), ${ }^{457,747,748}$ and the amino acid tyrosine. ${ }^{749}$ A few examples of sensitivity, high selectivity and strategies for excluding interfering analytes for graphene-based biosensors are provided here.

Ganganboina et al. ${ }^{750}$ reported a fluorescence turn-off/on biosensor base on nitrogen-doped GQD (N-GQD)-decorated $\mathrm{V}_{2} \mathrm{O}_{5}$ nanosheets for cysteine detection in human serum. $\mathrm{N}$ GQDs of 2-8 $\mathrm{nm}$ in diameter were deposited on few-layered $\mathrm{V}_{2} \mathrm{O}_{5}$ nanosheets to develop a fluorescence turn-off/on probe. The $\mathrm{V}_{2} \mathrm{O}_{5}$ nanosheets function as both cysteine detectors and fluorescence quenchers in the biosensor. The quenching of the fluorescence intensity of N-GQDs occurs after deposition onto the $\mathrm{V}_{2} \mathrm{O}_{5}$ nanosheets. Fig. 40 shows the fluorescence spectra of the N-GQD@ $\mathrm{V}_{2} \mathrm{O}_{5}$ nanocomposite-based sensor for detecting cysteine in the 0 to $125 \mu \mathrm{M}$ concentration range and the selectivity of cysteine in the presence of 22 common interfering electrolytes and biological analytes, including metal ions, inorganic salts, amino acids, sugar, reducing agents, proteins, and glucose oxidase. The addition of $60 \mu \mathrm{g} \mathrm{mL}^{-1}$ of $\mathrm{V}_{2} \mathrm{O}_{5}$ nanosheets was found to turn off the fluorescence intensity of N-GQDs, whereas the fluorescence subsequently turned on $4 \mathrm{~min}$ after the addition of cysteine in the $0-125 \mu \mathrm{M}$ concentration range. The inclusion of cysteine initiates the reduction of $\mathrm{V}_{2} \mathrm{O}_{5}$ to $\mathrm{V}^{4+}$ and the discharging of N-GQDs, which consequently causes the fluorescence to turn off/on for cysteine sensing. The N-GQDs@ $\mathrm{V}_{2} \mathrm{O}_{5}$ sensing probe showed a two-stage linear response for cysteine detection, a sharp rise in the 

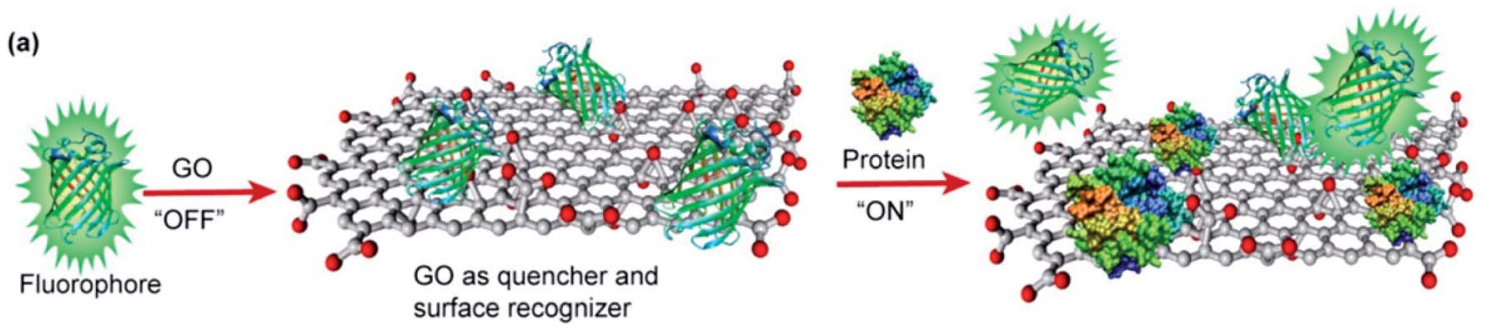

(b)

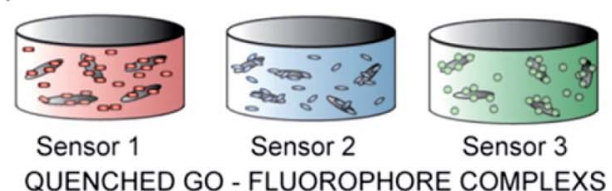

(c)
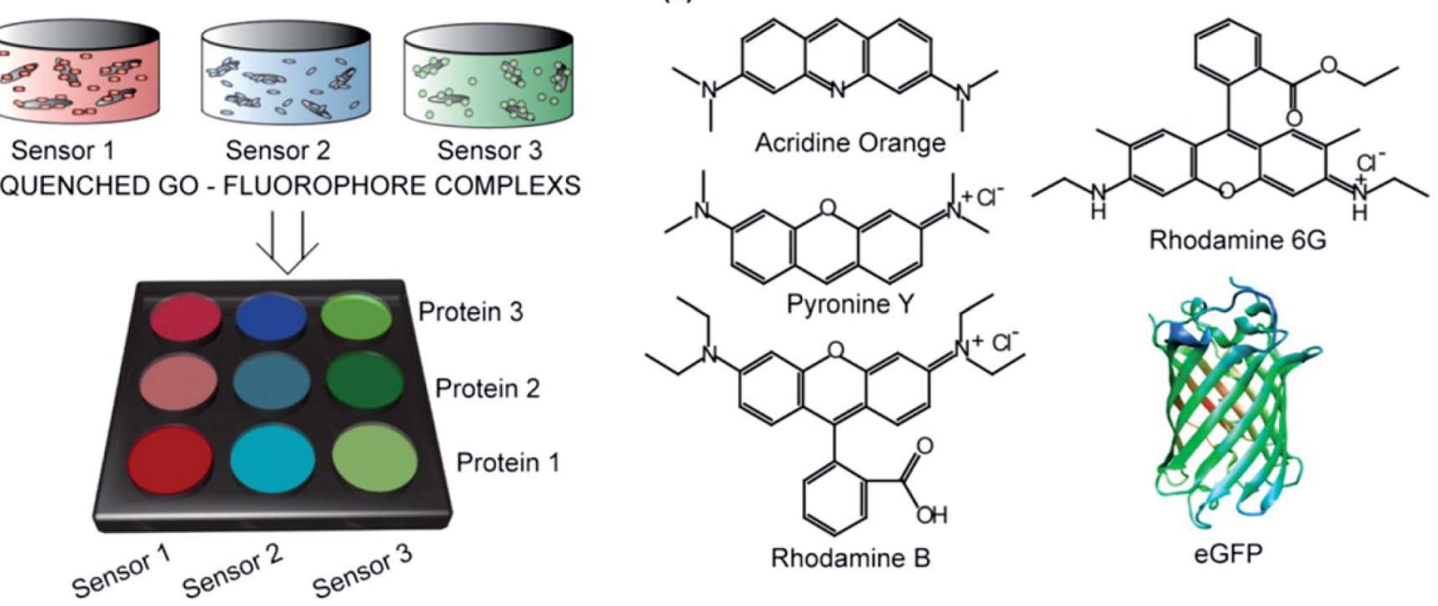

Rhodamine 6G Fig. 41 Schematic representation of $\mathrm{nGO}$-based biosensor array. (a) GO-protein binding and interactions showing GO as a quencher for
fluorophores. The fluorescence is restored by the displacement of quenched fluorophores due to the interactions between GO and the analyte proteins. Statistical analysis of the displaced fluorophores using LDA to examine differences in the GO-protein interactions between conventional GO and nGO flakes (20 nm diameter). (b) LDA patterns showed enhanced fluorescent restoration from a three sensor array of $\mathrm{nGO}$ flakes compared to that obtained with conventional GO. The nGO showed a high fluorescence response due to increased protein interactions compared with those observed for conventional GO flakes. (c) Chemical structures of the five fluorophores, including acridine orange, pyronine Y (PY), rhodamine B, rhodamine 6G (R6G) and His-tagged emerald green fluorescent protein (eGFP), used for the fluorescent sensor array. Reprinted with permission from ref. 753, S. S. Chou, M. De, J. Luo, V. M. Rotello, J. Huang, V. P. Dravid, Nanoscale Graphene Oxide (nGO) as Artificial Receptors: Implications for Biomolecular Interactions and Sensing, J. Am. Chem. Soc., 2012, 134, 16725-16733. Copyright@ 2018 American Chemical Society.

fluorescence at the low concentration range of 0.1-15 $\mu \mathrm{M}$ and thereafter a slight increase in the $15-125 \mu \mathrm{M}$ range at $6.5 \mathrm{pH}$, and an LOD of $50 \mathrm{nM}$. A correlation coefficient of 0.994 was obtained in the $0.1-15 \mu \mathrm{M}$ concentration range. The surfacemediated reaction between cysteine and N-GQDs@ $\mathrm{V}_{2} \mathrm{O}_{5}$ composites is fast in the low cysteine concentration range of 0.1-15 $\mu \mathrm{M}$ range due to the abundance of reactive sites on the $\mathrm{N}$ GQD@V $V_{2} \mathrm{O}_{5}$ nanosheets, whereas the availability of reactive sites on $\mathrm{V}_{2} \mathrm{O}_{5}$ nanosheets is significantly reduced over time in the high cysteine concentration range of $15-125 \mu \mathrm{M}$. The NGQD@V $\mathrm{V}_{2} \mathrm{O}_{5}$ nanocomposite-based sensor showed recoveries of $95 \%-108 \%$ for cysteine detection in human serum. The N$\mathrm{GQD}_{2} \mathrm{~V}_{2} \mathrm{O}_{5}$ nanocomposites showed high sensitivity and selectivity for detecting cysteine. The high selectivity toward cysteine was also demonstrated by the N-GQD@V $\mathrm{V}_{2} \mathrm{O}_{5}$-based sensor in the presence of 22 common interfering analytes: $\mathrm{Na}^{+}$, $\mathrm{K}^{+}, \mathrm{Mg}^{2+}, \mathrm{Ca}^{2+}, \mathrm{Mn}^{2+}, \mathrm{Cu}^{2+}, \mathrm{Fe}^{3+}, \mathrm{Zn}^{2+}$, tyrosine, glutathione, methionine, aspartic acid, glucose, fructose, citric acid, AA, glutamic acid, UA, BSA, tyrosinase, acetylcholinesterase, and GOx. Protein concentrations were $100 \mu \mathrm{M}$ and other analyte concentrations were $500 \mu \mathrm{M}$ (the spiked concentration of cysteine was $50 \mu \mathrm{M})$. The $\mathrm{N}-\mathrm{GQD} @ \mathrm{~V}_{2} \mathrm{O}_{5}$ sensor fluorescence recovery was $<10 \%$ for most of the interfering analytes except for glutathione and aspartic acid, which showed 16\%-23\% fluorescence intensity; however, all 22 interfering species showed significantly lower fluorescence intensity than cysteine. The smaller thiol group on the cysteine molecule may interact more rapidly with the $\mathrm{V}_{2} \mathrm{O}_{5}$ nanosheet surface, resulting in enhanced fluorescence recovery compared with the larger glutathione molecule.

The highly sensitive and selective detection of 4-nitrophenol using AuNPs@sulfur-doped GQD sensors was reported. ${ }^{751}$ The AuNPs@S-GQD sensing probe showed a detection range of 0.01-50 $\mu \mathrm{M}$ for 4-nitrophenol with an LOD of $8.4 \mathrm{nM}$ in contaminated food wastewater and a dynamic range of 0.005-50 $\mu \mathrm{M}$ with an LOD of $3.5 \mathrm{nM}$ in deionized water. The AuNPs@SGQD sensor also showed high selectivity for 4-nitrophenol in the presence of 7 interfering analytes, including $50 \mu \mathrm{M}$ toluene, phenol, 2-nitrotoluene, 4-nitrotoluene, 2-nitrophenol, 2,4,6-trinitrophenol, and 1-chloro-4-nitrobenzene, under identical conditions. The recovery of 4-nitrophenol by the AuNPs@S-GQD nanocomposites in the $100 \mathrm{nM}$ to $20 \mu \mathrm{M}$ concentration range 
was found to be in a similar range, from $97 \pm 2 \%$ to $110 \pm 3 \%$, to that in the lake water. This study demonstrated the high sensitivity and selectivity of AuNPs@S-GQD-based optical sensor for 4-nitrophenol in complex mixed aqueous solutions.

Biosensor arrays fabricated using nanographene oxide (nGO) have been studied for the selective detection biomolecular species with pattern recognition techniques. ${ }^{752,753}$ Oligonucleotides or peptides that specifically bind to a target molecule have been used for such target identification. Chou et al. ${ }^{753}$ demonstrated a size-dependent effect in the use of GO flakes for the selective detection of different proteins by fluorescence response patterns. A schematic representation of the nGObased biosensor array and chemical structures of the five fluorophores used in this study are shown in Fig. 41. GO acts as a quencher for fluorophores, and fluorescence is observed due to the interactions between GO-analyte proteins after the displacement of quenched fluorophores. To demonstrate the size-dependent effect, LDA was used to analyze the displacement of fluorophores and to compare the GO-proteins formed with conventional GO and nGO flakes (20 nm diameter). A higher fluorescence response was measured for nGO than for conventional GO flakes due to increased protein interactions, showing the effect of flake size. Five different fluorophores, including acridine orange, pyronin $\mathrm{Y}(\mathrm{PY})$, rhodamine $\mathrm{B}$, rhodamine 6G (R6G), and histone-tagged emerald green fluorescent protein (His-eGFP), were used in the development of arrays. Three fluorophores, pyronine Y, rhodamine 6G, and HiseGFP, were found to be significantly effective in generating responses among these five fluorophores. Eight analyte proteins of different sizes were included in the test, including BSA, $\beta$ galactosidase ( $\beta$-Gal), hemoglobin (Hem), Histone (His), lipase (Lip), lysozyme (Lys), myoglobin, and ribonuclease A (Rib-A). The training matrix data of different proteins were obtained at 10 and $100 \mathrm{nM}$ protein concentrations with the conventional GO and nGO arrays. The correlation of the fluorescence response from the conventional GO and nGO arrays at 10 and $100 \mathrm{nM}$ concentrations of analyte proteins and their LDA scores were scrutinized, and a 10-fold increase was observed for the
(A)

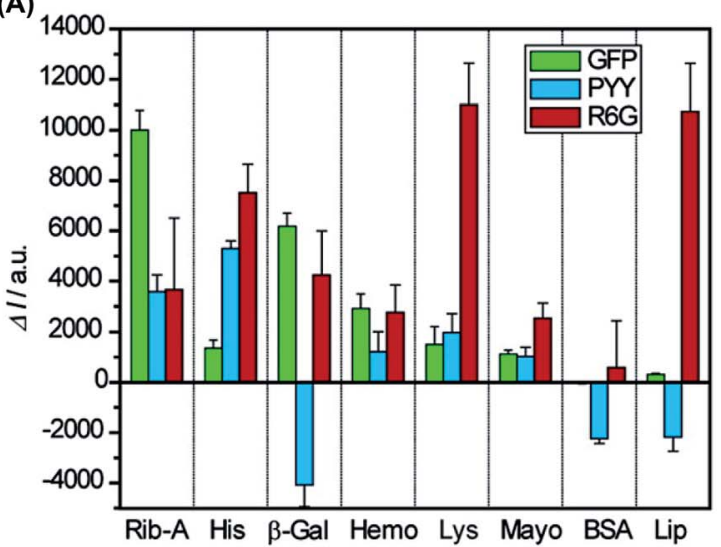

(B)

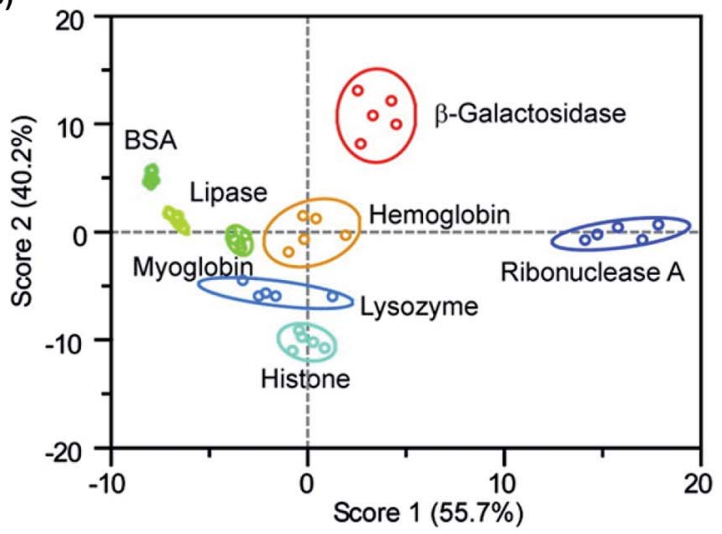

(C)

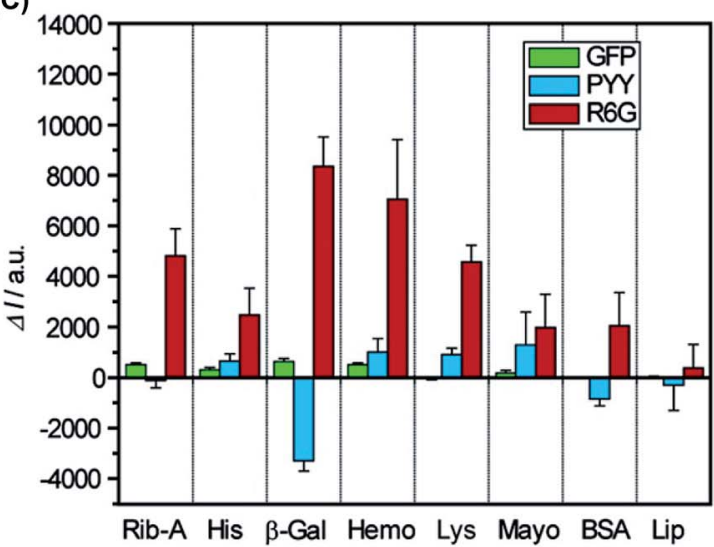

(D)

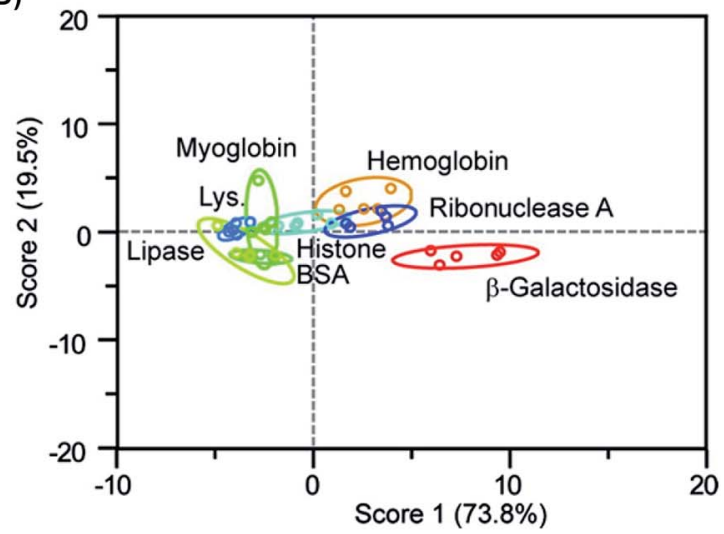

Fig. 42 (A) Fluorescence response patterns measured using nGO-based sensor arrays to selectively identify eight different analyte proteins, including ribonuclease A (Rib-A), histone (His), $\beta$-galactosidase ( $\beta$-Gal), hemoglobin (Hemo), lysozyme (Lys), myoglobin, lipase (Lip), and BSA at $10 \mathrm{nM}$ concentrations. Three different fluorophores, pyronine $Y$ (PY), rhodamine 6G (R6G), and His-tagged emerald green fluorescent protein (eGFP), were used in the biosensing arrays. (B) The canonical score plot obtained by the LDA method using the nGO sensor array showed 95\% accurate classification of all proteins. (C) The fluorescence response patterns measured using the conventional GO-based sensor array at $10 \mathrm{nM}$ protein concentration. (D) The corresponding canonical score plot for the conventional GO-based sensor array, revealing an unclear classification. Reprinted with permission from ref. 753, S. S. Chou, M. De, J. Luo, V. M. Rotello, J. Huang and V. P. Dravid, Nanoscale Graphene Oxide (nGO) as Artificial Receptors: Implications for Biomolecular Interactions and Sensing, J. Am. Chem. Soc., 2012, 134, 16725-16733. Copyright $\odot$ American Chemical Society. 
$10 \mathrm{nM}$ concentration compared with analogous sensor arrays. A success rate of $>95 \%$ was obtained for the identification of 48 unknown analytes at both 10 and $100 \mathrm{nM}$.

Biosensor arrays based on conventional GO and nGO were studied for the discrimination of analyte proteins with five fluorophores. With five sensors, each with eight proteins, and five replicates of each analyte protein present for each sensor, there were 200 data points for each biosensor array. The fluorescent signal intensity showed higher fluorophore displacement rates for the nGO-based array than for the conventional GO arrays because of the higher protein affinity for nGO surfaces resulting from the presence of edges. An LDA/jackknife accuracy of $100 \%$ was observed using only three fluorophore sensors (eGFP, R6G, and PY) in the nGO array at $100 \mathrm{nM}$. The canonical factors of $48.7,32.6$, and $18.7 \%$ were produced from training matrix data with 3 sensors $\times 8$ proteins $\times 5$ replicates in the LDA method. The jackknife analysis demonstrated $100 \%$ separation using acridine orange-, rhodamine B-, eGFP-, R6G-, and PY-based fluorophore sensors at $100 \mathrm{nM}$ and 98\% separation with eGFP, R6G, and PY fluorophores. The canonical charts were found to be overlapped and significantly compressed $(69.7 \%, 21.4 \%$, and $8.9 \%)$, showing an inability to identify unknown analytes. The GO and nGO-based arrays were also compared at $10 \mathrm{nM}$ protein concentrations under similar experimental conditions (Fig. 42). The canonical score plot obtained by the LDA method showed 95\% accuracy in the jackknife classification using the nGO sensor array at a $10 \mathrm{nM}$ protein concentration, while the corresponding canonical score plot exhibited an unclear classification for the conventional GObased sensor array. The nGO-based array showed high reproducibility of 24 out of 24 (protein concentration of $100 \mathrm{nM}$ ) and 22 out of $24(10 \mathrm{nM})$ compared with 17 out of $24(100 \mathrm{nM})$ and no ability to distinguish proteins $(10 \mathrm{nM})$ for conventional GO arrays. Analyte protein discrimination was clearly observed at a $10 \mathrm{nM}$ concentration for R6G, PY and eGFP fluorophores. The selectivity for different proteins depends upon the size scaling of GO flakes, where properties such as electrical conductivity, elasticity and viscosity may play important roles. Similar nGObased biosensor arrays were previously reported for the selective detection of ensemble aptamer proteins by Pei et al. ${ }^{752}$

Luminescent nanomaterials possessing aggregationinduced emission (AIE) characteristics have attracted attention as fluorescent sensors because of their high sensitivity and selectivity. ${ }^{665,754-759}$ Graphene-based fluorescent sensor arrays have been studied for biomolecule identification. Shen et al. ${ }^{760}$ used AIE and GO complexes to develop a fluorescent sensor array for detecting microbial lysates through their competitive biomolecular interactions (Fig. 43). Using AIE/GO complexbased sensor arrays, six microbial bioanalytes including two fungi (Candida albicans and Saccharomyces cerevisiae) and four bacteria including two Gram-positive bacteria (Staphylococcus aureus and Bacillus subtilis) and two Gram-negative bacteria (Pseudomonas aeruginosa and E. coli), were discriminated and identified. The reproducibility of AIE/GO complex sensor array was confirmed through PCA where each test was conducted five times and six microbial lysates were discriminated with $100 \%$ $\left(F_{1}=61.3 \%+F_{2}=29.9 \%\right)$ efficiency with $F_{1}$ and $F_{2}$ as the main contributing rates in the PCA method. The two fungi are located in the upper-left side whereas the two Gram-negative bacteria and two Gram-positive bacteria are situated in the upper-right side and bottom areas in the PCA plot. The high efficiency of identification and discrimination with AIE/GO sensor arrays resulted from the different molecular components of each microbial lysate and their competitive biomolecular interactions. Xu et al. $^{761}$ developed GO-based AIE biosensors with tetraphenylethene dye that exhibited high sensitivity and selectivity for detecting BSA protein. The fluorescence intensity recorded at an excitation wavelength of $350 \mathrm{~nm}$ increased
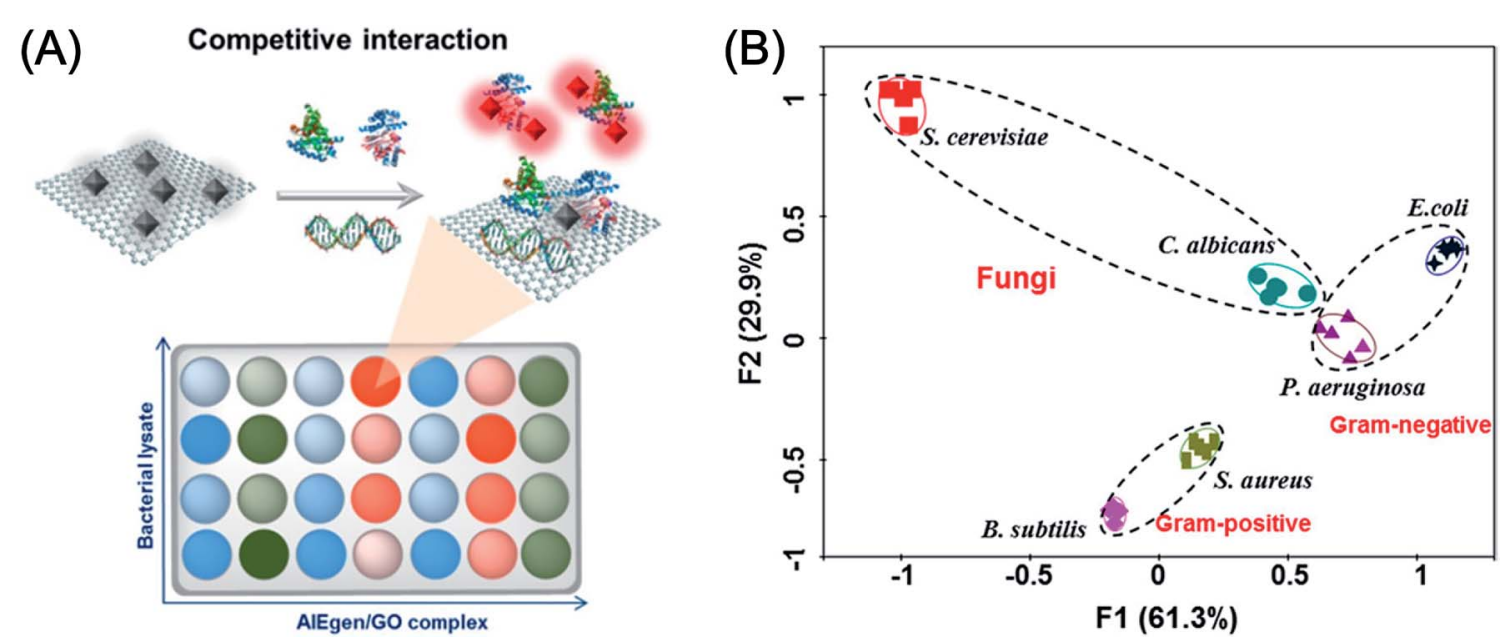

Fig. 43 (A) Schematic illustration of AIE/GO complex-based fluorescent sensor array showing competitive biomolecular interaction among AIE, microbes and GO. AIE is represented as AIEgen in the illustration. (B) Principal component analysis (PCA) plot showing three separate patterns formed from six microbial lysates where each test was conducted five times. The AIE/GO complex-based fluorescent sensor array identified six microbial lysates with $100 \%\left(F_{1}=61.3 \%+F_{2}=29.9 \%\right)$ efficiency. Reprinted with permission from ref. 760, J. Shen, R. Hu, T. Zhou, Z. Wang, Y. Zhang, S. Li, C. Gui, M. Jiang, A. Qin and B. Z. Tang, Fluorescent Sensor Array for Highly Efficient Microbial Lysate Identification through Competitive Interactions, ACS Sensors, 2018, 3, 2218-2222. Copyright@ American Chemical Society. 
dramatically for BSA molecules compared with the response to interfering lysozyme, papain, pepsin and trypsin proteins at 200 $\mu \mathrm{g} \mathrm{mL} \mathrm{m}^{-1}$. Similar PL intensity enhancement in the fluorescence spectra was observed for BSA compared to other proteins under the same experimental conditions. Therefore, GO-based AIE biosensors are highly selective for BSA.

Huang et al. ${ }^{762}$ used PtNPs-decorated graphene, cyclodextrin prepolymer (CDP) and MWCNT nanocomposite-based biosensors to simultaneously detect hydroquinone (HQ) and catechol (CC). The PtNP-graphene/CDP-MWCNT/GCE electrochemical sensor showed linear ranges of 0.05 to $27.2 \mu \mathrm{M}$ and 0.1 to 27.2 $\mu \mathrm{M}$ and LOD values of $0.015 \mu \mathrm{M}$ and $0.03 \mu \mathrm{M}$ for the simultaneous detection of HQ and CC, respectively. The HQ and CC measured in local river water showed recoveries ranging from $98.0 \%$ to $102.0 \%$ for HQ and from $99.3 \%$ to $101.5 \%$ for CC. The selectivity for HQ and CC was studied against 21 different interfering inorganic ions and organic analytes. The addition of 1000-fold concentrations of $\mathrm{Al}^{3+}, \mathrm{Cu}^{2+}, \mathrm{Zn}^{2+}, \mathrm{Mg}^{2+}, \mathrm{Na}^{+}, \mathrm{K}^{+}$, $\mathrm{NH}_{4}{ }^{+}, \mathrm{SO}_{4}{ }^{2-}, \mathrm{Cl}^{-}$, and $\mathrm{NO}_{3}{ }^{-}, 100$-fold of $\mathrm{Fe}^{3+}, \mathrm{Ni}^{2+}, \mathrm{Ca}^{2+}, \mathrm{SO}_{3}{ }^{2-}$, $\mathrm{S}^{2-}$, and $\mathrm{Br}^{-}$, 10-fold of resorcinol (RC), phenol, nitrophenol (NP), glucose and UA showed no interference with the detection of $50 \mu \mathrm{M}$ HQ and CC (Fig. 44). Therefore, the PtNP-graphene/ CDP-MWCNT/GCE electrochemical biosensor is highly selective and sensitive to the presence of HQ and CC.

Hybrids or nanocomposites of graphene with other semiconducting nanoparticles and polymers have been used to develop highly selective sensors for differentiating chemical species. ${ }^{48,206,763,764}$ Toxic metal ions, including $\mathrm{Hg}^{2+}$ ions, ${ }^{693,696,765}$
$\mathrm{Mn}^{2+},{ }^{766} \mathrm{~Pb}^{2+},{ }^{689,692} \mathrm{Cu}^{2+},{ }^{694,767} \mathrm{Fe}^{3+},{ }^{768} \mathrm{Fe}^{3+}, \mathrm{Cd}^{2+}$ and $\mathrm{Pb}^{2+},{ }^{769}$ $\mathrm{Ce}^{3+}, 770 \mathrm{~Pb}^{2+}, \mathrm{Zn}^{2+}$ and $\mathrm{Cd}^{2+}, 771 \mathrm{Tl}^{+}, \mathrm{Pb}^{2+}$ and $\mathrm{Hg}^{2+},{ }^{772}$ and $\mathrm{Ag}^{+},{ }^{702,730,773}$ and the mycotoxin OTA, ${ }^{683}$ have been selectively detected using graphene-based sensors.

Mao et al. ${ }^{766}$ developed a fluorescent sensor based on 1,2-bis(2-pyren-1-ylmethylamino-ethoxy) ethane (NPEY) and graphene nanosheets (GNs) for the detection of $\mathrm{Mn}^{2+}$ both in vitro and in living cells utilizing $\pi-\pi$ interactions. Fig. 45 shows the fluorescence intensity of NPEY/GNs hybrid system to different metal ions, schematic illustration of fluorescence mechanism for detecting $\mathrm{Mn}^{2+}$ with NPEY/GNs hybrid, and confocal fluorescence microscopy images of HeLa cells with NPEY, NPEY/GNs hybrid with and without $\mathrm{Mn}^{2+}$ ions. HRTEM image of NPEY/ GNs hybrid confirmed the NPEY decorated GNs. The GNs quenched fluorescence emission occurring between NPEY molecules and GNs due to the $\pi-\pi$ stacking interactions facilitated by the photoinduced electron transfer (PET) process. NPEY/GNs hybrid-based sensor showed high sensitivity and selectivity toward $\mathrm{Mn}^{2+}$ ions against other interfering heavy metal ions including $\mathrm{Ag}^{+}, \mathrm{Hg}^{2+}, \mathrm{Zn}^{2+}, \mathrm{Cr}^{2+}, \mathrm{Ca}^{2+}, \mathrm{Ni}^{2+}, \mathrm{Cd}^{2+}$, and $\mathrm{Pb}^{2+}$. The $\mathrm{Mn}^{2+}$ ions lead to a significant increase in the fluorescence intensity of NPEY/GNs hybrid. AFM showed no interaction between $\mathrm{Mn}^{2+}$ ions and GNs demonstrating the nonsensitivity of GNs. The ethylene glycol and imino groups of NPEY assist in binding with $\mathrm{Mn}^{2+}$ ions. The NPEY/GNs hybridbased sensor also showed the imaging and detection of $\mathrm{Mn}^{2+}$ ions in living cells.

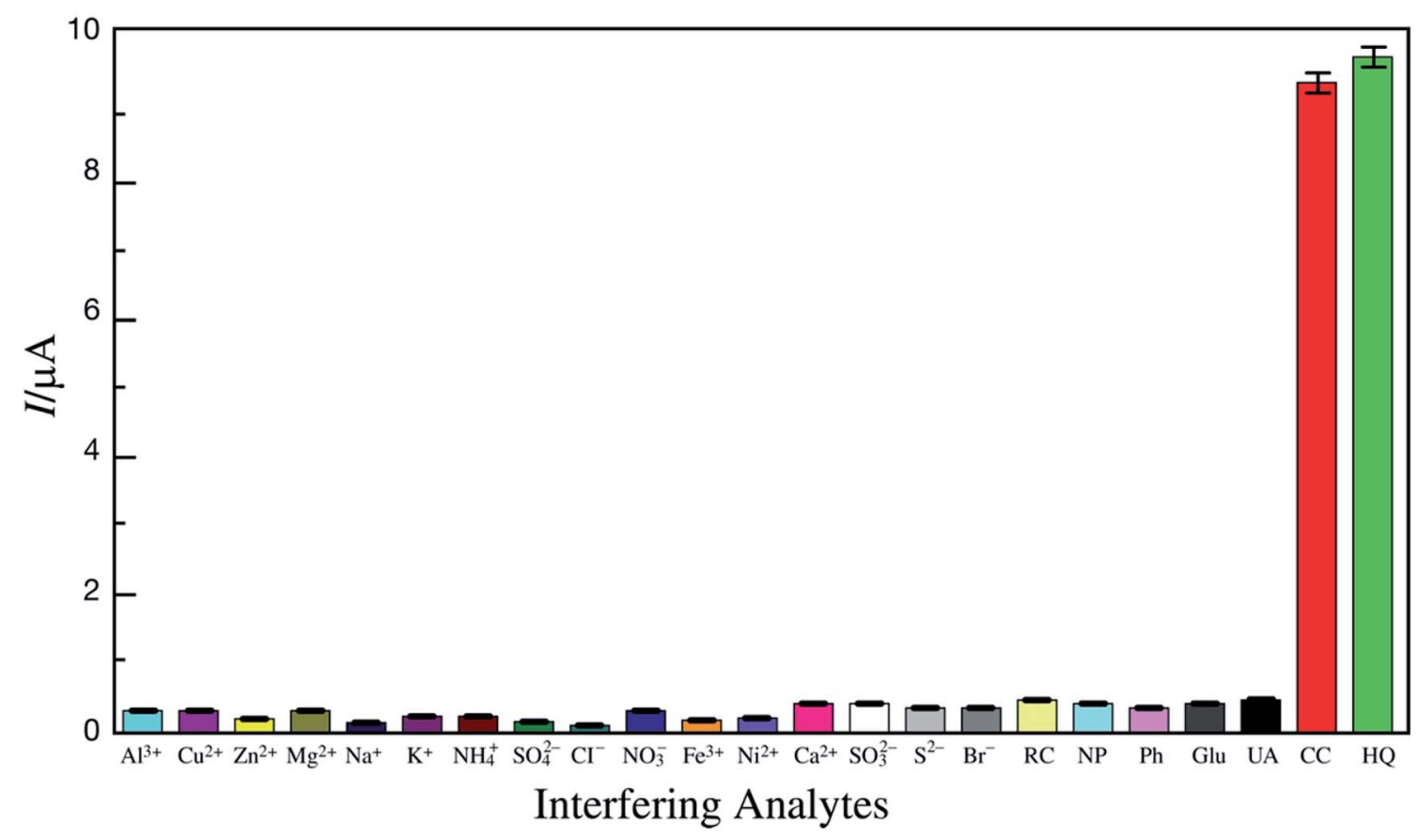

Fig. 44 Selectivity of PtNPs-graphene/CDP-MWCNTs/GCE electrochemical biosensor toward catechol (CC) and hydroquinone (HQ) against 21 different interfering analytes. The buffer was $0.1 \mathrm{M} \mathrm{PBS}\left(\mathrm{pH}\right.$ 6.0) at $50 \mathrm{mV} \mathrm{s}^{-1}$ scan rate. The concentrations of HQ and CC were $50 \mu \mathrm{M}$. Reprinted with permission from ref. 762, X. Huang, X. Deng, W. Qi and D. Wu, Simultaneous Detection of Hydroquinone and Catechol Using Platinum Nanoparticles Decorated Graphene/Poly-Cyclodextrin/Multiwalled Carbon Nanotubes (MWCNTs) Nanocomposite Based Biosensor, J. Nanosci. Nanotechnol., 2018, 18, 8118-8123. Copyright@ American Scientific Publishers. 
(A)

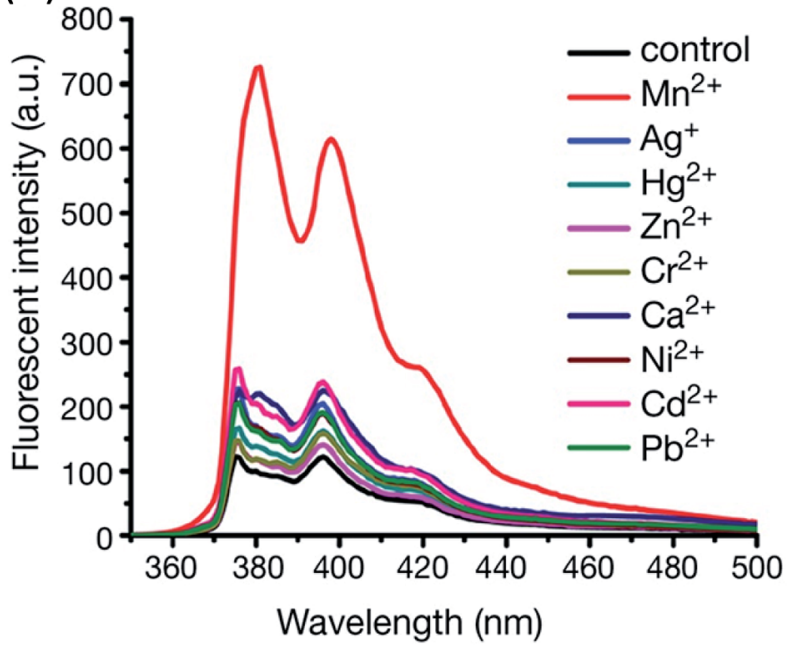

(C)

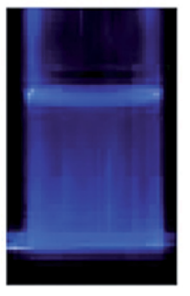

OFF

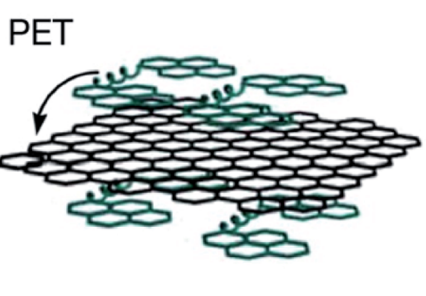

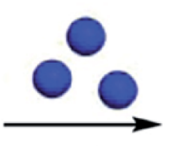

$\mathrm{Mn}^{2+}$
(B)

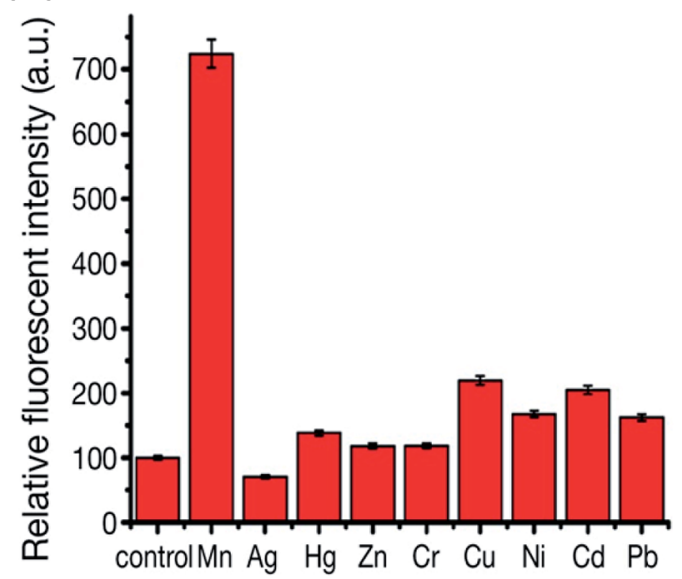

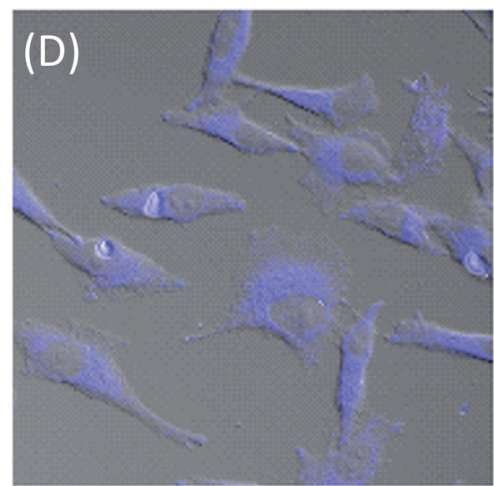

ON
PET Blocking

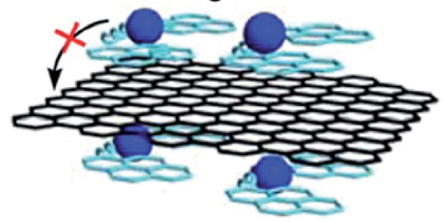

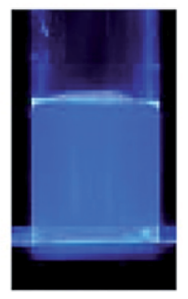

ON

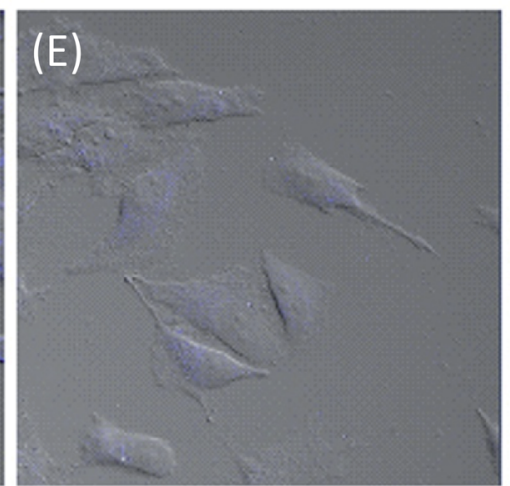

OFF

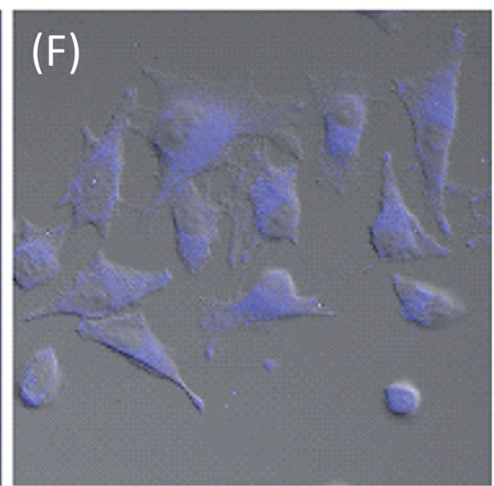

ON

Fig. 45 (A) Fluorescence intensity of 1,2-bis-(2-pyren-1-ylmethylamino-ethoxy)ethane (NPEY)/graphene nanosheets (GNs) hybrid system to different metal ions. (B) The changes of fluorescence intensity of NPEY/GNs hybrid measured at $376 \mathrm{~nm}$ after adding different heavy metal ions. (C) Schematic illustration of fluorescence "Turn-On" mechanism for detecting $\mathrm{Mn}^{2+}$ using NPEY/GNs hybrid; change in fluorescence emission with NPEY/GNs (left) and NPEY/GNs in the presence of $\mathrm{Mn}^{2+}$ (right) under $365 \mathrm{~nm}$ UV light illumination. The GNs quenched fluorescence emission due to the $\pi-\pi$ stacking interactions between NPEY molecules and GNs via photoinduced electron transfer (PET) process. (D) Confocal fluorescence microscopy images of HeLa cells with NPEY only (E) NPEY/GNs, and (F) NPEY/GN with Mn ${ }^{2+}$ after extensive washing of HeLa cells with PBS. [Reprinted with permission from ref. 766, X. Mao, H. Su, D. Tian, H. Li and R. Yang, Bipyrene-functionalized graphene as a "turn-on" fluorescence sensor for manganese(॥) ions in living cells, ACS Appl. Mater. Interfaces, 2013, 5, 592-597. Copyright@ American Chemical Society.]

Niu et al. ${ }^{\text {704 }}$ developed a "turn-on" fluorescence sensor using GQD and AuNP conjugates for $\mathrm{Pb}^{2+}$ detection. The GQD/AuNPbased fluorescence sensor showed a $\mathrm{Pb}^{2+}$ detection range of $50 \mathrm{nM}$ to $4 \mu \mathrm{M}$ with an LOD of $16.7 \mathrm{nM}$ and very high sensitivity and selectivity for $\mathrm{Pb}^{2+}$ ions in the presence of ten other interfering metal ions. Fig. 46 shows the fluorescence intensity response measured for $200 \mathrm{nM}$ concentrations of $\mathrm{Mn}^{2+}, \mathrm{Fe}^{3+}, \mathrm{K}^{+}$, $\mathrm{Hg}^{2+}, \mathrm{Cu}^{2+} \mathrm{Mg}^{2+}, \mathrm{Ca}^{2+}, \mathrm{Zn}^{2+}, \mathrm{Cd}^{2+}$, and $\mathrm{Ag}^{+}$ions and $50 \mathrm{nM} \mathrm{Pb}^{2+}$ ions. The fluorescence increased sharply with the addition of $50 \mathrm{nM} \mathrm{Pb}^{2+}$, whereas no significant change in fluorescence was observed after the addition of $200 \mathrm{nM}$ concentrations of ten other interfering metal ions. The $\mathrm{Pb}^{2+}$ ions showed almost 3- 


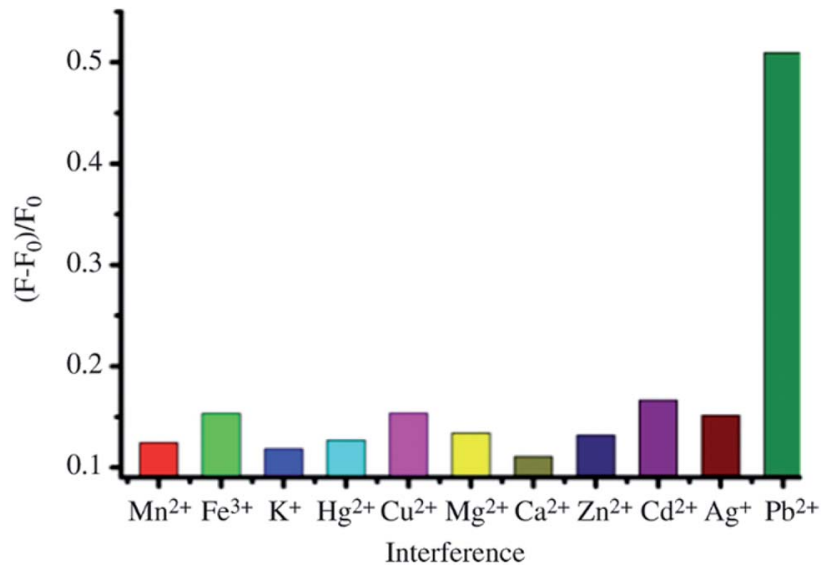

Fig. 46 Selectivity for $\mathrm{Pb}^{2+}$ ions (50 nM) using a "turn-on" fluorescence sensor based on GQD/AuNP conjugates in the presence of interfering analytes including $\mathrm{Mn}^{2+}, \mathrm{Fe}^{3+}, \mathrm{K}^{+}, \mathrm{Hg}^{2+}, \mathrm{Cu}^{2+} \mathrm{Mg}^{2+}, \mathrm{Ca}^{2+}$ $\mathrm{Zn}^{2+}, \mathrm{Cd}^{2+}$, and $\mathrm{Ag}^{+}$ions (200 nM). Reprinted with permission from ref. 704, X. Niu, Y. Zhong, R. Chen, F. Wang, Y. Liu, D. A. Luo, "Turn-on" Fluorescence Sensor for $\mathrm{Pb}^{2+}$ Detection Based on Graphene Quantum Dots and Gold Nanoparticles, Sens. Actuators, B, 2018, 255, 15771581. Copyright $\odot$ Elsevier. fold higher restoration efficiency than the other interfering metal ions, clearly differentiating $\mathrm{Pb}^{2+}$ from all metal ions. Other metal ions were not able to cleave the catalytic DNA strand and did not separate the GQDs and AuNPs; therefore, no quenching recovery was observed except for $\mathrm{Pb}^{2+}$ ions. The high sensitivity and selectivity of the fluorescent sensor toward $\mathrm{Pb}^{2+}$ ions occurred due to the FRET between GQDs and AuNPs. The strong fluorescence quenching and fluorescence recovery resulted from the combination of GQDs and AuNPs and their deaggregation, which was induced by the catalytic DNA strand and $\mathrm{Pb}^{2+}$ ions.

Jiang et al. ${ }^{774}$ fabricated electrochemical immunosensors using GO/AgNPs composites for the detection of $E$. coli SEM images of mica sheets coated with AuNPs/gold, BSA/antibody(Ab)/AuNPs/gold, E. coli/BSA/Ab/AuNPs/gold, and P-GOAgNPs-Ab/E. coli/BSA/Ab/AuNPs/gold were compared, and the selectivity of the detection of different bacteria was measured by analyzing current signals. Current responses of $0.946 \mu \mathrm{A}$ for $B$. subtilis $\left(1.8 \times 10^{4} \mathrm{CFU} \mathrm{\textrm {mL } ^ { - 1 }}\right), 1.124 \mu \mathrm{A}$ for Enterobacter aerogenes, $\left(2.0 \times 10^{4} \mathrm{CFU} \mathrm{m} \mathrm{L}{ }^{-1}\right), 1.505 \mu \mathrm{A}$ for Enterobacter dissolvens $\left(2.5 \times 10^{4} \mathrm{CFU} \mathrm{mL}^{-1}\right)$, and (d) $17.3000 \mu \mathrm{A}$ for $(E$. coli $(1.0$ $\times 10^{4} \mathrm{CFU} \mathrm{\textrm {mL } ^ { - 1 } )}$ were observed. Zhang et al. ${ }^{775}$ developed a fluorescent aptasensor assay using GO for detecting mucin 1 protein (MUC1) by employing deoxyribonuclease I (DNase I)-

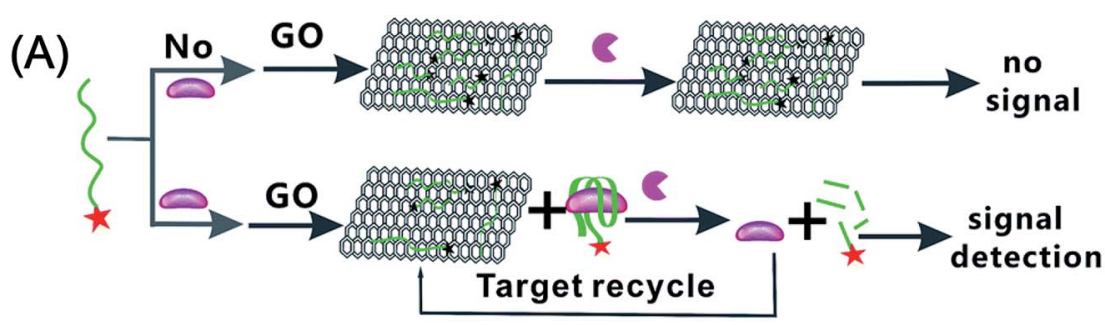

MUC1 D Dnase I $*$ FAM $\leadsto$ MUC1 aptamer

(B)

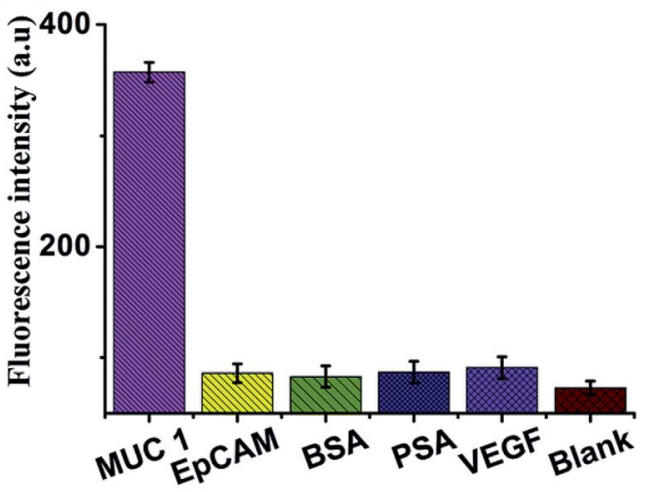

(C)

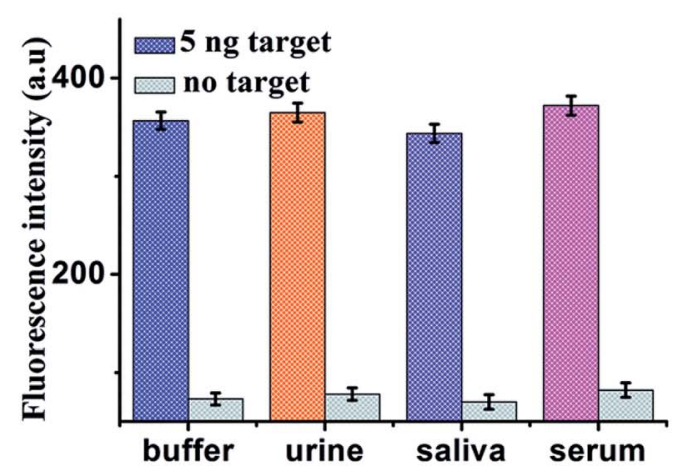

Fig. 47 (A) Schematic representation of a fluorescent aptasensor assay based on GO-probe for detecting mucin 1 protein (MUC1) (5'-FAMCCCGTCTTCCAGACAAGAGTGCAGGG-3') by using deoxyribonuclease I (DNase I)-mediated target cyclic amplification. The formation of GOprobe/MUC1 complex results in the fluorescence signal detection. (B) Fluorescence intensity of the GO-based aptasensor in the presence of MUC1 ( $\left.5 \mathrm{ng} \mathrm{mL}^{-1}\right)$, epithelial cell adhesion molecule (EpCAM) $\left(50 \mathrm{ng} \mathrm{mL}^{-1}\right)$, serum albumin (BSA) (50 ng mL $\left.{ }^{-1}\right)$, prostate-specific antigen (PSA) (50 $\mathrm{ng} \mathrm{m \textrm {m } ^ { - 1 }}$ ), vascular endothelial growth factor (VEGF) $\left(50 \mathrm{ng} \mathrm{mL}^{-1}\right)$, and black, respectively. (C) Fluorescence intensity of the GO-based aptasensor for detection of MUC1 protein in buffer and blank biological samples of human urine, saliva and serum. Reprinted with permission from ref. $775, \mathrm{~J}$. Zhang, F. Ran, W. Zhou, B. Shang, F. Yu, L. Wu, W. Hu, X. He and Q. Chen, Ultrasensitive fluorescent aptasensor for MUC1 detection based on deoxyribonuclease I-aided target recycling signal amplification, RSC Adv., 2018, 8, 32009-32015. Copyright@ The Royal Society of Chemistry. 
mediated target cyclic amplification. Fig. 47 shows the schematics of the GO-based fluorescent aptasensor assay, fluorescence intensity of the GO-based aptasensor for detection of MUC1 protein in the presence of different interfering proteins including epithelial cell adhesion molecule (EpCAM), BSA, prostate-specific antigen (PSA), vascular endothelial growth factor (VEGF and the biological samples of human urine, saliva and serum. The GO-probe/MUC1 complex formation plays an important role for the fluorescence detection. The GO strongly adsorbs deoxyribonuclease I (DNase I) in the absence of MUC1 protein so no fluorescence signal is observed whereas a fluorescence signal is turn-on when the GO-probe/MUC1 complexes are formed in the presence of MUC1. In this process, the DNA sequences are damaged after adding DNase which releases MUC1 and FAM fluorophores. The GO-based aptasensor showed a dramatic increase in the fluorescence intensity as the concentration of MUC1 was increased from 0 to $300 \mathrm{ng} \mathrm{mL}^{-1}$ with a linear range from $50 \mathrm{pg} \mathrm{mL}^{-1}$ to $100 \mathrm{ng} \mathrm{mL}^{-1}$ and an LOD

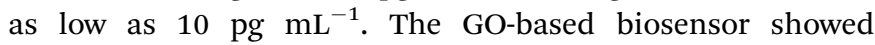
a significant increase in fluorescence intensity for $5 \mathrm{ng} \mathrm{mL}^{-1}$

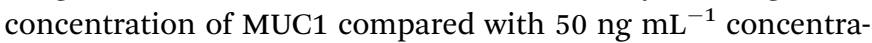
tion of each interfering EpCAM, BSA, PSA, and VEGF analytes. The GO biosensor demonstrated high selectivity and sensitivity toward MUC1 protein in the presence of EpCAM, BSA, PSA, VEGF proteins and MUC1 spiked biological samples of human serum, urine and saliva. The high sensitivity of the GO biosensor originated from the fluorescence signal amplification by DNase I-mediated target recycling process and the accumulation of FAM fluorophores.

The selectivity of graphene-based biosensors has been discussed throughout this article, which showed that the selectivity is governed by a number of factors, including morphological nanostructures, structural defects, interlayer spacing, and the nature and concentrations of dopants, and interfering analytes. Graphene-based biosensor arrays could accurately identify and classify a group of bioanalytes from interferents with the help of LDA and PCA pattern recognition methods. Furthermore, the nature of GBMs such as graphene, GO, and rGO, their functional reactive groups, such as $-\mathrm{OH},-\mathrm{COOH},-\mathrm{CO}$, and $-\mathrm{C}-\mathrm{O}-\mathrm{C}$, and their interactions with biomolecules are equally crucial for selectivity at the nanomolar level.

New graphene-based electrochemical and fluorescent biosensors are emerging rapidly for the detection of pathogenic E. coli O157:H7, ${ }^{564,776}$ Typhoidal Salmonella, ${ }^{777}$ S. aureus, ${ }^{778}$ Johne disease,${ }^{779}$ insulin, ${ }^{780}$ protein kinase activity, ${ }^{781}$ bisphenol $\mathrm{A},{ }^{782}$ AFB1, ${ }^{686}$ fenitrothion pesticide, ${ }^{783}$ circulating tumor cells (CTCs) ${ }^{784}$ breast cancer gene, ${ }^{785}$ Zika virus, ${ }^{786} \mathrm{M}$. tuberculosis ${ }^{787}$ glucose ${ }^{788}$ and microRNA in living cells. ${ }^{789}$ corrosion sulfatereducing bacteria (SRB), ${ }^{790}$ pyrophosphate, ${ }^{791}$ florfenicol in milk, ${ }^{792}$ foodborne mycotoxin, ${ }^{793}$ anticancer drug regorafenib, ${ }^{794}$ anticancer chemotherapy drug bleomycin, ${ }^{795}$ Alzheimer disease biomarker, ${ }^{796}$ tumor cells $,{ }^{797} \mathrm{HPV},{ }^{798}$ dengue virus ${ }^{799} \mathrm{HBV},{ }^{800,801}$ hepatitis C virus, ${ }^{\mathbf{8 0 2}}$ liver cancer cells, ${ }^{\mathbf{8 0 3}}$ vascular endothelial growth factor $165\left(\right.$ VEGF $\left._{165}\right)$ for cancer diagnosis, ${ }^{\mathbf{8 0 4}}$ human protein of cardiac troponin I (cTnI) biomarker for acute myocardial infarction, ${ }^{\mathbf{8 0 5}, \mathbf{8 0 6}}$ progesterone hormone, ${ }^{\mathbf{8 0 7}} 17 \beta$ - estradiol, ${ }^{\mathbf{8 0 8}}$ stem cell differentiation, ${ }^{\mathbf{8 0 9}}$ adenosine $5^{\prime}$-triphosphate (ATP) ${ }^{\mathbf{8 1 0}}$ acetaminophen, ${ }^{\mathbf{8 1 1}}$ tetracyclines,${ }^{\mathbf{8 1 2}}$ and lectins. ${ }^{\mathbf{8 1 3}}$

The strong quenching ability of GO and rGO compared with graphene offers exciting opportunities for the development of fluorescent biosensors with significantly improved sensitivity, selectivity, and low detection limits. The integration of graphene nanomaterials with inorganic nanoparticles and UCNPs combine the advantages of high carrier mobility and greatly enhanced quenching for the amplified detection of biomolecules. Finally, the high sensitivity and selectivity of the electrochemical and fluorescent biosensor platforms have already been used in clinical testing ${ }^{\mathbf{8 1 4}}$ and cancer biomarkers, ${ }^{\mathbf{8 1 5}-\mathbf{8 2 1}}$ demonstrating the promising future of inexpensive biosensing platforms.

\section{Conclusions}

Significant advances have been made in developing new biosensors using graphene-based nanomaterials for the detection of a wide variety of biological analytes, including glucose, $\mathrm{H}_{2} \mathrm{O}_{2}$, cholesterol, NAs, cancer biomarkers, food pathogens, heavy metal ions and pesticides, with desirable levels of sensitivity, low detection limits and good stability. Importantly, the successful integration of nanoscale materials such as inorganic nanoparticles (metals, metal oxides and semiconductors), organic polymers and hydrogels with graphene, GO and rGO creates unprecedented capabilities for detecting a very wide variety of bioanalytes, yielding biosensors with superior performance. Furthermore, the controlled fabrication of materials into different $2 \mathrm{D}$ and $3 \mathrm{D}$ nanostructures further helps to improve the sensing capabilities. The design of nano- or microscale devices such as microfluidic chips that incorporate multiple graphene-based nanocomposite materials is particularly promising for the detection of multiple biomarkers in a single chip. These advances in biosensor research provide new and promising platforms for the sensitive detection of various biomarkers, which can provide potential solutions not only in the biomedical field but also in other applications such as the food, water, agriculture industries and environmental monitoring.

\section{Outlook and future perspectives}

This review summarized recent advances in designing $2 \mathrm{D}$ and 3D graphene, graphene/polymer and graphene/inorganic nanoparticle hybrid architectures for diverse electrochemical biosensing applications. Integrating these hybrid nanostructures with biocompatible biopolymers, conducting polymers and hydrogels further amplifies the potential of these materials with greatly improved sensitivity, selectivity and operational stability, particularly for enzymatic biosensors. Over the last decade, despite recent developments in the controlled design of graphene and graphene-based hybrid nanocomposites, the understanding of the functional properties of graphene, GO, rGO and their interactions with surfacemodified polymers or inorganic nanoparticles remains a great challenge. The current synthesis approaches still lack controlled uniform deposition, assembly onto graphene surfaces and stability on the surfaces. More research efforts 
focusing on new and cost-effective synthetic approaches are needed to achieve precisely controlled synthesis and an indepth understanding of the structure-property relationships of graphene nanomaterials.

The large-scale processability, biocompatibility, large surface-to-volume ratio and oxygen-terminated functional groups of graphene facilitate surface modification with diverse functionalities and the site-specific conjugation of enzymes. The preparation of graphene-based hybrid nanocomposites and fine-tuning of their structural features are promising for developing novel biosensors to solve the existing stability issues and efficiently enhance the electron transfer rate in enzymatic biosensors. For instance, 3D architectures such as highly porous graphene, graphene foam, graphene scaffolds and graphene/polymer hydrogels have been explored, which trap enzymes inside pores and cavities that are similar or matching in size to different biomolecules (e.g., enzymes, other proteins, and aptamers), which helps to retain their biocatalytic functions for an extended time, to improve their stability by avoiding leaching effects and, importantly, to enhance the electron transfer rates. Specifically, the structural features of graphenebased derivatives such as GO and rGO and the integration of inorganic nanoparticles increase the electronic conductivity and electrocatalytic activity of these hybrid nanostructures by several orders of magnitude. In the case of enzymatic biosensors, the achievement of DET with both an enhanced electron transfer rate and operational stability simultaneously still faces challenges in third-generation enzymatic biosensors. The selectivity of graphene-based biosensors has been analyzed and showed dependence on a number of factors including morphology, defects, dopants and interferents.

Graphene-based microfluidic devices have attracted increasing interest due to their many advantages over the common electrochemical approach. In particular, the devices fabricated using microporous 3D architecture that interacts with nanoparticle systems seem to be highly efficient for the detection of multiple cancer biomarkers in a single-chip device with enhanced sensitivity and detection limits reaching subpicomolar concentrations. Similarly, graphene-based porous nanostructures have had major impacts on the detection of pathogenic bacteria and heavy metal ions. Numerous studies have demonstrated the detection of biomolecules down to the picomolar concentration range by the exploitation of signal amplification strategies. Despite this progress and advances in the fabrication of electrodes and their characterization, long-term studies on the toxicity of graphene-based materials for use in clinical industry, ${ }^{\mathbf{8 2 2 - 8 2 7}}$ there are still several challenges related to practical implementation, including limited lifetime, operational stability and reproducibility.

\section{Conflicts of interest}

The authors declare no competing financial interest.

\section{References}

1 B. D. Malhotra and A. Chaubey, Biosensors for Clinical Diagnostics Industry, Sens. Actuators, B, 2003, 91, 117-127.
$2 \mathrm{M}$. N. Velasco-Garcia and T. Mottram, Biosensor Technology Addressing Agricultural Problems, Biosyst. Eng., 2003, 84, 1-12.

3 M. S. Thakur and K. V. Ragavan, Biosensors in Food Processing, J. Food Sci. Technol., 2013, 50, 625-641.

4 S. Rodriguezmozaz, M. Alda, M. Marco and D. Barcelo, Biosensors for Environmental Monitoring A Global Perspective, Talanta, 2005, 65, 291-297.

5 S. Su, S. Chen and C. Fan, Recent Advances in TwoDimensional Nanomaterials-Based Electrochemical Sensors for Environmental Analysis, Green Energy \& Environment, 2018, 3, 97-106.

6 V. Gubala, L. F. Harris, A. J. Ricco, M. X. Tan and D. E. Williams, Point of Care Diagnostics: Status and Future, Anal. Chem., 2012, 84, 487-515.

7 Nanomaterial-Based Flexible and Multifunctional Sensors, ed. E. Singh and H. S. Nalwa, American Scientific Publishers, Los Angeles, CA, 2019.

8 S. O. Kelley, C. A. Mirkin, D. R. Walt, R. F. Ismagilov, M. Toner and E. H. Sargent, Advancing the Speed, Sensitivity and Accuracy of Biomolecular Detection Using Multi-Length-Scale Engineering, Nat. Nanotechnol., 2014, 9, 969-980.

9 Q. Liu, C. Wu, H. Cai, N. Hu, J. Zhou and P. Wang, CellBased Biosensors and Their Application in Biomedicine, Chem. Rev., 2014, 114, 6423-6461.

10 G. Rong, S. R. Corrie and H. A. Clark, In Vivo Biosensing: Progress and Perspectives, ACS Sens., 2017, 2, 327-338.

11 Y. Du and S. Dong, Nucleic Acid Biosensors: Recent Advances and Perspectives, Anal. Chem., 2017, 89, 189-215.

$12 \mathrm{X}$. Huang, Y. Liu, B. Yung, Y. Xiong and X. Chen, Nanotechnology-Enhanced No-Wash Biosensors for In vitro Diagnostics of Cancer, ACS Nano, 2017, 11, 5238-5292.

13 L. T. Bereza-Malcolm, G. Mann and A. E. Franks, Environmental Sensing of Heavy Metals Through Whole Cell Microbial Biosensors: A Synthetic Biology Approach, ACS Synth. Biol., 2014, 4, 535-546.

14 D. Gaddes, W. B. Reeves and S. Tadigadapa, Calorimetric Biosensing System for Quantification of Urinary Creatinine, ACS Sens., 2017, 2, 796-802.

15 K. De Wael, D. Daems, G. Van Camp and L. J. Nagels, Use of Potentiometric Sensors To Study (Bio)molecular Interactions, Anal. Chem., 2012, 84, 4921-4927.

16 A. Heller and B. Feldman, Electrochemical Glucose Sensors and Their Applications in Diabetes Management, Chem. Rev., 2008, 108, 2482-2505.

17 T. Tamura and I. Hamachi, Recent Progress in Design of Protein-Based Fluorescent Biosensors and Their Cellular Applications, ACS Chem. Biol., 2014, 9, 2708-2717.

18 Z. Wang, S. Zong, L. Wu, D. Zhu and Y. Cui, SERS-Activated Platforms for Immunoassay: Probes, Encoding Methods, and Applications, Chem. Rev., 2017, 117, 7910-7963.

19 J. N. Tiwari, V. Vij, K. C. Kemp and K. S. Kim, Engineered Carbon-Nanomaterial-Based Electrochemical Sensors for Biomolecules, ACS Nano, 2016, 10, 46-80.

20 G. Maduraiveeran, M. Sasidharan and V. Ganesan, Electrochemical Sensor and Biosensor Platforms Based on 
Advanced Nanomaterials for Biological and Biomedical Applications, Biosens. Bioelectron., 2018, 103, 113-129.

21 H. Zhang, H. Zhang, A. Aldalbahi, X. Zuo, C. Fan and X. Mi, Fluorescent Biosensors Enabled by Graphene and Graphene Oxide, Biosens. Bioelectron., 2017, 89, 96-106.

22 C. Zhu, G. Yang, H. Li, D. Du and Y. Lin, Electrochemical Sensors and Biosensors Based on Nanomaterials and Nanostructures, Anal. Chem., 2015, 87, 230-249.

23 (a) Handbook of Nanostructured Materials and Nanotechnology, ed. H. S. Nalwa, Academic Press, San Diego, 2000, vol. 1-5; (b) Encyclopedia of Nanoscience and Nanotechnology, ed. H. S. Nalwa, American Scientific Publishers, Los Angeles, 2004, vol. 1-10; (c) Handbook of Nanostructured Biomaterials and Their Applications in Nanobiotechnology, ed. H. S. Nalwa, American Scientific Publishers, Los Angeles, 2005, vol. 1-2; (d) V. Georgakilas, J. A. Perman, J. Tucek and R. Zboril, Broad Family of Carbon Nanoallotropes: Classification, Chemistry, and Applications of Fullerenes, Carbon Dots, Nanotubes, Graphene, Nanodiamonds, and Combined Superstructures, Chem. Rev., 2015, 115, 4744-4822.

24 C. M. Cobley, J. Chen, E. C. Cho, L. V. Wang and Y. Xia, Gold nanostructures: a class of multifunctional materials for biomedical applications, Chem. Soc. Rev., 2011, 40, 44-56.

25 S.-K. Sun, H.-F. Wang and X.-P. Yan, Engineering Persistent Luminescence Nanoparticles for Biological Applications: From Biosensing/Bioimaging to Theranostics, Acc. Chem. Res., 2018, 51, 1131-1143.

26 S. Nandini, S. Nalini, M. B. M. Reddy, G. S. Suresh, J. S. Melo, P. Niranjana, J. Sanetuntikul and S. Shanmugam, Synthesis of One-Dimensional Gold Nanostructures and the Electrochemical Application of the Nanohybrid Containing Functionalized Graphene Oxide for Cholesterol Biosensing, Bioelectrochemistry, 2016, 110, 79-90.

27 C. Tan, X. Cao, X.-J. Wu, Q. He, J. Yang, X. Zhang, J. Chen, W. Zhao, S. Han, G.-H. Nam, M. Sindoro and H. Zhang, Recent Advances in Ultrathin Two-Dimensional Nanomaterials, Chem. Rev., 2017, 117, 6225-6331.

28 (a) E. Singh, K. S. Kim, G. Y. Yeom and H. S. Nalwa, Atomically thin-layered molybdenum disulfide $\left(\mathrm{MoS}_{2}\right)$ for bulk-heterojunction solar cells, ACS Appl. Mater. Interfaces, 2017, 9, 3223-3245; (b) E. Singh, K. S. Kim, G. Y. Yeom and H. S. Nalwa, Two-dimensional transition metal dichalcogenide-based counter electrodes for dyesensitized solar cells, RSC Adv., 2017, 7, 28234-28290; (c) E. Singh, P. Singh, K. S. Kim, G. Y. Yeom and H. S. Nalwa, Flexible molybdenum disulfide $\left(\mathrm{MoS}_{2}\right)$ atomic layers for wearable electronics and optoelectronics, ACS Appl. Mater. Interfaces, 2019, DOI: 10.1021/acsami.8b19859; (d) R. Singh, E. Singh and H. S. Nalwa, Inkjet printed nanomaterial based flexible radio frequency identification (RFID) tag sensors for the internet of nano things, $R S C$ Adv., 2017, 7, 48597-48630.

29 N. P. Dasgupta, J. Sun, C. Liu, S. Brittman, S. C. Andrews, J. Lim, H. Gao, R. Yan and P. Yang, 25th Anniversary Article: Semiconductor Nanowires - Synthesis,
Characterization, and Applications, Adv. Mater., 2014, 26, 2137-2184.

30 K. E. Sapsford, W. R. Algar, L. Berti, K. B. Gemmill, B. J. Casey, E. Oh, M. H. Stewart and I. L. Medintz, Functionalizing Nanoparticles with Biological Molecules: Developing Chemistries that Facilitate Nanotechnology, Chem. Rev., 2013, 113, 1904-2074.

31 T. R. Fadel, D. F. Farrell, L. E. Friedersdorf, M. H. Griep, M. D. Hoover, M. A. Meador and M. Meyyappan, Toward the Responsible Development and Commercialization of Sensor Nanotechnologies, ACS Sens., 2016, 1, 207-216.

32 K. S. Novoselov, A. K. Geim, S. V. Morozov, D. Jiang, Y. Zhang, S. V. Dubonos, I. V. Grigorieva and A. A. Firsov, Electric Field Effect in Atomically Thin Carbon Films, Science, 2004, 306, 666-669.

33 C. Cheng, S. Li, A. Thomas, N. A. Kotov and R. Haag, Functional Graphene Nanomaterials Based Architectures: Biointeractions, Fabrications, and Emerging Biological Applications, Chem. Rev., 2017, 117, 1826-1914.

34 A. K. Geim and K. S. Novoselov, The Rise of Graphene, Nat. Mater., 2007, 6, 183-191.

35 Y. Zhu, S. Murali, W. Cai, X. Li, J. W. Suk, J. R. Potts and R. S. Ruoff, Graphene and Graphene Oxide: Synthesis, Properties, and Applications, Adv. Mater., 2010, 22, 39063924.

36 P. Avouris and C. Dimitrakopoulos, Graphene: Synthesis and Applications, Mater. Today, 2012, 15, 86-97.

37 H. Y. Mao, S. Laurent, W. Chen, O. Akhavan, M. Imani, A. A. Ashkarran and M. Mahmoudi, Graphene: Promises, Facts, Opportunities, and Challenges in Nanomedicine, Chem. Rev., 2013, 113, 3407-3424.

38 J. C. Meyer, A. K. Geim, M. I. Katsnelson, K. S. Novoselov, T. J. Booth and S. Roth, The Structure of Suspended Graphene Sheets, Nature, 2007, 446, 60-63.

39 Y. Xu, H. Bai, G. Lu, C. Li and G. Shi, Flexible Graphene Films via the Filtration of Water-Soluble Noncovalent Functionalized Graphene Sheets, J. Am. Chem. Soc., 2008, 130, 5856-5857.

40 H. Chen, M. B. Müller, K. J. Gilmore, G. G. Wallace and D. Li, Mechanically Strong, Electrically Conductive, and Biocompatible Graphene Paper, Adv. Mater., 2008, 20, 3557-3561.

41 X. Li, X. Wang, L. Zhang, S. Lee and H. Dai, Chemically Derived, Ultrasmooth Graphene Nanoribbon Semiconductors, Science, 2008, 319, 1229-1232.

42 Q. Xi, X. Chen, D. G. Evans and W. Yang, Gold NanoparticleEmbedded Porous Graphene Thin Films Fabricated via Layer-by-Layer Self-Assembly and Subsequent Thermal Annealing for Electrochemical Sensing, Langmuir, 2012, 28, 9885-9892.

43 C. Hu, J. Xue, L. Dong, Y. Jiang, X. Wang, L. Qu and L. Dai, Scalable Preparation of Multifunctional Fire-Retardant Ultralight Graphene Foams, ACS Nano, 2016, 10, 13251332.

44 A. A. Balandin, S. Ghosh, W. Bao, I. Calizo, D. Teweldebrhan, F. Miao and C. N. Lau, Superior 
Thermal Conductivity of Single-Layer Graphene, Nano Lett., 2008, 8, 902-907.

45 S. V. Morozov, K. S. Novoselov, M. I. Katsnelson, F. Schedin, D. C. Elias, J. A. Jaszczak and A. K. Geim, Giant Intrinsic Carrier Mobilities in Graphene and Its Bilayer, Phys. Rev. Lett., 2008, 100, 11-14.

46 R. R. Nair, P. Blake, A. N. Grigorenko, K. S. Novoselov, T. J. Booth, T. Stauber, N. M. R. Peres and A. K. Geim, Fine Structure Constant Defines Visual Transparency of Graphene, Science, 2008, 320, 1308.

47 M. D. Stoller, S. Park, Y. Zhu, J. An and R. S. Ruoff, Graphene-Based Ultracapacitors, Nano Lett., 2008, 8, 3498-3502.

48 E. Singh and H. S. Nalwa, Graphene-Based BulkHeterojunction Solar Cells: A Review, J. Nanosci. Nanotechnol., 2015, 15, 6237-6278.

49 E. Singh and H. S. Nalwa, Graphene-Based Dye-Sensitized Solar Cells: A Review, Sci. Adv. Mater., 2015, 7, 1863-1912.

50 W. Lv, Z. Li, Y. Deng, Q.-H. Yang and F. Kang, GrapheneBased Materials for Electrochemical Energy Storage Devices: Opportunities and Challenges, Energy Storage Materials, 2016, 2, 107-138.

51 U. Khan, T.-H. Kim, H. Ryu, W. Seung and S.-W. Kim, Graphene Tribotronics for Electronic Skin and Touch Screen Applications, Adv. Mater., 2017, 29, 2-8.

52 F. Schwierz, Graphene Transistors, Nat. Nanotechnol., 2010, 5, 487-496.

53 T.-H. Han, Y. Lee, M.-R. Choi, S.-H. Woo, S.-H. Bae, B. H. Hong, J.-H. Ahn and T.-W. Lee, Extremely Efficient Flexible Organic Light-Emitting Diodes with Modified Graphene Anode, Nat. Photonics, 2012, 6, 105-110.

54 E. Singh, M. Meyyappan and H. S. Nalwa, Flexible Graphene-Based Wearable Gas and Chemical Sensors, ACS Appl. Mater. Interfaces, 2017, 9, 34544-34586.

55 D. Iannazzo, A. Pistone, M. Salamò, S. Galvagno, R. Romeo, S. V. Giofré, C. Branca, G. Visalli and A. Di Pietro, Graphene Quantum Dots for Cancer Targeted Drug Delivery, Int. J. Pharm., 2017, 518, 185-192.

56 V. Kumar, K.-H. Kim, J.-W. Park, J. Hong and S. Kumar, Graphene and Its Nanocomposites as a Platform for Environmental Applications, Chem. Eng. J., 2017, 315, 210-232.

57 D. Chen, H. Feng and J. Li, Graphene Oxide: Preparation, Functionalization, and Electrochemical Applications, Chem. Rev., 2012, 112, 6027-6053.

58 P. T. Yin, S. Shah, M. Chhowalla and K.-B. Lee, Design, Synthesis, and Characterization of GrapheneNanoparticle Hybrid Materials for Bioapplications, Chem. Rev., 2015, 115, 2483-2531.

59 X. Zhao, P. Zhang, Y. Chen, Z. Su and G. Wei, Recent Advances in the Fabrication and Structure-Specific Applications of Graphene-Based Inorganic Hybrid Membranes, Nanoscale, 2015, 7, 5080-5093.

60 L. Qiu, D. Li and H.-M. Cheng, Structural Control of Graphene-Based Materials for Unprecedented Performance, ACS Nano, 2018, 12, 5085-5092.
61 S. Nardecchia, D. Carriazo, M. L. Ferrer, M. C. Gutiérrez and F. del Monte, Three Dimensional Macroporous Architectures And Aerogels Built of Carbon Nanotubes and/or Graphene: Synthesis and Applications, Chem. Soc. Rev., 2013, 42, 794-830.

62 F. Liu, Y. Piao, J. S. Choi and T. S. Seo, Three-Dimensional Graphene Micropillar Based Electrochemical Sensor for Phenol Detection, Biosens. Bioelectron., 2013, 50, 387-392.

63 J. Liu, S. Fu, B. Yuan, Y. Li and Z. Deng, Toward a Universal "Adhesive Nanosheet" for the Assembly of Multiple Nanoparticles Based on a Protein-Induced Reduction/ Decoration of Graphene Oxide, J. Am. Chem. Soc., 2010, 132, 7279-7281.

64 M. Wang, X. Duan, Y. Xu and X. Duan, Functional ThreeDimensional Graphene/Polymer Composites, ACS Nano, 2016, 10, 7231-7247.

65 W. K. Chee, H. N. Lim, N. M. Huang and I. Harrison, Nanocomposites of Graphene/Polymers: A Review, RSC Adv., 2015, 5, 68014-68051.

66 R. Zhang and W. Chen, Recent Advances in GrapheneBased Nanomaterials for Fabricating Electrochemical Hydrogen Peroxide Sensors, Biosens. Bioelectron., 2017, 89, 249-268.

67 P. Bollella, G. Fusco, C. Tortolini, G. Sanzò, G. Favero, L. Gorton and R. Antiochia, Beyond Graphene: Electrochemical Sensors and Biosensors for Biomarkers Detection, Biosens. Bioelectron., 2017, 89, 152-166.

68 L. Wang, Q. Xiong, F. Xiao and H. Duan, 2D Nanomaterials Based Electrochemical Biosensors for Cancer Diagnosis, Biosens. Bioelectron., 2017, 89, 136-151.

69 B. V. Chikkaveeraiah, A. A. Bhirde, N. Y. Morgan, H. S. Eden and $\mathrm{X}$. Chen, Electrochemical Immunosensors for Detection of Cancer Protein Biomarkers, ACS Nano, 2012, 6, 6546-6561.

70 Z. Farka, T. Juřík, D. Kovář, L. Trnková and P. Skládal, Nanoparticle-Based Immunochemical Biosensors and Assays: Recent Advances and Challenges, Chem. Rev., 2017, 117, 9973-10042.

71 M. Li, H. Gou, I. Al-Ogaidi and N. Wu, Nanostructured Sensors for Detection of Heavy Metals: A Review, ACS Sustainable Chem. Eng., 2013, 1, 713-723.

72 M. R. Saidur, A. R. A. Aziz and W. J. Basirun, Recent Advances in DNA-Based Electrochemical Biosensors for Heavy Metal Ion Detection: A Review, Biosens. Bioelectron., 2017, 90, 125-139.

73 N. Reta, C. P. Saint, A. Michelmore, B. Prieto-Simon and N. H. Voelcker, Nanostructured Electrochemical Biosensors for Label-Free Detection of Water- and FoodBorne Pathogens, ACS Appl. Mater. Interfaces, 2018, 10, 6055-6072.

74 Y. Zhang, J. Shen, H. Li, L. Wang, D. Cao, X. Feng, Y. Liu, Y. Ma and L. Wang, Recent Progress on Graphene-based Electrochemical Biosensors, Chem. Rec., 2016, 16, 273-294.

75 A. T. Lawal, Progress in Utilisation of Graphene for Electrochemical Biosensors, Biosens. Bioelectron., 2018, 106, 149-178. 
76 Y. Liu, X. Dong and P. Chen, Biological and Chemical Sensors Based on Graphene Materials, Chem. Soc. Rev., 2012, 41, 2283-2307.

77 P. A. Rasheed and N. Sandhyarani, Carbon Nanostructures as Immobilization Platform for DNA: A Review on Current Progress in Electrochemical DNA Sensors, Biosens. Bioelectron., 2017, 97, 226-237.

78 C. Chung, Y.-K. Kim, D. Shin, S.-R. Ryoo, B. H. Hong and D.-H. Min, Biomedical Applications of Graphene and Graphene Oxide, Acc. Chem. Res., 2013, 46, 2211-2224.

79 V. Georgakilas, M. Otyepka, A. B. Bourlinos, V. Chandra, N. Kim, K. C. Kemp, P. Hobza, R. Zboril and K. S. Kim, Functionalization of Graphene: Covalent and NonCovalent Approaches, Derivatives and Applications, Chem. Rev., 2012, 112, 6156-6214.

80 V. Georgakilas, J. N. Tiwari, K. C. Kemp, J. A. Perman, A. B. Bourlinos, K. S. Kim and R. Zboril, Noncovalent Functionalization of Graphene and Graphene Oxide for Energy Materials, Biosensing, Catalytic, and Biomedical Applications, Chem. Rev., 2016, 116, 5464-5519.

81 C. K. Chua and M. Pumera, Chemical Reduction of Graphene Oxide: A Synthetic Chemistry Viewpoint, Chem. Soc. Rev., 2014, 43, 291-312.

82 P. W. Sutter, J.-I. Flege and E. A. Sutter, Epitaxial Graphene on Ruthenium, Nat. Mater., 2008, 7, 406-411.

83 Y. Hernandez, V. Nicolosi, M. Lotya, F. M. Blighe, Z. Sun, S. De, I. T. McGovern, B. Holland, M. Byrne, Y. K. Gun'Ko, J. J. Boland, P. Niraj, G. Duesberg, S. Krishnamurthy, R. Goodhue, J. Hutchison, V. Scardaci, A. C. Ferrari and J. N. Coleman, High-Yield Production of Graphene by Liquid-Phase Exfoliation of Graphite, Nat. Nanotechnol., 2008, 3, 563-568.

84 A. Reina, X. Jia, J. Ho, D. Nezich, H. Son, V. Bulovic, M. S. Dresselhaus and J. Kong, Large Area, Few-Layer Graphene Films on Arbitrary Substrates by Chemical Vapor Deposition, Nano Lett., 2009, 9, 30-35.

85 N. Liu, F. Luo, H. Wu, Y. Liu, C. Zhang and J. Chen, OneStep Ionic-Liquid-Assisted Electrochemical Synthesis of Ionic-Liquid-Functionalized Graphene Sheets Directly from Graphite, Adv. Funct. Mater., 2008, 18, 1518-1525.

86 K. S. Subrahmanyam, L. S. Panchakarla, A. Govindaraj and C. N. R. Rao, Simple Method of Preparing Graphene Flakes by an Arc-Discharge Method, J. Phys. Chem. C, 2009, 113, 4257-4259.

87 Z.-S. Wu, W. Ren, L. Gao, J. Zhao, Z. Chen, B. Liu, D. Tang, B. Yu, C. Jiang and H.-M. Cheng, Synthesis of Graphene Sheets with High Electrical Conductivity and Good Thermal Stability by Hydrogen Arc Discharge Exfoliation, ACS Nano, 2009, 3, 411-417.

88 X. Wang, G. Sun, P. Routh, D.-H. Kim, W. Huang and P. Chen, Heteroatom-Doped Graphene Materials: Syntheses, Properties and Applications, Chem. Soc. Rev., 2014, 43, 7067-7098.

89 J. Filip and J. Tkac, Is Graphene Worth Using in Biofuel Cells?, Electrochim. Acta, 2014, 136, 340-354.

90 J. Xu, J. Shui, J. Wang, M. Wang, H.-K. Liu, S. X. Dou, I.-Y. Jeon, J.-M. Seo, J.-B. Baek and L. Dai, Sulfur-
Graphene Nanostructured Cathodes via Ball-Milling for High-Performance Lithium-Sulfur Batteries, ACS Nano, 2014, 8, 10920-10930.

91 S. Park and R. S. Ruoff, Chemical Methods for the Production of Graphenes, Nat. Nanotechnol., 2009, 4, 217224.

92 K. Erickson, R. Erni, Z. Lee, N. Alem, W. Gannett and A. Zettl, Determination of the Local Chemical Structure of Graphene Oxide and Reduced Graphene Oxide, Adv. Mater., 2010, 22, 4467-4472.

93 L. Gao, C. Lian, Y. Zhou, L. Yan, Q. Li, C. Zhang, L. Chen and K. Chen, Graphene Oxide-DNA Based Sensors, Biosens. Bioelectron., 2014, 60, 22-29.

94 J. H. Jung, D. S. Cheon, F. Liu, K. B. Lee and T. S. Seo, A Graphene Oxide Based Immuno-Biosensor for Pathogen Detection, Angew. Chem., Int. Ed., 2010, 49, 5708-5711.

95 W. S. Hummers and R. E. Offeman, Preparation of Graphitic Oxide, J. Am. Chem. Soc., 1958, 80, 1339.

96 D. R. Dreyer, S. Park, C. W. Bielawski and R. S. Ruoff, The Chemistry of Graphene Oxide, Chem. Soc. Rev., 2010, 39, 228-240.

97 M. J. McAllister, J.-L. Li, D. H. Adamson, H. C. Schniepp, A. A. Abdala, J. Liu, M. Herrera-Alonso, D. L. Milius, R. Car, R. K. Prud'homme and I. A. Aksay, Single Sheet Functionalized Graphene by Oxidation and Thermal Expansion of Graphite, Chem. Mater., 2007, 19, 4396-4404.

98 G. Williams, B. Seger and P. V. Kamat, $\mathrm{TiO}_{2}$-Graphene Nanocomposites. UV-Assisted Photocatalytic Reduction of Graphene Oxide, ACS Nano, 2008, 2, 1487-1491.

99 O. C. Compton and S. T. Nguyen, Graphene Oxide, Highly Reduced Graphene Oxide, and Graphene: Versatile Building Blocks for Carbon-Based Materials, Small, 2010, 6, 711-723.

100 M. Bacon, S. J. Bradley and T. Nann, Graphene Quantum Dots, Part. Part. Syst. Charact., 2014, 31, 415-428.

101 L.-s. Li and X. Yan, Colloidal Graphene Quantum Dots, J. Phys. Chem. Lett., 2010, 1, 2572-2576.

102 S. Zhou, H. Xu, W. Gan and Q. Yuan, Graphene Quantum Dots: Recent Progress in Preparation and Fluorescence Sensing Applications, RSC Adv., 2016, 6, 110775-110788.

103 H. Sun, L. Wu, W. Wei and X. Qu, Recent Advances in Graphene Quantum Dots for Sensing, Mater. Today, 2013, 16, 433-442.

104 D. Pan, J. Zhang, Z. Li and M. Wu, Hydrothermal Route for Cutting Graphene Sheets into Blue-Luminescent Graphene Quantum Dots, Adv. Mater., 2010, 22, 734-738.

105 N. Suzuki, Y. Wang, P. Elvati, Z.-B. Qu, K. Kim, S. Jiang, E. Baumeister, J. Lee, B. Yeom, J. H. Bahng, J. Lee, A. Violi and N. A. Kotov, Chiral Graphene Quantum Dots, ACS Nano, 2016, 10, 1744-1755.

106 J. Lu, P. S. E. Yeo, C. K. Gan, P. Wu and K. P. Loh, Transforming $\mathrm{C}_{60}$ Molecules Into Graphene Quantum Dots, Nat. Nanotechnol., 2011, 6, 247-252.

107 Z. Zhang, J. Zhang, N. Chen and L. Qu, Graphene Quantum Dots: An Emerging Material for Energy-Related Applications and Beyond, Energy Environ. Sci., 2012, 5, 8869-8890. 
108 Y. Li, Y. Zhao, H. Cheng, Y. Hu, G. Shi, L. Dai and L. Qu, Nitrogen-Doped Graphene Quantum Dots with OxygenRich Functional Groups, J. Am. Chem. Soc., 2012, 134, 1518.

109 Y. Guo, Y. Han, S. Shuang and C. Dong, Rational Synthesis of Graphene-Metal Coordination Polymer Composite Nanosheet as Enhanced Materials for Electrochemical Biosensing, J. Mater. Chem., 2012, 22, 13166-13173.

110 P. T. Yin, T.-H. Kim, J.-W. Choi and K.-B. Lee, Prospects for Graphene-Nanoparticle-Based Hybrid Sensors, Phys. Chem. Chem. Phys., 2013, 15, 12785-12799.

111 International Diabetes Federation, https:/www.idf.org/ aboutdiabetes/what-is-diabetes/facts-figures.html.

112 L. C. Clark and C. Lyons, Electrode Systems for Continuous Monitoring in Cardiovascular Surgery, Ann. N. Y. Acad. Sci., 1962, 102, 29-45.

113 E. Witkowska Nery, M. Kundys, P. S. Jeleń and M. JönssonNiedziółka, Electrochemical Glucose Sensing: Is There Still Room for Improvement?, Anal. Chem., 2016, 88, 1127111282.

114 J. Wang, Electrochemical Glucose Biosensors, Electrochem. Sensors, Biosens. their Biomed. Appl., 2008, pp. 57-69.

115 C. Chen, Q. Xie, D. Yang, H. Xiao, Y. Fu, Y. Tan and S. Yao, Recent Advances in Electrochemical Glucose Biosensors: A Review, RSC Adv., 2013, 3, 4473-4491.

116 H. Muguruma, H. Iwasa, H. Hidaka, A. Hiratsuka and H. Uzawa, Mediatorless Direct Electron Transfer between Flavin Adenine Dinucleotide-Dependent Glucose Dehydrogenase and Single-Walled Carbon Nanotubes, ACS Catal., 2017, 7, 725-734.

117 M. Zhao, Y. Gao, J. Sun and F. Gao, Mediatorless Glucose Biosensor and Direct Electron Transfer Type Glucose/Air Biofuel Cell Enabled with Carbon Nanodots, Anal. Chem., 2015, 87, 2615-2622.

118 Y.-F. Bai, T.-B. Xu, J. H. T. Luong and H.-F. Cui, Direct Electron Transfer of Glucose Oxidase-Boron Doped Diamond Interface: A New Solution for a Classical Problem, Anal. Chem., 2014, 86, 4910-4918.

119 A. Chen and S. Chatterjee, Nanomaterials Based Electrochemical Sensors for Biomedical Applications, Chem. Soc. Rev., 2013, 42, 5425-5438.

120 C. Cai and J. Chen, Direct Electron Transfer of Glucose Oxidase Promoted by Carbon Nanotubes, Anal. Biochem., 2004, 332, 75-83.

121 J. M. Goran, S. M. Mantilla and K. J. Stevenson, Influence of Surface Adsorption on the Interfacial Electron Transfer of Flavin Adenine Dinucleotide and Glucose Oxidase at Carbon Nanotube and Nitrogen-Doped Carbon Nanotube Electrodes, Anal. Chem., 2013, 85, 1571-1581.

122 Y. Lin, F. Lu, Y. Tu and Z. Ren, Glucose Biosensors Based on Carbon Nanotube Nanoelectrode Ensembles, Nano Lett., 2004, 4, 191-195.

123 M. Wooten, S. Karra, M. Zhang and W. Gorski, On the Direct Electron Transfer, Sensing, and Enzyme Activity in the Glucose Oxidase/Carbon Nanotubes System, Anal. Chem., 2013, 86, 752-757.
124 S. Tsujimura, K. Murata and W. Akatsuka, Exceptionally High Glucose Current on a Hierarchically Structured Porous Carbon Electrode with "Wired" Flavin Adenine Dinucleotide-Dependent Glucose Dehydrogenase, J. Am. Chem. Soc., 2014, 136, 14432-14437.

125 P. Rafighi, M. Tavahodi and B. Haghighi, Fabrication of a Third-Generation Glucose Biosensor Using GraphenePolyethyleneimine-Gold Nanoparticles Hybrid, Sens. Actuators, B, 2016, 232, 454-461.

126 H. Razmi and R. Mohammad-Rezaei, Graphene Quantum Dots as a New Substrate for Immobilization and Direct Electrochemistry of Glucose Oxidase: Application to Sensitive Glucose Determination, Biosens. Bioelectron., 2013, 41, 498-504.

127 A. Karimi, A. Othman, A. Uzunoglu, L. Stanciu and S. Andreescu, Graphene Based Enzymatic Bioelectrodes and Biofuel Cells, Nanoscale, 2015, 7, 6909-6923.

128 J. Kim, S.-J. Park and D.-H. Min, Emerging Approaches for Graphene Oxide Biosensor, Anal. Chem., 2017, 89, 232-248.

129 Y. Song, K. Qu, C. Zhao, J. Ren and X. Qu, Graphene Oxide: Intrinsic Peroxidase Catalytic Activity and Its Application to Glucose Detection, Adv. Mater., 2010, 22, 2206-2210.

130 L. Lipani, B. G. R. Dupont, F. Doungmene, F. Marken, R. M. Tyrrell, R. H. Guy and A. Ilie, Non-Invasive, Transdermal, Path-Selective and Specific Glucose Monitoring via a Graphene-Based Platform, Nat. Nanotechnol., 2018, 13, 504-511.

131 M. Zhou, Y. Zhai and S. Dong, Electrochemical Sensing and Biosensing Platform Based on Chemically Reduced Graphene Oxide, Anal. Chem., 2009, 81, 5603-5613.

132 H. Gao and H. Duan, 2D and 3D Graphene Materials: Preparation and Bioelectrochemical Applications, Biosens. Bioelectron., 2015, 65, 404-419.

133 Y. Wang, Y. Shao, D. W. Matson, J. Li and Y. Lin, NitrogenDoped Graphene and Its Application in Electrochemical Biosensing, ACS Nano, 2010, 4, 1790-1798.

134 A. Küchler, M. Yoshimoto, S. Luginbühl, F. Mavelli and P. Walde, Enzymatic Reactions in Confined Environments, Nat. Nanotechnol., 2016, 11, 409-420.

135 Z. Zhu, L. Garcia-Gancedo, A. J. Flewitt, H. Xie, F. Moussy and W. I. Milne, A Critical Review of Glucose Biosensors Based on Carbon Nanomaterials: Carbon Nanotubes and Graphene, Sensors, 2012, 12, 5996-6022.

136 J. Zhang, F. Zhang, H. Yang, X. Huang, H. Liu, J. Zhang and S. Guo, Graphene Oxide as a Matrix for Enzyme Immobilization, Langmuir, 2010, 26, 6083-6085.

137 L. Jin, K. Yang, K. Yao, S. Zhang, H. Tao, S.-T. Lee, Z. Liu and R. Peng, Functionalized Graphene Oxide in Enzyme Engineering: A Selective Modulator for Enzyme Activity and Thermostability, ACS Nano, 2012, 6, 4864-4875.

138 I. V. Pavlidis, M. Patila, U. T. Bornscheuer, D. Gournis and H. Stamatis, Graphene-Based Nanobiocatalytic Systems: Recent Advances and Future Prospects, Trends Biotechnol., 2014, 32, 312-320.

139 S. K. Singh, M. K. Singh, P. P. Kulkarni, V. K. Sonkar, J. J. A. Grácio and D. Dash, Amine-Modified Graphene: 
Thrombo-Protective Safer Alternative to Graphene Oxide for Biomedical Applications, ACS Nano, 2012, 6, 2731-2740. 140 Z. Wang, X. Zhou, J. Zhang, F. Boey and H. Zhang, Direct Electrochemical Reduction of Single-Layer Graphene Oxide and Subsequent Functionalization with Glucose Oxidase, J. Phys. Chem. C, 2009, 113, 14071-14075.

141 Y. Liu, D. Yu, C. Zeng, Z. Miao and L. Dai, Biocompatible Graphene Oxide-Based Glucose Biosensors, Langmuir, 2010, 26, 6158-6160.

142 Q. Li, F. Fan, Y. Wang, W. Feng and P. Ji, Enzyme Immobilization on Carboxyl-Functionalized Graphene Oxide for Catalysis in Organic Solvent, Ind. Eng. Chem. Res., 2013, 52, 6343-6348.

143 J. F. Rusling and R. J. Forster, Electrochemical Catalysis with Redox Polymer and Polyion-Protein Films, J. Colloid Interface Sci., 2003, 262, 1-15.

144 J. D. Newman and A. P. F. Turner, Home Blood Glucose Biosensors: A Commercial Perspective, Biosens. Bioelectron., 2005, 20, 2435-2453.

145 D. Li, W. Zhang, X. Yu, Z. Wang, Z. Su and G. Wei, When Biomolecules Meet Graphene: From Molecular Level Interactions to Material Design and Applications, Nanoscale, 2016, 8, 19491-19509.

146 T. S. Sreeprasad and V. Berry, How do the Electrical Properties of Graphene Change with its Functionalization?, Small, 2013, 9, 341-350.

147 X. Kang, J. Wang, H. Wu, I. A. Aksay, J. Liu and Y. Lin, Glucose Oxidase-Graphene-Chitosan Modified Electrode for Direct Electrochemistry and Glucose Sensing, Biosens. Bioelectron., 2009, 25, 901-905.

148 M. Guler and Y. Dilmac, Palladium nanoparticles decorated (3-aminopropyl) triethoxysilane functionalized reduced graphene oxide for electrochemical determination of glucose and hydrogen peroxide, J. Electroanal. Chem., 2019, 834, 49-55.

149 C. Shan, H. Yang, D. Han, Q. Zhang, A. Ivaska and L. Niu, Graphene/AuNPs/Chitosan Nanocomposites Film for Glucose Biosensing, Biosens. Bioelectron., 2010, 25, 10701074.

150 J. Singh, P. Khanra, T. Kuila, M. Srivastava, A. K. Das, N. H. Kim, B. J. Jung, D. Y. Kim, S. H. Lee, D. W. Lee, D.-G. Kim and J. H. Lee, Preparation of Sulfonated Poly(Ether-Ether-Ketone) Functionalized Ternary Graphene/AuNPs/Chitosan Nanocomposite for Efficient Glucose Biosensor, Process Biochem., 2013, 48, 1724-1735.

151 J. Zheng, Y. He, Q. Sheng and H. Zhang, DNA as a Linker for Biocatalytic Deposition of Au Nanoparticles on Graphene and Its Application in Glucose Detection, J. Mater. Chem., 2011, 21, 12873-12879.

152 B. Liang, L. Fang, G. Yang, Y. Hu, X. Guo and X. Ye, Direct Electron Transfer Glucose Biosensor Based on Glucose Oxidase Self-Assembled on Electrochemically Reduced Carboxyl Graphene, Biosens. Bioelectron., 2013, 43, 131-136.

153 M. Cui, B. Xu, C. Hu, H. B. Shao and L. Qu, Direct electrochemistry and electrocatalysis of glucose oxidase on three-dimensional interpenetrating, porous graphene modified electrode, Electrochim. Acta, 2013, 98, 48-53.
154 L. Qian and L. Lu, Three Dimensional Porous GrapheneChitosan Composites from Ice-Induced Assembly for Direct Electron Transfer and Electrocatalysis of Glucose Oxidase, RSC Adv., 2014, 4, 38273-38280.

155 R. S. Dey and C. R. Raj, A Hybrid Functional Nanoscaffold Based on Reduced Graphene Oxide-ZnO for the Development of an Amperometric Biosensing Platform, RSC Adv., 2013, 3, 25858-25864.

156 A. T. E. Vilian, S.-M. Chen, M. A. Ali and F. M. A. Al-Hemaid, Direct Electrochemistry of Glucose Oxidase Immobilized on $\mathrm{ZrO}_{2}$ Nanoparticles-Decorated Reduced Graphene Oxide Sheets for a Glucose Biosensor, RSC Adv., 2014, 4, 30358-30367.

157 M. F. Hossain and J. Y. Park, Amperometric Glucose Biosensor Based on Pt-Pd Nanoparticles Supported by Reduced Graphene Oxide and Integrated with Glucose Oxidase, Electroanalysis, 2014, 26, 940-951.

158 S. Palanisamy, C. Karuppiah and S.-M. Chen, Direct Electrochemistry and Electrocatalysis of Glucose Oxidase Immobilized on Reduced Graphene Oxide and Silver Nanoparticles Nanocomposite Modified Electrode, Colloids Surf., B, 2014, 114, 164-169.

159 Y. Yu, Z. Chen, S. He, B. Zhang, X. Li and M. Yao, Direct Electron Transfer of Glucose Oxidase and Biosensing For Glucose Based on PDDA-Capped Gold Nanoparticle Modified Graphene/Multi-Walled Carbon Nanotubes Electrode, Biosens. Bioelectron., 2014, 52, 147-152.

160 M. A. Tabrizi and J. N. Varkani, Green Synthesis of Reduced Graphene Oxide Decorated with Gold Nanoparticles and its Glucose Sensing Application, Sens. Actuators, B, 2014, 202, 475-482.

$161 \mathrm{H}$. Teymourian, A. Salimi and S. Firoozi, A High Performance Electrochemical Biosensing Platform for Glucose Detection and IgE Aptasensing Based on $\mathrm{Fe}_{3} \mathrm{O}_{4} /$ Reduced Graphene Oxide Nanocomposite, Electroanalysis, 2014, 26, 129-138.

162 A. T. E. Vilian, V. Mani, S.-M. Chen, B. Dinesh and S.-T. Huang, The Immobilization of Glucose Oxidase at Manganese Dioxide Particles-Decorated Reduced Graphene Oxide Sheets for the Fabrication of a Glucose Biosensor, Ind. Eng. Chem. Res., 2014, 53, 15582-15589.

163 K.-Y. Hwa and B. Subramani, Synthesis of Zinc Oxide Nanoparticles on Graphene-Carbon Nanotube Hybrid for Glucose Biosensor Applications, Biosens. Bioelectron., 2014, 62, 127-133.

164 S. Palanisamy, S. Cheemalapati and S.-M. Chen, Amperometric Glucose Biosensor Based on Glucose Oxidase Dispersed in Multiwalled Carbon Nanotubes/ Graphene Oxide Hybrid Biocomposite, Mater. Sci. Eng., C, 2014, 34, 207-213.

165 Y. Piao, D. J. Han and T. S. Seo, Highly Conductive Graphite Nanoparticle Based Enzyme Biosensor for Electrochemical Glucose Detection, Sens. Actuators, B, 2014, 194, 454-459.

166 R. Devasenathipathy, V. Mani, S.-M. Chen, S.-T. Huang, T.-T. Huang, C.-M. Lin, K.-Y. Hwa, T.-Y. Chen and B.-J. Chen, Glucose Biosensor Based On Glucose Oxidase Immobilized At Gold Nanoparticles Decorated Graphene- 
Carbon Nanotubes, Enzyme Microb. Technol., 2015, 78, 4045.

167 P. Nayak, P. N. Santhosh and S. Ramaprabhu, Synthesis of Au-MWCNT-Graphene Hybrid Composite for the Rapid Detection of $\mathrm{H}_{2} \mathrm{O}_{2}$ and Glucose, RSC Adv., 2014, 4, 4167041677.

168 T. Terse-Thakoor, K. Komori, P. Ramnani, I. Lee and A. Mulchandani, Electrochemically Functionalized Seamless Three-Dimensional Graphene-Carbon Nanotube Hybrid for Direct Electron Transfer of Glucose Oxidase and Bioelectrocatalysis, Langmuir, 2015, 31, 13054-13061.

169 B. Liang, L. Fang, Y. Hu, G. Yang, Q. Zhu and X. Ye, Fabrication and Application of Flexible Graphene Silk Composite Film Electrodes Decorated with Spiky Pt Nanospheres, Nanoscale, 2014, 6, 4264-4274.

170 Y. Fang, D. Zhang, Y. Guo, Y. Guo and Q. Chen, Simple OnePot Preparation of Chitosan-Reduced Graphene Oxide-Au Nanoparticles Hybrids for Glucose Sensing, Sens. Actuators, B, 2015, 221, 265-272.

171 W. Zhang, X. Li, R. Zou, H. Wu, H. Shi, S. Yu and Y. Liu, Multifunctional Glucose Biosensors from $\mathrm{Fe}_{3} \mathrm{O}_{4}$ Nanoparticles Modified Chitosan/Graphene Nanocomposites, Sci. Rep., 2015, 5, 11129.

172 M. F. Hossain and J. Y. Park, Plain to Point Network Reduced Graphene Oxide-Activated Carbon Composites Decorated with Platinum Nanoparticles for Urine Glucose Detection, Sci. Rep., 2016, 6, 21009.

173 S. Palanisamy, R. Devasenathipathy, S.-M. Chen, M. Ajmal Ali, C. Karuppiah, V. Balakumar, P. Prakash, M. S. Elshikh and F. M. A. Al-Hemaid, Direct Electrochemistry of Glucose Oxidase at Reduced Graphene Oxide and $\beta$-Cyclodextrin Composite Modified Electrode and Application for Glucose Biosensing, Electroanalysis, 2015, 27, 2412-2420.

174 B. Thirumalraj, S. Palanisamy, S.-M. Chen, C.-Y. Yang, P. Periakaruppan and B.-S. Lou, Direct Electrochemistry of Glucose Oxidase and Sensing of Glucose at a Glassy Carbon Electrode Modified with a Reduced Graphene Oxide/Fullerene-C ${ }_{60}$ Composite, RSC Adv., 2015, 5, 7765177657.

175 M. Qi, Y. Zhang, C. Cao, Y. Lu and G. Liu, Increased Sensitivity of Extracellular Glucose Monitoring Based on AuNP Decorated GO Nanocomposites, RSC Adv., 2016, 6, 39180-39187.

176 F. Tehrani and B. Bavarian, Facile and Scalable Disposable Sensor Based on Laser Engraved Graphene for Electrochemical Detection of Glucose, Sci. Rep., 2016, 6, 27975.

177 K. Vijayaraj, S. W. Hong, S.-H. Jin, S.-C. Chang and D.-S. Park, Fabrication of a Novel Disposable Glucose Biosensor Using an Electrochemically Reduced Graphene Oxide-Glucose Oxidase Biocomposite, Anal. Methods, 2016, 8, 6974-6981.

178 D. B. T. Mascagni, C. M. Miyazaki, N. C. da Cruz, M. L. de Moraes, A. Riul and M. Ferreira, Layer-by-Layer Assembly of Functionalized Reduced Graphene Oxide for Direct
Electrochemistry and Glucose Detection, Mater. Sci. Eng., $C, 2016,68,739-745$.

179 S. Wu, F. Su, X. Dong, C. Ma, L. Pang, D. Peng, M. Wang, L. He and Z. Zhang, Development of Glucose Biosensors Based on Plasma Polymerization-Assisted Nanocomposites of Polyaniline, Tin Oxide, and ThreeDimensional Reduced Graphene Oxide, Appl. Surf. Sci., 2017, 401, 262-270.

180 Y. Liu, X. Zhang, D. He, F. Ma, Q. Fu and Y. Hu, An Amperometric Glucose Biosensor Based on a $\mathrm{MnO}_{2} /$ Graphene Composite Modified Electrode, RSC Adv., 2016, 6, 18654-18661.

181 A. Halder, M. Zhang and Q. Chi, Electroactive and Biocompatible Functionalization of Graphene for The Development of Biosensing Platforms, Biosens. Bioelectron., 2017, 87, 764-771.

182 A. O. Osikoya, O. Parlak, N. A. Murugan, E. D. Dikio, H. Moloto, L. Uzun, A. P. F. Turner and A. Tiwari, Acetylene-Sourced CVD-Synthesised Catalytically Active Graphene for Electrochemical Biosensing, Biosens. Bioelectron., 2017, 89, 496-504.

183 S. Pakapongpan and R. P. Poo-arporn, Self-Assembly of Glucose Oxidase on Reduced Graphene Oxide-Magnetic Nanoparticles Nanocomposite-Based Direct Electrochemistry for Reagentless Glucose Biosensor, Mater. Sci. Eng., C, 2017, 76, 398-405.

184 J. Liu, X. Wang, T. Wang, D. Li, F. Xi, J. Wang and E. Wang, Functionalization of Monolithic and Porous ThreeDimensional Graphene by One-Step Chitosan Electrodeposition for Enzymatic Biosensor, ACS Appl. Mater. Interfaces, 2014, 6, 19997-20002.

185 Y. Wang, X.-y. Liu, X. Xu, Y. Yang, L.-h. Huang, Z.-y. He, Y.-h. Xu, J.-j. Chen and Z.-s. Feng, Preparation and Characterization of Reduced Graphene Oxide/ $\mathrm{Fe}_{3} \mathrm{O}_{4}$ Nanocomposite by a Facile In situ Deposition Method for Glucose Biosensor Applications, Mater. Res. Bull., 2018, 101, 340-346.

186 B. Çakıroğlu and M. Özacar, Tannic Acid Modified Electrochemical Biosensor for Glucose Sensing Based on Direct Electrochemistry, Electroanalysis, 2017, 29, 27192726.

187 B. Akkaya, B. Çakiroğlu and M. Özacar, Tannic AcidReduced Graphene Oxide Deposited with Pt Nanoparticles for Switchable Bioelectronics and Biosensors Based on Direct Electrochemistry, ACS Sustainable Chem. Eng., 2018, 6, 3805-3814.

188 R. M. Ianniello, T. J. Lindsay and A. M. Yacynych, Differential Pulse Voltammetric Study of Direct Electron Transfer in Glucose Oxidase Chemically Modified Graphite Electrodes, Anal. Chem., 1982, 54, 1098-1101.

189 G. S. Wilson, Native Glucose Oxidase does not Undergo Direct Electron Transfer, Biosens. Bioelectron., 2016, 82, 7-8.

190 B. Liang, X. Guo, L. Fang, Y. Hu, G. Yang, Q. Zhu, J. Wei and X. Ye, Study of Direct Electron Transfer and Enzyme Activity of Glucose Oxidase on Graphene Surface, Electrochem. Commun., 2015, 50, 1-5. 
191 E. Laviron, General Expression of the Linear Potential Sweep Voltammogram in the Case Of Diffusionless Electrochemical Systems, J. Electroanal. Chem. Interfacial Electrochem., 1979, 101, 19-28.

192 C. Shan, H. Yang, J. Song, D. Han, A. Ivaska and L. Niu, Direct Electrochemistry of Glucose Oxidase and Biosensing for Glucose Based on Graphene, Anal. Chem., 2009, 81, 2378-2382.

193 M. Guler, V. Turkoglu and M. R. Kivanc, A Novel Enzymatic Glucose Biosensor and Nonenzymatic Hydrogen Peroxide Sensor Based on (3-Aminopropyl) Triethoxysilane Functionalized Reduced Graphene Oxide, Electroanalysis, 2017, 29, 2507-2515.

194 B. Unnikrishnan, S. Palanisamy and S.-M. Chen, A Simple Electrochemical Approach to Fabricate a Glucose Biosensor Based on Graphene-Glucose Oxidase Biocomposite, Biosens. Bioelectron., 2013, 39, 70-75.

195 Y. Ravenna, L. Xia, J. Gun, A. A. Mikhaylov, A. G. Medvedev, O. Lev and L. Alfonta, Biocomposite Based on Reduced Graphene Oxide Film Modified with Phenothiazone and Flavin Adenine Dinucleotide-Dependent Glucose Dehydrogenase for Glucose Sensing and Biofuel Cell Applications, Anal. Chem., 2015, 87, 9567-9571.

196 J. Liu, N. Kong, A. Li, X. Luo, L. Cui, R. Wang and S. Feng, Graphene Bridged Enzyme Electrodes for Glucose Biosensing Application, Analyst, 2013, 138, 2567-2575.

197 X. Zhang, Q. Liao, M. Chu, S. Liu and Y. Zhang, Structure Effect on Graphene-Modified Enzyme Electrode Glucose Sensors, Biosens. Bioelectron., 2014, 52, 281-287.

198 J. Guo, T. Zhang, C. Hu and L. Fu, A Three-Dimensional Nitrogen-Doped Graphene Structure: A Highly Efficient Carrier of Enzymes for Biosensors, Nanoscale, 2015, 7, 1290-1295.

199 D. Manoj, K. Theyagarajan, D. Saravanakumar, S. Senthilkumar and K. Thenmozhi, Aldehyde Functionalized Ionic Liquid on Electrochemically Reduced Graphene Oxide as a Versatile Platform for Covalent Immobilization of Biomolecules and Biosensing, Biosens. Bioelectron., 2018, 103, 104-112.

200 G. Bharath, R. Madhu, S.-M. Chen, V. Veeramani, A. Balamurugan, D. Mangalaraj, C. Viswanathan and N. Ponpandian, Enzymatic Electrochemical Glucose Biosensors by Mesoporous 1D Hydroxyapatite-on-2D Reduced Graphene Oxide, J. Mater. Chem. B, 2015, 3, 1360-1370.

$201 \mathrm{H}$. Al-Sagur, S. Komathi, M. A. Khan, A. G. Gurek and A. Hassan, A Novel Glucose Sensor Using Lutetium Phthalocyanine as Redox Mediator in Reduced Graphene Oxide Conducting Polymer Multifunctional Hydrogel, Biosens. Bioelectron., 2017, 92, 638-645.

202 K. T. Nguyen and Y. Zhao, Integrated Graphene/ Nanoparticle Hybrids for Biological and Electronic Applications, Nanoscale, 2014, 6, 6245-6266.

203 J. Lu, I. Do, L. T. Drzal, R. M. Worden and I. Lee, Nanometal-Decorated Exfoliated Graphite Nanoplatelet Based Glucose Biosensors with High Sensitivity and Fast Response, ACS Nano, 2008, 2, 1825-1832.
204 L. Jin, Z. Meng, Y. Zhang, S. Cai, Z. Zhang, C. Li, L. Shang and Y. Shen, Ultrasmall Pt Nanoclusters as Robust Peroxidase Mimics for Colorimetric Detection of Glucose in Human Serum, ACS Appl. Mater. Interfaces, 2017, 9, 10027-10033.

205 Q. Wang, X. Cui, J. Chen, X. Zheng, C. Liu, T. Xue, H. Wang, Z. Jin, L. Qiao and W. Zheng, Well-Dispersed Palladium Nanoparticles on Graphene Oxide as a Non-Enzymatic Glucose Sensor, RSC Adv., 2012, 2, 6245-6249.

206 J. Luo, S. Jiang, H. Zhang, J. Jiang and X. Liu, A Novel NonEnzymatic Glucose Sensor Based On $\mathrm{Cu}$ Nanoparticle Modified Graphene Sheets Electrode, Anal. Chim. Acta, 2012, 709, 47-53.

207 S. Palanisamy, A. E. Vilian and S.-M. Chen, Direct Electrochemistry of Glucose Oxidase at Reduced Graphene Oxide/Zinc Oxide Composite Modified Electrode for Glucose Sensor, Int. J. Electrochem. Sci., 2012, 7, 2153-2163.

208 Y. Zhao, W. Li, L. Pan, D. Zhai, Y. Wang, L. Li, W. Cheng, W. Yin, X. Wang, J.-B. Xu and Y. Shi, ZnO-Nanorods/ Graphene Heterostructure: A Direct Electron Transfer Glucose Biosensor, Sci. Rep., 2016, 6, 32327.

209 J. Song, L. Xu, C. Zhou, R. Xing, Q. Dai, D. Liu and H. Song, Synthesis of Graphene Oxide Based CuO Nanoparticles Composite Electrode for Highly Enhanced Nonenzymatic Glucose Detection, ACS Appl. Mater. Interfaces, 2013, 5, 12928-12934.

210 B. Yuan, C. Xu, D. Deng, Y. Xing, L. Liu, H. Pang and D. Zhang, Graphene Oxide/Nickel Oxide Modified Glassy Carbon Electrode for Supercapacitor and Nonenzymatic Glucose Sensor, Electrochim. Acta, 2013, 88, 708-712.

$211 \mathrm{H}$. Teymourian, A. Salimi and S. Khezrian, $\mathrm{Fe}_{3} \mathrm{O}_{4}$ Magnetic Nanoparticles/Reduced Graphene Oxide Nanosheets as a Novel Electrochemical and Bioeletrochemical Sensing Platform, Biosens. Bioelectron., 2013, 49, 1-8.

212 Q. Wang, X. Zhang, L. Huang, Z. Zhang and S. Dong, OnePot Synthesis of $\mathrm{Fe}_{3} \mathrm{O}_{4}$ Nanoparticle Loaded 3D Porous Graphene Nanocomposites with Enhanced Nanozyme Activity for Glucose Detection, ACS Appl. Mater. Interfaces, 2017, 9, 7465-7471.

213 A. Devadoss, P. Sudhagar, S. Das, S. Y. Lee, C. Terashima, K. Nakata, A. Fujishima, W. Choi, Y. S. Kang and U. Paik, Synergistic Metal-Metal Oxide Nanoparticles Supported Electrocatalytic Graphene for Improved Photoelectrochemical Glucose Oxidation, ACS Appl. Mater. Interfaces, 2014, 6, 4864-4871.

214 J. T. Holland, C. Lau, S. Brozik, P. Atanassov and S. Banta, Engineering of Glucose Oxidase for Direct Electron Transfer via Site-Specific Gold Nanoparticle Conjugation, J. Am. Chem. Soc., 2011, 133, 19262-19265.

215 O. Yehezkeli, R. Tel-Vered, S. Raichlin and I. Willner, NanoEngineered Flavin-Dependent Glucose Dehydrogenase/ Gold Nanoparticle-Modified Electrodes for Glucose Sensing and Biofuel Cell Applications, ACS Nano, 2011, 5, 2385-2391. 
216 Y. Xiao, F. Patolsky, E. Katz, J. F. Hainfeld and I. Willner, "Plugging into Enzymes": Nanowiring of Redox Enzymes by a Gold Nanoparticle, Science, 2003, 299, 1877-1881.

$217 \mathrm{X}$. Wang and X. Zhang, Electrochemical Co-Reduction Synthesis of Graphene/Nano-Gold Composites and its Application to Electrochemical Glucose Biosensor, Electrochim. Acta, 2013, 112, 774-782.

218 J. C. Claussen, A. Kumar, D. B. Jaroch, M. H. Khawaja, A. B. Hibbard, D. M. Porterfield and T. S. Fisher, Nanostructuring Platinum Nanoparticles on Multilayered Graphene Petal Nanosheets for Electrochemical Biosensing, Adv. Funct. Mater., 2012, 22, 3399-3405.

219 A. Zaleska-Medynska, M. Marchelek, M. Diak and E. Grabowska, Noble Metal-Based Bimetallic Nanoparticles: The Effect of the Structure on the Optical, Catalytic and Photocatalytic Properties, Adv. Colloid Interface Sci., 2016, 229, 80-107.

220 S. Park, H. Boo and T. D. Chung, Electrochemical NonEnzymatic Glucose Sensors, Anal. Chim. Acta, 2006, 556, 46-57.

221 G. Zoldák, A. Zubrik, A. Musatov, M. Stupák and E. Sedlák, Irreversible Thermal Denaturation of Glucose Oxidase from Aspergillus niger is the Transition to the Denatured State with Residual Structure, J. Biol. Chem., 2004, 279, 4760147609.

222 K. Tian, M. Prestgard and A. Tiwari, A Review of Recent Advances in Nonenzymatic Glucose Sensors, Mater. Sci. Eng., C, 2014, 41, 100-118.

223 (a) S. Y. Tee, C. P. Teng and E. Ye, Metal Nanostructures for Non-Enzymatic Glucose Sensing, Mater. Sci. Eng., C, 2017, 70, 1018-1030; (b) S. A. Zaidi and J. H. Shin, Recent Developments in Nanostructure Based Electrochemical Glucose Sensors, Talanta, 2016, 149, 30-42.

224 T. Deepalakshmi, D. T. Tran, N. H. Kim, K. T. Chong and J. H. Lee, Nitrogen-Doped Graphene-Encapsulated Nickel Cobalt Nitride as a Highly Sensitive and Selective Electrode for Glucose and Hydrogen Peroxide Sensing Applications, ACS Appl. Mater. Interfaces, 2018, 10, 3584735858.

225 L. T. Hoa, K. G. Sun and S. H. Hur, Highly Sensitive NonEnzymatic Glucose Sensor Based on Pt Nanoparticle Decorated Graphene Oxide Hydrogel, Sens. Actuators, B, 2015, 210, 618-623.

226 D. Jiang, Q. Liu, K. Wang, J. Qian, X. Dong, Z. Yang, X. Du and B. Qiu, Enhanced Non-Enzymatic Glucose Sensing Based on Copper Nanoparticles Decorated NitrogenDoped Graphene, Biosens. Bioelectron., 2014, 54, 273-278.

227 L. Ju, G. Wu, B. Lu, X. Li, H. Wu and A. Liu, Non-Enzymatic Amperometric Glucose Sensor Based on Copper Nanowires Decorated Reduced Graphene Oxide, Electroanalysis, 2016, 28, 2543-2551.

228 N. S. K. Gowthaman, M. A. Raj and S. A. John, NitrogenDoped Graphene as a Robust Scaffold for the Homogeneous Deposition of Copper Nanostructures: A Nonenzymatic Disposable Glucose Sensor, ACS Sustainable Chem. Eng., 2017, 5, 1648-1658.
229 H. Gao, F. Xiao, C. B. Ching and H. Duan, One-Step Electrochemical Synthesis of PtNi Nanoparticle-Graphene Nanocomposites for Nonenzymatic Amperometric Glucose Detection, ACS Appl. Mater. Interfaces, 2011, 3, 3049-3057.

230 Q. Sheng, D. Liu and J. Zheng, NiCo Alloy Nanoparticles Anchored on Polypyrrole/Reduced Graphene Oxide Nanocomposites for Nonenzymatic Glucose Sensing, New J. Chem., 2016, 40, 6658-6665.

231 Y.-W. Hsu, T.-K. Hsu, C.-L. Sun, Y.-T. Nien, N.-W. Pu and M.-D. Ger, Synthesis of CuO/Graphene Nanocomposites for Nonenzymatic Electrochemical Glucose Biosensor Applications, Electrochim. Acta, 2012, 82, 152-157.

232 X.-C. Dong, H. Xu, X.-W. Wang, Y.-X. Huang, M. B. ChanPark, H. Zhang, L.-H. Wang, W. Huang and P. Chen, 3D Graphene-Cobalt Oxide Electrode for High-Performance Supercapacitor and Enzymeless Glucose Detection, ACS Nano, 2012, 6, 3206-3213.

233 X. Wang, X. Dong, Y. Wen, C. Li, Q. Xiong and P. Chen, A Graphene-Cobalt Oxide Based Needle Electrode for NonEnzymatic Glucose Detection in Micro-Droplets, Chem. Commun., 2012, 48, 6490-6492.

234 F. Xiao, Y. Li, H. Gao, S. Ge and H. Duan, Growth of CoralLike PtAu- $\mathrm{MnO}_{2}$ Binary Nanocomposites on Free-Standing Graphene Paper for Flexible Nonenzymatic Glucose Sensors, Biosens. Bioelectron., 2013, 41, 417-423.

235 M. Liu, R. Liu and W. Chen, Graphene Wrapped $\mathrm{Cu}_{2} \mathrm{O}$ Nanocubes: Non-Enzymatic Electrochemical Sensors for the Detection of Glucose and Hydrogen Peroxide with Enhanced Stability, Biosens. Bioelectron., 2013, 45, 206-212.

236 Y. He and J. Zheng, One-Pot Ultrasonic-Electrodeposition of Copper-Graphene Nanoflowers in Ethaline for Glucose Sensing, Anal. Methods, 2013, 5, 767-772.

237 G.-h. Wu, X.-h. Song, Y.-F. Wu, X.-m. Chen, F. Luo and X. Chen, Non-Enzymatic Electrochemical Glucose Sensor Based on Platinum Nanoflowers Supported on Graphene Oxide, Talanta, 2013, 105, 379-385.

238 P. Si, X.-C. Dong, P. Chen and D.-H. Kim, A Hierarchically Structured Composite of $\mathrm{Mn}_{3} \mathrm{O}_{4} / 3 \mathrm{D}$ Graphene Foam for Flexible Nonenzymatic Biosensors, J. Mater. Chem. B, 2013, 1, 110-115.

239 K. Dhara, J. Stanley, T. Ramachandran, B. G. Nair and T. G. S. Babu, Pt-CuO Nanoparticles Decorated Reduced Graphene Oxide for the Fabrication of Highly Sensitive Non-Enzymatic Disposable Glucose Sensor, Sens. Actuators, B, 2014, 195, 197-205.

240 P. Subramanian, J. Niedziolka-Jonsson, A. Lesniewski, Q. Wang, M. Li, R. Boukherroub and S. Szunerits, Preparation of Reduced Graphene Oxide-Ni(OH $)_{2}$ Composites by Electrophoretic Deposition: Application for Non-Enzymatic Glucose Sensing, J. Mater. Chem. A, 2014, 2, 5525-5533.

241 M. Li, X. Bo, Y. Zhang, C. Han and L. Guo, One-Pot Ionic Liquid-Assisted Synthesis of Highly Dispersed PtPd Nanoparticles/Reduced Graphene Oxide Composites for Nonenzymatic Glucose Detection, Biosens. Bioelectron., 2014, 56, 223-230. 
242 B. Zhan, C. Liu, H. Chen, H. Shi, L. Wang, P. Chen, W. Huang and X. Dong, Free-Standing Electrochemical Electrode Based on $\mathrm{Ni}(\mathrm{OH})_{2} / 3 \mathrm{D}$ Graphene Foam for Nonenzymatic Glucose Detection, Nanoscale, 2014, 6, 7424-7429.

243 Y. Hu, F. He, A. Ben and C. Chen, Synthesis of Hollow PtNi-Graphene Nanostructures for Nonenzymatic Glucose Detection, J. Electroanal. Chem., 2014, 726, 55-61.

244 T. Alizadeh and S. Mirzagholipur, A Nafion-Free NonEnzymatic Amperometric Glucose Sensor Based on Copper Oxide Nanoparticles-Graphene Nanocomposite, Sens. Actuators, B, 2014, 198, 438-447.

245 M. Yuan, A. Liu, M. Zhao, W. Dong, T. Zhao, J. Wang and W. Tang, Bimetallic PdCu Nanoparticle Decorated ThreeDimensional Graphene Hydrogel for Non-Enzymatic Amperometric Glucose Sensor, Sens. Actuators, B, 2014, 190, 707-714.

246 N. S. Ismail, Q. H. Le, H. Yoshikawa, M. Saito and E. Tamiya, Development of Non-enzymatic Electrochemical Glucose Sensor Based on Graphene Oxide Nanoribbon-Gold Nanoparticle Hybrid, Electrochim. Acta, 2014, 146, 98-105.

247 W. Gao, W. W. Tjiu, J. Wei and T. Liu, Highly Sensitive Nonenzymatic Glucose and $\mathrm{H}_{2} \mathrm{O}_{2}$ Sensor Based on $\mathrm{Ni}(\mathrm{OH})_{2} /$ Electroreduced Graphene Oxide-Multiwalled Carbon Nanotube Film Modified Glass Carbon Electrode, Talanta, 2014, 120, 484-490.

248 Y. Ye, P. Wang, E. Dai, J. Liu, Z. Tian, C. Liang and G. Shao, A Novel Reduction Approach to Fabricate Quantum-Sized $\mathrm{SnO}_{2}$-Conjugated Reduced Graphene Oxide Nanocomposites as Non-Enzymatic Glucose Sensors, Phys. Chem. Chem. Phys., 2014, 16, 8801-8807.

249 Z. Liu, Y. Guo and C. Dong, A High Performance Nonenzymatic Electrochemical Glucose Sensor Based on Polyvinylpyrrolidone-Graphene Nanosheets-Nickel Nanoparticles-Chitosan Nanocomposite, Talanta, 2015, 137, 87-93.

250 K. Dhara, T. Ramachandran, B. G. Nair and T. G. Satheesh Babu, Single Step Synthesis of $\mathrm{Au}-\mathrm{CuO}$ Nanoparticles Decorated Reduced Graphene Oxide for High Performance Disposable Nonenzymatic Glucose Sensor, $J$. Electroanal. Chem., 2015, 743, 1-9.

251 S. Fu, G. Fan, L. Yang and F. Li, Non-Enzymatic Glucose Sensor Based on Au Nanoparticles Decorated Ternary NiAl Layered Double Hydroxide/Single-Walled Carbon Nanotubes/Graphene Nanocomposite, Electrochim. Acta, 2015, 152, 146-154.

252 Y. Tian, Y. Liu, W.-p. Wang, X. Zhang and W. Peng, CuO Nanoparticles on Sulfur-Doped Graphene for Nonenzymatic Glucose Sensing, Electrochim. Acta, 2015, 156, 244-251.

253 S. Yang, L. Liu, G. Wang, G. Li, D. Deng and L. Qu, One-Pot Synthesis of $\mathrm{Mn}_{3} \mathrm{O}_{4}$ Nanoparticles Decorated with Nitrogen-Doped Reduced Graphene Oxide for Sensitive Nonenzymatic Glucose Sensing, J. Electroanal. Chem., 2015, 755, 15-21.
254 X. Zhang, Q. Liao, S. Liu, W. Xu, Y. Liu and Y. Zhang, CuNiO Nanoparticles Assembled on Graphene as an Effective Platform for Enzyme-Free Glucose Sensing, Anal. Chim. Acta, 2015, 858, 49-54.

255 G. Chang, H. Shu, Q. Huang, M. Oyama, K. Ji, X. Liu and Y. He, Synthesis of Highly Dispersed Pt Nanoclusters Anchored Graphene Composites and Their Application for Non-Enzymatic Glucose Sensing, Electrochim. Acta, 2015, 157, 149-157.

256 Q. Wang, Q. Wang, M. Li, S. Szunerits and R. Boukherroub, Preparation of Reduced Graphene Oxide/Cu Nanoparticle Composites Through Electrophoretic Deposition: Application for Nonenzymatic Glucose Sensing, RSC Adv., 2015, 5, 15861-15869.

257 L. Wang, X. Lu, C. Wen, Y. Xie, L. Miao, S. Chen, H. Li, P. Li and Y. Song, One-Step Synthesis of Pt-NiO Nanoplate Array/ Reduced Graphene Oxide Nanocomposites for Nonenzymatic Glucose Sensing, J. Mater. Chem. A, 2015, 3, 608-616.

258 M. Wu, S. Meng, Q. Wang, W. Si, W. Huang and X. Dong, Nickel-Cobalt Oxide Decorated Three-Dimensional Graphene as an Enzyme Mimic for Glucose and Calcium Detection, ACS Appl. Mater. Interfaces, 2015, 7, 2108921094.

259 X. Zhuang, C. Tian, F. Luan, X. Wu and L. Chen, One-Step Electrochemical Fabrication of a Nickel Oxide Nanoparticle/Polyaniline Nanowire/Graphene Oxide Hybrid on a Glassy Carbon Electrode for use as a NonEnzymatic Glucose Biosensor, RSC Adv., 2016, 6, 9254192546.

260 Z. Yang, X. Yan, Z. Li, X. Zheng and J. Zheng, Synthesis of $\mathrm{Cu}_{2} \mathrm{O}$ on $\mathrm{AlOOH} /$ Reduced Graphene Oxide for NonEnzymatic Amperometric Glucose Sensing, Anal. Methods, 2016, 8, 1527-1531.

261 G. Ma, M. Yang, C. Li, H. Tan, L. Deng, S. Xie, F. Xu, L. Wang and Y. Song, Preparation of Spinel Nickel-Cobalt Oxide Nanowrinkles/Reduced Graphene Oxide Hybrid for Nonenzymatic Glucose Detection at Physiological Level, Electrochim. Acta, 2016, 220, 545-553.

262 S. Pourbeyram and K. Mehdizadeh, Nonenzymatic Glucose Sensor Based on Disposable Pencil Graphite Electrode Modified by Copper Nanoparticles, J. Food Drug Anal., 2016, 24, 894-902.

263 S. Liu, K. S. Hui and K. N. Hui, Flower-Like Copper Cobaltite Nanosheets on Graphite Paper as HighPerformance Supercapacitor Electrodes and Enzymeless Glucose Sensors, ACS Appl. Mater. Interfaces, 2016, 8, 3258-3267.

264 C. Wang, Y. Sun, X. Yu, D. Ma, J. Zheng, P. Dou, Z. Cao and X. Xu, Ag-Pt Hollow Nanoparticles Anchored Reduced Graphene Oxide Composites for Non-Enzymatic Glucose Biosensor, J. Mater. Sci.: Mater. Electron., 2016, 27, 93709378.

265 J. Yang, H. Ye, Z. Zhang, F. Zhao and B. Zeng, MetalOrganic Framework Derived Hollow Polyhedron $\mathrm{CuCo}_{2} \mathrm{O}_{4}$ Functionalized Porous Graphene for Sensitive Glucose Sensing, Sens. Actuators, B, 2017, 242, 728-735. 
266 S. Samuei, J. Fakkar, Z. Rezvani, A. Shomali and B. Habibi, Synthesis and Characterization of Graphene Quantum Dots/CoNiAl-Layered Double-Hydroxide Nanocomposite: Application as a Glucose Sensor, Anal. Biochem., 2017, 521, 31-39.

267 A. I. Gopalan, N. Muthuchamy and K. P. Lee, A Novel Bismuth Oxychloride-Graphene Hybrid Nanosheets Based Non-Enzymatic Photoelectrochemical Glucose Sensing Platform for High Performances, Biosens. Bioelectron., 2017, 89, 352-360.

268 T. D. Thanh, J. Balamurugan, S. H. Lee, N. H. Kim and J. H. Lee, Effective Seed-Assisted Synthesis of Gold Nanoparticles Anchored Nitrogen-Doped Graphene for Electrochemical Detection of Glucose and Dopamine, Biosens. Bioelectron., 2016, 81, 259-267.

269 N. Karikalan, R. Karthik, S.-M. Chen, C. Karuppiah and A. Elangovan, Sonochemical Synthesis of Sulfur Doped Reduced Graphene Oxide Supported CuS Nanoparticles for the Non-Enzymatic Glucose Sensor Applications, Sci. Rep., 2017, 7, 2494.

270 H. Rao, Z. Zhang, H. Ge, X. Liu, P. Zou, X. Wang and Y. Wang, Enhanced Amperometric Sensing Using a $\mathrm{NiCo}_{2} \mathrm{O}_{4} /$ Nitrogen-Doped Reduced Graphene Oxide/ Ionic Liquid Ternary Composite for Enzyme-Free Detection of Glucose, New J. Chem., 2017, 41, 3667-3676.

271 P. Balasubramanian, M. Velmurugan, S.-M. Chen and K.-Y. Hwa, Optimized Electrochemical Synthesis of Copper Nanoparticles Decorated Reduced Graphene Oxide: Application for Enzymeless Determination of Glucose in Human Blood, J. Electroanal. Chem., 2017, 807, 128-136.

272 N. Hui and J. Wang, Electrodeposited Honeycomb-Like Cobalt Nanostructures on Graphene Oxide Doped Polypyrrole Nanocomposite for High Performance Enzymeless Glucose Sensing, J. Electroanal. Chem., 2017, 798, 9-16.

273 M. Mazaheri, H. Aashuri and A. Simchi, Three-Dimensional Hybrid Graphene/Nickel Electrodes on Zinc Oxide Nanorod Arrays as Non-Enzymatic Glucose Biosensors, Sens. Actuators, B, 2017, 251, 462-471.

274 S. Yang, G. Li, D. Wang, Z. Qiao and L. Qu, Synthesis of Nanoneedle-Like Copper Oxide on N-Doped Reduced Graphene Oxide: A Three-Dimensional Hybrid for Nonenzymatic Glucose Sensor, Sens. Actuators, B, 2017, 238, 588-595.

275 H. Wu, Y. Yu, W. Gao, A. Gao, A. M. Qasim, F. Zhang, J. Wang, K. Ding, G. Wu and P. K. Chu, Nickel Plasma Modification of Graphene for High-Performance NonEnzymatic Glucose Sensing, Sens. Actuators, B, 2017, 251, 842-850.

276 X. Yan, Y. Gu, C. Li, B. Zheng, Y. Li, T. Zhang, Z. Zhang and M. Yang, A Non-Enzymatic Glucose Sensor Based on the CuS Nanoflakes-Reduced Graphene Oxide Nanocomposite, Anal. Methods, 2018, 10, 381-388.

277 K. J. Babu, S. Sheet, Y. S. Lee and G. G. Kumar, ThreeDimensional Dendrite $\mathrm{Cu}-\mathrm{Co} /$ Reduced Graphene Oxide Architectures on a Disposable Pencil Graphite Electrode as an Electrochemical Sensor for Nonenzymatic Glucose Detection, ACS Sustainable Chem. Eng., 2018, 6, 1909-1918. 278 L. Jothi, N. Jayakumar, S. K. Jaganathan and G. Nageswaran, Ultrasensitive and Selective NonEnzymatic Electrochemical Glucose Sensor Based on Hybrid Material of Graphene Nanosheets/Graphene Nanoribbons/Nickel Nanoparticle, Mater. Res. Bull., 2018, 98, 300-307.

279 Q. Zhang, Q. Luo, Z. Qin, L. Liu, Z. Wu, B. Shen and W. Hu, Self-Assembly of Graphene-Encapsulated $\mathrm{Cu}$ Composites for Nonenzymatic Glucose Sensing, ACS Omega, 2018, 3, 3420-3428.

280 M. Li, X. Bo, Z. Mu, Y. Zhang and L. Guo, Electrodeposition of Nickel Oxide and Platinum Nanoparticles on Electrochemically Reduced Graphene Oxide Film as a Nonenzymatic Glucose Sensor, Sens. Actuators, B, 2014, 192, 261-268.

281 L. T. Hoa, J. S. Chung and S. H. Hur, A Highly Sensitive Enzyme-Free Glucose Sensor Based on $\mathrm{Co}_{3} \mathrm{O}_{4}$ Nanoflowers and 3D Graphene Oxide Hydrogel Fabricated via Hydrothermal Synthesis, Sens. Actuators, B, 2016, 223, 7682.

282 A. Ahmadalinezhad and A. Chen, High-Performance Electrochemical Biosensor for the Detection of Total Cholesterol, Biosens. Bioelectron., 2011, 26, 4508-4513.

283 A. N. Sekretaryova, V. Beni, M. Eriksson, A. A. Karyakin, A. P. F. Turner and M. Y. Vagin, Cholesterol Self-Powered Biosensor, Anal. Chem., 2014, 86, 9540-9547.

284 R. S. Dey and C. R. Raj, Redox-Functionalized Graphene Oxide Architecture for the Development of Amperometric Biosensing Platform, ACS Appl. Mater. Interfaces, 2013, 5, 4791-4798.

285 Y.-J. Lee and J.-Y. Park, Nonenzymatic Free-Cholesterol Detection via a Modified Highly Sensitive Macroporous Gold Electrode with Platinum Nanoparticles, Biosens. Bioelectron., 2010, 26, 1353-1358.

286 U. Saxena and A. B. Das, Nanomaterials Towards Fabrication of Cholesterol Biosensors: Key Roles and Design Approaches, Biosens. Bioelectron., 2016, 75, 196-205. 287 R. S. Dey and C. R. Raj, Development of an Amperometric Cholesterol Biosensor Based on Graphene-Pt Nanoparticle Hybrid Material, J. Phys. Chem. C, 2010, 114, 21427-21433.

288 R. Manjunatha, G. Shivappa Suresh, J. Savio Melo, S. F. D'Souza and T. Venkatarangaiah Venkatesha, An Amperometric Bienzymatic Cholesterol Biosensor Based on Functionalized Graphene Modified Electrode and its Electrocatalytic Activity Towards Total Cholesterol Determination, Talanta, 2012, 99, 302-309.

289 M. Zhang, R. Yuan, Y. Chai, S. Chen, X. Zhong, H. Zhong and C. Wang, A Cathodic Electrogenerated Chemiluminescence Biosensor Based on Luminol and Hemin-Graphene Nanosheets for Cholesterol Detection, RSC Adv., 2012, 2, 4639-4641.

290 M. Zhang, R. Yuan, Y. Chai, C. Wang and X. Wu, Cerium Oxide-Graphene as the Matrix for Cholesterol Sensor, Anal. Biochem., 2013, 436, 69-74. 
291 S. Cao, L. Zhang, Y. Chai and R. Yuan, An Integrated Sensing System for Detection of Cholesterol Based on $\mathrm{TiO}_{2}$-Graphene-Pt-Pd Hybrid Nanocomposites, Biosens. Bioelectron., 2013, 42, 532-538.

292 S. Cao, L. Zhang, Y. Chai and R. Yuan, Electrochemistry of Cholesterol Biosensor Based on a Novel Pt-Pd Bimetallic Nanoparticle Decorated Graphene Catalyst, Talanta, 2013, 109, 167-172.

293 X. Wu, Y. Chai, R. Yuan, X. Zhong and J. Zhang, Synthesis of Multiwall Carbon Nanotubes-Graphene Oxide-Thionine-Au Nanocomposites for Electrochemiluminescence Detection of Cholesterol, Electrochim. Acta, 2014, 129, 441-449.

294 N. Ruecha, R. Rangkupan, N. Rodthongkum and O. Chailapakul, Novel Paper-Based Cholesterol Biosensor Using Graphene/Polyvinylpyrrolidone/Polyaniline Nanocomposite, Biosens. Bioelectron., 2014, 52, 13-19.

295 O. Parlak, A. Tiwari, A. P. F. Turner and A. Tiwari, TemplateDirected Hierarchical Self-Assembly of Graphene Based Hybrid Structure for Electrochemical Biosensing, Biosens. Bioelectron., 2013, 49, 53-62.

296 R. S. Dey and C. R. Raj, Enzyme-integrated cholesterol biosensing scaffold based on in situ synthesized reduced graphene oxide and dendritic Pd nanostructure, Biosens. Bioelectron., 2014, 62, 357-364.

297 M. B. Gholivand and M. Khodadadian, Amperometric Cholesterol Biosensor Based on the Direct Electrochemistry of Cholesterol Oxidase and Catalase on a Graphene/Ionic Liquid-Modified Glassy Carbon Electrode, Biosens. Bioelectron., 2014, 53, 472-478.

298 Z. Li, C. Xie, J. Wang, A. Meng and F. Zhang, Direct Electrochemistry of Cholesterol Oxidase Immobilized on Chitosan-Graphene and Cholesterol Sensing, Sens. Actuators, B, 2015, 208, 505-511.

299 Q. Wu, Y. Hou, M. Zhang, X. Hou, L. Xu, N. Wang, J. Wang and W. Huang, Amperometric Cholesterol Biosensor Based on Zinc Oxide Films on a Silver Nanowire-Graphene Oxide Modified Electrode, Anal. Methods, 2016, 8, 1806-1812.

300 L. Yang, H. Zhao, Y. Li, X. Ran, G. Deng, Y. Zhang, H. Ye, G. Zhao and C.-P. Li, Indicator Displacement Assay for Cholesterol Electrochemical Sensing Using a Calix[6]arene Functionalized Graphene-Modified Electrode, Analyst, 2016, 141, 270-278.

301 L. Xu, Y. Hou, M. Zhang, T. Cheng, W. Huang, C. Yao and Q. Wu, Electrochemical Sensor Based on a Silver Nanowires Modified Electrode for the Determination of Cholesterol, Anal. Methods, 2015, 7, 5649-5653.

302 J. Huan, Q. Liu, A. Fei, J. Qian, X. Dong, B. Qiu, H. Mao and K. Wang, Amplified Solid-State Electrochemiluminescence Detection of Cholesterol in Near-Infrared Range Based on CDTE Quantum Dots Decorated Multiwalled Carbon Nanotubes@Reduced Graphene Oxide Nanoribbons, Biosens. Bioelectron., 2015, 73, 221-227.

303 S. Abraham, S. Srivastava, V. Kumar, S. Pandey, P. K. Rastogi, N. R. Nirala, S. Kashyap, S. K. Srivastava, V. N. Singh, V. Ganesan, P. S. Saxena and A. Srivastava, Enhanced Electrochemical Biosensing Efficiency of Silica Particles Supported on Partially Reduced Graphene Oxide for Sensitive Detection of Cholesterol, J. Electroanal. Chem., 2015, 757, 65-72.

304 A. Rengaraj, Y. Haldorai, C. H. Kwak, S. Ahn, K.-J. Jeon, S. H. Park, Y.-K. Han and Y. S. Huh, Electrodeposition of Flower-Like Nickel Oxide on CVD-Grown Graphene to Develop an Electrochemical Non-Enzymatic Biosensor, $J$. Mater. Chem. B, 2015, 3, 6301-6309.

305 S. Wu, Y. Wang, H. Mao, C. Wang, L. Xia, Y. Zhang, H. Ge and X.-M. Song, Direct Electrochemistry of Cholesterol Oxidase and Biosensing of Cholesterol Based on PSS/ Polymeric Ionic Liquid-Graphene Nanocomposite, RSC Adv., 2016, 6, 59487-59496.

306 K. Pramanik, P. Sarkar, D. Bhattacharyay and P. Majumdar, One Step Electrode Fabrication for Direct Electron Transfer Cholesterol Biosensor Based on Composite of Polypyrrole, Green Reduced Graphene Oxide and Cholesterol Oxidase, Electroanalysis, 2018, 30, 2719-2730.

307 S. Komathi, N. Muthuchamy, K. P. Lee and A. I. Gopalan, Fabrication of a Novel Dual Mode Cholesterol Biosensor Using Titanium Dioxide Nanowire Bridged 3D Graphene Nanostacks, Biosens. Bioelectron., 2016, 84, 64-71.

308 X. Du, D. Jiang, S. Chen, L. Dai, L. Zhou, N. Hao, T. You, $\mathrm{H}$. Mao and $\mathrm{K}$. Wang, $\mathrm{CeO}_{2}$ Nanocrystallines Ensembleon-Nitrogen-Doped Graphene Nanocomposites: One-Pot, Rapid Synthesis and Excellent Electrocatalytic Activity for Enzymatic Biosensing, Biosens. Bioelectron., 2017, 89, 681688.

309 Y. Huang, J. Tan, L. Cui, Z. Zhou, S. Zhou, Z. Zhang, R. Zheng, Y. Xue, M. Zhang, S. Li, N. Zhu, J. Liang, G. Li, L. Zhong and Y. Zhao, Graphene and Au NPs co-Mediated Enzymatic Silver Deposition for the Ultrasensitive Electrochemical Detection of Cholesterol, Biosens. Bioelectron., 2018, 102, 560-567.

310 S. Karimi, H. Ghourchian, P. Rahimi and H.-A. Rafiee-Pour, A Nanocomposite Based Biosensor for Cholesterol Determination, Anal. Methods, 2012, 4, 3225-3231.

311 N. Agnihotri, A. D. Chowdhury and A. De, Non-Enzymatic Electrochemical Detection of Cholesterol Using $\beta$ Cyclodextrin Functionalized Graphene, Biosens. Bioelectron., 2015, 63, 212-217.

312 G. B. V. S. Lakshmi, A. Sharma, P. R. Solanki and D. K. Avasthi, Mesoporous Polyaniline Nanofiber Decorated Graphene Micro-Flowers for Enzyme-Less Cholesterol Biosensors, Nanotechnology, 2016, 27, 345101.

313 S. Alexander, P. Baraneedharan, S. Balasubrahmanyan and S. Ramaprabhu, Modified Graphene Based Molecular Imprinted Polymer for Electrochemical Non-Enzymatic Cholesterol Biosensor, Eur. Polym. J., 2017, 86, 106-116.

314 W. Chen, S. Cai, Q.-Q. Ren, W. Wen and Y.-D. Zhao, Recent Advances in Electrochemical Sensing for Hydrogen Peroxide: A Review, Analyst, 2012, 137, 49-58.

315 B. Hou, H. Liu, S. Qi, Y. Zhu, B. Zhou, X. Jiang and L. Zhu, Preparation of Pristine Graphene in Ethanol Assisted by Organic Salts for Nonenzymatic Detection of Hydrogen Peroxide, J. Colloid Interface Sci., 2018, 510, 103-110.

316 Z. Yu, L. Zou, Y. Chen and J. Jiang, (Pc)Eu(Pc)Eu[trans$\left.\mathrm{T}\left(\mathrm{COOCH}_{3}\right)_{2} \mathrm{PP}\right] / \mathrm{GO}$ Hybrid Film-Based Nonenzymatic 
$\mathrm{H}_{2} \mathrm{O}_{2}$ Electrochemical Sensor with Excellent Performance, ACS Appl. Mater. Interfaces, 2016, 8, 30398-30406.

317 R. Mohammad-Rezaei and H. Razmi, Reduced Graphene Oxide|Carbon Ceramic Electrode Modified with CdSHemoglobin as a Sensitive Hydrogen Peroxide Biosensor, Electroanalysis, 2012, 24, 2094-2101.

318 W. Sun, X. Ju, Y. Zhang, X. Sun, G. Li and Z. Sun, Application of Carboxyl Functionalized Graphene Oxide as Mimetic Peroxidase for Sensitive Voltammetric Detection of $\mathrm{H}_{2} \mathrm{O}_{2}$ with $3,3^{\prime}, 5,5^{\prime}$-Tetramethylbenzidine, Electrochem. Commun., 2013, 26, 113-116.

319 S. K. Jha, C. N. Kumar, R. P. Raj, N. S. Jha and S. Mohan, Synthesis of $3 \mathrm{D}$ Porous $\mathrm{CeO}_{2} /$ Reduced Graphene Oxide Xerogel Composite and Low Level Detection of $\mathrm{H}_{2} \mathrm{O}_{2}$, Electrochim. Acta, 2014, 120, 308-313.

320 F. Xu, Y. Sun, Y. Zhang, Y. Shi, Z. Wen and Z. Li, GraphenePt Nanocomposite for Nonenzymatic Detection of Hydrogen Peroxide with Enhanced Sensitivity, Electrochem. Commun., 2011, 13, 1131-1134.

321 X. Dong, Y. Ma, G. Zhu, Y. Huang, J. Wang, M. B. ChanPark, L. Wang, W. Huang and P. Chen, Synthesis of Graphene-Carbon Nanotube Hybrid Foam and its use as a Novel Three-Dimensional Electrode for Electrochemical Sensing, J. Mater. Chem., 2012, 22, 17044-17048.

322 F. Xiao, Y. Li, X. Zan, K. Liao, R. Xu and H. Duan, Growth of Metal-Metal Oxide Nanostructures on Freestanding Graphene Paper for Flexible Biosensors, Adv. Funct. Mater., 2012, 22, 2487-2494.

323 F. Xiao, J. Song, H. Gao, X. Zan, R. Xu and H. Duan, Coating Graphene Paper with 2D-Assembly of Electrocatalytic Nanoparticles: A Modular Approach toward HighPerformance Flexible Electrodes, ACS Nano, 2012, 6, 100110.

324 J.-H. Yang, N. Myoung and H.-G. Hong, Facile and Controllable Synthesis of Prussian Blue on ChitosanFunctionalized Graphene Nanosheets for the Electrochemical Detection of Hydrogen Peroxide, Electrochim. Acta, 2012, 81, 37-43.

325 L. Zhong, S. Gan, X. Fu, F. Li, D. Han, L. Guo and L. Niu, Electrochemically Controlled Growth of Silver Nanocrystals on Graphene Thin Film and Applications for Efficient Nonenzymatic $\mathrm{H}_{2} \mathrm{O}_{2}$ Biosensor, Electrochim. Acta, 2013, 89, 222-228.

326 N. Zhu, S. Han, S. Gan, J. Ulstrup and Q. Chi, Graphene Paper Doped with Chemically Compatible Prussian Blue Nanoparticles as Nanohybrid Electrocatalyst, Adv. Funct. Mater., 2013, 23, 5297-5306.

327 P. Zhang, X. Zhang, S. Zhang, X. Lu, Q. Li, Z. Su and G. Wei, One-Pot Green Synthesis, Characterizations, and Biosensor Application of Self-Assembled Reduced Graphene OxideGold Nanoparticle Hybrid Membranes, J. Mater. Chem. B, 2013, 1, 6525-6531.

328 H. Teymourian, A. Salimi, S. Firoozi, A. Korani and S. Soltanian, One-Pot Hydrothermal Synthesis of Zirconium Dioxide Nanoparticles Decorated Reduced Graphene Oxide Composite as High Performance
Electrochemical Sensing and Biosensing Platform, Electrochim. Acta, 2014, 143, 196-206.

329 Q. Wang, M. Li, S. Szunerits and R. Boukherroub, Environmentally Friendly Reduction of Graphene Oxide Using Tyrosine for Nonenzymatic Amperometric $\mathrm{H}_{2} \mathrm{O}_{2}$ Detection, Electroanalysis, 2014, 26, 156-163.

330 S. K. Maji, S. Sreejith, A. K. Mandal, X. Ma and Y. Zhao, Immobilizing Gold Nanoparticles in Mesoporous Silica Covered Reduced Graphene Oxide: A Hybrid Material for Cancer Cell Detection through Hydrogen Peroxide Sensing, ACS Appl. Mater. Interfaces, 2014, 6, 13648-13656.

331 M. R. Mahmoudian, Y. Alias, W. J. Basirun, P. M. Woi and M. Sookhakian, Facile Preparation of $\mathrm{MnO}_{2}$ Nanotubes/ Reduced Graphene Oxide Nanocomposite for Electrochemical Sensing of Hydrogen Peroxide, Sens. Actuators, B, 2014, 201, 526-534.

332 B. Wu, N. Zhao, S. Hou and C. Zhang, Electrochemical Synthesis of Polypyrrole, Reduced Graphene Oxide, and Gold Nanoparticles Composite and its Application to Hydrogen Peroxide Biosensor, Nanomaterials, 2016, 6, 220.

333 Y. Sun, K. He, Z. Zhang, A. Zhou and H. Duan, Real-Time Electrochemical Detection of Hydrogen Peroxide Secretion in Live Cells by Pt Nanoparticles Decorated Graphene-Carbon Nanotube Hybrid Paper Electrode, Biosens. Bioelectron., 2015, 68, 358-364.

334 Y. Zhang, Z. Wang, Y. Ji, S. Liu and T. Zhang, Synthesis of $\mathrm{Ag}$ Nanoparticle-Carbon Nanotube-Reduced Graphene Oxide Hybrids for Highly Sensitive Non-Enzymatic Hydrogen Peroxide Detection, RSC Adv., 2015, 5, 3903739041.

335 J. Liu, X. Bo, Z. Zhao and L. Guo, Highly Exposed Pt Nanoparticles Supported on Porous Graphene for Electrochemical Detection of Hydrogen Peroxide in Living Cells, Biosens. Bioelectron., 2015, 74, 71-77.

336 M. Zhang, A. Halder, C. Hou, J. Ulstrup and Q. Chi, FreeStanding and Flexible Graphene Papers as Disposable Non-Enzymatic Electrochemical Sensors, Bioelectrochemistry, 2016, 109, 87-94.

337 H. Liu, K. Guo, C. Duan, X. Dong and J. Gao, Hollow $\mathrm{TiO}_{2}$ Modified Reduced Graphene Oxide Microspheres Encapsulating Hemoglobin for a Mediator-Free Biosensor, Biosens. Bioelectron., 2017, 87, 473-479.

338 V. Sharma and S. M. Mobin, Cytocompatible Peroxidase Mimic CuO:Graphene Nanosphere Composite as Colorimetric Dual Sensor for Hydrogen Peroxide and Cholesterol With its Logic Gate Implementation, Sens. Actuators, B, 2017, 240, 338-348.

339 Y. Zhao, D. Huo, J. Bao, M. Yang, M. Chen, J. Hou, H. Fa and C. Hou, Biosensor Based on 3D Graphene-Supported $\mathrm{Fe}_{3} \mathrm{O}_{4}$ Quantum Dots as Biomimetic Enzyme for In situ Detection of $\mathrm{H}_{2} \mathrm{O}_{2}$ Released from Living Cells, Sens. Actuators, B, 2017, 244, 1037-1044.

340 B. Dinesh, V. Mani, R. Saraswathi and S.-M. Chen, Direct electrochemistry of cytochrome $\mathrm{c}$ immobilized on a graphene oxide-carbon nanotube composite for picomolar detection of hydrogen peroxide, RSC Adv., 2014, 4, 28229-28237. 
341 E. Zor, M. E. Saglam, I. Akin, A. O. Saf, H. Bingol and M. Ersoz, Green Synthesis of Reduced Graphene Oxide/ Nanopolypyrrole Composite: Characterization and $\mathrm{H}_{2} \mathrm{O}_{2}$ Determination in Urine, RSC Adv., 2014, 4, 12457-12466.

342 M. Sadhukhan, T. Bhowmik, M. K. Kundu and S. Barman, Facile Synthesis of Carbon Quantum Dots and Thin Graphene Sheets for Non-Enzymatic Sensing of Hydrogen Peroxide, RSC Adv., 2014, 4, 4998-5005.

343 A. T. E. Vilian and S.-M. Chen, Simple Approach for the Immobilization of Horseradish Peroxidase on Poly-LHistidine Modified Reduced Graphene Oxide for Amperometric Determination of Dopamine and $\mathrm{H}_{2} \mathrm{O}_{2}$, RSC Adv., 2014, 4, 55867-55876.

344 W. Lei, L. Wu, W. Huang, Q. Hao, Y. Zhang and X. Xia, Microwave-Assisted Synthesis of Hemin-Graphene/ Poly(3,4-Ethylenedioxythiophene) Nanocomposite for a Biomimetic Hydrogen Peroxide Biosensor, J. Mater. Chem. B, 2014, 2, 4324-4330.

345 M. Zhang and Z. Wang, Nanostructured Silver NanowiresGraphene Hybrids for Enhanced Electrochemical Detection of Hydrogen Peroxide, Appl. Phys. Lett., 2013, 102, 213104.

346 L. Shang, B. Zeng and F. Zhao, Fabrication of Novel Nitrogen-Doped Graphene-Hollow AuPd Nanoparticle Hybrid Films for the Highly Efficient Electrocatalytic Reduction of $\mathrm{H}_{2} \mathrm{O}_{2}$, ACS Appl. Mater. Interfaces, 2015, 7, 122-128.

347 S. Li, J. Xiong, J. Shen, Y. Qin, J. Li, F. Chu, Y. Kong and L. Deng, A Novel Hydrogen Peroxide Sensor Based on Ag Nanoparticles Decorated Polyaniline/Graphene Composites, J. Appl. Polym. Sci., 2015, 132, 4-9.

348 S. Radhakrishnan and S. J. Kim, An Enzymatic Biosensor for Hydrogen Peroxide Based on One-Pot Preparation of $\mathrm{CeO}_{2}$-Reduced Graphene Oxide Nanocomposite, RSC Adv., 2015, 5, 12937-12943.

349 C. Karuppiah, S. Palanisamy, S.-M. Chen, V. Veeramani and P. Periakaruppan, A Novel Enzymatic Glucose Biosensor and Sensitive Non-Enzymatic Hydrogen Peroxide Sensor Based on Graphene and Cobalt Oxide Nanoparticles Composite Modified Glassy Carbon Electrode, Sens. Actuators, B, 2014, 196, 450-456.

350 Z. Yang, C. Qi, X. Zheng and J. Zheng, Facile Synthesis of Silver Nanoparticle-Decorated Graphene Oxide Nanocomposites and their Application for Electrochemical Sensing, New J. Chem., 2015, 39, 93589362.

351 S. Palanisamy, H. F. Lee, S.-M. Chen and B. Thirumalraj, An Electrochemical Facile Fabrication of Platinum Nanoparticle Decorated Reduced Graphene Oxide; Application for Enhanced Electrochemical Sensing of $\mathrm{H}_{2} \mathrm{O}_{2}$, RSC Adv., 2015, 5, 105567-105573.

352 J. Wang, H. Zhu, Y. Xu, W. Yang, A. Liu, F. Shan, M. Cao and J. Liu, Graphene Nanodots Encaged 3-D Gold Substrate as Enzyme Loading Platform for The Fabrication of High Performance Biosensors, Sens. Actuators, B, 2015, 220, 1186-1195.
353 J. Liu, M. Zhang, J. Liu and J. Zheng, Synthesis of Ag@Pt Core-Shell Nanoparticles Loaded onto Reduced Graphene Oxide and Investigation of its Electrosensing Properties, Anal. Methods, 2016, 8, 1084-1090.

354 J. Wang, H.-b. Sun, H.-y. Pan, Y.-y. Ding, J.-g. Wan, G.-h. Wang and M. Han, Detection of Hydrogen Peroxide at a Palladium Nanoparticle-Bilayer Graphene HybridModified Electrode, Sens. Actuators, B, 2016, 230, 690-696.

355 L. Ning, Y. Liu, J. Ma, X. Fan, G. Zhang, F. Zhang, W. Peng and Y. Li, Synthesis of Palladium, $\mathrm{ZnFe}_{2} \mathrm{O}_{4}$ Functionalized Reduced Graphene Oxide Nanocomposites as $\mathrm{H}_{2} \mathrm{O}_{2}$ Detector, Ind. Eng. Chem. Res., 2017, 56, 4327-4333.

356 V. Velusamy, S. Palanisamy, S.-M. Chen, T.-W. Chen, S. Selvam, S. K. Ramaraj and B.-S. Lou, Graphene Dispersed Cellulose Microfibers Composite for Efficient Immobilization of Hemoglobin and Selective Biosensor for Detection of Hydrogen Peroxide, Sens. Actuators, B, 2017, 252, 175-182.

357 Y. Qi, J. Bai, X. Ding and H.-M. Zhang, Electrochemically Prepared Three-dimensional Porous Nitrogen-doped Graphene Modified Electrode for Non-enzymatic Detection of Hydrogen Peroxide, Electroanalysis, 2017, 29, 2083-2089.

358 Y. Zhu, S. Lu, A. Gowri Manohari, X. Dong, F. Chen, W. Xu, Z. Shi and C. Xu, Polydopamine Interconnected Graphene Quantum Dots and Gold Nanoparticles for Enzymeless $\mathrm{H}_{2} \mathrm{O}_{2}$ Detection, J. Electroanal. Chem., 2017, 796, 75-81.

359 Y. Liu, X. Liu, Z. Guo, Z. Hu, Z. Xue and X. Lu, Horseradish Peroxidase Supported on Porous Graphene as a Novel Sensing Platform for Detection of Hydrogen Peroxide in Living Cells Sensitively, Biosens. Bioelectron., 2017, 87, 101-107.

360 M. Sookhakian, E. Zalnezhad and Y. Alias, Layer-by-Layer Electrodeposited Nanowall-Like Palladium-Reduced Graphene Oxide Film as a Highly-Sensitive Electrochemical Non-Enzymatic Sensor, Sens. Actuators, B, 2017, 241, 1-7.

361 L. A. Mercante, M. H. M. Facure, R. C. Sanfelice, F. L. Migliorini, L. H. C. Mattoso and D. S. Correa, OnePot Preparation of PEDOT:PSS-Reduced Graphene Decorated with $\mathrm{Au}$ Nanoparticles for Enzymatic Electrochemical Sensing of $\mathrm{H}_{2} \mathrm{O}_{2}$, Appl. Surf. Sci., 2017, 407, 162-170.

362 N. Yusoff, P. Rameshkumar, M. S. Mehmood, A. Pandikumar, H. W. Lee and N. M. Huang, Ternary Nanohybrid of Reduced Graphene Oxide-Nafion@Silver Nanoparticles for Boosting the Sensor Performance in Non-Enzymatic Amperometric Detection of Hydrogen Peroxide, Biosens. Bioelectron., 2017, 87, 1020-1028.

363 C. Karthikeyan, K. Ramachandran, S. Sheet, D. J. Yoo, Y. S. Lee, Y. S. kumar, A. R. Kim and G. G. kumar, Pigeon-Excreta-Mediated Synthesis of Reduced Graphene Oxide (rGO)/CuFe ${ }_{2} \mathrm{O}_{4}$ Nanocomposite and Its Catalytic Activity toward Sensitive and Selective Hydrogen Peroxide Detection, ACS Sustainable Chem. Eng., 2017, 5, 4897-4905.

364 C. Cheng, C. Zhang, X. Gao, Z. Zhuang, C. Du and W. Chen, 3D Network and 2D Paper of Reduced Graphene Oxide/ 
$\mathrm{Cu}_{2} \mathrm{O}$ Composite for Electrochemical Sensing of Hydrogen Peroxide, Anal. Chem., 2018, 90, 1983-1991.

365 S. Kubendhiran, B. Thirumalraj, S.-M. Chen and C. Karuppiah, Electrochemical Co-Preparation of Cobalt Sulfide/Reduced Graphene Oxide Composite for Electrocatalytic Activity and Determination of $\mathrm{H}_{2} \mathrm{O}_{2}$ in Biological Samples, J. Colloid Interface Sci., 2018, 509, 153-162.

366 Z. Bai, W. Dong, Y. Ren, C. Zhang and Q. Chen, Preparation of Nano $\mathrm{Au}$ and Pt Alloy Microspheres Decorated with Reduced Graphene Oxide for Nonenzymatic Hydrogen Peroxide Sensing, Langmuir, 2018, 34, 2235-2244.

367 C.-L. Sun, J.-S. Su, S.-Y. Lai and Y.-J. Lu, Size Effects of Pt Nanoparticle/Graphene Composite Materials on the Electrochemical Sensing of Hydrogen Peroxide, $J$. Nanomater., 2015, 2015, 861061.

368 S. Chen, R. Yuan, Y. Chai and F. Hu, Electrochemical Sensing of Hydrogen Peroxide Using Metal Nanoparticles: A Review, Microchim. Acta, 2013, 180, 15-32.

369 S. Guo, D. Wen, Y. Zhai, S. Dong and E. Wang, Platinum Nanoparticle Ensemble-on-Graphene Hybrid Nanosheet: One-Pot, Rapid Synthesis, and Used as New Electrode Material for Electrochemical Sensing, ACS Nano, 2010, 4, 3959-3968.

370 B. Zhan, C. Liu, H. Shi, C. Li, L. Wang, W. Huang and X. Dong, A Hydrogen Peroxide Electrochemical Sensor Based on Silver Nanoparticles Decorated ThreeDimensional Graphene, Appl. Phys. Lett., 2014, 104, 243704.

371 B. Yu, J. Feng, S. Liu and T. Zhang, Preparation of Reduced Graphene Oxide Decorated with High Density Ag Nanorods for Non-Enzymatic Hydrogen Peroxide Detection, RSC Adv., 2013, 3, 14303-14307.

372 D. Chen, X. Zhuang, J. Zhai, Y. Zheng, H. Lu and L. Chen, Preparation of Highly Sensitive Pt Nanoparticles-Carbon Quantum Dots/Ionic Liquid Functionalized Graphene Oxide Nanocomposites and Application for $\mathrm{H}_{2} \mathrm{O}_{2}$ Detection, Sens. Actuators, B, 2018, 255, 1500-1506.

373 Y. Sun, M. Luo, Y. Qin, S. Zhu, Y. Li, N. Xu, X. Meng, Q. Ren, L. Wang and S. Guo, Atomic-Thick PtNi Nanowires Assembled on Graphene for High-Sensitivity Extracellular Hydrogen Peroxide Sensors, ACS Appl. Mater. Interfaces, 2017, 9, 34715-34721.

374 C. Chen, Q.-H. Yang, Y. Yang, W. Lv, Y. Wen, P.-X. Hou, M. Wang and H.-M. Cheng, Self-Assembled Free-Standing Graphite Oxide Membrane, Adv. Mater., 2009, 21, 30073011.

375 H.-J. Qiu, Y. Guan, P. Luo and Y. Wang, Recent Advance in Fabricating Monolithic 3D Porous Graphene and Their Applications in Biosensing and Biofuel Cells, Biosens. Bioelectron., 2017, 89, 85-95.

376 H.-P. Cong, J.-F. Chen and S.-H. Yu, Graphene-Based Macroscopic Assemblies and Architectures: An Emerging Material System, Chem. Soc. Rev., 2014, 43, 7295-7325.

377 D. Lin, Z. Su and G. Wei, Three-Dimensional Porous Reduced Graphene Oxide Decorated with $\mathrm{MoS}_{2}$ Quantum Dots for Electrochemical Determination of Hydrogen Peroxide, Mater. Today Chem., 2018, 7, 76-83.
378 T. Zhang, Y. Gu, C. Li, X. Yan, N. Lu, H. Liu, Z. Zhang and H. Zhang, Fabrication of Novel Electrochemical Biosensor Based on Graphene Nanohybrid to Detect $\mathrm{H}_{2} \mathrm{O}_{2}$ Released from Living Cells with Ultrahigh Performance, ACS Appl. Mater. Interfaces, 2017, 9, 37991-37999.

379 Y. Zhang, J. Xiao, Q. Lv, L. Wang, X. Dong, M. Asif, J. Ren, W. He, Y. Sun, F. Xiao and S. Wang, In situ Electrochemical Sensing and Real-Time Monitoring Live Cells Based on Freestanding Nanohybrid Paper Electrode Assembled from 3D Functionalized Graphene Framework, ACS Appl. Mater. Interfaces, 2017, 9, 38201-38210.

$380 \mathrm{~J}$. Ju and W. Chen, In situ Growth of Surfactant-Free Gold Nanoparticles on Nitrogen-Doped Graphene Quantum Dots for Electrochemical Detection of Hydrogen Peroxide in Biological Environments, Anal. Chem., 2015, 87, 19031910.

381 Y. Zhang, X. Bai, X. Wang, K.-K. Shiu, Y. Zhu and H. Jiang, Highly Sensitive Graphene-Pt Nanocomposites Amperometric Biosensor and Its Application in Living Cell $\mathrm{H}_{2} \mathrm{O}_{2}$ Detection, Anal. Chem., 2014, 86, 9459-9465.

382 L. Wang, Y. Zhang, C. Cheng, X. Liu, H. Jiang and X. Wang, Highly Sensitive Electrochemical Biosensor for Evaluation of Oxidative Stress Based on the Nanointerface of Graphene Nanocomposites Blended with Gold, $\mathrm{Fe}_{3} \mathrm{O}_{4}$, and Platinum Nanoparticles, ACS Appl. Mater. Interfaces, 2015, 7, 18441-18449.

383 Y. Sun, M. Luo, X. Meng, J. Xiang, L. Wang, Q. Ren and S. Guo, Graphene/Intermetallic PtPb Nanoplates Composites for Boosting Electrochemical Detection of $\mathrm{H}_{2} \mathrm{O}_{2}$ Released from Cells, Anal. Chem., 2017, 89, 37613767.

384 Y. He, G. Huang and H. Cui, Quenching the Chemiluminescence of Acridinium Ester by Graphene Oxide for Label-Free and Homogeneous DNA Detection, ACS Appl. Mater. Interfaces, 2013, 5, 11336-11340.

385 J. Cervenka, A. Budi, N. Dontschuk, A. Stacey, A. Tadich, K. J. Rietwyk, A. Schenk, M. T. Edmonds, Y. Yin, N. Medhekar, M. Kalbac and C. I. Pakes, Graphene Field Effect Transistor as a Probe of Electronic Structure and Charge Transfer at Organic Molecule-Graphene Interfaces, Nanoscale, 2015, 7, 1471-1478.

386 T. Premkumar and K. E. Geckeler, Graphene-DNA Hybrid Materials: Assembly, Applications, and Prospects, Prog. Polym. Sci., 2012, 37, 515-529.

387 X. Zhu, L. Sun, Y. Chen, Z. Ye, Z. Shen and G. Li, Combination of Cascade Chemical Reactions with Graphene-DNA Interaction to Develop New Strategy for Biosensor Fabrication, Biosens. Bioelectron., 2013, 47, 3237.

388 N. Dontschuk, A. Stacey, A. Tadich, K. J. Rietwyk, A. Schenk, M. T. Edmonds, O. Shimoni, C. I. Pakes, S. Prawer and J. Cervenka, A Graphene Field-Effect Transistor as a Molecule-Specific Probe of DNA Nucleobases, Nat. Commun., 2015, 6, 6563.

389 J.-H. Lee, Y.-K. Choi, H.-J. Kim, R. H. Scheicher and J.-H. Cho, Physisorption of DNA Nucleobases on h-BN 
and Graphene: vdW-Corrected DFT Calculations, J. Phys. Chem. C, 2013, 117, 13435-13441.

390 Y. Yin, J. Cervenka and N. V. Medhekar, Molecular DipoleDriven Electronic Structure Modifications of DNA/RNA Nucleobases on Graphene, J. Phys. Chem. Lett., 2017, 8, 3087-3094.

391 A.-L. Liu, G.-X. Zhong, J.-Y. Chen, S.-H. Weng, H.-N. Huang, W. Chen, L.-Q. Lin, Y. Lei, F.-H. Fu, Z.-l. Sun, X.-H. Lin, J.-H. Lin and S.-Y. Yang, A Sandwich-Type DNA Biosensor Based on Electrochemical Co-Reduction Synthesis of Graphene-Three Dimensional Nanostructure Gold Nanocomposite Films, Anal. Chim. Acta, 2013, 767, 50-58.

392 T. Yang, Q. Li, L. Meng, X. Wang, W. Chen and K. Jiao, Synchronous Electrosynthesis of Poly(xanthurenic acid)Reduced Graphene Oxide Nanocomposite for Highly Sensitive Impedimetric Detection of DNA, ACS Appl. Mater. Interfaces, 2013, 5, 3495-3499.

393 T. Yang, L. Meng, X. Wang, L. Wang and K. Jiao, Direct Electrochemical DNA Detection Originated from the SelfRedox Signal of Sulfonated Polyaniline Enhanced by Graphene Oxide in Neutral Solution, ACS Appl. Mater. Interfaces, 2013, 5, 10889-10894.

394 A. Benvidi, N. Rajabzadeh, M. Mazloum-Ardakani, M. M. Heidari and A. Mulchandani, Simple and label-free electrochemical impedance amelogenin gene hybridization biosensing based on reduced graphene oxide, Biosens. Bioelectron., 2014, 58, 145-152.

395 H.-P. Peng, Y. Hu, P. Liu, Y.-N. Deng, P. Wang, W. Chen, A.-L. Liu, Y.-Z. Chen and X.-H. Lin, Label-Free Electrochemical DNA Biosensor for Rapid Detection of Mutidrug Resistance Gene Based on $\mathrm{Au}$ Nanoparticles/ Toluidine Blue-Graphene Oxide Nanocomposites, Sens. Actuators, B, 2015, 207, 269-276.

396 H. Huang, W. Bai, C. Dong, R. Guo and Z. Liu, An Ultrasensitive Electrochemical DNA Biosensor Based on Graphene/Au Nanorod/Polythionine for Human Papillomavirus DNA Detection, Biosens. Bioelectron., 2015, 68, 442-446.

397 L.-X. Fang, J.-T. Cao and K.-J. Huang, A Sensitive Electrochemical Biosensor for Specific DNA Sequence Detection Based on Flower-Like VS2, Graphene and Au Nanoparticles Signal Amplification, J. Electroanal. Chem., 2015, 746, 1-8.

398 A. Benvidi, A. D. Firouzabadi, S. M. Moshtaghiun, M. Mazloum-Ardakani and M. D. Tezerjani, Ultrasensitive DNA Sensor Based on Gold Nanoparticles/Reduced Graphene Oxide/Glassy Carbon Electrode, Anal. Biochem., 2015, 484, 24-30.

399 Y. Wang, X. Bai, W. Wen, X. Zhang and S. Wang, Ultrasensitive Electrochemical Biosensor for HIV Gene Detection Based on Graphene Stabilized Gold Nanoclusters with Exonuclease Amplification, ACS Appl. Mater. Interfaces, 2015, 7, 18872-18879.

400 B. Li, G. Pan, N. D. Avent, R. B. Lowry, T. E. Madgett and P. L. Waines, Graphene Electrode Modified with Electrochemically Reduced Graphene Oxide for Label-Free DNA Detection, Biosens. Bioelectron., 2015, 72, 313-319.
401 W. Wang, T. Bao, X. Zeng, H. Xiong, W. Wen, X. Zhang and S. Wang, Ultrasensitive Electrochemical DNA Biosensor Based on Functionalized Gold Clusters/Graphene Nanohybrids Coupling with Exonuclease III-Aided Cascade Target Recycling, Biosens. Bioelectron., 2017, 91, 183-189.

402 Y. Z. Zhang and W. Jiang, Decorating Graphene Sheets with Gold Nanoparticles for the Detection of Sequence-Specific DNA, Electrochim. Acta, 2012, 71, 239-245.

403 C. Liu, D. Jiang, G. Xiang, L. Liu, F. Liu and X. Pu, An Electrochemical DNA Biosensor for the Detection of Mycobacterium tuberculosis, Based on Signal Amplification of Graphene and a Gold Nanoparticle-Polyaniline Nanocomposite, Analyst, 2014, 139, 5460-5465.

404 A. Wu, Q. Wang, Q. Zhu, J. Ni and F. Gao, A Facile and Highly Sensitive Impedimetric DNA Biosensor with Ultralow Background Response Based on In situ Reduced Graphene Oxide, RSC Adv., 2015, 5, 90983-90990.

405 M. Chen, C. Hou, D. Huo, J. Bao, H. Fa and C. Shen, An electrochemical DNA biosensor based on nitrogen-doped graphene/Au nanoparticles for human multidrug resistance gene detection, Biosens. Bioelectron., 2016, 85, 684-691.

406 K. Zhang, N. Zhang, L. Zhang, H. Wang, H. Shi and Q. Liu, Label-free impedimetric sensing platform for microRNA-21 based on $\mathrm{ZrO}_{2}$-reduced graphene oxide nanohybrids coupled with catalytic hairpin assembly amplification, RSC Adv., 2018, 8, 16146-16151.

407 N. Gao, F. Gao, S. He, Q. Zhu, J. Huang, H. Tanaka and Q. Wang, Graphene Oxide Directed in situ Deposition of Electroactive Silver Nanoparticles and its Electrochemical Sensing Application for DNA Analysis, Anal. Chim. Acta, 2017, 951, 58-67.

408 Q. Gong, Y. Wang and H. Yang, A Sensitive Impedimetric DNA Biosensor for the Determination of the HIV Gene Based on Graphene-Nafion Composite Film, Biosens. Bioelectron., 2017, 89, 565-569.

409 Q. Gong, H. Yang, Y. Dong and W. Zhang, A Sensitive Impedimetric DNA Biosensor for the Determination of the HIV Gene Based on Electrochemically Reduced Graphene Oxide, Anal. Methods, 2015, 7, 2554-2562.

410 X. Niu, W. Zheng, C. Yin, W. Weng, G. Li, W. Sun and Y. Men, Electrochemical DNA Biosensor Based on Gold Nanoparticles and Partially Reduced Graphene Oxide Modified Electrode for the Detection of Listeria monocytogenes Hly Gene Sequence, J. Electroanal. Chem., 2017, 806, 116-122.

411 T. Yang, H. Chen, Z. Qiu, R. Yu, S. Luo, W. Li and K. Jiao, Direct Electrochemical Vibrio DNA Sensing Adopting Highly Stable Graphene-Flavin Mononucleotide Aqueous Dispersion Modified Interface, ACS Appl. Mater. Interfaces, 2018, 10, 4540-4547.

412 M. Arvand, M. Sanayeei and S. Hemmati, Label-Free Electrochemical DNA Biosensor for Guanine and Adenine by ds-DNA/Poly(L-Cysteine)/ $\mathrm{Fe}_{3} \mathrm{O}_{4}$ Nanoparticles-Graphene Oxide Nanocomposite Modified Electrode, Biosens. Bioelectron., 2018, 102, 70-79. 
413 P. T. K. Loan, D. Wu, C. Ye, X. Li, V. T. Tra, Q. Wei, L. Fu, A. Yu, L.-J. Li and C.-T. Lin, Hall Effect Biosensors with Ultraclean Graphene Film for Improved Sensitivity of Label-Free DNA Detection, Biosens. Bioelectron., 2018, 99, 85-91.

414 H. Imran, P. N. Manikandan and V. Dharuman, Graphene Oxide Supported Liposomes for Efficient Label Free Electrochemical DNA Biosensing, Sens. Actuators, B, 2018, 260, 841-851.

415 Y. Ye, J. Xie, Y. Ye, X. Cao, H. Zheng, X. Xu and Q. Zhang, A Label-Free Electrochemical DNA Biosensor Based on Thionine Functionalized Reduced Graphene Oxide, Carbon, 2018, 129, 730-737.

416 R. Z. A. R. Jamaluddin, L. Y. Heng, L. L. Tan and K. F. Chong, A Biosensor for Genetic Modified Soybean DNA Determination via Adsorption of Anthraquinone-2Sulphonic Acid in Reduced Graphene Oxide, Electroanalysis, 2018, 30, 250-258.

417 Q. Feng, X. Zhao, Y. Guo, M. Liu and P. Wang, Stochastic DNA Walker for Electrochemical Biosensing Sensitized with Gold Nanocages@Graphene Nanoribbons, Biosens. Bioelectron., 2018, 108, 97-102.

418 T. Hu, L. Zhang, W. Wen, X. Zhang and S. Wang, Enzyme Catalytic Amplification of miRNA-155 Detection with Graphene Quantum Dot-Based Electrochemical Biosensor, Biosens. Bioelectron., 2016, 77, 451-456.

419 M. Azimzadeh, M. Rahaie, N. Nasirizadeh, K. Ashtari and H. Naderi-Manesh, An Electrochemical Nanobiosensor for Plasma miRNA-155, Based on Graphene Oxide and Gold Nanorod, for Early Detection of Breast Cancer, Biosens. Bioelectron., 2016, 77, 99-106.

420 H.-L. Shuai, K.-J. Huang, W.-J. Zhang, X. Cao and M.-P. Jia, Sandwich-Type microRNA Biosensor Based on Magnesium Oxide Nanoflower and Graphene Oxide-Gold Nanoparticles Hybrids Coupling with Enzyme Signal Amplification, Sens. Actuators, B, 2017, 243, 403-411.

421 E. Eksin, S. K. Bikkarolla, A. Erdem and P. Papakonstantinou, Chitosan/Nitrogen Doped Reduced Graphene Oxide Modified Biosensor for Impedimetric Detection of microRNA, Electroanalysis, 2018, 30, 551-560.

422 Y.-H. Wang, K.-J. Huang, X. Wu, Y.-Y. Ma, D.-L. Song, C.-Y. Du and S.-H. Chang, Ultrasensitive SupersandwichType Biosensor for Enzyme-Free Amplified microRNA Detection Based on N-Doped Graphene/Au Nanoparticles and Hemin/G-Quadruplexes, J. Mater. Chem. B, 2018, 6, 2134-2142.

423 P.-P. Gai, C.-E. Zhao, Y. Wang, E. S. Abdel-Halim, J.-R. Zhang and J.-J. Zhu, NADH Dehydrogenase-Like Behavior of Nitrogen-Doped Graphene and Its Application in $\mathrm{NAD}^{+}$-Dependent Dehydrogenase Biosensing, Biosens. Bioelectron., 2014, 62, 170-176.

424 Z. Li, W. Su, S. Liu and X. Ding, An Electrochemical Biosensor Based on DNA Tetrahedron/Graphene Composite Film for Highly Sensitive Detection of $\mathrm{NADH}$, Biosens. Bioelectron., 2015, 69, 287-293.

425 M. Govindhan, M. Amiri and A. Chen, Au Nanoparticle/ Graphene Nanocomposite as a Platform for the Sensitive
Detection of NADH in Human Urine, Biosens. Bioelectron., 2015, 66, 474-480.

426 D. Sangamithirai, V. Narayanan, B. Muthuraaman and A. Stephen, Investigations on the Performance of Poly(OAnisidine)/Graphene Nanocomposites for the Electrochemical Detection of NADH, Mater. Sci. Eng., C, 2015, 55, 579-591.

427 S. Mutyala and J. Mathiyarasu, A Highly Sensitive NADH Biosensor Using Nitrogen Doped Graphene Modified Electrodes, J. Electroanal. Chem., 2016, 775, 329-336.

428 O.-M. Istrate, L. Rotariu, V. E. Marinescu and C. Bala, NADH Sensing Platform Based on Electrochemically Generated Reduced Graphene Oxide-Gold Nanoparticles Composite Stabilized With Poly(Allylamine Hydrochloride), Sens. Actuators, B, 2016, 223, 697-704.

429 J. Balamurugan, T. D. Thanh, N. H. Kim and J. H. Lee, Facile Fabrication of FeN Nanoparticles/Nitrogen-Doped Graphene Core-Shell Hybrid and Its Use as a Platform for NADH Detection in Human Blood Serum, Biosens. Bioelectron., 2016, 83, 68-76.

430 S. Han, T. Du, H. Jiang and X. Wang, Synergistic Effect of Pyrroloquinoline Quinone and Graphene Nano-Interface for Facile Fabrication of Sensitive NADH Biosensor, Biosens. Bioelectron., 2017, 89, 422-429.

431 G. A. Tı̆̆, Highly Sensitive Amperometric Biosensor for Determination of $\mathrm{NADH}$ and Ethanol Based on $\mathrm{Au}-\mathrm{Ag}$ Nanoparticles/Poly(L-Cysteine)/Reduced Graphene Oxide Nanocomposite, Talanta, 2017, 175, 382-389.

432 J. Wang, A. Shi, X. Fang, X. Han and Y. Zhang, An Ultrasensitive Supersandwich Electrochemical DNA Biosensor Based on Gold Nanoparticles Decorated Reduced Graphene Oxide, Anal. Biochem., 2015, 469, 71-75.

433 Q. Wang, J. Lei, S. Deng, L. Zhang and H. Ju, GrapheneSupported Ferric Porphyrin as a Peroxidase Mimic for Electrochemical DNA Biosensing, Chem. Commun., 2013, 49, 916-918.

434 A. Singh, G. Sinsinbar, M. Choudhary, V. Kumar, R. Pasricha, H. N. Verma, S. P. Singh and K. Arora, Graphene Oxide-Chitosan Nanocomposite Based Electrochemical DNA Biosensor for Detection of Typhoid, Sens. Actuators, B, 2013, 185, 675-684.

435 X. Han, X. Fang, A. Shi, J. Wang and Y. Zhang, An Electrochemical DNA Biosensor Based on Gold Nanorods Decorated Graphene Oxide Sheets for Sensing Platform, Anal. Biochem., 2013, 443, 117-123.

436 K. Jayakumar, M. B. Camarada, V. Dharuman, R. Rajesh, R. Venkatesan, H. Ju, M. Maniraj, A. Rai, S. R. Barman and Y. wen, Layer-by-Layer-Assembled AuNPs-Decorated First-Generation Poly(amidoamine) Dendrimer with Reduced Graphene Oxide Core as Highly Sensitive Biosensing Platform with Controllable 3D Nanoarchitecture for Rapid Voltammetric Analysis of Ultratrace DNA Hybridization, ACS Appl. Mater. Interfaces, 2018, 10, 21541-21555.

437 F. Traversi, C. Raillon, S. M. Benameur, K. Liu, S. Khlybov, M. Tosun, D. Krasnozhon, A. Kis and A. Radenovic, Detecting the Translocation of DNA Through a Nanopore 
Using Graphene Nanoribbons, Nat. Nanotechnol., 2013, 8, 939-945.

438 S. K. Min, W. Y. Kim, Y. Cho and K. S. Kim, Fast DNA Sequencing with a Graphene-Based Nanochannel Device, Nat. Nanotechnol., 2011, 6, 162-165.

439 S. J. Heerema and C. Dekker, Graphene Nanodevices for DNA Sequencing, Nat. Nanotechnol., 2016, 11, 127-136.

440 S. M. Avdoshenko, D. Nozaki, C. G. da Rocha, J. W. González, M. H. Lee, R. Gutierrez and G. Cuniberti, Dynamic and Electronic Transport Properties of DNA Translocation through Graphene Nanopores, Nano Lett., 2013, 13, 1969-1976.

441 H. Sadeghi, L. Algaragholy, T. Pope, S. Bailey, D. Visontai, D. Manrique, J. Ferrer, V. Garcia-Suarez, S. Sangtarash and C. J. Lambert, Graphene Sculpturene Nanopores for DNA Nucleobase Sensing, J. Phys. Chem. B, 2014, 118, 6908-6914.

442 E. A. Hunt, D. Broyles, T. Head and S. K. Deo, MicroRNA Detection: Current Technology and Research Strategies, Annu. Rev. Anal. Chem., 2015, 8, 217-237.

443 Y.-X. Chen, K.-J. Huang and K.-X. Niu, Recent Advances in Signal Amplification Strategy Based on Oligonucleotide and Nanomaterials for MicroRNA Detection-A Review, Biosens. Bioelectron., 2018, 99, 612-624.

444 T. Kilic, A. Erdem, M. Ozsoz and S. Carrara, MicroRNA Biosensors: Opportunities and Challenges among Conventional and Commercially Available Techniques, Biosens. Bioelectron., 2018, 99, 525-546.

445 A. R. Cardoso, F. T. C. Moreira, R. Fernandes and M. G. F. Sales, Novel and Simple Electrochemical Biosensor Monitoring Attomolar Levels of miRNA-155 in Breast Cancer, Biosens. Bioelectron., 2016, 80, 621-630.

446 Z. Li, Y. Huang, L. Chen, X. Qin, Z. Huang, Y. Zhou, Y. Meng, J. Li, S. Huang, Y. Liu, W. Wang, Q. Xie and S. Yao, Amperometric Biosensor for $\mathrm{NADH}$ and Ethanol Based on Electroreduced Graphene Oxide-Polythionine Nanocomposite Film, Sens. Actuators, B, 2013, 181, 280287.

447 M. A. Tabrizi and Z. Zand, A Facile One-Step Method for the Synthesis of Reduced Graphene Oxide Nanocomposites by $\mathrm{NADH}$ as Reducing Agent and Its Application in NADH Sensing, Electroanalysis, 2013, 26, 171-177.

448 J. M. Savitt, V. L. Dawson and T. M. Dawson, Diagnosis and Treatment of Parkinson Disease: Molecules to Medicine, $J$. Clin. Invest., 2006, 116, 1744-1754.

449 T. Xiao, F. Wu, J. Hao, M. Zhang, P. Yu and L. Mao, In vivo Analysis with Electrochemical Sensors and Biosensors, Anal. Chem., 2017, 89, 300-313.

450 A. C. Schmidt, X. Wang, Y. Zhu and L. A. Sombers, Carbon Nanotube Yarn Electrodes for Enhanced Detection of Neurotransmitter Dynamics in Live Brain Tissue, ACS Nano, 2013, 7, 7864-7873.

451 M. Zhang, P. Yu and L. Mao, Rational Design of Surface/ Interface Chemistry for Quantitative In vivo Monitoring of Brain Chemistry, Acc. Chem. Res., 2012, 45, 533-543.

452 L. Falat and H. Y. Cheng, Voltammetric Differentiation of Ascorbic Acid and Dopamine at an Electrochemically
Treated Graphite/Epoxy Electrode, Anal. Chem., 1982, 54, 2108-2111.

453 L. Zhang, X. Lin and Y. Sun, Separation of Anodic Peaks of Ascorbic Acid and Dopamine at an A-Alanine Covalently Modified Glassy Carbon Electrode, Analyst, 2001, 126, 1760-1763.

454 X. Mao, G. C. Rutledge and T. A. Hatton, Nanocarbon-Based Electrochemical Systems for Sensing, Electrocatalysis, and Energy Storage, Nano Today, 2014, 9, 405-432.

455 A. Ambrosi, T. Sasaki and M. Pumera, Platelet Graphite Nanofibers for Electrochemical Sensing and Biosensing: The Influence of Graphene Sheet Orientation, Chem.Asian J., 2010, 5, 266-271.

456 S. Devaramani, M. Sreeramareddygari, M. R. Reddy and R. Thippeswamy, Covalently Anchored p-Aminobenzene Sulfonate Multilayer on a Graphite Pencil Lead Electrode: A Highly Selective Electrochemical Sensor for Dopamine, Electroanalysis, 2017, 29, 1410-1417.

457 F. Gao, X. Cai, X. Wang, C. Gao, S. Liu, F. Gao and Q. Wang, Highly Sensitive and Selective Detection of Dopamine in the Presence of Ascorbic Acid at Graphene Oxide Modified Electrode, Sens. Actuators, B, 2013, 186, 380-387.

458 S. Li, Y. Ma, Y. Liu, G. Xin, M. Wang, Z. Zhang and Z. Liu, Electrochemical sensor based on a three dimensional nanostructured $\mathrm{MoS}_{2}$ nanosphere-PANI/reduced graphene oxide composite for simultaneous detection of ascorbic acid, dopamine, and uric acid, $R S C A d v ., 2019$, 9, 29973003.

459 Y.-R. Kim, S. Bong, Y.-J. Kang, Y. Yang, R. K. Mahajan, J. S. Kim and H. Kim, Electrochemical Detection of Dopamine in the Presence of Ascorbic Acid Using Graphene Modified Electrodes, Biosens. Bioelectron., 2010, 25, 2366-2369.

460 C.-L. Sun, H.-H. Lee, J.-M. Yang and C.-C. Wu, The Simultaneous Electrochemical Detection of Ascorbic Acid, Dopamine, and Uric Acid Using Graphene/Size-Selected Pt Nanocomposites, Biosens. Bioelectron., 2011, 26, 3450-3455.

461 C.-L. Sun, C.-T. Chang, H.-H. Lee, J. Zhou, J. Wang, T.-K. Sham and W.-F. Pong, Microwave-Assisted Synthesis of a Core-Shell MWCNT/GONR Heterostructure for the Electrochemical Detection of Ascorbic Acid, Dopamine, and Uric Acid, ACS Nano, 2011, 5, 7788-7795.

462 Z.-H. Sheng, X.-Q. Zheng, J.-Y. Xu, W.-J. Bao, F.-B. Wang and X.-H. Xia, Electrochemical Sensor Based on Nitrogen Doped Graphene: Simultaneous Determination of Ascorbic Acid, Dopamine and Uric Acid, Biosens. Bioelectron., 2012, 34, 125-131.

463 J. Ping, J. Wu, Y. Wang and Y. Ying, Simultaneous Determination of Ascorbic Acid, Dopamine and Uric Acid Using High-Performance Screen-Printed Graphene Electrode, Biosens. Bioelectron., 2012, 34, 70-76.

464 X. Tian, C. Cheng, H. Yuan, J. Du, D. Xiao, S. Xie and M. M. F. Choi, Simultaneous Determination of L-Ascorbic Acid, Dopamine and Uric Acid With Gold Nanoparticles$\beta$-Cyclodextrin-Graphene-Modified Electrode by Square Wave Voltammetry, Talanta, 2012, 93, 79-85. 
465 X. Wang, M. Wu, W. Tang, Y. Zhu, L. Wang, Q. Wang, P. He and Y. Fang, Simultaneous Electrochemical Determination of Ascorbic Acid, Dopamine and Uric Acid Using a Palladium Nanoparticle/Graphene/Chitosan Modified Electrode, J. Electroanal. Chem., 2013, 695, 10-16.

466 H. Zhang, P. Gai, R. Cheng, L. Wu, X. Zhang and J. Chen, Self-Assembly Synthesis of a Hierarchical Structure Using Hollow Nitrogen-Doped Carbon Spheres as Spacers to Separate the Reduced Graphene Oxide for Simultaneous Electrochemical Determination of Ascorbic Acid, Dopamine and Uric Acid, Anal. Methods, 2013, 5, 3591-3600.

467 X. Weng, Q. Cao, L. Liang, J. Chen, C. You, Y. Ruan, H. Lin and $\mathrm{L} . \mathrm{Wu}$, Simultaneous Determination of Dopamine and Uric Acid Using Layer-by-Layer Graphene and Chitosan Assembled Multilayer Films, Talanta, 2013, 117, 359-365.

468 P. Manivel, M. Dhakshnamoorthy, A. Balamurugan, N. Ponpandian, D. Mangalaraj and C. Viswanathan, Conducting Polyaniline-Graphene Oxide Fibrous Nanocomposites: Preparation, Characterization and Simultaneous Electrochemical Detection of Ascorbic Acid, Dopamine and Uric Acid, RSC Adv., 2013, 3, 14428-14437.

469 Q. Lian, Z. He, Q. He, A. Luo, K. Yan, D. Zhang, X. Lu and X. Zhou, Simultaneous Determination of Ascorbic Acid, Dopamine and Uric Acid Based on Tryptophan Functionalized Graphene, Anal. Chim. Acta, 2014, 823, 3239.

470 X. Liu, L. Zhang, S. Wei, S. Chen, X. Ou and Q. Lu, Overoxidized Polyimidazole/Graphene Oxide Copolymer Modified Electrode for the Simultaneous Determination of Ascorbic Acid, Dopamine, Uric Acid, Guanine and Adenine, Biosens. Bioelectron., 2014, 57, 232-238.

471 Y. J. Yang and W. Li, CTAB Functionalized Graphene Oxide/ Multiwalled Carbon Nanotube Composite Modified Electrode for the Simultaneous Determination of Ascorbic Acid, Dopamine, Uric Acid and Nitrite, Biosens. Bioelectron., 2014, 56, 300-306.

472 S. Ramakrishnan, K. R. Pradeep, A. Raghul, R. Senthilkumar, M. Rangarajan and N. K. Kothurkar, One-Step Synthesis of Pt-Decorated Graphene-Carbon Nanotubes for the Electrochemical Sensing of Dopamine, Uric Acid and Ascorbic Acid, Anal. Methods, 2015, 7, 779786.

473 S. Qi, B. Zhao, H. Tang and X. Jiang, Determination of Ascorbic Acid, Dopamine, and Uric Acid by a Novel Electrochemical Sensor Based on Pristine Graphene, Electrochim. Acta, 2015, 161, 395-402.

474 Q. Wang and Q. Tang, Improved Sensing of Dopamine and Ascorbic Acid Using a Glassy Carbon Electrode Modified with Electrochemically Synthesized Nickel-Cobalt Hexacyanoferrate Microparticles Deposited on Graphene, Microchim. Acta, 2015, 182, 671-677.

475 Y.-T. Shieh and H.-F. Jiang, Graphene Oxide-Assisted Dispersion of Carbon Nanotubes in Sulfonated ChitosanModified Electrode for Selective Detections Of Dopamine, Uric Acid, and Ascorbic Acid, J. Electroanal. Chem., 2015, 736, 132-138.
476 S.-M. Li, Y.-S. Wang, S.-T. Hsiao, W.-H. Liao, C.-W. Lin, S.-Y. Yang, H.-W. Tien, C.-C. M. Ma and C.-C. Hu, Fabrication of a Silver Nanowire-Reduced Graphene OxideBased Electrochemical Biosensor and its Enhanced Sensitivity in the Simultaneous Determination of Ascorbic Acid, Dopamine, and Uric Acid, J. Mater. Chem. C, 2015, 3, 9444-9453.

477 Y. Zhang, Y. Ji, Z. Wang, S. Liu and T. Zhang, Electrodeposition Synthesis of Reduced Graphene Oxide-Carbon Nanotube Hybrids on Indium Tin Oxide Electrode for Simultaneous Electrochemical Detection of Ascorbic Acid, Dopamine and Uric Acid, RSC Adv., 2015, 5, 106307-106314.

478 J. Jiang and X. Du, Sensitive Electrochemical Sensors for Simultaneous Determination of Ascorbic Acid, Dopamine, and Uric Acid Based on Au@Pd-Reduced Graphene Oxide Nanocomposites, Nanoscale, 2014, 6, 11303-11309.

479 S. Biswas, R. Das, D. Chakraborty, R. Bandhyopadhyay and P. Pramanik, Synthesis of Nitrogen Doped Multilayered Graphene Flakes: Selective Non-enzymatic Electrochemical Determination of Dopamine and Uric Acid in presence of Ascorbic Acid, Electroanalysis, 2015, 27, 1253-1261.

480 H. L. Zou, B. L. Li, H. Q. Luo and N. B. Li, A Novel Electrochemical Biosensor Based on Hemin Functionalized Graphene Oxide Sheets for Simultaneous Determination of Ascorbic Acid, Dopamine and Uric Acid, Sens. Actuators, B, 2015, 207, 535-541.

481 L.-X. Chen, J.-N. Zheng, A.-J. Wang, L.-J. Wu, J.-R. Chen and J.-J. Feng, Facile Synthesis of Porous Bimetallic Alloyed PdAg Nanoflowers Supported on Reduced Graphene Oxide for Simultaneous Detection of Ascorbic Acid, Dopamine, and Uric Acid, Analyst, 2015, 140, 3183-3192.

482 W. Cai, J. Lai, T. Lai, H. Xie and J. Ye, Controlled Functionalization of Flexible Graphene Fibers for the Simultaneous Determination of Ascorbic Acid, Dopamine and Uric Acid, Sens. Actuators, B, 2016, 224, 225-232.

483 X. Zan, H. Bai, C. Wang, F. Zhao and H. Duan, Graphene Paper Decorated with a 2D Array of Dendritic Platinum Nanoparticles for Ultrasensitive Electrochemical Detection of Dopamine Secreted by Live Cells, Chem.-Eur. J., 2016, 22, 5204-5210.

484 X. Zhang, Y.-C. Zhang and L.-X. Ma, One-Pot Facile Fabrication of Graphene-Zinc Oxide Composite and its Enhanced Sensitivity for Simultaneous Electrochemical Detection of Ascorbic Acid, Dopamine and Uric Acid, Sens. Actuators, B, 2016, 227, 488-496.

485 H. Mao, C. Ji, M. Liu, Y. Sun, D. Liu, S. Wu, Y. Zhang and X.-M. Song, Hydrophilic Polymer/Polypyrrole/Graphene Oxide Nanosheets with Different Performances in Electrocatalytic Applications to Simultaneously Determine Dopamine and Ascorbic Acid, RSC Adv., 2016, 6, 111632111639.

486 P. Muthukumaran, C. Sumathi, J. Wilson and G. Ravi, Enzymeless Biosensor Based on $\beta$-NiS@rGO/Au Nanocomposites for Simultaneous Detection of Ascorbic Acid, Epinephrine and Uric Acid, RSC Adv., 2016, 6, 96467-96478. 
487 V. Vinoth, J. J. Wu and S. Anandan, Sensitive Electrochemical Determination of Dopamine and Uric Acid Using AuNPs(EDAS)-rGO Nanocomposites, Anal. Methods, 2016, 8, 4379-4390.

488 X. Wang, D. Gao, M. Li, H. Li, C. Li, X. Wu and B. Yang, CVD Graphene as an Electrochemical Sensing Platform for Simultaneous Detection of Biomolecules, Sci. Rep., 2017, 7, 7044.

489 Q. Zhu, J. Bao, D. Huo, M. Yang, C. Hou, J. Guo, M. Chen, H. Fa, X. Luo and Y. Ma, 3D Graphene Hydrogel-Gold Nanoparticles Nanocomposite Modified Glassy Carbon Electrode for the Simultaneous Determination of Ascorbic Acid, Dopamine and Uric Acid, Sens. Actuators, B, 2017, 238, 1316-1323.

490 Q. Zhu, J. Bao, D. Huo, M. Yang, H. Wu, C. Hou, Y. Zhao, X. Luo and H. Fa, 3DGH-Fc Based Electrochemical Sensor for the Simultaneous Determination of Ascorbic Acid, Dopamine and Uric Acid, J. Electroanal. Chem., 2017, 799, 459-467.

491 H. Begum, M. S. Ahmed and S. Jeon, New Approach for Porous Chitosan-Graphene Matrix Preparation through Enhanced Amidation for Synergic Detection of Dopamine and Uric Acid, ACS Omega, 2017, 2, 3043-3054.

492 S.-M. Li, S.-Y. Yang, Y.-S. Wang, C.-H. Lien, H.-W. Tien, S.-T. Hsiao, W.-H. Liao, H.-P. Tsai, C.-L. Chang, C.-C. M. Ma and C.-C. Hu, Controllable Synthesis of Nitrogen-Doped Graphene and its Effect on the Simultaneous Electrochemical Determination of Ascorbic Acid, Dopamine, and Uric Acid, Carbon, 2013, 59, 418-429.

493 H. Y. Yue, S. Huang, J. Chang, C. Heo, F. Yao, S. Adhikari, F. Gunes, L. C. Liu, T. H. Lee, E. S. Oh, B. Li, J. J. Zhang, T. Q. Huy, N. V. Luan and Y. H. Lee, ZnO Nanowire Arrays on 3D Hierachical Graphene Foam: Biomarker Detection of Parkinson's Disease, ACS Nano, 2014, 8, 1639-1646.

494 R. K. Srivastava, S. Srivastava, T. N. Narayanan, B. D. Mahlotra, R. Vajtai, P. M. Ajayan and A. Srivastava, Functionalized Multilayered Graphene Platform for Urea Sensor, ACS Nano, 2012, 6, 168-175.

495 D. Dutta, S. Chandra, A. K. Swain and D. Bahadur, $\mathrm{SnO}_{2}$ Quantum Dots-Reduced Graphene Oxide Composite for Enzyme-Free Ultrasensitive Electrochemical Detection of Urea, Anal. Chem., 2014, 86, 5914-5921.

496 L. Wu and X. Qu, Cancer Biomarker Detection: Recent Achievements and Challenges, Chem. Soc. Rev., 2015, 44, 2963-2997.

497 A. J. Vargas and C. C. Harris, Biomarker Development in the Precision Medicine Era: Lung Cancer as a Case Study, Nat. Rev. Cancer, 2016, 16, 525-537.

498 V. S. P. K. S. A. Jayanthi, A. B. Das and U. Saxena, Recent Advances in Biosensor Development for the Detection of Cancer Biomarkers, Biosens. Bioelectron., 2017, 91, 15-23.

499 M. Swierczewska, G. Liu, S. Lee and X. Chen, HighSensitivity Nanosensors for Biomarker Detection, Chem. Soc. Rev., 2012, 41, 2641-2655.

500 Biomarkers Definitions Working Group, A. J. Atkinson Jr, W. A. Colburn, V. G. DeGruttola, D. L. DeMets,
G. J. Downing, D. F. Hoth, J. A. Oates, C. C. Peck and R. T. Schooley, Biomarkers and surrogate endpoints: preferred definitions and conceptual framework, Clin. Pharmacol. Ther., 2001, 69, 89-95.

501 J. F. Rusling, C. V. Kumar, J. S. Gutkind and V. Patel, Measurement of Biomarker Proteins for Point-of-Care Early Detection and Monitoring of Cancer, Analyst, 2010, 135, 2496-2511.

502 C. M. Hartshorn, M. S. Bradbury, G. M. Lanza, A. E. Nel, J. Rao, A. Z. Wang, U. B. Wiesner, L. Yang and P. Grodzinski, Nanotechnology Strategies to Advance Outcomes in Clinical Cancer Care, ACS Nano, 2018, 12, 24-43.

503 A. Schroeder, D. A. Heller, M. M. Winslow, J. E. Dahlman, G. W. Pratt, R. Langer, T. Jacks and D. G. Anderson, Treating Metastatic Cancer with Nanotechnology, Nat. Rev. Cancer, 2012, 12, 39-50.

504 Y.-E. Choi, J.-W. Kwak and J. W. Park, Nanotechnology for Early Cancer Detection, Sensors, 2010, 10, 428-455.

505 T. Pasinszki, M. Krebsz, T. T. Tung and D. Losic, Carbon Nanomaterial Based Biosensors for Non-Invasive Detection of Cancer and Disease Biomarkers for Clinical Diagnosis, Sensors, 2017, 17, 1919.

506 H. J. Yoon, T. H. Kim, Z. Zhang, E. Azizi, T. M. Pham, C. Paoletti, J. Lin, N. Ramnath, M. S. Wicha, D. F. Hayes, D. M. Simeone and S. Nagrath, Sensitive Capture of Circulating Tumour Cells by Functionalized Graphene Oxide Nanosheets, Nat. Nanotechnol., 2013, 8, 735-741.

507 H. J. Yoon, A. Shanker, Y. Wang, M. Kozminsky, Q. Jin, N. Palanisamy, M. L. Burness, E. Azizi, D. M. Simeone, M. S. Wicha, J. Kim and S. Nagrath, Tunable ThermalSensitive Polymer-Graphene Oxide Composite for Efficient Capture and Release of Viable Circulating Tumor Cells, Adv. Mater., 2016, 28, 4891-4897.

508 M. A. Ali, C. Singh, S. Srivastava, P. Admane, V. V. Agrawal, G. Sumana, R. John, A. Panda, L. Dong and B. D. Malhotra, Graphene Oxide-Metal Nanocomposites for Cancer Biomarker Detection, RSC Adv., 2017, 7, 35982-35991.

509 Q. Zhu, H. Liu, J. Zhang, K. Wu, A. Deng and J. Li, Ultrasensitive QDs Based Electrochemiluminescent Immunosensor for Detecting Ractopamine Using AuNPs and $\mathrm{Au}$ Nanoparticles@PDDA-Graphene as Amplifier, Sens. Actuators, B, 2017, 243, 121-129.

510 V. Kumar, S. Srivastava, S. Umrao, R. Kumar, G. Nath, G. Sumana, P. S. Saxena and A. Srivastava, Nanostructured Palladium-Reduced Graphene Oxide Platform for High Sensitive, Label Free Detection of a Cancer Biomarker, RSC Adv., 2014, 4, 2267-2273.

511 X. Chen, X. Jia, J. Han, J. Ma and Z. Ma, Electrochemical immunosensor for simultaneous detection of multiplex cancer biomarkers based on graphene nanocomposites, Biosens. Bioelectron., 2013, 50, 356-361.

512 D. Lin, J. Wu, M. Wang, F. Yan and H. Ju, Triple Signal Amplification of Graphene Film, Polybead Carried Gold Nanoparticles as Tracing Tag and Silver Deposition for Ultrasensitive Electrochemical Immunosensing, Anal. Chem., 2012, 84, 3662-3668. 
513 B. Jin, P. Wang, H. Mao, B. Hu, H. Zhang, Z. Cheng, Z. Wu, X. Bian, C. Jia, F. Jing, Q. Jin and J. Zhao, MultiNanomaterial Electrochemical Biosensor Based on LabelFree Graphene for Detecting Cancer Biomarkers, Biosens. Bioelectron., 2014, 55, 464-469.

514 X. Jia, Z. Liu, N. Liu and Z. Ma, A Label-Free Immunosensor Based on Graphene Nanocomposites for Simultaneous Multiplexed Electrochemical Determination of Tumor Markers, Biosens. Bioelectron., 2014, 53, 160-166.

515 C. Song, G. Xie, L. Wang, L. Liu, G. Tian and H. Xiang, DNABased Hybridization Chain Reaction for an Ultrasensitive Cancer Marker EBNA-1 Electrochemical Immunosensor, Biosens. Bioelectron., 2014, 58, 68-74.

516 S. Teixeira, G. Burwell, A. Castaing, D. Gonzalez, R. S. Conlan and O. J. Guy, Epitaxial Graphene Immunosensor for Human Chorionic Gonadotropin, Sens. Actuators, B, 2014, 190, 723-729.

517 H. D. Jang, S. K. Kim, H. Chang and J.-W. Choi, 3D LabelFree Prostate Specific Antigen (PSA) Immunosensor Based on Graphene-Gold Composites, Biosens. Bioelectron., 2015, 63, 546-551.

518 M. A. Ali, K. Mondal, Y. Jiao, S. Oren, Z. Xu, A. Sharma and L. Dong, Microfluidic Immuno-Biochip for Detection of Breast Cancer Biomarkers Using Hierarchical Composite of Porous Graphene and Titanium Dioxide Nanofibers, ACS Appl. Mater. Interfaces, 2016, 8, 20570-20582.

519 S. Kumar, J. G. Sharma, S. Maji and B. D. Malhotra, Nanostructured Zirconia Decorated Reduced Graphene Oxide Based Efficient Biosensing Platform for NonInvasive Oral Cancer Detection, Biosens. Bioelectron., 2016, 78, 497-504.

520 T. Feng, X. Qiao, H. Wang, Z. Sun, Y. Qi and C. Hong, An Electrochemical Immunosensor for Simultaneous Pointof-Care Cancer Markers Based on the Host-Guest Inclusion of $\beta$-Cyclodextrin-Graphene Oxide, J. Mater. Chem. B, 2016, 4, 990-996.

521 G. Wang, Y. Li, J. Liu, Y. Yuan, Z. Shen and X. Mei, Ultrasensitive Multiplexed Immunoassay of Autophagic Biomarkers Based on $\mathrm{Au} / \mathrm{rGO}$ and $\mathrm{Au}$ Nanocages Amplifying Electrochemical Signal, Sci. Rep., 2017, 7, 2442.

522 M. Pal and R. Khan, Graphene Oxide Layer Decorated Gold Nanoparticles Based Immunosensor for the Detection of Prostate Cancer Risk Factor, Anal. Biochem., 2017, 536, 51-58.

523 L. Hao, L. Liu, X. Meng, H. Cui and Z. Wang, Electrochemical Analysis of Ki67 Protein As Pancreatic Cancer Biomarker Based on Graphene-Polydopamine Nanocomposite, Int. J. Electrochem. Sci., 2017, 12, 30403049.

524 H. Wang, Y. Zhang, Y. Wang, H. Ma, B. Du and Q. Wei, Facile Synthesis of Cuprous Oxide Nanowires Decorated Graphene Oxide Nanosheets Nanocomposites and its Application in Label-Free Electrochemical Immunosensor, Biosens. Bioelectron., 2017, 87, 745-751.

525 Z. Yang, Q. Lan, J. Li, J. Wu, Y. Tang and X. Hu, Efficient Streptavidin-Functionalized Nitrogen-Doped Graphene for the Development of Highly Sensitive Electrochemical Immunosensor, Biosens. Bioelectron., 2017, 89, 312-318.

526 Y. Yang, Q. Liu, Y. Liu, J. Cui, H. Liu, P. Wang, Y. Li, L. Chen, Z. Zhao and Y. Dong, A Novel Label-Free Electrochemical Immunosensor Based on Functionalized Nitrogen-Doped Graphene Quantum Dots for Carcinoembryonic Antigen Detection, Biosens. Bioelectron., 2017, 90, 31-38.

527 S. K. Tuteja, T. Duffield and S. Neethirajan, GrapheneBased Multiplexed Disposable Electrochemical Biosensor for Rapid on-Farm Monitoring of NEFA and $\beta$ HBA Dairy Biomarkers, J. Mater. Chem. B, 2017, 5, 6930-6940.

528 L.-H. Pan, S.-H. Kuo, T.-Y. Lin, C.-W. Lin, P.-Y. Fang and H.-W. Yang, An Electrochemical Biosensor to Simultaneously Detect VEGF and PSA for Early Prostate Cancer Diagnosis Based on Graphene Oxide/ssDNA/PLLA Nanoparticles, Biosens. Bioelectron., 2017, 89, 598-605.

529 J. Feng, Y. Li, M. Li, F. Li, J. Han, Y. Dong, Z. Chen, P. Wang, H. Liu and Q. Wei, A Novel Sandwich-Type Electrochemical Immunosensor for PSA Detection Based on PtCu Bimetallic Hybrid (2D/2D) rGO/g- $\mathrm{C}_{3} \mathrm{~N}_{4}$, Biosens. Bioelectron., 2017, 91, 441-448.

530 A. A. Saeed, J. L. A. Sánchez, C. K. O'Sullivan and M. N. Abbas, DNA Biosensors Based on Gold Nanoparticles-Modified Graphene Oxide for the Detection of Breast Cancer Biomarkers for Early Diagnosis, Bioelectrochemistry, 2017, 118, 91-99.

531 X. Ren, H. Ma, T. Zhang, Y. Zhang, T. Yan, B. Du and Q. Wei, Sulfur-Doped Graphene-Based Immunological Biosensing Platform for Multianalysis of Cancer Biomarkers, ACS Appl. Mater. Interfaces, 2017, 9, 3763737644.

532 S. X. Lee, H. N. Lim, I. Ibrahim, A. Jamil, A. Pandikumar and N. M. Huang, Horseradish Peroxidase-Labeled Silver/ Reduced Graphene Oxide Thin Film-Modified ScreenPrinted Electrode for Detection of Carcinoembryonic Antigen, Biosens. Bioelectron., 2017, 89, 673-680.

533 M. Li, P. Wang, F. Li, Q. Chu, Y. Li and Y. Dong, An Ultrasensitive Sandwich-Type Electrochemical Immunosensor Based on the Signal Amplification Strategy of Mesoporous Core-Shell Pd@Pt Nanoparticles/ Amino Group Functionalized Graphene Nanocomposite, Biosens. Bioelectron., 2017, 87, 752-759.

534 F. Shahzad, S. A. Zaidi and C. M. Koo, Highly Sensitive Electrochemical Sensor Based on Environmentally Friendly Biomass-Derived Sulfur-Doped Graphene For Cancer Biomarker Detection, Sens. Actuators, B, 2017, 241, 716-724.

535 M. Sharafeldin, G. W. Bishop, S. Bhakta, A. El-Sawy, S. L. Suib and J. F. Rusling, $\mathrm{Fe}_{3} \mathrm{O}_{4}$ Nanoparticles on Graphene Oxide Sheets for Isolation and Ultrasensitive Amperometric Detection of Cancer Biomarker Proteins, Biosens. Bioelectron., 2017, 91, 359-366.

536 J.-Y. Huang, L. Zhao, W. Lei, W. Wen, Y.-J. Wang, T. Bao, H.-Y. Xiong, X.-H. Zhang and S.-F. Wang, A HighSensitivity Electrochemical Aptasensor of Carcinoembryonic Antigen Based on Graphene Quantum 
Dots-Ionic Liquid-Nafion Nanomatrix and DNAzymeAssisted Signal Amplification Strategy, Biosens. Bioelectron., 2018, 99, 28-33.

537 M. Chen, Y. Wang, H. Su, L. Mao, X. Jiang, T. Zhang and X. Dai, Three-Dimensional Electrochemical DNA Biosensor Based on 3D Graphene-Ag Nanoparticles for Sensitive Detection of CYFRA21-1 in Non-Small Cell Lung Cancer, Sens. Actuators, B, 2018, 255, 2910-2918.

538 J.-T. Cao, J.-J. Yang, L.-Z. Zhao, Y.-L. Wang, H. Wang, Y.-M. Liu and S.-H. Ma, Graphene Oxide@Gold NanorodsBased Multiple-Assisted Electrochemiluminescence Signal Amplification Strategy for Sensitive Detection of Prostate Specific Antigen, Biosens. Bioelectron., 2018, 99, 92-98.

539 R. Wang, J.-J. Feng, Y. Xue, L. Wu and A.-J. Wang, A LabelFree Electrochemical Immunosensor Based on AgPt Nanorings Supported on Reduced Graphene Oxide for Ultrasensitive Analysis of Tumor Marker, Sens. Actuators, B, 2018, 254, 1174-1181.

540 J. Wang, X. Wang, H. Tang, Z. Gao, S. He, J. Li and S. Han, Ultrasensitive Electrochemical Detection of Tumor Cells Based on Multiple Layer CDS Quantum DotsFunctionalized Polystyrene Microspheres and Graphene Oxide-Polyaniline Composite, Biosens. Bioelectron., 2018, 100, 1-7.

541 V. K. Singh, S. Kumar, S. K. Pandey, S. Srivastava, M. Mishra, G. Gupta, B. D. Malhotra, R. S. Tiwari and A. Srivastava, Fabrication of Sensitive Bioelectrode Based on Atomically Thin CVD Grown Graphene for Cancer Biomarker Detection, Biosens. Bioelectron., 2018, 105, 173181.

542 M. Mazloum-Ardakani, B. Barazesh, A. Khoshroo, M. Moshtaghiun and M. H. Sheikhha, A New Composite Consisting of Electrosynthesized Conducting Polymers, Graphene Sheets and Biosynthesized Gold Nanoparticles for Biosensing Acute Lymphoblastic Leukemia, Bioelectrochemistry, 2018, 121, 38-45.

543 G. Zhang, Z. Liu, L. Fan and Y. Guo, Electrochemical Prostate Specific Antigen Aptasensor Based on Hemin Functionalized Graphene-Conjugated Palladium Nanocomposites, Microchim. Acta, 2018, 185, 159.

544 S. Rauf, G. K. Mishra, J. Azhar, R. K. Mishra, K. Y. Goud, M. A. H. Nawaz, J. L. Marty and A. Hayat, Carboxylic Group Riched Graphene Oxide Based Disposable Electrochemical Immunosensor for Cancer Biomarker Detection, Anal. Biochem., 2018, 545, 13-19.

545 S. Shahrokhian and R. Salimian, Ultrasensitive Detection of Cancer Biomarkers Using Conducting Polymer/ Electrochemically Reduced Graphene Oxide-Based Biosensor: Application Toward BRCA $_{1}$ Sensing, Sens. Actuators, B, 2018, 266, 160-169.

546 L. Wu, H. Ji, Y. Guan, X. Ran, J. Ren and X. Qu, A GrapheneBased Chemical Nose/Tongue Approach for the Identification of Normal, Cancerous and Circulating Tumor Cells, NPG Asia Mater., 2017, 9, e356.

547 G. M. Whitesides, The Origins and the Future of Microfluidics, Nature, 2006, 442, 368-373.
548 R. A. G. de Oliveira, E. M. Materon, M. E. Melendez, A. L. Carvalho and R. C. Faria, Disposable Microfluidic Immunoarray Device for Sensitive Breast Cancer Biomarker Detection, ACS Appl. Mater. Interfaces, 2017, 9, 27433-27440.

549 D. M. Cate, J. A. Adkins, J. Mettakoonpitak and C. S. Henry, Recent Developments in Paper-Based Microfluidic Devices, Anal. Chem., 2015, 87, 19-41.

550 Y. Wu, P. Xue, Y. Kang and K. M. Hui, Paper-Based Microfluidic Electrochemical Immunodevice Integrated with Nanobioprobes onto Graphene Film for Ultrasensitive Multiplexed Detection of Cancer Biomarkers, Anal. Chem., 2013, 85, 8661-8668.

551 N. Singh, M. A. Ali, P. Rai, A. Sharma, B. D. Malhotra and R. John, Microporous Nanocomposite Enabled Microfluidic Biochip for Cardiac Biomarker Detection, ACS Appl. Mater. Interfaces, 2017, 9, 33576-33588.

552 C. Dye, After 2015: Infectious Diseases in a New Era of Health and Development, Philos. Trans. R. Soc., B, 2014, 369, 20130426.

553 T. C. Paulitz and R. R. Bélanger, Biologicalcontrol in Greenhousesystems, Annu. Rev. Phytopathol., 2001, 39, 103-133.

554 E. C. Alocilja and S. M. Radke, Market Analysis of Biosensors for Food Safety, Biosens. Bioelectron., 2003, 18, 841-846.

555 M. Khater, A. de la Escosura-Muñiz and A. Merkoçi, Biosensors for Plant Pathogen Detection, Biosens. Bioelectron., 2017, 93, 72-86.

556 M. B. dos Santos, J. P. Agusil, B. Prieto-Simón, C. Sporer, V. Teixeira and J. Samitier, Highly Sensitive Detection of Pathogen Escherichia coli 0157:H7 by Electrochemical Impedance Spectroscopy, Biosens. Bioelectron., 2013, 45, 174-180.

557 L. Yang and R. Bashir, Electrical/Electrochemical Impedance for Rapid Detection of Foodborne Pathogenic Bacteria, Biotechnol. Adv., 2008, 26, 135-150.

558 M. Amiri, A. Bezaatpour, H. Jafari, R. Boukherroub and S. Szunerits, Electrochemical Methodologies for the Detection of Pathogens, ACS Sens., 2018, 3, 1069-1086.

559 I. Tiwari, M. Singh, C. M. Pandey and G. Sumana, Electrochemical Genosensor Based on Graphene Oxide Modified Iron Oxide-Chitosan Hybrid Nanocomposite for Pathogen Detection, Sens. Actuators, B, 2016, 206, 276-283.

560 I. Tiwari, M. Singh, C. M. Pandey and G. Sumana, Electrochemical Detection of a Pathogenic Escherichia coli Specific DNA Sequence Based on a Graphene OxideChitosan Composite Decorated with Nickel Ferrite Nanoparticles, RSC Adv., 2015, 5, 67115-67124.

561 Q. Zhou, G. Li, Y. Zhang, M. Zhu, Y. Wan and Y. Shen, Highly Selective and Sensitive Electrochemical Immunoassay of $\mathrm{Cry}_{1} \mathrm{C}$ Using Nanobody and $\pi-\pi$ Stacked Graphene Oxide/Thionine Assembly, Anal. Chem., 2016, 88, 9830-9836.

562 F. Jia, N. Duan, S. Wu, X. Ma, Y. Xia, Z. Wang and X. Wei, Impedimetric Aptasensor for Staphylococcus aureus based on Nanocomposite Prepared from Reduced Graphene 
Oxide and Gold Nanoparticles, Microchim. Acta, 2014, 181, 967-974.

563 C. M. Pandey, I. Tiwari, V. N. Singh, K. N. Sood, G. Sumana and B. D. Malhotra, Highly Sensitive Electrochemical Immunosensor Based on Graphene-Wrapped Copper Oxide-Cysteine Hierarchical Structure for Detection of Pathogenic Bacteria, Sens. Actuators, B, 2017, 238, 10601069.

564 A. Pandey, Y. Gurbuz, V. Ozguz, J. H. Niazi and A. Qureshi, Graphene-Interfaced Electrical Biosensor for Label-Free and Sensitive Detection of Foodborne Pathogenic E. coli O157:H7, Biosens. Bioelectron., 2017, 91, 225-231.

565 W. Ye, J. Guo, X. Bao, T. Chen, W. Weng, S. Chen and M. Yang, Rapid and Sensitive Detection of Bacteria Response to Antibiotics Using Nanoporous Membrane and Graphene Quantum Dot (GQDs)-Based Electrochemical Biosensors, Materials, 2017, 10, 603.

566 H. Kaur, M. Shorie, M. Sharma, A. K. Ganguli and P. Sabherwal, Bridged Rebar Graphene Functionalized Aptasensor for Pathogenic E. coli O78:K80:H11 Detection, Biosens. Bioelectron., 2017, 98, 486-493.

567 I. J. Dinshaw, S. Muniandy, S. J. Teh, F. Ibrahim, B. F. Leo and K. L. Thong, Development of an Aptasensor Using Reduced Graphene Oxide Chitosan Complex to Detect Salmonella, J. Electroanal. Chem., 2017, 806, 88-96.

568 J. Fei, W. Dou and G. Zhao, Amperometric Immunoassay for the Detection of Salmonella pullorum Using a Screen Printed Carbon Electrode Modified with Gold Nanoparticle-Coated Reduced Graphene Oxide and Immunomagnetic Beads, Microchim. Acta, 2016, 183, 757764.

569 S. Muniandy, I. J. Dinshaw, S. J. Teh, C. W. Lai, F. Ibrahim, K. L. Thong and B. F. Leo, Graphene-Based Label-Free Electrochemical Aptasensor for Rapid and Sensitive Detection of Foodborne Pathogen, Anal. Bioanal. Chem., 2017, 409, 6893-6905.

570 S. Xu, Y. Zhang, K. Dong, J. Wen, C. Zheng and S. Zhao, Electrochemical DNA Biosensor Based on Graphene Oxide-Chitosan Hybrid Nanocomposites for Detection of Escherichia coli 0157: H7, Int. J. Electrochem. Sci., 2017, 12, 3443-3458.

571 N. Kumar, S. Rosy and R. N. Goyal, Gold-Palladium Nanoparticles Aided Electrochemically Reduced Graphene Oxide Sensor for the Simultaneous Estimation of Lomefloxacin and Amoxicillin, Sens. Actuators, B, 2017, 243, 658-668.

572 S. Chen, Y. Frank Cheng and G. Voordouw, ThreeDimensional Graphene Nanosheet Doped with Gold Nanoparticles as Electrochemical DNA Biosensor for Bacterial Detection, Sens. Actuators, B, 2018, 262, 860-868.

573 S. Shukla, Y. Haldorai, V. K. Bajpai, A. Rengaraj, S. K. Hwang, X. Song, M. Kim, Y. S. Huh and Y.-K. Han, Electrochemical Coupled Immunosensing Platform Based on Graphene Oxide/Gold Nanocomposite for Sensitive Detection of Cronobacter Sakazakii in Powdered Infant Formula, Biosens. Bioelectron., 2018, 109, 139-149.
574 R. Jijie, K. Kahlouche, A. Barras, N. Yamakawa, J. Bouckaert, T. Gharbi, S. Szunerits and R. Boukherroub, Reduced Graphene Oxide/Polyethylenimine Based Immunosensor for the Selective and Sensitive Electrochemical Detection of Uropathogenic Escherichia coli, Sens. Actuators, B, 2018, 260, 255-263.

575 A. Krittayavathananon and M. Sawangphruk, Impedimetric Sensor of ss-HSDNA/Reduced Graphene Oxide Aerogel Electrode toward Aflatoxin B1 Detection: Effects of Redox Mediator Charges and Hydrodynamic Diffusion, Anal. Chem., 2017, 89, 13283-13289.

576 S. Srivastava, V. Kumar, M. A. Ali, P. R. Solanki, A. Srivastava, G. Sumana, P. S. Saxena, A. G. Joshi and B. D. Malhotra, Electrophoretically Deposited Reduced Graphene Oxide Platform for Food Toxin Detection, Nanoscale, 2013, 5, 3043-3051.

577 S. Srivastava, S. Abraham, C. Singh, M. A. Ali, A. Srivastava, G. Sumana and B. D. Malhotra, Protein Conjugated Carboxylated Gold@Reduced Graphene Oxide For Aflatoxin $\mathrm{B}_{1}$ Detection, RSC Adv., 2015, 5, 5406-5414.

578 D. Wang, W. Hu, Y. Xiong, Y. Xu and C. Ming Li, Multifunctionalized Reduced Graphene Oxide-Doped Polypyrrole/Pyrrolepropylic Acid Nanocomposite Impedimetric Immunosensor to Ultra-Sensitively Detect Small Molecular Aflatoxin $\mathrm{B}_{1}$, Biosens. Bioelectron., 2015, 63, 185-189.

579 S. Srivastava, V. Kumar, K. Arora, C. Singh, M. A. Ali, N. K. Puri and B. D. Malhotra, Antibody Conjugated Metal Nanoparticle Decorated Graphene Sheets for a Mycotoxin Sensor, RSC Adv., 2016, 6, 56518-56526.

580 A.-L. Sun, Y.-F. Zhang, G.-P. Sun, X.-N. Wang and D. Tang, Homogeneous Electrochemical Detection of Ochratoxin A in Foodstuff Using Aptamer-Graphene Oxide Nanosheets and Dnase I-Based Target Recycling Reaction, Biosens. Bioelectron., 2017, 89, 659-665.

581 S. Eissa and M. Zourob, In vitro Selection of DNA Aptamers Targeting $\beta$-Lactoglobulin and Their Integration in Graphene-Based Biosensor for the Detection of Milk Allergen, Biosens. Bioelectron., 2017, 91, 169-174.

582 L. Zhu, L. Xu, B. Huang, N. Jia, L. Tan and S. Yao, Simultaneous Determination of $\mathrm{Cd}(\mathrm{II})$ and $\mathrm{Pb}$ (II) Using Square Wave Anodic Stripping Voltammetry at a Gold Nanoparticle-Graphene-Cysteine Composite Modified Bismuth Film Electrode, Electrochim. Acta, 2014, 115, 471477.

583 X. Gong, Y. Bi, Y. Zhao, G. Liu and W. Y. Teoh, Graphene Oxide-Based Electrochemical Sensor: A Platform for Ultrasensitive Detection of Heavy Metal Ions, RSC Adv., 2014, 4, 24653-24657.

584 H. Zhu, Y. Xu, A. Liu, N. Kong, F. Shan, W. Yang, C. J. Barrow and J. Liu, Graphene Nanodots-Encaged Porous Gold Electrode Fabricated via Ion Beam Sputtering Deposition for Electrochemical Analysis of Heavy Metal Ions, Sens. Actuators, B, 2015, 206, 592-600.

585 S. Tang, P. Tong, X. You, W. Lu, J. Chen, G. Li and L. Zhang, Label free Electrochemical Sensor for $\mathrm{Pb}^{2+}$ Based on 
Graphene Oxide Mediated Deposition of Silver Nanoparticles, Electrochim. Acta, 2016, 187, 286-292.

586 S. Xue, P. Jing and W. Xu, Hemin on Graphene Nanosheets Functionalized with Flower-Like $\mathrm{MnO}_{2}$ and Hollow AuPd for the Electrochemical Sensing Lead Ion Based on the Specific DNAzyme, Biosens. Bioelectron., 2016, 86, 958-965.

587 Z. Zhang, X. Fu, K. Li, R. Liu, D. Peng, L. He, M. Wang, H. Zhang and L. Zhou, One-Step Fabrication of Electrochemical Biosensor Based on DNA-Modified ThreeDimensional Reduced Graphene Oxide and Chitosan Nanocomposite for Highly Sensitive Detection of $\mathrm{Hg}$ (II), Sens. Actuators, B, 2016, 225, 453-462.

588 N. Wang, M. Lin, H. Dai and H. Ma, Functionalized Gold Nanoparticles/Reduced Graphene Oxide Nanocomposites for Ultrasensitive Electrochemical Sensing of Mercury Ions Based on Thymine-Mercury-Thymine Structure, Biosens. Bioelectron., 2016, 79, 320-326.

589 T. Priya, N. Dhanalakshmi and N. Thinakaran, Electrochemical Behavior of $\mathrm{Pb}$ (II) on a Heparin Modified Chitosan/Graphene Nanocomposite Film Coated Glassy Carbon Electrode and its Sensitive Detection, Int. J. Biol. Macromol., 2017, 104, 672-680.

590 Y. Zhu, D. Pan, X. Hu, H. Han, M. Lin and C. Wang, An Electrochemical Sensor Based on Reduced Graphene Oxide/Gold Nanoparticles Modified Electrode for Determination of Iron in Coastal Waters, Sens. Actuators, $B, 2017,243,1-7$.

591 L. Yang, N. Huang, L. Huang, M. Liu, H. Li, Y. Zhang and S. Yao, An Electrochemical Sensor for Highly Sensitive Detection of Copper Ions Based on a New Molecular Probe Pi-A Decorated on Graphene, Anal. Methods, 2017, 9, 618-624.

592 O. Sadak, A. K. Sundramoorthy and S. Gunasekaran, Highly Selective Colorimetric and Electrochemical Sensing of Iron (III) Using Nile Red Functionalized Graphene Film, Biosens. Bioelectron., 2017, 89, 430-436.

593 Z. Guo, D.-d. Li, X.-k. Luo, Y.-h. Li, Q.-N. Zhao, M.-m. Li, Y.-t. Zhao, T.-s. Sun and C. Ma, Simultaneous Determination of Trace $\mathrm{Cd}(\mathrm{II}), \mathrm{Pb}(\mathrm{II})$ and $\mathrm{Cu}(\mathrm{II})$ by Differential Pulse Anodic Stripping Voltammetry Using a Reduced Graphene Oxide-Chitosan/Poly-L-Lysine Nanocomposite Modified Glassy Carbon Electrode, $J$. Colloid Interface Sci., 2017, 490, 11-22.

594 Y. Zhang, G. M. Zeng, L. Tang, J. Chen, Y. Zhu, X. X. He and Y. He, Electrochemical Sensor Based on Electrodeposited Graphene-Au Modified Electrode and NanoAu Carrier Amplified Signal Strategy for Attomolar Mercury Detection, Anal. Chem., 2015, 87, 989-996.

595 M. B. Gumpu, M. Veerapandian, U. M. Krishnan and J. B. B. Rayappan, Simultaneous Electrochemical Detection of $\mathrm{Cd}(\mathrm{II}), \mathrm{Pb}(\mathrm{II}), \mathrm{As}(\mathrm{III})$ and $\mathrm{Hg}$ (II) Ions Using Ruthenium(II)-Textured Graphene Oxide Nanocomposite, Talanta, 2017, 162, 574-582.

596 X. Yiwei, Z. Wen, H. Xiaowei, S. Jiyong, Z. Xiaobo, L. Yanxiao, C. Xueping, H. E. Tahir and L. Zhihua, A SelfAssembled L-Cysteine and Electrodeposited Gold Nanoparticles-reduced Graphene Oxide Modified
Electrode for Adsorptive Stripping Determination of Copper, Electroanalysis, 2018, 30, 194-203.

597 S.-F. Zhou, X.-J. Han, H.-L. Fan, J. Huang and Y.-Q. Liu, Enhanced Electrochemical Performance for Sensing Pb(II) Based on Graphene Oxide Incorporated Mesoporous $\mathrm{MnFe}_{2} \mathrm{O}_{4}$ Nanocomposites, J. Alloys Compd., 2018, 747, 447-454.

598 L. Lu, L. Zhou, J. Chen, F. Yan, J. Liu, X. Dong, F. Xi and P. Chen, Nanochannel-Confined Graphene Quantum Dots for Ultrasensitive Electrochemical Analysis of Complex Samples, ACS Nano, 2018, 12, 12673-12681.

599 L. Rotariu, F. Lagarde, N. Jaffrezic-Renault and C. Bala, Electrochemical Biosensors for Fast Detection of Food Contaminants-Trends and Perspective, TrAC, Trends Anal. Chem., 2016, 79, 80-87.

600 N. F. D. Silva, J. M. C. S. Magalhães, C. Freire and C. Delerue-Matos, Electrochemical Biosensors for Salmonella: State of the Art and Challenges in Food Safety Assessment, Biosens. Bioelectron., 2018, 99, 667-682.

601 A. Vasilescu and J.-L. Marty, Electrochemical Aptasensors for the Assessment of Food Quality and Safety, TrAC, Trends Anal. Chem., 2016, 79, 60-70.

602 J. Huang, X. Gao, J. Jia, J.-K. Kim and Z. Li, Graphene OxideBased Amplified Fluorescent Biosensor for $\mathrm{Hg}^{2+}$ Detection through Hybridization Chain Reactions, Anal. Chem., 2014, 86, 3209-3215.

603 L. Lan, Y. Yao, J. Ping and Y. Ying, Recent Progress in Nanomaterial-Based Optical Aptamer Assay for the Detection of Food Chemical Contaminants, ACS Appl. Mater. Interfaces, 2017, 9, 23287-23301.

604 N. Kaur, A. Bharti, S. Batra, S. Rana, S. Rana, A. Bhalla and N. Prabhakar, An electrochemical aptasensor based on graphene doped chitosan nanocomposites for determination of ochratoxin A, Microchem. J., 2019, 144, 102-109.

605 L. Jiang, J. Qian, X. Yang, Y. Yan, Q. Liu, K. Wang and K. Wang, Amplified Impedimetric Aptasensor Based on Gold Nanoparticles Covalently Bound Graphene Sheet for the Picomolar Detection of Ochratoxin A, Anal. Chim. Acta, 2014, 806, 128-135.

606 G. Aragay, J. Pons and A. Merkoçi, Recent Trends in Macro-, Micro-, and Nanomaterial-Based Tools and Strategies for Heavy-Metal Detection, Chem. Rev., 2011, 111, 3433-3458.

607 F. Fu and Q. Wang, Removal of Heavy Metal Ions from Wastewaters: A Review, J. Environ. Manage., 2011, 92, 407418.

608 B. Bansod, T. Kumar, R. Thakur, S. Rana and I. Singh, A Review on Various Electrochemical Techniques for Heavy Metal Ions Detection with Different Sensing Platforms, Biosens. Bioelectron., 2017, 94, 443-455.

609 X. Gong, G. Liu, Y. Li, D. Y. W. Yu and W. Y. Teoh, Functionalized-Graphene Composites: Fabrication and Applications in Sustainable Energy and Environment, Chem. Mater., 2016, 28, 8082-8118.

610 D. Vilela, J. Parmar, Y. Zeng, Y. Zhao and S. Sánchez, Graphene-Based Microbots for Toxic Heavy Metal 
Removal and Recovery from Water, Nano Lett., 2016, 16, 2860-2866.

611 S. Muralikrishna, K. Sureshkumar, T. S. Varley, D. H. Nagaraju and T. Ramakrishnappa, In situ Reduction and Functionalization of Graphene Oxide with l-Cysteine for Simultaneous Electrochemical Determination of Cadmium(ii), Lead(ii), Copper(ii), and Mercury(ii) Ions, Anal. Methods, 2014, 6, 8698-8705.

612 H. Zhang, S. Shuang, G. Wang, Y. Guo, X. Tong, P. Yang, A. Chen, C. Dong and Y. Qin, $\mathrm{TiO}_{2}-$ Graphene Hybrid Nanostructures by Atomic Layer Deposition With Enhanced Electrochemical Performance for $\mathrm{Pb}(\mathrm{ii})$ and Cd(ii) Detection, $R S C A d v$. , 2015, 5, 4343-4349.

613 S. Palanisamy, R. Madhu, S.-M. Chen and S. K. Ramaraj, A Highly Sensitive and Selective Electrochemical Determination of $\mathrm{Hg}$ (ii) Based on an Electrochemically Activated Graphite Modified Screen-Printed Carbon Electrode, Anal. Methods, 2014, 6, 8368-8373.

614 R. Seenivasan, W.-J. Chang and S. Gunasekaran, Highly Sensitive Detection and Removal of Lead Ions in Water Using Cysteine-Functionalized Graphene Oxide/ Polypyrrole Nanocomposite Film Electrode, ACS Appl. Mater. Interfaces, 2015, 7, 15935-15943.

615 C. Yu, Y. Guo, H. Liu, N. Yan, Z. Xu, G. Yu, Y. Fang and Y. Liu, Ultrasensitive and Selective Sensing of Heavy Metal Ions with Modified Graphene, Chem. Commun., 2013, 49, 6492-6494.

616 W. Aktar, D. Sengupta and A. Chowdhury, Impact of Pesticides Use in Agriculture: Their Benefits and Hazards, Interdiscip. Toxicol., 2009, 2, 1-12.

617 D. Gunnell, M. Eddleston, M. R. Phillips and F. Konradsen, The Global Distribution of Fatal Pesticide Self-Poisoning: Systematic Review, BMC Public Health, 2007, 7, 357.

618 J. Grutzendler and J. C. Morris, Cholinesterase Inhibitors for Alzheimer's Disease, Drugs, 2001, 61, 41-52.

619 W. J. Deardorff, E. Feen and G. T. Grossberg, The Use of Cholinesterase Inhibitors Across All Stages of Alzheimer's Disease, Drugs Aging, 2015, 32, 537-547.

620 S.-Y. Hung and W.-M. Fu, Drug Candidates in Clinical Trials for Alzheimer's Disease, J. Biomed. Sci., 2017, 24, 47.

621 D. M. Quinn, Acetylcholinesterase: Enzyme Structure, Reaction Dynamics, and Virtual Transition States, Chem. Rev., 1987, 87, 955-979.

622 V. Tougu, Acetylcholinesterase: Mechanism of Catalysis and Inhibition, Curr. Med. Chem.: Cent. Nerv. Syst. Agents, 2001, 1, 155-170.

623 M. B. Colovic, D. Z. Krstic, T. D. Lazarevic-Pasti, A. M. Bondzic and V. M. Vasic, Acetylcholinesterase Inhibitors: Pharmacology and Toxicology, Curr. Neuropharmacol., 2013, 11, 315-335.

624 N. Jaffrezic-Renault, New Trends in Biosensors for Organophosphorus Pesticides, Sensors, 2001, 1, 60-74.

625 B. B. Narakathu, W. Guo, S. O. Obare and M. Z. Atashbar, Novel Approach for Detection of Toxic Organophosphorus Compounds, Sens. Actuators, B, 2011, 158, 69-74.

626 S. Hassani, S. Momtaz, F. Vakhshiteh, A. S. Maghsoudi, M. R. Ganjali, P. Norouzi and M. Abdollahi, Biosensors and Their Applications in Detection of Organophosphorus Pesticides in the Environment, Arch. Toxicol., 2017, 91, 109-130.

627 X. Yan, H. Li and X. Su, Review of Optical Sensors for Pesticides, TrAC, Trends Anal. Chem., 2018, 103, 1-20.

628 S. Viswanathan, H. Radecka and J. Radecki, Electrochemical Biosensor for Pesticides Based on Acetylcholinesterase Immobilized on Polyaniline Deposited on Vertically Assembled Carbon Nanotubes Wrapped with ssDNA, Biosens. Bioelectron., 2009, 24, 2772-2777.

629 W. Zhang, A. M. Asiri, D. Liu, D. Du and Y. Lin, Nanomaterial-Based Biosensors for Environmental and Biological Monitoring of Organophosphorus Pesticides and Nerve Agents, TrAC, Trends Anal. Chem., 2014, 54, 1-10.

630 A. P. Periasamy, Y. Umasankar and S.-M. Chen, Nanomaterials-Acetylcholinesterase Enzyme Matrices for Organophosphorus Pesticides Electrochemical Sensors: A Review, Sensors, 2009, 9, 4034-4055.

631 J. Jiang, S. Zou, L. Ma, S. Wang, J. Liao and Z. Zhang, Surface-Enhanced Raman Scattering Detection of Pesticide Residues Using Transparent Adhesive Tapes and Coated Silver Nanorods, ACS Appl. Mater. Interfaces, 2018, 10, 9129-9135.

632 E. H. Koh, C. Mun, C. Kim, S.-G. Park, E. J. Choi, S. H. Kim, J. Dang, J. Choo, J.-W. Oh, D.-H. Kim and H. S. Jung, M13 Bacteriophage/Silver Nanowire Surface-Enhanced Raman Scattering Sensor for Sensitive and Selective Pesticide Detection, ACS Appl. Mater. Interfaces, 2018, 10, 1038810397.

633 D. Huo, Q. Li, Y. Zhang, C. Hou and Y. Lei, A Highly Efficient Organophosphorus Pesticides Sensor Based on CuO Nanowires-SWCNTs Hybrid Nanocomposite, Sens. Actuators, B, 2014, 199, 410-417.

634 P. Kumar, A. K. Paul and A. Deep, A Luminescent Nanocrystal Metal Organic Framework for Chemosensing of Nitro Group Containing Organophosphate Pesticides, Anal. Methods, 2014, 6, 4095-4101.

635 S. Wu, X. Lan, L. Cui, L. Zhang, S. Tao, H. Wang, M. Han, Z. Liu and C. Meng, Application of Graphene for Preconcentration and Highly Sensitive Stripping Voltammetric Analysis of Organophosphate Pesticide, Anal. Chim. Acta, 2011, 699, 170-176.

636 M. Z. M. Nasir, C. C. Mayorga-Martinez, Z. Sofer and M. Pumera, Two-Dimensional 1T-Phase Transition Metal Dichalcogenides as Nanocarriers to Enhance and Stabilize Enzyme Activity for Electrochemical Pesticide Detection, ACS Nano, 2017, 11, 5774-5784.

637 L. Yang, G. Wang and Y. Liu, An Acetylcholinesterase Biosensor Based on Platinum Nanoparticles-Carboxylic Graphene-Nafion-Modified Electrode for Detection of Pesticides, Anal. Biochem., 2013, 437, 144-149.

638 L. Yang, G. Wang, Y. Liu and M. Wang, Development of a Biosensor Based on Immobilization of Acetylcholinesterase on $\mathrm{NiO}$ Nanoparticles-Carboxylic Graphene-Nafion Modified Electrode for Detection of Pesticides, Talanta, 2013, 113, 135-141. 
639 Q. Zhou, L. Yang, G. Wang and Y. Yang, Acetylcholinesterase Biosensor Based on $\mathrm{SnO}_{2}$ Nanoparticles-Carboxylic Graphene-Nafion Modified Electrode for Detection of Pesticides, Biosens. Bioelectron., 2013, 49, 25-31.

640 X. Tan, Q. Hu, J. Wu, X. Li, P. Li, H. Yu, X. Li and F. Lei, Electrochemical Sensor Based on Molecularly Imprinted Polymer Reduced Graphene Oxide and Gold Nanoparticles Modified Electrode for Detection of Carbofuran, Sens. Actuators, B, 2015, 220, 216-221.

641 M. Zhang, H. T. Zhao, T. J. Xie, X. Yang, A. J. Dong, H. Zhang, J. Wang and Z. Y. Wang, Molecularly Imprinted Polymer on Graphene Surface for Selective and Sensitive Electrochemical Sensing Imidacloprid, Sens. Actuators, B, 2017, 252, 991-1002.

642 T. Jeyapragasam, R. Saraswathi, S.-M. Chen and T.-W. Chen, Acetylcholinesterase Biosensor for the Detection of Methyl Parathion at an Electrochemically Reduced Graphene Oxide-Nafion Modified Glassy Carbon Electrode, Int. J. Electrochem. Sci., 2017, 12, 4768-4781.

643 M. Chao and M. Chen, Electrochemical Determination of Phoxim in Food Samples Employing a Graphene-Modified Glassy Carbon Electrode, Food Anal. Methods, 2014, 7, 1729-1736.

644 L. Wu, W. Lei, Z. Han, Y. Zhang, M. Xia and Q. Hao, A Novel Non-Enzyme Amperometric Platform Based on Poly(3Methylthiophene)/Nitrogen Doped Graphene Modified Electrode for Determination of Trace Amounts of Pesticide Phoxim, Sens. Actuators, B, 2015, 206, 495-501.

645 Y. Zheng, A. Wang, H. Lin, L. Fu and W. Cai, A Sensitive Electrochemical Sensor for Direct Phoxim Detection Based on an Electrodeposited Reduced Graphene OxideGold Nanocomposite, RSC Adv., 2015, 5, 15425-15430.

646 N. Karimian, H. Fakhri, S. Amidi, A. Hajian, F. Arduini and H. Bagheri, A novel sensing layer based on metal-organic framework UiO-66 modified with $\mathrm{TiO}_{2}$-graphene oxide: application to rapid, sensitive and simultaneous determination of paraoxon and chlorpyrifos, New J. Chem., 2019, 43, 2600-2609.

647 Q. Liu, A. Fei, J. Huan, H. Mao and K. Wang, Effective Amperometric Biosensor for Carbaryl Detection Based on Covalent Immobilization Acetylcholinesterase on Multiwall Carbon Nanotubes/Graphene Oxide Nanoribbons Nanostructure, J. Electroanal. Chem., 2015, 740, 8-13.

648 Y. Liu, G. Wang, C. Li, Q. Zhou, M. Wang and L. Yang, A Novel Acetylcholinesterase Biosensor Based on Carboxylic Graphene Coated with Silver Nanoparticles for Pesticide Detection, Mater. Sci. Eng., C, 2014, 35, 253-258.

649 G. Wang, X. Tan, Q. Zhou, Y. Liu, M. Wang and L. Yang, Synthesis of Highly Dispersed Zinc Oxide Nanoparticles on Carboxylic Graphene for Development a Sensitive Acetylcholinesterase Biosensor, Sens. Actuators, B, 2014, 190, 730-736.

650 Y. Zheng, Z. Liu, Y. Jing, J. Li and H. Zhan, An Acetylcholinesterase Biosensor Based on Ionic Liquid Functionalized Graphene-Gelatin-Modified Electrode for
Sensitive Detection of Pesticides, Sens. Actuators, B, 2015, 210, 389-397.

651 L. Zhang, A. Zhang, D. Du and Y. Lin, Biosensor Based on Prussian blue Nanocubes/Reduced Graphene Oxide Nanocomposite for Detection of Organophosphorus Pesticides, Nanoscale, 2012, 4, 4674-4679.

652 H. Zhao, X. Ji, B. Wang, N. Wang, X. Li, R. Ni and J. Ren, An Ultra-Sensitive Acetylcholinesterase Biosensor Based on Reduced Graphene Oxide-Au Nanoparticles- $\beta$ Cyclodextrin/Prussian Blue-Chitosan Nanocomposites for Organophosphorus Pesticides Detection, Biosens. Bioelectron., 2015, 65, 23-30.

653 H.-F. Cui, W.-W. Wu, M.-M. Li, X. Song, Y. Lv and T.-T. Zhang, A Highly Stable Acetylcholinesterase Biosensor Based on Chitosan- $\mathrm{TiO}_{2}$-Graphene Nanocomposites for Detection of Organophosphate Pesticides, Biosens. Bioelectron., 2018, 99, 223-229.

654 A. A. Ensafi, R. Noroozi, N. Zandi-Atashbar and B. Rezaei, Cerium(IV) Oxide Decorated on Reduced Graphene Oxide, a Selective and Sensitive Electrochemical Sensor for Fenitrothion Determination, Sens. Actuators, B, 2017, 245, 980-987.

655 N. Shams, H. N. Lim, R. Hajian, N. A. Yusof, J. Abdullah, Y. Sulaiman, I. Ibrahim, N. M. Huang and A. Pandikumar, A Promising Electrochemical Sensor Based on Au Nanoparticles Decorated Reduced Graphene Oxide for Selective Detection of Herbicide Diuron in Natural Waters, J. Appl. Electrochem., 2016, 46, 655-666.

656 Y. Yang, A. M. Asiri, D. Du and Y. Lin, Acetylcholinesterase Biosensor Based on a Gold Nanoparticle-PolypyrroleReduced Graphene Oxide Nanocomposite Modified Electrode for the Amperometric Detection of Organophosphorus Pesticides, Analyst, 2014, 139, 30553060 .

657 Y. Li, Y. Zhang, G. Han, Y. Xiao, M. Li and W. Zhou, An Acetylcholinesterase Biosensor Based on Graphene/ Polyaniline Composite Film for Detection of Pesticides, Chin. J. Chem., 2016, 34, 82-88.

658 Y. Li, L. Shi, G. Han, Y. Xiao and W. Zhou, Electrochemical Biosensing of Carbaryl Based on Acetylcholinesterase Immobilized onto Electrochemically Inducing Porous Graphene Oxide Network, Sens. Actuators, B, 2017, 238, 945-953.

659 J. Bao, C. Hou, M. Chen, J. Li, D. Huo, M. Yang, X. Luo and Y. Lei, Plant Esterase-Chitosan/Gold NanoparticlesGraphene Nanosheet Composite-Based Biosensor for the Ultrasensitive Detection of Organophosphate Pesticides, $J$. Agric. Food Chem., 2015, 63, 10319-10326.

660 S. Wu, F. Huang, X. Lan, X. Wang, J. Wang and C. Meng, Electrochemically Reduced Graphene Oxide and Nafion Nanocomposite for Ultralow Potential Detection of Organophosphate Pesticide, Sens. Actuators, B, 2013, 177, 724-729.

661 Y. Li, R. Zhao, L. Shi, G. Han and Y. Xiao, Acetylcholinesterase Biosensor Based on Electrochemically Inducing 3D Graphene Oxide Network/ 
Multi-Walled Carbon Nanotube Composites for Detection of Pesticides, RSC Adv., 2017, 7, 53570-53577.

662 Y. Zheng, Z. Liu, H. Zhan, J. Li and C. Zhang, Studies on Electrochemical Organophosphate Pesticide (OP) Biosensor Design Based On Ionic Liquid Functionalized Graphene and $\mathrm{a} \mathrm{Co}_{3} \mathrm{O}_{4}$ Nanoparticle Modified Electrode, Anal. Methods, 2016, 8, 5288-5295.

663 J. A. Hondred, J. C. Breger, N. J. Alves, S. A. Trammell, S. A. Walper, I. L. Medintz and J. C. Claussen, Printed Graphene Electrochemical Biosensors Fabricated by Inkjet Maskless Lithography for Rapid and Sensitive Detection of Organophosphates, ACS Appl. Mater. Interfaces, 2018, 10, 11125-11134.

664 A. P. de Silva, H. Q. N. Gunaratne, T. Gunnlaugsson, A. J. M. Huxley, C. P. McCoy, J. T. Rademacher and T. E. Rice, Signaling Recognition Events with Fluorescent Sensors and Switches, Chem. Rev., 1997, 97, 1515-1566.

665 M. Gao and B. Z. Tang, Fluorescent Sensors Based on Aggregation-Induced Emission: Recent Advances and Perspectives, ACS Sens., 2017, 2, 1382-1399.

666 A. Kasry, A. A. Ardakani, G. S. Tulevski, B. Menges, M. Copel and L. Vyklicky, Highly Efficient Fluorescence Quenching with Graphene, J. Phys. Chem. C, 2012, 116, 2858-2862.

667 W. Lin, B. Tian, P. Zhuang, J. Yin, C. Zhang, Q. Li, T.-M. Shih and W. Cai, Graphene-Based FluorescenceQuenching-Related Fermi Level Elevation and ElectronConcentration Surge, Nano Lett., 2016, 16, 5737-5741.

668 F. Tian, J. Lyu, J. Shi and M. Yang, Graphene and GrapheneLike Two-Denominational Materials Based Fluorescence Resonance Energy Transfer (FRET) Assays for Biological Applications, Biosens. Bioelectron., 2017, 89, 123-135.

669 M. J. Deka and D. Chowdhury, Tuning Electrical Properties of Graphene with Different $\pi$-Stacking Organic Molecules, J. Phys. Chem. C, 2016, 120, 4121-4129.

670 N. Li, A. Than, X. Wang, S. Xu, L. Sun, H. Duan, C. Xu and P. Chen, Ultrasensitive Profiling of Metabolites Using Tyramine-Functionalized Graphene Quantum Dots, ACS Nano, 2016, 10, 3622-3629.

671 P. Zhang, X. Zhao, Y. Ji, Z. Ouyang, X. Wen, J. Li, Z. Su and G. Wei, Electrospinning Graphene Quantum Dots into a Nanofibrous Membrane for Dual-Purpose Fluorescent and Electrochemical Biosensors, J. Mater. Chem. B, 2015, 3, 2487-2496.

672 R. Liu, R. Yang, C. Qu, H. Mao, Y. Hu, J. Li and L. Qu, Synthesis of Glycine-Functionalized Graphene Quantum Dots as Highly Sensitive and Selective Fluorescent Sensor of Ascorbic Acid in Human Serum, Sens. Actuators, B, 2017, 241, 644-651.

673 Y. Tu, W. Li, P. Wu, H. Zhang and C. Cai, Fluorescence Quenching of Graphene Oxide Integrating with the SiteSpecific Cleavage of the Endonuclease for Sensitive and Selective MicroRNA Detection, Anal. Chem., 2013, 85, 2536-2542.

674 W. Li, T. Hou, M. Wu and F. Li, Label-Free Fluorescence Strategy for Sensitive MicroRNA Detection Based on Isothermal Exponential Amplification and Graphene Oxide, Talanta, 2016, 148, 116-121.
675 C. Tong, C. Zhao, B. Liu, B. Li, Z. Ai, J. Fan and W. Wang, Sensitive Detection of RNase A Activity and Collaborative Drug Screening Based on rGO and Fluorescence Probe, Anal. Chem., 2018, 90, 2655-2661.

676 D. Bhatnagar, V. Kumar, A. Kumar and I. Kaur, Graphene Quantum Dots FRET Based Sensor for Early Detection of Heart Attack in Human, Biosens. Bioelectron., 2016, 79, 495-499.

677 K. Furukawa, Y. Ueno, M. Takamura and H. Hibino, Graphene FRET Aptasensor, ACS Sens., 2016, 1, 710-716.

678 J. Shi, C. Chan, Y. Pang, W. Ye, F. Tian, J. Lyu, Y. Zhang and M. Yang, A Fluorescence Resonance Energy Transfer (FRET) Biosensor Based on Graphene Quantum Dots (GQDs) and Gold Nanoparticles (AuNPs) for the Detection of mecA Gene Sequence of Staphylococcus aureus, Biosens. Bioelectron., 2015, 67, 595-600.

679 H. N. Abdelhamid and H.-F. Wu, Multifunctional Graphene Magnetic Nanosheet Decorated with Chitosan for Highly Sensitive Detection of Pathogenic Bacteria, J. Mater. Chem. B, 2013, 1, 3950-3961.

680 F. Yuan, H. Zhao, Z. Zhang, L. Gao, J. Xu and X. Quan, Fluorescent Biosensor for Sensitive Analysis of Oxytetracycline Based on an Indirectly Labelled LongChain Aptamer, RSC Adv., 2015, 5, 58895-58901.

681 X. Liu, F. Wang, R. Aizen, O. Yehezkeli and I. Willner, Graphene Oxide/Nucleic-Acid-Stabilized Silver Nanoclusters: Functional Hybrid Materials for Optical Aptamer Sensing and Multiplexed Analysis of Pathogenic DNAs, J. Am. Chem. Soc., 2013, 135, 11832-11839.

682 S. Wu, N. Duan, X. Ma, Y. Xia, H. Wang, Z. Wang and Q. Zhang, Multiplexed Fluorescence Resonance Energy Transfer Aptasensor between Upconversion Nanoparticles and Graphene Oxide for the Simultaneous Determination of Mycotoxins, Anal. Chem., 2012, 84, 6263-6270.

683 L. Sheng, J. Ren, Y. Miao, J. Wang and E. Wang, PVP-Coated Graphene Oxide for Selective Determination of Ochratoxin A via Quenching Fluorescence of Free Aptamer, Biosens. Bioelectron., 2011, 26, 3494-3499.

684 Z. Lu, X. Chen, Y. Wang, X. Zheng and C. M. Li, Aptamer Based Fluorescence Recovery Assay for Aflatoxin $\mathrm{B}_{1}$ Using a Quencher System Composed of Quantum Dots and Graphene Oxide, Microchim. Acta, 2014, 182, 571-578.

685 S. Wang, Y. Zhang, G. Pang, Y. Zhang and S. Guo, Tuning the Aggregation/Disaggregation Behavior of Graphene Quantum Dots by Structure-Switching Aptamer for HighSensitivity Fluorescent Ochratoxin A Sensor, Anal. Chem., 2017, 89, 1704-1709.

686 Z. Li, N. Xue, H. Ma, Z. Cheng and X. Miao, An Ultrasensitive and Switch-on Platform for Aflatoxin $\mathrm{B}_{1}$ Detection in Peanut Based on the Fluorescence Quenching of Graphene Oxide-Gold Nanocomposites, Talanta, 2018, 181, 346-351.

687 M. Liu, H. Zhao, S. Chen, H. Yu, Y. Zhang and X. Quan, Label-Free Fluorescent Detection of $\mathrm{Cu}$ (ii) Ions Based on DNA Cleavage-Dependent Graphene-Quenched DNAzymes, Chem. Commun., 2011, 47, 7749-7751. 
688 M. Liu, H. Zhao, S. Chen, H. Yu, Y. Zhang and X. Quan, A "Turn-on" Fluorescent Copper Biosensor Based on DNA Cleavage-Dependent Graphene-Quenched DNAzyme, Biosens. Bioelectron., 2011, 26, 4111-4116.

689 Y. Wen, C. Peng, D. Li, L. Zhuo, S. He, L. Wang, Q. Huang, Q.-H. Xu and C. Fan, Metal ion-Modulated GrapheneDNAzyme Interactions: Design of a Nanoprobe for Fluorescent Detection of Lead(ii) Ions with High Sensitivity, Selectivity and Tunable Dynamic Range, Chem. Commun., 2011, 47, 6278-6280.

690 X. Zhao, J. Gao, X. He, L. Cong, H. Zhao, X. Li and F. Tan, DNA-Modified Graphene Quantum Dots as a Sensing Platform For Detection of $\mathrm{Hg}^{2+}$ in Living Cells, RSC Adv., 2015, 5, 39587-39591.

691 S. Pang, S. Liu and X. Su, An Ultrasensitive Sensing Strategy for the Detection of Lead (II) Ions Based on the Intermolecular G-Quadruplex and Graphene Oxide, Sens. Actuators, B, 2015, 208, 415-420.

692 M. Li, X. Zhou, S. Guo and N. Wu, Detection of Lead (II) with a "Turn-On" Fluorescent Biosensor Based on Energy Transfer from CdSe/ZnS Quantum Dots to Graphene Oxide, Biosens. Bioelectron., 2013, 43, 69-74.

693 M. Li, X. Zhou, W. Ding, S. Guo and N. Wu, Fluorescent Aptamer-Functionalized Graphene Oxide Biosensor for Label-Free Detection of Mercury(II), Biosens. Bioelectron., 2013, 41, 889-893.

694 F. Wang, Z. Gu, W. Lei, W. Wang, X. Xia and Q. Hao, Graphene Quantum Dots as a Fluorescent Sensing Platform for Highly Efficient Detection of Copper(II) Ions, Sens. Actuators, B, 2014, 190, 516-522.

695 Z. S. Qian, X. Y. Shan, L. J. Chai, J. R. Chen and H. Feng, A Fluorescent Nanosensor Based on Graphene Quantum Dots-Aptamer Probe and Graphene Oxide Platform for Detection of Lead (II) Ion, Biosens. Bioelectron., 2015, 68, 225-231.

696 X. Cui, L. Zhu, J. Wu, Y. Hou, P. Wang, Z. Wang and M. Yang, A Fluorescent Biosensor Based on Carbon DotsLabeled Oligodeoxyribonucleotide and Graphene Oxide for Mercury (II) Detection, Biosens. Bioelectron., 2015, 63, 506-512.

697 A. D. Chowdhury and R. A. Doong, Highly Sensitive and Selective Detection of Nanomolar Ferric Ions Using Dopamine Functionalized Graphene Quantum Dots, ACS Appl. Mater. Interfaces, 2016, 8, 21002-21010.

698 X. Shi, W. Gu, W. Peng, B. Li, N. Chen, K. Zhao and Y. Xian, Sensitive $\mathrm{Pb}^{2+}$ Probe Based on the Fluorescence Quenching by Graphene Oxide and Enhancement of the Leaching of Gold Nanoparticles, ACS Appl. Mater. Interfaces, 2014, 6, 2568-2575.

699 X. Fang, Y. Liu, L. Jimenez, Y. Duan, G. B. Adkins, L. Qiao, B. Liu and W. Zhong, Rapid Enrichment and Sensitive Detection of Multiple Metal Ions Enabled by Macroporous Graphene Foam, Anal. Chem., 2017, 89, 11758-11764.

700 X. Zhu, Z. Zhang, Z. Xue, C. Huang, Y. Shan, C. Liu, X. Qin, W. Yang, X. Chen and T. Wang, Understanding the Selective Detection of $\mathrm{Fe}^{3+}$ Based on Graphene Quantum
Dots as Fluorescent Probes: The $K_{\mathrm{sp}}$ of a Metal HydroxideAssisted Mechanism, Anal. Chem., 2017, 89, 12054-12058.

701 B.-Y. Fang, C. Li, Y.-Y. Song, F. Tan, Y.-C. Cao and Y.-D. Zhao, Nitrogen-Doped Graphene Quantum Dot for Direct Fluorescence Detection of $\mathrm{Al}^{3+}$ in Aqueous Media and Living Cells, Biosens. Bioelectron., 2018, 100, 41-48.

702 S. Bian, C. Shen, Y. Qian, J. Liu, F. Xi and X. Dong, Facile Synthesis of Sulfur-Doped Graphene Quantum Dots as Fluorescent Sensing Probes For $\mathrm{Ag}^{+}$Ions Detection, Sens. Actuators, B, 2017, 242, 231-237.

703 C. Wang, F. Yang, Y. Tang, W. Yang, H. Zhong, C. Yu, R. Li, H. Zhou, Y. Li and L. Mao, Graphene Quantum Dots Nanosensor Derived from 3D Nanomesh Graphene Frameworks and its Application For Fluorescent Sensing of $\mathrm{Cu}^{2+}$ in Rat Brain, Sens. Actuators, B, 2018, 258, 672-681.

704 X. Niu, Y. Zhong, R. Chen, F. Wang, Y. Liu and D. Luo, A "Turn-on" Fluorescence Sensor for $\mathrm{Pb}^{2+}$ Detection Based on Graphene Quantum Dots and Gold Nanoparticles, Sens. Actuators, B, 2018, 255, 1577-1581.

705 D. Peng, L. Zhang, R.-P. Liang and J.-D. Qiu, Rapid Detection of Mercury Ions Based on Nitrogen-Doped Graphene Quantum Dots Accelerating Formation of Manganese Porphyrin, ACS Sens., 2018, 3, 1040-1047.

706 C. Zhang, B. Lin, Y. Cao, M. Guo and Y. Yu, Fluorescence Determination of Omethoate Based on a Dual Strategy for Improving Sensitivity, J. Agric. Food Chem., 2017, 65, 3065-3073.

707 Y. Liu, N. Cao, W. Gui and Q. Ma, Nitrogen-Doped Graphene Quantum Dots-Based Fluorescence Molecularly Imprinted Sensor for Thiacloprid Detection, Talanta, 2018, 183, 339-344.

708 J.-L. Chen, X.-P. Yan, K. Meng and S.-F. Wang, Graphene Oxide Based Photoinduced Charge Transfer Label-Free Near-Infrared Fluorescent Biosensor for Dopamine, Anal. Chem., 2011, 83, 8787-8793.

709 R. Cheng, C. Ge, L. Qi, Z. Zhang, J. Ma, H. Huang, T. Pan, Q. Dai and L. Dai, Label-Free Graphene Oxide Förster Resonance Energy Transfer Sensors for Selective Detection of Dopamine in Human Serums and Cells, $J$. Phys. Chem. C, 2018, 122, 13314-13321.

710 X.-J. Xing, W.-L. Xiao, X.-G. Liu, Y. Zhou, D.-W. Pang and H.-W. Tang, A Fluorescent Aptasensor Using DoubleStranded DNA/Graphene Oxide as the Indicator Probe, Biosens. Bioelectron., 2016, 78, 431-437.

711 L. Chen, L. Song, Y. Zhang, P. Wang, Z. Xiao, Y. Guo and F. Cao, Nitrogen and Sulfur Codoped Reduced Graphene Oxide as a General Platform for Rapid and Sensitive Fluorescent Detection of Biological Species, ACS Appl. Mater. Interfaces, 2016, 8, 11255-11261.

712 H. Zhang, Y. Wang, D. Zhao, D. Zeng, J. Xia, A. Aldalbahi, C. Wang, L. San, C. Fan, X. Zuo and X. Mi, Universal Fluorescence Biosensor Platform Based on Graphene Quantum Dots and Pyrene-Functionalized Molecular Beacons for Detection of MicroRNAs, ACS Appl. Mater. Interfaces, 2015, 7, 16152-16156.

713 P. Alonso-Cristobal, P. Vilela, A. El-Sagheer, E. LopezCabarcos, T. Brown, O. L. Muskens, J. Rubio-Retama and 
A. G. Kanaras, Highly Sensitive DNA Sensor Based on Upconversion Nanoparticles and Graphene Oxide, ACS Appl. Mater. Interfaces, 2015, 7, 12422-12429.

714 L.-J. Huang, X. Tian, J.-T. Yi, R.-Q. Yu and X. Chu, A Turn-on Upconversion Fluorescence Resonance Energy Transfer Biosensor for Ultrasensitive Endonuclease Detection, Anal. Methods, 2015, 7, 7474-7479.

715 C. I. L. Justino, T. A. Rocha-Santos, A. C. Duarte and T. A. Rocha-Santos, Review of Analytical Figures of Merit of Sensors and Biosensors in Clinical Applications, TrAC, Trends Anal. Chem., 2010, 29, 1172-1183.

716 M. Liu, J. Song, S. Shuang, C. Dong, J. D. Brennan and Y. Li, A Graphene-Based Biosensing Platform Based on the Release of DNA Probes and Rolling Circle Amplification, ACS Nano, 2014, 8, 5564-5573.

717 C. Hong, A. Baek, S. S. Hah, W. Jung and D.-E. Kim, Fluorometric Detection of MicroRNA Using Isothermal Gene Amplification and Graphene Oxide, Anal. Chem., 2016, 88, 2999-3003.

718 X. Fan, Y. Qi, Z. Shi, Y. Lv and Y. Guo, A Graphene-Based Biosensor for Detecting microRNA with Augmented Sensitivity Through Helicase-Assisted Signal Amplification of Hybridization Chain Reaction, Sens. Actuators, B, 2018, 255, 1582-1586.

719 Z. Shi, X. Zhang, R. Cheng, B. Li and Y. Jin, Sensitive Detection of Intracellular RNA of Human Telomerase by Using Graphene Oxide as a Carrier to Deliver the Assembly Element of Hybridization Chain Reaction, Analyst, 2016, 141, 2727-2732.

720 L. Yang, C. Liu, W. Ren and Z. Li, Graphene SurfaceAnchored Fluorescence Sensor for Sensitive Detection of MicroRNA Coupled with Enzyme-Free Signal Amplification of Hybridization Chain Reaction, ACS Appl. Mater. Interfaces, 2012, 4, 6450-6453.

721 H. Yan, Y. Xu, Y. Lu and W. Xing, Reduced Graphene OxideBased Solid-Phase Extraction for the Enrichment and Detection of microRNA, Anal. Chem., 2017, 89, 1013710140.

722 Y. Huang, X. Liu, L. Zhang, K. Hu, S. Zhao, B. Fang, Z.-F. Chen and H. Liang, Nicking Enzyme and Graphene Oxide-Based Dual Signal Amplification for Ultrasensitive Aptamer-Based Fluorescence Polarization Assays, Biosens. Bioelectron., 2015, 63, 178-184.

723 X. Yan, W. Li, K. Liu and L. Deng, Highly Sensitive Fluorescent Aptasensor for Salmonella paratyphi A via DNase I-Mediated Cyclic Signal Amplification, Anal. Methods, 2015, 7, 10243-10250.

724 J. Ge, Z.-M. Huang, Q. Xi, R.-Q. Yu, J.-H. Jiang and X. Chu, A Novel Graphene Oxide Based Fluorescent Nanosensing Strategy with Hybridization Chain Reaction Signal Amplification for Highly Sensitive Biothiol Detection, Chem. Commun., 2014, 50, 11879-11882.

725 J. Wen, W. Li, J. Li, B. Tao, Y. Xu, H. Li, A. Lu and S. Sun, Study on Rolling Circle Amplification of Ebola Virus and Fluorescence Detection based on Graphene Oxide, Sens. Actuators, B, 2016, 227, 655-659.
726 X.-H. Zhao, R.-M. Kong, X.-B. Zhang, H.-M. Meng, W.-N. Liu, W. Tan, G.-L. Shen and R.-Q. Yu, GrapheneDNAzyme Based Biosensor for Amplified Fluorescence "Turn-on" Detection of $\mathrm{Pb}^{2+}$ with a High Selectivity, Anal. Chem., 2011, 83, 5062-5066.

727 (a) H. Peng, A. M. Newbigging, Z. Wang, J. Tao, W. Deng, X. C. Le and H. Zhang, DNAzyme-Mediated Assays for Amplified Detection of Nucleic Acids and Proteins, Anal. Chem., 2018, 90, 190-207; (b) R. R. Breaker and G. F. Joyce, A DNA enzyme that cleaves RNA, Chem. Biol., 1994, 1, 223-229.

728 Q. Xi, J.-J. Li, W.-F. Du, R.-Q. Yu and J.-H. Jiang, A Highly Sensitive Strategy for Base Excision Repair Enzyme Activity Detection Based on Graphene Oxide Mediated Fluorescence Quenching and Hybridization Chain Reaction, Analyst, 2016, 141, 96-99.

729 R. Mitra and A. Saha, Reduced Graphene Oxide Based "Turn-On" Fluorescence Sensor for Highly Reproducible and Sensitive Detection of Small Organic Pollutants, ACS Sustainable Chem. Eng., 2017, 5, 604-615.

730 Y. Wen, F. Xing, S. He, S. Song, L. Wang, Y. Long, D. Li and C. Fan, A Graphene-Based Fluorescent Nanoprobe for Silver(i) Ions Detection by Using Graphene Oxide and A Silver-Specific Oligonucleotide, Chem. Commun., 2010, 46, 2596-2598.

731 C. Wang, S. Yang, M. Yi, C. Liu, Y. Wang, J. Li, Y. Li and R. Yang, Graphene Oxide Assisted Fluorescent Chemodosimeter for High-Performance Sensing and Bioimaging of Fluoride Ions, ACS Appl. Mater. Interfaces, 2014, 6, 9768-9775.

732 A. Ananthanarayanan, X. Wang, P. Routh, B. Sana, S. Lim, D.-H. Kim, K.-H. Lim, J. Li and P. Chen, Facile Synthesis of Graphene Quantum Dots from 3D Graphene and their Application for $\mathrm{Fe}^{3+}$ Sensing, Adv. Funct. Mater., 2014, 24, 3021-3026.

733 R. Guo, S. Zhou, Y. Li, X. Li, L. Fan and N. H. Voelcker, Rhodamine-Functionalized Graphene Quantum Dots for Detection of $\mathrm{Fe}^{3+}$ in Cancer Stem Cells, ACS Appl. Mater. Interfaces, 2015, 7, 23958-23966.

734 S. Li, Y. Li, J. Cao, J. Zhu, L. Fan and X. Li, Sulfur-Doped Graphene Quantum Dots as a Novel Fluorescent Probe for Highly Selective and Sensitive Detection of $\mathrm{Fe}^{3+}$, Anal. Chem., 2014, 86, 10201-10207.

735 E. Zor, E. Morales-Narváez, A. Zamora-Gálvez, H. Bingol, M. Ersoz and A. Merkoçi, Graphene Quantum Dots-based Photoluminescent Sensor: A Multifunctional Composite for Pesticide Detection, ACS Appl. Mater. Interfaces, 2015, 7, 20272-20279.

736 L. Gao, L. Ju and H. Cui, Chemiluminescent and Fluorescent Dual-Signal Graphene Quantum Dots and Their Application in Pesticide Sensing Arrays, J. Mater. Chem. C, 2017, 5, 7753-7758.

737 X. Yan, Y. Song, C. Zhu, J. Song, D. Du, X. Su and Y. Lin, Graphene Quantum Dot- $\mathrm{MnO}_{2}$ Nanosheet Based Optical Sensing Platform: A Sensitive Fluorescence "Turn off-on" Nanosensor for Glutathione Detection and Intracellular Imaging, ACS Appl. Mater. Interfaces, 2016, 8, 21990-21996. 
738 F. Nemati, M. Hosseini, R. Zare-Dorabei and M. R. Ganjali, Sensitive Recognition of Ethion in Food Samples Using Turn-on Fluorescence $\mathrm{N}$ and $\mathrm{S}$ Co-Doped Graphene Quantum Dots, Anal. Methods, 2018, 10, 1760-1766.

739 C. Kotlowski, M. Larisika, P. M. Guerin, C. Kleber, T. Kröber, R. Mastrogiacomo, C. Nowak, P. Pelosi, S. Schütz, A. Schwaighofer and W. Knoll, Fine Discrimination of Volatile Compounds by GrapheneImmobilized Odorant-Binding Proteins, Sens. Actuators, B, 2018, 256, 564-572.

740 S.-J. Choi, S.-J. Kim and I.-D. Kim, Ultrafast Optical Reduction of Graphene Oxide Sheets on Colorless Polyimide Film for Wearable Chemical Sensors, NPG Asia Mater., 2016, 8, e315.

741 M. H. M. Facure, L. A. Mercante, L. H. C. Mattoso and D. S. Correa, Detection of Trace Levels of Organophosphate Pesticides Using an Electronic Tongue Based on Graphene Hybrid Nanocomposites, Talanta, 2017, 167, 59-66.

742 K. S. Kim, J.-r. Jang, W.-S. Choe and P. J. Yoo, Electrochemical Detection of Bisphenol A with High Sensitivity and Selectivity Using Recombinant ProteinImmobilized Graphene Electrodes, Biosens. Bioelectron., 2015, 71, 214-221.

743 S. Gupta and R. Wood, Development of FRET Biosensor Based on Aptamer/Functionalized Graphene for Ultrasensitive Detection of Bisphenol A and Discrimination from Analogs, Nano-Struct. Nano-Objects, 2017, 10, 131-140.

744 S. Myung, A. Solanki, C. Kim, J. Park, K. S. Kim and K.-B. Lee, Graphene-Encapsulated Nanoparticle-Based Biosensor for the Selective Detection of Cancer Biomarkers, Adv. Mater., 2011, 23, 2221-2225.

745 D. Khatayevich, T. Page, C. Gresswell, Y. Hayamizu, W. Grady and M. Sarikaya, Selective Detection of Target Proteins by Peptide-Enabled Graphene Biosensor, Small, 2014, 10, 1505-1513.

746 S. Tomita, S. Ishihara and R. Kurita, A Multi-Fluorescent DNA/Graphene Oxide Conjugate Sensor for SignatureBased Protein Discrimination, Sensors, 2017, 17, E2194.

747 P. Si, H. Chen, P. Kannan and D.-H. Kim, Selective and Sensitive Determination of Dopamine by Composites of Polypyrrole and Graphene Modified Electrodes, Analyst, 2011, 136, 5134-5138.

748 S. Hou, M. L. Kasner, S. Su, K. Patel and R. Cuellari, Highly Sensitive and Selective Dopamine Biosensor Fabricated with Silanized Graphene, J. Phys. Chem. C, 2010, 114, 14915-14921.

749 S. Dong, Q. Bi, C. Qiao, Y. Sun, X. Zhang, X. Lu and L. Zhao, Electrochemical Sensor for Discrimination Tyrosine Enantiomers Using Graphene Quantum Dots and BCyclodextrins Composites, Talanta, 2017, 173, 94-100.

750 A. B. Ganganboina, A. D. Chowdhury and R.-A. Doong, NDoped Graphene Quantum Dots-Decorated $\mathrm{V}_{2} \mathrm{O}_{5}$ Nanosheet for Fluorescence Turn Off-On Detection of Cysteine, ACS Appl. Mater. Interfaces, 2017, 10, 614-624.

751 N. T. N. Anh and R.-a. Doong, One-Step Synthesis of SizeTunable Gold@Sulfur-Doped Graphene Quantum Dot
Nanocomposites for Highly Selective and Sensitive Detection of Nanomolar 4-Nitrophenol in Aqueous Solutions with Complex Matrix, ACS Appl. Nano Mater., 2018, 1, 2153-2163.

752 H. Pei, J. Li, M. Lv, J. Wang, J. Gao, J. Lu, Y. Li, Q. Huang, J. Hu and C. Fan, A Graphene-Based Sensor Array for HighPrecision and Adaptive Target Identification with Ensemble Aptamers, J. Am. Chem. Soc., 2012, 134, 13843-13849.

753 S. S. Chou, M. De, J. Luo, V. M. Rotello, J. Huang and V. P. Dravid, Nanoscale Graphene Oxide (nGO) as Artificial Receptors: Implications for Biomolecular Interactions and Sensing, J. Am. Chem. Soc., 2012, 134, 16725-16733.

754 R. T. K. Kwok, C. W. T. Leung, J. W. Y. Lam and B. Z. Tang, Biosensing by Luminogens with Aggregation-Induced Emission Characteristics, Chem. Soc. Rev., 2015, 44, 42284238.

755 J. Mei, Y. Huang and H. Tian, Progress and Trends in AIEBased Bioprobes: A Brief Overview, ACS Appl. Mater. Interfaces, 2017, 10, 12217-12261.

756 Y. Hong, L. Meng, S. Chen, C. W. T. Leung, L.-T. Da, M. Faisal, D.-A. Silva, J. Liu, J. W. Y. Lam, X. Huang and B. Z. Tang, Monitoring and Inhibition of Insulin Fibrillation by a Small Organic Fluorogen with Aggregation-Induced Emission Characteristics, J. Am. Chem. Soc., 2012, 134, 1680-1689.

757 X. Li, K. Ma, S. Zhu, S. Yao, Z. Liu, B. Xu, B. Yang and W. Tian, Fluorescent Aptasensor Based on AggregationInduced Emission Probe and Graphene Oxide, Anal. Chem., 2013, 86, 298-303.

758 J.-W. Liu, Y. Luo, Y.-M. Wang, L.-Y. Duan, J.-H. Jiang and R.-Q. Yu, Graphitic Carbon Nitride Nanosheets-Based Ratiometric Fluorescent Probe for Highly Sensitive Detection of $\mathrm{H}_{2} \mathrm{O}_{2}$ and Glucose, ACS Appl. Mater. Interfaces, 2016, 8, 33439-33445.

759 L. Zhao, Y. Chen, J. Yuan, M. Chen, H. Zhang and X. Li, Electrospun Fibrous Mats with Conjugated Tetraphenylethylene and Mannose for Sensitive Turn-On Fluorescent Sensing of Escherichia coli, ACS Appl. Mater. Interfaces, 2015, 7, 5177-5186.

760 J. Shen, R. Hu, T. Zhou, Z. Wang, Y. Zhang, S. Li, C. Gui, M. Jiang, A. Qin and B. Z. Tang, Fluorescent Sensor Array for Highly Efficient Microbial Lysate Identification through Competitive Interactions, ACS Sens., 2018, 3, 2218-2222.

761 X. Xu, J. Huang, J. Li, J. Yan, J. Qin and Z. Li, A Graphene OxideBased AIE Biosensor with High Selectivity Toward Bovine Serum Albumin, Chem. Commun., 2011, 47, 12385-12387.

762 X. Huang, X. Deng, W. Qi and D. Wu, Simultaneous Detection of Hydroquinone and Catechol Using Platinum Nanoparticles Decorated Graphene/Poly-Cyclodextrin/ Multiwalled Carbon Nanotubes (MWCNTs) Nanocomposite Based Biosensor, J. Nanosci. Nanotechnol., 2018, 18, 8118-8123.

763 H. Chang, X. Wu, C. Wu, Y. Chen, H. Jiang and X. Wang, Catalytic oxidation and determination of $\beta-\mathrm{NADH}$ using 
self-assembly hybrid of gold nanoparticles and graphene, Analyst, 2011, 136, 2735-2740.

764 Y. Yan, Q. Liu, X. Du, J. Qian, H. Mao and K. Wang, Visible Light Photoelectrochemical Sensor for Ultrasensitive Determination of Dopamine Based on Synergistic Effect of Graphene Quantum Dots and $\mathrm{TiO}_{2}$ Nanoparticles, Anal. Chim. Acta, 2015, 853, 258-264.

765 Z.-Q. Zhao, X. Chen, Q. Yang, J.-H. Liu and X.-J. Huang, Selective Adsorption Toward Toxic Metal Ions Results in Selective Response: Electrochemical Studies on A Polypyrrole/Reduced Graphene Oxide Nanocomposite, Chem. Commun., 2012, 48, 2180-2182.

766 X. Mao, H. Su, D. Tian, H. Li and R. Yang, Bipyrenefunctionalized graphene as a "turn-on" fluorescence sensor for manganese (II) ions in living cells, ACS Appl. Mater. Interfaces, 2013, 5, 592-597.

767 H. Sun, N. Gao, L. Wu, J. Ren, W. Wei and X. Qu, Highly Photoluminescent Amino-Functionalized Graphene Quantum Dots Used for Sensing Copper Ions, Chem.-Eur. J., 2013, 19, 13362-13368.

768 L. Xu, W. Mao, J. Huang, S. Li, K. Huang, M. Li, J. Xia and Q. Chen, Economical, Green Route to Highly Fluorescence Intensity Carbon Materials Based on Ligninsulfonate/ Graphene Quantum Dots Composites: Application as Excellent Fluorescent Sensing Platform for Detection of $\mathrm{Fe}^{3+}$ Ions, Sens. Actuators, B, 2016, 230, 54-60.

769 C. Göde, M. L. Yola, A. Yılmaz, N. Atar and S. Wang, A Novel Electrochemical Sensor Based on Calixarene Functionalized Reduced Graphene Oxide: Application to Simultaneous Determination of $\mathrm{Fe}(\mathrm{III}), \mathrm{Cd}(\mathrm{II})$ and $\mathrm{Pb}(\mathrm{II})$ Ions, J. Colloid Interface Sci., 2017, 508, 525-531.

770 F. Salehnia, F. Faridbod, A. S. Dezfuli, M. R. Ganjali and P. Norouzi, Cerium(III) Ion Sensing Based on Graphene Quantum Dots Fluorescent Turn-Off, J. Fluoresc., 2017, 27, 331-338.

771 N. Ruecha, N. Rodthongkum, D. M. Cate, J. Volckens, O. Chailapakul and C. S. Henry, Sensitive Electrochemical Sensor Using a Graphene-Polyaniline Nanocomposite for Simultaneous Detection of $\mathrm{Zn}(\mathrm{II}), \mathrm{Cd}(\mathrm{II})$, and $\mathrm{Pb}(\mathrm{II})$, Anal. Chim. Acta, 2015, 874, 40-48.

772 H. Bagheri, A. Afkhami, H. Khoshsafar, M. Rezaei, S. J. Sabounchei and M. Sarlakifar, Simultaneous Electrochemical Sensing of Thallium, Lead and Mercury Using a Novel Ionic Liquid/Graphene Modified Electrode, Anal. Chim. Acta, 2015, 870, 56-66.

773 A. Afkhami, A. Shirzadmehr, T. Madrakian and H. Bagheri, New Nano-Composite Potentiometric Sensor Composed of Graphene Nanosheets/Thionine/Molecular Wire for Nanomolar Detection of Silver Ion in Various Real Samples, Talanta, 2015, 131, 548-555.

774 X. Jiang, K. Chen, J. Wang, K. Shao, T. Fu, F. Shao, D. Lu, J. Liang, M. F. Foda and H. Han, Solid-State VoltammetryBased Electrochemical Immunosensor for Escherichia coli Using Graphene Oxide-Ag Nanoparticle Composites as Labels, Analyst, 2013, 138, 3388-3393.

775 J. Zhang, F. Ran, W. Zhou, B. Shang, F. Yu, L. Wu, W. Hu, $\mathrm{X}$. He and Q. Chen, Ultrasensitive fluorescent aptasensor for MUC1 detection based on deoxyribonuclease I-aided target recycling signal amplification, $R S C$ Adv., 2018, 8, 32009-32015.

776 B. Thakur, G. Zhou, J. Chang, H. Pu, B. Jin, X. Sui, X. Yuan, C.-H. Yang, M. Magruder and J. Chen, Rapid Detection of Single E. coli Bacteria Using a Graphene-Based FieldEffect Transistor Device, Biosens. Bioelectron., 2018, 110, $16-22$.

777 S. Xu, H. Duo, C. Zheng, S. Zhao, S. Song and G. Simon, Novel approach to fabrication of DNA Biosensor Based on a Carboxylated Graphene Oxide Decorated with $\mathrm{Fe}_{3} \mathrm{O}_{4} \mathrm{NPs}$ for the Detection of Typhoidal Salmonella, Int. J. Electrochem. Sci., 2019, 14, 1248-1269.

778 A. Mathur, R. Gupta, S. Kondal, S. Wadhwa, R. N. Pudake, Shivani, R. Kansal, C. S. Pundir and J. Narang, A New Tactics for the Detection of $S$. aureus via Paper Based Geno-Interface Incorporated with Graphene Nano Dots And Zeolites, Int. J. Biol. Macromol., 2018, 112, 364-370.

779 R. Chand, Y. L. Wang, D. Kelton and S. Neethirajan, Isothermal DNA amplification with functionalized graphene and nanoparticle assisted electroanalysis for rapid detection of Johne's disease, Sens. Actuators, B, 2018, 261, 31-37.

780 J. Yang, Z. Zhang and G. Yan, An Aptamer-Mediated CdSe/ ZnS QDs@Graphene Oxid Composite Fluorescent Probe for Specific Detection of Insulin, Sens. Actuators, B, 2018, 255, 2339-2346.

781 M. Wang, Z. Lin, Q. Liu, S. Jiang, H. Liu and X. Su, DNAHosted Copper Nanoclusters/Graphene Oxide Based Fluorescent Biosensor for Protein Kinase Activity Detection, Anal. Chim. Acta, 2018, 1012, 66-73.

782 J. Zou, G. Q. Zhao, J. Teng, Q. Liu, X. Y. Jiang, F. P. Jiao and J. G. Yu, Highly sensitive detection of bisphenol A in real water samples based on in situ assembled graphene nanoplatelets and gold nanoparticles composite, Microchem. J., 2019, 145, 693-702.

783 V. Velusamy, S. Palanisamy, S. W. Chen, S. Balu, T. C. Yang and C. E. Banks, Novel electrochemical synthesis of cellulose microfiber entrapped reduced graphene oxide: a sensitive electrochemical assay for detection of fenitrothion organophosphorus pesticide, Talanta, 2019, 192, 471-477.

784 L. Tian, J. Qi, K. Qian, O. Oderinde, Y. Cai, C. Yao, W. Song and Y. Wang, An ultrasensitive electrochemical cytosensor based on the magnetic field assisted binanozymes synergistic catalysis of $\mathrm{Fe}_{3} \mathrm{O}_{4}$ nanozyme and reduced graphene oxide/molybdenum disulfide nanozyme, Sens. Actuators, B, 2018, 260, 676-684.

785 S. Shahrokhian and R. Salimian, Ultrasensitive detection of cancer biomarkers using conducting polymer/ electrochemically reduced graphene oxide-based biosensor: application toward BRCA1 sensing, Sens. Actuators, B, 2018, 266, 160-169.

786 S. Afsahi, M. B. Lerner, J. M. Goldstein, J. Lee, X. Tang, D. A. Bagarozzi Jr, D. Pan, L. Locascio, A. Walker, F. Barron and B. R. Goldsmith, Novel graphene-based 
biosensor for early detection of Zika virus infection, Biosens. Bioelectron., 2018, 100, 85-88.

787 L. Li, Y. Yuan, Y. Chen, P. Zhang, Y. Bai and L. Bai, Aptamer based voltammetric biosensor for Mycobacterium tuberculosis antigen ESAT-6 using a nanohybrid material composed of reduced graphene oxide and a metal-organic framework, Microchim. Acta, 2018, 185, 379.

788 X. Xuan, H. S. Yoon and J. Y. Park, A wearable electrochemical glucose sensor based on simple and lowcost fabrication supported micro-patterned reduced graphene oxide nanocomposite electrode on flexible substrate, Biosens. Bioelectron., 2018, 109, 75-82.

789 (a) L. Yang, B. Liu, M. Wang, J. Li, W. Pan, X. Gao, N. Li and B. Tang, A Highly Sensitive Strategy for Fluorescence Imaging of MicroRNA in Living Cells and In vivo Based on Graphene Oxide-Enhanced Signal Molecules Quenching of Molecular Beacon, ACS Appl. Mater. Interfaces, 2018, 10, 6982-6990; (b) X. Ou, S. Zhan, C. Sun, Y. Cheng, X. Wang, B. Liu, T. Zhai, X. Lou and F. Xia, Simultaneous detection of telomerase and miRNA with graphene oxide-based fluorescent aptasensor in living cells and tissue samples, Biosens. Bioelectron., 2019, 124, 199-204.

790 S. Chen, Y. F. Cheng and G. Voordouw, Three-dimensional graphene nanosheet doped with gold nanoparticles as electrochemical DNA biosensor for bacterial detection, Sens. Actuators, B, 2018, 262, 860-868.

791 W. Zou, F. Gong, T. Gu, Z. Cao and J. Xia, An efficient strategy for sensing pyrophosphate based on nitrogenrich quantum dots combined with graphene oxide, Microchem. J., 2018, 141, 466-472.

792 A. S. Sadeghi, M. Mohsenzadeh, K. Abnous, S. M. Taghdisi and M. Ramezani, Development and characterization of DNA aptamers against florfenicol: fabrication of a sensitive fluorescent aptasensor for specific detection of florfenicol in milk, Talanta, 2018, 182, 193-201.

793 J. Tian, W. Wei, J. Wang, S. Ji, G. Chen and J. Lu, Fluorescence resonance energy transfer aptasensor between nanoceria and graphene quantum dots for the determination of ochratoxin A, Anal. Chim. Acta, 2018, 1000, 265-272.

794 M. Venu, S. Venkateswarlu, Y. V. M. Reddy, A. Seshadri Reddy, V. K. Gupta, M. Yoon and G. Madhavi, Highly Sensitive Electrochemical Sensor for Anticancer Drug by a Zirconia Nanoparticle-Decorated Reduced Graphene Oxide Nanocomposite, ACS Omega, 2018, 3, 14597-14605.

795 X. Liu, W. Na, Q. Liu and X. Su, A novel label-free fluorescent sensor for highly sensitive detection of bleomycin based on nitrogen-doped graphene quantum dots, Anal. Chim. Acta, 2018, 1028, 45-49.

796 M. A. Tabrizi, J. Ferré-Borrull, P. Kapruwan and L. F. Marsal, A photoelectrochemical sandwich immunoassay for protein $\mathrm{S} 100 \beta$, a biomarker for Alzheimer's disease, using an ITO electrode modified with a reduced graphene oxide-gold conjugate and CdSlabeled secondary antibody, Microchim. Acta, 2019, 186, 117.
797 W. Gui, J. Zhang, X. Chen, D. Yu and Q. Ma, N-Doped graphene quantum dot@ mesoporous silica nanoparticles modified with hyaluronic acid for fluorescent imaging of tumor cells and drug delivery, Microchim. Acta, 2018, 185, 66.

798 F. Chekin, K. Bagga, P. Subramanian, R. Jijie, S. K. Singh, S. Kurungot, R. Boukherroub and S. Szunerits, Nucleic aptamer modified porous reduced graphene oxide $/ \mathrm{MoS}_{2}$ based electrodes for viral detection: application to human papillomavirus (HPV), Sens. Actuators, B, 2018, 262, 9911000.

799 K. Navakul, C. Warakulwit, P. T. Yenchitsomanus, A. Panya, P. A. Lieberzeit and C. Sangma, A novel method for dengue virus detection and antibody screening using a graphenepolymer based electrochemical biosensor, Nanomedicine, 2017, 13, 549-557.

800 Q. Xiang, J. Huang, H. Huang, W. Mao and Z. Ye, A labelfree electrochemical platform for the highly sensitive detection of hepatitis B virus DNA using graphene quantum dots, $R S C$ Adv., 2018, 8, 1820-1825.

801 M. F. A. Muain, K. H. Cheo, M. N. Omar, A. S. A. Hamzah, H. N. Lim, A. B. Salleh, W. S. Tan and A. A. Tajudin, Gold nanoparticle-decorated reduced-graphene oxide targeting anti hepatitis B virus core antigen, Bioelectrochemistry, 2018, 122, 199-205.

802 A. Valipour and M. Roushani, Using silver nanoparticle and thiol graphene quantum dots nanocomposite as a substratum to load antibody for detection of hepatitis $\mathrm{C}$ virus core antigen: electrochemical oxidation of riboflavin was used as redox probe, Biosens. Bioelectron., 2017, 89, 946-951.

803 L. Ruiyi, C. Fangchao, Z. Haiyan, S. Xiulan and L. Zaijun, Electrochemical sensor for detection of cancer cell based on folic acid and octadecylamine-functionalized graphene aerogel microspheres, Biosens. Bioelectron., 2018, 119, 156-162.

804 Q. Zhou, H. Yan, F. Ran, J. Cao, L. Chen, B. Shang, H. Chen, J. Wei and Q. Chen, Ultrasensitive enzyme-free fluorescent detection of VEGF 165 based on target-triggered hybridization chain reaction amplification, $R S C A d v$, 2018, 8(46), 25955-25960.

805 D. Liu, X. Lu, Y. Yang, Y. Zhai, J. Zhang and L. Li, A novel fluorescent aptasensor for the highly sensitive and selective detection of cardiac troponin I based on a graphene oxide platform, Anal. Bioanal. Chem., 2018, 410, 4285-4291.

806 D. Sandil, S. Srivastava, B. D. Malhotra, S. C. Sharma and N. K. Puri, Biofunctionalized tungsten trioxide-reduced graphene oxide nanocomposites for sensitive electrochemical immunosensing of cardiac biomarker, $J$. Alloys Compd., 2018, 763, 102-110.

807 A. Gevaerd, S. F. Blaskievicz, A. J. Zarbin, E. S. Orth, M. F. Bergamini and L. H. Marcolino-Junior, Nonenzymatic electrochemical sensor based on imidazole-functionalized graphene oxide for progesterone detection, Biosens. Bioelectron., 2018, 112, 108-113. 
808 M. Liu, H. Ke, C. Sun, G. Wang, Y. Wang and G. Zhao, A simple and highly selective electrochemical label-free aptasensor of $17 \beta$-estradiol based on signal amplification of bi-functional graphene, Talanta, 2019, 194, 266-272.

809 J. H. Lee, H. K. Choi, L. Yang, S. T. D. Chueng, J. W. Choi and K. B. Lee, Nondestructive Real-Time Monitoring of Enhanced Stem Cell Differentiation Using a Graphene-Au Hybrid Nanoelectrode Array, Adv. Mater., 2018, 30, 1802762.

810 F. Gao, J. Wu, Y. Yao, Y. Zhang, X. Liao, D. Geng and D. Tang, Proximity hybridization triggered strand displacement and DNAzyme assisted strand recycling for ATP fluorescence detection in vitro and imaging in living cells, RSC Adv., 2018, 8, 28161-28171.

811 S. Yu, H. Li, G. Li, L. Niu, W. Liu and X. Di, Reduced graphene oxide-supported gold dendrite for electrochemical sensing of acetaminophen, Talanta, 2018, 184, 244-250.

812 W. Li, J. Zhu, G. Xie, Y. Ren and Y. Q. Zheng, Ratiometric system based on graphene quantum dots and $\mathrm{Eu}^{3+}$ for selective detection of tetracyclines, Anal. Chim. Acta, 2018, 1022, 131-137.

813 T. Jiang, H. Tan, Y. Sun, J. Wang, Y. Hang, N. Lu, J. Yang, $\mathrm{X}$. Qu and J. Hua, Graphene oxide-based NIR fluorescence probe with aggregation-induced emission property for lectins detection and liver cells targeting, Sens. Actuators, $B, 2018,261,115-126$.

814 N. Siraj, B. El-Zahab, S. Hamdan, T. E. Karam, L. H. Haber, M. Li, S. O. Fakayode, S. Das, B. Valle, R. M. Strongin, G. Patonay, H. O. Sintim, G. A. Baker, A. Powe, M. Lowry, J. O. Karolin, C. D. Geddes and I. M. Warner, Fluorescence, Phosphorescence, and Chemiluminescence, Anal. Chem., 2015, 88, 170-202.

815 H. Zhang, S. Ba, D. Mahajan, J. Y. Lee, R. Ye, F. Shao, L. Lu and T. Li, Versatile Types of DNA-Based Nanobiosensors for Specific Detection of Cancer Biomarker FEN1 in Living Cells and Cell-Free Systems, Nano Lett., 2018, 18, 73837388.

816 Z. Shang, L. Qian, S. Liu, X. Niu, Z. Qiao, Y. Sun, Y. Zhang, L. Y. Fan, X. Guan, C. X. Cao and H. Xiao, Graphene OxideFacilitated Comprehensive Analysis of Cellular Nucleic Acid Binding Proteins for Lung Cancer, ACS Appl. Mater. Interfaces, 2018, 10, 17756-17770.

817 D. Jin, F. Yang, Y. Zhang, L. Liu, Y. Zhou, F. Wang and G. J. Zhang, ExoAPP: Exosome-Oriented, Aptamer Nanoprobe-Enabled Surface Proteins Profiling and Detection, Anal. Chem., 2018, 90, 14402-14411.
818 R. Heidari, J. Rashidiani, M. Abkar, R. A. Taheri, M. M. Moghaddam, S. A. Mirhosseini, R. Seidmoradi, M. R. Nourani, M. Mahboobi, A. H. Keihan and H. Kooshki, CdS nanocrystals/graphene oxide-AuNPs based electrochemiluminescence immunosensor in sensitive quantification of a cancer biomarker: p53, Biosens. Bioelectron., 2019, 126, 7-14.

819 V. Serafín, A. Valverde, G. Martínez-García, E. MartínezPeriñán, F. Comba, M. Garranzo-Asensio, R. Barderas, P. Yáñez-Sedeño, S. Campuzano and J. M. Pingarrón, Graphene quantum dots-functionalized multi-walled carbon nanotubes as nanocarriers in electrochemical immunosensing. Determination of IL-13 receptor $\alpha 2$ in colorectal cells and tumor tissues with different metastatic potential, Sens. Actuators, B, 2019, 284, 711-722.

820 C. Tian, L. Wang, F. Luan and X. Zhuang, An electrochemiluminescence sensor for the detection of prostate protein antigen based on the graphene quantum dots infilled $\mathrm{TiO}_{2}$ nanotube arrays, Talanta, 2019, 191, 103-108.

821 M. S. Khan, W. Zhu, A. Ali, S. M. Ahmad, X. Li, L. Yang, Y. Wang, H. Wang and Q. Wei, Electrochemiluminescent immunosensor for prostate specific antigen based upon luminol functionalized platinum nanoparticles loaded on graphene, Anal. Biochem., 2019, 566, 50-57.

822 S. Singh and H. S. Nalwa, Nanotechnology and health safety-toxicity and risk assessments of nanostructured materials on human health, J. Nanosci. Nanotechnol., 2007, 7, 3048-3070.

823 Nanotoxicology: Interactions of Nanomaterials with Biological Systems, ed. Y. Zhao and H. S. Nalwa, American Scientific Publishers, Los Angeles, 2007.

824 K. Yang, Y. Li, X. Tan, R. Peng and Z. Liu, Behavior and toxicity of graphene and its functionalized derivatives in biological systems, Small, 2013, 9, 1492-1503.

825 B. Zhang, P. Wei, Z. Zhou and T. Wei, Interactions of graphene with mammalian cells: molecular mechanisms and biomedical insights, Adv. Drug Delivery Rev., 2016, 105, 145-162.

826 M. Ema, M. Gamo and K. Honda, A review of toxicity studies on graphene-based nanomaterials in laboratory animals, Regul. Toxicol. Pharmacol., 2017, 85, 7-24.

827 T. P. D. Shareena, D. McShan, A. K. Dasmahapatra and P. B. Tchounwou, A review on graphene-based nanomaterials in biomedical applications and risks in environment and health, Nano-Micro Lett., 2018, 10, 53. 